Werner Steden

\title{
Finanzpolitik und Einkommensverteilung
}

Ein Wachstums- und Konjunkturmodell der Bundesrepublik Deutschland 


\section{Werner Steden}

\section{Finanzpolitik und Einkommensverteilung}

Im Rahmen eines ökonometrischen Makromodells für die Bundesrepublik wird der Einfluß der staatlichen Finanzpolitik auf die Einkommens- und Vermögensverteilung untersucht. Das Modell berücksichtigt die quantitativ erheblichen Änderungen im staatlichen Einnahmen- und Ausgabenverhalten der 60er Jahre. Am Beispiel der Reformen im Bereich der Einkommensbesteuerung werden die Aussagen der Kreislauftheorie der Steuerüberwälzung überprüft.

Werner Steden, 42, ist Professor für Finanzwissenschaft an der Freien Universität Berlin. Er studierte von 1956 bis 1961 Volkswirtschaftslehre an der Universität München und promovierte dort 1967 bei Prof. Dr. H. Möller. Von 1963 bis 1970 war er Assistent von Prof. Dr. A. Oberhauser am Institut für Finanzwissenschaft an der Universität Freiburg i. Br., wo er sich nach Beurlaubung aufgrund eines Habilitandenstipendiums der Deutschen Forschungsgemeinschaft 1975 habilitierte. 
Finanzpolitik und Einkommensverteilung 


\section{Finanzwissenschaftliche Schriften}

Herausgegeben

von den Professoren

Albers, Krause-Junk, Littmann, Oberhauser, Pohmer, Schmidt

Band 1

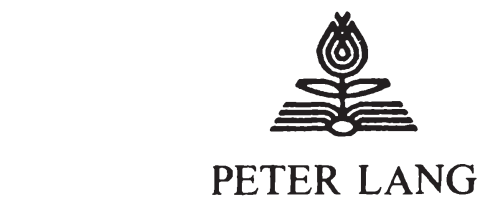

Frankfurt am Main - Bern - Las Vegas 
Werner Steden

\section{Finanzpolitik und Einkommensverteilung}

Ein Wachstums- und Konjunkturmodell der Bundesrepublik Deutschland

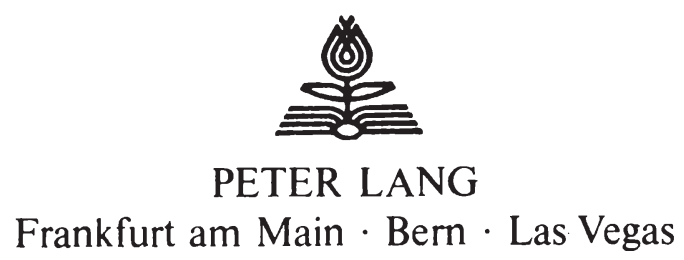


CIP-Kurztitelaufnahme der Deutschen Bibliothek

\section{Steden, Werner}

Finanzpolitik und Einkommensverteilung: e. Wachs-

tums- u. Konjunkturmodell d. Bundesrepublik

Deutschland/Werner Steden. - Frankfurt am Main,

Bern, Las Vegas: Lang, 1979.

(Finanzwissenschaftliche Schriften; Bd. 1)

Open Access: The online version of this publication is published on www.peterlang.com and www.econstor.eu under the international Creative Commons License CC-BY 4.0. Learn more on how you can use and share this work: http://creativecommons.org/licenses/ by/4.0.

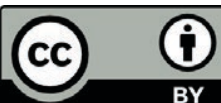

This book is available Open Access thanks to the kind support of ZBW - Leibniz-Informationszentrum Wirtschaft.

„Gedruckt mit Unterstützung der Deutschen Forschungsgemeinschaft“

ISBN 978-3-631-75224-1 (eBook)

(C) Verlag Peter Lang GmbH, Frankfurt am Main 1979

Alle Rechte vorbehalten.

Nachdruck oder Vervielfältigung, auch auszugsweise, in allen Formen wie Mikrofilm, Xerographie, Mikrofiche, Mikrocard, Offset verboten.

Druck: Fotokop Wilhelm Weihert KG, Darmstadt Titelsatz: Fotosatz Aragall GmbH, Wolfsgangstraße 92, Frankfurt am Main 
Die "Finanzwissenschaftlichen Schriften" bedürfen keiner besonderen Begründung. Mit der Ausdehnung der Staatstätigkeit haben die Probleme, die eine wissenschaftliche Durchdringung erfordern, zugenommen und werden weiter zunehmen. Bisher fehlte im deutschsprachigen Raum eine Reihe dieser speziellen Ausrichtung. Hier soll nun nicht zuletzt jüngeren Finanzwissenschaftlern eine Möglichkeit geboten werden, ihre Studien zu veröffentlichen; dabei ist an Monographien, vor allem an Habilitationsschriften und hervorragende Dissertationen gedacht. Schließlich sollen die Bände preiswert sein; sie erscheinen da her in bescheidenem äußeren Gewande.

Entscheidend für die Aufnahme in die Reihe, die allen Finanzwissenschaftlern offensteht, ist die Qualität der Arbeiten. Da bei lassen wir uns von der Erwartung leiten, mit den veröffent lichten Schriften, die wissenschaftliche Diskussion anzuregen.

Die Herausgeber 

Seite:

Vorbemerkungen $\quad 9$

1. Kapitel: Aufbau des Modells 17

$\$ 1$ Eine Grundskizze 17

$\uparrow 2$ Das System der Definitionsgleichungen 21

1. Stromgleichungen 21

2. Preisgleichungen 32

3. Bestandsgleichungen 35

4. Abschließende Bemerkungen $\mathrm{zu}$ den bisherigen 42

2. Kapitel: Das Verhalten der privaten Wirtschaftssubjekte 44

$\S 3$ Entwicklung und Struktur der Erwerbsbevölkerung 44

1. Entwicklung 44

2. Struktur 46

$\$ 4$ Die Kapazitätsplanung $\quad 49$

1. Vorbemerkung 49

2. Grundannahmen der langfristigen Produktions-
und Faktornachfrage

3. Die Definition der Faktorkosten $\quad 54$

4. Die Aufstellung der Erwartungswerte 64

5. Die dynamische Form der Anlagenplanungsfunktion, ihre empirische Überprüfung und Modifizierung $\quad 67$

6. Die dynamische Form der Beschäftigungsplanungs funktion, ihre empirische Überprüfung und $\begin{array}{ll}\text { Modifizierung } & 79\end{array}$

7. Die geplante Produktion 87

$\S 5$ Die kurzfristige Anpassung von Produktion und Beschäftigung 90

1. Die Grundannahmen der kurzfristigen Produktionsund Faktornachfragetheorie $\quad 90$

2. Eine Modifizierung der kurzfristigen Grenzproduktivitäts theorie

3. Schätzwerte für die Parameter der kurzfristigen Produktions - und Arbeitsnachfragefunktion 
$\$ 6$ Ergänzende Überlegungen zum privaten Investitionsverhalten

1. Anlagenabgang, Abschreibung für Kapitalverzehr und Preisindex für Anlageinvestitionen

2. Die Vorratsinvestitionen

$\$ 7$ Das Einkommen der Arbeitnehmer-Haushalte und seine Verwendung

1. Bestimmung der Geldlöhne

2. Die Vermögenserträge der Arbeitnehmerhaus halte

3. Der Konsum der Arbeitnehmer-Haushalte

$\$ 8$ Das Einkommen der Selbständigen-Haushalte und seine Verwendung

1. Der Unternehmerlohn

2. Das ausgeschüttete Besitzeinkommen der Selbständigen

3. Der Konsum der Selbständigen-Haushalte

\$ 9 Geldversorgung und Kassenhaltung

1. Eine Bemerkung zum Gleichgewicht auf dem Kreditmarkt

2. Das Geldangebot des Bankensektors

3. Die Geldnachfrage des privaten Sektors

4. Empirische Überprüfung des Verhaltens von Geldversorgung und privater Kassenhaltung

5. Die Aufteilung des Geldumlaufs auf die privaten Haushalte und Unternehmen

$\$ 10$ Die Kreditbeziehungen des privaten Sektors zur übrigen Welt

1. Die internationalen Kapitalbewegungen 166

2. Die privaten Vermögenserträge des Auslandes $\quad 169$

$\$ 11$ Der Warenverkehr mit der übrigen Welt 173

1. Vorbemerkung 173

2. Der Warenimport 174

3. Der Warenexport 178

4. Die Bestimmung der Wechselkurse und der Preise des Auslandes

3. Kapitel: Die Verhaltensinstrumente des Staates 185

$\$ 12$ Die indirekten Steuern 185

1. Die Verbrauchsteuer 185 
2. Die Gewerbeertragsteuer 187

3. Die Lohnsummensteuer 195

4. Die Gewerbekapitalsteuer 198

5. Die Importzölle 200

$\$ 13$ Die direkten Steuern 203

1. Die Lohnsteuer auf den Arbeitnehmerlohn 203

2. Die Lohnsteuer auf den rechnerischen Unternehmerlohn

3. Die Steuer auf das Besitzeinkommen der Selbständigen

4. Die Steuer auf das Selbständigen-Vermögen 219

$\$ 14$ Die sonstigen Einnahmen des Staates 225

1. Die Sozialversicherungsbeiträge 225

2. Die Vermögenserträge des Staates 227

3. Die staatliche Nettokreditaufnahme im privaten Sektor

$\$ 15$ Die staatlichen Lohn- und Gehaltszahlungen 235

1. Die Besoldung der öffentlichen Bediensteten 235

2. Das staatliche Beschäftigungsvolumen 237

$\$ 16$ Die Übertragungen des Staates 241

1. Die Übertragungen an die ArbeitnehmerHaushal te

2. Die Übertragungen des Staates an den Unternehmenssektor 243

3. Die Kapitalhilfe des Staates an die übrige Welt 245

$\$ 17$ Die staatlichen Güterkäufe $\quad 249$

1. Der Gesamtumfang 249

2. Der Anteil der öffentlichen Infrastrukturinvestitionen

3. Anlagenabgang und Abschreibungen für Kapitalverzehr des Staates $\quad 252$

4. Kapitel: Lösung und Auswertung der Modellergebnisse 255

$\$ 18$ Der rechnerische Lösungsweg 255

1. Zur Berechnungsmethode 255

2. Nachträgliche Korrekturen und Vereinfachungen 260

3. Die Liste der Symbole 263

4. Das Rechenprogramm INZID (FORTRAN IV) 285 
Seite:

$\$ 19$ Die Auswertung der Modellergebnisse 323

1. Der Standardablauf der Modellentwicklung

2. Die Steuerreformen 1962 und 1965 im Bereich der Steuer auf fundierte Selbständigen-Einkommen und ihre Inzidenzeffekte

$\$ 20 \quad$ Versuch einer abschließenden Beurteilung des makroökonomischen Überwälzungstheorems

342

Anhang 1: Bruttoanlagevermögen, Nettoanlagevermögen und Anlagenfinanzierung im langfristigen Wachstumsgleichgewicht des Unternehmenssektors

Anhang 2: Bemessung der Risikoprämie durch Ansatz einer verkürzten pay-off-Periode bei der Anlagenamortisation

Tabellenanhang

Literaturverzeichnis 


\section{VOR BEMER KUNG}

Mitte der 50er Jahre entfachte C. Föhl (1) in der finanzwissenschaftlichen Literatur eine Diskussion, die sich mit der Überwälzbarkeit von Gewinnsteuern befaßte und die in ihrem Verlauf zu einem provozierenden Ergebnis gelangte: Wenn in einem geschlossenen kapitalistischen Wirtschaftssystem bei Vollbeschäftigung und elastischem Geldangebot seitens des Bankensystems der Staat sich auf dem Wege der Gewinnbesteuerung Einnahmen aus dem Unternehmenssektor beschafft und in Höhe dieser Einnahmen nachfragewirksame Ausgaben tätigt und wenn auf grund der Besteuerung die Investitionen des Unternehmenssektors in ihrem realen Umfang nicht zurückgehen, sondern mit Hilfe einer zusätzlichen Kreditaufnahme beim Bankensektor ungestört fortgesetzt werden, wenn schließlich die Besteuerung die Unternehmerhaushalte nicht dazu veranlaßt, weniger als sonst zu konsumieren, und auch die Nicht-Unternehmerhaushalte nicht mehr als sonst sparen, dann werden, so lautete die Schlußfolgerung, die Gewinnsteuern vom Unternehmenssektor, ohne daß dieser das Ergebnis bewußt anstrebt, voll auf die Nicht-Unternehmerhaushalte überwälzt (2). - Dieses gesamtwirtschaftliche Überwälzungstheorem, das einige bis dahin herrschende Ansichten über die Steuerlastverteilung auf den Kopf stellte, auf jeden Fall zu einer Verunsicherung dieser Ansichten führte, wurde im Anschluß an die Diskussion auf andere finanzpolitische Maßnahmen des Staates wie öffentliche Verschuldung, staatliche Finanzinvestitionen, öffentliche Transferausgaben etc. angewendet (3); auch nahm sich die makroökonomische Verteilungstheorie des Problems an (4) und trug dazu bei, das Theorem zu einer makroökonomischen Inzidenztheorie des staatlichen Budgets zu verallgemeinern. Doch konnte der theoretische und empirische Nachweis seiner Gültigkeit letzten Endes nicht ausreichend geführt werden. So blieb das Theorem im Laufe der Diskussion heftig umstritten und hat bis heute weder im finanzwissenschaftlichen noch im finanzpolitischen Bereich allgemeine Anerkennung gefunden. Es liefert die theoretische Basis

1) Vgl. C. Föhl, Kritik der progressiven Einkommensbesteuerung, FA, 14, 1953/54, S. $88 \mathrm{ff}$.

2) Vgl. A. Oberhauser, Finanzpolitik und private Vermögensbildung, Köln-Opladen 1963, S. 63

3) Vgl. ebenda, Teil II. - Ferner: ders., Staatsschuldentilgung als finanzpolitisches Instrument, FA, 24, 1965, S. $283 \mathrm{ff}$.

4) Vgl. vor allem A. Stobbe, Untersuchungen zur makroökonomischen Theorie der Einkommensverteilung, Tübingen 1962, 6. Kap. 
lediglich für eine von mehreren finanzwissenschaftlichen Richtungen.

Der unbefriedigende Ausgang der Föhl-Diskussion ist zu einem großen Teil verteilungstheoretisch bedingt. Das abgeleitete Überwälzungstheorem bedient sich der Argumentationsweise einer von mehreren makroökonomischen Verteilungstheorien, die heute unvermittelt nebeneinander bestehen und miteinander im Wettstreit liegen. Es ist im Grunde eine finanzwissenschaftliche Anwendung der post-keynesianischen oder kaldorianischen Verteilungs theorie (1), ähnlich wie z. B. das HaavelmoTheorem eine finanzwissenschaftliche Anwendung der Keynes'schen Multiplikator theorie darstellt. Klarer formuliert: das makroökonomische Überwälzungstheorem bildet in seiner Verallgemeinerung die distributive Variante des Haavelmo-Theorems - genauso, wie der Kaldorsche Verteilungsmultiplikator die distributive Variante des Keynes'schen Einkommensmultiplikators bildet (2). - Aus dieser theoretischen Zuordnung wird ersichtlich, daß das Theorem sich auf eine verteilungstheoretische Richtung stützt, die ihre allgemeine Anerkennung eigentlich nur dort findet, wo sie sich als partielle Multiplikatortheorie gibt. Zur Zeit wird das Problem der makroökonomischen Steuerinzidenz auch von einer ganz anderen, freilich ebenso wenig allgemein akzeptierten Richtung, der neoklassischen Verteilungstheorie, untersucht (3). Hält man sich vor Augen, daß die finanzwissenschaftliche Überwälzungstheorie, sobald sie von der rein makroökonomischen Analyse abgeht, überwiegend neoklassisch argumentiert und daß die Skepsis gegenüber dem makroökonomischen Überwälzungstheorem sich zum guten Teil gerade von der Tradition nährt, auf der diese andere verteilungstheoretische Richtung aufbaut, dann gewinnt man den Eindruck, daß, solange diese beiden großen makroökonomischen Verteilungstheorien im Ansatz unver-

1) N. Kaldor hat diese Anwendungsmöglichkeit selbst gesehen. Vgl. N. Kaldor, Alternative Theories of Distribution, Rev. of Econ. Stud. , 23, 1955-56, wiederabgedruckt in: ders., Essays on Value and Distribution, London 1960, S. $209 \mathrm{ff}$. Dort insbes. S. 228 Fußn. 2 und S. 230 f.

2) Vgl. ebenda, S. 227 f. ferner auch H. Kolms, Finanzwissenschaft, Bd. IV, Berlin 1964, S. 129

3) Gedacht ist hierbei in erster Linie an die Beiträge von M. Krzyzaniak in dem von ihm herausgegebenen Sammelband Effects of Corporation Income Tax, Detroit 1966, in verschiedenen Jahrgängen der Zeitschrift Public Finance und im Finanzarchiv 1972. Vgl. aber auch den Überblicksartikel von P. Mieszkowsky, Tax Incidence Theory: The Effects of Taxes on the Distribution of Income, Journal of Economic Literature, 7, 1969, S. $1103 \mathrm{ff}$. 
knüpft nebeneinander stehen bleiben (1), ein befriedigender Abschluß der Föhl-Diskussion und die Formulierung einer allgemein anerkannten makroökonomischen Inzidenztheorie des öffentlichen Budgets nicht zu erwarten sind.

An der skizzier ten Situation setzt die vorliegende Untersuchung an. Sie wurde angefertigt mit dem Ziel, die Frage nach der Inzidenz der finanzpolitischen Aktivität des Staates in der makroökonomischen Verteilungs theorie weiter abzuklären, und mit dem Vorsatz, die Behandlung dieser Frage mit einer Konfrontation der postkeynesianischen und neoklassischen Verteilungstheorie zu verbinden. Dies sollte nicht durch Abwägen der den beiden Theorien zugrunde liegenden Prämissen und der aus ihnen folgenden Ergebnisse geschehen, sondern dadurch, daß die Annahmen beider Theorien aus allzu starrer Einseitigkeit herausgelöst und aufeinander abgestimmt werden. Das Ergebnis ist ein makroökonomisches Verteilungsmodell, das die wesentlichen Elemente der postkeynesianischen und der neoklassischen Verteilungstheorie als konsistente Bestandteile in sich vereinigt und in dem das Zusammenwirken beider Partialtheorien, d. h. letzten Endes der numerische Wert von Verhaltensparametern, über den Einfluß der beiden partiellen Gesetzmäßigkeiten auf die Einkommensverteilung und die Inzidenz staatlicher Maßnahmen entscheidet. Die Formulierung des Modells orientiert sich am ursprünglichen Aussagegehalt der beiden Theorien und geht davon aus, daß der wichtigste Schritt bei der Entwicklung einer aussagekräftigen gesamtwirtschaftlichen Verteilungstheorie in der Anwendung der dynamischen Wirtschaftstheorie liegt, was in der Diskussion der Überwälzungstheorie zu lange übersehen worden ist. In der Tat erlaubt erst die mit der Formulierung von "disequilibrium"-Modellen verbundene Unterscheidung zwischen Erscheinungen der kurzen, mittleren und langen Frist, die beiden Ansätze der Überwälzungstheorie zu verknüpfen, ohne vorab über ihren Erklärungswert zu befinden oder ihren ursprünglichen Aussagegehalt in der einen oder anderen Richtung einzuschränken.

Bei dem Versuch, mit einer Modellformulierung, wie sie hier skizziert wurde, wirksam in die Auseinandersetzung um das makroökonomische Überwälzungstheorem einzugreifen, ist zu beachten, da $\beta$ der unbefriedigende Ausgang der Föhl'schen Überwälzungsdiskussion zu einem Teil auf die unzulängliche Analyse der monetären Vorgänge im Zusammenhang mit der Investitionsfinanzierung und der Vorfinanzierung erhöhter Steuerzahlungen zurückzuführen ist. Die in der Diskussion angesprochenen kreislaufmäßigen Überwälzungsprozesse haben, wenn man sie auch nicht ausschließlich als monetär bedingt ansehen kann, einen ausgeprägt

1) Hiermit soll nicht die Existenz weiterer verteilungstheoretischer Ansätze geleugnet werden. Der Leser wird später feststellen, daß der hier unternommene Integrationsversuch einige interessante Aspekte auch hinsichtlich der Monopolgrad- und Zuschlagstheorien der Einkommensverteilung aufdeckt. 
monetären Charakter. Die notwendige Voraussetzung, die in der Sicherung der Investitionsfinanzierung durch ein ausreichendes Kreditangebot seitens der Banken besteht, wurde im Laufe der Diskussion nicht genügend ausdiskutiert; sie wurde auch in den später formulierten einschlägigen Modellen fast nie explizit und als endogener Bestandteil berücksichtigt (1), wodurch der Rahmen für die Auseinandersetzung mit dem Überwälzungstheorem zu eng wurde. Die vorliegende Modellformulierung geht daher über den üblichen Rahmen postkeynesianischer und neoklassischer Verteilungsmodelle hinaus: Monetäre und reale Größen werden ausdrücklich unterschieden; die wichtigsten gesamtwirtschaftlichen Finanzierungs vorgänge wie Geldschöpfung des Bankensektors und Kassenhaltung der privaten Nicht-Banken werden als endogene Bausteine des Modells berücksichtigt. Das Modell enthält damit, wenn auch nur in rudimentärer Form, eine Finanzierungsrechnung, die an die Stromrechnung des Einkommenskreislaufs anschließt und auf die Vermögensbestandsrechnung abgestimmt ist.

Damit sind die Ziele und die wichtigsten Grundzüge der Modellformulierung angedeutet. Um den Leser auf das, was ihn in Einzelheiten erwartet, vorzubereiten, sei darauf hingewiesen, daß die Arbeit einen umfangreichen empirischen Teil enthält, der die Lektüre belastet: Alle relevanten Verhaltenshypothesen der skizzierten Modellformulierung wurden mit Hilfe des statistischen Materials für die Bundesrepublik überprüft und quantifiziert; dort, wo das Material Lücken aufwies, wie z. B. in der Vermögensund Finanzierungsrechnung, wurden zuvor aufgrund plausibler Hilfsannahmen Modellrechnungen angestellt, mit deren Ergebnissen das Material für die Schätzungen ergänzt wurde (2). Der auf die Modellrechnungen und Schätzungen verwendete zeitraubende empirische Teil der Untersuchung war nicht zu vermeiden. Von einer allgemeinen mathematischen Lösung allein, wenn eine solche überhaupt existiert hätte, waren keine eindeutigen Aussagen zu erwarten, da innerhalb des Modells grundsätzlich die numerischen Werte von Verhaltensparametern über das Auftreten und den Charakter steuerlicher Überwälzungsprozesse entscheiden. Hinzu kam, daß sich bereits in einer frühen "einfachen" Version des Modells zeigte, daß im vorliegenden Fall eine allgemeine mathematische Lösung nicht existierte (3), so daß die Untersuchung auf jeden Fall auf eine Simulations-

1) Der gesamte geldtheoretische Komplex wurde in verteilungstheoretischen Überlegungen eigentlich nur bei S. Weintraub, An Approach to the Theory of Income Distribution, Philadelphia 1958, diskutiert. Vgl. allerdings auch $\mathrm{E}$. Scheele, Einkommensverteilung und Wirtschaftswachstum, Tübingen 1965.

2) Die Ergebnisse dieser Hilfsrechnungen finden sich im Anhang.

3) Hierauf wird im Paragraphen 18 noch ausführlicher eingegangen. 
rechnung angewiesen war (1); eine solche Simulationsrechnung verlangt die Vorgabe möglichst realistischer numerischer Parameterwerte, wozu die Literatur keine ausreichenden Angaben liefert. - Als Kompensation für die Belastung der Lektüre durch die zahlreichen Schätzungen erlaubte die Technik der Simulationsrechnung aber, auf viele Vereinfachungen der Verhaltensfunktionen durch Linearisierung etc. und ebenso auf die $\mathrm{Be}$ schreibung aller für die Modellösung erforderlichen rechnerischen Einzelschritte zu verzichten; auch bot sie die Gelegenheit, die Struktur des Modells stärker, als das sonst möglich gewesen wäre, auszubauen und damit auf die Verteilungssituation in der Bundesrepublik Deutschland anzupassen, was für die noch anstehende empirische Überprüfung des makroökonomischen Überwälzungstheorems von Bedeutung ist.

Die Untersuchung gliedert sich in vier Kapitel. Das erste Kapitel enthält einen Überblick über den nicht ganz einfachen Aufbau des Modells: Paragraph 1 macht in einer ersten verbalen Grundskizze mit der Struktur der analysierten Modellwirtschaft vertraut. Im folgenden Paragraphen 2 wird das System der Definitionsgleichungen aufgestellt und damit die Modellstruktur in größerer Ausführlichkeit beschrieben. Es handelt sich wohlverstanden um das definitorische Gerüst einer im Vergleich zu den empirischen Verhältnissen der Bundesrepublik vereinfachten Modellwirtschaft; eine ganze Reihe von vereinfachenden Annahmen, die im einzelnen nicht begründet werden und für das Untersuchungsziel unerheblich sind, schlägt sich bereits im definitorischen System nieder.

Im zweiten Kapitel wird das Verhalten des privaten Sektors, im dritten das des staatlichen Sektors in allen Einzelheiten beschrieben. In je einem Paragraphen werden eine oder mehrere zusammengehörende Verhaltenshypothesen theoretisch abgeleitet. Die Hypothesen berücksichtigen im Bereich des privaten Sektors nach Möglichkeit die Reaktionen der privaten Haushalte und Unternehmen auf das Verhalten des Staates, insbesondere auf die staatliche Steuerpolitik. Im staatlichen Sektor stellen sie vorwiegend auf die Formulierung von Steuerbelastungsfunktionen ab, die den gesetzlichen Tarifbestimmungen der Bundesrepublik lose angepaßt sind, sowie auf die Formulierung von aggregierten Ausgabefunktionen. Jeweils im Anschluß an die theoretische Ableitung werden die Verhaltenshypothesen empirisch überprüft. Die Bestimmung der Verhaltensparameter erfolgt fast ausschließlich mit Hilfe der Kleinst-Quadrat-Methode, und zwar im allgemeinen anhand der Jahresdaten, die die offizielle Volkswirtschaftliche Gesamtrechnung auf der Basis 1962 für die sechsziger

1) Mathematisch zugänglich ist die Analyse von Situationen des langfristigen Wachstumsgleichgewichts; ebenso besteht mathematisch die Möglichkeit, ganz kurzfristige Prozesse über eine Differentiation des gesamten Gleichungssystems nach Änderungen der staatlichen Verhaltensparameter zu analysieren. Die auf diese Weise erzielbaren Ergebnisse sind jedoch weniger informativ als die, die sich aus der Simulationsrechnung ergeben. 
Jahre der Bundesrepublik zur Verfügung stellt (1), sowie anhand der Banken-, Finanz- und Außenhandelsstatistik. Staatliche Verhaltensänderungen werden durch gesonderte Teilschätzungen, z. T. auch durch DummyVariablen für die Zeit vor und nach der Reform erfaßt. - Da die beiden Kapitel über das Verhalten des privaten und des staatlichen Sektors umfangmäßig den Großteil der vorliegenden Arbeit ausmachen und die Gefahr besteht, daß der Leser während der vielen Detailanalysen das eigentliche Ziel der Untersuchung aus den Augen verliert, wird nach der Formulierung und Überprüfung einer jeden Verhaltenshypothese ihre voraussichtliche Bedeutung für die Einkommens- und Vermögensverteilung und die Inzidenz finanzpolitischer Maßnahmen besprochen.

Das vierte Kapitel dient der Lösung und Auswertung des Modells. Das Kapitel enthält das in FORTRAN IV programmierte Simulationsprogramm INZID, mit dem das nicht-lineare dynamische Gleichungssystem über eine Newton-Iteration aufgelöst und über eine weitere Iteration korrigiert wird und mit dem in einer Sequenzenanalyse die zeitliche Entwicklung der Modellwirtschaft berechnet wird. Mit Hilfe des Programms wird zuerst ein Standardablauf des Modells dargestellt, in welchem der staatliche Sektor sich so verhält, wie das in der Bundesrepublik während der sechziger Jahre beobachtet und in den Parameterschätzungen zu erfassen versucht wurde. Dieser Standardablauf schafft die Vergleichssituation, anhand derer sich die Auswirkungen staatlicher Verhaltensreformen kontrollieren lassen. Im Anschluß folgen Modellabläufe, in denen jeweils mehrere zusammengehörende staatliche Verhaltensparameter im Vergleich zum Standardablauf geändert werden. Als einziges werden hier die Auswirkungen der Steueränderungsgesetze 1961/62 und der Einkommensteuerreform 1965 auf die Besteuerung der Besitzeinkommen der Selbständigen und Unternehmen analysiert; diese Analyse dient dazu, die Frage nach der Überwälzbarkeit von Gewinnsteuern aus der Sicht der Modellformulierung endgültig zu beantworten (2). Eine kurze zusammenfassende und verallgemeinernde Würdigung versucht, das makroökonomische Überwälzungstheorem aufgrund der erzielten Ergebnisse theoretisch neu einzuordnen, und schließt die Untersuchung.

Die vorliegende Arbeit ist die leicht überarbeitete und vervollständigte Fassung einer von der Wirtschaftswissenschaftlichen Fakultät der Univer sität Freiburg i. B. angenommenen Habilitationsschrift. Der theoretische Teil dieser Arbeit einschließlich der Regressionsschätzungen wurde Ende

1) Für die Jahre vor 1960 wurden die Daten durch eine Verkettung auf den Gebietsstand einschließlich Saarland und West-Berlin umgerechnet; ebenso wurde das ursprüngliche Material nach der Revision der Volks wirtschaftlichen Gesamtrechnung im Jahre 1970 mit den revidierten Daten durch eine Verkettung über das Jahr 1970 hinaus fortgeschrieben.

2) Hinsichtlich aller weiteren Reformen der staatlichen Finanzpolitik wird das Modell außerhalb dieser Arbeit ausgewertet. 
1973 abgeschlossen, weswegen die Datenbasis der meisten ursprünglichen Regressionsschätzungen nur bis zum Jahr 1972 reicht. Das Rechenprogramm wurde bis zum darauffolgenden Jahresende 1974 erstellt. Die Anregung zu der Arbeit ging von Professor Dr. Alois Oberhauser aus, als dessen Assistent ich während mehrerer Jahre Gelegenheit hatte, mich mit inzidenztheoretischen Fragen auseinanderzusetzen, und der die Untersuchung in vielen Diskussionen mit seinem Rat förderte. Wohlwollende Unterstützung kam der Untersuchung auch von verschiedenen anderen Seiten zugute. Mein ehemaliger Kollege Dr. Paul Hövelmann, der sich in seiner Dissertation ebenfalls mit der Inzidenzproblematik befaßte und zu ähnlichen Schlußfolgerungen gelangte, steuerte einige wichtige Anregungen bei; wir haben es bedauert, uns bei der Anfertigung unserer Arbeiten aus Gründen der Überprüfbarkeit von einander abgrenzen zu müssen. Die Deutsche Forschungsgemeinschaft gewährte eine großzügige finanzielle Unterstützung in Form eines Habilitandenstipendiums. Alle Berechnungen wurden in den Rechenzentren der Universität Freiburg i. Br. und der Freien Universität Berlin durchgeführt, wobei für die Regressionsschätzungen das vom Deutschen Rechenzentrum Darmstadt überlassene und von Dr. F. Gebhardt formulierte Regressionsprogramm REGT verwendet wurde.

Ursprünglich hatte ich gehofft, die Arbeit eher abschließen und veröffentlichen zu können. Leider ging diese Hoffnung wegen der fehlenden Stabilitätseigenschaften des Modells nicht auf. Der Versuch, mit Hilfe einer begrenzten Zahl partieller Korrekturen eine Parameterkonstellation zu finden, mit der sich über größere Periodenfolge einigermaßen stabile Entwicklungspfade der Modellwirtschaft errechnen ließen, erwies sich als äußerst zeitraubend und gelang bisher nur zum geringen Teil. Inzwischen habe ich eingesehen, daß die Untersuchung in dieser Hinsicht ungeachtet der Notwendigkeit besserer ökonometrischer Schätzungen in einigen Teilen theoretisch ungenügend ausformuliert ist und diesbezüglich einer grundlegenden Überarbeitung bedarf. Immerhin belegen die Ergebnisse der Modellrechnungen auch so andeutungsweise eine Reihe zuvor gezogener Schlußfolgerungen, was m. E. ihre Veröffentlichung rechtfertigt. Verlag und Herausgeber, die ich mit der eingetretenen Verzögerung auf eine harte Geduldsprobe gestellt habe bitte ich um Nachsicht; das gilt ebenso für die, denen ich wegen der absorbierenden Beschäftigung mit dieser Arbeit ein schlechter Freund gewesen bin.

W. St. 



\section{Kapitel}

\section{DER AUFBAU DES MODELLS}

\section{$\S 1$ Eine Grundskizze}

An den Anfang der Modellanalyse sei ein kurzer Überblick über die Gruppierung der in dem Modell auftretenden Wirtschaftssubjekte, über die den wirtschaftlichen Verkehr regulierenden Märkte und über die Art der Einkommensströme gestellt.

Das Modell erstreckt sich auf eine kapitalistische Volkswirtschaft, deren geringfügig wachsende Erwerbsbevölkerung sich in zwei Personengruppen aufteilt: in Selbständige und in Unselbständige oder Arbeitnehmer. Die Selbständigen sind die persönlichen Eigentümer bzw. Anteilseigner der in der Volkswirtschaft existierenden Unternehmen. Sie organisieren den Produktionsprozeß oder betrauen Arbeitnehmer mit der Leitung des Produktionsprozesses. Sie oder die von ihnen beauftragten Manager stellen Erwerbspersonen ein, die bereit sind, als unselbständig Beschäftigte die Bedienung der Produktionsanlagen im Unternehmenssektor oder Verwaltungsaufgaben im privaten oder staatlichen Bereich zu übernehmen.

Die Unternehmen, die allesamt dem privaten Sektor angehören, verfügen trotz aller eigentumsrechtlichen Bindungen an die Selbständigen bzw. Unternehmenseigner über eine gewisse wirtschaftliche Selbständigkeit. Sie erstellen das Inlandsprodukt des privaten Sektors und ergänzen es durch Importe. Ihr Güterangebot dient dem Konsum, der Anlageninvestition, dem Export und der Auffüllung von Warenlägern. Der Unternehmenssektor umfaßt ein Bankensystem, das die Volkswirtschaft mit einem Zahlungsmittel ausstattet, mit dem alle Transaktionen von Gütern und Diensten verrechnet und abgegolten werden. Das Bankensystem gliedert sich in eine Zentralbank und ein System von Geschäftsbanken. Die Zentralbank steuert die Liquiditätsversorgung des Geschäftsbankensystems und orientiert sich dabei an gesamtwirtschaftlichen Zielen. Das Geschäftsbankensystem betreibt auf der Basis der ihm zur Verfügung gestellten Liquidität Kreditschöpfung und sorgt für einen reibungslosen Ablauf des gesamten Zahlungsverkehrs.

Das übergeordnete Kollektivorgan der Volkswirtschaft ist der Staat. Er garantiert mit Hilfe von Transferzahlungen die soziale Sicherheit, gewährleistet mit Hilfe der von ihm gekauften Güter und Dienste eine ausreichende Infrastruktur und die Befriedigung der konsumtiven Kollektivbedürfnis se. Zur Finanzierung der Aufgabenerfüllung stehen ihm Steuern und Kredite des privaten Sektors zur Verfügung. 
Schließlich steht der gesamten Volkswirtschaft die "übrige Welt" gegenüber. Sie liefert einen Teil der im Inland abgesetzten und kauft einen Teil der im Inland produzierten Güter. Sie engagiert sich im inländischen Kapitalverkehr und erhält von den privaten und öffentlichen Haushalten des Inlands im allgemeinen positive unentgeltliche Leistungen.

Die marktwirtschaftlich organisierten Beziehungen des betrachteten Gesamtuniversums verlaufen über drei voll berücksichtigte Inlandsmärkte: Auf einem Arbeitsmarkt wird die Arbeitsleistung der Unselbständigen gehandelt. Dieser Markt ist organisiert, d. h. Gewerkschaften versuchen, mit der regelmäßigen Forderung tariflicher Lohnsteigerungen durchzusetzen, daß die Arbeitnehmer am steigenden Wohlstand angemessen beteiligt werden, und sorgen dafür, daß die Arbeitnehmer die wegen langfristiger Arbeitsverträge nicht direkt am laufenden Arbeitsmarktgeschehen beteiligt sind, dennoch in den Genuß der laufenden Lohnsteigerungen kommen.

Auf dem Kreditmarkt werden ausschließlich Forderungstitel der Produktionsunternehmen, des Staates und des Auslandes gehandelt. Diese Titel lauten auf bestimmte Geldbeträge inländischer Währung und sind mit einer variablen Nominalverzinsung, die sich an das laufende inländische Zinsniveau anpaßt, ausgestattet, allerdings nicht mit Wertsicherungs klauseln. Die Zinszahlung erfolgt jährlich und nachschüssig, so daß innerhalb einer Periode $t$ der Zinssatz festgelegt wird, der während der darauffolgenden Periode $t+1$ zur Auszahlung kommt. In diesen Kredittiteln werden alle Kreditbeziehungen abgewickelt, die Kreditbeziehungen zwischen Sparern und Investoren, die mit der Geldversorgung verbundenen Kreditbeziehungen zwischen Banken und Nichtbanken des privaten Sektors, die Kreditbeziehungen zwischen Staat und privatem Sektor und ebenso der gesamte internationale Kapitalverkehr. Auch die vermögensrechtlichen Ansprüche der Unternehmenseigner werden mit diesen Titeln geregelt. $\mathrm{Zu}$ diesem Zweck wird angenommen, daß die üblichen Eigentumsrechte der Anteilseigner in dem Modell aufgespalten sind: Ein Teil des Eigentumsrechtes, der auf dem Zurverfügungstellen von Kapital beruht, wird lediglich als Kreditbeziehung erfaßt und führt entsprechend den allgemeinen Kreditmarktbedingungen nur zu einer Verzinsung des zur Verfügung gestellten Kapitals; der andere Teil des Eigentums rechts beruht auf der Übernahme von Risiko und Haftung; er besteht unabhängig von Vermögens rechten und berechtigt die Anteilseigner zum Einbehalten aller über die Kapitalverzinsung und Amortisation hinaus anfallenden Profite.

Schließlich existiert im Inland der Gütermarkt, auf dem die Unternehmen ihre produzierten bzw. importierten Fertigwaren absetzen, soweit diese nicht für den Export bestimmt sind.

Alle drei Inlandsmärkte sind monopolistisch strukturiert. Dies folgt aus der Inhomogenität der gehandelten Güter und Dienste, aus der räumlichen Wirtschaftsstruktur und der Kostspieligkeit des Verkehrs, ferner aus der Größenstruktur des Unternehmenssektors und der unterschiedlichen 
rechtlichen Organisation der einzelnen Unternehmen.

Den inländischen Märkten entsprechen Märkte der übrigen Welt. Diese treten jedoch, wenn überhaupt, nur rudimentär in Erscheinung: Es bestehen ein Weltmarkt, von dem der Import bezogen wird, ferner ein ausländischer Kreditmarkt, über den alle privaten internationalen Kapitalbewegungen in der einen oder anderen Richtung laufen. Die Tauschverhältnisse dieser drei Märkte sind zum Teil beeinflußt von den Aktivitäten der Inländer bzw. von den Bedingungen auf den Inlandsmärkten der Modellwirtschaft. Ferner bestehen ein ausländischer Arbeitsmarkt und ein Devisenmarkt, von deren Existenz jedoch abgesehen wird, da laufende internationale Wanderungen der Arbeitskräfte und Änderungen der Währungsparitäten durch ausländische Staaten ausgeschlossen werden.

In dem betrachteten Wirtschaftssystem fließen folgende verteilungstheoretisch wichtigen Einkommensströme:

Arbeitnehmer beziehen aus ihrer unselbständigen Beschäftigung Lohneinkommen und aus der Kreditmarktanlage eines Großteils ihrer Ersparnisse Zinserträge. Sie beziehen neben dem Zinseinkommen keine Gewinneinkommen, da sie nicht Eigentümer, sondern nur Gläubiger des Unternehmenssektor sind.

Selbständige erhalten für die von ihnen zum Produktionsprozeß beigetragene Arbeitsleistung einen Unternehmerlohn und ebenso wie die Unselbständigen eine Verzinsung ihres in den Unternehmenssektor eingebrachten Finanzkapitals. Sie sind jedoch die alleinigen Eigentümer der Unternehmen; ihnen gehören daher die in dem Unternehmenssektor anfallenden Gewinne, die sich aus Monopol- und windfall-Profiten zusammensetzen.

In der betrachteten Volkswirtschaft verzichten die Eigentümer der Unternehmen aus Rentabilitätsgründen oder zur Sicherung des Unternehmensbestandes häufig auf eine Entnahme der ihnen zustehenden Kapitalerträge. Auch lassen die Unternehmen von sich aus einen Teil der erzielten Gewinne nicht zur Ausschüttung an die Unternehmenseigner gelangen. Aus diesen Gründen erscheint es sinnvoll, den Unternehmen trotz möglicherweise fehlender Rechtspersönlichkeit eine gewisse wirtschaftliche Selbständigkeit zuzusprechen und ihnen in Höhe der nichtausgeschütteten Zins - und Gewinneinkommen ein eigenes Einkommen zuzurechnen. Unter wirtschaftlich institutionellen Aspekten wird in der volkswirtschaftlichen Gesamtrechnung der Modellwirtschaft daher das letztlich den Selbständigen zufallende Einkommen zerlegt in das an die Selbständigen-Haushalte ausgeschüttete Einkommen, das auf jeden Fall den vollen Unternehmerlohn enthält und das hier in einem engeren Sinn als Selbständigeneinkommen bezeichnet wird, und in das nichtausgeschüttete Zins- und Gewinneinkommen, das sog. Unternehmenseinkommen (1).

(1) $\mathrm{Zu}$ dieser Isolierung des Unternehmenseinkommens vgl. ähnlich G. Bombach, Preisstabilität, Wirtschaftswachstum und Einkommensverteilung, Schweiz. Z.f. Volksw. u. Stat., 95, 1959, S. $1 \mathrm{ff}$. 
Der Staat nimmt an dem über die Märkte verlaufenden Prozeß der Einkommensverteilung nur insoweit teil, als er in der Kreditverflechtung der Sektoren eine Nettogläubigerposition einnimmt. Den größten Teil seiner Einnahmen erhält er in einem nicht über die Märkte verlaufenden Prozeß der Einkommensumverteilung, in dem als letztes die für die Struktur der verfügbaren Einkommen bedeutsame Kategorie der Transfereinkommen entsteht. 
Der Ablauf der Modellwirtschaft läßt sich mit allen Vereinfachungen anhand eines Systems von Definitionsgleichungen beschreiben. Dieses System wird am besten von der Stromrechnung und wie bei der kontenmäßigen Darstellung der volkswirtschaftlichen Gesamtrechnung vom laufenden Produktionsprozeß her entwickelt.

\section{Stromgleichungen}

Das zu Marktpreisen bewertete Bruttosozialprodukt der betrachteten Volkswirtschaft setzt sich zusammen aus der Bruttowertschöpfung des privaten und des staatlichen Sektors, $Y_{\mathrm{pr}, t}^{\star} \mathrm{bzw} . \mathrm{Y}_{\mathrm{St}, \mathrm{t}}^{\star}$, und aus dem Saldo der bewerteten Faktordienste im internationalen Verkehr. Da laufende internationale Wanderungen und ein grenzüberschreitender Pendlerverkehr von Arbeitskräften $z$ wischen den Arbeitsmärkten des In- und Auslandes ausgeschlossen sind, im internationalen Kapitalverkehr außerdem nur Forderungstitel gehandelt werden, ist der Saldo der bewerteten Faktordienste im Inland gleichzusetzen dem in der Zahlungsbilanz auftretenden Überschuß der von der übrigen Welt erhaltenen Zinserträge über die an das Ausland geleisteten Zinsaufwendungen, $Z_{R}, t$, und es gilt für die Entstehung des Bruttosozialprodukts zu Marktpreisen die Gleichung (1):

$$
Y_{t}^{b}=Y_{p r, t}^{*}+Y_{S t, t}^{*}{ }_{R, t}^{*}
$$

Der einkommensmäßige Gegenwert des Bruttosozialprodukts zu Marktpreisen ist das Bruttoinländereinkommen. Es entsteht durch die Nachfrage der selbständigen und unselbständigen Erwerbspersonen zur Deckung ihres Konsums, $\mathrm{C}_{S}, \mathrm{t}$ bzw. $\mathrm{C}_{\mathrm{A}}, \mathrm{t}$, die Güternachfrage der privaten Investoren, I $\mathrm{Ir}_{\mathrm{pr}} \mathrm{t}$, und die Nachfrage des Staates nach Gütern und Dienstleistungen, ASt, $t$, die Abschreibungen für staatlichen Kapitalverzehr, DSt, $t$, sowie durch den Überschuß der Exporterlöse über die Importaufwendungen den sog. Außenbeitrag. Der wirtschaftliche Verkehr mit der übrigen Welt erstreckt sich ausschließlich auf den Waren- und Kapitalverkehr. Daher setzt sich der Aussenbeitrag zusammen aus dem in der Zahlungsbilanz verzeichneten Saldo zwischen den bewerteten Warenexporten und den bewerteten Warenimporten, $Z_{\mathbf{X}}, t$, und aus dem bereits genannten Saldo der Kapitalertragsbilanz, $Z_{R}, t . F u ̈ r$ die Entstehung des Bruttoinländereinkommens gilt daher die Gleichung:

$$
Y_{t}^{b r}=C_{A, t}+C_{S, t}+I_{p r, t}^{b r}+A_{S t, t}+D_{S t, t}{ }^{+Z} x, t{ }_{R, t}
$$

(1) Ein Stern kennzeichnet in diesem Paragraphen die Abgrenzung der Variablen nach dem Inlandskonzept im Gegensatz zur Abgrenzung nach dem Inländerkonzept. 
Das Bruttoinländereinkommen wird um private und staatliche Abschreibungen, $D_{\mathrm{pr}, \mathrm{t}} \mathrm{bzw}$. DSt, $\mathrm{t}$, und um indirekte Steuerzahlungen an den Staat, $\mathrm{T}_{\text {ind, }} \mathrm{t}$, bereinigt und ergibt dann das Volkseinkommen, $\mathrm{Y}_{\mathrm{t}}$, das an die inländischen Wirtschaftssubjekte verteilt werden kann:

$$
\text { (2.3) } \quad Y_{t}=Y_{t}^{b r}-D_{p r}, t^{-D} \text { St, } t^{-T} \text { ind }, t
$$

Die Verteilung des Volkseinkommens erfolgt über drei funktionelle Einkommensströme, Arbeitseinkommen, $\mathrm{L}_{\mathrm{t}}$, Zinseinkommen, $\mathrm{R}_{\mathrm{t}}$, und Gewinneinkommen, $\mathrm{G}_{\mathrm{t}}$ :

$$
\text { (2.4) } \quad Y_{t}=L_{t}+R_{t}+G_{t}
$$

die sich alle drei nach dem Sektor, in dem sie entstanden sind, oder nach der Gruppe von Wirtschaftssubjekten, der sie zufallen, aufgliedern lassen.

Die Arbeitseinkommen entstehen im Unternehmenssektor, der vollständig dem privaten Sektor des Inlands angehört, und im Staatssektor des Inlands (1):

$$
L_{t}=L_{p r, t}^{*}+L_{S t, t}^{*}
$$

Die orginären Zinseinkommen entstehen in Höhe von $R_{p r}^{\star}, t$ im Unternehmenssektor und in Höhe von $Z_{R}, t$ im internationalen Kapitalverkehr mit dem Ausland. Ein Zins auf staatliches Anlagevermögen wird nicht verrechnet.

$$
\text { (2.6) } \quad R_{t}=R_{p r, t}^{+Z_{R, t}}
$$

Die Gewinneinkommen entstehen lediglich im inländischen Unternehmenssektor:

$$
(2.7) \quad G_{t}=G_{p r, t}^{*}
$$

(1) Wegen des Ausschlusses von Wanderungen und Pendlerverkehr über die nationalen Grenzen kann die Kennzeichnung der Variablen von (2.5) durch einen Stern entfallen. 
Alle Faktorzahlen des Unternehmenssektor stellen notwendigerweise konsolidierte Einkommensgrößen der Produktionsunternehmen und der Banken dar (1).

Die Arbeitseinkommen entfallen auf Einkommen aus unselbständiger Ar beit, $L_{A, t}$, und den aggregierten Unternehmerlohn, $L_{S, t}$ :

$$
\text { (2.8) } \quad L_{t}=L_{A, t}+L_{S, t}
$$

Die aus dem privaten Produktionsprozeß und dem internationalen Kapitalverkehr zugeflossenen Zinseinkommen verteilen sich entsprechend der Struktur des Inländervermögens auf die selbständigen und unselbständigen Erwerbspersonen, $\mathbf{R}_{S, t}$ bzw. $R_{A, t}$, und den Staat, $R_{S t, t}$ :

$$
R_{t}=R_{A, t}+R_{S, t}+R_{S t, t}
$$

Dabei ist zu beachten, daß der durch $R_{S t, t}$ erfaßte Einkommensstrom ausschließlich aus dem Kreditverkehr zwischen dem Staat und dem privaten Sektor stammt. Kredite im staatlichen Kapitalverkehr mit der übrigen Welt, die ohnehin nur in Richtung eines internationalen Kapitalexports

(1) Schöpfen die Banken auf der Basis der von der Zentralbank zur Verfügung gestellten Liquidität ein Vielfaches an Krediten und dienen diese den Produktionsunternehmen im engen Sinne als produzierte Produktionsmittel, dann können die entsprechenden Zinserträge im vorliegenden Modell nicht als Verrechnungs- oder Umverteilungsposten zwischen zwei Untersektoren des Unternehmenssektors verstanden werden. Sie haben im Prinzip auch nichts mit den übrigen Dienstleistungsgeschäften der Banken zu tun, wie z. B. der Abwicklung des Zahlungsverkehrs, von denen angenommen werden kann, daß sie kostenmäßig durch Verrechnung von Provisionen abgedeckt werden. Die Zinserträge sind vielmehr Entgelt für einen von den Banken geleisteten spezifischen Dienst, der mit der Übernahme und Kontrolle des bei der Kreditvergabe entstandenen Liquiditätsrisikos zusammenhängt, den Einsatz von Produktionsfaktoren erfordert und damit Kosten verursacht. Mit der Gewährung neugeschaffener Kredite ist also zwangsläufig ein Prozeß der Produktion verbunden, und die entsprechenden Zinszahlungen setzen zwangsläufig einen Prozeß der Einkommensentstehung und anschließenden Einkommensverteilung in $\mathbf{G}$ ang. In der volkswirtschaftlichen Gesamtrechnung dieses Modells kann wohl der Kreditverkehr $\mathrm{z}$ wischen Produktionsunternehmen und Banken durch die Verrechnung entsprechender Forderungen und Verbindlichkeiten einigermaßen gesondert berücksichtigt werden - vgl. hierzu R. Richter, Die Rolle der Banken in makroökonomischen Modellen, Jahrbücher für Nationalökonomie und Statistik, 180, 1967, S. $444 \mathrm{ff}$. -; dies gilt jedoch nicht für den mit der Kreditschöpfung verbundenen Prozeß der Produktion, der Einkommensentstehung sowie der anschließenden Einkommensverteilung. 
verlaufen, sind, obwohl nicht unbefristet, unentgeltlich. Der Posten $\mathrm{R}_{\mathrm{St}, \mathrm{t}}$ erhält im Fall einer Nettoschuldnerposition des Staates gegenüber dem privaten Sektor einen negativen Wert; er stellt dann einen Zinstransfer dar, der die Zinseinkommen des privaten Sektors über die im Produktionsprozeß entstandenen Zinseinkommen hinaus erhöht (1).

Die Gewinne des Unternehmenssektors gehören in der betrachteten Volkswirtschaft allein den Selbständigen:

$$
\text { (2.10) } \quad G_{t}=G_{S, t}
$$

Die Gleichungen (2.8) bis (2.10) kennzeichnen die Verteilung der funktionellen Einkommensströme nach der letzten rechtlichen Zugehörigkeit der Faktoren. Ähnlich lassen sich die den Faktorbesitzern letztlich gehörenden Einkommen auch nach funktionellen Einkommenskategorien unterteilen. Für den tatsächlichen Ablauf des Systems ist aber weniger die Aufteilung nach der letzten rechtlichen Zugehörigkeit der Gruppeneinkommen als vielmehr die Aufteilung nach wirtschaftlich-institutionellen Gesichtspunkten von Bedeutung. Unter diesem Gesichtspunkt ergeben sich vier institutionelle Einkommenskategorien:

Das Einkommen der unselbständigen Erwerbspersonen, $\mathrm{Y}_{\mathrm{A}, t}$, setzt sich zusammen aus dem Einkommen aus unselbständiger Arbeit und dem Zinsertrag des Unselbständigen-Vermögens, $R_{A, t}$ :

$$
\text { (2.11) } \quad Y_{A, t}=L_{A, t}+R_{A, t}
$$

Die letztlich den Selbständigen zuzurechnende Summe aus Unternehmerlohn, Verzinsung des Selbständigenvermögens und Unternehmergewinn entfällt in Höhe des ausgezahlten Unternehmerlohns und der an die Selbständigen verteilten Besitzeinkommen, $\mathrm{V}_{t}$, auf das persönliche Einkommen der Selbständigen, $\mathrm{Y}_{\mathrm{S}, \mathrm{t}}$ :

$$
Y_{S, t}=L_{S, t}+V_{S, t}
$$

in Höhe der verbleibenden, nichtausgeschütteten Besitzeinkommen (2)

(1) Dies würde sich ändernn, sobald alle aufgenommen Schuldbeträge der Finanzierung öffentlicher Anlageinvestitionen dienten und in der volks wirtschaftlichen Gesamtrechnung des Systems auf staatliche Anlagevermögen eine Kapitalverzinsung verrechnet würde, die zu einer Erhöhung des im Produktionsprozeß entstandenen erfaßten Zinseinkommen $R_{t}$ führte.

(2) Durch die Abgrenzung nach verteilten und unverteilten Besitzeinkommen der Selbständigen wird im Sinne der allgemein praktizierten volkswirtschaftlichen Gesamtrechnung die Verteilungsrechnung mit der Umverteilungsrechnung vermischt, was hier aber nicht stören soll. 
bildet sie das Unternehmenseinkommen, $\mathrm{Y}_{\mathrm{U}, \mathrm{t}}$ :

(2.13) $\quad Y_{U, t}=R_{S, t}+G_{S, t}-V_{S, t}$

Der übrige Teil des Volkseinkommens fällt, sofern er positiv ist, in Form von Zinszahlungen des privaten Sektors dem Staat zu:

(2.14) $\quad Y_{S t, t}=R_{S t, t}$

Nach der Entstehung und der funktionellen und institutionellen Verteilung der Einkommen setzt mit Hilfe von einseitigen Übertragungen der Prozeß der Einkommensumverteilung ein, der zum größten Teil über den Staatshaushalt verläuft:

Die unselbständigen Erwerbspersonen haben neben Lohnsteuern, $T_{L, t}$, an den Staat Beiträge zur Finanzierung ihrer sozialen Sicherung zu leisten, $\mathrm{T}_{\mathrm{SV}}$, t. Ihre Vermögen und Vermögenserträge sind aus sozialpolitischen Gründen steuerfrei. Im Gegenzug für ihre Sozialversicherungsbeiträge erhalten die Unselbständigen für sich und ihre Familienangehörigen die Leistungen der staatlichen Sozialinstitutionen, USt, A, t. Ein Teil ihres Einkommens wird in Form von Unterstützungszahlungen ehemals zugewanderter ausländischer Arbeitskräfte an die übrige Welt überwiesen, $U_{A}$, Ausl, t. Für das verfügbare Einkommen der unselbständigen Haushalte, $\mathrm{Y}_{\mathrm{A}, \mathrm{t}}^{\mathrm{n}}$ gilt:

(2.14a) $Y_{A, t}^{n}=Y_{A, t^{-T}}{ }_{L A, t^{-T}} S V, t^{+U_{S t}, A, t^{-U}}$ A, Aus I, $t$

Entsprechend der institutionellen Gliederung der Verteilungsrechnung werden auch die Übertragungen, die das letztlich den Selbständigen zustehende verfügbare Einkommen verändern, aufgeteilt in Übertragungen, die das persönliche Einkommen der Selbständigen, und in solche, die das Unternehmenseinkommen betreffen. Diese Aufteilung orientiert sich wie die institutionelle Aufteilung der über den Markt verteilten Einkommen an der tatsächlichen wirtschaftlichen Verfügungsmacht über Einkommensteile; sie berücksichtigt dabei einige Besonderheiten des betrachteten Wirtschaftssystems: Ein großer Teil der in der Volkswirtschaft lebenden Selbständigen, der sich aus Handwerkern und Bauern zusammensetzt, erzielt in etwa ein Einkommen, das dem Durchschnittsverdienst eines Arbeitnehmers entspricht und das sich vorwiegend aus dem Unternehmerlohn und nur zu einem kleinen Teil aus der Kapitalverzinsung zusammensetzt. Dieser Teil der Selbständigen kommt ähnlich den Arbeitnehmern in den Genuß staatlicher Vergünstigungen bei der Besteuerung der verhältnismäßig kleinen Vermögen und Vermögenserträge; insoweit ihn Einkommensteuern treffen, ist er zu einer Einschränkung seiner Lebens haltung gezwungen, da wegen des schwierigen Zugangs zum Kreditmarkt und der geringen Neigung zur Fremdfinanzierung die Kapitalver- 
zinsung zur Finanzierung dringender Investitionen benötigt wird. - Der andere Teil der Selbständigen verfügt im Durchschnitt über ein solches Vermögen und damit über einen solchen Einfluß über den Unternehmenssektor, daß ihm eine "Vorwälzung" der persönlichen Steuern auf den Unternehmenssektor gelingt. Soweit er Eigentümer von Personalunternehmen ist, erhöht er die Zins - und Gewinnentnahmen im Umfang der persönlichen Steuern, so daß diese letzten Endes von den Unternehmen gezahlt werden und zulasten der Selbstfinanzierung gehen; im Fall des Eigentums an Kapitalgesellschaften erreicht er ein ähnliches Ergebnis über Änderungen in der Vermögensanlagestruktur oder über einen aufgrund von Anteilsmehrheiten ausgeübten Einfluß auf die Ausschüttungspolitik .

Diese Besonderheiten führen dazu, daß in einer institutionellen Gliederung der betrachteten Volkswirtschaft die Gruppe der Selbständigenhaushalte auf ihre persönliches Einkommen lediglich Lohnsteuern auf den Unternehmerlohn, $T_{L S, t}$, zugerechnet bekommt; die Selbständigen wer den weder zu Sozialversicherungsbeiträgen herangezogen noch erhalten sie Leistungen der staatlichen Sozialinstitutionen:

$$
\text { (2.15) } \quad Y_{S, t}^{n}=Y_{S, t}{ }^{-T_{L S}, t}
$$

Vom Unternehmenseinkommen, $Y_{U}, t$ werden entsprechend eine Steuer auf ausgeschüttete und einbehaltene Zins - und Gewinneinkommen der Selbständigen, $T_{R G S}, t$, und eine Steuer auf das über den Kreditmarkt oder auf dem Wege der Selbstfinanzierung in den Unternehmenssektor eingebrachte Selbständigenvermögen, $T_{F}, t$, in Abzug gebracht; hinzuzuzählen sind die Übertragungen des Staates an den Unternehmenssektor, $U_{S t, U, t}$, die als direkte Subventionen aus wachstums-, konjunktur - oder strukturpolitischen Gründen gewährt werden:

$$
\text { (2.16) } \quad Y_{U, t}^{n}=Y_{U, t^{-T}} T_{R S, t^{-T}} T_{t}+U_{S t, U, t}
$$

n

Das verfügbare Einkommen des Staates, $\mathrm{Y}_{\mathrm{St}, \mathrm{t}}$, bildet sich aus dessen Anteil am Volkseinkommen, den indirekten Steuerzahlungen des privaten Sektors, der Lohnsteuer und den Sozialversicherungsbeiträgen der Arbeitnehmer, der Steuer auf den Unternehmerlohn der Selbständigen, der Steuer auf ausgeschüttete und einbehaltene Besitzeinkommen der Selbständigen und der Steuer auf die im Unternehmenssektor angelegten Ver mögen der Selbständigen abzüglich des Sozialtransfers des Staates an die Arbeitnehmer und seiner Übertragungen an die Unternehmen. Zusätzlich ist bei der Bildung seines verfügbaren Einkommens zu berücksichtigen, daß der Staat der übrigen Welt Unterstützungen in Form von unentgeltlichen Einkommenstransfers, U $\mathrm{U}_{\mathrm{St}}$, Ausl, $t$, gewährt:

$$
\begin{aligned}
& \text { (2.17) } \quad Y_{S t, t}^{n}=Y_{S t, t}{ }^{+T_{\text {ind }, t}}{ }^{+T_{L A}, t}{ }^{+T_{S V}, t}{ }^{+T_{L S}, t}{ }^{+T_{R G S}, t}{ }^{+T_{F, t}}
\end{aligned}
$$

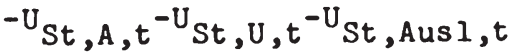


Die in (2.17) angeführten indirekten Steuern erstrecken sich, das sei hier nachgetragen, auf zwei spezielle Wertschöpfungsteuern, eine Gewerbeertragsteuer, $T_{G R, t}$, die alle im Unternehmenssektor entstandenen Zins - und Gewinneinkommen belastet, und eine Lohnsummensteuer, $\mathrm{T}_{\mathrm{L}, \mathrm{t}}$, die die im Unternehmenssektor entstandenen Arbeitseinkommen belastet, ferner auf eine Gewerbekapitalsteuer, $T_{k, t}$, die am Anlagevermögen des Unternehmenssektors ansetzt, sowie eine indirekte Verbrauchsteuer, $T_{C}, t$. Daneben umfassen sie die Zölle, die allein auf Importe erhoben werden, $\mathrm{T}_{\mathrm{Im}, \mathrm{t}}$ :

$$
T_{\text {ind }, t}=T_{R G, t}+T_{L, t}+T_{k, t}+T_{C, t}+T_{I m, t}
$$

Auf der Basis der verfügbaren Einkommen vollzieht sich der Prozeß der letzten Verwendung des Einkommens, in dem zwischen Konsum und Ersparnisbildung entschieden wird:

Die verfügbaren Einkommen der Arbeitnehmer - und Selbständigenhaus halte zerfallen in Konsum, $C_{A, t}$ bzw. $C_{S, t}$, und Ersparnis, $S_{A, t} b z w$. $\mathrm{S}_{\mathrm{S}, \mathrm{t}}$ :

$$
\begin{aligned}
& \text { (2.19) } \mathrm{Y}_{\mathrm{A}, \mathrm{t}}^{\mathrm{n}}=\mathrm{C}_{\mathrm{A}, \mathrm{t}}{ }^{+\mathrm{S}_{\mathrm{A}, \mathrm{t}}} \\
& (2.20) \quad \mathrm{Y}_{\mathrm{S}, \mathrm{t}}^{\mathrm{n}}=\mathrm{C}_{\mathrm{S}, \mathrm{t}}{ }^{+S_{S, t}}
\end{aligned}
$$

Die im Unternehmenssektor verbleibenden Besitzeinkommen der Selbständigen stellen in vollem Umfang Ersparnisder Unternehmen dar:

(2.21) $\quad Y_{U, t}^{n}=S_{U, t}$

Beim staatlichen Sektor wird an dieser Stelle noch nicht zwischen konsumtiver Verwendung und Vermögensbildung unterschieden. Sein verfügbares Einkommen wird zerlegt in Ausgaben für die laufenden Käufe von Gütern und Dienstleistungen, $A_{S t, t}$, und den Finanzierungssaldo des Budgets, BSt, $t$, der lediglich eine positive oder negative Geldvermögensbildung des Staates darstellt:

(2.22) $\quad Y_{S t, t}^{n}=A_{S t, t}{ }^{+B_{S t, t}}$

Da die Analyse des Wirtschaftsablaufs in dem Modell sich ausdrücklich auch auf monetäre Phänomene erstreckt, werden in der volkswirtschaftlichen Gesamtrechnung des Modells auch die Entscheidungen über die Anlage der Ersparnis verzeichnet:

Die Ersparnisse der privaten Haushalte dienen dem laufenden Kauf von Forderungstiteln, $\Delta \mathrm{F}_{1 \mathrm{~A}, \mathrm{t}} \mathrm{bzw}, \Delta \mathrm{F}_{1 \mathrm{~S}, \mathrm{t}}$, und dem laufenden Aufbau der Kassenhaltung, $\Delta M_{1 A, t}$ bzw. $\Delta M_{1 S, t}$ : 
(2.23) $\quad S_{A, t}=\Delta F_{1 A, t}+\Delta M_{1 A, t}$

(2.24) $\quad S_{S, t}=\Delta F_{1 S, t}+\Delta M_{1 S, t}$

Der Unternehmenssektor legt die Gesam theit seiner Ersparnisse in Forderungstiteln des inländischen Kreditmarktes, $\Delta F_{U, t}$, an (1). Der Staat tätigt in Höhe seines Finanzierungssaldos Nettokäufe von Forderungsti teln am inländischen Kreditmarkt, $\Delta F_{S t}$, Inl, t, und gewährt der übrigen Welt Kapitalhilfe in Form zinsloser Kredite, die hier nach Bereinigung um entsprechende Kreditrückflüsse als nicht über den inländischen Kreditmarkt verlaufende staatliche Nettokäufe von ausländischen Forderungstiteln, $\Delta \mathrm{F}_{\mathrm{St}}$, Ausl, $\mathrm{t}$, erfaßt werden:

$$
S_{U, t}=\Delta F_{U, t}
$$

$$
B_{S t, t}=\Delta F_{S t, I n l, t}+\Delta F_{S t, A u s l, t}
$$

Mit den Gleichungen (2.23) bis (2.26) für die Verwendung der Ersparnisbildung ist zugleich die Verteilung der Ersparnisbildung bestimmt. Ähnlich wie die über den Markt verlaufende Einkommensverteilung wird die Verteilung der Ersparnisbildung aber noch durch einen anschließenden Prozeß der Umverteilung überlagert, der allein innerhalb des privaten Sektors stattfindet.

Der erste Umverteilungsvorgang vollzieht sich zwischen Unternehmen und Selbständigenhaushalten: Nachdem der Unternehmenssektor durch eine Kreditmarktanlage seiner Ersparnisse dafür gesorgt hat, daß das angelegte Finanzkapital über eine Kreditmarktverschuldung weiterhin zu Investitionszwecken zur Verfügung steht, überträgt er die erworbenen Forderungstitel nach Art von "Gratisaktien" an die Selbständigenhaushal te (2) und gibt damit sein aus Ersparnissen gebildetes positives Geldvermögen auf.

(1) Die Annahme einer Kreditmarktanlage der Unternehmensersparnisse ist hier nur technisch bedingt und dient einer bestimmten Art der Vermögensrechnung.

(2) Es wird hier also entsprechend der wirtschaftlich-institutionellen $A b-$ grenzung genau umgekehrt verfahren wie üblicherweise in der volks wirtschaftlichen Gesamtrechnung, wo entsprechend der strengen rechtlichen Zuordnung ein Teil der Einkommen erst den privaten Haus halten als den Eigentümern der angebotenen Faktorleistungen zugerechnet wird und wo anschließend in Höhe der nichtentnommenen Gewinne der Personalunternehmen und des von den privaten Haushalten geleisteten Finanzierungsbeitrags zu den Wohnungsbauinvestitionen Vermögens übertragungen an den Unternehmenssektor verrechnet werden. 
Der zweite Umverteilungsvorgang vollzieht sich zwischen den selbständigen und unselbständigen Haushalten: In der betrachteten Volkswirtschaft findet eine ständige Änderung der Erwerbsstruktur statt. Per Saldo geben stets selbständige Erwerbspersonen ihre Selbständigkeit auf und wechseln in den Arbeitnehmersektor. Bei diesem Wechsel verzichten sie auf das Eigentum an Unternehmen (1), nehmen aber stets ihre rein vermögensrechlichen Ansprüche mit in den Unselbständigensektor. Der Erwerbsstrukturänderung entspricht daher eine Vermögensstrukturänderung, die hier in Form von rechnerischen Vermögensübertragungen zwischen den beiden Haushaltsgruppen berücksichtigt werden muß und die sich auf den Bestand an Forderungstiteln und den Kassenbestand der Haushalte erstreckt.

Die beiden Arten von Vermögensübertragungen sind die einzigen, die in die volkswirtschaftliche Gesamtrechnung der Modellwirtschaft eingehen. Praktisch müßten als dritte Art mindestens noch die Vermögensüber tragungen berücksichtigt werden, die aus Erbschaften entstehen. Eine angenommene Eigenschaft der Modellwirtschaft besteht jedoch darin, da $\beta$ der Großteil der Erbschaften sich innerhalb der beiden Haushalts gruppen vollzjeht und daß der Erbverkehr zwischen den Selbständigen- und Unselbständigen-Haushalten per Saldo stets ausgeglichen ist. Ferner sind hier die in den meisten Volkswirtschaften erhobenen Erbschaftsteuern in das System der laufenden Vermögensbesteuerung eingebaut und werden daher implizit als Einkommensübertragungen verrechnet.

Nach Berücksichtigung der Vermögensübertragungen läßt sich die Gesamtänderung der in den Gruppen gehaltenen Bestände an Forderungstiteln und Kassenhaltung angeben:

Der Bestand der Unselbständigen-Haushalte an Forderungstiteln erfährt eine Änderung durch die aus Arbeitnehmerersparnissen finanzierten laufenden Käufe von Forderungstiteln, $\Delta F_{1 A}, t$, und dadurch, daß die ihre Selbständigkeit aufgebenden Erwerbspersonen einen zusätzlichen Bestand von Forderungstiteln als Vermögensübertragungen in den Kreis der Unselbständigen-Haushalte einbringen, $\dot{U}_{\mathrm{FSA}, t}$ :

$$
\text { (2.27) } \Delta F_{A, t}=\Delta F_{1 A, t}+U_{F S A, t}
$$

Der Bestand der Selbständigen-Haushalte an Forderungstiteln erfährt eine Änderung durch die laufenden Titelkäufe der Selbständigen, die aus Haushaltsersparnis finanziert werden, $\Delta F_{1 S}, t$ durch die Übertragung neu erworbener Forderungstitel des Unternehmenssektors an die Selbständigen-Haushalte, $\ddot{U}_{\mathrm{FUS}} \mathrm{t}$, und durch die mit dem Ausscheiden von Erwerbspersonen aus der Selbständigengruppe verbundenen Übertragungen von Titeln, $\dot{U}_{F S A}, \dot{t}$

(1) Das heißt praktisch: Sie verkaufen ihr Unternehmen oder die oben i. e. S. definierten Eigentumsrechte an ihrem Unternehmen. 


\section{(2.28) $\quad \Delta F_{S, t}=\Delta F_{1 S, t}+U_{F U S, t}{ }^{-U_{F S A} t}$}

Die laufenden Käufe von Forderungstiteln durch die Unternehmen und die anschließende, wie eine Gewährung von Gratisaktien vollzogene Übertragung dieser Titel an die Selbständigen-Haushalte gleichen sich aus:

$$
\text { (2.29) } \Delta F_{U, t}=U_{F U S, t}
$$

Ähnlich erfahren die Kassenbestände der Unselbständigen- und Selbstän digen-Haushalte,eine Änderung durch die laufende Aufstockung des Kassenbestandes aus Arbeitnehmer- bzw. Selbständigenersparnissen, $\Delta M_{1 A, t}$ bzw. $\Delta \mathrm{M}_{1 \mathrm{~S}, \mathrm{t}}$, und durch die im Zuge der Erwerbsstrukturänderungen vollzogene Übertragung von Kassenbeständen, ÜMSA, $\mathrm{t}$ zwischen den beiden Haushaltsgruppen:

(2.30) $\quad \Delta M_{A, t}=\Delta M_{1 A, t}+U_{M S A, t}$

(2.31) $\quad \Delta M_{S, t}=\Delta M_{1 S, t}{ }^{-U} M_{S A, t}$

Die Übertragung von Forderungs - und Kassenbeständen zwischen den beiden Sektoren der Selbständigen - und Unselbständigenhaushalte ergeben zusammen den Wert der entsprechenden Vermögensübertragungen, ÜWSA, $\mathrm{t}$ :

(2.32) $\quad U_{W S A, t}=U_{F S A, t}+U_{M S A, t}$

Addiert man die am Kreditmarkt angelegten Ersparnisse des privaten und staatlichen Sektors und fügt ihnen die mit einer Kreditschöpfung verbundenen Käufe von Forderungstiteln durch inländische Banken, $\Delta \mathrm{F}_{\mathrm{Bk}, \mathrm{t}}$, hinzu, gelangt man zur gesamten über den Kreditmarkt der Volkswirtschaft verlaufenden Kreditvergabe des Inlands:

$(2.33)$

$\Delta F_{t}=\Delta F_{A, t}+\Delta F_{S, t}+\Delta F_{S t, I n l, t}+\Delta F_{B k, t}$

Diese inländische Kreditvergabe wird ergänzt um den in der Zahlungsbi lanz erfaßten Saldo des privaten Kapitalverkehrs mit der übrigen Welt bzw. um die privaten ausländischen Nettokäufe, $\Delta F_{\text {Ausl, }} t$, von Forderungstiteln am inländischen Kreditmarkt:

(2.34) $\quad \Delta F_{t}^{*}=\Delta F_{t}+\Delta F_{\text {Ausl, } t}$

und ergibt die gesamte Kreditvergabe an den inländischen Unternehmenssektor, $\Delta \mathrm{F}_{\mathrm{t}}^{\star}$.

Die Unternehmen, die im Umfang von $\Delta \mathrm{F}_{\mathrm{t}}^{\star}$ Forderungstitel neu emittiert haben, verwenden einen kleinen Teil der aufgenommenen Kredite zur 
Aufstockung ihrer Kassenhaltung; der Großteil des aufgenommenen Finanzkapitals dient zusammen mit den in den Unternehmen verbliebenen Abschreibungserlösen zur Finanzierung der privaten Investitionstätigkeit:

$$
\text { (2.35) } \quad I_{p r, t}^{b r}=\Delta F_{t}^{-}-\Delta M_{U, t}+D_{p r, t}
$$

die wiederum der Anlageninvestition, $\mathrm{I}_{\mathrm{pr}}^{\mathrm{br}}, \mathrm{Anl}, \mathrm{t}$, und der Vorratsinvestition, $I_{v}, t$, dient:

(2.36) $\quad I_{p r, t}^{b r}=I_{p r, A n l, t}^{b r}+I_{v, t}$

An dieser Stelle ist eine Klärung hinsichtlich der Aufteilung der staatlichen Güter - und Dienstleistungskäufe sinnvoll: Wie der private Sektor verwendet der Staat einen Teil der laufend gekauften Güter und Dienstleistungen zur Anlageninvestition. Die Kapazitätseffekte der erstellten öffentlichen Anlagen dienen, sofern sie nicht ausschließlich der Befriedigung konsumtiver Kollektivbedürfnisse zugute kommen, zu einem Teil als unmittelbare Vorleistungen für den Produktionsprozeß des privaten Sektors; zu einem anderen Teil führen sie erst über weitere produktive Umwege, also mittelbar, zu einer Beeinflussung des privaten Produktionsprozesses. Im Anschluß an diese Unterscheidung wird aus dem Gesamtbetrag der öffentlichen Anlageinvestitionen der Teil ausgesondert, der in der Produktionsfunktion des privaten Sektors als eigener Produktionsfaktor berücksichtigt werden muß und der im folgenden kurz als Infrastrukturinvestition bezeichnet wird, $\mathrm{I}_{\mathrm{St}}^{\mathrm{b}} \mathrm{t}$. Diesem Teil werden die sonstigen öffentlichen Anlageinvestitionen gegenübergestellt, I $\mathrm{St}$, sonst, $t$, deren Kapazitätseffekte während der Modellanalyse nicht explizit untersucht werden.

Im Anschluß an Gleichung (2.35) ergibt sich daher eine Definitionsgleichung für die staatlichen Infrastrukturinvestitionen:

(2.37) $\quad I_{S t, t}^{b r}=A_{S t, t}-\left(C_{S t, t}{ }^{-D} S_{S t, t}\right)-I_{S t, \text { sonst }, t}^{b r}$

wobei ( $\mathrm{C}_{S t, t}$ - DSt, $t$ die Käufe für staatlichen Konsum und $\mathrm{C}_{\mathrm{St}}, \mathrm{t}$ den öffentlichen Konsum einschließlich öffentlichen Kapitalverzehr kennzeichnet.

Mit Gleichung (2.37) ist der in der Volkswirtschaft ablaufende Wirtschafts prozeß, der von der Güterproduktion bis zur Verwendung der entstandenen Einkommen für Käufe von Gütern und Diensten reicht, einmal gedanklich durchgespielt worden. Der Überblick über den Kreislaufprozeß bedarf nur noch hinsichtlich des Finanzierungssystems einer Ergänzung:

Faßt man den Überschuß der Kapitalertragsbilanz, der Warenverkehrsbilanz und der Kapitalbilanz im internationalen Verkehr mit der übrigen Welt zusammen und zieht von ihnen die Unterstützungszahlungen der Arbeitnehmer und des Staates an die übrige Welt ab, so zeigt sich, daß 
die Bilanz des gesamten internationalen Zahlungsverkehrs mit der übrigen Welt nicht notwendigerweise ausgeglichen ist. Es bleibt eine Differenz, in deren Höhe ein Nettozu- oder - abfluß an internationalen Zahlungsmitteln stattfindet. Da der gesamte internationale Zahlungsverkehr in ausländischen Währungseinheiten abgewickelt wird, stellt dieser Zahlungsstrom einen Nettozu- oder -abfluß an Devisen, $Z_{\text {Dev, }} t$, dar, dessen Wert in inländischen Währungseinheiten erfaßt wird:

$$
\begin{aligned}
& (2.38) \\
& Z_{\text {Dev, } t}=Z_{R, t}{ }^{+Z_{x, t}}+\Delta F_{\text {Ausl }, t^{-\Delta}} F_{\text {St, Ausl }, t} \\
& { }^{-U} \mathrm{~A}, \text { Aus } 1, \mathrm{t}^{-\mathrm{U}_{\mathrm{St}}, \text { Aus } 1, t}
\end{aligned}
$$

Findet ein Zufluß an internationalen Zahlungsmitteln statt, so werden die Devisenerlöse im Bankensystem gegen inländische Zahlungsmittel eingetauscht und stellen eine Quelle für inländische Geldversorgung neben der Kreditschöpfung des Bankensystems dar:

$$
\Delta M_{t}=\Delta F_{B k}, t+Z_{\text {Dev }, t}
$$

Da die Zahlungseingänge und Zahlungsausgänge auf den staatlichen Girokonten ziemlich gleichmäßig anfallen, der Staat außerdem die Möglichkeit hat, bei Liquiditätsengpässen Kassenkredite des Bankensystems in Anspruch zu nehmen, ist der Kassenbestand des Staates in der betrachteten Volkswirtschaft stets gleich Null, und die in (2.39) angegebene Änderung des inländischen Geldumlaufs entspricht der Änderung der Kassenhaltung der privaten Wirtschaftssubjekte:

$$
\text { (2.40) } \quad \Delta M_{t}=\Delta M_{A, t}+\Delta M_{S, t}+\Delta M_{U, t}
$$

\section{Preisgleichungen}

Die bisherige Beschreibung der Modellwirtschaft stellte ausschließlich auf die nominellen, d. h. zu laufenden Preisen bewerteten Stromgrößen des Wirtschaftskreislaufs ab. Um den Anschluß an eine güterwirtschaftliche Betrachtung des Wirtschaftsprozesses zu gewinnen, werden die nominellen oder Wertgrößen zerlegt in ihre Mengen- und ihre Preiskomponente, wodurch weitere Eigenschaften der Modellwirtschaft verdeutlicht werden. Die Aufspaltung geschieht in einem System von definitorischen Preisgleichungen.

Da der Staat in der Modellwirtschaft sein Inlandsprodukt der Bevölkerung nicht verkauft, sondern als freies Gut zur Verfügung stellt, existiert nur für das Bruttoinlandsprodukt des privaten Sektors ein Preis bzw. ein Preisindex. Bezeichnet $x_{t}$ das reale Bruttoinlandsprodukt des privaten Sektors und $\mathrm{p}_{\mathrm{x}, \mathrm{t}}$ den Preisindex dieses Produkts, dann gilt:

$$
\mathrm{Y}_{\mathrm{pr}, \mathrm{t}}^{\mathrm{H}}=\mathrm{x}_{\mathrm{t}} \cdot \mathrm{p}_{\mathrm{x}, \mathrm{t}}
$$


Der Preisindex $p_{x, t}$ ist der allgemeinste Preisindex in der Modellformulierung. Er dient aus Gründen der rechnerischen Vereinfachung als Indikator für das Preisniveau der Lebenshaltungskosten im Inland.

Gesondert erfaßt wird in der Modellformulierung der Preisindex für Bruttoanlageinvestitionen, $p_{k}, t$, der wegen seiner starken konjunkturellen Schwankungen von Bedeutung ist und der auch die Funktion eines Index der Wiederbeschaffungspreise in der Anlagenvermögensrechnung der inländischen Unternehmen übernimmt. Es gilt für die privaten Anlageinvestitionen und die staatlichen Infrastrukturinvestitionen:

$$
\begin{aligned}
& \text { (2.42) } \quad I_{p r, A n l, t}^{b r}=\Delta k_{p r, t}^{+} \cdot p_{k, t} \\
& (2.43) \quad I_{S t, t}^{b r}=\Delta k_{S t, t}^{+} \cdot p_{k, t}
\end{aligned}
$$

wobei $\Delta_{\mathrm{k}}^{+}$jeweils den Zugang an neuen Anlagen bzw. den Bruttoanlagenzugang angibt, ferner für die Abschreibungen des privaten und staatlichen Sektors:

$$
\begin{aligned}
& \text { (2.44) } D_{p r, t}=d_{p r, t} \cdot p_{k, t} \\
& (2.45) D_{S t, t}=d_{S t, t} \cdot p_{k, t}
\end{aligned}
$$

in denen das Symbol d den realen Kapitalverzehr angibt.

Ebenso wird in der Modellformulierung ein Preisindex für Vorratsinvestitionen berücksichtigt, die sich z. T. aus importierten Rohstoffen und Halbfabrikaten zusammensetzen und deren Preisentwicklung beträchtlich von der für Konsumgüter oder Anlageninvestitionen abweicht. Bezeichnet $\Delta v_{t}$ den Nettozugang an realen Vorratsbeständen und $\mathrm{p}_{\mathrm{v}, \mathrm{t}}$ den Preisindex für Vorratsinvestitionen, dann gilt:

$$
\text { (2.46) } I_{v, t}=\Delta v_{t} \cdot p_{v, t}
$$

Schließlich müssen von dem Preisniveau des privaten Inlandsprodukts die Preisniveaus $\mathrm{pIm}, \mathrm{t}$ bzw. $\mathrm{p}_{\mathrm{Ex}}, \mathrm{t}$ unterschieden werden, $\mathrm{zu}$ denen die Importgüter, $\mathrm{x}_{\mathrm{Im}, \mathrm{t}}$, beschafft und die Exportgüter, $\mathrm{x}_{\mathrm{Ex}, \mathrm{t}}$, abgesetzt werden und die außerhalb des Inlands gebildet werden. Für den Überschuß der Exporterlöse über die Importaufwendungen im internationalen Warenverkehr gilt die Preisgleichung:

$$
\text { (2.47) } z_{x, t}=x_{E x, t} \cdot p_{E x, t}{ }^{-x_{I m}, t} \cdot p_{I m, t}
$$

Alle vier Güterpreise verstehen sich als Preisindices mit einem einheitlichen Basisjahr.

Auf dem Arbeitsmarkt des privaten Sektors wird durch die Kollektivorganisationen der Arbeitnehmer und Arbeitgeber das Standardlohnniveau für das ganze Wirtschaftssystem festgelegt, das die Höhe der Brutto- 
arbeitseinkommen einschließlich aller lohnbezogenen Beiträge zur staatlichen Sozialversicherung regelt. $\mathrm{Zu}$ diesem Lohnniveau, $\mathrm{l}_{\mathrm{pr}}, \mathrm{t}$, werden die Arbeitnehmer des privaten Sektors, $a_{p r}, t$, entlohnt; an diesem Lohnniveau orientiert sich auch die Besoldung, l $\mathrm{St}, t$, der öffentlichen Bediensteten, ast, t. Für die Entlohnung der Arbeitnéhmer gelten daher die Lohngleichungen:

$$
\begin{aligned}
& \text { (2.48) } \mathrm{L}_{A, t}{ }^{-L_{S t, t}}=a_{p r, t} \cdot I_{p r, t} \\
& \text { (2.49) } L_{\text {St }, t}=a_{S t, t} \cdot{ }^{I_{S t, t}}
\end{aligned}
$$

Darüber hinaus ist das Standardlohnniveau bestimmend für die Ermittlung eines rechnerischen Unternehmerlohnsatzes, $1_{S t, t}$, der selbständigen Erwerbstätigen, $s_{t}$. Dieser Lohn bildet sich zwar nicht konkret an einem Markt heraus, da jedoch der Großteil der Selbständigen Funktionen ausübt, die mit denen der unselbständig Beschäftigten vergleichbar sind, und da die Selbständigen die Vor- und Nachteile ihrer Selbständigkeit laufend gegen die Vor- und Nachteile einer unselbständigen Beschäftigung abwägen, um dann über einen etwaigen Wechsel in der Art der Erwerbstätigkeit zu entscheiden, kann in der Modellwirtschaft seine Höhe annähern ausgemacht werden:

$$
\text { (2.50) } \quad L_{S, t}=s_{t} \cdot I_{S, t}
$$

Die drei Lohnsätze sind als absolute Geldbeträge definiert und geben das während der Periode verdiente durchschnittliche Arbeitseinkommen eines Arbeitnehmers im privaten bzw. Öffentlichen Dienst sowie eines Selbständigen im Unternehmensbereich an.

Die dritte Gruppe von definitorischen Preisgleichungen erstreckt sich auf die Zinserträge der Forderungsbestände. Ähnlich wie auf dem Arbeitsmarkt, freilich aus anderen Gründen, bildet sich auf dem Kreditmarkt ein Standardzins, $r_{t}$, heraus, der die Verzinsung des größten Teils der in inländischen Forderungstiteln angelegten Vermögen bestimmt. Er gibt den Ertrag des Selbständigen-Vermögens, $F_{S}$, t, und den Ertrag auf den positiven oder negativen Nettoforderungsbestand des Staates gegenüber dem inländischen Unternehmenssektor, $F_{S t, I n l, t}$, an. $F u ̈ r$ den Forderungsbestand der Unselbständigen, $F_{A, t}$, gilt als Folge des besonderen Anlageverhaltens ein abweichender Ertragssatz, $\mathrm{r}_{\mathrm{A}, \mathrm{t}}$, der sich allerdings sehr stark am Standardzins orientiert. Ebenso gilt für das im Inland angelegte positive oder negative Nettovermögen des Auslandes, $F_{\text {Ausl, } t}$, das zu einem Teil aus typischen Geldmarktanlagen besteht, ein abweichender Ertragssatz, $r_{z, t}$.

Aufgrund der nachschüssigen Verzinsung der umlaufenden Forderungstitel hängen die Zinseinkommensströme in der Modellwirtschaft von den Forderungsbeständen und dem Zinsniveau der jeweiligen Vorperiode ab. Dies soll bei der Aufstellung der Zinsgleichungen berücksichtigt werden, obwohl damit bereits Elemente von Verhaltensannahmen in das 
System der Definitionsgleichungen Eingang finden.

$$
\begin{array}{ll}
(2.51) & R_{A, t}=F_{A, t-1} \cdot r_{A, t-1} \\
(2.52) & R_{S, t}=F_{S, t-1} \cdot r_{t-1} \\
(2.53) & R_{S t, t}=F_{S t, I n l, t-1} \cdot r_{t-1} \\
(2.54) & z_{R, t}=F_{\text {Aus } 1, t-1} \cdot r_{z, t-1}
\end{array}
$$

3. Bestandsgleichungen

Da die Produktion bzw. Entstehung des Realeinkommens und die Einkommensverteilung in der Modellwirtschaft durch die Größe der vorhandenen Faktorbestände mitbestimmt werden, bedarf das definitorische Gerippe des Modells notwendigerweise der Ergänzung durch ein System von Bestandsdefinitionen. Einige Bestandsgrößen sind bereits genannt worden, müssen hier zur Präzisierung aber noch einmal systematisch angeführt werden.

Am Anfang stehen die Bestandsdefinitionen, die sich auf die Bevölkerung der Modellwirtschaft erstrecken: Während einer Teilperiode $t$ stellt die inländische Wohnbevölkerung eine Erwerbsbevölkerung im Umfang von $e_{v}, t$. Diese Erwerbsbevölkerung bildet das absolute Vollbeschäftigungs potential an Arbeitskräften; sie findet aber nur im Umfang von et, der Erwerbstätigenzahl, ein Unterkommen im Erwerbsprozeß; ein Teil von ihr, der stets der Gruppe der unselbständigen Erwerbspersonen angehört, bleibt aus konjunkturellen oder strukturellen Gründen arbeitslos. Bezeichnet $\mathrm{a}_{\mathrm{v}, \mathrm{t}}$ die Zahl der unselbständigen Erwerbspersonen, die prinzipiell zu einer unselbständigen Beschäftigung bereit sind, und at die Zahl der Unselbständigen, die tatsächlich eine Beschäftigung als Arbeitnehmer gefunden haben, dann gilt:

$$
e_{v, t}=e_{t}+\left(a_{v, t}-a_{t}\right)
$$

wobei der Index v den Vollbeschäftigungsumfang der jeweiligen Größen kennzeichnet.

Die Gesamtzahl der Erwerbstätigen setzt sich zusammen aus der Zahl der Arbeitnehmer, $a_{t}$, und der Zahl der Selbständigen, $s_{t}$ :

$$
\text { (2.56) } \quad e_{t}=a_{t}+s_{t}
$$

Eine Beschäftigung als Selbständiger gibt es ex-definitione nur im privaten Sektor. Unselbständige finden ihre Beschäftigung als Arbeitnehmer des privaten oder staatlichen Sektors, $a_{\mathrm{pr}, t}$ bzw. ${ }^{\mathrm{S} t, t} \mathrm{t}$ :

$$
\text { (2.57) } \quad a_{t}=a_{p r, t}{ }^{+a_{S t, t}}
$$


Für den Umfang der Erwerbstätigkeit im privaten Sektor, $e_{p r, t}$, gilt:

$$
\text { (2.58) } \quad e_{p r, t}=a_{p r, t}+s_{t}
$$

Alle übrigen Bestandsgrößen bilden sich aus einem mit dem Wirtschaftsprozeß verbundenen Akkumulationsvorgang und finden daher mit Hilfe von rekursiven Definitionen den Anschluß an entsprechende Größen der Stromrechnung.

Verzichten die privaten Haushalte auf eine konsumtive Verwendung von Einkommen und ebenso auf eine Kreditmarktanlage der gesparten Einkommen oder nehmen die Unternehmen Kassenkredite auf, so erhöht sich dadurch unmittelbar ihr jeweiliger Kassenbestand. Die Kassenbestände der privaten Wirtschaftssubjekte während einer Periode $t, M_{i}, t$, lassen sich daher definieren als der jeweilige Bestand der Vorperiode $M_{i, t-1}$, plus der laufenden Kassenaufstockung, $\Delta M_{i, t}$ :

$$
\text { (2.59) } \quad M_{i, t}=M_{i, t-1}+\Delta M_{i, t} \quad(i=A, S, U)
$$

Aus (2.59) zusammen mit (2.40) folgt logisch eine Gleichung für die Verteilung der inländischen Kassenbestände:

$$
M_{t}=M_{A, t}+M_{S, t}+M_{U, t}
$$

wobei zu beachten ist, daß die staatliche Kassenhaltung annahmegemäß gleich Null ist und daß, da der gesamte internationale Zahlungsverkehr in ausländischen Währungseinheiten abgewickelt wird, die übrige Welt keinerlei Bestände an Inlandswährung hält.

Ähnlich führt die Kreditgewährung zu einer unmittelbaren Erhöhung der Forderungsbesiände. Die von den Sektoren i während einer Periode $t$ gehaltenen Forderungsbestände, $F_{i, t}$ lassen sich daher definieren als Titelbestand der Vorperiode plus dem laufenden Zugang an Titeln aufgrund von Kauf oder Übertragung (1):

$$
\text { (2.61) } \quad F_{i, t}=F_{i, t-1}+\Delta F_{i, t} \quad(i=A, S, B k, \text { St, Aus } 1)
$$

Aus (2.60) und (2.61) zusammen mit (2.33) und (2.34) folgen logisch zwei Gleichungen für die Besitzverteilung der am inländischen Kreditmarkt erworbenen Forderungstitel auf die beiden privaten Haushaltsgruppen, den Bankensektor, den Staat und das Ausland:

(2.62) $\quad F_{t}=F_{A, t}+F_{S, t}+F_{S t, \text { Inl }, t}+F_{B k, t}$

(2.63) $\quad F_{t}^{*}=F_{t}+F_{\text {Aus } 1, t}$

(1) Der zweite Index zur Kennzeichnung der staatlichen Titelbestände, "Inl" bzw. "Ausl", wird hier vernachlässigt. 
Der Nettodevisenbestand, von dem angenommen wird, daß er ausreichend groß ist, um nachhaltige Zahlungsbilanzdefizite zu überbrücken, wird vernachlässigt. Stattdessen wird die Summe aller in der Vergangenheit eingetretenen Devisenzu- und -abflüsse, bewertet in inländischen Währungs einheiten, gebildet (1). Es gilt:

$$
\text { (2.64) } \quad M_{\text {Dev,t }}=M_{\text {Dev }, t-1}+Z_{\text {Dev, }}
$$

Aus (2.64) und (2.39) ergibt sich eine Bestandsgleichung für die Quellen der inländischen Geldversorgung, wonach der gesamte inländische Geldumlauf gleich ist dem im Zuge der Kreditschöpfung aufgebauten Forderungsbestand der Inlandsbanken plus dem Gegenwert der in der Vergangenheit eingetretenen Devisenzu- und -abflüsse:

$$
\text { (2.65) } \quad M_{t}=F_{B k, t}+M_{D e v, t}
$$

Mit den Gleichungen (2.59) bis (2.65) ist die gesamte Finanzierungsstruktur der Modellwirtschaft samt deren Ände rungen von der Seite der Forderungsansprüche her dargestellt. Um keine Unklarheiten entstehen zu lassen, sei daran erinnert, daß die Forderungsbestände bzw. die nichtliquide gehaltenen Ersparnisse der privaten Haushalte insgesamt in Kreditmarkttiteln angelegt sind, die von Banken oder Nicht-Banken emittiert wurden. Wurden diese Kredittitel von Banken emittiert, stehen den entsprechenden Verbindlichkeiten in der aggregierten Bilanz des Bankensektors in gleichem Umfang (2) Forderungen der Banken an Nicht-Banken, also vor allem an Produktionsunternehmen gegenüber. Sieht man von dem Prozeß der Fristentransformation ab, handelt es sich für den Bankensektor also lediglich um durchlaufende Kredite zwischen Sparern und Investoren. Diese durchlaufenden Kreditbeziehungen werden hier nicht explizit erfaßt (3). Soweit Forderungsbestände der Banken hier explizit erfaßt werden, stellen sie einen bilanzmäßigen Gegenposten allein zu den unverzinslichen Sichtverbindlichkeiten des Bankensektors

(1) Die Zu- bzw. Abflüsse einer Periode werden zum gerade herrschenden Wechselkurs in inländische Währungseinheiten umgerechnet. Bewertungsprinzip ist also der Anschaffungspreis. Damit gehen die kumulierten Aufwertungsverluste und Abwertungsgewinne auf die im Bankensystem vorhandenen Devisenbestände nicht in die Größe $\mathrm{M}_{\text {Dev , } t}$ ein.

(2) Genau genommen muß es heißen "in annähernd gleichem Umfang". Ein gewisser Prozentsatz des aufgenommenen Finanzkapitals dient der Realvermögensfinanzierung im Bankensektor selbst.

(3) Nur am Rande der Modellformulierung wird im Paragraphen 9 das gesamte Einlagenvolumen der Banken zusammen mit dem Liquiditätsvolumen als Bestimmungsfaktor ihres Geldschöpfungsangebotes erfaßt. 
dar, die im Zuge einer Kreditschöpfung entstanden sind. Damit wird eine klare gedankliche Trennung vollzogen $\mathrm{z}$ wischen der Kreditvermittlung $z$ wischen Sparern und Investoren und der echten Kreditschöpfung, die hier neben dem Umtausch von Devisen als einzige Funktion des Bankensystems ausdrücklich und gesondert berücksichtigt wird.

Dem am Kreditmarkt angelegten Finanzkapital $\mathrm{F}_{\mathrm{t}}$ entsprechen, soweit die aufgenommenen Kredite dem Unternehmenssektor nicht als Kassenkredite dienen, reale Vermögensanlagen, also Produktionsanlagen und Lagerbestände.

Investiert der Unternehmenssektor in Bauten oder Ausrüstungen, so bedarf es einer gewissen Zeit, bis die Anlagen produktionsreif sind. Werden daher die Anlagen durch den Umfang der ausgereiften Produktionskapazität gemessen, dann muß berücksichtigt werden, daß die laufenden Anlageinvestitionen die Produktionskapazität noch nicht in der Periode der Investitionsgüterkäufe, sondern erst später erhöhen. Da für die betrachtete Modellwirtschaft eine Gestationsperiode von einem Jahr anzusetzen ist - wiederum eine Quasiverhaltensannahme wie die Annahme einer nachschüssigen Verzinsung von Forderungstiteln - kann das reale Bruttoanlagevermögen des Unternehmenssektors während einer Periode $t$, $\mathrm{k}_{\mathrm{pr}, \mathrm{t}}$, definiert werden als der entsprechende Bestand der Vorperiode plus dem Zugang an neuen Anlagen aufgrund von Investitionsgüterkäufen

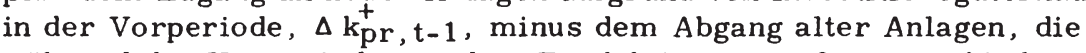
während der Vorperiode aus dem Produktionsprozeß ausgeschieden sind, $\Delta \mathrm{k} \dot{\mathrm{p}} \mathrm{r}, \mathrm{t}-1$ :

$$
\text { (2.66) } \quad k_{p r, t}=k_{p r, t-1}+\Delta k_{p r, t-1}^{+}-\Delta k_{p r, t-1}^{\div}
$$

Dieser Typ einer rekursiven Definition gilt auch für den realen Bestand an Infrastrukturanlagen im staatlichen Sektor, $\mathrm{k}_{\mathrm{St}, \mathrm{t}}$ :

$$
\text { (2.67) } \quad \mathrm{k}_{\text {St, } t}=\mathrm{k}_{\mathrm{St}, t-1}+\Delta \mathrm{k}_{\mathrm{St}, t-1}^{+}-\Delta \mathrm{k}_{\mathrm{St}, t-1}^{\circ}
$$

und er wird auch dann zugrunde gelegt, wenn die um den realen Kapitalverzehr, $d_{p r}, t$, bereinigte Wertgröße des Anlagevermögens, das reale Nettoanlagevermögen des Unternehmenssektors, $k_{p r}, t$, erfaßt werden soll:

$$
k_{p r, t}^{n}=k_{p r, t-1}^{n}+\Delta k_{p r, t-1}^{+}-d_{p r, t-1}
$$

Da Lagerinvestitionen sich sofort bestandserhöhend auswirken, gilt für den realen Lagerbestand:

$$
v_{t}=v_{t-1}+\Delta v_{t}
$$


Da die Produktionsunternehmen der betrachteten Volkswirtschaft die einbehaltenen Gewinne bzw. die Unternehmensersparnis am Kreditmarkt in Forderungstiteln anlegen und diese Titel anschließend an die Selbständigenhaushalte übertragen, befindet sich, wenn man ihre Kassenbestände mit ihren Kassenkrediten saldiert, das gesamte verbleibende positive Geldvermögen außerhalb des Sektors der Produktionsunternehmen (1). Diesem positiven Geldvermögen außerhalb entspricht ein negatives Geldvermögen innerhalb der Produktionsunternehmen, das in deren konsolidierter Bilanz auf der Passivseite verzeichnet ist. Soll die Anlagen- und Vorratsvermögensrechnung der Produktionsunternehmen über eine $\mathrm{Bi}$ lanzgleichung den Anschluß an die gesamtwirtschaftliche Finanzierungsrechnung finden, die von der Seite der Forderungsansprüche her aufgezogen wurde, muß berücksichtigt werden, daß sich in dieser Bilanzgleichung die Bewertungspraxis der Unternehmen niederschlägt: Das von den Produktionsunternehmen aufgenommene Finanzkapital wird auf der Passivseite ihrer konsolidierten Bilanz zum Nennwert angesetzt. Wegen der variablen Verzinsung werden die Forderungstitel auf dem Kreditmarkt ebenfalls stets zum Nennwert gehandelt (2). Das insgesamt aufgenommene Finanzkapital der Produktionsunternehmen entspricht damit dem Nominalwert des aggregierten Titelbestandes $F_{t}^{*}$. - Auf der anderen Seite verfügen die Produktionsunternehmen nicht mehr über die ursprünglich mit der laufenden Kreditaufnahme finanzierten Anlagen und Vorräte. Der größte Teil der Anlagen ist verbraucht und abgeschrieben bzw. längst aus dem Produktionsprozeß ausgeschieden; ebenso sind die Lagerbestände im Lauf der Vergangenheit viele Male umgeschlagen worden. Die Tat-

(1) Die Abgrenzung eines Untersektors "Produktionsunternehmen" darf nicht darüber hinwegtäuschen, daß die Banken, die zur Produktion und zur Einkommensentstehung des Unternehmenssektors beitragen, auch an den kreditmarktfinanzierten Anlagen (z. B. Bauten) dieses Untersektors teilhaben. Die Abgrenzung zwischen Banken und Produktionsunternehmen ist, wie bereits angedeutet, rein rechnerisch-funktional. Sie entspricht einer gesonderten Berücksichtigung des Finanzierungs kontos des Bankensektors in der kontenmäßigen Darstellung der volkswirtschaftlichen Gesamtrechnung; alle übrigen Konten des Bankensektors wären in einer solchen Darstellung in den konsolidierten Konten des gesamten Unternehmenssektors enthalten, und zwar in der Strom- wie in der Bestandsrechnung.

(2) Im Modell brauchen also keine vom Nennwert abweichenden Wertpapierkurse berücksichtigt zu werden. Auch die Gewinnerwartungen der Wirtschaftssubjekte erzwingen eine derartige Berücksichtigung nicht. Die Selbständigen kommen aufgrund der spezifischen im Paragraphen 1 beschriebenen vermögens - bzw. eigentumsrechtlichen Annahmen ohnehin in den Genuß steigender Gewinne. Wollen Arbeitnehmer sich bei günstigen Gewinnerwartungen in die Verteilung der Gewinne einschalten, müssen sie selbständig werden, um die erwarteten Gewinnchancen realisieren zu können. 
sache, daß an die Stelle alter Anlagen und Vorräte laufend neue getreten sind und noch treten, die in der Regel nur zu höheren Preisen als die ursprünglichen beschafft werden können, veranlaßt die Produktionsunternehmen zu einer Bewertung der vorhandenen Güterbestände zu Wiederbeschaffungspreisen. Diese Bewertungspraxis verursacht, daß in der konsolidierten Bilanz der Produktionsunternehmen die Summe der so bewerteten Vermögensanlagen und der Kassenbestände auf der Aktivseite das zum Nennwert angesetzte aufgenommene Finanzkapital auf der Passivseite übersteigt, und zwingt die Unternehmen, zur Wahrung der Bilanzgleichheit Wertberichtigungsposten in Form von Substanzerhaltungsrücklagen $\mathrm{zu}$ veranschlagen.

Da die Vorratsbestände der Produktionsunternehmen in der Modellwirtschaft keinerlei reale Wertminderung erfahren und nicht abgeschrieben werden, lautet die Bilanzgleichung für den Anschluß der Anlagen- und Vorratsvermögensrechnung des Sektors Produktionsunternehmen an die bestandsmäßig aufgestellte gesamtwirtschaftliche Finanzierungsrechnung nach den bisherigen Definitionen:

$$
M_{U, t}+v_{t} \cdot p_{v, t}+k_{p r, t+1}^{n} \cdot p_{k, t}=F_{t}^{+}+E_{t}
$$

wobei $E_{t}$ das Symbol für die bis zum Ende der Periode $t$ aufgelaufene aggregierte Substanzerhaltungsrücklage der Produktionsunternehmen ist.

Als letztes sind die Nominalvermögensbestände zu berücksichtigen, anhand derer die Vermögensverteilung innerhalb der Modellwirtschaft dargestellt wird: Das bis zum Ende einer Periode $t$ gebildete Nominalvermögen der Arbeitnehmerhaushalte, $W_{A, t}$, setzt sich zusammen aus dem Kassenbestand und dem Bestand an Forderungstiteln der Arbeitnehmerhaushalte:

$$
\text { (2.71) } \quad W_{A, t}=M_{A, t}+F_{A, t}
$$

Ähnliches gilt für das Nominalvermögen der Selbständigenhaushalte, $\mathrm{W}_{\mathrm{S}, \mathrm{t}}$ :

$$
\text { (2.72) } \quad w_{S, t}=M_{S, t}+F_{S, t}
$$

Um das Nominalvermögen des Staates, $W_{S t, t}$, darzustellen, muß noch einmal auf eine rekursive Definition zurückgegriffen werden. Es gilt:

$$
\text { (2.73) } \begin{aligned}
W_{S t, t}= & w_{S t, t-1}+\Delta F_{S t, I n l, t}+\Delta F_{S t, A u s l, t} \\
& +I_{S t, t}^{b r}+I_{S t, \text { sonst }, t}^{b r}
\end{aligned}
$$

Der Staat besitzt im Gegensatz zu den privaten Haushalten also Geldvermögen und Sachvermögen. Ersteres ist in inländischen und ausländischen 
Forderungstiteln angelegt, letzteres besteht aus den öffentlichen Infrastrukturanlagen und dem sonstigen Anlagevermögen.

Das Vermögen des Unternehmenssektors, der sich in einer Nettoschuldnerposition befindet, besteht ausschließlich aus Sachvermögen und Auslandsforderungen: Seine in Forderungstiteln angelegte Ersparnis hat er den Selbständigenhaushalten übertragen. Die im Zuge der Kreditschöpfung aufgebauten Forderungsbestände des Untersektors "Banken" gleichen sich mit Verbindlichkeiten der Produktionsunternehmen aus; etwas Ähnliches gilt für die Kassenbestände der Produktionsunternehmen, denen entsprechende Verbindlichkeiten der Banken gegenüberstehen. Macht man für den gesamten Unternehmenssektor die um die internen Kreditbeziehungen bereinigte konsolidierte Bilanz auf, so finden sich auf deren Aktivseite allein die zu Wiederbeschaffungspreisen bewerteten Anlagen und Vorräte sowie der Devisenbestand, auf der Passivseite das durch Emmission von Titeln bei den privaten und öffentlichen Haushalten und im Ausland (1) aufgenommene und zum Nennwert angesetzte Finanzkapital, Verbindlichkeiten in Höhe der von den privaten Haushalten (2) gehaltenen Kassenbestände sowie die aggregierte Substanzerhaltungsrücklage. Die aggregierte Substanzerhaltungsrücklage enthält nun auch alle durch Wechselkursänderungen bedingten Wertberichtigungen auf den Devisenbestand der Notenbank, die im Unternehmenssektor erfaßt ist. Nur insoweit die Aktiva nicht durch Nettoansprüche der übrigen Sektoren auf der Passivseite abgedeckt sind, also in Höhe der Substanzerhaltungsrücklage, wird dem Unternehmenssektor ein Nominalvermögen zugerechnet. Da die Aufwertungsverluste bzw. Abwertungsgewinne der Notenbank in der Modellbetrachtung vernachlässigt werden und die Untersuchung allein an der Vermögensrechnung des privaten Sektors interessiert ist, wird dem (privaten) Unternehmenssektor als Nominalvermögen, $W_{U, t}$, lediglich die Substanzerhaltungsrücklage der Produktionsunternehmen zugerechnet:

$$
(2.74) \quad W_{U, t}=E_{t}
$$

die in letzter eigentumsrechtlicher Konsequenz allerdings den Selbständigen-Haushalten gehört.

(1) Im Fall des Staates oder des Auslandes wird das aufgenommene Finanzkapital als eine Nettogröße angesehen, die ein negatives Vorzeichen haben kann.

(2) Annahmegemäß halten der Staat und die übrige Welt keine Bestände an Inlandswährung. 
4. Abschließende Bemerkung zu den bisherigen Abgrenzungen und Definitionen

Das hiermit fertiggestellte definitorische Gerüst der Modellwirtschaft ist so aufgebaut worden, daß sich mit seiner Hilfe das Wirtschaftssystem der Bundesrepublik in seinen wichtigsten wirtschaftlichen Grundtatbeständen abbilden läßt. In einem wesentlichen Bereich, dem der Finanzierungsund Vermögensrechnung, weist das Modell jedoch schon von den definitorischen Annahmen her Eigenschaften auf, die sich mit den empirischen Verhältnissen der Bundesrepublik nicht decken bzw. diese nur unzulänglich wiedergeben. Diese Eigenschaften bedürfen nachträglich einer gewissen Rechtfertigung.

Als erstes bleibt festzuhalten, daß die genannten definitorischen Modelleigenschaften, die sich auf die Finanzierung und Vermögensverteilung beziehen, in dem vorliegenden Modell unvermeidbar sind. Es wird hier mit einfachen Mitteln der Versuch unternommen, die gesamtwirtschaftliche Finanzierungsrechnung in ein makroökonomisches Verteilungsmodell einzubeziehen und dieses Modell auf die Strom- und Bestandsrechnung der Bundesrepublik abzustimmen. Wäre bei diesem Versuch eine größere Annäherung an die Wirklichkeit angestrebt worden, hätten in dem Modell mehrere Finanzierungsinstrumente bzw. mehrere Arten einer Geldvermögensanlage und bewegliche Wertpapierkurse berücksichtigt werden müssen, und hinsichtlich dieser Tatbestände hätten später Annahmen über das Finanzierungsverhalten der Unternehmen und über das Anlageverhalten der Geldvermögensbesitzer aufgestellt werden müssen, was alles mit zusätzlichem hohen Zeitaufwand und weiterer Komplikation des Modells verbunden gewesen wäre. Unter diesem Gesichtspunkt sind die von den empirischen Verhältnissen der Bundesrepublik abweichenden Annahmen - Konstruktion eines von der Unternehmensfinanzierung losgelösten Eigentums - bzw. Verfügungsrechtes an den Unternehmen; Abwicklung des gesamtwirtschaftlichen Finanzierungsprozesses über eine einzige Art rein schuldrechtlicher Geldvermögenstitel; eigentumsmäßige Zurechnung des gesamten Unternehmenssektors zu den SelbständigenHaushalten - der Preis für eine einfache Darstellung. Jede andere einfache Darstellungsweise hätte ähnlich große Probleme verursacht (1).

(1) Zum Beispiel wären bei einer gesonderten Berücksichtigung der Kapitalgesellschaften und bei einem Verzicht auf Zurechnung ihrer einbehaltenen und thesaurierten Gewinne zu den privaten Haushalten die Fragen entstanden, inwieweit sich die Erhöhung des inneren Unternehmenswertes durch Selbstfinanzierung in den Aktienkursen und damit doch wieder in der Vermögensrechnung der privaten Haushalte niederschlägt, und in Zusammenhang damit wäre wiederum die Frage aufgetaucht, wie sich der bestehende und laufend neu gebildete Aktienbesitz auf die Haushaltsgruppen verteilt. 
Zweitens ist nicht beabsichtigt, mit der vorliegenden Untersuchung ein ökonometrisches Totalmodell mit möglichst hohem statistischen Erklärungswert für das Wirtschaftssystem der Bundesrepublik zu liefern. Die Zielrichtung ist primär theoretischer Natur; eine Orientierung an den empirischen Verhältnissen dient vor allem zur Absicherung der zentralen Verhaltensannahmen, einer Aufgabe, die im Laufe der Föhlschen Überwälzungsdiskussion zu kurz gekommen ist. Insoweit stört es im Prinzip nicht, wenn der aus dem Modell resultierende Wirtschaftsablauf in Bezug auf konjunkturelle Schwankungen und Trendentwicklungen von den tatsächlichen Verhältnissen der Bundesrepublik abweicht; insoweit stören auch gewisse Abweichungen in der definitorischen Struktur der volkswirtschaftlichen Gesamtrechnung im Prinzip nicht, solange die verfochtenen zentralen Aussagen dadurch in ihrem theoretischen und empirischen Gehalt nicht wesentlich beeinträchtigt werden.

Von großer Bedeutung ist schließlich ein Argument, das sich aus den für die Untersuchung angestellten empirischen Beobachtungen ergibt: Was in der Untersuchung als Vermögen der Sektoren Arbeitnehmerbzw. Selbständigen-Haushalte und Staat bezeichnet wird $\left(W_{A, t}, W_{S, t}\right.$, $\left.W_{S t, t}\right)$, ist die laufende bzw. zeitlich aggregierte Nominalvermögensbildung dieser Sektoren im Sinne der volkswirtschaftlichen Gesamtrechnung. In diesem Sinn ist das Vermögensaggregat für den Sektor der Arbeitnehmerhaushalte, auf den es verteilungspolitisch vor allem ankommt, definitorisch korrekt abgegrenzt und läßt sich durch Wahl eines geeigneten Ausgangswertes und einer geeigneten Annahme über die Vermögensübertragungen quantitativ abschätzen. Das so definierte und ermittelte Vermögensaggregat für den Sektor "Arbeitnehmerhaushalte" eignet sich, wie noch gezeigt wird, ganz gut zur Schätzung der Vermögenseinkommen der Arbeitnehmer. Damit leistet ein sonst begrenzt aussagekräftiges Konzept der Vermögenszurechnung dem eigentlichen Ziel der Untersuchung, der Erklärung der Einkommensverteilung, einen wertvollen Dienst und erfährt allein schon von dorther eine ausreichende Rechtfertigung. 


\section{Kapitel}

\section{DAS VERHAL TEN DER PRIVATEN WIRTSCHAFTSSUBJEKTE}

$\$ 3$ Entwicklung und Struktur der Erwerbsbevölkerung

\section{Die Entwicklung}

Die langfristige Entwicklung der Erwerbsbevölkerung folgt innerhalb des Modells einem exogen vorgegebenen zeitlichen Trend, der durch die natürliche Bevölkerungsentwicklung bestimmt ist. Der Trend steigt mit sinkenden prozentualen Zuwachsraten gegen ein stationäres. Niveau an (1).

Kurzfristig reagiert die Erwerbsbevölkerung bzw. das Vollbeschäftigungsarbeitsangebot auf die Arbeitsmarktlage. Steigt im konjunkturellen Auf schwung die Arbeitsnachfrage über das mittelfristige Niveau hinaus, dann sinkt die Zahl der angemeldeten Arbeitslosen, ebenso steigt die Zahl der ausgewiesenen offenen Stellen, und die Lohnsteigerungsraten werden mit einer gewissen zeitlichen Verzögerung höher. Diese Ereignisse locken Arbeitskräfte aus versteckter Arbeitslosigkeit heraus (Landwirtschaft) und machen sonstige Teile der Wohnbevölkerung (Hausfrauen) zu einem zusätzlichen Arbeitsangebot bereit. - Umgekehrt verläuft die Reaktion bei rückläufiger Konjunktur.

Erfaßt man die kurzfristige Arbeitsmarktlage durch den Nominallohnanstieg im privaten Sektor, so lassen sich die lang- und kurzfristigen Reaktionen des Vollbeschäftigungsarbeitsangebots durch Funktion (3.1) beschreiben:

$$
\frac{e_{v, t}}{e_{v, t-1}}=\varepsilon_{1} \cdot \varepsilon_{2}^{\frac{1}{t-1950}} \cdot\left[\frac{1 p r, t}{1 p r, t-1}\right] \varepsilon_{3} \cdot\left[\frac{p_{x, t-1}}{p_{x, t-2}}\right] \varepsilon_{4}
$$

Nach (3.1) ist die Änderungsrate der Erwerbsbevölkerung bestimmt durch die zeitliche Trendvariable $t$, die Lohnsteigerungsrate des privaten Sektors und, zur Ausschaltung von Geldillusion, durch den Preisanstieg des privaten Bruttoinlandsprodukts in der Vorperiode.

Die Parameterschätzung erfolgt nach einer logarithmischen Transformation aufgrund von Jahresdaten für die Bundesrepublik Deutschland (1960 -

(1) Vgl. dagegen W. Krelle, D. Beckerhoff, H. G. Langner und H. Fuß, Ein Prognosesystem für die wirtschaftliche Entwicklung der Bundesrepublik Deutschland, Meisenheim a. Gl. 1969, S. 314, Gleichung (12.3.2), wo die Erwerbsbevölkerung als ständig steigend angenommen wird. 
1970) und mit Hilfe der Methode der kleinsten Quadrate. Die Schätzung ergab folgende Parameterwerte (1):

$$
\text { (3.2) } \begin{aligned}
& \lg \varepsilon_{1}=-0,00947( \pm 0,00417) \\
& \lg \varepsilon_{2}=+0,06068( \pm 0,04336) \\
& \varepsilon_{3}=+0,23767 \quad( \pm 0,05696) \\
& \varepsilon_{4}=-0,14709 \quad( \pm 0,11081) \\
& R_{1 \cdot 2,3,4}^{2}=0,70962 \quad \text { (Irrt.-Wahrsch.:<2\%) } \\
& R_{2 \cdot 3,4}^{2}=0,07011 \\
& R_{3 \cdot 2,4}^{2}=0,03823 \\
& R_{4 \cdot 2,3}^{2}=0,08785 \\
& v N Q
\end{aligned}
$$

Zwischen dem theoretischen Fundament von Funktion (3.1) und den zur Schätzung herangezogenen empirischen Verhältnissen der Bundesrepublik besteht ein gravierender Unterschied: Die kurz - und langfristige Entwicklung der Erwerbsbevölkerung im Modell ist stark geprägt durch den Zustrom von Arbeitskräften aus inländischen Arbeitskräftereservoirs. In der Bundesrepublik fließt dieser Strom aus dem Ausland, was hier vernachlässigt wird. Der Gastarbeiterzustrom macht den altersstrukturbedingten Rückgang der heimischen Erwerbsbevölkerung wett und verleiht dem Vollbeschäftigungsarbeitsangebot eine hohe konjunkturelle Reagibilität. Es kann bezweifelt werden, daß diese Konjunkturreagibilität des Gesamtarbeitsangebotes in der Bundesrepublik rein angebots bedingt ist. Vorstellbar ist, daß die Erscheinung auf Reaktionen der Arbeitsnachfrage zurückzuführen ist, die im Boom verstärkt auf ein von den inländischen Lohnsteigerungsraten unabhängiges ausländisches Arbeitsangebot zurückgreift. Insofern wäre Funktion (3.1), die die Konjunkturreagibilität mit dem Einfluß des Nominallohnanstiegs erfaßt, ökonometrisch auf jeden Fall falsch spezifiziert - ein möglicher Mangel, der hier nicht weiter stören soll.

(1) Die Variablen von (3.1) sind in ihrer Reihenfolge durchnummeriert. Die abhängige Variable ist Variable No. 1 und so fort. Der multiple Korrelationskoeffizient $R_{i}$. jk. . bezieht sich auf die Korrelation der Variablen i gegenüber den Variablen j, k und so fort. - Das Symbol vNQ bezeichnet den Wert des von-Neumann-Quotienten, der hier als Testgröße auf Autokorrelation dient. 


\section{Die Struktur}

Die Erwerbsbevölkerung ist in dem Modell einer ständigen Umstrukturierung $\mathrm{z}$ wischen den beiden Gruppen der selbständigen und unselbständigen Erwerbspersonen ausgesetzt. Ständig geben Selbständige ihre Selbständigkeit auf, verkaufen oder liquidieren ihre Unternehmen und nehmen eine unselbständige Beschäftigung an. Dieser Umstrukturierungsprozeß folgt langfristig ähnlich wie die absolute Bevölkerungsentwicklung einem exogen vorgegebenen Trend, wird in konjunkturellen Rezessicnsphasen aber hinausgezögert, da die marginalen Selbständigen bei einem Erwerbswechsel dann mit dem Risiko der Arbeitslosigkeit rechnen müssen, und beschleunigt sich in Boomphasen, in denen sich von den Einkommensrelationen her der Anreiz zu einem Erwerbswechsel verstärkt (1) und dieser sich auch reibungslos vollzieht.

Bezieht man die Zahl der Selbständigen auf die Gesamtheit aller Erwerbs personen, so erhält man die Selbständigenquote $s_{t} / e_{v}, t$, anhand derer sich der Umstrukturierungsprozeß am besten veranschaulichen läßt. Für die Entwicklung dieser Selbständigenquote gilt die Verhaltensfunktion (3. 1) (2):

$$
\left.\frac{s_{t}}{e_{v, t}}=\varepsilon_{1}^{\prime} \cdot \varepsilon_{2}^{\prime}\right]^{t-1960} \cdot\left[\frac{e_{v, t}-e_{t}}{a_{t}+\left(e_{v, t}-e_{t}\right)}\right]^{\varepsilon_{3}^{\prime}} \cdot\left[\frac{e_{p r, t-1} \cdot{ }_{p r, t-1}}{x_{t-1} \cdot p_{x, t-1}}\right]^{\varepsilon_{\dot{4}}^{\prime}}
$$

Die Funktion macht die Selbständigenquote also abhängig von einem zeitlichen Trend, von der Arbeitslosenquote der laufenden Periode und von dem Anteil der Arbeitseinkommen an der Bruttowertschöpfung des privaten Sektors in der vergangenen Periode. Die Parameterschätzung erfolgte wie oben nach logarithmischer Transformation und aufgrund der Jahresdaten für die Bundesrepublik (1960 - 1970) und ergab folgende Parameterwerte:

(1) Es ist hier vor allem an die Bedeutung der Einkommensdisparität zwischen dem landwirtschaftlichen und dem industriellen Sektor für die Abwanderung selbständiger Landwirte in die Industrie zu denken.

(2) W. Krelle und Mitarbeiter machen die Entwicklung der Selbständigenquote bzw. ihrer Gegengröße, der Unselbständigenquote, allein von der Entwicklung der Kapitalintensität abhängig. Vgl. a. a. O. , S. $251 \mathrm{f}$. 


$$
\text { (3.4) } \begin{array}{rll}
\text { lg } \varepsilon_{1}^{1}=-0,73196 & (\text { signifikant })^{1)} \\
\lg \varepsilon_{2}^{1}=-0,01087 & ( \pm 0,00021) \\
\varepsilon_{3}^{1}=+0,02843 & ( \pm 0,00469) \\
\varepsilon_{4}^{1} & =-0,58988 \quad( \pm 0,13327) \\
\mathrm{R}_{1 \cdot 2,3,4}^{2} & =0,99715 \quad \text { (Irrt.-Wahrsch.:<0,1\%) } \\
\mathrm{R}_{2 \cdot 3,4}^{2}=0,06487 \\
\mathrm{R}_{3 \cdot 2,4}^{2}=0,01071 \\
\mathrm{R}_{4}^{2} \cdot 2,3=0,07284 \\
\mathrm{vNQ}=1,673
\end{array}
$$

Nach bisherigen Untersuchungen (2) scheint die Änderung der Erwerbsstruktur einen Einfluß auf die Entwicklung der Einkommensverteilung in der Bundesrepublik zu haben. Fällt den Selbständigen im Laufe des Wirtschaftsprozesses neben dem Besitzeinkommen ein spezifischer Unternehmerlohn zu, so wird dieser in der volkswirtschaftlichen Gesamtrechnung innerhalb der Einkommen aus Unternehmertätigkeit und Vermögen erfaßt. Geben Selbständige die Selbständigkeit zugunsten einer unselbständigen Beschäftigung auf, tritt an die Stelle des Unternehmerlohns Einkommen aus unselbständiger Arbeit. Die Verschiebung der Erwerbsstruktur verursacht dann eine Verschiebung der gesamtwirtschaftlichen Einkommensverteilungsquoten in der volkswirtschaftlichen Gesamtrechnung; sie führt zu einer Erhöhung der "Lohn"-quote, die sich bei Fehlen kompensierender Effekte statistisch als entsprechende Trendbewegung bemerkbar macht.

(i) In einer ganzen Reihe von Fällen wurden die Regressionsschätzungen wegen vermuteter Singularität der Kovarianzmatrix und dadurch ver ursachter Berechnungsfehler mit transformierten Werten der Variablen (Abweichungen vom Durchschnitt) wiederholt. In diesen Fällen wurde der Schätzwert des Absolutgliedes nachberechnet; die Signifikanzaussage konnte den ursprünglichen Berechnungen entnommen werden und bezieht sich jeweils auf eine Irrtumswahrscheinlichkeit von $5 \%$.

(2) Vgl. A. Jeck, The Trends of Income Distribution in West-Germany, in: J. Marshal und B. Ducros (Hrsg.), The Distribution of National Income, London u. a. 1968 , S. 78 ff. Der Gedanke wird weiterverfolgt in: ders., Wachstum und Verteilung des Volkseinkommens, Tübingen 1970 . 
Der Einfluß der Erwerbsstrukturänderungen ist nicht der einzige denkbare Bestimmungsfaktor für die langfristige Entwicklung der Arbeitnehmerlohnquote in der Bundesrepublik. Wahrscheinlich handelt es sich aber um den wichtigsten Bestimmungsfaktor. Dies ist zumindest die hier akzeptierte Hypothese. Insoweit ist es angebracht, darauf hinzuweisen, daß mit der Quantifizierung von (3.3) bereits eine erste Entscheidung über die langfristige Verteilungsentwicklung in der Modellwirtschaft getroffen wird. Gäbe es in der Modellwirtschaft keine anderen Faktoren, die eine längerfristige Verschiebung $\mathrm{z}$ wischen den Einkommen aus unselbständiger Arbeit und den Einkommen aus Unternehmertätigkeit und Vermögen verursachten, so wäre damit zu rechnen, daß die Arbeitnehmerlohnquote asymptotisch gegen ein im Vergleich zum Ausgangsstand höheres stationäres Niveau anstiege. 
1. Vorbemerkung

Innerhalb des Modells werden zwei Aspekte des privatwirtschaftlichen Produktionsprozesses unterschieden: Lang-oder mittelfristig planen die Unternehmen unter Wirtschaftlichkeitsgesichtspunkten die optimale Anlagenkapazität; sie entfalten eine entsprechende Investitionsgüternachfrage und verfügen nach Ablauf der Gestationsperiode über entsprechende Produktionsmöglichkeiten. Sind die erstellten Anlagen voll ausgereift und weicht die Güternachfrage vom erwarteten Umfang in der einen oder anderen Richtung ab, stellt sich kurzfristig (1) den Unternehmen das Problem der Kapazitätsauslastung, und auch dieses Anpassungsproblem wird, soweit möglich, unter Wirtschaftlichkeitsgesichtspunkten gelöst. - Beide Aspekte der Produktionssteuerung lassen sich neoklassich und anhand einer einzigen Produktionsfunktion übersichtlich darstellen, sofern im Sinne von H. Uzawa (2) von der Möglichkeit Gebrauch gemacht wird, die Faktorinputs in bestimmter Gruppierung zu erfassen und mit Hilfe dieser Gruppierung einen langfristigen und einen kurzfristigen Term der Produktionsfunktion zu unterscheiden.

Der nun folgende Paragraph befaßt sich allein mit dem Problem der Kapazitätsvorausplanung oder, technisch gesprochen, mit dem langfristigen Term der Produktionsfunktion. Die Überlegungen verlaufen in rein neoklassischen Bahnen. Es wird davon ausgegangen, daß die Güter - und Faktormärkte der Modellwirtschaft monopolistisch strukturiert sind, daß aber die strukturellen Wettbewerbsverhältnisse stabil sind und daher für die Unternehmen im Durchschnitt der Gesamtwirtschaft mit konstanten Preis-Bezugs- und Preis-Absatz-Elastizitäten gerechnet werden kann (3).

(1) Die Grenze zwischen langer bzw. mittlerer Frist und kurzer Frist ist also bestimmt durch die Länge der Gestationsperiode, die hier mit einem Jahr angesetzt wird.

(2) Vgl. H. Uzawa, Production Functions with Constant Elasticities of Substitution, Review of Economic Studies, XXIV, 1962, S. $294 \mathrm{ff}$.

(3) Die Annahme findet sich auch bei W. Krelle, Verteilungs theorie, Tübingen 1962, S. 137 
2. Die Grundannahmen der langfristigen Produktionsund Faktornachfrage theorie

Wenn die Unternehmen der Modellwirtschaft die Produktionskapazität für eine bestimmte Zukunftsperiode $t$ vorausplanen, dann liegen ihrer Planung bestimmte Erwartungen hinsichtlich der zukünftigen Güternachfrage bzw. der zukünftigen Absatzchancen zugrunde, an die sie sich mit ihren Kapazitätsplänen anzupassen versuchen. Hinzu kommen bestimmte, nach aller Erfahrung zutreffende Vorstellungen über den Stand und die Art der Produktionstechnik, die sich in einer Produktionsfunktion formulieren lassen. Nach diesen Vorstellungen erfordert die vorgesehene Produktion, $x_{0}, t$, bei dem für die Periode $t$ erwarteten $B e-$ stand an öffentlichen Infrastrukturanlagen, $k_{o}, S t, t$, und dem dann herrschenden technischen Effizienzniveau einen bestimmten Arbeitseinsatz (1), $\mathbf{e}_{\mathrm{o}, \mathrm{pr}, t}$, und einen bestimmten realen Anlagenbestand (2), $\mathrm{k}_{\mathrm{o}, \mathrm{pr}, \mathrm{t}}$. Die den Vorstellungen zugrunde liegende Produktionsfunktion ist linearhomogen; sie zeichnet sich durch einen mit konstanter Rate $f_{a}$ verlaufenden autonomen und Harrod-neutralen technischen Fortschritt sowie durch konstante partielle Substitutionselastizitäten aus. Da die Unternehmen aus Erfahrung wissen, daß die Substitutionselastizität zwischen dem staatlichen Infrastrukturkapitalbestand einerseits und den beiden anderen in konstanter Relation eingesetzten Faktoren andererseits kleiner ist als die partielle Substitutionselastizität zwischen eingesetzter Arbeit und den privaten Anlagenbestand, hat die Funktion die Gestalt einer asymetrisch geschachtelten CES-Funktion (3).

(4.1) $x_{0, t}=\left[a_{1} \cdot\left(a_{2} \cdot\left\{a_{3} \cdot e_{0, p r, t} \cdot\left\langle 1+r_{a}\right\rangle^{t-1960}\right\}^{-a_{4}}\right.\right.$

$$
\left.\left.+\left\{1-a_{2}\right\} \cdot k_{0, p r, t}^{-a_{4}}\right)^{\frac{a_{5}}{a_{4}}}+\left(1-a_{1}\right) k_{0, s t, t}^{-a_{5}}\right]^{-\frac{1}{a_{5}}}
$$

In dieser Funktion sind $\alpha_{1}$ der Verteilungsparameter der äußeren Funktionsschachtel, $\alpha 2$ der Verteilungsparameter der inneren Funktionsschachtel, $\alpha_{3}$ ein Effizienzparameter $\alpha_{4}$ und $a_{5}$ die Substitutionsparameter und tie Zeitvariable.

(1) Hierbei wird der Arbeitseinsatz der Arbeitnehmer und Selbständigen zusammengenommen betrachtet.

(2) Der Index o kennzeichnet Erwartungs - oder Planwerte im Kalkül der Unternehmen.

(3) Vgl. W. Scheper, Produktionsfunktionen mit konstanten Substitutionselastizitäten, Jahrbücher für Nationalökonomie und Statistik, 177 , i 965 , S. $1 \mathrm{ff}$. 
Zur Erleichterung der Interpretation wird die Funktion umgeformt zu:

$$
\begin{aligned}
x_{0, t}= & {\left[a_{2} \cdot\left(a_{3} \cdot e_{0, p r, t} \cdot\left\{1+r_{a}\right\}^{t-1960}\right)^{-a_{4}}+\left(1-a_{2}\right) \cdot\right.} \\
& \left.k_{0, p r, t}^{-a_{4}}\right]^{-\frac{1}{a_{4}}} \cdot k_{0, s t, t}^{*}
\end{aligned}
$$

mit (1)

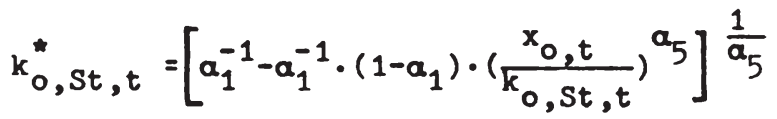

Könnten die Unternehmen davon ausgehen, daß der Staat langfristig öffentliche Infrastrukturanlagen stets in einer konstanten Relation zur privaten Produktion zur Verfügung stellte, reduzierte sich die verschachtelte Produktionsfunktion (4.1) also zu einer einfachen CES-Funktion, die durch einen konstanten Faktor $k_{O}^{\star}, S t, t$ modifiziert wäre.

Bei der Planung der künftigen Produktionskapazität versuchen die Unternehmen, den erwarteten Zukunftsgewinn der investierten Anlagen zu maximieren. Dieses Wirtschaftslichkeitskalkül wird zumeist auf dem Wege der Abdiskontierung der erwarteten Zukunftserträge und Zukunfts kosten auf den Investitionszeitpunkt angestellt. In der hier angewendeten neoklassischen Theorie findet es seinen Ausdruck zeitlich verkürzt durch den Vergleich der marginalen Faktorerträge und marginalen Faktorkosten, mit denen im Investitionszeitpunkt für eine einzige angesteuerte Zukunftsperiode $t$ gerechnet wird. Die Unternehmen stellen also für die Periode $t$ geltende Erwartungswerte von Absatz, Güter- und Faktorpreisen auf und berechnen auf der Basis dieser Erwartungswerte und mit Hilfe der als konstant angenommenen strukturellen Preis-Bezugsund Preis-Absatz-Elastizitäten die erwarteten Grenzerlösprodukte der

(1) Gleichung (4.3) ist von einiger Bedeutung für die Art, in der im weiteren der Einfluß der staatlichen Infrastrukturanlagen auf die Faktorplanung sowie auf Produktion und Beschäftigung des privaten Sektors erfaßt wird. Als erstes sei hier zur Ergänzung angeführt, daß die primären Zukunftserwartungen der Unternehmen sich in der Modellwirtschaft auf die Größe von $\mathrm{k}_{\mathrm{O}}^{\star}$, St, $\mathrm{t}$ beziehen, also auf den Einfluß, der von den staatlichen Infrastrukturanlagen auf privaten Produktionsmöglichkeiten ausgeht. Der von den Unternehmen erwartete Infrastrukturanlagenbestand selbst, $\mathrm{k}_{\mathrm{O}}, \mathrm{St}, \mathrm{t}$, ist eine aus $\mathrm{k}_{\mathrm{O}}^{\star}, \mathrm{St}, \mathrm{t}$ über (4.3) abgeleitete sekundäre Erwartungsgröße. Vgl. $(4.19),(4.24),(4.29)$ und (4.36) sowie die aus ihnen abgeleiteten Gleichungen (4.52) und (4.53) samt den dazu gehörenden Bemerkungen. 
Faktoren und die erwarteten Grenzfaktorausgaben, und sie planen die Faktorbestände, bei denen die so errechneten Grenzerlösprodukte und Grenzfaktorausgaben einander gleich sind (1).

Wendet man die Überlegungen der statischen Grenzproduktivitätstheorie auf Produktionsfunktion (4.1) an und berücksichtigt hierbei die Konstanz der strukturellen Preis-Bezugs - und Preis-Absatz-Elastizitäten, erhält man zwei Funktionen für die Faktoreinsatzplanung, die in folgender Weise formuliert werden können:

$$
\begin{aligned}
& \text { (4.4) } \quad k_{o, p r, t}=\left[\mu_{k} \cdot a_{1} \cdot\left(1-a_{2}\right)\right]^{\frac{1}{1+a_{4}}} \cdot r_{0, t}^{*}-\frac{1}{1+a_{4}} . \\
& -\frac{a_{4}-a_{5}}{1+a_{4}} \\
& e_{o, p r, t}=\left[\mu_{a} \cdot a_{1} \alpha_{2} a_{3}^{-\alpha_{4}}\right]^{\frac{1}{1+a_{4}}} \cdot\left[\frac{1_{0, p r, t}^{*}}{p_{0, x, t}}\right]^{-\frac{1}{1+a_{4}}} . \\
& \begin{aligned}
-\frac{\alpha_{4}}{1+\alpha_{4}}(t-1960) & -\frac{\alpha_{4}-\alpha_{5}}{1+\alpha_{4}} \\
{\left[1+r_{a}\right.} & \cdot k_{0, S t, t}^{*} \cdot x_{0, t}
\end{aligned}
\end{aligned}
$$

Hierbei ist $\mathbf{r}_{o, t}^{\star} t$ der erwartete reale Kapitalkostensatz pro Anlageneinheit, $1_{o}^{*}, p r, t$ der erwartete nominelle Arbeitskostensatz und $p_{0, x, t}$ der erwartete Güterpreis. $k_{0}^{*}$. St, $t$ ist wieder entsprechend (4.3) definiert. $\mu_{\mathrm{k}}$ und $\mu_{\mathrm{a}}$ sind als konstant angenommene Monopolgradfaktoren (2), an

(1) Der Einbau der Erwartungen bringt ein typisch keynesianisches Element in die neoklassische Theorie des Unternehmensverhaltens. Vgl. J. M. Keynes, A General Theory of Employment, Interest and Money, London 1936, Kapitel 5 und 12.

(2) Die beiden Monopolgradfaktoren lassen sich als mit den Faktorlohnsummen gewogene Durchschnitte der einzelwirtschaftlichen AmorosoRobinson-Quotienten vorstellen. In Wirklichkeit ist der Faktor $\mu_{k}$ noch etwas komplexer zusammengesetzt, da hier die Verhältnisse auf dem Absatzmarkt, dem Markt für Kapitalgüter wie auf dem Kreditmarkt von Bedeutung sind. 
die sich die Unternehmen im Laufe der Zeit angepaßt haben.

Die Umformung dieser Funktionen zu entsprechenden Funktionen für die Faktorkosten - und Monopolprofitanteile zeigt, daß die aus dem geplanten Einsatz der Faktoren Arbeit und privater Anlagenbestand entstehenden Kostensummen zuzüglich Monopolprofit den veranschlagten Produktionserlös nicht ausschöpfen. Da die private Produktion sich auf die öffentlichen Infrastrukturanlagen als dritten Produktionsfaktor stützt, dieser vom Staat aber kostenlos zur Verfügung gestellt wird, verbleibt dem Unternehmenssektor in der Vorauskalkulation ein zusätzlicher Profit in Höhe des nicht vergüteten Grenzbeitrags der Infrastrukturanlagen zum privaten Produktionserlös (1).

Von den anderen bekannten neoklassischen Eigenschaften der Faktorplanungsfunktionen sei hier nur auf eine abgestellt, die in mehrfacher Hinsicht von Bedeutung ist:

Der Exponent des Kapital- bzw. Arbeitskostensatzes in (4.4) bzw. (4.5), $-1 /\left(1+a_{4}\right)$, entspricht der partiellen Substitutionselastizität zwischen geplantem Arbeitseinsatz und geplantem privaten Anlagenbestand. In der Modellwirtschaft ist diese Elastizität absolut genommen größer als Null. Nach (4.4) reagiert der private Anlagenbestand daher auf Änderungen des erwarteten Kapitalkostensatzes; mit anderen Worten, die geplanten privaten Anlageinvestitionen sind grundsätzlich zinselastisch (2). Mit dieser Eigenschaft des Investitionsverhaltens setzt sich das Modell a priori deutlich von der üblichen Annahme post-keynesianischer Verteilungsmodelle ab, die die Investitionstätigkeit allein vom Nachfrageniveau abhängig machen und eine annähernde Konstanz der Investitionsquote am Volkseinkommen behaupten. Die Annahme wird jedoch später wegen ihrer großen inzidenz-theoretischen Bedeutung noch empirisch überprüft.

Die Größe der Substitutionselastizität hat natürlich im Zusammenhang mit der Anwendung der statischen Grenzproduktivitäts theorie auf das Faktoreinsatzverhalten der Unternehmen verteilungstheoretische Konsequenzen. Je nachdem, ob der Wert der Elastizität absolut genommen kleiner, gleich oder größer eins ist, machen die kalkulierten Arbeitskosten im Laufe des Wachstumsprozesses und vor Erreichen eines langfristigen Gleichgewichtspfades einen steigenden, konstanten oder sinkenden Anteil an der vom Unternehmenssektor veranschlagten Faktor kostensumme aus. Dies ist nichts anderes als das bekannte, aus der Anwendung der Grenzproduktivitätstheorie resultierende Verteilungs -

(1) Vgl. E. Streißler, Long Term Structural Changes in the Distribution of Income, Zeitschrift für Nationalökonomie, 29, 1969, S. $81 \mathrm{ff}$.

(2) Vgl. H. König und V. Timmermann, Ein ökonometrisches Modell für die Bundesrepublik Deutschland 1950 - 1960, Zeitschrift für die gesamte Staatswissenschaft, 118, 1962, S. 638 
ergebnis. Der gravierende Unterschied zu der üblichen Formulierung liegt hier nur darin, daß die Erscheinung allein in den Plänen des Unternehmenssektors auftritt und nicht unmittelbar wirksam wird. Das Modell enthält rein theoretisch keinen Mechanismus, der sicherstellt, daß die Erwartungen und Pläne der Unternehmen stets erfüllt werden; rein theoretisch ist nicht einmal gesichert, daß die Modellwirtschaft eine Tendenz zu einem Zustand aufweist, in dem die Pläne restlos aufgehen. Insofern trägt die hier vorgenommene Anwendung der statischen Grenzproduktivitätstheorie allenfalls dazu bei, den mittel- bzw. langfristigen Verlauf der erwarteten Arbeits - und Kapitalkostenanteile zu bestimmen, ohne jedoch das entsprechende mittel- bzw. langfristige Niveau der tatsächlichen Quoten schon von sich aus eindeutig zu fixieren.

Diese Überlegungen lassen sich auch auf die grenzproduktivitätstheoretischen Inzidenzeffekte steuerpolitischer Maßnahmen des Staates anwenden, die sich in der durch die Kapazitätsplanung der Unternehmen angesteuerten Einkommensverteilung auswirken. Je nachdem, ob die Substitutionselastizität $-1 /\left(1+\alpha_{4}\right)$ der Produktionsfunktion (4.1) absolut genommen kleiner, gleich oder größer eins ist, wirkt sich eine Erhöhung des erwarteten realen Kapitalkostensatzes $r_{0}^{\star}, t$ durch Unternehmenssteuern aufgrund der induzierten Korrektur der Faktoreinsatzpläne derart aus, daß der Anteil der kalkulierten Kapitalkosten an der veranschlagten Faktorkostensumme steigt, konstant bleibt oder fällt (1); ebenso wird eine steuerbedingte Erhöhung des Arbeitskostensatzes $l_{0, t}$ den Anteil der kalkulierten Arbeitskosten steigen, konstant bleiben oder sinken lassen. Dies sind aber nur die Verteilungseffekte, die sich nach der ersten steuerbedingten Korrektur in den Kapazitätsplänen der Unternehmen einstellen. Werden die Pläne realisiert, wirken sich die Planrevisionen auf die Kreislaufströme, die Güter - und Faktorpreise aus und erzwingen auf diesem Wege erneute Plankorrekturen; mit anderen Worten, es setzt ein Prozeß sukzessiver Planrevisionen ein, währenddessen mit einem Aufgehen der durch die Kapazitätspläne jeweils implizierten Inzidenzeffekte der Unternehmensbesteuerung nicht gerechnet werden kann.

\section{Die Definition der Faktorkosten}

In neoklassischen Modellen werden als Kosten des privaten Kapital- und Arbeitseinsatzes meist nur der Kapitalmarktzins und der Lohnsatz

(1) Ist die Substitutionselastizität absolut genommen kleiner als eins, kommt es grenzproduktivitäts theoretisch zu einer Verschiebung bzw. Rückwälzung der Steuerlast auf den Faktor Arbeit. Die Verschiebung kann je nach Größe der Substitutionselastizität über das Ausmaß der Besteuerung hinausgehen. 
angesehen. In der beschriebenen Modellwirtschaft verursacht der Einsatz der Produktionsfaktoren aber Kosten über den bloßen Zins- bzw. Lohnsatz hinaus. Dies hat erhebliche Bedeutung für die Formulierung des Modells.

Eine der wesentlichen kapitalkostenverursachenden Eigenschaften der Modellwirtschaft ist die begrenzte Lebensdauer der einmal investierten Anlagen. Sie führt dazu, daß das Anlagevermögen der Unternehmen ständigen Wertminderungen ausgesetzt ist und daß aus Gründen der Vermögenserhaltung entsprechende Abschreibungen für Kapitalverzehr als Kosten kalkuliert und erlöst werden müssen. Werden die Abschreibungserlöse am Kapitalmarkt angelegt, so tragen sie mit ihrer Verzinsung zur Bedienung der ursprünglich aufgenommenen Finanzkapitalsumme bei; die angelegten Abschreibungserlöse werden von anderen Unternehmen wieder als Kredite zur Investitionsfinanzierung aufgenommen, diese Unternehmen erwirtschaften die entsprechende Kapitalverzinsung mit ihren Anlagen. Eliminiert man die Doppelzählung (1), die bei der Betrachtung des gesamten Unternehmenssektors aus der Kapitalmarktanlage der Abschreibungserlöse und ihrer Wiederaufnahme durch andere Unternehmen entsteht, dann sind die Kapitalkosten des Unternehmenssektors in einer Periode $t$ gleich dem Zins auf das gesamte zur Anlagenfinanzierung aus Ersparnis und Bankkredit aufgenommene Finanzkapital $\mathrm{F}_{A \mathrm{~A} l, \mathrm{t}}^{\star}$ plus den in der Periode fälligen Abschreibungs kosten $D_{\text {pr , t }}$

Der reale Kapitalkostensatz je Einheit des im Unternehmenssektor existierenden Anlagenbestandes ist bei Berücksichtigung einer begrenzten Lebensdauer der Anlagen definiert als der nominelle Kapitalkostensatz je realer Anlageneinheit, deflationiert mit dem allgemeinen Preisindex der privaten Produktion. Es müßte daher gelten:

$$
r_{t}^{*}=r_{t} \cdot \frac{F_{\text {Anl }, t-1}^{*}}{k_{p r, t} \cdot p_{x, t}}+\frac{D_{p r, t}}{k_{p r, t} \cdot p_{x, t}}
$$

bzw. nach einer Umformung:

$$
r_{t}^{*}=r_{t} \cdot \frac{F_{A n 1, t-1}^{*}}{k_{p r, t}^{n} \cdot p_{x, t}} \cdot \frac{k_{p r, t}^{n}}{k_{p r, t}}+\frac{D_{p r, t}}{k_{p r, t} \cdot p_{x, t}}
$$

Gleichung (4.7) zerlegt die Definition des realen Kapitalkostensatzes in vier Teilkompenenten, von denen drei aufgrund des für die Bundesrepublik verfügbaren statistischen Materials quantifiziert werden können.

(1) Vgl. J.E. Meade, A Neo-classical Theory of Economic Growth, 2. Aufl., London 1962, S. $84 \mathrm{f}$. 
$r_{\mathrm{t}}$ ist der Kapitalmarktzins. Sein Niveau und seine Zeitentwicklung werden anhand von Angaben über die Umlaufsrendite festverzinslicher Wertpapiere in der Bundesrepublik bestimmt (1).

Der Quotient aus dem deflationierten Abschreibungsbetrag und dem zu konstanten Preisen bewerteten Bruttoanlagevermögen ist der Abschreibungssatz. Die Abschreibungen für realen Kapitalverzehr werden in der Modellwirtschaft linear von dem zu Wiederbeschaffungspreisen bewerteten Bruttoanlagevermögen berechnet. Daher ist der Abschreibungssatz gleich dem Quotienten eins dividiert durch durchschnittliche Lebensdauer der Anlagen (2). Er wird aufgrund der Anlagevermögensrechnung des Statistischen Bundesamtes bestimmt (3) und entspricht weitgehend den Verhältnissen in der volkswirtschaftlichen Gesamtrechnung des Statistischen Bundesamtes.

Der Quotient aus dem linear abgeschriebenen realen Nettoanlagevermögen und dem realen Bruttoanlagenbestand, $k_{\mathrm{pr}, t}^{\mathrm{n}} / \mathrm{k}_{\mathrm{pr}, \mathrm{t}}$, der das zentrale Verknüpfungsstück zwischen dem Wertphänomen Zins und dem Realphänomen Grenzproduktivität des Anlagenbestandes darstellt und über den der Kapazitätserweiterungseffekt der wiederangelegten Abschreibungsbeträge in die Grenzproduktivitätsformel Eingang findet, wird ebenfalls aufgrund der genannten Anlagevermögensrechnung bestimmt (4).

(1) Vgl. N. K. A. Läufer, Der revisionstheoretische Gegensatz zwischen Loanable-Funds - und Liquiditätstheorie des Zinses mit ökonometrischem Test, in: G. Bombach (Hrsg.), Studien zur Geldtheorie und monetäre Ökonometrie, Schriften des Vereins für Socialpolitik, Bd. 66, Berlin 1972, S. 136 sowie Monatsberichte der Deutschen Bundesbank

(2) Diese Annahme ist nicht unproblematisch. Es gibt Argumente für die Annahme eines progressiven und Argumente für die Annahme eines degressiven Abschreibungsverlaufs. Würden die Anlagen einer wachsenden Wirtschaft generell progressiv abgeschrieben, ergäbe sich in der gesamtwirtschaftlichen Stromrechnung ein durchschnittlicher Abschreibungssatz der unter dem Quotienten eins dividiert durch (durchschnittliche) Lebensdauer der Anlagen läge; würde allgemein degressiv abgeschrieben, läge der durchschnittliche Abschreibungssatz über dem Wert dieses Quotienten. Im Fall eines degressiven Abschreibungsverlaufs kann der durchschnittliche Abschreibungssatz der Gesamtwirtschaft eine bestimmte Obergrenze nicht überschreiten; vgl. Anhang 2.

(3) Vgl. H. Lützel, Das reproduzierbare Anlagevermögen in Preisen von 1962, Wirtschaft und Statistik, 1971, S. $593 \mathrm{ff}$.

(4) Bei nicht zu starken Schwankungen der Wachstums - und Inflationsraten läßt sich der Quotient aufgrund einer Modellrechnung für langfristige Gleichgewichtssituationen annähern, die im Anschluß an das Vorgehen 
Lediglich für den Quotienten aus dem durch Ersparnis bzw. Kreditschöpfung aufgebrachten und zur Anlagenfinanzierung verwendeten Finanzkapital, $F_{A}^{\star} n l, t-1$, und dem damit aufgebauten und mit dem allgemeinen Preisindex bewerteten Nettoanlagevermögen, $k_{p r^{n}}^{n} t^{\bullet} p_{x}, t$, finden sich empirisch keine eindeutigen Anhaltspunkte. Der Quotient dient zur Berücksichtigung des kapitalkostensenkenden Effektes von Preissteigerungen und ist damit, zusammen mit dem Kapitalmarktzins, ein Substitut für den in anderen Untersuchungen als Kostenfaktor berücksichtigten Realzins, $r_{t}-\Delta p_{t} / p_{t}$. Zur numerischen Bestimmung wird auf den Wert zuzückgegriffen, den der Quotient in einem steady-state bei einer mit konstanter Rate wachsenden Nettokreditaufnahme des Unternehmenssektors, gnom, und bei einem konstanten Anstieg der Wiederbeschaffungspreise, gp, einnehmen würde (2).

$$
\text { (4.8) }\left[\frac{\mathrm{F}_{\mathrm{AnI}}^{*} \mathrm{t}}{\mathrm{k}_{\mathrm{pr}, t}^{\mathrm{n}} \cdot \mathrm{p}_{\mathrm{k}, t}}\right]_{\text {steady-state }}=\frac{\mathrm{g}_{\mathrm{nom}}-\mathrm{g}_{\mathrm{p}}}{\mathrm{B}_{\text {nom }}}
$$

Durch Einsetzen der für die Bundesrepublik vorgefundenen durchschnittlichen Anstiegsraten von Preisniveau und nomineller Anlageinvestition in Gleichung (4.8) wird für ein Jahr, nämlich 1960, der Wert des Quotienten festgesetzt. Multipliziert mit dem statistischen Wert für das Nettoanlagevermögen zu Wiederbeschaffungspreisen des privaten Sektors zu Beginn des Jahres 1960 ergibt der Quotient einen hypothetischen FAnl, 1959-Wert, der für das Jahresende 1959 gilt. Von diesem Wert ausgehend kann mit Hilfe der volkswirtschaftlichen Gesamtrechnung gemäß Definition

$$
\text { (4.8a) } F_{A n l, t}^{\star}=F_{A n l, t-1}^{\star}+I_{p r, A n l, t}^{b r}{ }_{p r, t}
$$

eine Zeitreihe der FAnl, $\mathrm{F}$ - Werte für die übrigen Jahre errechnet wer den, die schließlich mit Hilfe der Anlagevermögensrechnung des Statistischen Bundesamtes die Ermittlung einer Zeitreihe des Quotienten $\mathrm{F}$ Anl, $\mathrm{t}-1 /\left(\mathrm{k}_{\mathrm{pr}}^{\mathrm{n}} \mathrm{t} \cdot \mathrm{p}_{\mathrm{x}}, \mathrm{t}\right)$ erlaubt.

Gleichung (4.7) gibt den realen Kapitalkostensatz je Anlageneinheit bei Berücksichtigung einer begrenzten Lebensdauer der Anlagen an. Als modifizierendes Moment tritt hinzu, daß die Unternehmen ihre Inve-

von R. F. Harrod, Replacements, Net Investment, Amortisation Funds, Economic Journal, 80,1970 , S. $24 \mathrm{ff}$. , berechnet wurde. - Vgl. Anhang 1.

(1) Das aufgenommene Finanzkapital ist zum Nennwert angesetzt und unterliegt bei Preissteigerungen keiner inflationistischen Aufblähung.

(2) Zur Ermittlung dieser Formel vgl. Anhang 1. 
stitionsentscheidungen in Unsicherheit über die zukünftige Wirtschaftsentwicklung treffen und aus diesem Grund dem Zinssatz eine Risikoprämie zuschlagen oder mit einer kalkulatorisch verkürzten Lebens dauer der Anlagen und entsprechend überhöhtem Abschreibungssatz rechnen. (4.7) wird daher durch den Zuschlag einer langfristig konstanten Risikoprämie, $r_{R i s}$, zum Kapitalmarktzins erweitert; allerdings wird die Höhe der Risikoprämie über die Fixierung einer verkürzten pay-off-Periode bei der Anlagenamortisation ermittelt, indem die dadurch verursachte Erhöhung des Abschreibungssatzes in einen Zinszuschlag umgerechnet wird (1).

Neben den "natürlichen" Kostenbestandteilen enthalten die Kapitalkosten je nach Art und tariflicher Bemessungsgrundlage auch die dem Unternehmenssektor aufgebürdeten Steuern. Es handelt sich um die Steuern, die den Einsatz bzw. die Vergütung des Produktionsfaktors Kapital betreffen; da der Produktionsertrag gemä $\beta$ Produktionsfunktion und Grenzproduktivitätskalkül den Faktoren zugerechnet wird, handelt es sich ferner um die Steuern auf den Produktionserlös, die anteilig dem Faktor Kapital zugerechnet werden (2):

Nicht unter die Kapitalkosten fällt in der Modellwirtschaft die indirekte Steuer auf den Verbrauch, die in Form einer Nettoumsatzsteuer mit Vorabzug der Investitionen erhoben wird. Diese Steuer reduziert zwar im Vergleich zur Bruttogröße den pro Outputeinheit erzielten Nettopreis, in dem gleichen prozentualen Umfang aber auch den Nettoeinstandspreis der neu investierten Anlagen, der als Wiederbeschaffungspreis zur Bewertung der vorhandenen Anlagenbestände dient (3). Wird der reale Kapitalkostensatz so definiert, daß er zum Vergleich mit der physischen Grenzproduktivität des Anlagenbestandes herangezogen werden kann, dann kommt es zu einer Kompensation beider Effekte.

Die in der Modellwirtschaft erhobene Gewerbeertragsteuer belastet die gesamte Nettowertschöpfung des Unternehmenssektors nach Abzug der

(1) Zum Einfluß der verkürzten pay-off-Periode auf die Höhe des gesamtwirtschaftlichen Abschreibungssatzes vgl. Anhang 2.

(2) Die in diesem Sinne vorgenommene Einbeziehung von Steuern auf den Ertrag in den Bereich der Kosten-bzw. Faktoreinsatzbesteuerung ist nicht ganz korrekt, da im Prinzip steuerpflichtige Erträge anfallen können, ohne zuvor einen Einsatz von Produktionsfaktoren und damit Kosten verursacht zu haben. Das Vorgehen dient dem Anschluß an Gleichung (4.7) und zur Vereinfachung weiterer Überlegungen.

(3) Vgl. Wissenschaftlicher Beirat beim Bundesministerium der Finanzen, Probleme einer Netto-Umsatzbesteuerung, Schriftenreihe des Bundesministeriums der Finanzen, Heft 2, Bonn o. J. , S. $27 \mathrm{ff}$. 
Arbeitskosten, Importzölle und Verbrauchsteuerbelastung. Insoweit die Nettowertschöpfung aus vorauskalkulierbaren Kapitalkosten, nämlich Zins - und Risikoprämie, besteht, wird auch die Gewerbeertragsteuer anteilig als Kostenfaktor veranschlagt. Sie erhöht den Kapitalkostensatz in der Weise, daß die Gesam theit von Zins und Risikozuschlag (1) um den Faktor eins plus gewerbeertragsteuerliche Durchschnittsbelastung (2), $1+t_{R G, t}$, erhöht wird, wobei:

(4.9) $\quad t_{R G, t}=\frac{T_{R G, t}}{R_{t}^{\star}+G_{t}}$

Die Gewerbekapitalsteuer, $T_{k, t}$, belastet in der Modellwirtschaft das zu Wiederbeschaffungspreisen bewertete Nettoanlagevermögen des Unternehmenssektors. Sie führt ebenfalls zu einer Erhöhung des realen Kapitalkostensatzes.

Nicht in die Kapitalkosten einbezogen wird die direkte Steuer auf das Vermögen der Selbständigen, die in Form einer Sollertragsteuer erhoben wird. Sie ist eine persönliche Steuer der Selbständigen, die diese unabhängig von der Art der Vermögensanlage, also unabhängig davon, ob die Vermögen am Kapitalmarkt oder in eigenen Unternehmungen angelegt sind, belastet und die nach den späteren Annahmen hinsichtlich der Konsumfunktion, abgesehen vom Einkommenseffekt, keinen Einfluß auf die Konsum-Spar-Entscheidungen der Selbständigen hat. Die Steuerzahlung wird zwar de facto von den Unternehmen übernommen, bevor die Vermögenserträge netto an die Eigentümer bzw. Anteilseigner ausgeschüttet werden, doch berührt sie die Wirtschaftlichkeitsüberlegung der Unternehmen nicht, da sie keinen direkten Einfluß auf die Höhe der

(1) Da die Möglichkeit einer steuerlichen Verlustübertragung über die Periodengrenzen hinweg beschränkt ist, der Risikozuschlag außerdem eine subjektiv bestimmte Größe ist, die über den Satz der Schadensversicherungsprämie hinausgeht, wird die steuerliche Belastung auch auf den Zuschlag kalkuliert.

(2) Die Art, in der hier und im folgenden die steuerlichen Faktorkosten angesetzt werden, ist nur ein notdürftiger Behelf, der allerdings ganz auf der Linie des verwendeten neoklassischen Modells liegt. Die Entscheidungen der Wirtschaftssubjekte werden allein durch die individuellen steuerlichen Marginalbelastungen bestimmt. Der Versuch, einen exakten Durchschnitt dieser individuellen Marginalsätze als endogene Variable in das Modell einzuführen, muß bei der Annahme eines Steuersystems mit Freigrenzen, Freibeträgen und direkten Tarifprogressionen zwangsläufig scheitern. Aus diesem Grund werden statt der durchschnittlichen Marginalsätze die oben definierten steuerlichen Durchschnittsbelastungen angesetzt. 
alternativen Finanzierungskosten ausübt.

Ähnliche Überlegungen gelten im Prinzip für die Steuer auf das ausgeschüttete bzw. einbehaltene Besitzeinkommen der Selbständigen, soweit dieses lediglich eine Normalverzinsung des Selbständigenvermögens darstellt (1). Doch trifft diese Steuer auch die von den Unternehmen erlöste Risikoprämie bzw. die bei einer verkürzten pay-off-Periode anfallenden überhöhten Abschreibungserlöse. Damit diese Erlöse in vollem Umfang netto zur Verfügung stehen, wird in der Modellwirtschaft der auf sie entfallende Anteil der Steuer als zusätzlicher Kostenfaktor kalkuliert, und zwàr wird die Risikoprämie noch einmal um den Prozentsatz, den die Einkommensteuer auf Besitzeinkommen der Selbständigen, $t_{R G S, t}$, ausmacht, erhöht:

(4.10) $\quad t_{R G S, t}=\frac{T_{R G S, t}}{R_{S, t}+G_{t}}$

Faßt man alle genannten Kapitalkostenbestandteile zusammen, so gelangt man zu der endgültigen Definition des realen Kapitalkostensatzes je realer Anlageneinheit. Bei der Definition ist darauf zu achten, daß sich die Berechnung der steuerlichen Kapitalkostenbestandteile an der jeweiligen Steuerzahlung und nicht an der jeweiligen Steuerschuld orientiert (2) und daß in der Modellwirtschaft kein Unterschied zwischen der kalkulatorischen und der steuergesetzlich vorgeschriebenen Abschreibungsermittlung besteht (3):

(1) Soweit die Steuer auf den Gewinn der Unternehmen entfällt, stellt sie unter strengen Wirtschaftlichkeitsüberlegungen ohnehin keinen Bestandteil der Kapitalkosten dar. - Es sei hier im übrigen darauf hingewiesen, daß der Staat der Modellwirtschaft das Prinzip der realen Substanzerhaltung anerkennt und Bewertungsgewinne aus der Unternehmensbestandsrechnung nicht besteuert.

(2) Die Annahme einer derartigen Orientierung ist geeignet, dem Finanzierungs - bzw. Liquiditätsaspekt der Kapitalkosten Rechnung zu tragen, hat letzten Endes aber ökonometrische Gründe und dient der Vereinfachung.

(3) In dem Modell lassen sich ohne weiteres steuerliche Abschreibungsvorschriften berücksichtigen, mit denen der Staat zur Investitionsförderung eine überhöhte steuerliche Abschreibungsbemessung zuläßt. Auf eine derartige Berücksichtigung wurde verzichtet, weil die Auswirkungen steuerrechtlicher Abschreibungsvorschriften und ihrer Variation in der Bundesrepublik hier nicht ausreichend analysiert bzw. quantifiziert werden konnten. - R. R. Hall und D. W. Jorgensen, Tax Policy and Investment Behavior, American Economic Review, 57 , 1967, S. $389 \mathrm{ff}$, haben ein ähnliches neoklassisches Modell wie 


$$
\begin{aligned}
r^{*}= & \left\{\left[r_{t} \cdot\left(1+t_{R G, t}\right)+r_{R i s} \cdot\left(1+t_{R G, t}+t_{R G S, t}\right)\right] \cdot F_{A n}^{\star}, t-1\right. \\
& \left.+T_{k, t}+D_{p r, t}\right\} \frac{1}{k_{p r, t} \cdot p_{x, t}}
\end{aligned}
$$

Der Inhalt der geschlängelten Klammer von (4.11) bezeichnet endgültig die nominelle Kapitalkostensumme, wie sie in der Modellwirtschaft von den Unternehmen veranschlagt wird (1). Der Gesamtausdruck gibt den nominellen Kapitalkostensatz je realer Anlageneinheit, deflationiert mit dem allgemeinen Preisindex der privaten Produktion, an. Der erwartete Wert $r_{0}^{\star}, t$ dieser Größe ist in die Grenzproduktivitätsformel bzw. in die Planungsfunktion (4.4) für den Kapitalbestand einzusetzen.

Aus Gründen, die hier nicht dargestellt werden können, und die mit einer weiteren ökonometrischen Kontrolle des Investitionsverhaltens, insbesondere hinsichtlich der Preisentwicklung zusammenhängen, wurde der Kapitalkostensatz bei den Schätzungen und in der Modellrechnung abweichend von (4.11) nicht mit dem allgemeinen Preisindex $p_{x}, t$, sondern mit dem Preisindex für Investitionsgüter $p_{k, t}$, deflationiert. Der reale Kapitalkostensatz ist daher im weiteren definiert als (2):

$$
\text { (4.11a) } \begin{aligned}
r^{*}= & \left\{\left[r_{t} \cdot\left(1+t_{R G, t}\right)+r_{R i s} \cdot\left(1+t_{R G, t}+t_{R G S, t}\right)\right] \cdot F_{A n l, t-1}^{*}\right. \\
& \left.+T_{k, t}+D_{p r, t}\right\} \cdot \frac{1}{k_{p r, t} \cdot p_{k, t}}
\end{aligned}
$$

das hier aufgestellte benutzt, um den Einfluß neu eingeführter steuerlicher Abschreibungsvorschriften auf das Investitionsverhalten in den USA zu überprüfen. Das zugrunde liegende Modell wurde bereits in D. W. Jorgensen, Capital Theory and Investment Behavior, American Economic Review, 53, 1963, Papers and Proceedings, S. $247 \mathrm{ff}$., entwickelt.

(1) Die Definition (4.11) des Kapitalkostensatzes impliziert, daß die Unternehmen keinen rechnerischen Zins auf das in der Substanzerhaltungsrücklage gebundene Finanzierungskapital verrechnen.

(2) Eine nach (4.11a) berechnete Zeitreihe für den realen Kapitalkostensatz des privaten Sektors in der Bundesrepublik findet sich in Tabelle 1 des Tabellenanhangs. 
Die Tatsache, daß die mit dem Kapitaleinsatz verbundenen Kosten in der Modellwirtschaft über den Zinssatz hinausgehen, verweist noch einmal zurück auf die Behauptung eines zinselastischen Investitionsverhaltens, das zu den wesentlichen Eigenschaften der Modellwirtschaft gehört, und auf einen Einwand, der häufig gegen die Annahme eines derartigen Verhaltens angeführt wird. Je höher nämlich der Abschreibungssatz ist, mit dem die Unternehmen in der Modellwirtschaft rechnen, um so geringer ist der Einfluß, den der Zinssatz auf das angenommene Investitionsverhalten ausübt (1), umso größer wird dagegen der Einfluß der amortisationsbedingten Kapitalkosten. Diese Feststellung, sonst in extremer Formulierung abseits von neoklassischen Überlegungen abgeleitet, ergibt sich hier aus diesen Überlegungen selbst und kann damit nicht ohne weiteres als Gegeneinwand gegen sie angeführt werden. Ähnliche Rückwirkungen können sich im Prinzip aus der steuerbedingten Erhöhung des Kapitalkostensatzes ergeben; doch muß hier differenziert werden. Die Erhebung der Gewerbekapitalsteuer, $T_{k, t}$, und der Steuer auf einbehaltene Besitzeinkommen der Selbständigen, $T_{\text {RGS, }}$, mindert den Einfluß, den der Zinssatz auf das Investitionsverhalten der Unternehmen ausübt; die Gewerbeertragsteuer, $T_{R G}, t$, erhöht dagegen den Einfluß des Zinssatzes (2) (3). Wegen der Kompensation

(1) Diese Auswirkung zeigt sich auch bei der Ermittlung der Substitutionselastizität. Bei den hier getroffenen Annahmen über das Unternehmerverhalten gilt die Substitutionselastizität-1/(1+ $\left.\alpha_{4}\right)$ der angenommenen Produktionsfunktion (4.1) nicht hinsichtlich der Lohn-Zins-Relation, sondern hinsichtlich der Relation des eben definierten Kapital- und Arbeitskostensatzes. Berechnet man den entsprechenden Wert der Substitutionselastizität bezogen auf zinsbedingte Änderungen der Lohn-Zins-Relation, so ist deren Wert je nach Anteil der abschreibungsbedingten Kapitalkosten absolut genommen wesentlich kleiner als $1 /\left(1+a_{4}\right)$ und nicht konstant.

(2) Das gleiche gilt für die hier nicht berücksichtigten überhöhten $\mathrm{Ab}-$ schreibungen bei der Bemessung des steuerpflichtigen Unternehmens gewinnes.

(3) Die Elastizität des Realkapitalbestandes bezogen auf den Zinssatz $\mathbf{r}_{\mathrm{t}}$ läßt sich zerlegen gemäß

$$
\frac{\delta k_{p r, t}}{\delta r_{t}} \cdot \frac{r_{t}}{k_{p r, t}}=\left[\frac{\delta k_{p r, t}}{\delta r_{t}^{\star}} \cdot \frac{r_{t}^{\star}}{k_{p r, t}}\right] \cdot\left[\frac{\delta r_{t}^{*}}{\delta r_{t}} \cdot \frac{r_{t}}{r_{t}^{*}}\right]
$$

Die erste Komponente nimmt die Stelle ein, die der Gesamtausdruck in einem Modell mit unendlicher Lebensdauer der Anlagen und Fehlen steuerbedingter Kapitalkosten hätte. Zur Beurteilung der oben genannten Aussagen genügt es, im Anschluß an (4.11) die zweite Teilkomponente, also die Elastizität des Kapitalkostensatzes bezogen auf Änderungen des Zinssatzes zu berechnen. 
beider Effekte braucht in der Modellwirtschaft mit einer nennenswerten Einschränkung der Zinselastizität der Investition durch die Unternehmensbesteuerung nicht gerechnet $\mathrm{zu}$ werden.

Die Definition des Arbeitskostensatzes ist wesentlich einfacher und unproblematischer als die des Kapitalkostensatzes:

Der private Sektor zahlt an seine Beschäftigten den nominellen Lohnsatz $l_{\mathrm{pr}, \mathrm{t}}$. Da vom Staat auf die Summe der gezahlten Arbeitseinkommen die Lohnsummensteuer, $\mathrm{T}_{\mathrm{L}}$, erhoben wird, wird der Lohnsatz um den Prozentsatz der Lohnsummensteuer-Durchschnittsbelastung, $t_{L, t}$, erhöht (1): (4.12) $\quad t_{L, t}=\frac{T_{L, t}}{e_{p r, t} \cdot I_{p r, t}}$

Gleichzeitig wirkt sich, anders als bei den Kapitalkosten, die Belastung der Absatzerlöse durch die indirekte Verbrauchsteuer aus; der tarifliche Satz der in Form der Nettoumsatzsteuer erhobenen Abgabe wird angenähert als $t_{C}, t$ :

$$
\text { (4.13) } \quad t_{c, t}=\frac{T_{C, t}}{C_{A, t}+C_{S, t}{ }^{+A_{S t, t}}{ }^{-L_{S t, t}}}
$$

Werden die Kosten, die aus dem Einsatz und Ertrag eines Beschäftigten entstehen, so definiert, daß sie sich zu einem Vergleich mit der zu Bruttoabsatzpreisen bewerteten Grenzproduktivität des Faktors Arbeit eignen, dann gilt (2):

$$
\text { (4.14) } I_{\text {pr, }}^{*}=I_{\text {pr, }} \cdot \frac{1+t_{L, t}}{1-t_{C, t}}
$$

Der erwartete Wert $1_{0}^{\star}$, pr $t$ dieser Größe ist in die Planungsfunktion (4.5) der privaten Beschäftigung einzusetzen.

(1) Die Beiträge zur staatlichen Sozialversicherung werden in der Modellwirtschaft ausschließlich von Seiten der Arbeitnehmer gezahlt.

(2) Wird die in Form einer Nettoumsatzsteuer erhobene Verbrauchsteuer bei ihrer Einführung sofort in die Preise überwälzt, was entsprechende geldpolitische Maßnahmen voraussetzt, dann steht der durch sie bewirkten Erhöhung des Arbeitskostensatzes eine entsprechende Erhöhung des Bruttoabsatzpreises gegenüber, und man gelangt aufgrund von (4.11) und (4.14) zu dem Ergebnis, daß die optimale Faktoreinsatzrelation nicht tangiert wird. 
Um zu den Erwartungswerten für den in Periode t erzielbaren Absatz und das Niveau von Kapitalkostensatz, Arbeitskostensatz und Güterpreis zu gelangen, greifen die Unternehmen auf entsprechende Wert der Vergangenheit zurück. Aus laufenden Informationen und früheren Kalkulations unterlagen stellen sie jeweils eine Reihe von Erfahrungswerten für den Absatz, den Kapital- und Arbeitskostensatz und den Güterpreis zusammen, die in Planungsperiode $t-1$ beginnt und in die Vergangenheit t- $. \tau-1$ zurückreicht:

(4.15) $x_{t-1}, x_{t-2}, \ldots \ldots \ldots, x_{t-\tau-1}$

(4.16) $r_{t-1}^{*}, r_{t-2}^{*}, \ldots \ldots, \ldots, r_{t-\tau-1}^{*}$

(4.17) $\quad 1_{\mathrm{pr}, t-1}^{*}, 1_{\mathrm{pr}, t-2}^{\star}, \cdots, 1_{\mathrm{pr}, t-\tau-1}^{\star}$

(4.18) $\quad p_{x, t-1}, p_{x, t-2} \cdots \cdots \cdots, p_{x, t-\tau-1}$

Ähnlich versuchen sie, näherungsweise eine in die Vergangenheit reichende Reihe von Erfahrungswerten, $k_{S t, t-v}(v=1,2, \ldots, \tau+1)$, uber den Einfluß der staatlichen Infrastrukturanlagen auf die privaten Produktionsmöglichkeiten aufzustellen. Die Reihe dieser $k_{S t}^{\star} t-v-W e r t e$ wird, da die Unternehmen die langfristigen Substitutionsmöglichkeiten richtig einschätzen, durch Einsetzen der tatsächlichen Werte von privater Produktion, $x_{t-v}$, und staatlichem Infrastrukturanlagenbestand,

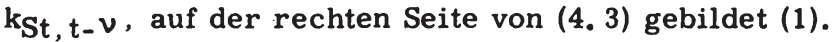

(4.19) $\quad k_{S t, t-1}^{\star}, k_{S t, t-2}^{\star} \cdots \cdots, k_{S t, t-\tau-1}^{\star}$

Im Anschluß werden die Reihenwerte in Wachstumsraten bzw. in Meßziffern bezogen auf den Vorjahreswert transformiert:

(1) Gleichung (4.3) hat damit eine doppelte Funktion: die annähernde Bestimmung eines für die Vergangenheit geltenden Erfahrungswertes, $k_{S t, t-v}^{\star}$, aufgrund der durch $(5.27)$ und $(2.42),(2.65),(17.2)$ bestimmten Erfahrungswerte $x_{t-v}$ und $k_{S t, t-v}$; die Reihe dieser $\mathbf{k}_{\text {St, } t-v}^{\star}$-Werte dient als Information bei der Bildung der entsprechenden Erwartungswerte, $k_{o}^{\star}, S t, t$, gemäß (4.53) - ferner die Ableitung eines für die Zukunft geltenden Erwartungswertes, $k_{0}, S_{t}, t$, aufgrund der durch (4.53) und (4.51) bestimmten Erwartungs - bzw. Planwe rte $k_{o, S t, t}^{\star}$ und $x_{0, t}$. 


$$
\begin{aligned}
& \text { (4.20) } \quad 1+g_{x, t-1}, 1+g_{x, t-2}, \ldots . ., 1+g_{x, t-\tau} \\
& \text { (4.21) } 1+g_{r^{*}, t-1}, 1+g_{r^{*}, t-2}, \ldots ., 1+g_{r^{*}, t-\tau} \\
& \text { (4.22) } 1+g_{1 *, t-1}, 1+g_{1 *, t-2}, \ldots, ., 1+g_{1 *}, t-\tau \\
& \text { (4.23) } \quad 1+g_{p, t-1}, 1+g_{p, t-2}, \ldots, \ldots, 1+g_{p, t-\tau} \\
& \text { (4.24) } \quad 1+g_{k^{*}, t-1}, 1+g_{k^{*}, t-2}, \ldots, 1+g_{k *}, t-\tau
\end{aligned}
$$

Die Zeitreihen für die so erhaltenen Wachstumsfaktoren der Vergangenheit werden mit einer einheitlichen distributed-lag-Funktion zu einem erwarteten Wachstumsfaktor zusammengefaßt (1):

$$
\begin{array}{ll}
\text { (4.25) } & 1+\hat{g}_{x, t}=\prod_{v=1}^{\tau}\left[1+g_{x, t-v}\right]^{\lambda} \\
\text { (4.26) } & 1+\hat{g}_{r^{*}, t}=\prod_{v=1}^{\tau}\left[1+g_{r^{*}, t-v}\right]^{\lambda} \\
\text { (4.27) } & 1+\hat{g}_{1 *, t}=\prod_{v=1}^{\tau}\left[1+g_{1 *}, t-v\right]^{\lambda} v \\
\text { (4.28) } & \left.1+\hat{g}_{p, t}=\prod_{v=1}^{\tau}\left[1+g_{p, t}\right]^{\lambda}\right]^{\lambda} \\
\text { (4.29) } & 1+\hat{g}_{k^{*}, 1}=\prod_{v=1}^{\tau}\left[1+g_{k *}, t-v\right]^{\lambda}
\end{array}
$$

(1) Die Annahme einer einheitlichen distributed-lag-Funktion bei der Verarbeitung aller Vergangenheitsinformationen durch die Unternehmen scheint im allgemeinen sinnvoll zu sein; sie impliziert aber, daß die Unternehmen sich hinsichtlich der zeitlichen Geltungsdauer der herrschenden Steuertarife in der gleichen subjektiven Unsicherheit befinden wie hinsichtlich der Entwicklung der Güter- und Faktorpreise. Wegen der nachhaltigen Geltungsdauer der Steuertarife im Vergleich zu der Entwicklung der Güter- und Faktorpreise mag man die Annahme kritisieren; in Hinsicht auf die von Zeit zu Zeit anfallenden Steuerreformen und die Art der herrschenden Tarifprogression im Bereich der direkten Steuern kann man ihr eine gewisse Rechtfertigung nicht absprechen. Zur Kritik der Annahme vgl. im übrigen R. Eisner, Tax Policy and Investment Behavior. Further Comment, American Economic Review, 60, 1970, S. 746 ff. kritisiert, wo auf die Unter suchung von R. E. Hall und D. W. Jorgensen, Tax Policy and Investment Behavior, a. a. O. , Bezug genommen wird. 
wobei die Informationen der ferneren Vergangenheit jeweils mit einem schwächeren exponentiellen Gewichtungsfaktor $\lambda_{v}$ berücksichtigt werden als die Informationen der näheren Vergangenheit und wobei das Gewichtungssystem $\left(\lambda_{1}, \lambda_{2}, \ldots, \lambda_{\tau}\right)$ durch folgende Eigenschaften gekennzeichnet ist (1):

$$
\text { (4.30) } \sum_{v=1}^{\tau} \lambda_{v}=1 \quad(\tau=\infty)
$$$$
\text { (4.31) } \quad \lambda_{v}=\lambda \cdot(1-\lambda)^{\nu-1}
$$

Durch Fortschreibung der in der Planungsperiode $t-1$ vorgefundenen tatsächlichen Werte von $x_{t-1}, r_{t-1}^{\star}, 1_{p r, t-1}^{\star}, p_{x, t-1}$ und $k_{S t, t-1}^{\star}$ mit den durch (4.25) bis (4.29) definierten Wachstumsfaktoren schätzen die Unternehmen die Erwartungswerte für den Abșatz, den Kapital- und Arbeitskostensatz, das Preisniveau und den $\mathrm{k}_{\mathrm{St}}$-Faktor, mit denen sie in ihrer Kapazitätsplanung für Periode $t$ rechnen:

$$
\begin{aligned}
& \text { (4.32) } x_{0, t}=x_{t-1} \cdot\left[1+\hat{g}_{x, t}\right] \\
& \text { (4.33) } r_{0, t}^{\star} \quad=r_{t-1}^{\star} \cdot\left[1+\hat{\delta}_{r^{\star}, t}\right] \\
& \text { (4.34) } I_{o, p r, t}^{\star}=I_{\mathrm{pr}, t-1}^{*} \cdot\left[1+\hat{\mathrm{g}}_{1 *, t}\right] \\
& \text { (4.35) } \mathrm{p}_{\mathrm{o}, \mathrm{x}, t}=\mathrm{p}_{\mathrm{x}, t-1} \cdot\left[1+\hat{\mathrm{g}}_{\mathrm{p}, t}\right] \\
& \text { (4.36) } k_{o, s t, t}^{*}=k_{S t, t-1}^{*} \cdot\left[1+\hat{g}_{k}^{*}, t\right]
\end{aligned}
$$

(1) Es handelt sich also um einen distributed-lag des Koyck-Typs. Vgl. L. M. Koyck, Distributed Lags and Investment Analysis, Amsterdam 1954 
5. Die dynamische Form der Anlagenplanungsfunktion, ihre empirische Überprüfung und Modifizierung

Löst man die in der Anlagenplanungsfunktion (4.4) enthaltenen Erwartungswerte $x_{0, t}, r_{o, t}^{\star}$ und $k_{o}^{\star}, S t, t$ gemäß $(4.32),(4.33)$ und $(4.36)$ sowie gemäß $(4.25),(4.26)$ und $(4.29)$ auf, gewinnt die Funktion eine ziemlich unübersichtliche Gestalt. Die angenommenen Eigenschaften (4.30) und (4. 31) der distributed-lag-Funktion erlauben jedoch durch bloße Umformung eine wesentliche Vereinfachung. Nach dieser Umformung lautet die Anlagenplanungsfunktion:

$$
\begin{aligned}
& \text { (4.37) } k_{o, p r, t}=k_{0, p r, t-1}^{1-\lambda}\left\{\left(\mu_{k} \cdot a_{1} \cdot\left\{1-a_{2}\right\}\right)^{\frac{1}{1+\alpha_{4}}} \cdot r_{t-1}^{*}-\frac{1}{1+\alpha_{4}} \cdot\right. \\
& \left.k_{S t, t-1}^{-\frac{a_{4}-a_{5}}{1+a_{4}}} \cdot x_{t-1}\right]^{\lambda} \cdot\left[\left(1+g_{r}, t-1\right)^{-\frac{1}{1+a_{4}}} .\right. \\
& \left.\left(1+g_{k \star t-1}\right)^{-\frac{a_{4}-a_{5}}{1+a_{4}}} \cdot\left(1+g_{x, t-1}\right)\right]
\end{aligned}
$$

Es ist $z u$ beachten, daß $k_{0}, p r, t$ in (4.37) den Anlagenbestand angibt, den die Unternehmen für Periode $t$ anstreben. Die Bestandsplanung wird aber auch mit Hilfe der in Periode $t-1$ vorgenommenen Anlageninvestitionen realisiert. Da alle begonnenen Projekte eine Ausreifungszeit von einem $\mathrm{J}$ ahr haben, kennzeichnet $\mathrm{k}_{\mathrm{o}, \mathrm{pr}} \mathrm{t}$ also auch den Anlagenbestand, der in Periode $t$ tatsächlich für produktive Zwecke zur Verfügung steht und dann kurzfristig nicht mehr variiert werden kann:

(4.38) $k_{o, p r, t}=k_{p r, t}$

Die Anlagenplanungsfunktion kennzeichnet daher auch den Entwicklungspfad des privaten Anlagenbestandes im Wachstumsprozeß. Nach Berücksichtigung von (4.38) und einer Transformation gilt: 


$$
\begin{aligned}
& \text { (4.39) } \frac{k_{p r, t}}{k_{p r, t-1}}=\left[\left(\mu_{k} \cdot a_{1} \cdot\left\{1-a_{2}\right\}\right)^{\frac{1}{1+a_{4}}} \cdot r_{t-1}^{*}{ }^{-\frac{1}{1+a_{4}}}\right. \text {. }
\end{aligned}
$$

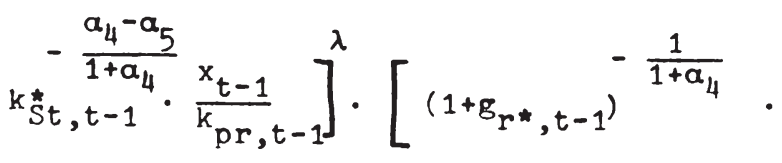

$$
\begin{aligned}
& \left.\left(1+g_{k *, t-1}\right)^{-\frac{a_{4}-a_{5}}{1+a_{4}}} \cdot\left(1+g_{x, t-1}\right)\right]
\end{aligned}
$$

Nach (4.39) wird die Entwicklung des privaten Anlagenbestandes vor allem durch die Wachstumsrate des Güterabsatzes sowie die Änderungsrate des Kapitalkostensatzes bestimmt (1). Außerdem wird sie durch das absolute Niveau des Kapitalkostensatzes und des Kapitalkoeffizienten beeinflußt; allerdings wirkt sich der Einfluß dieses Niveaus kurzfristig nur in begrenztem Umfang aus und benötigt einen langen Zeitraum, um seine volle Wirksamkeit zu entfalten.

Um die Bedeutung der hier aufgestellten Investitionstheorie für den Ablauf der Modellwirtschaft bereits vor Abschluß der gesamten Modellkonstruktion zu skizzieren, wird die Anlagenplanungsfunktion (4.37) mit (4.38) durch Einsetzen der Produktionsfunktion (4.1) zu einer Differenzengleichung für den privaten Anlagenbestand umgeformt (2). Zusätzlich werden folgende Vereinfachungen unterstellt: 1. Die Erwerbsbevölkerung ist konstant. - 2. Es herrscht Vollbeschäftigung. 3. Der Kapitalkostensatz verharrt auf konstantem Niveau. - 4. Der Staat stellt öffentliche Infrastrukturanlagen in stets gleicher Relation zur privaten Produktion zur Verfügung. - Unter diesen Annahmen

(1) Der Einfluß, der von mengenmäßigen Änderungen der öffentlichen Infrastrukturanlagen ausgeht, ist, da es sich hier nur um einen bestimmten Teil des gesamten staatlichen Anlagenbestandes handelt, nämlich um die Anlagen des staatlichen Verkehrssektors, begrenzt. Rechnerisch wirkt sich das über einen sehr niedrigen Wert von $\left(1-a_{1}\right)$ in $(4.3)$ aus.

(2) Die angestellte $Z$ wischenüberlegung beruht also weder auf der üblichen neoklassischen Annahme, die Investition werde stets über den Umfang der freiwilligen Ersparnis bestimmt, eine Annahme, die der gesamten Modellkonzeption widerspricht, noch auf der keynesianischen Multiplikatortheorie. 
enthält die Differenzengleichung als einzige Variable die Werte des privaten Anlagenbestandes in drei aufeinanderfolgenden Perioden. Für den Fall, daß die partielle Substitutionselastizität zwischen eingesetzter Arbeit und privatem Anlagenbestand, $-1 /\left(1+\alpha_{4}\right)$, null oder minus eins ist, also für den Leontief- oder Douglas-Cobb-Fall, ist die Funktion linear in den Logarithmen dieser Variablen und läßt sich als lineare inhomogene Differenzengleichung zweiten Grades auf dem üblichen Wege lösen.

Zur Skizzierung sei hier nur der Douglas-Cobb-Fall mit einer Substitutionselastizität von minus eins betrachtet: In diesem Fall ist der durch die Differenzengleichung beschriebene Pfad der privaten Kapitalakkumulation stets stabil in dem Sinn, daß er mit oder ohne zyklische Schwankungen gegen einen mit der Harrod-neutralen technischen Fortschrittsrate ansteigenden Gleichgewichtspfad tendiert (1). Bei realistischen Werten für die Produktionselastizität des privaten Anlagenbestandes und den Parameter $\lambda$ der Erwartungsfunktionen konvergiert der Wachstumspfad ohne Schwankungen gegen den Gleichgewichtspfad (2). Auf jeden Fall ist der Zeitbedarf für die volle Anpassung an den Gleichgewichts pfad sehr groß.

Hat die private Kapitalakkumulation den durch die Lösung der Differenzgleichung beschriebenen Gleichgewichtspfad einmal erreicht, dann ist die Wachstumsrate des privaten Anlagenbestandes gleich der Wachstumsrate der Produktion. Daraus folgt, daß dann in (4.39) der erste eckige Klammerausdruck der rechten Gleichungsseite gleich eins ist. Die Tatsache, daß der Klammerwert gleich eins ist, bedeutet aber analog zu (4.4) nichts anderes, als daß der jeweilige private Anlagenbestand im Sinne der statischen Grenzproduktivitätstheorie optimal bemessen ist. Das Grenzerlösprodukt des privaten Anlagenbestandes ist gleich den privaten Grenzkapitalausgaben. Das Investitionskalkül der Unternehmen geht auf.

(1) Die erforderliche Bedingung für Stabilität in diesem weiteren Sinn ist allein, daß die Produktionselastizität des privaten Anlagenbestandes, die im Anschluß an (4.1) mit $\left(1-\alpha_{2}\right)$ bezeichnet werden kann, kleiner als eins ist. Der kritische Punkt bezüglich der Stabilitätseigenschaft ist, daß die Schwingungsamplitude der Anpassung erheblich ausfallen dürfte.

(2) Die zusätzliche erforderliche Bedingung für Stabilität in diesem engeren Sinne ist:

$$
1>\frac{4 \cdot\left(1-a_{2}\right)}{\left[1-\lambda+(1+\lambda) \cdot\left(1-a_{2}\right)\right]^{2}}
$$

wobei wiederum $\left(1-\alpha_{2}\right)$ die Produktionselastizität des privaten Anlagenbestandes ist. 
Hat die private Kapitalakkumulation dagegen den Gleichgewichtspfad noch nicht erreicht, dann fallen die Wachstumsraten von privatem Anlagenbestand und privater Produktion auseinander. Dann ist der erste eckige Klammerausdruck von (4.39) ungleich eins, und dies bedeutet, daß der private Anlagenbestand seine jeweilige Optimalgröße im Sinne der statischen Grenzproduktivitätstheorie noch nicht erreicht hat. Solange der private Anlagenbestand z. B. suboptimal ist, versuchen die Unternehmer dadurch, daß sie den geplanten Anlagenbestand schneller als die laufende Produktion steigen lassen, einen optimalen Kapitalkoeffizienten zu erreichen. Aber dies gelingt ihnen wegen des durch die Erwartungsfunktionen implizierten Verzögerungsmechanismus erst nach einer sehr langen Anpassungszeit, und, worauf neoklassische Wachstumsmodelle fast nie aufmerksam machen, während dieser Anpassungszeit übersteigt das bewertete Grenzprodukt des jeweiligen privaten Anlagenbestandes stets die veranschlagten Grenzkapitalausgaben, wie sie bei der Kapazitätsplanung angesetzt und bei der Vornahme der Investitionen realisiert werden.

Versucht man, die Akkumulationsfunktion (4.39) empirisch zu überprüfen, entsteht eine gewisse Schwierigkeit dadurch, daß die Substitutionselastizität $\mathrm{z}$ wischen dem öffentlichen Infrastrukturanlagenbestand und den beiden anderen Produktionsfaktoren, $-1 /\left(1+\alpha_{5}\right)$, unbekannt ist, der Substitutionsparameter $\alpha_{5}$ nach (4.3) aber bereits zur Bildung der $\mathbf{k}_{S}^{\star} t, t-1-$ Werte benötigt wird. An diesem Problem braucht die Überprü-

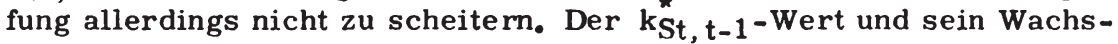
tumsfaktor liegen auf jeden Fall nahe eins und haben praktisch nur einen geringen Einfluß auf das Wachstum des privaten Anlagenbestandes. Um zu Vorstellungen über den numerischen Wert der Funktionsparameter zu gelangen, ist es daher am einfachsten, beide Größen zuerst einmal ganz zu vernachlässigen. Man gelangt dann zu einer vereinfachten Version von (4.39), die nach einer logarithmischen Transformation als Regressionsgleichung für Kleinst-Quadrat-Schätzungen geeignet ist:

$$
\text { (4.40) } \begin{aligned}
\lg \frac{k_{p r, t}}{k_{p r, t-1}} & =a_{1}^{\prime} \\
& +a_{2}^{\prime} \cdot \lg r_{t-1}^{*} \\
& +a_{3}^{\prime} \cdot \lg \left(x_{t-1} / k_{p r, t-1}\right) \\
& +a_{4}^{\prime} \cdot \lg \left(1+g_{r *, t-1}\right) \\
& +a_{5}^{\prime} \cdot \lg \left(1+g_{x, t-1}\right)
\end{aligned}
$$

Als Material zur Schätzung dieser Funktion dienten die Angaben des Statistischen Bundesamtes über das Bruttoanlagevermögen des privaten 
Sektors (1), die Angaben der volkswirtschaftlichen Gesamtrechnung über den Bruttoinlandsprodukt-Beitrag des privaten Sektors (2) sowie die bei Annahme einer Risikoprämie von 1,5\% berechnete Zeitreihe für den privaten Kapitalkostensatz (3).

In den vorgenommenen Regressionsschätzungen ließ sich der statistische Erklärungswert von (4.40) dadurch verbessern, daß die Verzögerung, mit der sich die Einflußfaktoren auf das Wachstum des privaten Anlagenbestandes auswirken, um eine Periode verlängert wurde. Diese Verbesserung ergab sich insbesondere in Hinsicht auf den Parameter des reziproken Kapitalkoeffizienten; nicht ganz so deutlich war sie beim Kapitalkostensatz und den beiden Wachstumsfaktoren, was aber auf die starke Autokorrelation der entsprechenden Zeitreihen zurückzuführen ist. Die Berücksichtigung des Kapitalkostensatzes der Periode t-1 als zusätzliche Erklärungsvariable neben dem Kapitalkostensatz der Periode t-2 brachte keine Verbesserung des Regressionsergebnisses.

Berücksichtigt man dies durch eine Umformulierung von (4.40):

$$
\text { (4.41) } \begin{aligned}
\lg \frac{k_{p r, t}}{k_{p r, t-1}} & =a_{1}^{\prime} \\
& +a_{2}^{\prime} \cdot 1 g r_{t-2}^{*} \\
& +a_{3}^{\prime} \cdot \lg \left(x_{t-2} / k_{p r, t-2}\right) \\
& +a_{4}^{\prime} \cdot \lg \left(1+g_{r *, t-2}\right) \\
& +a_{5}^{\prime} \cdot \lg \left(1+g_{x, t-2}\right)
\end{aligned}
$$

dann lauten die Ergebnisse der Regressionsschätzung ( $t=1958$, ..., 1971):

(1) Vgl. H. Lützel, Das reproduzierbare Anlagevermögen in Preisen von 1962 , aaO., S. $593 \mathrm{ff}$.

(2) Der Bruttoinlandsprodukt-Beitrag des privaten Sektors wurde zur Abstimmung auf die volkswirtschaftliche Gesamtrechnung des Modells um den Saldo der vom Ausland erhaltenen Einkommen aus unselbständiger Arbeit ergänzt.

(3) Vgl. Tabelle 1 des Tabellenanhangs. 


$$
\begin{aligned}
& \left.\alpha_{1}^{\prime}=-0,00128 \text { (nicht signifikant) }{ }^{1}\right) \\
& \alpha_{2}^{\prime}=-0,03967( \pm 0,04589) \\
& \alpha_{3}^{1}=+0,04416( \pm 0,06135) \\
& \alpha_{4}^{\prime}=-0,02406( \pm 0,04262) \\
& a_{5}^{\prime}=+0,05177( \pm 0,03741) \\
& \mathrm{R}_{1 \cdot 2,3,4,5}^{2}=0,82238 \text { (Irrt.-Wahrsch.: }<1 \% \text { ) } \\
& \mathrm{R}_{2 \cdot 3,4,5}^{2}=0,92428 \\
& \mathrm{R}_{3 \cdot 2,4,5}^{2}=0,92948 \\
& R_{4 \cdot 2,3,5}^{2}=0,74338 \\
& \mathrm{R}_{5 \cdot 2,3,4}^{2}=0,21114 \\
& =1,334
\end{aligned}
$$

Abgesehen von der angeregten Modifikation der Regressionsfunktion ist das zweite wichtige Ergebnis der Schätzungen, daß die ermittelten Parameter $a_{1}^{j}$ bis $\alpha_{5}^{j}$ alle sehr gering ausgefallen sind. Nach der theoretischen Ableitung hätte man für $\alpha_{5}^{\prime}$ einen Wert von eins erwartet; dies hätte aber starke Reaktionen der Anlageinvestition bedeutet, wie sie in der Bundesrepublik nicht anzutreffen sind (2).

Das dritte wichtige Ergebnis betrifft die Größe der Substitutionselastizität zwischen Arbeitseinsatz und privatem Anlagenbestand. Interpretiert man die Schätzwerte der Parameter durch die entsprechenden Exponenten von Kapitalkostensatz, Kapitalkoeffizient und Wachstumsfaktoren in (4.39), dann müßte, abgesehen von einigen ökonometrischen Einschränkungen (3), gelten:

(1) Als signifikant gilt hier und im folgenden eine Irrtumswahrscheinlichkeit von weniger als $5 \%$.

(2) Vgl. hierzu die jährlichen Wachstumsraten des privaten Bruttoanlagevermögens in der Bundesrepublik zwischen 1950 und 1970 in H. Lützel, Das reproduzierbare Anlagevermögen in Preisen von 1962 , a. a. O., S. 602 . -

(3) Selbst wenn die geschätzten Parameter $\alpha_{2}^{\prime}$ bis $\alpha_{5}^{\prime}$ als erwartungstreu im Sinne von (4.39) angesehen werden müssen, stellt der Quotient von (4.43) keine unverzerrte Schätzung der Substitutionselastizität dar. 


$$
\text { (4.43) } \frac{a_{2}^{\prime}}{\alpha_{3}^{\prime}}=\frac{\alpha_{4}^{\prime}}{a_{5}^{\prime}}=-\frac{1}{1+\alpha_{4}}
$$

Das heißt, der Quotient aus den Parametern $\alpha_{2}^{\prime}$ und $\alpha_{3}^{\prime} b z w$. aus den Parametern $\alpha_{4}^{\prime}$ und $\alpha_{5}^{\prime}$ wäre mit dem Wert der Substitutionselastizität gleichzusetzen. Bildet man den Quotienten aus den Schätzwerten der beiden ersten Parametern, die allein wegen der hohen Multikollinearität nicht signifikant ermittelt werden konnten, dann erhält man einen Wert von -0,91. Dieses Ergebnis ist natürlich $u_{\text {. }} \mathrm{a}_{\text {. durch die Berechnungs- }}$ methode für den Kapitalkostensatz, insbesondere durch die angenommene Höhe der Risikoprämie bedingt; wäre bei der Berechnung die Risikoprämie auf 1,4\% statt auf 1,5\% angesetzt worden, hätte der absolute Wert des Quotienten statt unter knapp über dem Wert von eins gelegen. Andererseits könnte der Quotient aus den Parametern $\alpha_{4}^{\prime}$ und $\alpha_{5}^{\text {? mit }}$ einem Wert von $-0,46$ als Indiz dafür angesehen werden, daß mit einer Substitutionselastizität von wesentlich unter eins zu rechnen ist.

Insgesamt zeigt die empirische Überprüfung, daß mit der gebotenen theoretischen Ableitung das Investitionsverhalten der Unternehmen nicht ganz zufriedenstellend erfaßt wird. Der nicht sehr hohe Wert des vonNeumann-Quotienten in (4.42) legt die Vermutung von Autokorrelation nahe. Hierfür könnte man eine ganze Reihe von Faktoren verantwortlich machen: die vorgenommene Abgrenzung des Kapitalkostensatzes, die Verwendung eines einfachen geometrisch verteilten distributed-lag oder ganz allgemein einer falschen Funktionsform bei der Aufstellung der Erwartungsfunktion u. a. m. Immerhin deutet der hohe statistische Erklärungswert von $82 \%$ darauf hin, daß der gewählte Ansatz grundsätzlich dazu geeignet ist, das Verhalten der Unternehmen hinsichtlich ihrer Anlageinvestitionen zu beschreiben.

Das Ergebnis der Regressionsschätzungen legt es nahe, in der bisherigen Formulierung des Unternehmensverhaltens unabhängig von der gebotenen theoretischen Ableitung eine entsprechende Änderung vorzunehmen. Als erstes wird für die Modellwirtschaft daher angenommen, daß die Unternehmen die Anlagenkapazität für Periode $\mathrm{t} z \mathrm{war}$ in Periode $\mathrm{t}-1$ bestimmen, daß sie aber bei der Aufstellung der Kapazitätspläne nicht die laufenden Ereignisse der Planungsperiode berücksichtigen, die z. T. das Ergebnis des geplanten Investitionsverhaltens sind und im Planungsstadium noch nicht vorliegen. Die Kapazitätspläne werden also in $\mathrm{Pe}-$ riode $t-1$ unabhängig von den gerade herrschenden Absatzbedingungen, Kapitalmarktverhältnissen und Steuervorschriften aufgestellt und im geplanten realen Umfang über entsprechende Investitionen realisiert (1).

(1) Durch die Veränderung des Verzögerungsmechanismus verliert die oben genannte Differenzengleichung für den privaten Anlagenbestand nicht ihre Stabilitätseigenschaft im weiteren Sinn, sofern für den Parameter der Erwartungsfunktion der $a_{3}^{\prime}$-Wert von (4.42) und für 
Mit dieser ersten Modifikation der Investitionshypothese, die in einer gewissen Übereinstimmung zu den Ansichten der modernen Geldtheorie über die Wirkungsverzögerungen der Notenbankplitik steht, ist in der Auseinandersetzung mit dem Föhlschen Überwälzungstheorem natürlich ein ganz entscheidender Schritt getan. In der Modellwirtschaft ist nun garantiert, daß die Anlageninvestitionen auf eine etwaige Erhöhung irgendwelcher Steuersätze kurzfristig nicht reagieren, sofern nur das Kreditangebot des Bankensektors eine genügende Elastizität aufweist, um eine Vorfinanzierung der steuerlichen Mehrbelastung zu ermöglichen. Allerdings ist mit der Annahme dieser für das Föhlsche Überwälzungstheorem wesentlichen Hypothese noch keine fertige Antwort auf die Frage nach der Steuerinzidenz diesseits oder jenseits der kurzen Frist erreicht, und es muß bei der Beurteilung der endgültigen Modellergebnisse auch stets bedacht werden, daß die zugrunde liegende Investitions hypothese noch weiterer empirischer Überprüfungen bedarf, ehe man sich auf ihren empirischen Gehalt verlassen karın.

Als zweites wird in der Modellformulierung die partielle Substitutionselastizität zwischen geplantem Arbeitseinsatz und geplantem privaten Anlagenbestand der Produktionsfunktion (4.1), $-1 /\left(1+a_{4}\right)$, auf minus eins festgesetzt (1). Diese Fixierung entspricht in ihrer verteilungstheoretischen Konsequenz den empirischen Verhältnissen der Bundesrepublik: Ermittelt man für die Bundesrepublik eine rechnerische Arbeitseinkommensquote am Beitrag des privaten Sektors zum Bruttoinlandsprodukt, so erhält man für die Quote in der Zeit von 1952 bis

die Produktionselastizität des Anlagenbestandes ein Wert von etwa 0,30 angenommen wird.

(1) R. E. Hall und D. W. Jorgenson, Taxes and Investment Behavior, a. a. O., hatten dies aufgrund der Konstruktion ihrer distributedlag-Funktion a priori unterstellen müssen. Die Unterstellung wurde von R. Eisner in: Tax Policy and Investment Behavior: Further Comment, a. a. O., S. 746 f., kritisiert. Auch die oben angestellte Regressionsschätzung entgeht dem erdrückenden Gewicht produktions theoretischer Einwände nur insofern, als sie bei der Messung der Substitutionselastizität die zeitlich verteilte Dauer des Anpassungsprozesses im Unternehmenssektor und den Einfluß amortisations - und steuerbedingter Kapitalkostenbestandteile berücksichtigt. 
heute einen annähernd stationären Trendverlauf (1), was unter den Annahmen der Grenzproduktivitätstheorie und bei Harrod-Neutralität des technischen Fortschritts mit einem starken Abweichen der langfristigen Substitutionselastizität vom Wert minus eins nicht vereinbar ist (2). Zumindest in der durch die Kapazitätsplanung des Unternehmenssektors angestrebten Einkommensverteilung tritt dieser stationäre Trendver lauf nun auch innerhalb der Modellwirtschaft auf: In der durch die Kapazitätspläne angesteuerten Einkommensverteilung entfallen alle die Verteilungseffekte, die sich bei einer von minus eins abweichenden Größe der Substitutionselastizität aus der privaten Kapitalakkumulation ergeben, ebenso die Verteilungseffekte, die bei einer von minus eins abweichenden Größe der Substitutionselastizität von der Unternehmensbesteuerung durch den Staat ausgehen und sich über die Planrealisierung auf den tatsächlichen Verteilungsprozeß auswirken.

Die Tatsache, daß durch die vorgenommene Fixierung die von M. Krzyzaniak und anderen untersuchten typisch neoklassischen Inzidenzeffekte der Unternehmensbesteuerung $z$ wangsläufig vernachlässigt werden, ist sicher ein gewisser Nachteil. Dafür erfahren die Produktionsfunktion (4.1) und die Planungsfunktionen für den Faktoreinsatz eine wesentlich formale Vereinfachung, und der aus dem Ablauf der Modellwirtschaft resultierende Verteilungsprozeß wird leichter kontrollierbar. Hierin liegt, ganz abgesehen von der gewonnenen Übereinstimmung mit den Verhältnissen der Bundesrepublik, der entscheidende

(1) Die Quote wurde berechnet unter der Annahme, daß die Selbständigen pro Kopf das gleiche durchschnittliche Arbeitseinkommen erhielten wie die Unselbständig-Beschäftigten des privaten Sektors. Siehe Tab. 2 des Tabellenanhangs. - Vgl. auch W. Krelle, Verteilungstheorie, Tübingen 1962, S. $16 \mathrm{f}$; ders., Macht und ökonomisches Gesetz in der Verteilung, in: Macht und ökonomisches Gesetz, Schriften des Vereins für Socialpolitik, N. F. 74, Berlin 1973, S. 77 ff. sowie Jahresgutachten 1972 des Sachverständigenrates zur Begutachtung der gesamtwirtschaftlichen Entwicklung, Bundesrats-Drucksache $612 / 72$ vom 6.12.1972, Tab. 38 .

(2) Die wirtschaftliche Entwicklung der Bundesrepublik weist in den 50 er Jahren abgesehen vom nachkriegsbedingten Wiederaufbauprozeß deutliche Züge eines asymptotischen Anpassungsprozesses auf, was sich am Verlauf des Kapitalkoeffizienten wie auch am Verlauf der Investitionsgüterpreise ablesen läßt. Insofern läßt sich auf sie nicht die Vorstellung eines steady-state anwenden, in dem wegen HarrodNeutralität des technischen Fortschritts etc. die Verteilungsquoten unabhängig von der Größe der Substitutionselastizität ein langfristig stabiles Gleichgewichtsniveau erreicht hätten. 
Vorteil für die Auseinandersetzung mit der kreislauftheoretischen Überwälzungstheorie.

Zusätzlich zu diesen empirisch angeregten Annahmen werden in die Modellformulierung zwei miteinander zusarnmenhängende Annahmen eingeführt, für die keine empirischen Anhaltspunkte vorliegen: Die langfristige Substitutionselastizität $z$ wischen öffentlıchem Inf rastrukturanlagenbestand und den beiden anderen Produktionsfaktoren wird willkürlich mit $-1 /\left(1+\alpha_{5}\right)=-0,50$ angesetzt. Unterstellt man, der Staat hätte Infrastrukturanlagen stets in einem solchen Umfang zur Verfügung gestellt, daß deren Grenzbeitrag zur Bruttowertschöpfung des privaten Sektors gleich dem Kapitalmarktzins sei, dann erlaubt die Fixierung der Substitutionselastizität $-1 /\left(1+a_{5}\right)$ zugleich, den numerischen Wert des Verteilungsparameters $\alpha_{1}$ von (4.1) festzulegen; bei Verwendung der Zeitreihen für den Infrastrukturanlagenbestand (1) und die private Produktion in der Bundesrepublik gelangt man so zu einem Wert von $a_{1}=+0,99$. - Aikzeptiert man diese beiden Annahmen bzw. die a-priori-Festlegung der beiden Parameter $a_{1}$ und $a_{5}$, erfährt die langfristige Produktions - und Faktornachfragetheorie nochmals eine Vereinfachung; vor allem läßt sich dann der $\mathrm{k}_{\mathrm{St}, \mathrm{t}}^{\star}$-Faktor wie für (4.19) angegeben, quartifizieren und bei der empirischen Überprüfung der Produktions- und Faktorplanungsfunktionen mit berücksichtigen.

Nach der vorgenommenen Modifizierung und Parameterfestlegung lautet die Funktion für die private Anlagenakkumulation in ihrer endgültigen Fassung:

$$
\begin{aligned}
\text { (4.44) } \quad l g \frac{k_{p r, t}}{k_{p r, t-1}=} & \alpha_{1}^{\prime \prime}+ \\
& \alpha_{2}^{\prime \prime} \cdot \lg \left[\frac{x_{t-2}}{k_{p r, t-2} \cdot r_{t-2}^{\star}} \cdot k_{S t, t-2}^{\star}\right]+ \\
& \alpha_{3}^{\prime \prime} \cdot \lg \left[\frac{\left(1+g_{x, t-2}\right)\left(1+g_{k}{ }_{k}, t-2\right)}{\left(1+g_{r \star}, t-2\right)}\right]
\end{aligned}
$$

wobei $k_{S t, t-2}^{\star}$ unter Berücksichtigung von $\alpha_{1}=+0,99$ und $\alpha_{5}=+1,00$ gemäß (4.3) definiert ist. Die endgültigen Werte der Parameter $\alpha_{1}^{\mu}$, $\alpha_{2}^{\prime \prime}$ und $\alpha_{3}^{\prime \prime}$ wurden durch eine Regressionsschätzung von (4.44) für die Jahre 1958 bis 1971 mit dem Ergebnis

(1) Dieser wurde mit $50 \%$ des gesamten öffentlichen Bruttoanlagevermögens in der Bundesrepublik angesetzt. 


$$
\text { (4.45) } \begin{aligned}
& \alpha_{1}^{\prime \prime}=-0,00274( \pm 0,00440) \\
& \alpha_{2}^{\prime \prime}=+0,04399( \pm 0,00715) \\
& \alpha_{3}^{\prime \prime}= \pm 0,02800( \pm 0,01917) \\
& R_{1 \cdot 2,3}^{2}=0,80938 \text { (Irrt.-Wahrsch.: <0,1\%) } \\
& r_{2 \cdot 3}^{2}=0,05410 \\
& \text { vNQ }=1,345
\end{aligned}
$$

bestimmt. Dabei wurde der zur Schätzung verwendete Kapitalkostensatz bei einer Risikoprämie von 1,4\% statt 1,5\% ermittelt, womit, wie im Anschluß an Regressionsergebnis (4.42) angedeutet, eine gewisse Anpassung an die auf minus eins angesetzte Substitutionselastizität zwischen den beiden privaten Produktionsfaktoren erreicht wurde (1).

(1) Mit der Quantifizierung der Parameter $\alpha_{1}^{\prime \prime}, \alpha_{2}^{\prime \prime}$ und $\alpha_{3}^{\prime \prime}$ läßt sich noch einmal für den konkreten Fall die Frage beantworten, ob dann, wenn die Modellwirtschaft ein langfristiges Wachstumsgleichgewicht erreicht hat, der von den Unternehmern erwartete reale Kapitalkostensatz gleich der physischen Grenzproduktivität des Anlagenbestandes ist bzw. ob dann die Unternehmererwartungen hinsichtlich der Anlagenplanung aufgehen.

Im langfristigen Wachstumsgleichgewicht gilt:

$$
\begin{aligned}
& r_{t}^{*}=\text { konstant } \\
& k_{S t, t}^{\star}=\text { konstant }
\end{aligned}
$$

daher

$$
\begin{aligned}
& 1+g_{r \star t}=1 \\
& 1+g_{k \star, t}=1
\end{aligned}
$$

sowie

$$
1+g_{x, t}=1+f_{a}
$$

Der Anteil der Kapitalkosten am Produktionserlös des privaten Sektors beträgt dann nach $(4.44)$ :

$$
\frac{k_{p r, t} \cdot r_{t}^{\star}}{x_{t}}=\left[\frac{a_{1}^{\prime \prime}}{\left(1+f_{a}\right)^{1-a_{3}^{\prime \prime}}}\right]^{\frac{1}{\alpha_{2}^{\prime \prime}}} \cdot k_{S t, t}^{\star}
$$


Aufgrund der Anlagenplanungsfunktion (4.44) und der zugehörigen Regressionsergebnisse (4.45) ergibt sich eine kurzfristige Elastizität des für Periode $t$ geplanten Anlagenbestandes bezogen auf die Höhe des Kapitalkostensatzes von Periode $t-2$ in Höhe von $-\left(\alpha_{2}^{\prime \prime}+a_{3}^{\prime \prime}\right)=-0,072$. $\mathrm{Da}$ das Verhältnis von realen Bruttoanlageinvestitionen zu realem Bruttoanlagenbestand während der Beobachtungsperiode etwa 7,5 \% betrug, läßt sich daraus eine Elastizität der Bruttoanlageinvestitionen bezogen auf den Kapitalkostensatz der Vorperiode von etwa minus eins ableiten. Die entsprechende Elastizität der Bruttoanlageinvestitionen bezogen auf den Zinssatz der Vorperiode beträgt ungefähr ein Viertel dieses Wertes (1).

Nach (4.4) beträgt die durch die Unternehmenskalkulation angesteuerte und grenzproduktivitätstheoretisch bestimmte Kapitalkostenquote für $a_{4}=0$ und $\alpha_{5}=+1,00$ :

$$
\frac{k_{0, p r, t} \cdot r_{0, t}^{\star}}{x_{0, t}}=\mu_{k} \alpha_{1} \cdot\left(1-a_{2}\right) \cdot k_{0, s t, t}^{\star}
$$

Ist der Wert der hier a priori festgesetzten Parameter richtig bemessen, sind die Parameter $\alpha_{1}^{\prime \prime}, \alpha_{2}^{\prime \prime}$ und $\alpha_{3}^{\prime \prime}$ zuverlässig geschätzt und stimmen die zwei Quoten in etwa miteinander überein, kann mit einem Aufgehen der Unternehmererwartungen im angesteuerten langfristigen Wachstumsgleichgewicht gerechnet werden.

(1) H. König und V. Timmermann, Ein ökonometrisches Modell für die Bundesrepublik Deutschland 1950-1960, a. a. O. , S. 638, ermittelten eine Zinselastizität der Ausrüstungsinvestitionen von -0,52 und der Wohnungsbauinvestitionen von $-0,75$. Diese Elastizitäten sind ohne Lag definiert. 
6. Die dynamische Form der Beschäftigungsplanungsfunktion, ihre empirische Überprüfung und Modifizierung

Analog zur Anlagenplanungsfunktion (4.37) gewinnt man aus (4.5) durch Auflösung der Erwartungswerte $x_{0}, t, l_{o}^{*}, p r, t, p_{o}, x, t$ und $k_{O}^{*}, S t, t$ eine dynamische Form der Beschäftigungsplanungsfunktion:

$$
\begin{aligned}
& \text { (4.46) } e_{o, p r, t}=e_{0, p r, t-1}^{1-\lambda} \cdot\left[\left(\mu_{a} \alpha_{1} \alpha_{2} \alpha_{3}^{-a_{4}}\right)^{\frac{1}{1+\alpha_{4}}} \cdot\left(\frac{1 p_{p, t-1}^{*}}{p_{x, t-1}}\right)^{\frac{1}{1+\alpha_{4}}} .\right. \\
& \left.-\frac{a_{4}}{1+a_{4}}(t-1-1960)-\frac{a_{4}-a_{5}}{1+a_{4}} \cdot x_{t-1}\right]^{\lambda} \text {. } \\
& {\left[\left(1+f_{a}\right)^{-\frac{\alpha_{4}}{1+\alpha_{4}}} \cdot\left(\frac{1+g_{1} \star t-1}{1+g_{p, t-1}}\right)^{-\frac{1}{1+\alpha_{4}}}\right.} \\
& \left.\left(1+g_{k *, t-1}\right)^{-\frac{a_{4}-a_{5}}{1+a_{4}}} \cdot\left(1+g_{x, t-1}\right)\right]
\end{aligned}
$$

Setzt man in diese Funktion die für die Anlagenplanungsfunktion akzeptierten Substitutionsparameter $\alpha_{4}=0,00$ und $\alpha_{5}=+1,00$ ein, so erhält man:

$$
\begin{aligned}
\text { (4.47) } e_{o, p r, t}= & e_{0, p r, t-1}^{1-\lambda} \cdot\left[\left(\mu_{\dot{a}} a_{1} \cdot a_{2}\right) \cdot \frac{x_{t-T^{p}, t-1}}{I_{p r, t-1}^{\hbar}} .\right. \\
& \left.k_{S t, t-1}^{\star}\right]^{\lambda} \cdot\left[\left(1+g_{x, t-1}\right) \cdot \frac{1+g_{p, t-1}}{1+g_{1} \star t-1} .\right. \\
& \left.\left(1+g_{k \star t-1}\right)\right]
\end{aligned}
$$

wobei $k_{S t, t-1}^{\star}$ gemäß (4.3) definiert ist.

Sieht man einmal von dem Einfluß der staatlichen Inf rastrukturanlagen $a b$, die sich über $k_{S t, t-1}^{\star}$ und $1+g_{k}^{\star}, t-1$ auswirken, enthält (4.47) eine einfache Aussage, die dann besonders deutlich wird, wenn man die ganze 


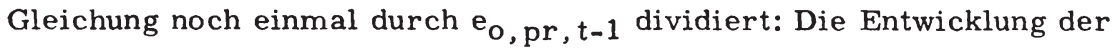
geplanten Beschäftigung wird bestimmt durch die Wachstumsrate der Produktion und den Anstieg des Reallohns bzw. des realen Arbeitskostensatzes, die den Wert der zweiten eckigen Klammer bestimmen. Außerdem ist sie abhängig von dem Umfang, in dem die für Periode t-1 geplante Beschäftigung sich aus der Sicht der Unternehmen im nachhinein als falsch erweist, bzw. von dem Umfang, in dem die für t-1 geplante Beschäftigung von dem Wert der in t-1 realisierten Beschäftigung abweicht (1).

Nach Erweiterung mit epr,t-1, dem Umfang der tatsächlichen Beschäftigung in Periode $t-1$, ergibt $(4.47)$ :

$$
\begin{aligned}
& \text { (4.48) } e_{o, p r, t}=e_{o, p r, t-1} \cdot\left[\frac{e_{p r, t-1}}{e_{o, p r, t-1}}\right]^{-\lambda} \text {. } \\
& {\left[\left(\mu_{a} a_{1} a_{2}\right) \cdot \frac{x_{t-1 \cdot{ }_{x, t-1}}}{e_{p r, t-1} \cdot{ }_{p r, t-1}^{\star}} \cdot k_{S t, t-1}^{\star}\right]^{\lambda} .} \\
& {\left[\left(1+g_{x, t-1}\right) \cdot \frac{1+g_{p, t-1}}{1+g_{I} \star t-1} \cdot\left(1+g_{k}, t-1\right)\right]}
\end{aligned}
$$

Da es sich bei der Anlagenplanungsfunktion als zweckmäßig erweist, davon auszugehen, daß das Planungsverhalten unabhängig von den laufenden Ereignissen der Planungsperiode ist, wird die Verzögerung in der $z$ weiten und dritten Klammer von (4.48) um eine Periode verlängert. Die Verzögerung innerhalb der ersten eckigen Klammer wird jedoch unverändert gelassen, und zwar aus folgendem Grund:

Eine ganze Reihe empirischer und theoretischer Untersuchungen kommt zu dem Ergebnis, daß die Beschäftigung von Arbeitskräften im privaten Sektor bei konjunkturellen Schwankungen von Produktion und Güterabsatz kurzfristig nicht so stark variiert, wie man das aufgrund makroökono-

(1) Nach (4,5) ist $e_{0}, \mathrm{pr}, \mathrm{t}-1$ für $\alpha_{4}=0$ und $\alpha_{5}=1$ gleich dem Wert des Ausdrucks innerhalb der zweiten eckigen Klammer von (4.48), wenn sich die Beschăftigungsplanung als richtig erweist. 
mischer grenzproduktivitätstheoretischer Überlegungen erwartet (1). Für die Beobachtung werden mehrere Gründe genannt: der kurzfristig fixe Charakter eines Teils der eingesetzten Arbeit, Kündigungsschutz, Vorherrschen längerfristigen Gewinnmaximierungsstrebens und die Möglichkeit, daß freie Arbeitskraftreserven als Substitut für Güterläger angesehen werden und zum Teil deren Funktionen übernehmen, sowie die Schwierigkeit, in der Rezession entlassene Arbeitskräfte im Boom zurückzugewinnen. - Die Beobachtung ist von elementarer Bedeutung für die im nächsten Paragraphen entwickelte Theorie des kurzfristigen Unternehmensverhaltens, sie muß konsequenterweise aber auch hier berücksichtigt werden. Wenn nämlich Unternehmen in einer Rezession überschüssige Arbeitskräfte entlassen möchten, dies aber aus Gründen des

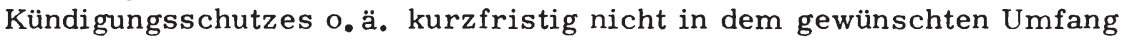
tun, dann kann damit gerechnet werden, daß sie die kurzfristig unterlassene Beschäftigungsanpassung zumindest in der Beschäftigungsplanung für die nächste Periode ganz oder zum Teil nachholen (2). - In der Behauptung eines derartigen Nachholeffektes besteht die Zusatzhypothese, die bei der endgültigen Formulierung der Beschäftigungsplanungsfunktion akzeptiert wird, wenn die Verzögerung in der ersten eckigen Klammer von (4.48) nicht um eine weitere Periode verlängert wird.

Die Beschäftigungsplanungsfunktion hat daher in ihrer endgültigen Formulierung folgende Gestalt, in der sie einer empirischen Kontrolle unterzogen wird:

(1) Vgl. W. G. Wilson, The Relationship between Output and Employment, Review of Economics and Statistics, 42, 1960, S. 37 ff. - Th. A. Wilson und O. Eckstein, Short Run Productivity Behavior in U.S. Manufacturing Review of Economics and Statistics, 46, 1964, S. $41 \mathrm{ff}$. - R. M. Solow, Distribution in the Long and Short Run, in: J. Marchal und B. Ducros (Hrsg.), The Distribution of National Income, London 1968, S. 449 ff. - R. L. Miller, The Reserve Labour Hypothesis: Some Tests of its Implications, Economic Journal, 81 , 1971 , S. $17 \mathrm{ff}$.

(2) Anhaltspunkte für einen derartigen Nachholeffekt ergeben sich aus den Untersuchungsergebnissen des Sachverständigenrates in seinem Jahresgutachten 1972, BR-Drucksache 612/72, Bonn 1972, Tz. 457 sowie aus den Ergebnissen des Ifo-Konjunkturtests in Ifo-Schnelldienst 1974 , Heft 19 , S. $4 \mathrm{f}$. 
(4.49) $e_{o, p r, t}=e_{0, p r, t-1} \cdot\left[\frac{e_{p r, t-1}}{e_{0, p r, t-1}}\right]^{B_{1}}$.

$$
\begin{aligned}
& {\left[\beta_{0} \cdot \frac{x_{t-2 \cdot p_{x, t-2}}}{e_{p r, t-2} \cdot 1_{p r, t-2}^{\star}} \cdot k_{S t, t-2}^{\star}\right]^{B_{2}} .} \\
& {\left[\left(1+g_{x, t-2}\right) \cdot \frac{1+g_{p, t-2}}{1+g_{1 *, t-2}} \cdot\left(1+g_{k *, t-2}\right)\right]^{B_{3}}}
\end{aligned}
$$

In der empirischen Überprüfung liegt das eigentliche Problem der Beschäftigungsplanungsfunktion: Wegen des Fehlens einer der Gestationshypothese (4.38) analogen Annahme enthält die Beschäftigungsplanungsfunktion bzw. Gleichung (4.49) unbekannte Erwartungsgrößen (1) und entzieht sich damit einer direkten Überprüfung mit Hilfe von Regressionsschätzungen. Die empirische Kontrolle ist daher auf ein indirektes Verfahren angewiesen, das einen Vorgriff auf die im folgenden Paragraphen für die kurze Frist entwickelte Produktions- und Faktornachfragetheorie erfordert:

Als Funktionsparameter werden zuerst die in (4.45) für die Anlagenplanungsfunktion geschätzten Parameterwerte eingesetzt; die Werte von $\beta_{1}$ und $\beta_{2}$ werden also dem dortigen Wert von $\alpha_{2}$, derWert von $\beta_{3}$ dem Wert von $\alpha_{3}^{\prime \prime}$ gleichgesetzt, der Wert von $\beta_{0}$ muß a priori bestimmt werden. Zusätzlich wird für ein Ausgangsjahr ein konjunkturbereinigter Wert der tatsächlichen privaten Beschäftigung anstelle des Wertes der geplanten Beschäftigung in die Funktion eingesetzt.

Mit Hilfe dieses Basiswertes der Planbeschäftigung, der angenommenen Parameterwerte und des statistischen Materials für die übrigen Funktionsvariablen wird dann eine Sequenz der $e_{0}, \mathrm{pr}, \mathrm{t}$-Werte berechnet. Die erhaltenen $e_{o}, p r, t-$ Werte werden durch entsprechende Werte für den tatsächlichen Umfang der privaten Beschäftigung in den verschiedenen Jahren des Beobachtungszeitraumes dividiert und ergeben eine Zeitreihe für die Abweichung der tatsächlichen privaten Beschäftigung

(1) Einen empirischen Zugang zur weiteren Erforschung der Erwartungsbzw. Plangröße "Planbeschäftigung" eröffnen möglicherweise die Angaben der Erwerbsstatistik über die Zahl der offenen Stellen. Die Zahl der offenen Stellen ist jedoch definitionsgemäß positiv und daher nicht gleichzusetzen mit der Differenz zwischen geplanter und tatsächlicher Beschäftigung, die positiv oder negativ sein kann. Ein direkterRückschluß von der Zahl der offenen Stellen auf den Umfang der Planbeschäftigung ist also nicht möglich. 
von der hypothetisch ermittelten privaten Planbeschäftigung, $e_{p r, t} / e_{o, p r, t}$. Stimmt die hypothetisch ermittelte Planbeschäftigung mit der tatsächlichen Planbeschäftigung der Unternehmen überein, muß nach der im folgenden Paragraphen noch zu entwickelnden kurzfristigen Produktionsund Faktornachfragetheorie der Quotient $e_{\mathrm{pr}, \mathrm{t}} / \mathrm{e}_{\mathrm{o}}, \mathrm{pr}, \mathrm{t}$ mit dem Arbeitskostenanteil am Produktionserlös des Unternehmenssektors korrelieren. Es wird daher die Korrelation zwischen beiden Zeitreihen ermittelt.

Das ganze Verfahren wird bei systematischer Variation der Funktionsparameter von (4.47) und des Ausgangswertes der Planbeschäftigung solange wiederholt, bis sich ein maximaler Wert des Korrelationskoeffizienten ergibt.

Es sei darauf hingewiesen, daß bei den vorgenommenen Berechnungen der Arbeitskostensatz $l_{\mathrm{p} r}^{\star}, t$ noch abweichend von (4.12) bis (4.14) definiert worden war (1) und daß die erzielten Parameterwerte daher wahrscheinlich geringfügig von den Werten abweichen, die bei einer Abgrenzung gemäß (4.12) bis (4.14) erzielt worden wären. Die Parameterwerte werden demnach, abgesehen von einer Korrektur des Niveauparameters ${ }^{\beta_{0}}$, unverändert der späteren Modellrechnung zugrundegelegt.

Außerdem wurde als Kontrollkriterium die rechnerische Arbeitseinkommensquote und zusätzlich der Anstieg der Arbeitsproduktivität im privaten Sektor der Bundesrepublik gewählt, der nach den Modellvorstellungen über das kurzfristige Unternehmensverhalten ebenfalls ein sinnvoller Indikator für die Abweichungen der Beschäftigung von ihrer Plangröße ist. Zur Kontrolle wurden also zwei Korrelationen berechnet (2). Die absoluten Werte beider Korrelationskoeffizienten wurden addiert; akzeptiert wurden die Parameterwerte, bei denen die so erhaltene Koeffizientensumme ein Maximum aufwies. Dieses Verfahren wurde nur zur Bestimmung der Parameter $B_{1}, B_{2}$ und $B_{3}$ sowie zur Bestimmung des "optimalen" Ausgangswertes der Planbeschäftigung für das Jahr 1952 angewandt. Parameter $\beta_{0}$ wurde mit dem Durchschnitt der modifizierten rechnerischen Arbeitskostenquote $\left(e_{\mathrm{pr}, t} \cdot \mathrm{l}_{\mathrm{pr}, t}\right) /\left(\mathrm{x}_{\mathrm{t}} \cdot \mathrm{p}_{\mathrm{x}, \mathrm{t}} \cdot \mathrm{k}_{\mathrm{S}} \mathrm{t}, \mathrm{t}\right)$

(1) Statt wie in (4.13) wurde $t C, t$ in (4.14) als $T_{C, t} /\left(x_{t} \cdot p_{x, t}\right)$ angesetzt, was in etwa der Erhebung der Bruttoumsatzsteuer in der Bundesrepublik vor 1968 Rechnung trug.

(2) Es wurden Spearman'sche Rangkorrelationskoeffizienten berechnet. Der Grad der erreichten Annäherung dürfte deswegen nicht ganz so hoch wie bei Verwendung des Bravais-Pearson'schen Korrelationskoeffizienten sein. Das gilt vor allem hinsichtlich Parameter $B_{2}$, für den der Rangkorrelationskoeffizient im Intervall $0,034 \leq \mathrm{B}_{2} \leq$ 0,048 den gleichen, maximalen Wert annahm. 
während der Jahre 1952 bis 1971 angesetzt und nicht variiert.

Die aufgrund des Annäherungsverfahrens akzeptierten Parameterwerte der Beschäftigungsplanungsfunktion (4.49) sind (1):

$$
\text { (4.50) } \begin{aligned}
& B_{0}=+0,667 \\
& B_{1}=+0,820 \\
& B_{2}=+0,040 \\
& B_{3}=+0,690 \\
& \frac{e_{\text {pr, } 1952}}{e_{o, p r}, 1952}=1,000
\end{aligned}
$$

Die entsprechende Reihe des Quotienten aus tatsächlicher privater Beschäftigung und hypothetischer privater Beschäftigung für die Jahre 1952 bis 1971 korreliert mit der rechnerischen Arbeitseinkommensquote des privaten Sektors zu $\mathrm{r}^{2} \approx 0,162$ und mit dem jährlichen Anstieg der Arbeitsproduktivität im privaten Sektor zu $\mathrm{r}^{2}=0,409$ (2).

Die niedrigen Werte der Korrelationskoeffizienten legen die Vermutung nahe, daß noch keine zufriedenstellende Formulierung der Beschäftigungsplanungstheorie oder noch keine zufriedenstellende Methode ihrer empirischen Überprüfung gefunden worden ist (3). Das Gesamtergebnis der Berechnungen wirft jedoch ein überraschendes Licht auf einen stark vernachlässigten Sektor der makroökonomischen Theorie. Während der Berechnungen ergaben sich deutliche Anzeichen dafür, daß die bei der Aufstellung der Beschäftigungsplanungsfunktion (4.49) formulierte Zusatzhypothese ein wichtiger Bestandteil der Theorie der Beschäftigungsplanung ist und daß man nicht von einer vollen Symmetrie in der formalen Struktur der beiden Faktorplanungsfunktionen ausgehen kann: Erst, wenn die Reaktionen der Planungsbeschäftigung auf Fehl-

(1) Zur Beurteilung des erzielten Wertes von $e_{p r}, 1952 / e_{o}, p r, 1952$ und der damit behaupteten Situation vgl. die Bemerkung des Wissenschaftlichen Beirats beim Bundesministerium für Wirtschaft in seinem Gutachten vom 18.1.1953 zum Thema "Sicherung der wirtschaftlichen Expansion": "Die jetzige Wirtschaftslage der Bundesrepublik zeigt keine Kennzeichen ausgesprochen inflationistischen oder deflationistischen Charakters. "Vgl. derselbe, Sammelband der Gutachten von 1948 bis 1972, Göttingen 1973, S. 169.

(2) Die Reihe findet sich in Tabelle 3 des Tabellenanhangs.

(3) Mit einer abgeänderten Formulierung der Beschäftigungsplanungsfunktion wurden bei Vernachlässigung der steuerlichen Arbeitskostenbestandteile höhere Korrelationskoeffizienten erzielt, aber die Reihe der $e_{p r, t} / e_{o}, p r, t-W e r t e$ oszillierte mit zunehmender Amplitude. 
anpassungen in der Vor-bzw. Planungsperiode berücksichtigt werden, spiegelt sich die konjunkturelle Entwicklung der Bundesrepublik in den Abweichungen der tatsächlichen Beschäftigung von der hypothetischen Beschäftigung so wieder, wie das a priori vermutet wurde.

Die durch die Zusatzhypothese behaupteten Reaktionen der Beschäftigungsplanung auf Fehlanpassungen in der Planungsperiode erweisen sich als unerwartet stark. Entsprechend der geschätzten Größe des Parameters $\beta_{1}$ veranlassen kurzfristige Beschäftigungsabweichungen von der Plangröße die Unternehmen dazu, die Beschäftigungsplanung für die nächstfolgende Periode in der gleichen Richtung und fast in dem gleichen Umfang zu korrigieren; die Plankorrektur beträgt ungefähr $82 \%$ der kurzfristigen Abweichung. Wegen dieser unerwarteten Stärke der Reaktionen kennzeichnen die Beschäftigungspolitik der Unternehmen zwei Eigenschaften, die für den Ablauf des Wirtschaftsprozesses, zumindest im Modell, folgenreich sind:

Hauptsächlich als Folge der starken, mit einer Verzögerung von einem Jahr wirksamen Reaktionen weist die Planungsbeschäftigung einen ausgesprochen prozyklischen Charakter auf. Ihre konjunkturellen Schwankungen verlaufen genau in umgekehrter Richtung wie die konjunkturellen Schwankungen der tatsächlichen Beschäftigung (1). Da die konjunkturellen Schwankungen der Planbeschäftigung zu einem erheblichen Teil für die Abweichungen zwischen der tatsächlichen Beschäftigung und der Planbeschäftigung verantwortlich sind und diese Abweichungen im Konjunkturzyklus ein erhebliches Ausmaß annehmen, muß vermutet werden, daß die über die kurze Frist von einem Jahr hinausgehende Beschäftigungspolitik der Unternehmen mit ihren Nachholwirkungen zu den starken konjunkturerhaltenden Kräften des Wirtschaftssystems gehört.

Schwerwiegender ist die andere Konsequenz: Wahrscheinlich treten die starken Reaktionen der Planbeschäftigung auf Fehlanpassungen in der Vor- bzw. Planungsperiode nicht nur in den konjunkturellen Wechsellagen, sondern auch bei fundamentalen Störungen des Beschäftigungsgleichgewichts in Erscheinung. Die Entwicklung der rechnerischen Arbeitseinkommensquote im privaten Sektor der Bundesrepublik, die hier als Indikator für Abweichungen der privaten Beschäftigung von der Plangröße angesehen wird, liefert dazu einen ersten empirischen Anhaltspunkt: Der Verlauf der Quote hat sich in der Nachkriegszeit bereits 1952 auf sein mittelfristig stabiles Niveau eingespielt (2) und deutet aus

(1) Vgl. Tabelle 3 des Tabellenanhangs.

(2) Der Verlauf der Quote in den Jahren 1950 und 1951 wurde nicht zur Überprüfung der Beschäftigungsplanungsfunktion mit Hilfe der Korrelationskoeffizienten herangezogen und kann daher durchaus als Argument dienen. 
der Sicht der hier aufgestellten Theorie an, daß zu diesem Zeitpunkt der durch die unmittelbare Nachkriegsentwicklung bedingte Anpassungsproze $\beta$ der privaten Beschäftigungsplanung abgeschlossen war (1). Im Gegensatz dazu deutet die Entwicklung des Kapitalkoeffizienten im privaten Sektor darauf hin, daß die private Kapitalakkumulation nur einen allmählichen Anpassungsprozeß vollzogen hat, der heute noch andauert. Wahrscheinlich wird man davon ausgehen müssen, daß die Beschäftigungsplanung sich grundsätzlich wesentlich schneller als die Anlagenplanung an die laufenden Preis- und Nachfrageverhältnisse anpaßt, und den Grund für das unterschiedliche Anpassungstempo darin suchen, daß Fehlentscheidungen in der Investitionspolitik der Unternehmen sich nur schwer korrigieren lassen und die Rentabilität der Unternehmen möglicherweise auf Jahre belasten, während Fehlentscheidungen in der Beschäftigungspolitik in verhältnismäßig kurzer Zeit behoben werden können. Da die Unternehmen sich in der Beschäftigungsplanung weniger vorsichtig zu verhalten brauchen als in der Planung des Anlagenbestandes, bauen sie Ungleichgewichte im Faktoreinsatz beim Faktor Arbeit schneller ab als beim Faktor Kapital.

Das hohe Anpassungstempo der Beschäftigungsplanung hat unmittelbare Auswirkungen auf den Prozeß der Einkommensverteilung. Wenn die Anpassung der Beschäftigungsplanung an die laufenden Marktverhältnisse sich im Vergleich zur Anlagenplanung sehr rasch vollzieht, dann muß sich auch die durch Ungleichgewichtssituationen entstandene Schere zwischen realisiertem Faktorlohn und dem in der Vorausplanung der Unternehmen angesetzten langfristigen Grenzerlösprodukt beim Faktor Arbeit wesentlich schneller schließen als beim Faktor Kapital. Geht das Wirtschaftssystem daher von einem Wachstumsungleichgewicht aus und paßt es sich einem Gleichgewichtspfad des langfristigen Wirtschaftswachstums an, dann fallen nur ganz zu Beginn dieses Anpassungsprozesses im Unternehmenssektor Anpassungsgewinne oder Anpassungsverluste an, die den Anteil der gesamten Besitzeinkommen am Produktionserlös zulasten oder zugunsten der Arbeitseinkommensquote ändern. Soweit bis zum Ende des Anpassungsprozesses Anpassungsgewinne oder Anpassungsverluste bestehenbleiben, gehen diese zulasten oder zugunsten der Zinseinkommensquote und verändern damit lediglich die funktionale Struktur der Besitzeinkommen (2).

Dieses Ergebnis mag rein verteilungstheoretisch schon von einiger Bedeutung sein; das hohe Anpassungstempo der Arbeitseinsatzplanung

(1) Das heißt nicht zugleich, daß die Arbeitslosigkeit der deutschen Nachkriegswirtschaft absorbiert war.

(2) Eine entsprechende Umstrukturierung der Besitzeinkommen zwischen Zins- und Gewinneinkommen scheint sich während der Nachkriegszeit in der Bundesrepublik vollzogen zu haben. Vgl. S. $135 \mathrm{f}$. 
hat zugleich aber eine ganz schwerwiegende inzidenztheoretische Konsequenz: Man unterstelle einmal, der Staat erhöhe in einem Jahr die Gewinnsteuersätze, er verausgabe das Steuermehraufkommen nachfragewirksam und wegen der verzögerten Reaktionen der Unternehmen in der Vorausplanung der Faktoreinsätze käme es innerhalb des Jahres kreislaufbedingt zu einer vollen Überwälzung der Steuererhöhung auf die Bezieher kontraktbestimmter Faktoreinkommen - so sieht der vollzogene kreislaufmäßige Überwälzungsprozeß aus der Sicht der aufgestellten neoklassischen Theorie ja aus! Dann führt die Anpassung der Faktoreinsätze an die gestiegene Nachfrage auf die Dauer dazu, daß der kreislauf - bzw. verzögerungsbedingte Überwälzungseffekt abgebaut wird und sich stattdessen die Inzidenzeffekte durchsetzen, die der Unternehmenssektor nach Einbeziehung der Steuererhöhung in sein grenzproduktivitätsorientiertes Planungskalkül ansteuert. Dieser Abbau des Überwälzungseffektes vollzieht sich auf Seiten des Faktors Kapital wegen der verhältnis mäßig langsamen Anpassung der Anlagenplanung nur allmählich und dauert über Jahrzehnte, sofern nicht ein Anstieg des Kapitalmarktzinses den Prozeß abkürzt. Auf Seiten des Faktors Arbeit vollzieht er sich wegen der raschen Anpassung der Beschäftigungsplanung innerhalb weniger Jahre; nach Ablauf dieser Jahre ist der Überwälzungseffekt bzw. die Minderung der Arbeitseinkommensquote wieder verschwunden (1). Konfrontiert man diese Schlußfolgerung mit der kreislauf theoretischen Überwälzungstheorie Föhlscher Prägung, kommt man zu dem Ergebnis, daß diese Theorie in der Modellwirtschaft ihre Gültigkeit nur für eine Übergangszeit von wenigen Jahren behält; jenseits dieser Übergangszeit hat sie ihre Bedeutung zugunsten der neoklassischen Inzidenztheorie eingebüßt. Mit anderen Worten, eine Schwäche der kreislauftheoretischen Überwälzungstheorie, so wie sie in der Föhlschen Überwälzungsdiskus sion aufgestellt wurde, ist die Vernachlässigung des längerfristigen Faktornachfrageverhaltens, insbesondere des längerfristigen Arbeitsnachfrageverhaltens.

\section{Die geplante Produktion}

Während der bisherigen Überlegungen wurden die Substitutionselastizität zwischen geplantem privatem Arbeitseinsatz und geplantem privatem Anlagenbestand auf minus eins, die Substitutionselastizität $z$ wischen diesen beiden Faktoren und dem öfentlichen Infrastrukturanlagenbestand auf minus einhalb und der Verteilungsparameter $\alpha 1$ auf t0,99 festgesetzt. Aufgrund dieser numerischen Fixierung erhält die Produktionsfunktion

(1) Die geschätzte Größe des Parameters $\beta_{1}$ deutet bereits an, daß die Anpassung der Beschäftigungsplanung und damit der Abbau des Überwälzungseffektes sich zum überwiegenden Teil im ersten Folgejahr vollzieht. 
(4.1) bzw. (4.2), die die langfristigen Produktionsmögiichkeiten der Modellwirtschaft so beschreibt, wie sie auch von den Unternehmen richtig eingeschätzt werden, folgende vereinfachte Gestalt:

(4.51) $x_{o, t}=\left[a_{3} \cdot e_{0, p r, t} \cdot\left(1+f_{a}\right)^{t-1960}\right]^{\alpha_{2}} \cdot k_{o, p r, t}^{1-\alpha_{2}} \cdot k_{o, s t, t}^{\star}$ wobei $\mathrm{k}_{\mathrm{O}}^{\star}$, St, $\mathrm{t}$ gemäß $(4.3)$ definiert ist.

Die drei in (4.51) eingehenden unabhängigen Variablen sind inzwischen bestimmt. Die Plangrößen von privatem Anlagenbestand und privater Beschäftigung wurden in den beiden vorangegangenen Abschnitten behandelt. Die Erwartungsgröße $k_{0}^{\star}, \mathrm{St}, \mathrm{t}$ ergibt sich aus den Gleichungen (4.29) und (4.36) bzw, aus der von diesen abgeleiteten Gleichung (4.52):

(4.52) $k_{0, S t, t}^{\star}=k_{0, S t, t-1}^{\star} \cdot\left[1+g_{k}, t-1\right] \cdot\left[\frac{k_{S t, t-1}^{\star} t}{k_{0, S t, t-1}^{\star}}\right]^{\lambda}$

Nach Verlängerung des lags in den eckigen Klammern von (4.52) um eine weitere Periode lautet ihre Bestimmungsfunktion:

(4.53) $k_{0, s t, t}^{*}=k_{0, S t, t-1}^{*} \cdot\left[1+g_{k}, t-2\right] \cdot\left[\frac{k_{S t, t-2}^{*}}{k_{0, S t, t-2}^{*}}\right]^{\lambda}$

wobei für $\lambda$ der für die Anlagenplanungsfunktion in (4.45) angegebene Wert von $\alpha_{2}^{\prime \prime}$ angenommen wird.

Mit der Gleichung wurde eine zeitliche Sequenz von Modellwerten für $\mathrm{k}_{\mathrm{O}, \mathrm{St}, \mathrm{t}}^{*}(\mathrm{t}=1952, \ldots)$ berechnet. Dabei wurde auf die Reihe der $\mathrm{k}_{\mathrm{S} t, \mathrm{t}^{-}}$ Werte zurückgegriffen, die bereits zur Schätzung der Anlagenplanungsfunktion (4.44) benötigt und dort gemäß (4.3) unter der Annahme $\alpha_{1}=$ $+0,99$ und $\alpha_{5}=+1,00$ aus den statistisch belegten Werten von $x_{t}$ und $\mathrm{k}_{\mathrm{St}, \mathrm{t}}$ ermittelt wurde (1). Anstelle der entsprechenden Erwartungswerte für die Ausgangsjahre 1950 und 1951 wurden konjunkturbereinigte Werte der Reihe eingesetzt.

Damit lagen für alle drei unabhängigen Variablen der Produktionsfunktion (4.51) Modellwerte für die Jahre 1950 bis 1971 vor.

Der noch unbekannte Wert der Produktionselastizität $\alpha_{2}$ wurde a priori

(1) Es sei noch einmal darauf hingewiesen, daß dabei ein öffentlicher Infrastrukturanlagenbestand in Höhe von $50 \%$ des gesamten staatlichen Bruttoanlagevermögens in der Bundesrepublik unterstellt wurde. 
auf 0,75 festgesetzt (1). Bei Verwendung dieses Wertes wurden schließlich der Wert des Effizienzparameters $\alpha_{3}$ und die Rate des technischen Fortschritts, $f_{a}$, durch eine einfache Trendanpassung an den Verlauf der privaten Produktion ermittelt.

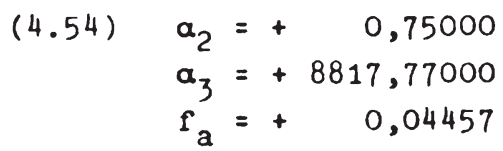

Nachdem für den zeitlichen Verlauf der unabhängigen Variablen und für die Parameter der Produktionsfunktion Modellwerte vorlagen, konnte aus (4.51) eine Zeitreihe für die Plankapazität bzw. den geplanten Output $\mathbf{x}_{\mathbf{0}, \mathrm{t}}$ des Unternehmenssektor berechnet werden. Die Werte dieser Reihe liegen im Boom unter und in Rezessionsphasen über den entsprechenden Werten für die private Produktion der Bundesrepublik und zeigen damit bereits, daß in der Modellformulierung die Plankapazität nicht ein kurzfristig starres Kapazitätsmaximum setzt, sondern lediglich ein Optimum der mittelfristigen Kapazitätsauslastung kennzeichnet, von dem die Produktion des Unternehmenssektors kurzfristig in beiden Richtungen abweichen kann.

Bezieht man den Beitrag des privaten Sektors zum Bruttoinlandsprodukt der Bundesrepublik auf die hypothetisch ermittelte Plankapazität, so erhält man einen Quotienten für die Kapazitätsauslastung des Sektors. Der Quotient (2) korreliert mit dem vom Deutschen Institut für Wirtschaftsforschung für die Industrie geschätzten Kapazitätsausnutzungsgrad (3) während der Jahre 1950 bis $1971 \mathrm{zu} \mathrm{r} \mathrm{r}^{2}=+0,733$.

(1) Unter der Annahme, daß der rechnerische Arbeitskostenanteil an der Bruttowertschöpfung des privaten Sektors im Durchschnitt der Jahre 1952 bis 1971 dem Grenzerlösproduktanteil des Faktors Arbeit gleich war, und bei dem unterstellten Einfluß des staatlichen Infrastrukturkapitals auf die private Produktionskapazität entspricht die Festsetzung einem Monopolgradfaktor von $\mu_{a}=0,889$. - Zur Größe der Produktions elastizität vgl. Gehrig und K. C. Kuhlo, Ökonometrische Analyse des Produktionsprozesses, Ifo-Studien, 7, 1961, S. $222 \mathrm{ff}$.

(2) Die Zeitreihe des Quotienten findet sich in Tabelle 4 des Tabellenanhangs.

(3) Die Angaben für die Jahre 1950 bis 1965 wurden entnommen aus G. Menges und J. Großmann, Ökonometrische Untersuchungen der Preisentwicklung in der Bundesrepublik Deutschland, Schriftenreihe der Wirtschaftsvereinigung Eisen- u. Stahlindustrie zur Wirtschafts- und Industriepolitik, Heft 9, Düsseldorf 1968, S. 87. Die Angaben für die Jahre 1965 bis 1971 entstammen dem Wochenbericht des DIW, 1972, Heft 32, S. 283. Beide Reihen wurden 1965 verknüpft. 
5 Die kurzfristige Anpassung von

Produktion und Beschäftigung

1. Die Grundannahmen der kurzfristigen Produktions und Faktornachfragetheorie

In diesem Paragraphen wird das logische Ergänzungsstück zur Theorie der Kapazitätsplanung entwickelt, die Theorie der kurzfristigen Kapazitätsauslastung: Wenn die Unternehmen der Modellwirtschaft die Faktoreinsätze vorausplanen und bereits während der Planungsperiode die entsprechenden Anlageninvestitionen vornehmen, dann ist wegen der Gestationsperiode von einem Jahr und wegen des hier fest vorgegebenen Anlagenabgangs der Anlagenbestand für die nächstfolgende Periode bestimmt und kann während dieser Periode kurzfristig nicht mehr geändert werden. Sind die neuen Anlagen erstellt und produktionsbereit, besteht der nächste Schritt der Unternehmen darin, die Beschäftigung kurzfristig dem geplanten Niveau anzupassen. Bei dieser Anpassung stellen die Unternehmen möglicherweise fest, daß ihre Erwartungen hinsichtlich der Güternachfrage, der Faktorpreise etc. nicht völlig aufgehen und daß deswegen kurzfristig Änderungen im Produktionsprogramm erforderlich sind. Da der Anlagenbestand kurzfristig fix vorgegeben ist, werden sie ihre mengenmäßigen Korrekturen allein bei der Durchführung der geplanten Beschäftigungsanpassung anbringen und mit der Änderung allein des Arbeitseinsatzes der Gefahr einer Überproduktion zu entgehen oder nicht vorhergesehene Produktionschancen auszunutzen suchen. Diese Korrekturen werden - das ist eine wichtige Eigenschaft des kurzfristigen Unternehmensverhaltens hinsichtlich Produktion und Beschäftigung stets in Hinsicht auf das geplante mittelfristige Optimum der Kapazitätsauslastung vorgenommen. Die hier entwickelte Theorie der Kapazitätsauslastung ist also eine Theorie der Abweichung von Produktion und Beschäftigung von dem vorausgeplanten Optimalniveau.

Den Kern der kurzfristigen Produktions- und Beschäftigungstheorie bildet die kurzfristige Produktionsfunktion, die sich mathematisch mit der langfristigen Produktionsfunktion (4.1) bzw. (4.2) zu einer einzigen Funktion verknüpfen läßt.

$$
\begin{aligned}
x_{t}= & x_{0, t} \cdot\left[\delta_{1} \cdot\left(\frac{e_{p r, t}}{e_{0, p r, t}}\right)^{-\delta_{4}}+\delta_{2} \cdot\left(\frac{k_{p r, t}}{k_{0, p r, t}}\right)^{-\delta_{4}}+\right. \\
& \delta_{3} \cdot\left(\frac{k_{S t, t}}{k_{0, s t, t}}\right)
\end{aligned}
$$

Bringt man die vorausgeplante Größe $x_{0, t}$ auf die linke Gleichungsseite, entpuppt sich (5.1) als eine gewöhnliche CES-Produktionsfunktion mit 90 
einer Substitutionselastizität von $-1 /\left(1+\delta_{4}\right)$, in die die Variablen, also Output und Faktoreinsätze, nicht, wie gewohnt, mit ihrem absoluten Umfang eingehen, sondern nur in Form von Meßziffern, die auf die jeweiligen Plan- bzw. Erwartungsgrößen basiert sind (1). Die Funktion enthält also nur die Abweichungen der Produktion und Faktoreinsätze von ihrem Plan- bzw. Erwartungsniveau als Variable.

Da der private Anlagenbestand wegen der Gestationsannahme (4.38) kurzfristig nicht geändert werden kann und damit ex definitione mit seiner Plangröße übereinstimmt, entfällt in (5.1) die mittlere runde Klammer.

$$
\frac{\mathrm{k}_{\mathrm{pr}, t}}{\mathrm{k}_{\mathrm{o}, \mathrm{pr}, \mathrm{t}}}=1
$$

Der öffentliche Infrastrukturanlagenbestand kann wegen der Ausreifungszeit der öffentlichen Anlagenzugänge, die hier, wie bei den privaten Anlagen mit einem Jahr angesetzt wird, ebenfalls nicht kurzfristig variiert werden. Er ist aber nicht zwangsläufig so groß, wie die Unternehmen das erwartet haben. Die dritte runde Klammer von (5.1) enthält daher eine kurzfristig konstante, meist geringfügig von eins abweichende Größe:

$$
\frac{k_{S t, t}}{k_{0, S t, t}}=\text { kurzfristig vorgegeben }
$$

Die einzigen kurzfristig variablen Größen von (5.1) sind die private Produktion $x t$ und die private Beschäftigung epr, $t$ bzw. deren Meßziffern, $x_{t} / x_{0}, t$ und $e_{p r}, t / e_{o}, p r, t$ deren Verlauf für die Bundesrepublik im letzten Paragraphen geschätzt wurde.

Eine notwendige Eigenschaft von (5.1) besteht darin, daß sich die Verteilungsparameter $\delta_{1}, \delta_{2}$ und $\delta_{3}$ zu eins addieren:

$$
\text { (5.4) } \quad \delta_{1}+\delta_{2}+\delta_{3}=1
$$

Nur unter dieser Bedingung behält die Funktion ihre Gültigkeit auch dann, wenn alle Erwartungen und Pläne der Unternehmen aufgehen und die Meß. ziffern von Produktion, Arbeitseinsatz und öffentlichem Infrastrukturanlagenbestand gleich eins sind.

Eine weitere Eigenschaft der Produktionsfunktion zeigt sich bei der Ableitung der kurzfristigen Arbeitsnachfragefunktion. Da die Unternehmen nicht nur die Kapazitätsvorausplanung, sondern auch die kurzfristige

(1) Nur unter dem Strich sei darauf hingewiesen, daß die Annahme einer kurzfristig einheitlichen Substitutionselastizität für alle drei Produktionsfaktoren sicher nicht realistisch ist, bei dem geringen Wert der kurzfristigen Substitutionselastizitäten aber vielleicht keine allzu restriktive Annahme ist. 
Anpassung von Produktion und Beschäftigung unter Wirtschaftlichkeits gesichtspunkten vornehmen, werden sie die Beschäftigung realisieren, bei der das kurzfristige Grenzerlösprodukt des Faktors Arbeit dem in der Periode herrschenden nominellen Arbeitskostensatz entspricht. Im Anschluß an (5.1) gilt daher:

(5.5) $\quad l_{\text {pr }, t}^{\star}=\mu_{a} \cdot \delta_{1}\left[\frac{x_{0, t}}{e_{0, p r, t}}\right]^{-\delta_{4}} \cdot\left[\frac{x_{t}}{e_{p r, t}}\right]^{1+\delta_{4}} \cdot p_{x, t}$

woraus sich die für die kurze Frist geltende grenzproduktivitätstheoretische Arbeitsnachfragefunktion ergibt:

(5.6) $\quad e_{p r, t}=\left[\mu_{a} \delta_{1}\right]^{\frac{1}{1+\delta_{4}}} \cdot\left[\frac{x_{0, t}}{e_{0, p r, t}}\right]^{\frac{\delta_{4}}{1+\delta_{4}}} \cdot\left[\frac{1 \text { pr,t }}{p_{x, t}^{*}}\right]^{-\frac{1}{1+\delta_{4}}}$

Der grenzproduktivitätstheoretischen Entlohnungsfunktion (5.5) entspricht eine grenzproduktivitätstheoretische Verteilungsfunktion, nach der der Anteil der Arbeitskosten an der Bruttowertschöpfung bestimmt ist durch:

(5.7) $\frac{e_{p r, t} \cdot{ }_{p r}^{*}, t}{x_{t} \cdot p_{x, t}}=\mu_{a} \cdot \delta_{1} \cdot\left[\left(\frac{x_{t}}{e_{p r, t}}\right) /\left(\frac{x_{0, t}}{e_{0, p r, t}}\right)\right] \delta_{4}$

Weichen Produktion und Beschäftigung des Unternehmenssektors und damit die Arbeitsproduktivität nicht von dem erwarteten bzw. geplanten Niveau ab, dann entspricht der kurzfristig realisierten Arbeitsnachfrage nach (5.7) ein Arbeitskostenanteil an der Bruttowertschöpfung von

$$
\frac{e_{p r, t} \cdot{ }_{p r, t}^{*}}{x_{t} \cdot p_{x, t}}=\mu_{a} \cdot \delta_{1} \quad \text { für } \frac{x_{t}}{e_{p r, t}}=\frac{x_{0, t}}{e_{o, p r, t}}
$$

In der Kapazitätsplanung hatte der Unternehmenssektor nach (4.5) aber eine Arbeitskostenquote von

$$
\frac{e_{0, p r, t} \cdot l_{0, p r, t}^{*}}{x_{0, t} \cdot p_{0, x, t}}=\mu_{a} \cdot \alpha_{1} \cdot a_{2} \cdot a_{3}^{-\alpha_{4}} \cdot\left(1+f_{a}\right)^{-\alpha_{4} \cdot(t-1960)}
$$

$$
\left[\frac{x_{0, t}}{e_{0, p r, t}}\right]^{a_{4}} \cdot k_{0, s t, t}^{-\left(a_{4}-a_{5}\right)}
$$

angesteuert. 
Soll die realisierte Arbeitsnachfrage beim Aufgehen der Unternehmenserwartungen und Unternehmenspläne zu der gleichen Arbeitskostenquote und zu dem gleichen mengenmäßigen Arbeitseinsatz führen wie die Beschäftigungsplanung, sollen also Produktionsplanung und Produktionsvollzug des Unternehmenssektors miteinander konsistent sein, dann muß offensichtlich gelten:

$$
\text { (5.10) } \begin{aligned}
\sigma_{1}= & \alpha_{1} \cdot \alpha_{2} \cdot a_{3}^{-\alpha_{4}} \cdot\left(1+f_{a}\right)^{-\alpha_{4}(t-1960)} \cdot\left[\frac{x_{0, t}}{e_{0, p r, t}}\right]^{a_{4}} . \\
& -\left(\alpha_{4}-a_{5}\right) \\
& k_{0, s t, t}^{*}, s
\end{aligned}
$$

Ähnlich muß dann für die beiden anderen Verteilungsparameter $\delta_{2}$ und $\delta_{3}$ gelten:

$$
\sigma_{2}=a_{1} \cdot\left(1-a_{2}\right) \cdot\left[\frac{x_{0, t}}{k_{0, p r, t}}\right]^{a_{4} \cdot k_{0, s t, t}^{-\left(a_{4}-a_{5}\right)}}
$$

$$
\delta_{3}=1-a_{1} \cdot k_{0, s t, t}^{a_{5}}
$$

wobei $\mathrm{k}_{\mathrm{O}}^{\star}$, St, $\mathrm{t}$ gemäß $(4.3)$ definiert ist.

Unter den getroffenen Annahmen einer langfristigen Substitutionselastizität $z$ wischen den beiden privaten Faktoren von minus eins und einer langfristigen Substitutionselastizität $z$ wischen diesen beiden Faktoren und dem öffentlichen Infrastrukturanlagenbestand von minus einhalb wird aus (5.10) bis (5.12):

$$
\left.\begin{array}{ll}
(5.13) & \delta_{1}=a_{1} \cdot a_{2} \cdot k_{0, s t, t}^{*} \\
(5.14) & \delta_{2}=a_{1} \cdot\left(1-a_{2}\right) \cdot k_{0, s t, t}^{*} \\
(5.15) & \delta_{3}=1-k_{0, s t, t}^{*}
\end{array}\right\} \text { fur } \begin{array}{r}
a_{4}=0,00 \\
a_{5}=+1,00
\end{array}
$$

Die Verteilungsparameter der kurzfristigen Produktionsfunktion sind kurzfristig also invariant, aber sie erfahren jenseits der kurzen Frist über die Erwartungen, die sich auf den Einfluß der staatlichen Infrastruktur erstrecken, bzw. über $\mathrm{k}_{\mathrm{O}}^{\star}, \mathrm{St}, \mathrm{t}$ durchaus gewisse Änderungen.

Aus den vorstehenden Überlegungen ist bereits das verteilungstheoretische Grundkonzept der ganzen Untersuchung ersichtlich geworden: Die Anwendung der Grenzproduktivitätstheorie der Faktornachfrage vollzieht sich in zweifacher Weise. Das langfristige Grenzproduktivitätskalkül 
bestimmt die Planung der Faktoreinsätze. Da sich die Planung der privaten Anlagenbestände bereits während der Planungsperiode in entsprechenden Anlageninvestitionen niederschlägt, ist auch die Realisierung des geplanten Kapitaleinsatzes durch das langfristige Grenzproduktivitätskalkül bestimmt, und das zu Anlageninvestitionen aufgenommene Finanzkapital wird in der nächsten Periode mit dem Kapitalmarktzins bedient, der aufgrund dieses langfristigen Grenzproduktivitätskalküls vereinbart wurde bzw. der sich aufgrund des entsprechenden Investitionsverhaltens herausgebildet hat (1). Im Gegensatz dazu wird die geplante Beschäftigung von Arbeitskräften erst in der auf die Planungsperiode folgenden Periode realisiert und, da hierbei zugleich die kurzfristig sinnvollen Anpassungskorrekturen vorgenommen werden, richtet sich die Entlohnung des Faktors Arbeit nach dem kurzfristigen Grenzproduktivitätsprinzip. Weichen Produktion und Beschäftiguing von dem geplanten Niveau ab, dann kommt es zu Abweichungen der kurzfristigen Grenzproduktivitäten von den langfristigen Grenzproduktivitäten und beim Faktor Arbeit und nur bei diesem zwangsläufig zu einem Abweichen der tatsächlichen, durch das kurzfristige Grenzproduktivitätskalkül bestimmten Faktorkosten von den geplanten, durch das langfristige Grenzproduktivitäts kalkül bestimmten Faktorkosten.

Nach allgemeiner Ansicht muß angenommen werden, daß die kurzfristige Substitutionselastizität absolut genommen wesentlich kleiner als eins ist. Damit tritt von der kurzfristigen Grenzproduktivitätstheorie her etwas ein, was auch von der Kreislauftheorie der Einkommensverteilung her zu erwarten ist. Überschreiten die Güternachfrage und daher Produktion und Beschäftigung einmal das im voraus erwartete bzw. geplante Niveau und orientiertsich der tatsächliche Arbeitseinsatz streng am kurzfristigen Grenzproduktivitätsprinzip, dann miüssen nach (5.1) und (5.7) die Arbeitsproduktivität und die Arbeitskostenquote unter das vorauskalkulierte Niveau sinken. Es passiert also folgendes: die Arbeitskosten steigen dem absoluten Umfang nach, aber sie sinken quotal; die Kapitalkosten, die in ihrem absoluten Niveau weitgehend vorherbestimmt sind (2), sinken ebenfalls quotal; die einzige Einkommenskomponente, die absolut und quotal gestiegen ist, ist der aggregierte Unternehmensgewinn. Das Mehr an Unternehmensgewinn ist in dem angeführten Beispiel ein typischer windfall-Profit; ohne die unerwartet hohe Güternachfrage wäre er

(1) Aufgrund der variablen Verzinsung aller Titel gilt dieser Kapitalmarktzins für den gesamten Bestand des aufgenommenen Finanzkapitals.

(2) Das heißt nicht, daß die Kapitalkosten absolut konstant sind; z. B. werden die Abschreibungen für Kapitalverzehr von der laufenden Entwicklung der Wiederbeschaffungspreise mitbestimmt und steigen $u_{\bullet} U$. stärker als erwartet. 
nicht zustandegekommen (1).

Es dürfen jedoch keine Mißverständnisse auftreten. Nach (5.7) zusammen mit (5.1) entspricht jedem Kapazitätsauslastungsgrad $x_{\forall} / x_{0}, t$ bzw. jedem Beschäftigungsgrad $e_{p r, t} / e_{o}, p r, t$ von der kurzfristigen Grenzproduktivitätstheorie her eine bestimmte Höhe der Arbeitskosten- bzw. - einkommensquote. Ebenso ergibt sich für jeden Kapazitätsauslastungsgrad xt/ $x_{0, t}$ bzw. Beschäftigungsgrad $e_{p r, t} / e_{o, p r, t}$ vom Verhalten der Arbeits nachfrage und der gewerkschaftlichen Lohnplitik her eine bestimmte Lohnsumme und vom Verhalten der Konsumenten, der Investoren, des Staates etc. her eine bestimmte Nachfrage nach Produkten des Unternehmenssektors, damit auch eine bestimmte kreislaufbedingte Höhe der Lohn-bzw. Arbeitseinkommensquote. Kurzfristig spielt sich ein Zustand ein, bei dem beide Niveaus der Arbeitskosten- bzw. -einkommensquote miteinander übereinstimmen und sich auch ein bestimmter Anteil an Unternehmensprofiten einstellt. Der dazu erforderliche Anpassungsprozeß vollzieht sich jedoch auf beiden Seiten (2). Die Frage, ob sich die grenzproduktivitätstheoretisch bestimmte Verteilung der kreislaufbestimmten Verteilung angepaßt habe oder umgekehrt, und noch mehr die Frage, ob die Verteilungsquoten grenzproduktivitätstheoretsich oder kreislauftheoretisch bestimmt seien, sind daher bereits vom Ansatz her verfehlt, zumindest in der vorliegenden Modellformulierung, in der die Parameter der kurzfristigen Produktionsfunktion das Verteilungsergebnis auf jeden Fall mitbestimmen.

Im Sinne der vorstehenden Überlegungen sind auch alle makroökonomischen Überwälzungsphänomene, die innerhalb des Modells kurzfristig als Folge von Steueränderungen in Erscheinung treten, sowohl neoklassicher wie keynesianischer Natur: Erhöht der Staat der Modellwirtschaft die steuerliche Belastung der Unternehmensgewinne, so bleibt die Planbeschäftigung, $e_{o}, p r, t$, und ebenso die Plankapazität der Unternehmen, $x_{0, t}$ in der laufenden Periode davon unberührt. Man setze nun eine genügende Zinselastizität des Angebots an Investitionsfinanzierungsmitteln und eine nachfragewirksame Wiederverausgabung des Steuermehraufkommens durch den Staat voraus. Dann steigen nach allen bisherigen Überlegungen die monetäre Gesamtnachfrage, damit die Nachfrage nach Gütern des Unternehmenssektors und die private Produktion, bei einer Größe der kurzfristigen Substitutionselastizität, $1 /\left(1+\delta_{4}\right)$, von kleiner als eins nach (5.1) und (5.7) schließlich auch der Wertschöpfungsanteil der Bruttobesitzeinkommen vor Abzug der Steuern.

(1) Wegen der grundsätzlichen Homogenitätseigenschaft von (5.1) vor Berücksichtigung von (5.2) entspricht die Summe aus Kapitalkosten und windfall-Profit genau dem kurzfristigen Grenzerlösproduktanteil des eingesetzten Privatkapitals an der Bruttowertschöpfung.

(2) Vgl. R. Lüdeke, Synthesemöglichkeiten des nachfrage- und angebotstheoretischen Ansatzes in der makroökonomischen Verteilungstheorie, Jahrbücher f. Nationalökonomie und Statistik, 186, 1972, S. $309 \mathrm{ff}$. 
Die Gewinnsteuererhöhung wird also überwälzt - ob zu mehr oder weniger als $100 \%$, das hängt unter anderem von der Höhe der kurzfristigen Substitutionselastizität ab.

Ähnlich verläuft die Argumentation im Fall der indirekten Steuern: Die Arbeitsnachfragefunktion (5.6) enthält als Argument den Arbeitskostensatz und damit nach $(4.12)$ bis $(4.14)$ den Einfluß der indirekten Verbrauchsteuer und der Lohnsummensteuer. Eine Erhöhung des Arbeitskostensatzes durch die beiden Steuern führt ceteris paribus zu einer Verringerung der kurzfristigen Arbeitsnachfrage und einer mengenmäßigen Angebotsverknappung auf dem Gütermarkt, die sich in steigenden Preisen auswirkt. Der Zusammenhang zwischen der indirekten Steuerbelastung und dem Güterpreis läßt sich auch anders darstellen: Formt man die kurzfristige Arbeitsnachfragefunktion (5.6) zu einer Funktion für das aggregierte Preisverhalten der Unternehmen um, wie das später in (5.21) bzw. (5.23) getan wird, so veranlaßt die steuerlich bedingte Erhöhung des Arbeitskostensatzes die Unternehmen nach dieser Funktion ceteris paribus zu einer proportionalen Erhöhung des Güterangebotspreises, d. h. zu einem bewußten Überwälzungsversuch. - Verwendet der Staat das durch die Steuererhöhung erhaltene Mehraufkommen zur zusätzlichen Güternachfrage im Unternehmenssektor und verhindert er die steuerbedingten Arbeitsnachfrage- und Beschäftigungseffekte, setzt sich diese Preissteigerungstendenz, wie man sie auch interpretiert, durch, d. h. es tritt eine volle Überwälzung der Steuererhöhung ein, der Arbeits kostenanteil am laufenden Produktionswert steigt trotz der Erhöhung des Arbeitskostensatzes nicht. Legt der Staat dagegen das Mehraufkommen still, und erhöht sich die monetäre Güternachfrage nicht, kommt es wegen des über die Preise vorgenommenen Überwälzungsversuchs $z u$ Produktions- und Beschäftigungseinschränkungen. Bei einer kurzfristigen Substitutionselastizität von kleiner als eins sind diese Einschränkungen nach (5.1) und (5.7) mit einer Erhöhung des Arbeitskostenanteils verbunden. Die angestrebte volle Überwälzung der erhöhten Verbrauchbzw. Lohnsummensteuer gelingt hier also kurzfristig, je nach Größe der kurzfristigen Substitutionselastizität, nur zum Teil oder überhaupt nicht.

\section{Eine Modifizierung der kurzfristigen Grenzproduktivitätstheorie}

Will die Grenzproduktivitätstheorie Gültigkeit für die kurzfristige Anpassung von Produktion und Beschäftigung beanspruchen, muß sie sich eine Modifikation gefallen lassen. Das ergibt sich aus folgender Überlegung. Nach der für die kurze Frist aufgestellten Grenzproduktivitäts theorie weist der private Produktionsprozeß im Laufe der Konjunkturzyklen vier Merkmale auf: 1. Die jährlichen Anstiegsraten von Reallohn und Arbeitsproduktivität des privaten Sektors schwanken nach (5.5) gleichläufig. - 2. Wegen der abnehmenden Durchschnitts - und Grenzpro- 
duktivitäten des Produktionsfaktors Arbeit liegen sie im Boom niedriger als in der Rezession. - 3. Nach (5.7) besteht eine positive Korrelation zwischen der Arbeiteinkommens- bzw. der Arbeitskostenquote und den Abweichungen der Arbeitsproduktivität von dem in der Kapazitätsplanung angestrebten Normalniveau. - 4. Ebenso korreliert die Arbeits einkommens- bzw. Arbeitskostenquote an der Bruttowertschöpfung des privaten Sektors positiv mit dem jährlichen Reallohnanstieg. - Von diesen vier Erscheinungen läßt sich in Wirklichkeit nur die erste empirisch beobachten (1). In den anderen drei Punkten stellt man genau das Gegenteil von dem fest, was nach der aufgestellten Theorie zu erwarten ist (2). Man gelangt daher zu dem Ergebnis, daß die Grenzproduktivitätstheorie so, wie sie im vorigen Abschnitt für die kurze Frist formuliert wurde, nicht gelten kann.

Über eine erste Richtung, in der die kurzfristige Grenzproduktivitätstheorie zu modifizieren ist, geben die Aussagen der Literatur über das kurzfristige Arbeitsnachfrageverhalten Auskunft, die bereits bei der Aufstellung der Beschäftigungsplanungsfunktion zur Formulierung einer Ergänzungshypothese herangezogen wurde (3). Diese Aussagen decken zwei Schwächen der kurzfristigen Grenzproduktivitätstheorie auf: Die aufgestellte Theorie vernachlässigt die Inhomogenität des Arbeitseinsatzes, der nur zum Teil kurzfristig variiert werden kann. Sie muß daher bei dem Versuch, die Produktivitätsentwicklung für die eine als homogen angesehene Faktorgruppe zu erklären, zwangsläufig zu falschen Ergebnissen kommen und übertreibt auf jeden Fall die konjunkturellen $\mathrm{Be}$ schäftigungsschwankungen. - Sie sieht ferner davon ab, daß eine kurzfristige Gewinnmaximierungspolitik unter dem Gesichtspunkt der längerfristigen Gewinnmaximierung unwirtschaftlich sein kann und aus sozialpolitischen Gründen häufig auch gar nicht zu realisi eren ist.

Um die Aussagekraft der aufgestellten Theorie zu sichern, wird im folgenden eine der vernachlässigten Eigenschaften des kurzfristigen Arbeitsnachfrageverhaltens zusätzlich in die Modellformulierung aufgenommen. Es wird davon ausgegangen, daß das kurzfristige Gewinnmaximierungsprinzip wegen längerfristigen Rentabilitätsstrebens und sozialpolitischer Hemmnisse eingeschränkt gilt und daß allein aus diesem Grund kurzfristige Abweichungen der tatsächlichen Arbeitsnachfrage von der geplanten Beschäftigung nur in begrenztem Umfang in Erscheinung treten. Um das zusätzliche Argument zu berücksichtigen, wird wie bisher

(1) Vgl. z. B. Jahresgutachten 1971 des Sachverständigenrates zur Begutachtung der gesamtwirtschaftlichen Entwicklung, Bundestagsdrucks. VI/2847, Bonn 1971, S. 37, Tab. 10, Spalten (1) und (7)

(2) Vgl. ebenda, S. 37, Tab. 10, Spalten (1) und (7) sowie S. 36, Tab. 9

(3) Vgl. Paragraph 4, Abschnitt 6 und die dort angegebenen Beiträge der Literatur 
unterschieden zwischen der geplanten Beschäftigung, $e_{o} p r, t$, und der Arbeitsnachfrage, die bei den gerade herrschenden Marktbedingungen und bei uneingeschränkter Gültigkeit des kurzfristigen Grenzproduktivitäts prinzips von den Unternehmern entfaltet worden wäre. Letztere wird als die kurzfristig optimale Arbeitsnachfrage bzw. Beschäftigung bezeichnet und, anders als bisher, durch $e_{\mathbf{x}}, \mathrm{pr}, \mathrm{t}$ symbolisiert. Die Bezeichnung deutet bereits an, daß die Abweichungen der tatsächlichen Beschäftigung von dem kurzfristig optimalen Beschäftigungsumfang das absolute Produktionsniveau in der Modellformulierung nicht beeinflussen, sondern allein durch ein Dehnen oder Pressen der Arbeitsintensität kompensiert werden. Die tatsächliche Arbeitsnachfrage bzw. Beschäftigung, $e_{p r, t}$, liegt $\mathrm{z}$ wischen den beiden genannten hypothetischen Beschäftigungsmengen, also $z$ wischen der geplanten und der kurzfristig optimalen

Beschäftigung, und wird nach Art eines gewogenen geometrischen Durchschnitts aus diesen beiden Größen ermittelt:

(5.16) $\quad e_{p r, t}=e_{0, p r, t}^{1-\gamma_{1}} \cdot e_{x, p r, t}^{\gamma_{1}} \quad\left(0<\gamma_{1}<1\right)$

Dabei gilt nun, wie beschrieben, statt $(5.6)$ :

(5.17) $e_{x, p r, t}=\left[\mu_{a} \cdot \delta_{1}\right]^{\frac{1}{1+\delta_{4}}} \cdot\left[\frac{x_{o, t}}{e_{o, p r, t}}\right]^{-\frac{\delta_{4}}{1+\delta_{4}}} \cdot\left[\frac{1_{p r, t}^{*}}{p_{x, t}}\right]^{-\frac{1}{1+\delta_{4}}} \cdot x_{t}$

und statt $(5.1)$ bei Berücksichtigung von (5.2):

$$
x_{t}=x_{0, t} \cdot\left[\delta_{1} \cdot\left(\frac{e_{x, p r, t}}{e_{0, p r, t}}\right)^{-\delta_{4}}+\delta_{2}+\delta_{3} \cdot\left(\frac{k_{S t, t}}{k_{0, S t, t}}\right)^{-\delta_{4}}\right]^{-\frac{1}{\delta_{4}}}
$$

Setzt man (5.16) in (5.18) ein, löst nach $\mathrm{x}_{t} / \mathrm{e}_{\mathrm{pr}, t}$, der Arbeitsproduktivität, auf und differenziert partiell nach $x_{t}$, dann zeigt sich, daß der Verhaltensparameter $\gamma_{1}$ von $(5,16)$, der die Gültigkeit des kurzfristigen Gewinnmaximierungsprinzips für das kurzfristige Arbeitsnachfragever halten im Modell einschränkt, eine bestimmte Obergrenze haben muß, damit die Arbeitsproduktivität bei kurzfristigen Schwankungen der Produktion sich in der Richtung bewegt, die die empirischen Beobachtungen angeben. Diese Obergrenze ist durch

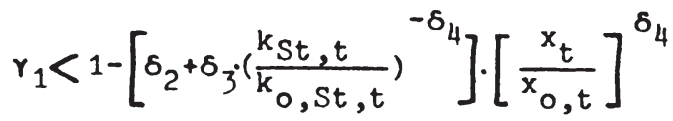


bestimmt. Unterschreitet $\gamma_{1}$ die Obergrenze, dann tritt das ein, was sich während der Konjunkturzyklen beobachten läßt: Mit steigender konjunktureller Auslastung der Anlagenkapazitäten steigt die Produktivität des Faktors Arbeit. Gleichung (5.19) deutet allerdings an, daß die Obergrenze variabel ist. Jenseits einer bestimmten Höhe der Kapazitätsauslastung dürfte die Bedingung nicht mehr erfüllt sein und die Arbeitsproduktivität bei weiterer Ausnutzung des noch vorhandenen Kapazitätsspielraumes zu sinken beginnen.

Nach der mit $(5.16)$ und (5.17) vorgenommenen Modifizierung läßt sich das kurzfristige Arbeitsnachfrageverhalten der Unternehmen ausdrücken durch die Funktion:

$$
\frac{e_{p r, t}}{e_{o, p r, t}}=\left[\mu_{a^{-} \delta_{1}} \cdot \frac{x_{0, t}}{e_{o, p r, t}}\right]^{\frac{r_{1}}{1+\delta_{4}}} \cdot\left[\frac{l_{p r, t}^{*}}{p_{x, t}}\right]^{-\frac{r_{1}}{1+\delta_{4}}} \cdot\left[\frac{x_{t}}{x_{0, t}}\right]^{Y_{1}}
$$

Die Modellformulierung bedarf noch einer zweiten Modifizierung. Löst man (5.17) nach $1_{\mathrm{pr}, t}^{\star} / \mathrm{p}_{\mathrm{x}, \mathrm{t}}$, dem realen Arbeitskostensatz, auf, ebenso (5.18) nach $x_{t} / e_{x}, p r, t$ und setzt man die umgeformte Gleichung (5.18) in die umgeformte Gleichung (5.17) ein, berechnet man schließlich das partielle Differential von $1_{p r}^{\star}, t / p_{x, t}$ nach Änderungen von $x_{t}$, dann sieht man, daß die vorgenommene erste Modifikation zwar die kurzfristige Entwicklung der Arbeitsproduktivität im Modell korrigieren kann, nicht aber die kurzfristige Entwicklung des Reallohnes. Die kurzfristige Reallohnentwicklung ist völlig unabhängig von der Größe des Parameters $\gamma_{1}$. Steigt im Boom die Produktion und werden mehr Arbeitskräfte als geplant eingesetzt, so sinkt im Modell der reale Lohn- bzw. Arbeitskostensatz genauso wie die Grenzproduktivität unter das erwartete Normalniveau und verläuft damit weiterhin anders, als das für die kurze Frist empirisch zu beobachten ist.

Der Grund hierfür ist in folgendem zu suchen: Wenn in einem konjunkturellen Aufschwung die Güternachfrage die Erwartungen der Unternehmen übersteigt und diese bei kurzfristig konstantem Anlagenbestand die Produktion durch einen vermehrten Einsatz von Arbeitskräften zu erhöhen trachten, dann führt die zu diesem Zweck erhöhte Arbeitsnachfrage der Unternehmen zu einem zusätzlichen Anstieg des Nominallohnniveaus. Gleichzeitig sinkt nach Produktionsfunktion (5.1) das Grenzprodukt der Arbeit und bei strenger Grenzproduktivitätsorientierung des Arbeitsnachfrageverhaltens der Reallohn, da vermehrt Arbeitskräfte eingesetzt werden. Ein Sinken des Reallohnes bei steigendem Nominallohn müßte nun bedeuten, daß die Unternehmen die auf dem Gütermarkt geforderten Preise im Boom stärker heraufsetzen als die auf dem Arbeitsmarkt gebotenen Nominallohnsätze.

Ein derartiges preispolitisches Verhalten der Unternehmen läßt sich aber empirisch nicht beobachten. Vergleicht man die Entwicklung der 
Güterpreise und Nominallöhne, so stellt man fest, daß die konjunkturellen Schwankungen der Güterpreise, zumindest in der Bundesrepublik wesentlich geringer als die konjunkturellen Schwankungen der Nominallöhne sind (1). Die Unternehmen verhalten sich preispolitisch also offensichtlich nicht so, wie man das bei Gültigkeit der kurzfristigen Grenzproduktivitätstheorie erwarten muß, und hierin liegt die Ursache für die von der kurzfristigen Grenzproduktivitätstheorie abweichende Entwicklung des Reallohns bzw. des realen Arbeitskostensatzes im Konjunkturzyklus. Es liegt daher nahe, eine zusätzliche Ergänzungshypothese derart anzunehmen, daß das kurzfristige Grenzproduktivitätsprinzip nicht allein hinsichtlich des Arbeitsnachfrageverhaltens, sondern auch hinsichtlich der kurzfristigen Preispolitik der Unternehmen auf dem Gütermarkt nur eingeschränkt gilt, und diese Hypothese stimmt in etwa mit dem Keynes'schen Argument von der begrenzten Flexibilität der Güterpreise überein.

Das kurzfristige preispolitische Verhalten der Unternehmen läßt sich im Anschluß an die für die kurze Frist abgeleitete Grenzproduktivitätstheorie genauer analysieren. Dabei stellt man als erstes fest, daß die vorgenommene erste Modifikation des Arbeitsnachfrageverhaltens sich nicht auf die implizierte Preis-Angebots-Funktion des Unternehmenssektors auf dem Gütermarkt auswirkt. Setzt man (5.20) über (5.16) in (5.18) ein, so gelangt man zu derselben Preis-Angebots-Funktion, wie wenn man die Entlohnungsfunktion (5.5) nach $e_{\mathrm{pr}, \mathrm{t}} / \mathrm{e}_{\mathrm{o}}, \mathrm{pr}, \mathrm{t}$ auflöst und in die Produktionsfunktion $(5.1)$ bei Berücksichtigung von (5.2) einsetzt:

$$
\begin{aligned}
p_{x, t}= & {\left[\mu_{a} \cdot \delta_{1}\right]^{-1} \cdot\left[1_{p r, t}^{*} /\left(\frac{x_{0, t}}{e_{0, p r, t}}\right)\right] \cdot } \\
& {\left.\left[\frac{1}{\delta_{1}}-\frac{\delta_{2}+\delta_{3} \cdot\left(k_{s t, t} / k_{0, s t, t}\right)}{\delta_{1}} \cdot\left(\frac{x_{t}}{x_{0, t}}\right)\right]^{-\delta_{4}}\right]^{-\frac{\delta_{4}}{\delta_{4}}} }
\end{aligned}
$$

Die einzigen kurzfristig variablen und für die Preiskalkulation der Unternehmen relevanten Bestimmungsfaktoren sind nach dieser Funktion der Umfang, indem der Arbeitskostensatz von der vorausgeplanten Arbeitsproduktivität abweicht, und die Abweichung der realen Güternachfrage bzw. der Produktion von dem erwarteten bzw. geplanten Niveau. Die Funktion enthält also ein Kostenargument und ein Nachfrageargument

(1) Vgl. z. B. Jahresgutachten 1971 des Sachverständigenrates. a. a. O. , S. 37, Tab. 10, Spalten (3) und (5) bzw. (6). Die durchschnittliche Abweichung der dort in Spalte (3) angegebenen jährlichen Lohnsteigerungsrate beträgt $\pm 2,2$ Prozentpunkte, die der in Spalte (6) angegebenen jährlichen Preissteigerungsrate beträgt $\pm 1,0$ Prozentpunkte. 
als laufende Bestimmungsfaktoren des Angebotspreises. Der Angebotspreis verhält sich proportional zur Größe des Arbeitskostensatzes; Änderungen der realen Güternachfrage wirken sich auf ihn stets in gleicher Richtung und mit zunehmender (abnehmender) Höhe der Kapazitätsauslastung immer stärker (schwächer) aus (Figur 5.1).

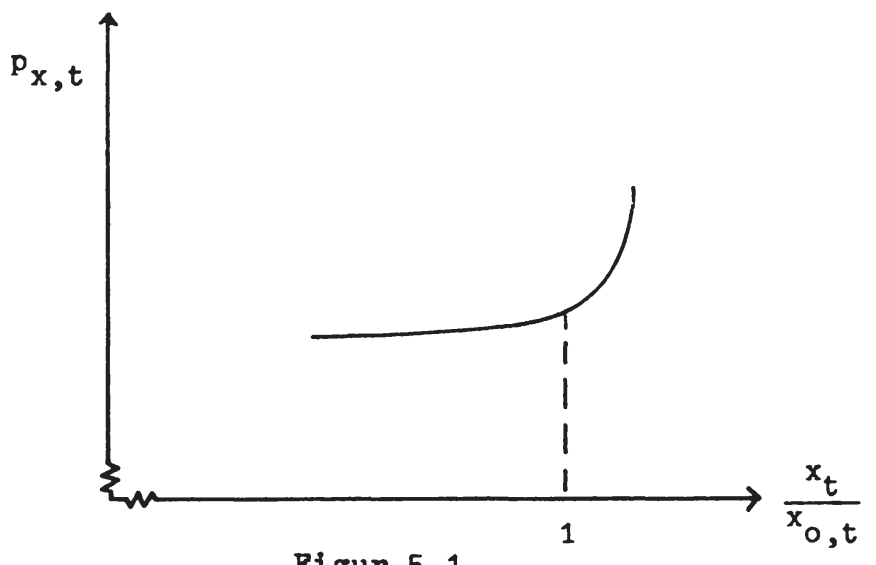

Figur 5.1

Um diesen Verlauf der Preis-Angebots-Funktion zu überprüfen, wurde die Preisentwicklung des privaten Sektors in der Bundesrepublik einer eingehenden Analyse unterzogen, bei der auch die geschätzten Werte von $\mathrm{e}_{\mathrm{O}, \mathrm{pr}, \mathrm{t}}$ und $\mathrm{x}_{\mathrm{O}, \mathrm{t}}$ als Erklärungsgrößen und alle Erklärungsgrößen mit unterschiedlichen lags berücksichtigt wurden. Es zeigte sich, daß für die Zeit nach 1955 der bei weitem beste statistische Erklärungsfaktor der Preisentwicklung der Quotient aus Lohn- und Produktivitätsanstieg der laufenden Periode ist. Zwischen den Preisanstiegsraten des privaten BIP-Beitrages und der Größe des Quotienten besteht für die Jahre 1955 bis 1970 eine Korrelation von $r^{2}=0$, 831; für die Jahre 1960 bis 1971 wurde sogar eine Korrelation von $\mathrm{r}^{2}=0,932$ ermittelt. Dabei treten die stärksten Preissteigerungsraten in der Regel im Konjunkturabschwung auf, und zwar infolge des lags der gewerkschaftlichen Lohnpolitik und was hier vor allem von Bedeutung ist - infolge der konjunkturbedingten Verlangsamung des Produktivitätsanstieges (1), die wiederum auf die gedämpften konjunkturellen Beschäftigungsreaktionen der Unternehmen zurückzuführen ist. Diese Beobachtung stimmt nun keineswegs mit dem in Figur 5.1 angegebenen Verlauf der Preis-Angebots-Funktion des Unternehmenssektors überein; ihr entspricht vielmehr ein Verlauf der aggregierten Preis-Angebots-Kurve, wie er in Figur 5.2 abgebildet ist:

(1) Die konjunkturellen Schwankungen der Lohnsteigerungsrate für sich genommen erklären die konjunkturellen Schwankungen des Preisanstiegs wesentlich schlechter. 


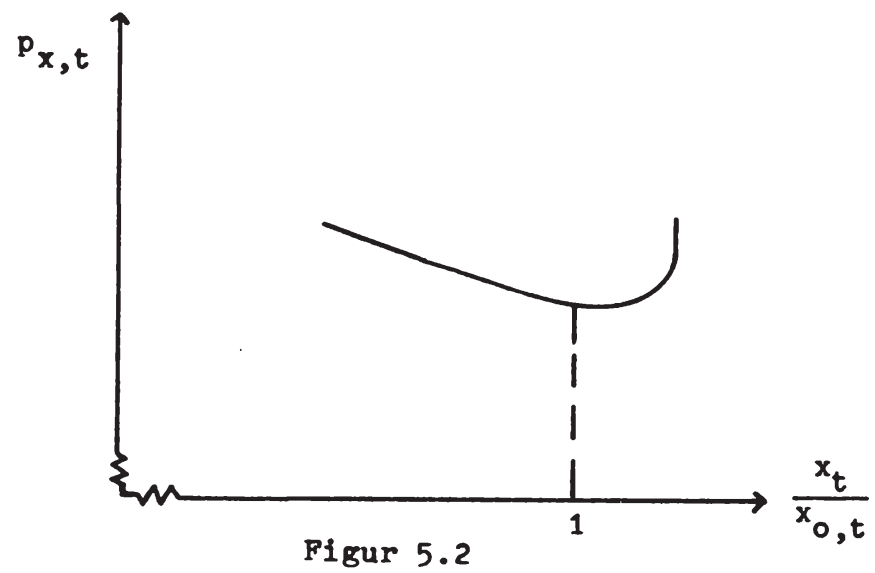

Damit erinnert die Beobachtung an Theorien, die ein Vorherrschen der Zuschlagskalkulation bei der kurzfristigen Preisgestaltung behaupten und diese Behauptung gelegentlich auch in den Mittelpunkt verteilungstheoretischer Erklärungsversuche gestellt haben (1). Man wird daher eine Preisgestaltung, die auf Marginalüberlegungen beruht, vor allem für das längerfristige Unternehmensverhalten in wachstums - und wettbewerbspolitischen Entscheidungen annehmen und diese Annahme hinsichtlich der kurzfristigen Preisgestaltung im Konjunkturverlauf graduell zugunsten der Annahme einer gröberen Kalkulationspraxis aufgeben, wie sie z. B. die Zuschlagskalkulation darstellt (2).

In dieser Richtung wird die hier aufgestellte kurzfristige Grenzproduktivitätstheorie zusätzlich modifiziert. Es wird davon ausgegangen, daß

(1) Vgl. vor allem M. Kalecki, The Theory of Economic Dynamics. An Essay on Cyclical and Long-Run Changes in Capitalist Economy, London 1954 sowie E. Preiser, Wachstum und Einkommensverteilung, Sitzungsberichte der Heidelberger Akademie der Wissenschaften, Philosophisch-historische Klasse, Jahrgang 1961 - 5. Abhandlung, Heidelberg 1961.

(2) Aus den oben genannten Gründen könnte man geneigt sein, die Grenzproduktivitätstheorie für die kurze Frist bzw. für die Beschreibung des kurzfristigen Arbeitsnachfrage- bzw. Güterangebotsverhaltens ganz aufzugeben. Es ist jedoch der Vorteil der oben formulierten modifizier ten Grenzproduktivitätstheorie, daß sie zur Preisbestimmung nicht einfach von der Annahme konstanter Prozentzuschläge zu den Arbeitsstückkosten ausgeht, was wiederum den empirischen Erfahrungen widersprechen würde, sondern die Höhe derartiger Zuschläge selbst zum Gegenstand ihrer Aussage macht. 
die produktionstechnisch überschüssigen Beschäftigten mit steigender Kapazitätsauslastung zunehmend zur Produktion benötigt werden. Ihre produktive Verwendung läßt die Arbeitsproduktivität steigen und die Stücklohnkosten sinken und verursacht von dort her ein Sinken des Angebotspreises. Erst von einer bestimmten Kapazitätsauslastung an machen sich bei zunehmender Güternachfrage bzw. Produktion die bekannten Engpaßerscheinungen bemerkbar und veranlassen die Unternehmen zu einer Erhöhung des Angebotspreise:.

Für die zusätzliche Modifizierung wird die Preis-Angebots-Funktion (5.21) umgeformt zu:

$$
\begin{aligned}
p_{x, t}= & {\left[\mu_{\dot{a}_{1} \delta_{1}}\right]^{-1} \cdot\left[I_{\mathrm{pr}, t}^{*} /\left(\frac{x_{0, t}}{e_{0, p r, t}}\right)\right] \cdot\left[\frac{x_{t}}{x_{0, t}}\right]^{-\left(1+\delta_{4}\right)} . } \\
& {\left[\frac{1}{\delta_{1}} \cdot\left(\frac{x_{t}}{x_{0, t}}\right)^{-\delta_{4}}-\frac{\delta_{2}+\delta_{j}\left(k_{s t, t} / k_{0, s t, t}\right)^{-\delta_{4}}}{\delta_{1}}\right]^{-\frac{1+\delta_{4}}{\delta_{4}}} }
\end{aligned}
$$

In (5.22) taucht die Kapazitätsauslastung $x_{t} / x_{0, t}$ in zweifacher Hinsicht als Bestimmungsfaktor des Angebotspreises auf: Eine sinkende Kapazitätsauslastung läßt den Angebotspreis aufgrund des dritten eckigen Klammerausdrucks steigen; sie läßt ihn aufgrund des vierten eckigen Klammerausdrucks sinken.

Die Modifizierung besteht nun darin, den Einfluß der vierten eckigen Klammer von (5.22) zu schwächen, so daß der Einfluß der dritten eckigen Klammer die Preisgestaltung stärker als zuvor bestimmt. Dies geschieht dadurch, daß der Exponent der vierten eckigen Klammer durch Multiplikation mit einem neu eingeführten Faktor $\gamma_{2}\left(0<\gamma_{2}<\gamma 1\right)$ verkleinert wird:

$$
\begin{aligned}
p_{x, t}= & {\left[\mu_{a} \delta_{1}\right]^{-1} \cdot\left[l_{p r, t}^{*} /\left(\frac{x_{0, t}}{e_{0, p r}, t}\right)\right] \cdot\left[\frac{x_{t}}{x_{0, t}}\right]^{-\left(1+\delta_{4}\right)} } \\
& {\left[\frac{1}{\delta_{1}}\left(\frac{x_{t}}{x_{0, t}}\right)^{-\delta_{4}}-\frac{\delta_{2}+\delta_{3}\left(k_{S t, t} / k_{0, S t, t}\right)^{-\delta_{4}}}{\delta_{1}}\right]^{-\frac{1+\delta_{4}}{\delta_{4}} \cdot r_{2}} }
\end{aligned}
$$

Würde man auf die Formulierung (5.1) der kurzfristigen Produktionsfunktion zurückgreifen, müßte bei Berücksichtigung von (5.2) gelten:

$$
\frac{e_{p r, t}}{e_{0, p r, t}}=\left[\frac{1}{\delta_{1}} \cdot\left(\frac{x_{t}}{x_{0, t}}\right)^{-\delta_{4}}-\frac{\delta_{2}+\delta_{3} \cdot\left(k_{s t, t} / k_{0, s t, t}\right)^{-\delta_{4}}}{\delta_{1}}\right]^{-\frac{1}{\delta_{4}}}
$$


Setzt man (5.24) in (5.23) ein, erhält man:

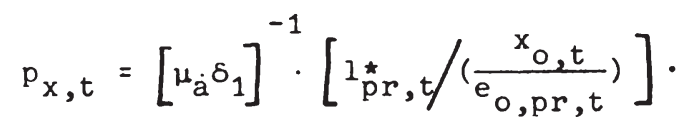

$$
\left[\frac{x_{t}}{x_{0, t}}\right]^{-\left(1+\delta_{4}\right)} \cdot\left[\frac{e_{p r, t}}{e_{0, p r, t}}\right]^{\left(1+\delta_{4}\right) \cdot r_{2}}
$$

woraus sich durch Umformung

$$
\begin{aligned}
\frac{e_{p r, t}}{e_{o, p r, t}}=\left[\mu_{a} \cdot \delta_{1}\right]^{\frac{1}{\left(1+\delta_{4}\right) r_{2}}} \cdot\left[\frac{x_{0, t}}{e_{0, p r, t}}\right]^{\frac{1}{\left(1+\delta_{4}\right) \cdot r_{2}}} \\
{\left[\frac{l_{p r, t}^{*}}{p_{x, t}}\right]^{-\frac{1}{\left(1+\delta_{4}\right) r_{2}}} \cdot\left[\frac{x_{t}}{x_{0, t}}\right]^{\frac{1}{r_{2}}} }
\end{aligned}
$$

ergibt.

Gleichung (5.26) zeigt, daß von der Modifizierung des angenommenen preispolitischen Verhaltens Rückwirkungen auf das kurzfristige Arbeitsnachfrageverhalten der Unternehmen ausgehen. Wäre Parameter $\gamma_{2}$ gleich eins, entspräche (5.26) der ursprünglich aufgestellten kuzrfristigen Arbeitsnachfragefunktion (5.6).

Die beiden Korrekturen an der kurzfristigen Grenzproduktivitätstheorie lassen sich kurz zusammenfassen:

Wegen der mit der ersten Modifikation eingeführten Annahmen (5.16) und (5.18) gilt statt der ursprünglich formulierten Produktionsfunktion (5.1) bei Berücksichtigung von (5.2) die kurzfristige Produktionsfunktion

$$
\left.-\frac{\delta_{4}}{\gamma_{1}}+\delta_{2}+\delta_{3}\left(\frac{k_{S t, t}}{k_{0, S t, t}}\right)-\delta_{4}\right]^{-\frac{1}{\delta_{4}}}
$$

in der die Produktion "'abhängig" gemacht wird von der - im Vergleich zur kurzfristigen Optimalgröße zu reichlich oder zu knapp bemessenen tatsächlichen Beschäftigung.

Da beide Modifizierungen der kurzfristigen Grenzproduktivitäts theorie sich nach (5.20) und (5.26) auf das kurzfristige Arbeitsnachfrageverhalten in ähnlicher Weise auswirken, läßt sich auch ihr Zusammenwirken 
einfach darstellen. Statt der ursprünglich aufgestellten kurzfristigen Arbeitsnachfragefunktion (5.6) gilt nun die kurzfristige Arbeitsnachfragefunktion

$$
\frac{e_{p r, t}}{e_{o, p r, t}}=\left[\mu_{\dot{a}} \delta_{1} \cdot\left(\frac{x_{0, t}}{e_{0, p r, t}}\right) \cdot\left(\frac{1_{p r, t}^{\star}}{p_{x, t}}\right)^{-1}\right]^{\frac{Y_{1}}{\left(1+\delta_{4}\right) \cdot r_{2}}} \cdot\left[\frac{x_{t}}{x_{0, t}}\right]^{\frac{Y_{1}}{Y_{2}}}
$$

In dieser modifizierten Form ist die Theorie der Produktion und des Arbeitsnachfrageverhaltens konsistent mit allen empirischen Beobachtungen hinsichtlich der konjunkturellen Entwicklung von Kapazitätsauslastung, Arbeitsproduktivität, Nominallohn, Güterpreis und Arbeitseinkommensquote.

Ihrem Charakter nach orientiert sich die aufgestellte Theorie nach der vorgenommenen Modifikation weiterhin an der kurzfristigen Grenzproduktivitätstheorie. Mit den Werten $Y_{1}=1$ und $Y_{2}=1$ enthält sie die ur sprüngliche strenge Formulierung der kurzfristigen Grenzproduktivitätstheorie als Spezialfall. Mit $0<\gamma_{1}<1$ und $0<\gamma_{2}<1$ räumt sie aber ein, daß das kurzfristige Grenzproduktivitätsprinzip nur eingeschränkte Gültigkeit hat. Dies gilt auch in verteilungstheoretischer Hinsicht. Formt man (5.28) um, erhält man die Funktion für den Arbeitskostenanteil an der Bruttowertschöpfung des privaten Sektors:

$$
\frac{e_{p r, t} \cdot 1_{p r, t}^{\star}}{x_{t} \cdot p_{x, t}}=\mu_{a^{\prime}} \delta_{1} \cdot\left[\frac{e_{p r, t}}{e_{0, p r, t}}\right]^{\frac{r_{1}-\left(1+\delta_{4}\right) \cdot r_{2}}{r_{1}}} \cdot\left[\frac{x_{t}}{x_{0, t}}\right]^{\delta_{4}}
$$

Nach (5.29) beeinflussen beide Modifikationen das Verteilungsergebnis, allerdings in entgegengesetzter Richtung, so daß eine Kompensation beider Effekte eintritt. Das Verteilungsergebnis stimmt vollständig mit dem Ergebnis in der ursprünglichen Formulierung der kurzfristigen Grenzproduktivitätstheorie überein, wenn $\gamma_{1}=\gamma_{2}$. Beide Ergebnisse fallen auch dann zusammen, wenn $\gamma_{1} \neq \gamma_{2}$, aber $x_{t}=x_{0, t}$ und $e_{p r}, t=e_{0}, p r, t$, also im Fall einer kurzfristig ausgeglichenen Produktions - und Beschäftigungssituation. Die einzige Situation, in der sich die beiden Modifikationen auf das Verteilungsergebnis auswirken, ist also durch $\gamma_{1} \neq \gamma_{2}$, $x_{t} \neq x_{o, t}$ und $e_{p r, t} \neq e_{o, p r, t}$ gekennzeichnet; hier ist mit einer Änderung im Ausmaß der Verteilungseffekte zu rechnen, u. U. auch mit einer Richtungsänderung - das hängt von der Stärke beider Modifikationen bzw. dem Verhältnis $\gamma_{2} / \gamma_{1}$ ab. Die Berechnungen des folgenden Abschnitts deuten jedoch darauf hin, daß eine Umkehrung der grenzproduktivitätstheoretischen Verteilungsergebnisse nicht in Betracht kommt, d. h. , praktisch berühren die Modifikationen allein die Stärke der kurzfristigen bzw. konjunkturellen Abweichungen vom mittelfristigen Normalniveau der funktionalen Verteilungsquoten. Entsprechend beeinflussen sie auch 
nur die Stärke kurzfristiger Überwälzungsprozesse, die durch finanzpolitische Aktionen des Staates ausgelöst werden, nicht aber deren prinzipiellen Charakter, wie er am Ende des vorigen Abschnitts beschrieben wurde (1).

3. Schätzwerte für die Parameter der kurzfristigen Produktions - und Arbeitsnachfragefunktion

Der letzte Schritt bei der Aufstellung der kurzfristigen Produktions- und Arbeitsnachfragetheorie besteht in der Quantifizierung der in Produktionsfunktion (5.27) und Arbeitsnachfragefunktion (5.28) enthaltenen und noch nicht bestimmten Modellparameter. Zu diesem Zweck wurden zwei Berechnungen angestellt. Zuerst wurde die Arbeitsnachfragefunktion $(5.27)$ bei Berücksichtigung von (5.13) zu

$$
\frac{e_{p r, t}}{e_{o, p r, t}}=\left[\mu_{a} \cdot a_{1} a_{2}\right]^{\frac{r_{1}}{\left(1+\delta_{4}\right) r_{2}}} .
$$

$$
\left[\frac{x_{0, t} \cdot p_{x, t} \cdot k_{0, S t, t}^{*}}{e_{0, p r, t} \cdot l_{p r, t}^{*}}\right]^{\frac{r_{1}}{\left(1+\delta_{4}\right) \cdot r_{2}}} \cdot\left[\frac{x_{t}}{x_{0, t}}\right]^{\frac{r_{1}}{r_{2}}}
$$

umgeformt und logarithmisch transformiert. Eine Kleinst-QuadratSchätzung der transformierten Gleichung für die Jahre 1960 bis 1971 lieferte als Ergebnis:

(1) Entgegen allen bisherigen konjunkturellen Erfahrungen lag die bereinigte Lohnquote während der Rezessionsjahre 1966/67 über dem mittelfristigen Trendniveau. Vgl. Tabelle 2 des Tabellenanhangs. Diese Erscheinung wäre im Anschluß an die aufgestellte Theorie so zu erklären, daß das kurzfristige Unternehmensverhalten bezüglich Arbeitsnachfrage und Preiskalkulation von dem Normalverhalten abwich, wie es oben durch die modifizierte Grenzproduktivitätstheorie beschrieben wurde. 


$$
\begin{aligned}
& \frac{Y_{1}}{\left(1+\delta_{4}\right) Y_{2}} \\
& \lg \left[\mu_{a} \alpha_{1} \alpha_{2}\right]^{-1+\sigma_{4} \gamma_{2}}=-0,05160( \pm 0,01418) \\
& \frac{\gamma_{1}}{\left(1+\delta_{4}\right) \cdot r_{2}} \quad=0,28917( \pm 0,08105) \\
& \frac{r_{1}}{r_{2}} \quad=0,66954( \pm 0,06486) \\
& \mathrm{R}_{1 \cdot 2,3}^{2}=0,92220\left(\text { Irrt. }-\mathrm{W}_{0}:<0,1 \%\right) \\
& \mathbf{r}_{2 \cdot 3}^{2}=0,09897 \\
& \text { VNQ }=1,864
\end{aligned}
$$

Die Auflösung der Regressionsparameter von (5.31) ergab indirekte Schätzwerte für $\left(\mu_{\mathrm{a}} \cdot \alpha_{1} \cdot \alpha_{2}\right)$ und $\delta_{4}$ :

$$
\begin{array}{cl}
{\left[\mu_{\dot{a}} a_{1} \cdot a_{2}\right]} & =0,663068 \\
\delta_{4} & =1,315385
\end{array}
$$

Da die Parameter $\alpha_{1}$ und $\alpha_{2}$ a priori auf +0,99 bzw. +0,75 festgelegt wurden, entspricht dem $\left(\mu_{\mathrm{a}} \cdot a_{1} \cdot \alpha_{2}\right)$ - Wert von (5.32) ein Monopolgradfaktor von $\mu_{a}=0,893$. Dieser Wert stimmt ziemlich genau mit den Erwartungen überein, die sich als Nebenprodukt bei der a-priori-Fixierung der langfristigen Produktionselastizität herausbildeten (1).

(1) Vgl. Fußnote 1 von S. 95 - Die Übereinstimmung ist wohl lediglich als ein Zeichen für die Konsistenz der vorgenommenen Berechnungsschritte anzusehen. Insofern ist der über (5.32) erhaltene $\mu_{a}$-Wert nur Ausdruck der getroffenen Annahmen und enthält keine empirischen Informationen darüber, wie hoch der Wert des Monopolgradfaktors tatsächlich zu veranschlagen ist.

Jeder der mit der Materie einigermaßen vertraut ist, weiß im übrigen, wie schwierig es ist, über den gesamtwirtschaftlichen Durchschnitt der einzel-wirtschaftlichen Monopolgradfaktoren überhaupt irgendwelche verläßlichen größenmäßigen a-priori-Vorstellungen zu entwickeln. Das wird z. B. deutlich an den unterschiedlichen Annahmen über die durchschnittliche Größe der einzelwirtschaftlichen Preis-Absatz- Elastizität bei W. Krelle, Verteilungstheorie, a. a. O. , S. 137 u. S. 169 Fußnote 3, S. 176 Fußnote 1, S. 199 Fußnote 1, S. 220 Fußnote 2 sowie bei J. Niehans, Ein Beitrag zum Verständnis von Krelles Makromodell, Weltwirtschaftliches Archiv, 92, 1964, S. 238 ff. und bei 
Dem indirekten Schätzwert von $\delta_{4}$ entspricht eine Substitutionselastizität von $-1 /\left(1+\delta_{4}\right)=-0,431$. Die kurzfristige Substitutionselastizität ist also absolut genommen wesentlich kleiner als die "langfristige" Substitutionselastizität, die die Vorausplanung der Faktoreinsätze für die jeweils folgende Periode bestimmt und die aufgrund der Schätzung (4.42) der Anlagenplanungsfunktion mit minus eins angesetzt wurde. Ihr Wert weicht jedoch noch verhältnismäßig stark von der Annahme einer kurzfristigen Substitutionselastizität von null ab, die in makroökonomischen Modellen häufig für die kurze Frist zugrundegelegt wird und die dann die Anwendung der Grenzproduktivitätstheorie für die Erklärung des kurzfristigen Verteilungsprozesses unbrauchbar zu machen scheint (1).

Um Schätzwerte für die beiden Parameter $\gamma_{1}$ und $\gamma_{2}$ zu erhalten, wurde auf die Produktionsfunktion (5.27) zurückgegriffen. Für diese Funktion sind mit dem in (5.32) angegebenen $\delta_{4}$-Wert alle Größen bis auf Parameter $Y_{1}$ bestimmt. Verwendet man nun einen willkürlich gewählten hypothetischen $\gamma_{1}$-Wert und die sonstigen auf der rechten Gleichungsseite von (5.27) in Erscheinung tretenden Größen, die alle bereits bestimmt sind, so kann eine Zeitreihe hypothetischer $x_{t} / x_{0}, t-$ Werte berechnet werden. Diese Reihe kann auf ihre Übereinstimmung mit den in Tabelle 4 angegebenen Schätzwerten von $x_{t} / x_{0, t}$ überprüft werden. Verfährt man so für alternative hypothetische $\gamma_{1}$-Werte, läßt sich der $\gamma_{1}$-Wert bestimmen, bei dem die Übereinstimmung zwischen der Zeitreihe der hypothetisch ermittelten $x_{t} / x_{0}, t-$ Werte mit den in Tabelle 4 angegebenen Schätzwerten von $x_{t} / x_{o}, t a m$ größten ist. Dieser "optimale" Wert beträgt +0,536 und führt für die Jahre 1960 bis $1971 \mathrm{zu}$ einer

E. Heuß, Krelles Verteilungstheorie, Zeitschrift f.d. gesamte Staatswissenschaft, 122, 1966, S. $163 \mathrm{ff}$, insbes. S. $174 \mathrm{f}$. und der Erwiderung von W. Krelle, Eine vereinfachte Version der "Verteilungstheorie", ebenda, S. $511 \mathrm{ff}$., insbes. S. 515 Fußnote 1, S. 516 und S. 519 Fußnote 2.

(1) Die oben entwickelte Grenzproduktivitätstheorie des kurzfristigen Arbeitsnachfrageverhaltens ist in erster Linie eine Theorie des Faktornachfrage-bzw. Güterangebotsverhaltens. - Vgl. E. Preiser, Erkenntniswert und Grenzen der Grenzproduktivitätstheorie, Schweiz. Zeitschr. f. Volkswirtschaft u. Statistik, 89, 1953, S. 25 ff. , wiederabgedruckt in: derselbe, Bildung und Verteilung des Volkseinkommens, 2. Aufl., Göttingen 1961, S. 265 ff. - Als solche hat sie auch bei einer Größe der kurzfristigen Substitutionselastizität von null volle Gültigkeit und bestimmt zusammen mit den Annahmen über das Güternachfrage- und Arbeitsangebotsverhalten bzw. das Verhalten der gewerkschaftlichen Lohnpolitik das Ergebnis des kurzfristigen Verteilungsprozesses. 
Übereinstimmung der beiden $x_{t} / x_{O}, t$-Reihen von $r^{2}=0,796$ (1). Dividiert man seine Größe durch den in (5.31) angegebenen Schätzwert von $\gamma_{1} / \gamma_{2}$, erhält man eine Schätzung für $\gamma_{2}$.

Aufgrund des angestellten Verfahrens gilt also:

$$
\text { (5.33) } \begin{aligned}
r_{1} & =0,536 \\
r_{2} & =0,800
\end{aligned}
$$

Beide Parameter zeigen die Bedeutung, die der Modifizierung der kurzfristigen Grenzproduktivitätstheorie bei der Anwendung auf die empirischen Verhältnisse beigemessen werden muß.

(1) Bezeichnet man die in Tabelle 4 angegebenen Schätzwerte von $x_{t} / x_{0}, t$ mit $X_{t}$ und die mit Hilfe der Funktion (5.27) ermittelten hypothetischen Werte von $x_{t} / x_{0}$, mit $\hat{x}_{t}$, dann ist das oben ermittelte Maß $r^{2}$ definiert als das "Bestimmtheitsmaß"

$$
r^{2}=1-\frac{\operatorname{var}\left(x_{t}-\hat{x}_{t}\right)}{\operatorname{var}\left(x_{t}\right)}
$$


$\$ 6$ Ergänzende Überlegungen zum privaten Investitionsverhalten

1. Anlagenabgang, Abschreibungen für Kapitalverzehr und Preisindex für Anlageinvestitionen

In den beiden vorausgegangenen Paragraphen wurden die Probleme behandelt, die mit der Grenzproduktivitätstheorie des Faktoreinsatzes zusammenhängen. Soweit sich die Überlegungen auf den Einsatz des Faktors Kapital erstreckten, bezogen sie sich allein auf die Größe des realen Anlagenbestandes. Soll die aufgestellte Theorie der Anlagenbestandsplanung zu einer vollständigen Theorie des privaten Investitionsverhaltens ausgebaut werden, bedarf sie notwendigerweise der Ergänzung durch eine Theorie des Anlagenabgangs und des Kapitalverzehrs.

In der Modellwirtschaft ist die Lebensdauer der Anlagen wegen technischer Abnutzung und wirtschaftlicher Veraltung begrenzt. Ständig scheiden alte Anlagen aus dem Produktionsprozeß aus und müssen durch neue Anlagen ersetzt werden. Die dazu erforderlichen Ersatzinvestitionen sind Bestandteil der laufenden Bruttoinvestitionen des Unternehmenssektors und bestimmen daher dessen Investitionsneigung. Wegen technischer Abnutzung und wirtschaftlicher Veraltung erfährt der Anlagenbestand zugleich ständige Wertminderungen und wird in der Vermögensrechnung des Unternehmenssektors abgeschrieben. Die zum Ausgleich dieser Wertminderungen von den Unternehmen einbehaltenen Abschreibungserlöse machen einen wesentlichen Teil des zur Investition verfügbaren Finanzierungsspielraumes aus (1) und sind daher mitentscheidend für die Kapitalmarktrückwirkungen der privaten Investitionstätigkeit.

Das vorliegende Modell geht der Einfachheit halber von einem altersmäßig homogenen Anlagenbestand aus und macht die technische Effizienz und die wirtschaftliche Ertragskraft der Anlagen nicht vom Zeitpunkt der Neuanschaffung abhängig. Eine endogene Bestimmung der wirtschaftlichen Lebensdauer der Anlagen scheidet für die Modellformulierung damit praktisch aus. Stattdessen wird die durchschnittliche Lebensdauer der Anlagen, die der zentrale Bestimmungsfaktor von Anlagenabgang und Abschreibungen ist, exogen vorgegeben.

An sich würde man der Einfachheit halber für den Anlagenzugang der einzelnen Perioden eine rechteckige Überlebensfunktion annehmen; bei der exogenen Vorgabe der durchschnittlichen Lebensdauer, die hier mit $\mathrm{T}_{\mathrm{t}}$ bezeichnet wird, wäre dann der Anlagenabgang der Periode $t, \mathrm{k}_{\mathrm{pr}}^{-} \mathrm{t}$, bestimmt durch den Anlagenzugang der Periode $t-T_{t}, k_{p r}^{+}, t-T_{t}$ :

(6.1)

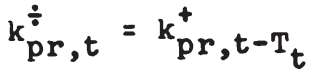

(1) Vgl. E. Domar, Depreciation, Replacement and Growth, Economic Journal, 63, 1953, S. $1 \mathrm{ff}$. 
Da die durchschnittliche Lebensdauer der Anlagen nach der Anlagenvermögensrechnung des Statistischen Bundesamtes für die relevanten Jahre mit mehr als $25 \mathrm{Jahren}$ anzusetzen ist und sie damit fast stets über die Grenzen des hier gesteckten Untersuchungszeitraums hinausreicht, ist diese Verhaltensgleichung aber bedeutungslos (1). In der Modellformulierung wird daher nicht nur die durchschnittliche Lebensdauer der Anlagen, sondern auch der Anlagenabgang selbst exogen vorgegeben. Praktisch wird wie folgt vorgegangen: Die Entwicklung des privaten Anlagenabgangs wird im Modell für die Jahre 1960 bis 1972 durch die entsprechenden Schätzwerte in der Anlagenvermögensrechnung des Statistischen Bundesamtes (2) exogen vorgegeben. Für die Jahre danach gilt eine Trendfunktion, die die Wachstumsrate des Anlagenabgangs wie in der zweiten Hälfte der 60 er Jahre weiterhin sinken und gleichmäßig gegen einen Wert von $5,5 \%$ streben läßt:

$$
\text { (6.2) } \quad 18 \mathrm{k}_{\mathrm{pr}, \mathrm{t}}^{\div}=\xi_{1}+\xi_{2} \cdot[t-1960]+\xi_{3} \cdot\left[\frac{1}{t-1965}\right]
$$

In (6.2) wurde der Parameter $\xi_{2}$ mit $\lg 1,055$ vorgegeben. Durch Einsetzen der Variablenwerte für die Jahre 1966 und 1971 ergaben sich zwei Gleichungen, deren Auflösung zur Bestimmung der Parameter $\xi_{1}$ und $\xi_{3}$ führte:

$$
\begin{array}{ll}
\text { (6.3) } \quad E_{1}=+1,26653 & \text { Ig Mrd. DM } \\
E_{2}=+0,02325 & \\
E_{3} & =-0,07820
\end{array}
$$

Da im Umfang des exogen vorgegebenen Anlagenabgangs Ersatzinvestitionen vorgenommen werden, enthalten die Bruttoinvestitionen in der Modellwirtschaft eine autonome Komponente. Diese Komponente weist aufgrund von (6.2) ab 1971 einen völlig gleichmäßig ansteigenden Verlauf auf und verursacht damit eine Glättung in der Entwicklung der Bruttoinvestitionen. Wegen des geringen Anteils der Ersatzinvestitionen ist der Glättungseffekt nicht erheblich.

Zur Bestimmung des Abschreibungsverhaltens im privaten Sektor wird aus den Angaben der Anlag evermögensrechnung des Statistischen Bundesamtes der Abschreibungssatz berechnet. Dieser ist definiert als Quotient aus dem realen Kapitalverzehr des privaten Sektors und dem privaten Bruttoanlagevermögen, beide in Preisen von $1962, \mathrm{~d}_{\mathrm{pr}}, \mathrm{t} / \mathrm{k}_{\mathrm{pr}}, \mathrm{t}$. Da die Abschreibungen im Modell wie in der Vermögensrechnung des Bundes-

(1) Es ist nicht vorgesehen, den Modellablauf für mehr als 30 Jahre durchzurechnen.

(2) Vgl. H. Lützel, a. a. O. , S. $607^{\star}$ ff. sowie ders., Das reproduzierbare Sachvermögen zu Anschaffungs- und Wiederbeschaffungspreisen, Wirtschaft und Statistik, 1972, S. $689^{\star}$ 
amtes linear vom Bruttoanlagevermögen berechnet werden, entspricht der Abschreibungssatz dem reziproken Wert der durchschnittlichen Lebensdauer der Anlagen, $T_{t}$. Es gilt also:

(6.4) $\quad \frac{d_{p r, t}}{k_{p r, t}}=\frac{1}{T_{t}}$

Für die Jahre 1960 bis 1971 wird die Höhe des Abschreibungssatzes in der Modellformulierung durch die für die Bundesrepublik ermittelten Werte exogen vorgegeben. Für die Jahre $1972 \mathrm{ff}$. wird seine Höhe ebenfalls exogen vorgegeben, und zwar durch die Trendfunktion (6.5):

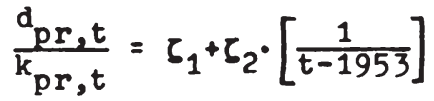

deren Parameter durch eine Regression über die Jahre 1960 bis 1971 geschätzt wurden:

$$
\begin{aligned}
& \zeta_{1}=+0,04073 \quad( \pm 0,00020) \\
& \zeta_{2}=-0,07177 \quad( \pm 0,00224) \\
& r_{1 \cdot 2}^{2}=0,99035 \quad(\text { Irrt.-Wahrsch.: }<0,1 \%) \\
& \text { vNQ }=1,001
\end{aligned}
$$

Infolge der laufenden Umstrukturierung des Anlagenbestandes in Richtung auf einen zunehmenden Anteil der verhältnismäßig kurzlebigen Ausrüstungsanlagen steigt in der Bundesrepublik der Abschreibungssatz bzw. sinkt die durchschnittliche Lebensdauer der Anlagen. Die Entwicklung weist jedoch Sättigungserscheinungen auf, weswegen der Abschreibungssatz auf den reziproken Zeitabstand vom Jahr 1953 regressiert wurde (1).

Durch Anwendung des exogen vorgegebenen Abschreibungssatzes auf das endogen bestimmte, zu Wiederbeschaffungspreisen bewertete Bruttoanlagevermögen ergeben sich die Abschreibungserlöse des Unternehmenssektors:

$$
D_{p r, t}=\frac{1}{T_{t}} \cdot k_{p r, t} \cdot p_{k, t}
$$

Als Index der Wiederbeschaffungspreise zur Bewertung des Anlagevermögens dient in der Modellformulierung der Preisindex für Anlageinvestitionen, $p_{k, t}$. Das Preisniveau für Güter der Anlageinvestition hängt

(1) Die Verwendung jedes anderen Basisjahres hätte das Regressionsergebnis verschlechtert. 
in der Modellwirtschaft vom allgemeinen Preisniveau der privaten Produktion, $p_{x, t}$, ab und vom Anteil der realen Investitionsgüternachfrage an der realen Gesamtnachfrage nach Produkten des privaten Sektors in Periode $t$ und $t-1,\left(\Delta \mathrm{k}_{\mathrm{pr}, \mathrm{t}}{ }^{+2} \Delta \mathrm{k}_{\mathrm{St}, \mathrm{t}}\right) / \mathrm{x}_{\mathrm{t}}$. Hierbei ist $\mathrm{zu}$ berücksichtigen, daß die staatlichen Bruttoanlageinvestitionen in der Modellwirtschaft nur zur Hälfte zum Ausbau der öffentlichen Infrastruktur dienen und daher insgesamt das Doppelte des Bruttozugangs an Infrastrukturanlagen, $\mathrm{k}_{\mathrm{St}, \mathrm{t}}$, ausmachen:

$$
\begin{aligned}
p_{k, t}= & u_{1} \cdot\left[\frac{\Delta k_{p r, t}^{+}+2 \cdot \Delta k_{S t, t}^{+}}{x_{t}}\right]^{u_{2}} \cdot \\
& {\left[\frac{\Delta k_{p r, t-1}^{+}+2 \cdot \Delta k_{S t, t-1}^{+}}{x_{t-1}}\right]^{u_{3}} \cdot p_{x, t} }
\end{aligned}
$$

Funktion (6.8) ist geeignet, den typisch asymptotischen Trendverlauf der Preisrelation $\mathrm{p}_{\mathrm{k}, \mathrm{t}} / \mathrm{p}_{\mathrm{x}, \mathrm{t}}$ in den Nachkriegsjahren der Bundesrepublik zu beschreiben, der mit dem asymptotischen Trendanstieg des Kapitalkoeffizienten im privaten Sektor einhergeht; ferner bringt sie die konjunkturellen Schwankungen der Investitionsgüterpreise zum Ausdruck, die vor allem auf die konjunkturellen Schwankungen der Investitionstätigkeit zurückzuführen sind.

Für die Schätzung wurde die Gleichung auf beiden Seiten durch $\mathrm{p}_{\mathbf{x}, t}$ dividiert, logarithmiert und, um etwaige Einflüsse der Umsatzsteuerreform von $1968 \mathrm{zu}$ berücksichtigen, um eine Dummy-Variable ergänzt, die für die Jahre bis einschließlich 1967 den Wert null, anschließend den Wert eins erhielt:

$$
\begin{aligned}
\lg \frac{p_{k, t}}{p_{x, t}}= & 1 g v_{1}+v_{2} \cdot 1 g\left[\frac{\Delta k_{p r, t}^{+}+2 \cdot \Delta k_{S t, t}^{+}}{x_{t}}\right]+ \\
& v_{3} \cdot 1 g\left[\frac{\Delta k_{p r, t-1}^{+}+2 \cdot \Delta k_{S t, t-1}^{+}}{x_{t-1}}\right]+ \\
& v_{4} \cdot \text { Dummy }_{p_{k}, t}
\end{aligned}
$$

Als statistisches Material dienten die Werte des privaten und öfentlichen Bruttoanlagenzugangs, die sich aus der Anlagenvermögensrechnung des Statistischen Bundesamtes ergeben, sowie die auf Basis 1962 berechneten Preisindices für den privaten BIP-Beitrag und für Anlageinvestitionen der Volkswirtschaftlichen Gesamtrechnung. 
Das Regressionsergebnis zu (6.9) für die Jahre 1958 bis 1971 lautet (1):

$$
\text { (6.10) } \begin{aligned}
& \lg u_{1}=+0,24767( \pm 0,02724) \\
& u_{2}= \pm 0,36411 \quad( \pm 0,07655) \\
& u_{3}=+0,08498 \quad( \pm 0,06665) \\
& u_{4}= \pm 0,00578 \quad( \pm 0,00218) \\
& R_{1 \cdot 2,3,4}^{2}=0,90896 \quad \text { (Irrt.-Wahrs ch.: <0,1\%) } \\
& R_{2 \cdot 3,4}^{2}=0,51962 \\
& R_{3 \cdot 2,4}^{2}=0,51748 \\
& R_{4 \cdot 2,3}^{2}=0,06120 \\
& v_{N Q}^{2}=1,861
\end{aligned}
$$

\section{Die Vorratsinvestitionen}

Als letzte Komponente der Bruttoinvestitionen sind in der Modellwirtschaft die Vorratsinvestitionen zu berücksichtigen. Die Bedeutung der Vorratsinvestitionen für die gesamte Entwicklung der Modellwirtschaft liegt in ihrem destabilisierenden Charakter. Sie verstärken auftretende Änderungen der monetären Gesamtnachfrage bzw. die davon ausgehenden Auswirkungen. Sie bestimmen daher nicht nur ähnlich wie die Kapazitätsplanung des Unternehmenssektors den konjunkturellen Ablauf der Modellwirtschaft mit, sondern auch die kurzfristigen Inzidenzeffekte finanzpolitischer Maßnahmen, die von Budgetmaßnahmen des Staates ausgehen.

Da die Vorratsbestände in der Formulierung des Modells nicht als Produktionsmittel angesehen werden, wi rd auf die Lagerhaltung des Unternehmenssektors nicht das Grenzproduktivitätsprinzip angewendet. Die Vorratsinvestitionen werden durch das sonst mit der Grenzproduktivitätstheorie bei der Erklärung des Investitionsverhaltens konkurrierende Akzelerationsprinzip bestimmt. Dieses Prinzip wird jedoch in der abge-

(1) Der Wert von Parameter $v_{4}$ deutet darauf hin, daß die Investitionsgüterpreise infolge der Umsatzsteuerreform von 1968 um 1, $3 \%$ stärker stiegen als der Preisindex des privaten BIP-Beitrages. 
wandelten Form des flexiblen Akzelerators (1) angenommen. Der Lagerbestand ist eine Funktion des entsprechenden Vorperiodenbestandes und des privaten Produktionsniveaus sowie der Gewinnsituation, repräsentiert durch die Arbeitseinkommensquote an der privaten Bruttowertschöpfung, und der Inflationsrate. Änderungen des Produktionsniveaus wirken sich bereits in der laufenden Periode aus, Änderungen der Arbeitseinkommensquote und der Inflationsrate werden erst nach Ablauf der Periode wirksam.

Ursprünglich wurde davon ausgegangen, daß sich Änderungen der Inflationsrate ebenfalls in der laufenden Periode auswirkten, und zwar über eine Beeinflussung der spekulativen Absatzpreiserwartungen. Ein entsprechender "Einfluß" ließ sich statistisch auch bei einer Irrtumswahrscheinlichkeit von $6 \%$ nachweisen. Dabei war das Bestimmtheitsmaß der Regressionsschätzung geringfügig besser als bei der hier akzeptierten Schätzung. Der zugehörige Regressionsparameter hatte jedoch einen derart hohen positiven Wert, daß, wie sich erst im nachhinein herausstellte, die Preiselastizität der gesamtwirtschaftlichen Nachfrage in der Modellrechnung positiv wurde und die Gesamtnachfragefunktion selbst in Situationen der Voll- und Überbeschäftigung häufig keinen Schnittpunkt mit der Gesamtangebotsfunktion mehr aufwies (2). Da das gelegentliche Fehlen einer kurzfristigen Gleichgewichtslösung von der übrigen Modellformulierung her nicht hingenommen werden kann, wurde ein ausschließlich verzögerter Einfluß der Inflationsrate angenommen, was dazu führte, das pathologische Ungleichgewichtssituationen in der Modellrechnung kurzfristig nicht mehr in Erscheinung traten.

Der reale Lagerbestand der Modellwirtschaft am Ende der Periode $t, v_{t}$, wird also bestimmt durch die Funktion:

$$
\frac{v_{t}}{v_{t-1}}=n_{1} \cdot\left[\frac{x_{t}}{v_{t-1}}\right]^{n_{2}} \cdot\left[\frac{p_{x, t-1}}{p_{x, t-2}}\right]^{n_{3}} \cdot\left[\frac{e_{p r, t-1} \cdot 1_{p r, t-1}}{x_{t-1} \cdot p_{x, t-1}}\right]^{n_{4}}
$$

Nach logarithmischer Transformation wurden für die Parameter dieser Gleichung innerhalb der Schätzperiode 1960-1971 die folgenden Schätz-

(1) Vgl. D. W. Jorgenson, Capital Theory and Investment Behavior, a. a. $\mathrm{O}$.

(2) Vgl. dazu die Schwierigkeiten, die im Zusammenhang mit der Bestimmung der Lagerinvestitionen im Krelle'schen Prognosemodell auftraten. Vgl. W. Krelle, D. Beckerhoff, H. G. Langer, H. Fuß, Ein Prognosesystem für die wirtschaftliche Entwicklung der Bundesrepublik Deutschland, a. a. O., S. $298 \mathrm{ff}$. - Im übrigen vgl. die Bemerkungen des folgenden Paragraphen 18. 
ergebnisse erzielt (1):

$$
\begin{aligned}
& \text { (6.12) } \lg , n_{1}=-0,84105 \quad( \pm 0,14439) \\
& n_{2}=+0,84523( \pm 0,16476) \\
& n_{3}=+0,93685( \pm 0,48667) \\
& n_{4}=-1,67348 \quad( \pm 0,35499) \\
& \left.\mathrm{R}_{1 \cdot 2,3,4}^{2}=0,90744 \text { (Irrt. -Wahrsch.: }<0,1 \%\right) \\
& R_{2 \cdot 3,4}^{2}=0,66618 \\
& R_{3 \cdot 2,4}^{2}=0,74097 \\
& R_{4 \cdot 2,3}^{2}=0,34292 \\
& \operatorname{VNQ}=2,316
\end{aligned}
$$

Aufgrund des Schätzwertes von Parameter $\eta_{2}$ verursacht eine einprozentige reale Nachfrageerhöhung kurzfristig eine 0,84-prozentige gleichgerichtete Änderung des Vorratsbestandes. Da der gesamtwirtschaftliche Vorratsbestand etwa $30 \%$ der Jahresproduktion ausmacht, beträgt der von der Vorratsveränderung ausgehende reale Nachfrageeffekt mehr als ein viertel Prozent $\left(\Delta v / x=n_{2}[v / x] \cdot[\Delta x / x]\right)$ und muß in dieser Höhe

(1) Der Lagerbestand für das Jahresende 1960 wurde mit dem 0, 302fachen des auf Basis 1962 preisbereinigten privaten Bruttoinlandsproduktbeitrages von 1960 angesetzt. - Vgl. Jahresgutachten 1969 des Sachverständigenrates zur Begutachtung der gesamtwirtschaftlichen Entwicklung, Bundestagsdrucks. VI/100 vom 1.12.1969, Bonn 1969, S. 11, Schaubild 8. - Anschließend wurde mit Hilfe der Angaben der volkswirtschaftlichen Gesamtrechnung über die Entwicklung der preisbereinigten Vorratsinvestitionen vor - und zurückgerechnet. - Leider hat sich an dieser Stelle ein unverzeihlicher Fehler in die gesamte Bestandsrechnung des Modells eingeschlichen. Die genannte Relation von 0,302 bezieht sich im Sachverständigengutachten auf das Verhältnis von Lagerbestand zu realem Bruttosozialprodukt; sie wurde hier fälschlicherweise auf den realen BIP-Beitrag des privaten Sektors angewandt. Die Folge ist eine um $7 \%$ zu niedrige Bemessung des Lagerbestandes zum Jahresende 1960 und eine entsprechende Unterschätzung aller übrigen Jahreswerte. Der Fehler schlägt sich in Regressionsergebnis (6.12) nieder und wirkt sich auf die modellmäßig aufgestellte Vermögensrechnung aus, die wichtigen Aussagetendenzen bleiben von ihm aber unberührt. 
zu der ursprünglichen einprozentigen Nachfrageerhöhung hinzuaddiert werden. Aufgrund der Interdependenz von Nachfrageänderung und Vorratsänderung ergibt sich im Endeffekt eine Verstärkung des ursprünglichen einprozentigen Nachfrageeffektes um etwa ein drittel Prozentpunkt.

Der destabilisierende Charakter der Vorratsinvestitionen wird allerdings gedämpft durch die Preisentwicklung für Vorratsinvestitionen. Wie bereits bei der Darstellung der volkswirtschaftlichen Gesamtrechnung im Paragraphen 2 angedeutet wurde, unterscheidet sich die Preisentwicklung für Vorratsinvestitionen in der Bundesrepublik wegen der Entwicklung der internationalen Rohstoffpreise und der Entwicklung der inländischen Erzeugerpreise wesentlich von der allgemeinen Preisentwicklung für Konsum- oder Investitionsgüter. Die Preisentwicklung weist bei den Vorratsinvestitionen seit Mitte der fünfziger Jahre einen fast stationären Trend auf, während der Preisindex des privaten Bruttoinlandsprodukts ständig gestiegen ist. Gleichzeitig verhalten sich ihre konjunkturellen Schwankungen gegenläufig zur konjunkturellen Nachfrageentwicklung, was sich auch beim Preisindex des privaten Bruttoinlandsprodukts beobachten läßt. Aus diesem Grund wird für Vorratsinvestitionen innerhalb der Modellwirtschaft ein eigener Preisindex berücksichtigt.

Der Preisindex der Vorratsinvestitionen wird bestimmt durch das allgemeine Preisniveau, $p_{x, t}$, und das Preisniveau für Importe, $p_{I m, t}$, in der laufenden Periode. Um die Auswirkungen der Umsatzsteuer reform von 1968 auf den Preisindex für Vorratsinvestitionen zu neutralisieren (1), wird zusätzlich eine Dummy-Variable berücksichtigt, die bis einschließlich 1967 den Wert 10 und anschließend den Wert 1 erhält. Es gilt also:

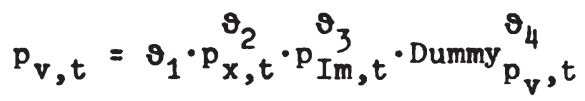

mit

$$
\text { Dummy }_{t}=\left\{\begin{array}{l}
10 \text { fur } t=\ldots, 1966,1967 \\
1 \text { fur } t=1968,1968,1969, \ldots
\end{array}\right.
$$

(1) Vgl. G. Hamer, Die Behandlung der Umsatz- (Mehrwert-)-steuer in den volkswirtschaftlichen Gesamtrechnungen, Wirtschaft und Statistik, 1968 , S. $439 \mathrm{ff}$. 
Für diese Preisgleichung wurden nach logarithmischer Transformation die folgenden Regressionsergebnisse erzielt (1958-1971) (1):

$$
\text { (6.14) } \begin{aligned}
18 \vartheta_{1}=-0,03057 \quad( \pm 0,00489) \\
\vartheta_{2}=+0,29884 \quad( \pm 0,03880) \\
\vartheta_{3}= \pm 0,86556 \quad( \pm 0,16737) \\
\vartheta_{4}=+0,02788 \quad( \pm 0,00435) \\
R_{1 \cdot 2,3,4}^{2}=0,93363 \quad \text { (Irrt.-Wahrsch.: <0,1\%) } \\
R_{2 \cdot 3,4}^{2}=0,62995 \\
R_{3 \cdot 2,4}^{2}=0,41499 \\
R_{4 \cdot 2,3}^{2}=0,59830 \\
v_{N Q}^{2}=1,799
\end{aligned}
$$

Per Saldo, also auch nach Berücksichtigung der Preisentwicklung, bleibt der destabilisierende Charakter der Vorratsirvestitionen erhalten. Auch muß in der Modellwirtschaft mit der genannten Stärkung der kurzfristigen, kreislaufbestimmten Inzidenzeffekte gerechnet werden, die von den budgetären Maßnahmen des Staates ausgehen: Erhöht der Staat die Gewinnsteuern und verausgabt er das erzielte Steueraufkommen in der gleichen Periode nachfragewirksam, so bleiben kurzfristig nicht nur die vorausbestimmten Anlageinvestitionen in ihrem realen Umfang von dieser Maßnahme unberührt, sondern es steigen auch die Vorratsinvestitionen, so daß von hier aus eine für steuerliche Überwälzungsprozesse durchaus günstige Situation entsteht.

(1) Die Größenrelation der Schätzwerte für $\vartheta_{2}$ und $\vartheta_{3}$ ist u. U. ein guter Anhaltspunkt für den Anteil inländischer bzw. ausländischer Güter an der Gesamtheit der Vorratsinvestitionen. - Im übrigen gibt der für die Dummy-Variable geschätzte Parameterwert $\left(\vartheta_{4}\right)$ den "Einfluß" der Umsatzsteuerreform auf das Preisniveau für Vorratsinvestitionen mit $-6,6 \%$ an. 
$\$ 7$ Das Einkommen der Arbeitnehmer-Haushalte und seine Verwendung

\section{Die Bestimmung der Geldlöhne}

In den meisten kapitalistischen Wirtschaftssystemen wird der Lohnsatz der Arbeitnehmer auf zwei Marktebenen bestimmt. Die Kollektivorganisationen der Arbeitnehmer und Arbeitgeber handeln miteinander tarifliche Mindestlöhne aus; auf der Basis dieser Mindestlöhne schließen die Arbeitnehmer und Arbeitgeber die individuellen Arbeitsverträge ab. Das Zusammenwirken der beiden Marktebenen läßt sich nicht einfach erfassen und kann hier nur behelfsmäßig dargestellt werden. Die Behelfslösung ist so konstruiert, daß sie der Möglichkeit eines Produktionsund Einkommensgleichgewichts bei Unterbeschäftigung Rechnung trägt.

Das aggregierte Verhalten der Arbeitgeber, soweit es ihre individuelle Arbeitsnachfrage betrifft, wurde durch die Beschäftigungsplanungsfunktion (4.49) und die kurzfristige Arbeitsnachfragefunktion (5. 25) formuliert. Für das aggregierte Verhalten des individuellen Arbeitsangebotes gilt die Funktion für die Entwicklung der Erwerbsbevölkerung (3.1). Beiden Funktionen wird nun eine Lohnsatzbestimmungsfunktion hinzu-

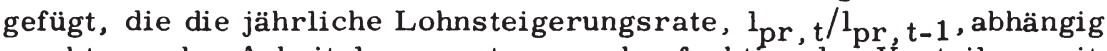
macht von der Arbeitslosenquote, von der funktionalen Verteilungssituation und dem Preisanstieg. Die Funktion ist eine erweiterte ex-anteFormulierung der Phillips-Kurve (1) und berücksichtigt mit der Abhängigkeit von Größen der Vergangenheit die empirisch vielfach beobachtete Erscheinung eines Lohn-lags (2). Es wird davon ausgegangen, daß die Funktion das Ergebnis der kollektiven Lohnverhandlungen in seiner Auswirkung auf das Effektivlohnniveau beschreibt (3).

(1) Vgl. A. W. Phillips, The Relation between Unemployment and the Rate of Change of Money Wages in the United Kingdom, 1861-1957, Economica, 25, 1958 , S. $283 \mathrm{ff}$.

(2) Vgl. z. B. die Beobachtungen zum Lohn-lag in den Jahresgutachten des Sachverständigenrates zur Begutachtung der gesamtwirtschaftlichen Entwicklung, $1958 \mathrm{ff}$.

(3) Gegen diese Interpretation der Phillips-Kurve lassen sich sicher einige Einwände vorbringen, da sich in ihr wahrscheinlich auch das aggregierte Verhalten des individuellen Arbeitsangebotes und der individuellen Arbeitsnachfrage niederschlägt. 
Die Funktion hat folgende Gestalt:

$$
\text { (7.1) } \begin{aligned}
\frac{1_{p r, t}}{I_{p r, t-1}}= & x_{1} \cdot\left[\frac{e_{v, t}-e_{t}}{a_{t}+\left(e_{v, t} e_{t}\right)}\right]^{x_{2}} \cdot\left[\frac{e_{p r, t-1} 1_{p r, t-1}}{x_{t-1} \cdot p_{x, t-1}}\right]^{x_{3}} \\
& {\left[\frac{p_{x, t-1}}{p_{x, t-2}}\right]^{x_{4}} \cdot\left[\frac{p_{x, t-2}}{p_{x, t-3}}\right]^{x_{5}} \cdot\left[\frac{p_{x, t-3}}{p_{x, t-4}}\right]^{x_{6}} \cdot } \\
& {\left[\frac{p_{x, t-1}}{p_{x, t-6}}\right]^{x_{7}} \cdot \text { Dummy }_{1, t} }
\end{aligned}
$$

mit

$$
\text { Dummy }_{1, t}=\left\{\begin{array}{l}
10 \text { für } t=1969 \\
1 \text { für } t \neq 1969
\end{array}\right.
$$

Dabei bezeichnet der Lohnsatz, $1_{\mathrm{pr}} \mathrm{t}$, das Bruttoarbeitseinkommen eines Beschäftigten einschließlich aller lohnbezogenen Sozialabgaben, die in der Modellwirtschaft auf der Arbeitnehmerseite erhoben werden. Neben der Arbeitslosenquote (1) der laufenden Periode, $\left(e_{v}, t-e_{t}\right) /\left(a_{t}+e_{v}, t-e_{t}\right)$, und der rechnerischen Arbeitseinkommensquote der Vorperiode, $\left(e_{\mathrm{pr}, \mathrm{t}-1^{\bullet}} 1_{\mathrm{pr}, \mathrm{t}-1}\right) /\left(\mathrm{x}_{\mathrm{t}-1} \mathbf{p}_{\mathrm{x}, \mathrm{t}-1}\right)$, die die funktionelle Verteilungssituation kennzeichnet, enthält die Funktion als Bestimmungsfaktoren des Lohnanstiegs die kurzfristigen Preisanstiegsraten der drei vorausgegangenen Perioden sowie die mittelfristige Preisanstiegsrate für die Zeit des ver-

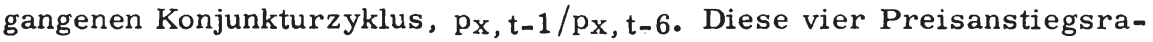
ten werden als für die Preiserwartungen der Gewerkschaften grundlegende Informationen angesehen. Schließlich wird mit Hilfe der Dummy-Variablen der Einfluß erfaßt, der von der Konzertierten Aktion des Staates und der

(1) Die Zahl der offenen Stellen wurde nicht als endogene Variable in die Modellformulierung aufgenommen. Grundsätzlich erscheint es jedoch sinrıvoll, diese Größe in der Lohnsatzrunktion mitzuberücksichtigen, und zwar dadurch, daß die Arbeitslosenquote durch die Quote (abhängige Erwerbspersonen minus Arbeitslose plus offene Stellen)/(abhängige Erwerbspersonen) ersetzt wird. 
Sozialpartner in der Bundesrepublik auf die Lohnentwicklung des Jahres 1969 ausging.

In Hinsicht auf die analysierten Verteilungsvorgänge ist die Verwendung der Arbeitseinkommensquote als Argument der Lohnbestimmungsfunktion nicht unproblematisch. Es sei deswegen auf zwei Implikationen hingewiesen. Als erstes ist zu beachten, daß die Gegengröße zu der Quote sich auf die Bruttozins - und -gewinneinkommen vor Abzug aller Unternehmenssteuern bezieht. Die Funktion läuft also darauf hinaus, daß sich die Gewerkschaften in ihrer Lohnpolitik an den im Unternehmenssektor entstandenen Bruttobesitzeinkommen orientieren und steuerliche Minderungen der Bruttobesitzeinkommen gegen sich nicht gelten lassen. Für die gegenwärtige Situation der Bundesrepublik ist eine solche Annahme wahrscheinlich zu vertreten. Hat der Staat jedoch eine weitgehende Umverteilung zu Lasten der Besitzeinkommen und insbesondere der Unternehmensgewinne durchgesetzt, dürfte die Funktion das lohnpolitische Verhalten der Gewerkschaften nicht ganz richtig beschreiben, weil dann u. U. ihr gesamtwirtschaftliches funktionales Verteilungsziel erreicht ist.

Schwerwiegender ist, daß das Argument nicht auf den Anteil der Arbeitskosten einschließlich der dem Produktionsfaktor Arbeit zurechenbaren steuerlichen Arbeitskostenbestandteile, sondern auf den Anteil der Arbeitseinkommen an der Bruttowertschöpfung des privaten Sektors abstellt. Erhöht der Staat die Sätze der im Unternehmenssektor erhobenen direkten oder indirekten Steuern und gelingt es den Unternehmen wegen der vollen nachfragewirksamen Verausgabung des Mehraufkommens durch den Staat, die Steuererhöhung kurzfristig zu überwälzen, so sinkt diese Arbeitseinkommensquote. Die Quotenänderung löst nach (7.1) Reaktionen der Gewerkschaften aus, die in der Folgeperiode einen erhöhten Lohnanstieg durchsetzen und damit für eine Verstärkung des überwälzungsbedingten monetären Expansionsprozesses sorgen. Im Fall der direkten Unternehmensbesteuerung und der indirekten Besteuerung des Faktors Kapital beschleunigen dabei der erhöhte Lohnanstieg und der durch ihn verstärkte monetäre Expansionsprozeß mit seinen Kreditmarktrückwirkungen den ohnehin zu erwartenden Abbau (1) der kurzfristig in Erscheinung getretenen Überwälzungseffekte. Im Fall der allgemeinen indirekten Steuern und der indirekten Besteuerung des Faktors Arbeit sind jedoch aufgrund der aufgestellten Verteilungshypothese endgültige Überwälzungseffekte zu erwarten. Wenn die Gewerkschaften auch hier mit der Durchsetzung eines erhöhten Lohnanstiegs reagieren, setzen sie damit einen längeranhaltenden und von inflatorischen Prozessen begleiteten Verteilungskampf in Gang, während dessen es ihnen u. U. gelingt, die Überwälzungseffekte vorübergehend abzuwehren, an dessen Ende jedoch wahrscheinlich eine auf Dauer erhöhte Arbeitslosenquote zu verzeichnen ist, weil die geldpolitischen Instanzen inflatorische Prozes-

(1) Vgl. Paragraph 5 Ziffer 6. 
se stärkeren Ausmaßes auf Dauer nicht hinnehmen. Zu dieser letzten Konsequenz käme es in der Modellwirtschaft nicht, wenn die Gewerkschaften sich statt an der Arbeitseinkommensquote am Anteil der Arbeitskosten einschließlich der zurechenbaren steuerlichen Arbeitskostenbestandteile orientierten, um die funktionale Verteilungssituation $\mathrm{zu}$ beurteilen, d. $h$. wenn sie die vom Staat vorgesehene Überwälzung der indirekten Steuern akzeptierten.

Die Regressionsergebnisse für die logarithmisch transformierte Gleichung $(7.1)$ sind $(t=1960, \ldots, 1971)$ :

$$
\text { (7.2) } \begin{array}{ll}
x_{1}=-0,71220 & ( \pm 0,06773) \\
x_{2}=-0,03645 & ( \pm 0,00481) \\
x_{3}=-2,81775 & ( \pm 0,26073) \\
x_{4}=+1,82998 & ( \pm 0,20352) \\
x_{5}= \pm 1,36596 & ( \pm 0,18615) \\
x_{6}=+1,15316 & ( \pm 0,26623) \\
x_{7}=-0,78873 & ( \pm 0,14226) \\
x_{8}=-0,01694 & ( \pm 0,00283) \\
R_{1 \cdot 2,3,4,5,6,7,8}^{2}=0,98791 \quad(\text { Irrt. }- \text { Wahrsch. }:<0,1 \%) \\
R_{2 \cdot 3,4,5,6,7,8}^{2}=0,35212 \\
R_{3 \cdot 2,4,5,6,7,8}^{2}=0,87310 \\
R_{4 \cdot 2,3,5,6,7,8}^{2}=0,86397 \\
R_{5 \cdot 2,3,4,6,7,8}^{2}=0,75550 \\
R_{6 \cdot 2,3,4,5,7,8}^{2}=0,71402 \\
R_{7 \cdot 2,3,4,5,6,8}^{2}=0,81414 \\
R_{8 \cdot 2,3,4,5,6,7}^{2}=0,43705 \\
v N Q=3,162 & \\
&
\end{array}
$$


Trotz der starken Mulitkollinearität sind alle Regressionsparameter bei einer Irrtumswahrscheinlichkeit von $1 \%$ signifikant von null zu unterscheiden. Der hohe Wert des von-Neumann-Quotienten ist verursacht durch starke Fehlereinflüsse in den Jahren 1960 und 1961, in denen sich offensichtlich ein Bruch im lohnplitischen Verhalten der Tarifpartner vollzog (1).

Nach den Schätzergebnissen wurden die Lohnsteigerungen in der Bundesrepublik sehr stark durch die Preisentwicklung in den vorausgegangenen Jahren bestimmt. Weniger stark reagierten sie auf kurzfristige Änderungen der Arbeitsnachfrage während der gerade laufenden Periode; z. B. hätte bei den vorgefundenen durchschnittlichen Preissteigerungsraten die Arbeitslosenquote auf über $9 \%$ steigen müssen, um den Lohnanstieg auf null Prozent zu reduzieren.

\section{Die Vermögenserträge der Arbeitnehmer-Haushalte}

Um die Entwicklung der den Arbeitnehmer-Haushalten in der Modellwirtschaft zufallenden Vermögenserträge zu bestimmen, wird von Definitionsgleichung (2.51) ausgegangen, wonach die Vermögenserträge sich ausdrücken lassen als das Produkt aus dem Forderungsbestand der Unselbständigen, $F_{A}$, und der Ertragsrate auf diesem Forderungsbestand, $\mathrm{r}_{\mathrm{A}}$. Die Ertragsrate, $r_{A}$, wird in $z$ wei Komponenten zerlegt, den allgemeinen Kapitalmarktzins, $r$, und die für das Vermögensanlageverhalten der Unselbständigen typische Relation von Vermögensertragsrate zu Kapitalmarktzins, $r_{A} / r$. Für die Vermögenserträge der Unselbständigen in Periode $t$ gilt daher:

$$
R_{A, t}=\left[\frac{r_{A, t-1}}{r_{t-1}}\right] \cdot r_{t-1} \cdot F_{A, t-1}
$$

d. h. die Erträge sind bestimmt durch den Forderungsbestand am Ende der Periode t-1 bzw. zu Beginn der Periode $t$ sowie durch das allgemeine Zinsniveau und die Ertragsrate/Zins-Relation in Periode $t-1$.

Von den in (7.3) enthaltenen vier Größen sind für die Bundesrepublik zwei bekannt: Der Kapitalmarktzins, $r_{t}$, wird im Modell durch die Umlaufrendite festverzinslicher Wertpapiere repräsentiert. Für die aggregierten Vermögenseinkünfte der Unselbständigen haben W. Kaiser und

(1) Die Existenz eines solchen Bruches zeigt sich deutlich in getrennten Regressionsschätzungen für die Jahre 1952 bis 1961 einerseits und 1962 bis 1971 andererseits. 
A. Zerwass (1) eine von 1950 bis 1967 reichende Zeitreihe berechnet. - Die beiden anderen Größen sind für die Bundesrepublik empirisch noch nicht belegt. Es wird daher der Versuch unternommen, ihre Größe und Entwicklung modellmäßig festzulegen.

Der erste Schritt der Modellrechnung besteht in der Bestimmung des aggregierten Nominalvermögens, das dem privaten Sektor der Modellwirtschaft zuzurechnen ist. Hierzu wurde die vom Statistischen Bundesamt veröffentlichte Zeitreihe für das Nettoanlagevermögen des privaten Sektors in der Bundesrepublik (2) sowie die eigene Berechnung des im Unternehmenssektor der Bundesrepublik vorhandenen Vorratsbestandes herangezogen. Aus dem 1960er-Wert beider Zeitreihen wurde nach der im Textanhang 1 angegebenen Methode das von den Unternehmen bis Anfang 1960 zur Finanzierung von Anlagen und Vorräten aufgenommene Finanzkapital, $F_{t}^{\star}$, geschätzt und mit Hilfe der Angaben über die private Nettoinvestition anschließend vor- und zurückgerechnet. Durch Addition des gesamtwirtschaftlichen Devisenbestandes und durch Abzug der geschätzten Nettoforderungsbestände von Staat und Ausland gegenüber dem privaten Sektor ergaben sich schätzwerte für das Nominalvermögen des privaten Sektors, das der Summe der in der Vergangenheit gebildeten privaten Nominalersparnis entspricht (3).

Die eigentliche Willkür und Problematik der Modellrechnung liegt in dem folgenden Schritt. Nach einer Untersuchung von W. Krelle, J. Schunk und J. Siebke (4) betrug der Anteil der Unselbständigen-Haushalte an der seit 1950 aufaddierten Nominalvermögensbildung des privaten Sektors in der Bundesrepublik $196025 \%$ und $196335 \%$ (5). In loser Anlehnung an

(1) Vgl. W. Kaiser und A. Zerwass, Die Struktur des Sparens in der Bundesrepublik Deutschland von 1950 bis 1967, Berlin 1970, Tabelle 23. Die Tabelle enthält die Vermögenseinkünfte nach Abzug entsprechender Steuern. Für die vorliegenden $Z$ wecke wurden die Angaben für Arbeitnehmer - und Rentner - bzw. Pensionärshaushalte zusammengezogen. Vgl. ebenda, S. 47.

(2) Vgl. H. Lützel, Das reproduzierbare Anlagevermögen in Preisen von 1962 , a. a. O. , S. $607^{\star}$

(3) Die Zeitreihe für das geschätzte Nominalvermögen des privaten Sektors findet sich in Tabelle 5 des Tabellenanhangs.

(4) Vgl. W. Krelle, J. Schunk u. J. Siebke, Überbetriebliche Ertragsbeteiligung der Arbeitnehmer, Tübingen 1968, Bd. II, S. $326 \mathrm{f}$.

(5) Aufgrund der revidierten Ergebnisse der volkswirtschaftlichen Gesamtrechnung (Basis 1962) sind die Anteile wahrscheinlich etwas niedriger anzusetzen, weil dort die einbehaltenen Gewinne der Unternehmen höher bemessen wurden als vor der Revision. 
diese Werte wurde angenommen, der Anteil der Unselbständigen am insgesamt vorhandenen Nominalvermögen des privaten Sektors habe zu Beginn des Jahres 195025 \% betragen (1). Eine weitere Problematik entsteht bei der Fortrechnung der Vermögensbestände (2). Könnte man von der Annahme einer konstanten Erwerbsstruktur ausgehen, also von einer gleichbleibenden Aufteilung der Erwerbsbevölkerung in Selbständige und Unselbständige, ließen sich die aggregierten Vermögensbestände der beiden Personengruppen bei der getroffenen Annahme über die Vermögensverteilung im Ausgangsjahr 1950 mit Hilfe von Angaben über die Nominalersparnis der Selbständigen und Unselbständigen sowie der Unternehmen ohne allzu große Schwierigkeit fortrechnen. Tatsächlich vollzog sich aber eine ständige Änderung der Erwerbsstruktur in Richtung auf einen zunehmenden Anteil der Unselbständigen, und es muß damit gerechnet werden, daß die aus dem Sektor der Selbständigen abwandernden Erwerbspersonen ihre Vermögensbestände mit in den UnselbständigenSektor einbrachten. Die Schwierigkeit besteht darin, entsprechende rechnerische Vermögensübertragungen zwischen beiden Sektoren größenmäßig richtig anzusetzen.

Zwei einfache Alternativen bieten sich zu einer realistischen modellmäßigen Erfassung der Vermögensübertragungen an: 1. Die gerade abwandernden Selbständigen besitzen pro Kopf genau so viel Vermögen wie der Durchschnitt der unselbständigen Erwerbspersonen. - 2. Die abwandern-

(1) Vgl. dazu auch die Vermögensverteilungsangaben in den Tabellen von H. J. Krupp, Theorie der personellen Einkommensverteilung, Berlin 1968, S. $171 \mathrm{ff}$, die auf einen Vermögensanteil der Arbeitnehmer von 0,2327 im Jahre 1955 und von 0,2635 im Jahre 1960 hinauslaufen, freilich bei Verwendung eines anderen Vermögenskonzeptes.

(2) Den Hinweis darauf, daß sich Erwerbsstrukturänderungen auf die Vermögensverteilung auswirken und daß die im folgenden akzeptierte Modellannahme 1 wahrscheinlich die am meisten realisitische Annahme für eine einfache Erfassung der entsprechenden Vermögensüber tragungen ist, verdanke ich Dr. Paul Hövelmann. Seiner Meinung nach könnte es sein, daß ein Teil der aus dem Sektor der Selbständigen abwandernden Erwerbspersonen sein Betriebsvermögen verkauft, also "Produktivvermögen" gegen "Geldvermögen" tauscht; unter diesen Umständen entstüncle eine Tendenz zur Konzentration von "Produktivvermögen" bei den im Selbständigen-Sektor verbleibenden Erwerbspersonen und eine entsprechende Tendenz zur Konzentration von "Geldvermögen" im Unselbständigen-Sektor, Tendenzen, die sich als solche in der Bundesrepublik durchaus empirisch nachweisen lassen. Erwerbsstrukturänderungen beeinflussen die gesamtwirtschaftliche Vermögensstruktur also in mehrfacher Hinsicht. 
den Selbständigen besitzen pro Kopf genau so viel Vermögen wie der Durchschnitt aller selbständigen und unselbständigen Erwerbspersonen. Die tatsächlichen Verhältnisse halten sich wahrscheinlich $\mathrm{z}$ wischen diesen beiden Modellannahmen. Es ist jedoch schwierig, irgendwelche genaueren Annahmen zu treffen. Aus diesem Grund wird für die Modellwirtschaft die Annahme 1 akzeptiert (1).

Die durch Änderungen der Erwerbsstruktur bedingten Vermögensüber tragungen zwischen dem Sektor der Selbständigen und dem Sektor der Unselbständigen, ÜWSA, $t$, werden in der Modellwirtschaft also, wie folgt, bestimmt: $s_{t} / e_{v, t}$ gibt die Selbständigenquote, $1-s_{t} / e_{v, t}$ die Unselbständigenquote an der Erwerbsbevölkerung der Modellwirtschaft an. $\left(s_{t-1} / e_{v, t-1}-s_{t} / e_{v, t}\right) /\left(1-s_{t-1} / e_{v, t-1}\right)$ kennzeichnet die prozentuale Änderung der Unselbständigenquote gegenüber dem Vorjahr. Für die Vermögensübertragungen gilt:

$$
U_{W S A, t}=w_{A, t-1} \cdot \frac{\left(s_{t-1} / e_{v, t-1}\right)-\left(s_{t} / e_{v, t}\right)}{1-\left(s_{t-1} / e v_{t-1}\right)} .
$$

d. h. die Übertragungen entsprechen dem Vorjahresendbestand am Nominalvermögen der Unselbständigen multipliziert mit der prozentualen Änderung der Unselbständigenquote.

Ausgehend von dem für Anfang 1950 angenommenen Unselbständigenvermögen in Höhe von $25 \%$ des gesamten privaten Nominalvermögens wurde durch Zuschlag der mit Gleichung (7.4) bestimmten rechnerischen Vermögensübertragungen zwischen Selbständigen und Unselbständigen und durch Zuschlag der jährlichen Unselbständigen-Ersparnis (2) eine Zeit-

(1) Die Annahme ähnelt der Annahme, mit der die rechnerische Arbeitseinkommensquote an der Bruttowertschöfung des privaten Sektors ermittelt wurde. Im Gegensatz zu jener eignet sie sich aber nicht für eine sehr langfristige Fortrechnung des Modells, da sie auf die Dauer sicher zu einer Unterschätzung der zeitlich kumulierten Vermögensübertragungen führt.

(2) Die Angaben hierzu wurden berechnet nach W. Kaiser und A. Zerwass, Die Struktur des Sparens in der Bundesrepublik Deutschland von 1950 bis 1967 , a. a. O. , Tab. 22, wobei die dort getrennt ausgewiesenen Arbeitnehmer - und Rentner - bzw. Pensionärshaushalte zusammengefaßt wurden. - Das dort geschätzte Größenverhältnis von Unselbständigenersparnis zu Selbständigenersparnis (ohne einbehaltene Gewinne der Personalgesellschaft) wurde auf die Ersparnis der privaten Haushalte (ebenfalls ohne einbehaltene Gewinne der Personalgesellschaften) in der revidierten volkswirtschaftlichen Gesamtrechnung (Basis 1962) angewendet. 
reihe für das Vermögen der Unsєlbständigen in der Modellwirtschaft berechnet (1). Nach Abzug der geschätzten Kassenhaltung ergab sich eine entsprechende Reihe für den Forderungsbestand der Unselbständigen. Damit war die dritte Größe der Gleichung (7.3) bestimmt (2).

Die Berechnung der vierten Größe von (7.3) bereitete nach den bisherigen Schritten keine Schwierigkeiten mehr. Die Division der Vermögenseinkünfte der Unselbständigen durch den modellmäßig berechneten Forderungsbestand ergab die Ertragsrate auf das Forderungsvermögen der Unselbständigen; die nochmalige Division durch die Umlaufsrendíte festverzinslicher Wertpapiere führte zu der Relation aus Ertragsrate auf Unselbständigenvermögen und Kapitalmarktzins (3). Die Zeitreihe der so berechneten Relation weist einen erstaunlich regelmäßigen Verlauf auf, der sich in etwa durch den Verlauf einer logistischen Funktion beschreiben läßt. Trotz des starken Modellcharakters der aufgestellten Vermögensrechnung könnte man den Verlauf der Relation als eine Illustration zu der vom DIW getroffenen Feststellung ansehen, wonach sich im Vermögensanlageverhalten der privaten Haushalte in der Nachkriegszeit eine Wandlung in Richtung auf ein zunehmendes Renditebewußtsein vollzogen habe (4). Entsprechend dem zeitlichen Verlauf der berechneten Relation müßte diese Wandlung gegen Ende der 60er Jahre fast abgeschlossen sein; die Ertragsrate auf den Forderungsbestand der Unselbständigen hätte dann aufgrund des sich wandelnden Anlageverhaltens (5)

(1) Die Reihe findet sich in Tab. 6 des Tabellananhangs.

(2) Der Vermögensertrag der Unselbständigen wird durch Zuschlag des Ertrages $r_{A, t-1}$ auf den noch nicht um die Vermögensübertragungen erhöhten Forderungsbestand vom Vorjahresende, $F_{A, t-1}$, berechnet. Dies impliziert, daß der Zins auf die während der laufenden Periode übertragenen Forderungsbestände $\ddot{U}_{F S A} t$ noch bei den Zinseinkommen der Selbständigen erfaßt wird. Außerdem erstrecken sich die Vermögensübertragungen gemäß (7.4) nicht auf die Substanzerhaltungsrücklagen. Zur Rechtfertigung wird davon ausgegangen, daß die ihre Selbständigkeit aufgebenden Erwerbspersonen ihre Betriebe mit einem Verlust in Höhe der laufenden Kapitalverzinsung und der Substanzerhaltungsrücklage verkaufen und daß dieser Verlust den Käufern der Betriebe zufällt.

(3) Die Modellwerte für die Ertragsrate auf das nicht in Form von Kasse gehaltene Nominalvermögen der Unselbständigen findet sich in Tab. 7 des Tabellenanhangs.

(4) Vgl. Die Vermögenseinkünfte der privaten Haushalte in der Bundesrepublik Deutschland, in: Wochenbericht des Deutschen Instituts für Wirtschaftsforschung, Nr. 20/21, vom 16.5.1958, S. 119

(5) Wegen der großen Bedeutung des Kontensparens dürfte die Rendite des Unselbständigen-Vermögens unter der des Selbständigen-Vermögens liegen. 
eine Obergrenze von etwa $60 \%$ der Umlaufsrendite festverzinslicher Wertpapiere erreicht.

Der Verlauf der Relation wird in der Modellwirtschaft durch eine einfache Trendfunktion exogen vorgegeben:

$$
\frac{R_{A, t}}{r_{t-1} \cdot F_{A, t-1}}=\iota_{1}+\iota_{2} \cdot\left[\frac{1}{t-1955}\right]
$$

Die Parameter dieser Funktion wurden durch eine Regression für die Jahre 1958 bis 1967 geschätzt:

$$
\begin{aligned}
& \text { (7.6) } \quad l_{1}=0,70714 \quad( \pm 0,02883) \\
& l_{2}=-1,29258 \quad( \pm 0,16245) \\
& \left.r_{1.2}^{2}=0,88782 \quad \text { (Irrt.-Wahrsch.: }<0,1 \%\right) \\
& \text { vNQ }=2,249
\end{aligned}
$$

Das bei der Schätzung anfallende Bestimmtheitsmaß hat einen verhältnismäßig lohen Wert. Bezieht man die errechnete Ertragsrate des Unselbständigen-Vermögens jedoch nicht auf den Kapitalmarktzins der Vorperiode, sondern auf den der laufenden Periode, so verbessert sich das Ergebnis noch einmal um $10 \%$ (1). U. U. ist dies darauf zurückzuführen, daß die Schätzung der von W. Kaiser und A. Zerwass bzw. vom DIW (2) errechneten Vermögenseinkünfte selbst sehr stark auf der Verwendung der Umlaufsrendite festverzinslicher Wertpapiere für die laufende Periode beruht. Möglicherweise zeigt sich hierin aber auch, daß die Annahme, die Forderungstitel würden mit dem Kapitalmarktzins der jeweiligen Vorperiode bedient, keine gute Verzinsungsannahme ist. In der Modellwirtschaft

(1) Die entsprechenden Regressionsergebnisse lauten:

$$
\begin{aligned}
& \text { (7.6a) } \frac{R_{A, t}}{r_{t} \cdot F_{A, t-1}}=\frac{0,66029}{(0,00904)}-\left(\begin{array}{l}
1,00992 \\
(0,05091)
\end{array} \cdot\left[\frac{1}{t-1955}\right]\right. \\
& \left.\mathbf{r}_{1 \cdot 2}^{2}=0,98007 \text { (Irrt.-Wahrsch.: }<0,1 \%\right) \\
& \text { VNQ } \quad=1,997
\end{aligned}
$$

(2) Die Berechnungen von W. Kaiser und A. Zerwass beruhen auf Berechnungen des DIW. Vgl. W. Kaiser und A. Zerwass, DieStruktur des Sparens in der Bundesrepublik Deutschland 1950 bis 1967, a. a. O. , Tabelle 23 
wird der Einfachheit halber dennoch von dieser Annahme ausgegangen; um die Ertragsrate des nicht in Kasse angelegten UnselbständigenVermögens in Abhängigkeit vom allgemeinen Kapitalmarktzins der Modellwirtschaft zu bestimmen, wird also auf (7.5) zurückgegriffen.

\section{Der Konsum der Unselbständigen-Haushalte}

Neben den im privaten Sektor des Inlandes verdienten Arbeits- und Besitzeinkommen beziehen die Unselbständigen-Haushalte Einkommen aus unselbständiger Beschäftigung im staatlichen Sektor. Korrigiert man die Summe dieser drei Einkommenskomponenten um die Belastung durch Lohnsteuer und Sozialabgaben und um die Summe der vom Staat empfangenen Transferzahlungen (1), gelangt man zu dem Einkommen, über dessen Verwendung die Unselbständigen Haushalte frei entscheiden können.

Einen Teil ihres Einkommens verwenden die Unselbständigen in der Modellwirtschaft als Unterstützungszahlungen an die übrige Welt (2). Es handelt sich vor allem um die Überweisungen ehemals zugewanderter ausländischer Arbeitskräfte an die im Ausland gebliebenen Familienangehörigen. Dieser Teil, $U_{A}$, Ausl, $t$, wird bestimmt durch die lohnsteuerfreien Nettoeinkommen aus unselbständiger Arbeit in der laufenden Periode, die hier mit $L_{A, t}^{n}$ bezeichnet seien. Es gilt:

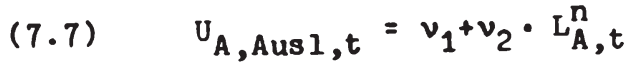

mit den Regressionsergebnissen für die Jahre 1960 bis 1971:

$$
\begin{aligned}
& v_{1}=-2,73495 \quad( \pm 0,18042) \text { Mrd.DM } \\
& v_{2}=0,02557 \quad( \pm 0,00078) \\
& r_{1 \cdot 2}^{2}=0,99087 \quad(\text { Irrt.-Wahrsch.: }<0,1 \%) \\
& \text { vNQ }=1,307
\end{aligned}
$$

(1) Zur statistischen Erfassung wurde angenommen, alle Transferzahlungen des Staates an die privaten Haushalte, wie sie in der volkswirtschaftlichen Gesamtrechnung der Bundesrepublik Deutschland angewiesen werden, seien ausschließlich an die UnselbständigenHaushalte geflossen.

(2) Als statistisches Material wurde der Saldo der unentgeltlichen Leistungen bzw. Übertragungen $\mathrm{zwischen}$ dem privaten Sektor und der übrigen Welt verwendet. Vgl. Statistische Beihefte zu den Monatsberichten der Deutschen Bundesbank, Reihe 3, Zahlungsbilanzstatistik, No. 7, Juli 1972, Beilage, S. 2 f. 
Nach Abzug der Unterstützungszahlungen an die übrige Welt verbleibt den Unselbständigen das sog. "verfügbare Einkommen" (1), Y ist der Hauptbestimmungsfaktor für die Nachfrage nach Gütern und Diensten, die sie zur Deckung ihres laufenden Konsums entfalten.

Das Konsumverhalten der Unselbständigen wird in der Modellwirtschaft durch eine Brownsche Konsumfunktion (2) beschrieben. Danach ist der reale Pro-Kopf-Konsum eine lineare Funktion des verfügbaren realen Pro-Kopf-Einkommens der unselbständigen Erwerbspersonen sowie ihres Vorperiodenkonsums:

$$
\frac{c_{A, t}}{a_{v, t} \cdot p_{x, t}}=o_{A 1}+o_{A 2} \cdot\left[\frac{Y_{A, t}^{n}}{a_{v, t} p_{x, t}^{p_{x}}}\right]+o_{A 3} \cdot\left[\frac{c_{A, t-1}}{a_{v, t-1} p_{x, t-1}}\right]
$$

Die Regressionsergebnisse der für (7.9) angestellten Kleinst-QuadratSchätzung

$$
\text { (7.10) } \begin{aligned}
& o_{A 1}=+514,31836( \pm 46,49513) \text { DM p.c.real } \\
& o_{A 2}= \pm 0,68704( \pm 0,05927) \\
& o_{A 3}= \pm 0,19269( \pm 0,07145) \\
& \left.R_{1 \cdot 2,3}^{2}=0,99958 \text { (Irrt.-Wahrsch.: }<0,1 \%\right) \\
& r_{2 \cdot 3}^{2}=0,99400 \\
& h
\end{aligned}
$$

(1) Das verfügbare Einkommen der Unselbständigen wurde zum Zweck der empirischen Überprüfung der für die Modellwirtschaft geltenden Verhaltensfunktion also wie folgt abgegrenzt: Bruttoeinkommen aus unselbständiger Arbeit (gem. volkswirtschaftlicher Gesamtrechnung) plus Vermögenseinkünfte der Unselbständigen (gem. W. Kaiser und A. Zerwass) minus Lohnsteuer und Sozialabgaben plus Unterstützungszahlungen des Staates an die privaten Haushalte (gem. volkswirtschaftlicher Gesamtrechnung) minus unentgeltliche Leistungen des privaten Sektors an die übrige Welt (gem. Zahlungsbilanzstatistik der Deutschen Bundesbank).

(2) Vgl. T. M. Brown, Habit Persistence and Lags in Consumer Behavior, Econometrica, 20, 1952, S. $355 \mathrm{ff}$.

(3) Als Testgröße auf Autokorrelation wird wegen des Auftretens des Vorperiodenkonsums als verzögert-endogene Einflußgröße das Durbin'sche h-Maß verwendet. Vgl. J. Durbin, Testing for Serial Correlation in least-Squares Regressions when Some of the Regressors are Lagged Dependent Variables, Econometrica, 38, 1970, S. $410 \mathrm{ff}$. 
die sich auf die Periode 1951 bis 1967 beziehen (1) zeigen mit einem hohen Wert des Absolutgliedes o A1, daß das Konsumverhalten der Unselbständigen in der Bundesrepublik durch einen starken konsumtiven Nachholbedarf in der unmittelbaren Nachkriegszeit und einen anschließenden Rückgang der durchschnittlichen Konsumneigung gekennzeichnet ist. Der Schätzwert von Parameter o A3, der trotz der hohen Multikollinearität bei einer Irrtumswahrscheinlichkeit von $2 \%$ signifikant ist, deutet darauf hin, daß die Gewohnheitsausrichtung im Konsumverhalten der Arbeitnehmer für die Jahresperiode nur schwach ausgeprägt ist.

Die Bemühungen der deutschen Bundesregierung um eine Förderung der Vermögensbildung in Arbeitnehmerhand legen nahe zu überprüfen, ob das Spar- bzw. Konsumverhalten der Unselbständigen nicht von dorther einem zusätzlichen Einfluß ausgesetzt war, und, wenn ja, den Einfluß im Modell zu berücksichtigen. Zur Überprüfung wurden daher die staatlichen Ausgaben zur Förderung der Vermögensbildung nach dem Ersten und Zweiten Vermögensbildungsgesetz (2) als zusätzliche unabhängige Variable in die Schätzung einbezogen, und zwar als preisbereinigte Größen, umgelegt auf die Zahl der unselbständigen Erwerbspersonen. Ein Einfluß ließ sich in dieser Weise nicht signifikant ermitteln. Alternativ wurde in die Konsumfunktion eine Dummy-Variable aufgenommen, die bis zum Zeitpunkt, an dem das Erste Vermögensbildungsgesetz wirksam wurde, den Wert null, anschließend, also für die Jahre 1963 ff., den Wert eins erhielt. Bezeichnet man den zugehörigen Regressionsparameter mit dem Symbol oA4, so lauten die Regressionsergebnisse für die so erweiterte Konsumfunktion (7.9):

(1) Die Konsumausgaben der Unselbständigen wurden ermittelt durch Abzug der bereits im vorigen Abschnitt benötigten und im Anschluß an die Untersuchung von W. Kaiser und A. Zerwass berechneten Unselbständigen-Ersparnis vom verfügbaren unselbständigen Einkommen, $\mathrm{Y}_{\mathrm{A}, \mathrm{t}}^{\mathrm{n}}$.

(2) Die Angaben hierzu wurden entnommen dem Finanzbericht des Bundesministeriums der Finanzen 1966, S. 197 und 1967, S. 169 (für die Jahre 1963 und 1964) und 1970, S. 298 (für die Jahre 1965 ff). 


$$
\text { (7.11) } \begin{aligned}
O_{A 1} & =+369,84278 \quad( \pm 66,34917) \text { DM p.c. real } \\
O_{A 2}=+0,73948 \quad( \pm 0,07094) & O_{A 3}=+0,16203 \quad( \pm 0,08429) \\
O_{A 4} & =-137,67578 \quad( \pm 35,92504) \\
R_{1 \cdot 2,3,4}^{2} & =0,99947 \quad(\text { Irrt.-Wahrsch.: }<0,16) \\
R_{2 \cdot 3,4}^{2} & =0,99420 \\
R_{3 \cdot 2,4}^{2} & =0,99400 \\
R_{4 \cdot 2,3}^{2} & =0,60155 \\
h & =0,337
\end{aligned}
$$

Der Parameter der Dummy-Variablen ist bei einer Irrt umswahrscheinlichkeit von kleiner als $0,5 \%$ signifikant von null verschieden (1). Es ist also möglich bzw. sogar wahrscheinlich, daß das erste Vermögensbildungsgesetz der Bundesregierung Auswirkungen auf das Spar-bzw. Konsumverhalten der Unselbständigen hatte. Allerdings handelt es sich nur um einen Niveaueffekt, der hier signifikant festgestellt werden konnte, nicht um eine Änderung der marginalen Konsumneigung, die einen nachhaltigen Einfluß auf die Vermögensverteilung gehabt hätte (2). Im Gegensatz zu einer Vermutung von W. Krelle und J. Siebke (3) muß der bewirkte Vermögensbildungseffekt jedoch ein ganz beachtliches Ausmaß gehabt haben und betragsmäßig die staatlichen Auf wendungen zur Förderung der Vermögensbildung während der gesamten Untersuchungs-

(1) Die Einführung der Dummy-Variablen reduziert das standard-normalverteilte $h-M a \beta$ beträchtlich und hat daher die in (7.10) festgestellte Autokorrelation stark zum Verschwinden gebracht.

(2) Die Auswirkungen des geänderten Konsum- bzw. Sparverhaltens der Unselbständigen auf die Verteilung des Nominalvermögens lassen sich anhand der modellmäßig aufgestellten Vermögensverteilungsrechnung von Tabelle 6 des Tabellenanhangs verfolgen. Die dort angegebene Entwicklung des Vermögensanteil der Unselbständigen weist zwischen Anfang 1963 und Anfang 1964 einen deutlichen Bruch auf.

(3) Vgl. W. Krelle und J. Siebke, Vermögensverteilung und Vermögenspolitik in der Bundesrepublik Deutschland. Ein Überblick, Zeitschrift f. d. gesamte Staatswissenschaft, 129, 1973, S. $478 \mathrm{ff}$. 
periode um ein Vielfaches überschritten haben (1) (2).

(1) Zur Abschätzung des Erfolges der vermögenspolitischen Maßnahmen diene folgende Übersicht:

\begin{tabular}{rllr} 
Jahr & $\begin{array}{l}\text { Vermögens- } \\
\text { bildungs- } \\
\text { effekt a) }\end{array}$ & $\begin{array}{l}\text { staatliche Lei- } \\
\text { stungen zur För }-\end{array}$ & $\frac{(1)}{(2)}$ \\
derung der Ver- & & \\
mögensbildung b) & & \\
\hline & Mrd. DM & $(2)$ & $(3)$ \\
\hline 1963 & $(1)$ & 0,008 & 380,2 \\
64 & 3,041 & 0,012 & 305,0 \\
65 & 3,660 & 0,140 & 27,9 \\
66 & 3,910 & 0,180 & 22,5 \\
67 & 4,058 & 0,200 & 20,1 \\
68 & 4,027 & 0,230 & 17,7 \\
69 & 4,088 & 0,265 & 16,2
\end{tabular}

a) Geschätzt im Anschluß an (7.11)

b) Quelle: Bundesministerium der Finanzen, Finanzbericht 1967,

S. 169; Finanzbericht 1970, S. 298; Finanzbericht 1971, S. 60.

(2) Die Ergebnisse von Proberechnungen mit dem Simulationsmodell legen die Vermutung nahe, daß das plötzliche Auftreten und die beachtliche Größe des 1963 ausgelösten Vermögensbildungseffektes in erheblichem Umfang zu den unerwarteten Rezessionstendenzen des Jahres 1963 beigetragen haben. 
$\$ 8$ Das Einkommen der Selbständigen-Haushalte und seine Verwendung

\section{Der Unternehmerlohn}

Wie bereits bei der Darstellung des Systems der Definitionsgleichungen ausgeführt (1), überprüfen in der Modellwirtschaft die selbständigen Erwerbspersonen ständig, ob sich die spezifischen Mühen der selbständigen Beschäftigung im Vergleich zu einer alternativen Beschäftigung lohnt. Als Folge dieser ständigen Kontrolle und als Folge ihrer Arbeitgeberfunktion haben sie eine genaue Kenntnis der durch unselbständige Beschäftigung erzielbaren Alternativeinkommen und rechnen sich in dessen Höhe einen rechnerischen Unternehmerlohn zu.

Für den Unternehmerlohn, ${ }^{1}, t$, gilt:

$$
\text { (8.1) } \quad 1_{S, t}=1_{p r, t}
$$

und für das gesamtwirtschaftliche Aggregat der verrechneten Unternehmerlöhne:

$$
L_{S, t}=s_{t} \cdot{ }^{1} s, t
$$

Der rechnerische Unternehmerlohn wird von den Unternehmen auf jeden Fall an die Selbständigen-Haushalte ausgeschüttet und bildet nach Abzug der in der Modellwirtschaft erhobenen Unternehmerlohnsteuer das Einkommen, aus dem diese ihre Lebenshaltung hauptsächlich bestreiten.

\section{Das ausgeschüttete Besitzeinkommen der Selbständigen}

Um die zweite Komponente des verfügbaren Selbständigeneinkommens, das ausgeschüttete Besitzeinkommen der Selbständigen, für die Modellwirtschaft festzulegen, wurde im Anschluß an die volkswirtschaftliche Gesamtrechnung der Bundesrepublik folgende Berechnung vorgenommen: Durch Abzug von Abschreibungen und indirekten Steuern wurde aus der Bruttowertschöpfung zu Marktpreisen die Nettowertschöpfung zu Faktorkosten des privaten Sektors ermittelt. Die anschließende Subtraktion des rechnerischen Arbeitseinkommens ergab das im privaten Sektor der Bundesrepublik entstandene rechnerische Besitzeinkommen. Ein Teil

(1) Vgl. Paragraph 2 Abschnitt 2 
dieses Besitzeinkommens entfiel auf den Staat (1) und das Ausland, die dem privaten Sektor gegenüber fast stets eine Nettoforderungsposition einnahmen, ein anderer Teil auf die Vermögenseinkünfte der Unselbständigen-Haushalte. Der verbleibende Rest stellt das Besitzeinkommen der Selbständigen und Unternehmen dar, das in der Modellformulierung insgesamt den Selbständigen als Eigentümern des Unternehmenssektors zugerechnet wird $\left(R_{S, t}+G_{t}\right)$.

Anschließend wurde das den Selbständigen zugerechnete Besitzeinkommen in Kapitalverzinsung, $\mathrm{R}_{\mathrm{S}, \mathrm{t}}$, und Unternehmensgewinn, $\mathrm{G}_{t}$, aufgespalten. Hierzu wurden der im vorigen Paragraphen modellmäßig aufgestellten Vermögensverteilungsrechnung die Werte für das nicht den Arbeitnehmer-Haushalten gehörende Nominalvermögen des privaten Sektors entnommen, das den Selbständigen in der Modellformulierung ebenfalls insgesamt zugerechnet wird, WS, t. Durch Abzug der geschätzten Kassenhaltung der Selbständigen-Haushalte ergab sich der in Titeln des Unternehmenssektors angelegte Bestand des Selbständigen-Vermögens, FS, t. Der auf die Kapitalverzinsung entfallende Teil des Besitzeinkommens der Selbständigen, $R_{S, t}$, wurde gemäß (2.52) durch Zuschlag des vollen Kapitalmarktzinses der Vorperiode berechnet.

Das Residuum der beschriebenen Berechnungen ist die Modellgröße für den aggregierten Bruttounternehmensgewinn (2). Sein Wert stieg in der Zeit nach 1960 mit einer Wachstumsrate, die unter der Wachstumsrate der Arbeitseinkommen und unter der Wachstumsrate der aggregierten Kapitalverzinsung lag. In der Bundesrepublik fand also, wenn man der Modellrechnung trauen kann, innerhalb der Besitzeinkommen eine Umstrukturierung zulasten des Anteil der Unternehmensgewinne statt. Eine derartige Umstrukturierung der Besitzeinkommen muß nach den im Paragraphen 4 entwickelten Überlegungen zur "langfristigen "Grenzproduktivitätstheorie bzw. zur Grenzproduktivitätstheorie der Faktoreinsatzplanung erwartet werden, da sich mit zunehmender Annäherung an einen Pfad ausgeglichenen Wirtschaftswachstums die Schere zwischen Kapitalkostensatz und "langfristigem" Grenzerlösprodukt des Faktors Privatkapital allmählich schließt und damit die durch die verzögerte

(1) Zur Eliminierung versteckter Subventionen wurde der Kapitalmarktzins der Vorperiode auf den geschätzten (Netto-)Forderungsbestand des Staates dem privaten Sektor gegenüber verrechnet. Die Differenz zwischen den so ermittelten Besitzeinkommen des Staates und den in der volkswirtschaftlichen Gesamtrechnung ausgewiesenen Einkommen des Staates aus Unternehmertätigkeit und Vermögen wurde den direkten Übertragungen des Staates an den Unternehmenssektor zugeschlagen.

(2) "Brutto" heißt hier: vor Einsetzen der Umverteilung durch direkte Steuern und Übertragungen. 
Anpassung der Anlagenplanung verursachten dynamischen Profite zum Verschwinden bringt. Die Vermutung einer derartigen Umstrukturierung wird aber auch durch das spezifische Finanzierungsverhalten der Unternehmen in der Bundesrepublik nahegelegt, daß durch eine sinkende

"Selbstfinanzierungsquote" gekennzeichnet ist.

Ergänzt man die Summe der den Selbständigen zugerechneten Besitzeinkommen, $\left(R_{S}, t+G_{t}\right)$, um die direkten Unterstützungszahlungen des Staates, USt, U, t, und berejnigt sie um die direkten Steuern auf das gesamte Besitzeinkommen der Selbständigen, TRGS, $t$, sowie auf das Vermögen der Selbständigen, $T_{F}, t$, die in der Modellwirtschaft alle vom Unternehmenssektor gezahlt werden, erhält man den Wert des an die Selbständigen-Haushalte verteilbaren Besitzeinkommens, über dessen Verwendung die Unternehmen- mehr oder weniger frei - zu entscheiden haben (1). Nach Abzug der von den Unternehmen einbehaltenen Gewinne (2), $Y_{U}^{n}, t$, verbleibt der Teil, der an die Selbständigen-Haushalte ausgeschüttet wird, $\mathrm{V}_{\mathrm{t}}$.

Bei der Bemessung der Ausschüttungen $V_{t}$ orientieren sich die Unternehmen der Modellwirtschaft vor allem am Kapitalmarktzins, der dem im Unternehmenssektor investierten Selbständigen-Vermögen zugerechnet werden muß. Der eigentliche Unternehmensüberschuß, der nach Abzug der gesamten Kapitalverzinsung und nach Verrechnung aller zwischen dem Unternehmenssektor und dem Staat verlaufenden Transferzahlungen übrigbleibt, beeinflußt die Ausschüttungen wesentlich weniger stark; er dient zum großen Teil der Selbstfinanzierung von Investitionen im Unternehmenssektor.

Die Ausschüttungsfunktion lautet:

$$
v_{S, t}=\pi_{1}+\pi_{2} \cdot R_{S, t}+\pi_{3} \cdot\left[G_{t}+U_{S t, U, t}-T_{R G S, t}-T_{F, t}\right]
$$

(1) Die genannten Posten, ihre statistische Abgrenzung und die entsprechenden Materialquellen werden im 3. Kapitel über das Verhalten des Staates besprochen.

(2) Die Werte für die einbehaltenen Gewinne der Unternehmen (nach Abzug der direkten Steuern) wurden der volkswirtschaftlichen Gesamtrechnung entnommen. 
Für die Jahre 1958 bis 1970 (1) ergab die Funktion als Regressionsergebnis:

$$
\begin{aligned}
& \text { (8.4) } \quad \pi_{1}=-12,55303( \pm 2,74483) \text { Mrd. DM } \\
& \pi_{2}=+0,88872( \pm 0,07860) \\
& \pi_{3}=+0,62164( \pm 0,14606) \\
& \mathrm{R}_{1 \cdot 2,3}^{2}=0,94221 \text { (Irrt.-Wahrsch.: }<0,1 \% \text { ) } \\
& r_{2 \cdot 3}^{2}=0,02363 \\
& \text { vNQ }=2,915
\end{aligned}
$$

Die Größenrelation der Parameter $\pi_{2}$ und $\pi_{3}$ und der im Vergleich zur Kapitalverzinsung niedrige Anstieg der Unternehmensgewinne, der durch den Einfluß der direkten Steuern noch vermindert wurde, trugen dazu bei, daß der Anteil der Ausschüttungen an den verteilbaren Besitzeinkommen stieg. Die Erhöhung der Ausschüttungsquote ist aber offensichtlich nicht allein oder vorwiegend auf die sich ändernde Struktur der den Selbständigen zufallenden Besitzeinkommen zurückzuführen, sondern vor allem darauf, daß, wie das negative Absolutglied $\pi_{1}$ von (8.4) anzeigt, die Neigung der Unternehmen zum Ausschütten der Besitzeinkommen gestiegen ist (2). Möglicherweise ist dies eine langfristige Auswirkung der Körperschaftsteuerreform von 1958, bei der der Satz für ausgeschüttete Gewinne der Kapitalgesellschaften reduziert wurde.

\section{Der Konsum der Selbständigen-Haushalte}

Addiert man das an die Selbständigen-Haushalte ausgeschüttete Besitzeinkommen zu dem um die Unternehmerlohnsteuer bereinigten aggregierten Unternehmerlohn, erhält man das verfügbare Einkommen der

(1) Aufgrund der Funktion (7.5) für die Rendite des nicht in Kasse angelegten Unselbständigen-Vermögens und (7.9) für den Unselbständigen-Konsum sowie der Regressionsergebnisse (7.6a) und (7.11) konnten die modellmäßig aufgestellte Vermögensverteilungsrechnung und damit die Größen $R_{S, t}$ und $G_{t}$ bis 1970 fortgerechnet werden.

(2) Vgl. hierzu die empirischen Untersuchungsergebnisse von P. Baumgarten, Selbstfinanzierung und Einkommensverteilung, Tübingen 1971 , S. 121 ff. 
Selbständigen-Haushalte, $Y_{S}^{n}, t$. Das verfügbare Einkommen ist der Hauptbestimmungsfaktor für das Konsumverhalten der Selbständigen. Es beeinflußt ihren Konsum in der gleichen Weise wie bei den UnselbständigenHaushalten (1).

Die Konsumfunktion der Selbständigen-Haushalte lautet:

$$
\frac{c_{S, t}}{s_{t} \cdot p_{x, t}}=o_{S 1}+o_{S 2} \cdot\left[\frac{r_{S, t}^{n}}{s_{t} \cdot p_{x, t}}\right]+o_{S 3} \cdot\left[\frac{c_{S, t-1}}{s_{t-1} \cdot p_{x, t-1}}\right]
$$

Die zugehörigen Regressionsergebnisse beziehen sich auf die Jahre 1959 bis 1967:

$$
\begin{aligned}
& \text { (8.6) } \\
& \begin{array}{l}
o_{S 1}=+810,53000( \pm 89,17000) \text { DM p.c. real } \\
o_{S 2}=+0,63253( \pm 0,04333) \\
o_{S 3}=+0,18046( \pm 0,05892)
\end{array} \\
& R_{1 \cdot 2,3}^{2}=0,99956 \text { (Irrt.-Wahrsch.: }<0,18 \text { ) } \\
& \mathbf{r}_{2 \cdot 3}^{2}=0,97733 \\
& \mathrm{~h}=0,218
\end{aligned}
$$

Vergleicht man das Konsumverhalten der Selbständigen-Haushalte mit dem der Unselbständigen-Haushalte und bezieht man in diesen Vergleich das Ausschüttungsverhalten der den Selbständigen gehörenden Unternehmen mit ein, so läßt sich bereits vor der Lösung des Modells eine wichtige verteilungsmäßige Entwicklungstendenz innerhalb der Modellwirtschaft feststellen. Nach (8.6) liegt die langfristige marginale Konsumneigung (2) der Selbständigen-Haushalte, bezogen auf das verfügbare Haushaltseinkommen, unter der entsprechenden marginalen Konsumneigung der Unselbständigen-Haushalte. Die Gegengröße, die langfristige marginale Sparneigung, ist bei den Selbständigen etwa doppelt so groß wie bei den Unselbständigen. Diese Struktur der Sparneigungen

(1) Die Angaben über den Konsum der Selbständigen wurden durch Abzug der Haushaltsersparnis der Selbständigen von dem oben abgegrenzten verfügbaren Selbständigen-Einkommen ermittelt. Zur Berechnung der Haushaltsersparnis der Selbständigen vgl. Fußnote 2 von S. 126 .

(2) Als langfristig werden hier die marginalen Konsum- bzw. Sparneigungen im langfristigen Wachstumsgleichgewicht angesehen, und zwar bei vollzogener Anpassung des Gewohnheitsverhaltens. 
verursacht, daß die Relation der durchschnittlichen Pro-Kopf-Vermögen zwischen Selbständigen und Unselbständigen sich in der Modellwirtschaft ständig zu Ungunsten der Unselbständigen entwickelt. Die Tendenz wird dadurch verstärkt, daß ein zusätzlicher und nicht unerheblicher Teil der Selbständigenersparnis sich außerhalb der eigentlichen Haushalts sphäre, nämlich in den Unternehmen, vollzieht, rein rechnerisch auch dadurch, daß es vor allem die Selbständigen der unteren Vermögensklassen sind, die mit ihrem Vermögen in den Sektor der Unselbständigen überwechseln, und sie kann sich entwickeln, ohne daß ihr das dargestellte Wirtschaftssystem mit einem endogenen Abwehrmechanismus begegnet. Die Struktur der Einkommensverwendungsentscheidungen, die sich auf die kurzfristige Nachfrageentwicklung innerhalb der Modellwirtschaft durchaus stabilisierend auswirkt, führt langfristig also dazu, daß die Pro-Kopf-Vermögensverteilung und damit die Pro-Kopf-Einkommensverteilung zwischen Selbständigen und Unselbständigen eine Entwicklung nimmt, die sich für den Bestand der Modellwirtschaft in einem über die formalisierten Modellannahmen hinausgehenden Sinn auf die Dauer bedrohlich auswirken kann.

Man muß sich aber klar vor Augen halten, worin der bedrohliche Charakter dieser Entwicklung liegt. Er liegt nicht im Bereich der Klassenverteilung als solcher. Das Verhältnis der beiden Aggregate kumulierte Nominalersparnis der Unselbständigen, $W_{A}$, und kumulierte Nominalersparnis der Selbständigen, $W_{S}$, zu einander verschiebt sich innerhalb der Modellwirtschaft im Trend ganz erheblich zugunsten des Sektors der Unselbständigen (1). Ganz langfristig ist aufgrund der Schätzungen mit einem Vermögensanteil der Unselbständigen zu rechnen, der etwa in der Größenordnung von 70 \% der gesamten kumulierten Nominalersparnis liegt. Der bedrohliche Charakter der Entwicklung liegt allein darin, daß innerhalb der Modellwirtschaft ein zahlenmäßig abnehmender Personenkreis existiert, dessen pro-Kopf-Vermögen und damit auch pro-KopfEinkommen im Vergleich zu den übrigen Personen ein Vielfaches beträgt und aufgrund der laufenden Spartätigkeit weiterhin ständig ansteigt, eine Erscheinung, die als personelle Einkommens- und Vermögenskonzentration bezeichnet werden muß.

(1) Siehe Tab. 6 des Tabellenanhangs. - Der oben angegebene langfristige Gleichgewichtswert, dem sich der Vermögensanteil $W_{A} /\left(W_{A}+W_{S}\right)$ der Unselbständigen asymptotisch nähert, wurde im Anschluß an die Überlegungen von L. Pasinetti, Rate of Profit and Income Distribution in Relation to the Rate of Economic Growth, Review of Economic Studies, 29, 1962, S. $267 \mathrm{ff}$. und J. E. Meade, The Rate of Profit in an Growing Economy, Economic Journal, 73, 1963, S. 665 ff. berechnet. Der Berechnung lagen die hier für die Einkommensverwendung der Arbeitnehmer, der Selbständigen und der Unternehmen geschätzten Parameter sowie die als konstant angenommenen steuerlichen Durchschnittsbelastungen und die ebenfalls als konstant angenommene Selbständigenquote des Jahres 1970 zugrunde. 
Vielleicht sollte diese Aussage durch eine kurze Bemerkung ergänzt werden: Ein kritischer Einfluß auf die Entwicklung der Vermögensverteilung geht in der Modellwirtschaft nicht allein von der Struktur der Sparneigungen aus, sondern auch von der vorhandenen allgemeinen Preissteigerungstendenz. Rechnet man dem Vermögen der Selbständigen die Bewertungsgewinne $\mathrm{zu}$, die in der Substanzerhaltungsrücklage des Unternehmenssektors erfaßt sind, $W_{U, t}$, so macht der Vermögensanteil der Arbeitnehmer, $W_{A, t} /\left(W_{A, t}+W_{S, t}+W_{U, t}\right)$, langfristig keineswegs $70 \%$ aus, sondern nur noch einen Bruchteil des langfristigen Gleichgewichtsanteils an der kumulierten Nominalersparnis, $W_{A, t} /\left(W_{A}, t+W_{S, t}\right)$. Dieser Bruchteil entspricht dem Quotienten (4.8), mit dem die inflationistische Erosion der Forderungsbestände im Wachstums - und Inflationsgleichgewicht zum Ausdruck gebracht wird. Bei einer Preissteigerungsrate von $3,5 \%$, wie sie während der sechziger Jahre in der Bundesrepublik für Investitionsgüter galt, und einer langfristigen Wachstumsrate von real $4,3 \%$ hat der Bruchteil einen Wert von 0,551, so daß man nach Art einer Daumenregel den langfristigen Anteil der Unselbständigen am Gesamtvermögen einschließlich der Bewertungsgewinne mit 0, $385(=0,70$. 0,551 ) ansetzen könnte. Der Bruchteil reagiert stark auf einen Anstieg der Inflationsrate (1). Von einer unsoliden Geld- und Finanzpolitik des Staates können in der Modellwirtschaft daher wesentlich stärkere Hemmnisse für die Herausbildung einer gleichmäßigen Vermögensverteilung ausgehen als von der angegebenen Struktur der Sparneigungen (2).

(1) Vgl. die Tabelle in Anhang 1.

(2) Diese Aussage bleibt in der Modellformulierung wahrscheinlich unberührt von der Tatsache, daß der Zins bei allgemeinen Preissteigerungstendenzen eine Inflationsprämie enthält. - Natürlich bewirkt eine Verringerung des allgemeinen Preisansteigs eine entsprechende Erhöhung des Anteils der Arbeitnehmer am Gesamtvermögen einschließlich der Bewertungsgewinne. Die Wirkung ist auch hier in der richtigen Relation zu sehen: Der langfristige Vermögensverteilungseffekt einer vollständigen Preisniveaustabilisierung geht in der Modellwirtschaft weit über den Verteilungseffekt hinaus, der aus der Sicht der Modellformulierung langfristig den beiden ersten Vermögensbildungsgesetzen und ihrem Einfluß auf das Sparverhalten der Arbeitnehmer in der Bundesrepublik zuzurechnen ist. Vgl. Paragraph 7, Abschnitt 3. In der Modellwirtschaft, in der die Arbeitnehmer kein Realvermögen besitzen, ist das wirksame erste Mittel einer staatlichen Verteilungspolitik also wahrscheinlich die Politik der Preisniveaustabilisierung. 
1. Eine Bemerkung zum Gleichgewicht auf dem Kreditmarkt

Die Modellformulierung unterscheidet sich von den üblichen neoklas sischen Verteilungsmodellen durch eine Eigenschaft, die sie mit der post-keynesianischen Verteilungstheorie gemeinsam hat: Sie berücksichtigt die Ungültigkeit des Say'schen Theorems. - Selbständige und Unselbständige bilden in der Modellwirtschaft Ersparnisse, ohne daß diese Ersparnisse in entsprechendem Umfang eine Nachfrage nach Investitionsgütern erzwingen; die Unternehmen entfalten eine Nachfrage nach Investitionsgütern, ohne daß in entsprechendem Umfang zuvor gesparte Einkommensteile als Finanzierungsmittel zur Verfügung gestellt werden müßten. Mit anderen Worten, Geldstillegung und Geldschöpfung spielen für den Ablauf der Modellwirtschaft und insbesondere auch für das Ergebnis des damit einhergehenden Verteilungsprozesses eine bedeutende Rolle.

Dies gilt nicht nur für kurzfristige Abweichungen vom mittelfristigen Normalverlauf des Wirtschaftssystems, sondern auch langfristig. Insoweit die währungspolitischen Instanzen der Modellwirtschaft sich am Ziel der Preisniveaustabilität orientieren, verlangt das wirtschaftliche Wachstum eine ständige Expansion der Geldmenge. Bei ausgeglichener Devisenbilanz und ausgeglichenem Staatsbudget vollzieht sich diese Expansion in der Weise, daß die Investoren die Investitionen nicht nur mit zuvor gesparten Einkommensteilen, sondern darüber hinaus mit der vom Bankensystem angebotenen zusätzlichen Kreditschöpfung finanzieren. Wenn in der volkswirtschaftlichen Gesamtrechnung der Modellwirtschaft dann ex-post festgestellt wird, daß die private Investitionstätigkeit insgesamt der privaten Ersparnisbildung entsprach, so darf nicht vergessen werden, daß ein Teil der privaten Ersparnis zum Aufbau der im Wachstum steigenden privaten Kassenhaltung diente und zur privaten Investitionsfinanzierung nicht zur Verfügung stand und daß in genau demselben Umfang die privaten Investitionen aus Kreditschöpfung finanziert wurden.

In der Modellwirtschaft existiert ein einziger Kreditmarkt, über den alle mit der Vermögensbildung und mit der Geldversorgung verbundenen Finanzierungsvorgänge verlaufen. In der Ausdrucksweise der sog. Leihfondstheorie setzt sich das Angebot dieses Marktes zusammen aus den privaten Ersparnissen, wozu hier auch die Unternehmensersparnisse zu zählen sind, dem Nettokreditangebot des Auslandes und dem Kreditschöpfungsangebot des Bankensektors. Dem Angebot steht die Nachfrage gegenüber, die sich aus der privaten Nachfrage nach Investitionsfinanzierungsmitteln, der Nettokreditnachfrage des Staates und der privaten Nachfrage nach zusätzlicher Kasse zusammensetzt. Setzt man das gesamte Angebot der gesamten Nachfrage gleich, so erhält man die leih- 
fondstheoretisch formulierte Kreditmarktgleichung. Diese Gleichung ist im Grunde nichts anderes als eine Zusammenfassung der aus der volkswirtschaftlichen Gesamtrechnung ableitbaren Vermögensbildungsgleichung, wonach die private Ersparnis gleich ist der privaten Investition plus dem laufenden staatlichen Budgetdefizit plus dem Außenbeitrag minus den unentgeltlichen Nettoleistungen der Modellwirtschaft an die übrige Welt, und der Geldversorgungsgleichung, wonach der Aufbau der privaten Kassenhaltung letzten Endes gespeist wird durch die Kreditschöpfung des Bankensektors und den Nettodevisenzustrom, und sie läßt sich wieder in diese beiden Gleichungen zerlegen.

Für die weiteren Überlegungen erweist es sich als Vorteil, daß in der Modellformulierung ein differenzierter Gebrauch von den Vorstellungen eines gesamtwirtschaftlichen Gleichgewichts gemacht wird. In einem kurzfristigen Sinn stellt sich ein gesamtwirtschaftliches Gleichgewicht in jeder Periode ein. In diesem Sinn hat die Vermögensbildungsgleichung in der Modellformulierung nicht nur einen definitorischen Gharakter, sondern kennzeichnet auch ein - kurzfristiges - Einkommensgleichgewicht. Wenn sich aufgrund der Marktkräfte in jeder Periode ein Gleichgewicht des gesamten Kreditmarktes einspielt, das durch die Räumung dieses Marktes gekennzeichnet ist, und wenn sich zugleich in jeder Periode ein kurzfristiges Einkommensgleichgewicht herausbildet, so folgt aber logisch, daß die Geldversorgungsgleichung, die sich durch Subtraktion der Vermögensbildungsgleichung von der loanable-fundsGleichung ergibt, ebenfalls ein Gleichgewicht kennzeichnet, das kurzfristige Gleichgewicht der Geldversorgung.

Obwohl letzten Endes das gesamte Angebot an und die gesamte Nachfrage nach Leihfonds den Kreditmarktzins und damit das kurzfristige Gleichgewicht des Wirtschaftsprozesses bestimmen, erlauben die genannten Zusammenhänge bei einigen Vereinfachungen, daß sich die Darstellung des gesamten Kreditmarktgeschehens im folgenden auf das Geldangebot des Bankensektors und die Geldnachfrage der privaten Wirtschaftssubjekte konzentriert und so tut, als ob die Zinsbestimmung allein aus dem Gleichgewicht zwischen diesen beiden resultiere. Es sei noch ergänzt, daß sich die Darstellung hierbei anstelle der Strombetrachtung der Gleichungen (2.38) und (2.39) der Bestandsbetrachtung von (2.57) und $(2,63)$ bedient.

\section{Das Geldangebot des Bankensektors}

Die Geldversorgung der Modellwirtschaft ist Funktion des gesamten Bankensystems; diese Funktion übt das Bankensystem mit verteilten Rollen aus: Die Zentralbank stattet das Geschäftsbankensystem in einem noch zu definierenden Sinn mit Liquidität aus; auf der Basis der verfügbaren Liquidität betreibt das Geschäftsbankensystem Geldschöpfung. - Entsprechend der Funktionteilung läßt sich das Geldangebotverhalten des 
aggregierten Bankensektors in zwei Stufen formulieren. Begonnen wird mit dem Verhalten des Geschäftsbankensystems bei Annahme einer gegebenen Liquiditätsausstattung.

Wenn das Geschäftsbankensystem auf der Basis der frei verfügbaren Liquiditätsreserven eine multiple Geldschöpfung betreibt, so geht es wegen des ständig drohenden Abrufs liquider Mittel von Seiten der Nicht-Banken ein spezifisches Liquiditätsrisiko ein. Dieses Risiko ist umso höher, je höher der Umfang der insgesamt vollzogenen Geldschöpfung im Vergleich zum vorhandenen Liquiditätsvolumen ist. Da aus der Überwachung und Abwehr des Liquiditätsrisikos Kosten entstehen und diese Kosten mit der Höhe des Liquiditätsrisikos steigen, da ferner die Banken versuchen, diese Kosten neben den Kosten der Liquiditätsbeschaffung bei der Notenbank auf die Kreditnehmer umzulegen, kann davon ausgegangen werden, daß das Geldschöpfungsangebot an die NichtBanken von der Höhe des jeweiligen Zinssatzes abhängig und auf jeden Fall begrenzt zinselastisch ist.

Das durch Kreditschöpfung entstandene Liquiditätsrisiko wird von den Geschäftsbanken nicht nur bei unterschiedlicher Liquiditätsanspannung verschieden hoch angesetzt; die Banken beurteilen das Risiko auch in den konjunkturellen Wechsellagen unterschiedlich. In Rezessionsphasen bieten sie bei gleicher Liquiditätsausstattung die gleiche Geldmenge nur zu höheren Zinssätzen an als in konjunkturellen Aufschwungsperioden. In die Geldangebotsfunktion wird daher der Krisenindikator Arbeitslosenquote als Funktionsargument für die Konjunkturabhängigkeit des Bankenverhaltens aufgenommen.

Für das Geldschöpfungsangebot des Geschäftsbankensystems läßt sich daher formulieren:

$$
M_{t}=\rho_{1} \cdot r_{t}^{\rho_{2}} \cdot Q_{t} \rho_{3} \cdot\left[\frac{e_{v, t}-e_{t}}{a_{t}+\left(e_{v}, t^{-e_{t}}\right)}\right] \rho_{4}
$$

wobei $Q_{\mathrm{t}}$ die Liquiditätsbasis des Bankensystems bezeichnet.

In der Funktion schlägt sich die Liquiditätspräferenz des Geschäftsbankensektors nieder, die abgesehen von Unterschieden in der Abgrenzung des Begriffs Liquidität im Keynes'schen Sinne das Gegenstück zur Liquiditätspräferenz der privaten Nicht-Banken ist. Die Annahme einer Konjunkturabhängigkeit des Geldangebotes läuft dabei in ihren Auswirkungen auf die Berücksichtigung der Keynes'schen "liquidity-trap" hinaus. - Dividiert man die Gleichung auf beiden Seiten durch Q, erhält man die Funktion für den vom Geschäftsbankensystem realisierten Geldschöpfungsmultiplikator, $M_{t} / Q_{t}$.

Funktion (9.1) kennzeichnet nur eine der beiden Quellen des Geldangebotes. Betreibt die Zentralbank im Inland eine restriktive Politik und 
trägt damit zu einer Erhöhung des inländischen Zinsniveaus im Vergleich zum ausländischen Zinsniveau bei, setzen Kapitalzuflüsse aus dem Ausland ein. Dabei handelt es sich zum Teil um echte Auslandsgelder, zum Teil nur um liquide Auslandsanlagen der Inlandsbanken, die repatriiert werden, um zu den günstigeren Bedingungen am inländischen Kreditmarkt angelegt $\mathrm{zu}$ werden. Insofern diese Einflüsse lediglich auf Liquiditätsumschichtungen der Inlandsbanken beruhen, erhöhen sie den inländischen Geldumlauf, ohne den Umfang der Bankenliquidität insgesamt zu erhöhen. Um diese zweite Quelle des inländischen Geldangebotes mitzuberücksichtigen, wird der Kreditmarktzins des Auslandes als zusätzliche Variable in (9.1) aufgenommen. Bei der empirischen Überprüfung wird außerdem noch eine Dummy-Variable, DummyQ, $t$, berücksichtigt, die 1960 den Wert 10, ansonsten den Wert 1 hat und mit der dem besonderen Verhalten der Bundesbank im Jahre 1960 und den entsprechenden Auswirkungen auf das inländische Geldangebot Rechnung getragen wird.

Die Geldangebots - oder Geldversorgungsfunktion lautet daher insgesamt:

$$
M_{t}=\rho_{1} \cdot r_{t}^{\rho_{2}} \cdot Q_{t}{ }^{\rho_{3}} \cdot\left[\frac{e_{v}, t^{-e_{t}}}{a_{t}+\left(e_{v}, t^{-e_{t}}\right)}\right]^{\rho_{4}} \cdot r_{\text {Aus } 1, t^{D u m m y}}^{\rho_{Q}}{ }_{Q}^{\rho_{6}}
$$

Der zweite Schritt in der Analyse des Geldangebots-Verhaltens besteht in der Bestimmung der den Banken zur Verfügung stehenden Liquiditäts reserven. Diese Liquiditätsreserven bestehen in der Modellwirtschaft aus dem Bestand an über das Mindestreservesoll hinausgehenden Zentralbankguthaben der Banken bzw. ihrer Barreserve, dem Bestand an unausgenutzten Rediskontkontingenten, dem Bestand an inländischen Geldmarktpapieren und dem Bestand an kurzfristigen Auslandsanlagen, abzüglich der Summe der bei der Zentralbank in Anspruch genommenen Lombardkredite (1). Sie sind - das geht aus der vorgenommenen Abgrenzung bereits hervor - zu einem großen Teil (2) bestimmt durch das Verhalten der Zentralbank. Die Banken haben es jedoch in der Hand, durch Aufstockung ihres haftenden Kapitals (3), durch Rückruf von Ausleihungen bzw. durch Verlangsamung der Kreditexpansion sowie durch Aufnahme ausländischer Bankkredite den Umfang ihres Liquiditätsspiel-

(1) Vgl. Deutsche Bundesbank, Erläuterungen zur Liquiditätsanalyse der Bundesbank, Monatsberichte der Deutschen Bundesbank, Juli 1970 , S. $30 \mathrm{f}$.

(2) In der Bundesrepublik lassen sich die zwischen 1958 und 1970 eingetretenen jährlichen Änderungen im Liquiditätsspielraum der Banken etwa zu $80 \%$ durch geldpolitische Maßnahmen der Bundesbank erklären.

(3) Der Umfang der Bruttorediskontkontingente richtet sich in der Bundesrepublik nach dem haftenden Kapital der Geschäftsbanken. 
raums selbst zu beeinflussen und damit in bestimmten Grenzen auch den Einwirkungen der Notenbankpolitik zu entgehen. Insoweit wirkt sich die Liquiditätspräferenz der Geschäftsbanken auch hier und nicht allein in der Geldangebotsfunktion (9.1) bzw. (9.2) aus (1). Außerdem besteht im Prinzip natürlich eine dritte Quelle der Bankenliquidität, und zwar in Form von Devisenzuflüssen, die nicht im Bankensektor ausgelöst werden, aber dort gegen Bankguthaben eingetauscht werden.

Für die so abgegrenzten Liquiditätsreserven der Banken in der Bundesrepublik stellt die Deutsche Bundesbank eine bis ins Jahr 1958 zurückreichende Zeitreihe zur Verfügung (2), deren sich die hier vorgenommene empirische Analyse bedient hat. Zur genauen Kontrolle des Einflusses, der von der Bundesbankpolitik auf die Bankenliquidität ausging, wurde für die Reihe zusätzlich eine eigene Schätzung angestellt, die eine durchgehende Zeitreihenanalyse der einzelnen Liquiditätskomponenten bzw. der entsprechenden liquiditätspolitischen Maßnahmen der Bundesbank zuließ (3).

Aufgrund der empirisch erzielten Untersuchungsergebnisse wird für die Modellwirtschaft davon ausgegangen, daß die Zentralbank den Liquiditätsspielraum der Banken im Trend fast parallel zum wachsenden Sozialprodukt steigen läßt. Ausweitungen des Liquiditätsspielraumes durch Devisenzuflüsse aus dem Ausland bzw. durch eigene Liquiditätsbeschaffungs aktionen der Geschäftsbanken werden dabei, soweit möglich, in Rechnung gestellt. Mit diesem Verhalten prägt die Notenbank bei den im Wachstum steigenden privaten Kassenhaltungswünschen dem geldpolitischen Ziel der Preisniveaustabilität Rechnung und überläßt das Ziel des Zahlungsbilanzausgleichs den durch den Staat vorgenommenen Wechselkurskorrekturen.

Man sollte annehmen, daß dann, wenn der jährliche Preisanstieg über einen gewissen kritischen Wert hinausgeht, die Zentralbank kurzfristig im restriktiven Sinn von dieser Verhaltensnorm abweicht, mit anderen Worten, daß der jährliche Preisniveauanstieg ein Hauptbestimmungsfaktor des kurzfristigen Zentralbankverhaltens sei. Dies trifft jedoch nicht zu. In der Modellwirtschaft treten, worauf bereits hingewiesen wurde (4),

(1) Das gilt (möglicherweise) auch hinsichtlich konjunkturbedingter Schwankungen der Liquiditätspräferenz.

(2) Vgl. Deutsche Bundesbank, Erläuterungen..., a. a. O., S. 33, sowie fortlaufend in den Monatsberichten der Deutschen Bundesbank.

(3) Die geschätzten Werte bezogen sich auf die Jahre 1958 bis 1971. Sie lagen im Durchschnitt um 20,3\% über den von der Deutschen Bundesbank ausgewiesenen Werten und korrelierten mit diesen $\mathrm{zu} \mathrm{r}^{2}=0,94$. Da der Niveauunterschied von $20 \%$ belanglos ist, stimmten beide Wertereihen für die vorliegenden $Z$ wecke ausreichend überein.

(4) Vgl. Paragraph 5, Abschnitt 2. 
die stärksten kurzfristigen Preissteigerungstendenzen als Folge des "lags" der gewerkschaftlichen Lohnpolitik und der konjunkturellen Produktivitätsentwicklung meist in Rezessionsphasen auf. Dies versetzt die Zentralbank in einen Konflikt zwischen preispolitischer und beschäftigungspolitischer Zielsetzung und läßt sie folgerichtig den Ausweg in dem Versuch der allgemeinen Konjunkturstabilisierung bzw. der Stabilisierung der monetären Nachfrage suchen. - Auch nachhaltigen Inflationstendenzen steht die Notenbankpolitik wegen der starren Wechselkurse und des ungehinderten internationalen Kapitalverkehrs machtlos gegenüber. Den Ausgleich derartiger Inflationstendenzen überläßt die Zentralbank dem Leistungsbilanzmechanismus und der staatlichen Wechselkurspolitik.

Bei dem Versuch der Konjunkturstabilisierung kann sich die Zentralbank kurzfristig mit Erfolg ihres geld- und liquiditätspolitischen Instrumentariums bedienen. $Z$ war setzen als Folge der verursachten Geldmarktanspannungs - oder - entspannungstendenzen internationale Kapitalbewegungen ein, doch gelingt es der Zentralbank, diese Kapitalströme, vor allem, soweit sie sich auf die Bankenliquidität auswirken, in gewissem Umfang zu neutralisieren. Da sich die konjunkturellen Ausschläge nach einem Jahr abschwächen und da die internationalen Kapitalbewegungen ihrem Umfang und Charakter nach den Erfolg der Stabilitätsbemühungen dann ernsthaft zu bedrohen beginnen, setzt die Zentralbank ihre Stabilisierungspolitik nach Verstreichen einer Frist von einem Jahr wieder ab (1).

Faßt man die Überlegungen zusammen, so kann man die Funktion zur endogenen Bestimmung der Bankenliquidität formulieren als

$(9.3)$

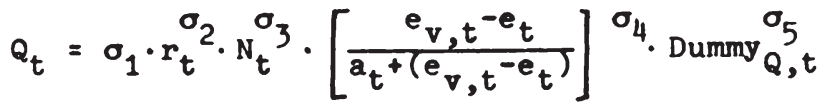

(1) In der statistischen Vorausanalyse wurde das Bundesbankverhalten gemessen als die durch Variationen der Mindestreservesätze und der Bruttorediskontkontingente verursachten Änderungen der Bankenliquidität. Für die Jahre 1958 bis 1970 erwiesen sich als beste Erklärungsgrößen für das so gemessene Bundesbankverhalten die Wachstumsrate des nominellen Sozialprodukts gegenüber dem Vorjahr und die Differenz zwischen dem Kapitalmarktzins der Bundesrepublik und dem Kapitalmarktzins der Vereinigten Staaten im jeweiligen Vorjahr; beide Größen sind allerdings miteinander korreliert. Erst mit Abstand folgt dann die Arbeitslosenquote. - Es sei darauf verwiesen, daß die Variationen der Rediskontkontingente sich wegen deren großen, nicht ausgeschöpften Umfangs als geldpolitisch unwirksam erwiesen; anders dagegen die Variationen der Mindestreservesätze: Vgl. J. Siebke und M. Willms, Das Geldangebot in der Bundesrepublik Deutschland. Eine empirische Analyse für die Periode von 1958 bis 1968, Zeitschrift f. d. ges. Staatswissenschaft, 126, 1970, S. $55 \mathrm{ff}$. 
Dabei bezeichnet $N_{t}$ das Einlagevolumen im Geschäftsbankensystem, über welches im Modell das Wachstum des gesamten Liquiditäts - und Kreditschöpfungspotentials gesteuert wird. Die Variable inländischer Kapitalmarktzins dient zur Berücksichtigung der Tatsache, daß die Bankenliquidität selbst von der Entwicklung im Kreditverkehr zwischen den Geschäftsbanken und den Nicht-Banken abhängig ist, da mit einer Kreditexpansion (-kontraktion) ein Abruf (Rückfluß) liquider Mittel aus dem Banken-Sektor verbunden ist. Die Stabilisierungsbemühungen der Zentralbank kommen in den Variablen Arbeitslosenquote (1) zum Ausdruck (2). Die DummyVariable hat im Jahr 1960 den Wert zehn, ansonsten den Wert eins, sie bringt den im Jahr 1960 unternommenen und im Frühjahr 1961 wieder aufgegebenen Versuch der Bundesbank zum Ausdruck, den starken internationalen Kapitalzufluß vor der DM-Aufwertung 1961 in seinen Auswirkungen auf das Geldangebot des Bankensektors durch eine restriktive Liquiditätspolitik voll zu kompensieren.

Das Einlagevolumen, $N_{t}$, der Geschäftsbanken (3) wird in einem rudimentären portfoliotheoretischen Ansatz abhängig gemacht von der Höhe des nominellen Bruttosozialprodukts, vom Vermögen der privaten Haushalte zu Beginn der laufenden Periode, ( $\left.W_{A, t-1}+W_{S}, t-1\right)$, und vom Kapitalmarktzins. Auch hier werden mit Hilfe der Dummy-Variablen Auswirkungen des Bundesbankverhaltens von 1960 erfaßt, die auf eine Abschrekkung von Ausländereinlagen im inländischen Bankensystem hinausliefen.

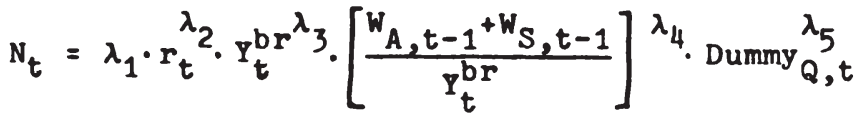

Nach Einsetzen der Einlagenfunktion (9.4) in die Liquiditätsbestimmungsfunktion (9.3) sowie der Liquiditätsbestimmungsfunktion in die Geldver -

(1) Laufen konjunkturbedingte Schwankungen in der Liquiditätspräferenz der Banken darauf hinaus, daß auch bei längerfristigen Einlagen, die hier nicht zur Geldmenge gezählt werden, trotz gleichbleibenden Zinsniveaus etc. geänderte Reserverelationen angestrebt werden, so wird dieser Einfluß in (9.3) ebenfalls durch das Funktionsargument Arbeitslosenquote erfaßt.

(2) Dividiert man (9.3) auf beiden Seiten durch das Liquiditätsvolumen, $N_{t}$, erhält man eine Funktion für die sog. Liquiditätsquote, durch deren Gegenüberstellung mit der Zinsentwicklung die Deutsche Bundesbank die Liquiditätssituation der Geschäftsbanken kontrolliert hat. Vgl. Geschäftsbericht der Deutschen Bundesbank für das Jahr 1970, S. 27 und 47 (Schaubilder).

(3) Die statistischen Werte für das Einlagenvolumen, $\mathrm{N}_{\mathrm{t}}$, wurden berechnet aus den Angaben der Deutschen Bundesbank, Erläuterungen .... a. a. O. , S. 33 
sorgungsfunktion (9.2) erhält man eine Funktion, nach der die Geldversorgung der Modellwirtschaft von den ausländischen Kreditmarktverhältnissen abhängt, ansonsten aber eine endogene Variable des inländischen Wirtschaftsprozesses ist (1).

\section{Die Geldnachfrage des privaten Sektors}

Im Vergleich zum Verhalten des aggregierten Geldangebotes bzw. der gesamtwirtschaftlichen Geldversorgung läßt sich das Verhalten der von den privaten Haushalten und Unternehmen ausgehenden aggregierten Geldnachfrage einfach formulieren: Hauptbestimmungsfaktor der Geldnachfrage ist das Einkommen der Geldnachfrager, in der aggregierten Funktion das nominelle Bruttosozialprodukt. Damit wird unterstellt, daß Kassenhaltung in der Modellwirtschaft hauptsächlich zur Abwicklung des laufenden mit der Einkommensentstehung und Einkommensverwendung verbundenen Zahlungsverkehrs dient.

Mit dem Einkommen bzw. dem abzuwickelnden Transaktionsvolumen ist die Geldnachfrage allerdings noch nicht determiniert. Ein im Umfang gleicher Zahlungsverkehr kann mit einem unterschiedlich hohen Kassenbestand abgewickelt werden. Hierbei entstehen je nach Höhe des gehaltenen Kassenbestandes unterschiedlich hohe Kosten und veranlassen die aggregierte Geldnachfrage der privaten Haushalte und Unternehmen, sich so zu verhalten, daß die durch Aufgabe von Liquidität verursachten marginalen Kosten und Mühen in etwa den durch Kassenhaltung entgangenen Zinserträgen entsprechen. Mit anderen Worten, die Nachfrage nach liquiden Mitteln - hier nach Geld - ist auf Seiten der privaten Haushalte und Unternehmen genauso wie auf Seiten der Banken zinsabhängig.

Der Zins als marktmäßig erzielbarer Preis für die Aufgabe von Liquidität ist nur ein Teil der mit Kassenhaltung verbundenen Alternativkosten. In Zeiten steigenden Preisniveaus sinkt der reale Wert der Kassenbestände jährlich um die Preissteigerungsrate. Diese Wertminderungen werden von den privaten Wirtschaftssubjekten ähnlich wie Abschreibungen auf das Anlagevermögen in Rechnung gestellt. Um das Kalkül der Kassenhalter richtig wiederzugeben, wird daher die Inflationsrate neben dem Zinssatz

(1) Indirekt über das Einlagevolumen wird die Liquiditätsversorgung der Banken vom nominellen Sozialprodukt abhängig gemacht. Haben Preissteigerungstendenzen sich einmal durchgesetzt, versucht die Zentralbank nicht, das Preisniveau von seinem neuen, höheren Niveau auf das ursprüngliche, niedrigere Niveau zurückzuführen, so daß eine langfristige Proportionalität zum realen Sozialprodukt bestünde. Die Annahme hat gewisse Implikationen für das längerfristige Verhalten des Geldangebotes bei Auftreten finanzpolitisch verursachter Überwälzungsprozesse. 
als eigene Variable berücksichtigt. Würden sich Preissteigerungen auf die Dauer in vollem Umfang auf das Niveau des Kapitalmarktzinses auswirken, und zwar derart, daß dieser die längerfristige Preisanstiegsrate sozusagen als Inflationsprämie mitenthält (1), müßte das zusätzliche Kostenargument, um Doppelzählungen auszuschalten, durch die Abweichungen von der längerfristigen Preisanstiegsrate erfaßt werden; es ist jedoch fraglich, ob die längerfristige Inflationsrate sich tatsächlich in vollem Umfang auf das Zinsniveau auswirkt (2).

Die Kassenhaltung der privaten Haushalte und Unternehmen ist in der Modellwirtschaft schließlich ähnlich wie das Kreditangebot der Banken konjunkturabhängig. Arbeitnehmerhaushalte müssen mit dem Risiko der Arbeitsentlastung und dadurch verursachter Dienstausfälle rechnen; sie halten bei hoher Arbeitslosenquote c. p. mehr Kasse als unter normalen Umständen. Ähnlich befürchten die Unternehmen bei Nachfragerückgängen Erlöseinbußen und Liquiditätsengpässe und haben unter diesen Umständen eine höhere Liquiditätspräferenz als bei ausgeglichener Konjunkturlage.

Aus diesem Grund wird die Funktion für das Verhalten der aggregierten Geldnachfrage in der Modellwirtschaft formuliert als (3)

(1) Vgl. J. Siebke und M. Willms, Zinsniveau, Geldpolitik und Inflation, Kredit und Kapital, 5, 1972, S. $171 \mathrm{ff}$.

(2) Da die Entscheidungen zwischen Sparen und Konsumieren sich aufgrund der Annahme einer Brown'schen Konsumfunktion nicht an den relativen Vorteilen der beiden Einkommensverwendungsalternativen orientieren, beruhen Änderungen der Kassenhaltung infolge von Zins- oder Preisniveauänderungen bei den privaten Haushalten der Modellwirtschaft lediglich auf einer Substitution von Kasse durch verzinsliche Forderungstitel. Zins und Inflationsrate bringen hier also nur die relative Vorteilhaftigkeit der beiden alternativen Vermögensanlageformen zum Ausdruck. Enthielte z. B. der Kapitalmarktzins eine Inflationsprämie in voller Höhe der allgemeinen Preissteigerungsrate, würde die Vorteilhaftigkeit der - im Modell mit einer variablen Verzinsung ausgestatteten Forderungstitel nicht, die Vorteilhaftigkeit der Kassenhaltung aber sehr wohl durch Inflation gemindert.

(3) Für das Geldnachfrageverhalten werden im Prinzip ebenso wie für das Konsum-/Sparverhalten des privaten Sektors unverzögerte Verhaltensweisen angenommen. Die Variable $\left(x_{t} \cdot p_{x, t}\right) /\left(x_{t-1} \cdot p_{x, t-1}\right)$ in (9.4) dient ausschließlich als konjunkturelles Argument. - Vgl. dagegen $\mathrm{H}_{\text {. }}$ König, Einkommenskreislaufgeschwindigkeit des Geldes und Zinssatzveränderungen: Eine ökonometrische Studie über die Geldnachfrage in der BRD, Zeitschr.f.d.ges. Staatswissenschaft, 124, 1968, S. $70 \mathrm{ff}$, wo mit Hilfe von Vierteljahresdaten (!) die Hypothese eines echt verzögerten Geldnachfrageverhaltens untersucht wird. 


$$
\begin{aligned}
M_{t}= & \tau_{1} \cdot r_{t}^{\tau_{2}} \cdot Y_{t}^{b r^{\tau}} \cdot\left[\frac{e_{v, t}-e_{t}}{a_{t}+\left(e_{v, t}-e_{t}\right)}\right]^{\tau_{4}} . \\
& {\left[\frac{x_{t} \cdot p_{x, t}}{x_{t-1} \cdot p_{x, t-1}}\right]^{\tau_{5}} \cdot\left[\frac{p_{x, t}}{p_{x, t-1}}\right]^{\tau_{6}} }
\end{aligned}
$$

4. Empirische Überprüfung des Verhaltens von Geldversorgung und privater Kassenhaltung

Die Gleichungen $(9.2)$ bis $(9.5)$ bilden nach einer logarithmischen Transformation ein lineares interdependentes Gleichungssystem. Die Verhaltensparameter dieses Systems können, da die einzelnen Gleichungen im ökonometrischen Sinn überidentifiziert sind, mit Hilfe einer zweistufigen Kleinst-Quadrat-Schätzung bestimmt werden (1). Zu diesem Zweck werden die vier Gleichungen in die Logarithmen transformiert und aufgelöst. Die Auflösung gibt Gleichgewichtswerte für den Kassenbestand, das Liquiditätsvolumen, das Einlagevolumen und den Zinssatz, die nunmehr lediglich eine Funktion exogen vorgegebener Variablen, nämlich der Arbeitslosenquote, des Auslandszinses, des Wachstums der privaten Bruttowertschöpfung, der Inflationsrate, des Bruttosozialproduktes und der Vermögens-Einkommens-Relation sind. Diese reduzierte Form des ursprünglichen Gleichungssystems läßt sich in Matrixform schreiben als:

(1) Zur Methode der zweistufigen Kleinst-Quadrat-Schätzung vgl. J. Johnston, Econometric Methods, New York u. a. 1963, S. 236 f. und 258 ff. sowie G. Tintner, Econometrics, New York 1965 (1952), S. $154 \mathrm{ff}$. 


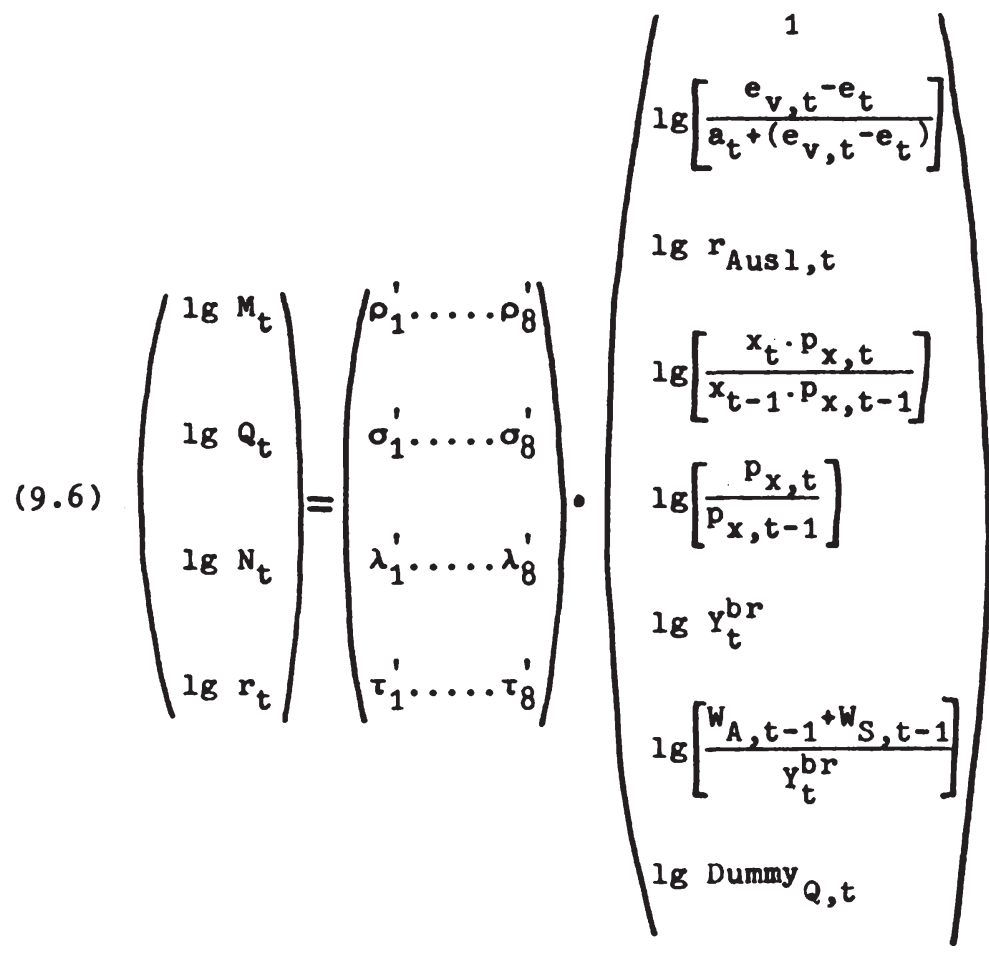

und dient als Regressionsmodell der Kleinst-Quadrat-Schätzungen erster Stufe.

Zur Schätzung der vier reduzierte-Form-Gleichungen von (9.6) wurde der Kassenbestand als Durchschnitt aus vier Vierteljahreswerten von Bargeld und Sichtguthaben der privaten Haushalte und Unternehmen in der Bundesrepublik errechnet (1). Das Liquiditätsvolumen der Banken wurde erfaßt als Durchschnitt aus den März-, Juni-, September- und Dezember-Angaben der Deutschen Bundesbank über die freien Liquiditätsreserven der Kreditinstitute (2). Das Einlagevolumen ist definiert als die Summe der Verbindlichkeiten der Banken gegenüber Nicht-Banken und ausländischen

(1) Vgl. Deutsche Bundesbank, Längerfristige Entwicklung des Geldvolumens, Monatsberichte der Deutschen Bundesbank, Juli 1971, S. 23 ff. , sowie deren Monatsberichte fortlaufend.

(2) Vgl. Deutsche Bundesbank, Erläuterungen zur Liquiditätsanalyse der Deutschen Bundesbank, Monatsberichte der Deutschen Bundesbank, Juli 1970, S. 33, sowie Monatsberichte fortlaufend. 
Kreditinstituten; es wurde indirekt aus den Angaben der Deutschen Bundesbank über das Liquiditätsvolumen und die sog. Liquiditätsquote bestimmt (1); bei beiden Werten handelte es sich wiederum um den Durchschnitt aus vier Vierteljahreswerten. Als Auslandszins diente der Durchschnitt aus vier Vierteljahreswerten der Ertragsrate aus US. Government-Bonds (2). Die Schätzperiode erstreckte sich auf die Jahre 1959 bis 1971. Die Schätzergebnisse befinden sich in Tab. 9.1.

(1) Vgl. ebenda; - die sog. Liquiditätsquote ist der Quotient aus Liquiditäts- und Einlagevolumen.

(2) Vgl. United Nations, Statistical Yearbook, New York, fortlaulend. Die Angaben für die Jahre 1972 bis 1975 wurden entnommen dem Federal Reserve Bulletin, Tabelle A 30. 
Tabelle 9.1:

Kleinst-Quadrat-Schätzung der reduzierten Form $(9,6)$ des Kreditmarktmodells

Regressionsparameter (1)

$\mathrm{zu}$
reduzierte-Form-Gleichung zur Bestimmung von

$\lg M_{t} \quad \lg Q_{t} \quad \lg N_{t} \quad \lg r_{t}$
Multi kollinearität (2)

$$
\begin{aligned}
& \text { Absolutglied } \\
& \begin{array}{llll}
-0,81865 & -2,30274 & -2,49976 & -1,20329
\end{array} \\
& (0,22402)(3,30268)(0,85593) \quad(1,30964) \\
& \lg \frac{e_{v, t}-e_{t}}{a_{t}+\left(e_{v, t}-e_{t}\right)} \\
& -0,00423+0,49560+0,05745 \\
& (0,01127)(0,16613)(0,04306) \quad(0,06588) \\
& 0,74701 \\
& \begin{array}{llll}
-0,08359 & -1,77211 & -0,16688 & +0,75599
\end{array} \\
& \lg r_{\text {Ausl, } t} \\
& (0,06607)(0,97411)(0,25245) \quad(0,38627) \\
& \lg \frac{x_{t} \cdot p_{x, t}}{x_{t-1} \cdot p_{x, t-1}} \\
& \begin{array}{lllll}
-0,05224+8,77735+0,33868 & -2,79700 & 0,80946
\end{array} \\
& (0,19890)(2,93233)(0,75994) \quad(1,16278) \\
& \lg \frac{p_{x, t}}{p_{x, t-1}} \\
& -1,35406+8,36154+1,99570 \quad-2,35809 \quad 0,91164 \\
& (0,47204)(6,95926)(1,80357) \quad(2,75962) \\
& \lg \mathrm{Y}_{\mathrm{t}}^{\mathrm{br}} \\
& +0,98292+0,61489+1,28212+0,24999 \quad 0,92582 \\
& (0,02996)(0,44164)(0,11446) \quad(0,17513) \\
& \lg \frac{W_{A, t-1}+W_{S, t-1}}{Y_{t}^{b r}} \\
& \begin{array}{llll}
-0,06852 & +9,24461 & +2,23958 & -2,56464 \\
(0,24113) & (3,55500) & (0,92132) & (1,40970)
\end{array} \\
& 0,94128 \\
& 0,95531 \\
& \begin{array}{lllll}
-0,01153 & -0,19132 & -0,02574 & +0,03829 & 0,30316
\end{array} \\
& (0,00477)(0,07034)(0,01823) \quad(0,02789)
\end{aligned}
$$

$\mathrm{R}_{1 \cdot 2,3,4,5,6,7,8}^{2}$

$$
0,99959
$$

0,93246

0,99766

0,92479

Irrt. - W.

$$
<0,1 \%
$$

$<2 \%$

$<0,1 \%$

$<2 \%$

vNQ

$$
3,076
$$

2,705

2,834

2,628

Bemerkungen:

(1) Die geklammerten Werte geben die Standardfehler der Regressionsparameter an.

(2) Korrelation $\left(\mathrm{R}^{2}\right) \mathrm{zwischen}$ den exogenen Variablen. 
Die vier Strukturgleichungen (9.2) bis (9.5) sind überidentifiziert (1); die Schätzwerte der Parameter $\rho_{i}^{\prime}, \sigma_{i}^{\prime}, \lambda_{i}^{\prime}$ und $\tau_{i}^{\prime}(i=1, \ldots 8)$ von (9.6) in Tabelle 9.1 erlauben keinen Rúckschluß auf die Größe der Verhaltensparameter $o_{i}^{\prime}, \sigma_{i}^{\prime}, \lambda_{i}^{\prime}$, und $\tau_{i}^{\prime}$. Aus diesem Grund wurden die Funktionen (9.2) bis (9.5) einer direkten Kleinst-Quadrat-Schätzung unterzogen, wobei das Liquiditäts- und Einlagevolumen sowie der Kreditmarktzins, soweit sie als Ursachenvariablen in Erscheinung treten, nicht mit ihren tatsächlichen, fehlerbehafteten Originalwerten berücksichtigt wurden, sondern - entsprechend der Methode der zweistufigen Kleinst-QuadratSchätzung - mit den fehlerbereinigten Werten, die als Ergebnis der reduzierten-Form-Schätzung anfielen.

Die so erzielten Schätzergebnisse zweiter Stufe sind im vorliegenden Fall beeinträchtigt durch die $z$ wischen den Ursachenvariablen auftretende Multikorrelation, die sich auf die Größe der geschätzten Standardfehler auswirkt; sie geben ansonsten eine brauchbare Vorstellung von der Größe der (9.2) bis (9.5) auftretenden Verhaltensparameter und einen gewissen Einblick in das Funktionieren des Kreditmarktmechanismus in der Bundesrepublik der sechziger Jahre.

Für die Geldangebotsfunktion (9.2) ergab die Schätzung zweiter Stufe:

$$
\begin{aligned}
& \text { (9.7) } \quad \text { ig } \rho_{1}=+3,33223( \pm 0,23219) \\
& \rho_{2}=+2,02975( \pm 0,10141) \\
& \rho_{3}=+0,64224( \pm 0,03893) \\
& \rho_{4}=-0,19815 \quad( \pm 0,01560) \\
& \rho_{5}=-0,55046 \quad( \pm 0,08627) \\
& \rho_{6}=+0,04096( \pm 0,01190) \\
& \mathrm{R}_{1 \cdot 2,3,4,5,6}^{2}=0,99771 \text { (Irrt.-Wahrsch.: }<0,18 \text { ) } \\
& \mathrm{R}_{2 \cdot 3,4,5,6}^{2}=0,81378 \\
& R_{3 \cdot 2,4,5,6}^{2}=0,82301 \\
& \mathrm{R}_{4 \cdot 2,3,5,6=0,46908}^{2} \\
& R_{5 \cdot 2,3,4,6}^{2}=0,89453 \\
& \mathrm{R}_{6 \cdot 2,3,4,5}^{2}=0,54922 \\
& \text { vNQ } \\
& =1,760
\end{aligned}
$$

(1) Zum Identifikationsproblem vgl. J. Johnston, Econometric Methods, a. a. O., S. $240 \mathrm{ff}$. sowie G. Tintner, Econometrics, a. a. O., S. $155 \mathrm{ff}$. 
Danach ist das Geldangebot des Bankensektors bei gegebenem Bestand an freien Liquiditätsreserven hoch zinselastisch. Der Schätzwert für die partielle Zinselastizität des Geldangebotes $\left(\rho_{2}\right)$ beträgt +2,03, eine Erhöhung des Zinssatzes um $1 \%$, also z. B. von $5 \%$ auf $5,05 \%$, würde die Banken zu einer Ausweitung des Kreditvolumens um 2,04\% bereit machen. Dies ist jedoch nur eine partielle Aussage. Endgültige Aussagen über die Zinselastiziät des Kreditangebotes sollten bei Einbeziehung der Rückwirkungen getroffen werden, die von den Zinseffekten auf den Liquiditätsstatus des Geschäftsbankensystems ausgehen und darüber das Geldangebot indirekt noch einmal beeinflussen. - Ein zweites Ergebnis von (9.7) ist, daß eine Ausweitung der sog. freien Liquiditätsreserven die Banken c.p. keineswegs zu einer proportionalen Ausweitung des Geldangebotes veranlaßt, was einen c.p. konstanten Geldschöpfungsmulti plikator implizieren würde. Der entsprechende Elastizitätsparameter $\left(\rho_{3}\right)$ beträgt nicht - wie im Proportionalitätsfall - eins, sondern nur +0,64. Dieses Ergebnis muß im Zusammenhang mit dem überproportionalen Anstieg der Bankdepositen und einem entsprechenden Anstieg der zur Risikoüberwachung erforderlichen Liquiditätsreserven im Laufe des Wachstumsprozesses der Bundesrepublik gesehen werden. - Bei einer gegebenen Liquiditätsausstattung reagiert das Geldangebot des Bankensektors sehr empfindlich auf Änderungen der konjunkturellen Situation. Eine kurzfristige Änderung der Arbeitslosenquote um $10 \%$, also z. B. von $2 \%$ auf $2,2 \%$, veranlaßt den Geschäftsbankensektor entsprechend dem Schätzwert von $\rho_{4}$ zu einer Einschränkung des Geldangebotes um 1,9\% der gesamten Geldmenge. Dieses ist jedoch wieder eine partielle Aussage, die ohne Berücksichtigung der Notenbankinterventionen und sonstiger Zins - und Liquiditätseffekte auf das Geldangebot gilt. - Verhältnismäßig hoch ist der Schätzwert von Parameter $\rho_{5}$, der die Elastizität des inländischen Geldangebotes, bezogen auf den Auslandszins, angibt. Der Parameter hat das richtige Vorzeichen und ist signifikant von null zu unterscheiden, so daß über den internationalen Kapitalverkehr eine starke Abhängigkeit des inländischen Geldangebotes vom (langfristigen) Kapitalmarktzins der USA angenommen werden muß.

Die vorstehenden Ergebnisse und Interpretationen werden sehr gut ergänzt durch das Schätzergebnis für die Liquiditätsbestimmungsfunktion (9. 3): 


$$
\begin{aligned}
& \text { Ig } \sigma_{1}=-4,32335 \quad( \pm 1,99362) \\
& \sigma_{2}=-2,61753( \pm 0,76456) \\
& \sigma_{3}=+1,16612( \pm 0,21091) \\
& \sigma_{4}=+0,27518( \pm 0,08441) \\
& \sigma_{5}=-0,03243 \quad( \pm 0,07102) \\
& \mathrm{R}_{1 \cdot 2,3,4,5}^{2}=0,90742 \text { (Irrt.-Wahrsch.: }<0,18 \text { ) } \\
& R_{2 \cdot 3,4,5}^{2}=0,84842 \\
& R_{3 \cdot 2,4,5}^{2}=0,86583 \\
& \mathrm{R}_{4 \cdot 2,3,5}^{2}=0,16056 \\
& \mathrm{R}_{5 \cdot 2,3,4}^{2}=0,41434 \\
& \text { vNQ }=1,921
\end{aligned}
$$

Hier zeigt nämlich der Schätzwert von $\sigma_{2}$, daß die Bereitschaft der Geschäftsbanken, bei gegebenem Einlagevolumen auf Liquiditätsreserven zu verzichten, durch geringfügige Zinsänderungen erheblich gesteigert werden kann, so daß in erhöhtem Umfang freigesetzte Liquidität als Deckungsreserve für zusätzliche Geldschöpfung und damit verbundenes Einlagewachstum zur Verfügung steht. Die hohe Zinsreagibilität der bei gegebenem Einlagevolumen etc. von den Banken angestrebten Liquiditätsreserven ist das kurzfristige Gegenstück zu dem niedrigen Wert der Geldschöpfungselastizität $\rho_{3}$ von (9.7). - Der Schätzwert von $\sigma_{3}$ deutet darauf hin, daß die Geschäftsbanken ihre Liquiditätsreserven in etwa proportional zum wachsenden Einlagevolumen steigen lassen, wenn alle übrigen Einflüsse gleich bleiben. - Der Schätzwert von Parameter $\sigma_{4}$ spiegelt mit einem verhältnismäßig hohen Wert von $+0,27$ vor allem den Einfluß der Notenbankpolitik, in gewissem Umfang möglicherweise auch konjunkturbedingte Schwankungen in der Liquiditätspräferenz der Banken wieder. Bei einer Erhöhung der Arbeitslosenquote um $10 \%$, etwa von $2 \%$ auf 2,2 \% führt der aggregierte Einfluß beider Faktoren zu einer 2, 7 \%igen Ausweitung der Bankenliquidität (1).

(1) In einer zusätzlichen Schätzung wurde überprüft, ob nicht das Wachstum der privaten Bruttowertschöpfung als Orientierungsgröße des Notenbankverhaltens und der konjunkturbedingten internationalen Kapital- 
Das Schätzergebnis für das Einlageverhalten der Nicht-Banken lautet im Anschluß an (9.4):

$$
\begin{aligned}
& \text { (9.9) Ig } \lambda_{1}=-2,02554 \quad( \pm 0,54237) \\
& \lambda_{2}=+0,04638 \quad( \pm 0,11874) \\
& \lambda_{3}=+1,25066 \quad( \pm 0,07056) \\
& \lambda_{4}=+1,62417 \quad( \pm 0,27398) \\
& \lambda_{5}=-0,02921 \quad( \pm 0,01672) \\
& R_{1 \cdot 2,3,4,5}^{2}=0,99650 \text { (Irrt.-Wahrsch.: }<0,18 \text { ) }
\end{aligned}
$$

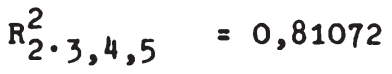

$$
\begin{aligned}
& R_{3 \cdot 2,4,5}^{2}=0,81703 \\
& \mathrm{R}_{4 \cdot 2,3,5}^{2}=0,37761 \\
& R_{5 \cdot 2,3,4}^{2}=0,22353 \\
& \text { vNQ } \quad=2,817
\end{aligned}
$$

Der Wert von Parameter $\lambda_{2}$ deutet eine positive Zinselastizität des Einlagenverhaltens auf Seiten der privaten Nicht-Banken an, ist aber kaum signifikant von null zu unterscheiden. Im übrigen bringt das Schätzergebnis vor allem das in den sechziger Jahren zu beobachtende starke Wachstum des gesamten Einlagevolumens zum Ausdruck, das von Bedeutung für die Liquiditätsdispositionen und Geldangebotsreaktionen der Geschäftsbanken in der Bundesrepublik und damit auch für eine entsprechend abgestimmte Geldpolitik der Bundesbank war. Dieses Einlagenwachstum ist

zuflüsse sowie der Auslandszins als Alternativertrag für kurzfristige Auslandsgelder einen Einflu $\beta$ auf die Bankenliquidität haben: Das Bestimmtheitsmaß erhöhte sich im Vergleich zu (9.8) von 0,907 auf 0,932 . Die Standardfehler gingen jedoch in beiden Fällen weit über den Wert des jeweiligen Regressionsparameters hinaus. Auch blieben die Schätzwerte der übrigen Parameter von der Einbeziehung der beiden Zinsvariablen weitgehend unberührt. Schließlich stellten sich im Rahmen der gesamten Modellrechnung falsche Reaktionen des Zinsniveaus auf simulierte Änderungen der monetären Gesamtnachfrage innerhalb einer Periode und im Laufe des Konjunkturzyklus ein. - Aus allen drei Gründen wurde die Vermutung derartiger $\mathrm{Zu}$ satzeinflüsse fallen gelassen. 
zu einem großen Teil durch den allgemeinen Einkommensanstieg veranlaßt und reagiert auf dessen Entwicklung überproportional $\left(\lambda_{3}\right)$. Zu einem anderen Teil resultiert es aus dem strukturellen Vermögensanlageverhalten der privaten Wirtschaftssubjekte. Während der sechziger Jahre näherte sich die Vermögens-Einkommens-Relation, $\left(W_{A, t-1}+W_{S, t-1}\right)$ $/ \mathrm{Y}_{\mathrm{t}}^{\mathrm{br}}$, aufgrund des Spar- und Investitionsverhaltens asymptotisch einer Obergrenze - eine Entwicklung, die an sich parallel zur Entwicklung des privaten Kapitalkoeffizienten verlief, ab Ende der sechziger Jahre aber durch starke inflationsbedingte Gegentendenzen (1) zurückgeworfen wurde. Wegen der geschätzten Größe von Parameter $\lambda_{4}$ muß davon ausgegangen werden, daß die privaten Wirtschaftssubjekte im Zuge des Aufbaus einer bestimmten Vermögens-Einkommens-Relation ihr Vermögen in Richtung auf einen höheren Anteil an Bankeinlagen umstrukturieren (2).

(1) Das Nominalvermögen der privaten Haushalte, das den Zähler des Quotienten bildet, enthält keine Bewertungsgewinne auf den vorhandenen Vermögensbestand und erfährt daher im Gegensatz zur Einkommensgröße im Nenner inflatorische Aufblähungen nur insoweit, wie sie in dem laufenden Nominalvermögenszug ang enthalten sind.

(2) Nur unter dem Strich und zusammengefaßt für die Schätzergebnisse $(9.7),(9.8)$ und $(9.9)$ sei auf die geschätzten Parameterwerte für die Dummy-Variable verwiesen, mit der die Auswirkungen des Bundesbankverhaltens im Jahr vor der DM-Aufwertung 1961 erfaßt wurden. Aufgrund der Schätzwerte laufen die Sondereinflüsse der damaligen Bundesbankpolitik im Endeffekt auf eine Erhöhung des Kapitalmarktzinses um 0,8\% und eine Verringerung der Geldmenge um $0,1 \%$ hinaus. 
Die Schätzergebnisse zur Geldnachfragefunktion (9.5) lauten:

$$
\begin{aligned}
& \text { (9.10) } \quad \lg \tau_{1}=-0,82185 \quad( \pm 0,22821) \\
& \tau_{2}=-0,12930 \quad( \pm 0,07643) \\
& \tau_{3}=+0,97130( \pm 0,02640) \\
& \tau_{4}=-0,01592 \quad( \pm 0,00980) \\
& \tau_{5}=-0,35036( \pm 0,13066) \\
& \tau_{6}=-0,96655 \quad( \pm 0,31262) \\
& \mathrm{R}_{1 \cdot 2,3,4,5,6}^{2}=0,99915 \text { (Irrt.-Wahrsch.: }<0,18 \text { ) } \\
& R_{2 \cdot 3,4,5,6}^{2}=0,87834 \\
& R_{3 \cdot 2,4,5,6=0,85729}^{2} \\
& R_{4.2,3,5,6}^{2}=0,50098 \\
& R_{5 \cdot 2,3,4,6=0,34082}^{2} \\
& R_{6 \cdot 2,3,4,5}^{2}=0,69923 \\
& \text { VNQ } \quad=2,199
\end{aligned}
$$

Der Schätzwert für die Zinselastizität der Geldnachfrage $\left(\tau_{2}\right)$ hat das richtige Vorzeichen und entspricht in etwa den vergleichbaren Werten anderer Untersuchungen (1). Der Wert ist statistisch wenig signifikant. Weniger stark als auf Zinsänderungen reagiert die private Geldnachfrage nach $(9.10)$ auf Änderungen der Inflationsrate. Um den Einfluß von Preisniveausteigerungen und Kapitalmarktzins gegeneinander abzuwägen, darf nicht auf einen direkten Vergleich des entsprechenden Parameters $\left(\tau_{6}\right)$ mit der Zinselastizität $\left(\tau_{2}\right)$ abgestellt werden, weil der Einfluß der Inflation durch die Meßziffer des Preisniveaus (Vorjahr $=1$ ) und nicht

(1) Vgl. H. König, Einkommenskreislaufgeschwindigkeit des Geldes und Zinssatzveränderungen: Eine ökonometrische Studie über die Geldnachfrage in der BRD, a. a. O., S. 74; U. Westphal, Theoretische und empirische Untersuchungen zur Geldnachfrage und zum Geldangebot, Tübingen 1970, S. 55, 67 und 71; sowie A. Woll, Zur Theorie der Geldnachfrage: Analytische Ansätze und statistische Ergebnisse für die Bundesrepublik Deutschland, Zeitschr.f.d. gesamte Staatswissenschaft, 125,1969 , S. 76 . 
durch die Preissteigerungsrate als solche erfaßt wurde und weil die relativen Änderungen der Meßziffer sich in ganz anderen Größenordnungen bewegen als die relativen Änderungen des Kapitalmarktzinses. Eine Erhöhung der Meßziffer des Preisniveaus um einen Prozentpunkt, also etwa von 1,02 auf 1,03 , bewirkt nach $(9,10)$ einen Rückgang der privaten Geldnachfrage um 0,9\% der gesamten Geldmenge; eine Erhöhung des Zinssatzes um einen Prozentpunkt, also etwa von 0,05 auf 0,06, bewirkt dagegen einen Rückgang um 2,3\%. - Von den beiden Parametern $\tau_{4}$ und $\tau_{5}$, mit denen die Konjunkturabhängigkeit der Geldnachfrage auf Seiten der privaten Haushalte einerseits und der Unternehmen andererseits erfaßt werden sollte, hat der erste, wie ein Vergleich mit dem späteren Schätzergebnis (9.15) zeigt, wohl infolge der Aggregation der Kassenbestände von Haushalten und Unternehmen das falsche Vorzeichen. - Schließlich entspricht der Schätzwert für die Einkommenselastizität der privaten Geldnachfrage mit einer Größe von nahe eins wieder den Ergebnissen anderer Untersuchungen, bei denen der Wert der Elastizität jedoch häufig geringfügig über eins liegt (1).

Insgesamt liefert die zweistufige Kleinst-Quadrat-Schätzung des Kreditmarktmodells die Informationen, die für die Beurteilung des Kreditmarktgeschehens und seiner Funktion innerhalb des gesamtwirtschaftlichen Verteilungsprozesses erforderlich sind. Die Schätzergebnisse des angebots theoretischen Teils erlauben eine Quantifizierung der in der Föhl'schen Überwälzungsdiskussion angesprochenen Elastizität des Geldangebotes (2), die hier mit der Zinselastizität des Geldangebotes bei Berücksichtigung aller indirekten Kapitalmarktwirkungen identifiziert wird. Setzt man die Funktion für das Einlageverhalten der Nicht-Banken (3.4) in die Liquiditätsbestimmungsfunktion (3.3) und diese wiederum in die Geldangebotsfunktion (3.2) ein, so berechnet sich diese Zinselastizität als

(1) Aufgrund der Schätzergebnisse (9.7), (9.8) und (9.9) beträgt die Einkommenselastizität des Geldangebotes

$$
\rho_{3} \cdot \sigma_{3} \cdot \lambda_{3}=+0,93665
$$

Ihr Wert stimmt also weitgehend mit der Einkommenselastizität der Geldnachfrage, $\tau_{3}$, überein. Damit ist sichergestellt, daß im Wachstumsprozeß Geldangebot und Geldnachfrage c. p. gleich stark expandieren und keine wesentlichen langfristigen Zinsänderungstendenzen auslösen.

(2) Vgl. R. Stucken und W. Ehrlicher, Zur Frage der Überwälzung der Unternehmungsteuern, Finanzarchiv, NF. 14, 1953/54, S. 374 f. 
und erhält aufgrund der Schätzergebnisse von (9.7) bis (9.9) einen Wert von $+0,383$. Der Wert ist kleiner als der Schätzwert von $\rho_{2}$ in (9.7), weist aber immer noch eine ganz beachtliche Höhe auf. Angenommen, der Staat würde den Unternehmen eine Gewinnsteuer in Höhe von ein Prozent des nominellen Bruttosozialproduktes auferlegen und die Unternehmen finanzierten diese neue eingeführte Gewinnsteuer in der ersten Runde durch eine entsprechende Kreditaufnahme im Bankensektor, die mit einer zusätzlichen Geldschöpfung gleicher Höhe verbunden wäre. Dann müßten die Banken zu einer Ausweitung der insgesamt angebotenen Geldmenge um ebenfalls ein Prozent veranlaßt werden. Hierzu wäre eine Erhöhung des Zinssatzes um 2,6\% erforderlich, also etwa von $5 \%$ auf $5,13 \%$.

Man muß vermuten, daß die beachtliche Höhe der Zinselastizität des Geldangebotes auf die zinsempfindlichen Reaktionen des internationalen Kapitalverkehrs zurückzuführen ist. In einer geschlossenen Wirtschaft bzw. in einem Wirtschaftssystem, das sich durch flexible Wechselkurse gegenüber kurzfristigen internationalen Kapitalbewegungen abgesichert hat, ist mit einem geringeren Wert der Geldangebotselastizität zu rechnen. Allerdings ist es grundsätzlich vorstellbar, daß die internationalen Kapitalbewegungen ganz kurzfristig vor allem auf Liquiditätsdispositionen der Inlandsbanken beruhen, die in einer Substitution von liquiden Auslandsanlagen gegen liquide Inlandsanlagen bei unveränderten Liquiditätsreserven insgesamt bestehen.

Die Größe der Geldangebotselastizität ist in verteilungs- bzw. inzidenztheoretischer Hinsicht von unterschiedlicher Bedeutung. Nachdem eine kurzfristige Zinsabhängigkeit der Investitionen nicht nachgewiesen werden konnte, für die Modellwirtschaft daher auch eine Unabhängigkeit der Investitionen vom laufenden Zinsniveau angenommen wurde, kann man davon ausgehen, daß die Höhe der Geldangebotselastizität für die kurzfristige Überwälzung von Steuermehrbelastungen gesamtwirtschaftlich unerheblich ist. Wichtig ist, daß das Geldangebot kurzfristig überhaupt eine gewisse Elastizität aufweist, um den durch Steuererhöhungen im Unternehmenssektor verursachten Liquiditätsentzug - bei welchem Zins auch immer - auszugleichen. Insofern sind die angestellten Berechnungen für die Diskussion der kurzfristigen Überwälzungsproblematik überflüssig.

Von Bedeutung sind die Berechnungen aber für die Überwälzungserscheinungen, die nach Verstreichen der kurzen Frist von einem Jahr verbleiben. Benötigen die Unternehmen zur Finanzierung der geplanten Investitionen wegen des Liquiditätsentzugs durch Steuererhöhungen zusätzliche Kredite, so verursachen sie einen Anstieg des Kreditmarktzinses. Die Stärke des Zinssatzes hängt von der Elastizität des Kreditangebotes 
ab. Von der Stärke des Zinsanstieges andererseits hängt die verzögerte Reaktion der Investitionen ab, und diese ist entscheidend für das Tempo, mit dem die kurzfristig aufgetretenen Überwälzungseffekte abgebaut werden. Wegen der zu vermutenden Unterschiede in der Zinselastizität des Geldangebotes ist das Tempo dieses Anpassungsprozesses in der geschlossenen Wirtschaft oder in einer offenen Wirtschaft mit flexiblen Wechselkursen wahrscheinlich höher als in der offenen Wirtschaft mit festen Wechselkursen (1).

Es sei hier zum Schluß nur darauf aufmerksam gemacht, daß die einzige Variable des Kreditmarktmodells, die auch im Rahmen des Gesamtmodells exogen vorgegeben wird, der Auslandszins ist. Seine Höhe wird in den Jahren 1960 bis 1975 durch die jeweilige Ertragsrate auf US-Government-Bonds vorgegeben; für die Jahre nach 1975 wird sie mit einem trendmäßig fortgeschriebenen Wert dieser Ertragsrate angesetzt. Zur Fortschreibung dient die Trendgleichung

$$
r_{\text {AUs } 1, t}=\rho_{1}^{n}+\rho_{2}^{n} \cdot r_{\text {AUs } 1, t-1}
$$

mit den frei Hand bestimmten Parameterwerten:

$$
\begin{aligned}
& \rho_{1}^{n}=+0,025 \\
& \rho_{2}^{n}=+0,500
\end{aligned}
$$

Gemäß (9.12) und (9.13) sinkt der Auslandszins von 0, $069 \mathrm{im} \mathrm{Jahr} 1975$ gleichmäßig auf ein Niveau von 0,05 .

5. Die Aufteilung des Geldumlaufs auf die privaten Haushalte und Unternehmen

Für die mathematische Auflösung des formulierten Modells erweist es sich als günstig, daß das Verhalten der privaten Geldnachfrage durch eine einzige aggregierte Funktion dargestellt wird. Wegen der Einbeziehung der Vermögensrechnung, die für die institutionelle Verteilung der

(1) Aus dem gleichen Grund sind die gesamtwirtschaftlichen Überwälzungsmöglichkeiten des Unternehmenssektors in der Bundesrepublik wahr scheinlich auch seit der Liberalisierung des internationalen Kapitalverkehrs im Jahre 1958 größer als zuvor. Es sei allerdings noch einmal darauf hingewiesen, daß nach den angestellten Überlegungen der vorigen Paragraphen die langfristigen Überwålzungserscheinungen sich fast ausschließlich auf die Funktionalstruktur der Besitzeinkommen, nicht auf das Verhältnis von Arbeitseinkommen zu Besitzeinkommen erstrecken. 
Besitzeinkommen von Bedeutung ist, ist es aber erforderlich, zumindest nach der mathematischen Auflösung des Modells für die jeweilige Periode zu bestimmen, wie sich die Kassenbestände auf die Selbständigen-Haushalte, die Unselbständigen-Haushalte und die Unternehmen verteilen. Erst dann kann nämlich bestimmt werden, welcher Anteil der Nominalersparnis der Arbeitnehmerhaushalte in verzinslichen Forderungstiteln angelegt wird und in der Folgeperiode zum Besitzeinkommen der Arbeitnehmer beiträgt.

Aus diesem Grund wird als erstes zusätzlich zur Funktion für das aggregierte Verhalten der privaten Geldnachfrage noch eine Funktion für die Geldnachfrage der privaten Haushalte formuliert. Diese Funktion macht die Kassenhaltung der privaten Haushalte abhängig von deren aggregiertem Bruttoarbeitseinkommen, vom Zinssatz und der Preissteigerungsrate als Kostenfaktoren und von der Arbeitslosenquote als Argument für die Konjunkturabhängigkeit der Kassenhaltungswünsche. Dabei wird die Kassenhaltung auf das rechnerische Bruttoarbeitseinkommen der privaten Haushalte bezogen und ergibt einen Kassenhaltungsquotienten, $\left(M_{A, t}+M_{S, t}\right) /\left(L_{A, t}+L_{S, t}\right)$. Anschließend wird die Kassenhaltungsfunktion der privaten Haushalte formuliert als

$$
\frac{M_{A, t}+M_{S, t}}{L_{A, t}+L_{S, t}}=\Phi_{1} \cdot r_{t}^{\varphi_{2}} \cdot\left[\frac{p_{x, t}}{p_{x, t-1}}\right]^{\Phi_{3}} \cdot\left[\frac{e_{v, t}-e_{t}}{a_{t}+\left(e_{v, t}-e_{t}\right)}\right]^{\Phi_{4}}
$$

Für den Kassenbestand der privaten Haushalte zum Jahresende 1969 und 1970 hat die Deutsche Bundesbank eine Schätzung veröffentlicht (1). Für die jährliche Ersparnisbildung der privaten Haushalte in Form von Kassenhaltung findet sich eine Zeitreihe bei W. Kaiser und A. Zerwass (2). Aus beiden Angaben wurde eine Zeitreihe für den Geldbestand der privaten Haushalte zum jeweiligen Jahresende errechnet. Der Durchschnitt aus dem Endbestand der Jahre t und t-1 wurde als Durchschnittsbestand des Jahres $t$ angesehen und auf das rechnerische Bruttoarbeitseinkommen der privaten Haushalte im Jahre $t$ bezogen. Für die Variable Kapitalmarktzins wurden die fehlerbereinigten Werte verwendet, die sich

(1) Vgl. Deutsche Bundesbank, Gesamtwirtschaftliche Finanzierungsrechnung sowie Bestand an Geldvermögen und Verpflichtungen 1970, Monatsberichte der Deutschen Bundesbank, Mai 1971, S. 29. - Die neuesten Schätzungen der Bundesbank weichen von diesen Angaben $\mathrm{ab}$, konnten aber nicht mehr berücksichtigt werden. Vgl. dieselbe, Entwicklung des Geldvermögens und der Verpflichtungen von 1950 bis 1971, Monatsberichte der Deutschen Bundesbank, März 1973, S. 28.

(2) Vgl. W. Kaiser und A. Zerwass, Die Struktur des Sparens in der Bundesrepublik Deutschland von 1950 bis 1967, a. a. O., Tabelle 18. 
aus der reduzierten-Form-Schätzung des Kreditmarktmodells ergaben.

Das Regressionsergebnis der Kleinst-Quadrat-Schätzung für die Gleichung (9.14) lautet $(t=1959, \ldots, 1971)$ :

$$
\text { (9.15) } \begin{aligned}
\text { Ig } \Phi_{1} & =-0,86230 \quad( \pm 0,04316) \\
\varphi_{2} & =-0,02975 \quad( \pm 0,03363) \\
\varphi_{3} & =-1,34781 \quad( \pm 0,27313) \\
\varphi_{4} & = \pm 0,01766 \quad( \pm 0,00938) \\
R_{1 \cdot 2,3,4}^{2} & =0,92399 \quad \text { (Irrt.-Wahrsch.: } 0,1 \%) \\
R_{2 \cdot 3,4}^{2} & =0,37307 \\
R_{3 \cdot 2,4}^{2} & =0,60684 \\
R_{4 \cdot 2,3}^{2} & =0,45643 \\
v_{N Q} & =1,642
\end{aligned}
$$

Sieht man davon $a b$, daß die Kassenhaltung der privaten Haushalte hier als unabhängig vom Wachstum der privaten Bruttowertschöpfung angesehen wird und daß die Einkommenselastizität der Geldnachfrage bezogen auf das Bruttoarbeitseinkommen mit eins vorgegeben wurde, stimmt das Ergebnis mit dem von (9.10) überein. Allerdings hat der Parameter für die Konjunkturabhängigkeit der von den Haushalten ausgehenden Geldnachfrage $\left(\varphi_{4}\right)$ anders als dort $\left(\tau_{4}\right)$ das Vorzeichen, das er zur Erfassung einer "liquidity-trap" haben müßte. Außerdem ist der Parameter für die Zinselastizität der Geldnachfrage in (9.15) wesentlich geringer als in (9.10). Damit führen die Schätzungen dieses Paragraphen insgesamt zu dem Ergebnis, daß die Zinselastizitäten dort, wo sie sich auf das Liquiditätsverhalten der privaten Haushalte beziehen, geringer sind als dort, wo sie sich auf das Liquiditätsverhalten des gesamten privaten Nicht-Banken-Sektors beziehen, und hier wiederum geringer als dort, wo sie sich auf das entsprechende Verhalten der Finanzinstitutionen bzw. des gesamten Bankensektors beziehen.

Regressionsergebnis (9.15) wurde mit den Jahresdurchschnittswerten (1) der Variablen erzielt. Da die Kassenbestände der privaten Haushalte in die aggregierte Vermögensrechnung der Modellwirtschaft einbezogen werden sollen, diese Rechnung aber zum Ende eines jeden Jahres aufgemacht wird, muß eine kleine Korrektur vorgenommen werden. In den

(1) Die Werte der Arbeitslosenquote beziehen sich jeweils auf die Jahresmitte. 
Jahren 1959 bis 1971 lagen die Kassenbestände der privaten Haushalte am Jahresende um durchschnittlich 4,1 \% über den Jahresdurchschnittsbeständen. Der Schätzwert des Niveauparameters $\varphi_{1}$ von (9.15) wird entsprechend um 4,1\% auf +0,143 erhöht.

Für die Modellwirtschaft wird angenommen, der Geldbestand der privaten Haushalte teile sich auf die beiden Haushaltsgruppen der Selbständigen- und Unselbständigenhaushalte gemäß ihrem Anteil am rechnerischen Bruttoarbeitseinkommen auf. Es gilt also:

(9.16) $\quad M_{A, t}=L_{A, t} \cdot\left[\frac{M_{A, t}{ }^{+M_{S}, t}}{L_{A, t}+L_{S, t}}\right]$

wobei für den in eckige Klammern gesetzten Kassenhaltungsquotienten (9.12) mit den Regressionsergebnissen (9.13) einzusetzen ist.

Mit dem Kreditmarktmodell (9.2) bis (9.5) sowie den Gleichungen (9.12) und (9.14) ist die Aufteilung des gesamtwirtschaftlichen Kassenbestandes auf die Arbeitnehmer- und Selbständigen-Haushalte sowie die Unternehmen der Modellwirtschaft bestimmt. 
$\$ 10$ Die Kreditbeziehungen des privaten Sektors zur übrigen Welt

1. Die internationalen Kapitalbewegungen

Der internationale Kapitalverkehr zwischen dem privaten Sektor und der übrigen Welt beeinflußt die inländische Bankenliquidität und die inländische Geldversorgung der Modellwirtschaft. Dies wurde im Kreditmarktmodell des vorigen Paragraphen implizit, aber doch ausreichend, berücksichtigt. Internationale Kapitalbewegungen verursachen außerdem internationale Einkommensströme in Form von Zinszahlungen und wirken sich dadurch auf die institutionelle Verteilung der im Inland entstandenen oder anfallenden Besitzeinkommen aus. Insofern ist es zweckmäßig, den internationalen Kapitalverkehr und die auf ihn entfallenden Zinszahlungen auch explizit zu berücksichtigen.

$\mathrm{Zu}$ diesem $\mathrm{Zweck}$ wird ein theoretischer Ansatz gewählt, mit dem der private Kapitalverkehr zwischen der Bundesrepublik und dem Ausland bereits mit Erfolg untersucht wurde (1). In dem Ansatz wurde der private Nettokapitalzu- bzw. - abfluß der Bundesrepublik ursprünglich abhängig gemacht vom Saldo der Handelsbilanz als Ausdruck der konjunkturellen Situation im Inland und vom Unterschied $\mathrm{zwischen}$ dem inländischen und ausländischen Geldmarktzins (2). Die zugehörigen Regressionsschätzungen auf der Basis von Vierteljahresdaten lieferten eine ziemlich genaue Beschreibung der Salden im privaten Kreditverkehr zwischen der Bundesrepublik und dem Ausland.

Zur Anpassung an die Modellformulierung wird dieser Ansatz einigen Änderungen unterzogen: Der Nettokapitalzu-bzw. - abfluß wird abhängig gemacht von der Bruttowertschöpfung des Privatsektors im laufenden Jahr und im dritten Vorjahr, an der sich die kurz- und längerfristigen Konjunkturerwartungen orientieren. Der Unterschied zwischen dem Geldmarktzins des In- und Auslandes, der auch bei der Analyse von Jahresdaten zweifellos der beste statistische Erklärungsfaktor des internationalen Kapitalverkehrs ist, wird durch das Produkt aus der Liquiditätsquote, $Q_{t} / N_{t}$, des inländischen Geschäftsbankensystems und der privaten Bruttowertschöpfung, $x_{t}{ }^{\circ} p_{x}, t$, ersetzt. Zusätzlich wird eine DummyVariable berücksichtigt, die im Jahr einer Aufwertung der Inlandswährung gegenüber dem Ausland den Wert +1 , im Jahr einer Abwertung den Wert

(1) Vgl. R. Erbé, Causes and Effects of Private Capital Movements in Germany, 1955-1969, Kyklos, XXII, 1970, S. 927 ff, insbes. S. 937 ff.

(2) Es handelt sich um den Zins für 3-Monats-Gelder in der Bundesrepublik und auf dem Euro-Dollar-Markt. 
-1 und in den übrigen Jahren den Wert null annimmt (1). Diese DummyVariable dient dazu, den auf Wechselkursänderungen hin eintretenden Rückfluß spekulativer Gelder über die nationalen Grenzen zu erfassen. Da der Umfang der spekulativen Kapitalbewegungen wegen des weltweiten Wachstumsprozesses von Mal zu Mal steigt, wird auch die DummyVariable mit der privaten Bruttowertschöpfung des Inlandes multipliziert.

Wird der Saldo des privaten Kapitalverkehrs mit dem Ausland ausgedrückt als eine Änderung der Nettoforderungsposition des Auslandes gegenüber dem inländischen Unternehmenssektor, $\Delta F_{\text {Ausl, } t}$, kann die Gleichung für den privaten Kapitalverkehr der Modellwirtschaft mit der übrigen Welt formuliert werden als:

(10.1)

$$
\begin{aligned}
\Delta F_{\text {Aus l }, t}= & \Psi_{1} \cdot\left[x_{t} \cdot p_{x, t}\right]+ \\
& \Psi_{2} \cdot\left[x_{t-3} \cdot p_{x, t-3}\right]+ \\
& \Psi_{3} \cdot\left[\frac{Q_{t}}{N_{t}} \cdot\left(x_{t} \cdot p_{x, t}\right)\right]+ \\
& \psi_{4} \cdot\left[\text { Dummy }_{t} \cdot\left(x_{t} \cdot p_{x, t}\right)\right]
\end{aligned}
$$

mit

$$
\text { Dummy }_{t}=\left\{\begin{aligned}
+1 & \text { im Jahr einer Währungsaufwertung } \\
0 & \text { im Jahr ohne Wechselkurskorrektur } \\
-1 & \text { im Jahr einer Währungsabwertung }
\end{aligned}\right.
$$

Zur Trendausschaltung und zur Vermeidung von Multikollinearität wird Gleichung (10.1) durch $\left(\mathrm{x}_{\mathrm{t}} \mathrm{p}_{\mathrm{x}, \mathrm{t}}\right)$ dividiert:

$$
\begin{aligned}
\frac{\Delta F_{\text {Aus } 1, t}}{x_{t} \cdot p_{x, t}}= & \Psi_{1}+\psi_{2} \cdot\left[\frac{x_{t-3} \cdot p_{x, t-3}}{x_{t} \cdot p_{x, t}}\right]+ \\
& \Psi_{3} \cdot\left[\frac{Q_{t}}{N_{t}}\right]+\psi_{4} \cdot \text { Dummy }_{t}
\end{aligned}
$$

(1) Die Annahme impliziert eine Symmetrie im Umfang der von Aufwertungs - und Abwertungsspekulationen ausgelösten Kapitalbewegungen. 
Für die transformierte Gleichung (10.2) wurden mit Hilfe der Daten für die Bundesrepublik (1) folgende Schätzergebnisse (2) erzielt $(t=1959, \ldots$, 1970):

$$
\text { (10.3) } \begin{aligned}
& \Psi_{1}=+0,27034 \quad( \pm 0,04114) \\
& \psi_{2}=-0,31172 \quad( \pm 0,05268) \\
& \Psi_{3}=-0,13181 \quad( \pm 0,05499) \\
& \Psi_{4}=-0,01968 \quad( \pm 0,00534) \\
& R_{1 \cdot 2,3,4}^{2}=0,87969 \quad(\text { Irrt. -Wahrsch.: }<0,1 \%) \\
& R_{2 \cdot 3,4}^{2}=0,03972 \\
& R_{3 \cdot 2,4}^{2}=0,03504 \\
& R_{4 \cdot 2,3}^{2}=0,01038 \\
& v_{N Q}^{2}=3,043
\end{aligned}
$$

Die Schätzergebnisse zeigen, daß die Salden des internationalen Kapitalverkehrs wahrscheinlich von der Geldmarktsituation bzw. der jeweiligen Bankenliquidität innerhalb der Bundesrepublik abhängig sind, und bestätigen damit die Aussagen von R. Erbé für die Analyse von Jahresdaten (3). Entsprechend dem negativen Vorzeichen von Parameter $\Psi_{3}$ setzen z. B. dann, wenn infolge einer kontraktiven Notenbankpolitik die Bankenliquidität abnimmt und der Geldmarkt im Inland sich anspannt, internationale

(1) Der Saldo des privaten Kapitalverkehrs wurde einschließlich der "errors and omissions" der Zahlungsbilanzstatistik erfaßt. Die Angaben wurden entnommen den Statistischen Beiheften zu den Monatsberichten der Deutschen Bundesbank, Reihe 3, Zahlungsbilanzstatistik, Juli 1972, Beilage "Die Zahlungsbilanz der Bundesrepublik im Jahr 1971 nach Regionen", S. 2 f. - Den Werten der Liquiditätsquote liegen ebenfalls die entsprechenden Angaben der Bundesbank, nicht die fehlerbereinigten Werte des vorigen Paragraphen zugrunde.

(2) Der hohe Wert des von-Neumann-Quotienten ist wahrscheinlich darauf zurückzuführen, daß der Einfluß der durch Wechselkursspekulation ausgelösten Kapitalbewegungen mit Hilfe der Dummy-Variablen nicht genügend genau erfaßt wird.

(3) Vgl. R. Erbé, a. a. O. , S. 938 
Kapitalzuflüsse ein; der Forderungsbestand des Auslandes gegenüber dem privaten Sektor des Inlandes nimmt zu. Die so induzierten Kapitalbewegungen sind in ihrem Umfang geringer als die Kapitalbewegungen, die durch Konjunkturerwartungen oder durch bevorstehende bzw. vollzogene Wechselkurskorrekturen ausgelöst werden (1). Es muß jedoch vermutet werden, daß auch der spekulative Kapitalverkehr zum großen Teil vom Bankensektor ausging. Insofern ist es gerechtfertigt, den laufenden Kapitalverkehr mit dem Ausland zu einem großen Teil als eine Erscheinung des internationalen Geldmarktverkehrs zwischen Banken anzusehen und seinen Einfluß auf das inländische Geldangebot in der im vorigen Paragraphen gewählten Weise zu berücksichtigen (2). Allerdings erfuhr die Bundesrepublik, was aus den Regressionsergebnissen nicht ohne weiteres zu ersehen ist, während der Beobachtungsperiode einen ständigen und ansteigenden Nettokapitalzufluß, der unabhängig von Geldmarktsituation, Konjunktur und Währungssituation war und über dessen Charakter hier noch keine eindeutigen Aussagen gemacht werden können.

\section{Die privaten Vermögenserträge des Auslandes}

Zur Analyse der Vermögenserträge, die per Saldo vom Ausland empfangen oder an das Ausland gezahlt werden, wäre es an sich sinnvoll, von einer getrennten Betrachtung der Auslandsforderungen und Auslandsverbindlichkeiten des inländischen Unternehmenssektors auszugehen. Nur so ließe sich der Einfluß, der von unterschiedlichen Zinsniveaus des Inund Auslandes und von Wechselkursänderungen auf den Saldo der internationalen Vermögensertragströme ausgeht, einwandfrei bestimmen. Dieses Vorgehen ist für die Modellformulierung aber zu aufwendig. Um die Entwicklung der Kapitalertragsbilanz der Modellwirtschaft gegenüber dem Ausland zu beschreiben, wird ein einfacher theoretischer Ansatz gewählt, der einigen Besonderheiten in der internationalen Kreditverflechtung der Bundesrepublik Rechnung trägt.

Nimmt man an, die Bilanz der privaten Auslandsforderungen und Auslandsverbindlichkeiten der Bundesrepublik bzw. die Bilanz der privaten inländischen Kapitalanlagen im Ausland und der ausländischen Kapital-

(1) Dies kommt am besten in der graphischen Darstellung der Regressionsergebnisse von R. Erbé zum Ausdruck. Vgl. ebenda, S. 939

(2) Aufgrund der Gleichungen (9.3), (9.4) und (10.2) samt den zugehörigen Regressionsergebnissen ermittelt man für den Bereich der Variablendurchschnitte eine Zinselastizität der privaten Nettokapitalbewegungen bezogen auf das inländische Zinsniveau von 8. Der Wert bringt die hohe Reagibilität der Salden im internationalen Kapitalverkehr zum Ausdruck. 
anlagen im Inland sei Anfang 1950 ausgeglichen gewesen, und addiert man die jährlichen Salden des internationalen privaten Kapitalverkehrs einschließlich der "errors and ommissions" der Zahlungsbilanzstatistik für die Bundesrepublik von 1950 an auf, so erhält man Werte, die hier als Modellwerte für die Nettoforderungs-bzw. -schuldenposition des Auslandes gegenüber dem privaten Sektor der betrachteten Modellwirtschaft, F Ausl, t, angesehen werden (1) (2). Die Werte geben für die sechziger Jahre fast durchweg eine (seit 1950 aufgelaufene) private Nettoschuldenposition dem Ausland gegenüber an. Wäre das System der starren Wechselkurse in den siebziger Jahren unverändert beibehalten worden und hätte man die weitere Entwicklung des internationalen Kapitalverkehrs zwischen der Bundesrepublik und dem Ausland mit Hilfe von Gleichung (10.2) und Regressionsergebnis (10.3) prognostiziert, hätte man auf jeden Fall für die weitere Zukunft mit dieser oder gar einer erhöhten Nettoschuldenposition gerechnet (3).

Nach den Angaben der volkswirtschaftlichen Gesamtrechnung wies der private Sektor der Bundesrepublik in den sechziger Jahren außerdem einen ständigen Nettoabfluß von Einkommen aus Unternehmertätigkeit und Vermögen gegenüber dem Ausland auf. Dieser Nettoabfluß stimmt mit der Vermutung einer privaten Nettoschuldenposition gegenüber dem Ausland überein.

Für die Modellwirtschaft wird ein Zusammenhang zwischen beiden Beobachtungen hergestellt. Es wird davon ausgegangen, daß die private Nettoforderungsposition des Auslandes erhalten bleibt und daß das Ausland auf seinen Nettoforderungsbestand eine Verzinsung erhält, die sich am inländischen Zinsniveau orientiert. Die privaten Vermögenserträge des Auslandes werden abhängig gemacht von dem Produkt aus dem inländischen Zinssatz der Vorperiode und der Nettoforderungsposition des Aus landes am Ende der vergangenen Periode, $r_{t-1} \cdot F_{\text {Ausl, }} t-1$. Um den Einflu $\beta$ der Wechselkursänderungen zu berücksichtigen, der sich über die vom

(1) Die Nettoforderungs - bzw. - schuldenposition ist hier unter Ausschluß der Devisenbestände definiert.

(2) Die Werte beziehen sich auf den jeweiligen Jahresanfang. Wegen der Einbeziehung der "errors and omissions", wahrscheinlich auch wegen einiger anderer Abgrenzungsunterschiede weichen sie erheblich von den neu veröffentlichen Angaben der Bundesbank über die Auslandsforderungen und Auslandsverbindlichkeiten bzw. die Nettoposition des Unternehmenssektors gegenüber dem Ausland ab. Vgl. Deutsche Bundesbank, Entwicklung des Geldvermögens und der Verpflichtungen von 1950 bis 1971 , a. a. O. , S. $37 \mathrm{ff}$.

(3) Natürlich hätte eine im Vergleich zum Ausland abnehmende innere Währungsdisziplin des Staates oder der Notenbank einen Strich durch diese Prognose gemacht. 
Ausland empfangenen in Inlandswährung bewerteten Vermögenserträge auswirkt, wird als zusätzlicher Bestimmungsfaktor die Meßziffer des Wechselkurses (Meßziffer für den Preis der Inlandswährung in ausländischen Währungseinheiten), wt, eingeführt. Da zwischen dem staatlichen Sektor der Modellwirtschaft und dem Ausland keine Vermögenseinkommen fließen, kann der Saldo der zwischen dem Unternehmenssektor und dem Ausland fließenden Vermögenserträge mit dem Saldo der Kapitalertragsbilanz der Modellwirtschaft, $Z_{R}, t$, gleichgesetzt werden. Die Funktion für die Bestimmung dieses Saldos lautet daher:

$$
z_{R, t}=\omega_{1}+\omega_{2} \cdot\left[r_{t-1} \cdot F_{\text {Aus } 1, t-1}\right]+\omega_{3} \cdot w_{t}
$$

Kehrt sich die Nettoforderungsposition des Auslandes gegenüber der Modellwirtschaft in eine Nettoschuldenposition um, was als Folge geld- und währungspolitischer Maßnahmen gelegentlich eintreten kann, sind mit diesem Ansatz einige zusätzliche Ungenauigkeiten verbunden, deren Ausmaß sich durch das Produkt aus der Zinsdifferenz zwischen In- und Ausland und der jeweiligen Nettoschuldenposition des Auslandes abschätzen läßt.

Für die Parameterschätzung wurde die Meßziffer des Wechselkurses, $w_{t}$, so berechnet, daß sie lediglich die von der Bundesrepublik vorgenommenen Änderungen der offiziellen Währungsparität berücksichtigt; es handelt sich jeweils um den Durchschnitt aus zwölf Monatswerten.

Die Regressionsergebnisse (1) lauten $(t=1959, \ldots, 1970)$ :

$$
\text { (10.5) } \begin{array}{ll}
\omega_{1}=+5,92392 & ( \pm 2,19531) \text { Mrd. DM } \\
\omega_{2}=-0,40561 \quad & ( \pm 0,12126) \\
\omega_{3}=-7,30807 \quad( \pm 2,08817) \\
\left.\mathrm{R}_{1.2,3}^{2}=0,72157 \quad \text { (Irrt.-Wahrsch.: }<1 \%\right) \\
\mathbf{r}_{2.3}^{2}=0,00003 \\
\text { vNQ }=1,793
\end{array}
$$

Die Parameter $\omega_{2}$ und $\omega_{3}$ sind statistisch signifikant und haben die zu erwartenden Vorzeichen. Parameter $\omega_{3}$ gibt an, daß infolge der beiden

(1) Die Verwendung des Kapitalmarktzinses der laufenden Periode, $r_{t}$, hätte das Regressionsergebnis für (10.4) ähnlich wie für Gleichung (7.5) besser ausfallen lassen. Aus Gründen der Modellformulierung wurde auf die Berücksichtigung der Verbesserungsmöglichkeit verzichtet. 
DM-Aufwertungen von 1961 und 1968 die vom Ausland empfangenen Einkommen aus Unternehmertätigkeit und Vermögen des privaten Sektors zurückgegangen und die privaten Nettovermögenserträge des Auslandes entsprechend gestiegen sind. - Nach Parameter $\omega_{2}$ besteht ein signifikanter Zusammenhang zwischen den Nettovermögenserträgen des Auslandes und der (seit 1950 aufgelaufenen) privaten Nettoschuldenposition des privaten Sektors der Bundesrepublik dem Ausland gegenüber. Der Parameter ist kleiner als eins und deutet an, daß das Ausland auf sein nach 1950 eingegangenes Nettokreditengagement weniger als die Hälfte des in der Bundesrepublik herrschenden langfristigen Zinssatzes erhielt. Dies stützt die Ansicht, daß es sich bei den seit 1950 von der Bundesrepublik aufgenommenen privaten Nettokapitalzuflüssen vorwiegend um kurzfristige oder Geldmarktanlagen bzw. um typische Portfolioinvestitionen des Auslandes handelt (1). Der niedrige Zinsertrag dieser Anlagen deckt im übrigen nur den geringeren Teil der per Saldo an das Ausland gezahlten Einkommen aus Unternehmertätigkeit und Vermögen. Gemäß (10.5) verbleibt ein während der sechziger Jahre ziemlich stabilei sonstiger Betrag von anderthalb Milliarden DM pro Jahr.

(1) Es ist wahrscheinlich etwas gewagt, derartige Aussagen über kumulierte Salden von Kapitalbewegungen zu treffen. 
1. Vorbemerkung

Im Gegensatz zum internationalen Kapitalverkehr bzw. den durch ihn verursachten internationalen Besitzeinkommensströmen beeinflußt der internationale Warenverkehr (1) unmittelbar die Entstehung der Inlandseinkommen und ihre funktionale Verteilung. Eine kurzfristige Erhöhung des Außenbeitrags verursacht z. B. gesamtwirtschaftlich einen Nachfrageüberschuß und läßt bei dem angenommenen Gestations-Lag der Anlageninvestitionen und bei den begrenzten kurzfristigen Substitutionsmöglichkeiten im Einsatz der Produktionsfaktoren den Anteil der Arbeitseinkommen an der privaten Bruttowertschöpfung kurzfristig sinken (2). Da Änderungen des Saldos im internationalen Waren- und Dienstleistungsverkehr durch die Einkommensentwicklung im In- und Ausland sowie durch die Preisentwicklung im In- und Ausland und auf den Weltmärkten hervorgerufen werden, treten derartige Verteilungseffekte in der Modellwirtschaft bei allen preis- und einkommenswirksamen Maßnahmen des Staates auf und überlagern die bisher betrachteten Effekte; d. h. z. B. , daß die kurzfristigen Überwälzungschancen im Fall einer Gewinnsteuererhöhung wegen der durch die Verausgabung verursachten Nachfrageexpansion und der damit verursachten defizitären Leistungsbilanzeffekte gemindert werden.

Umfang und Richtung der Verteilungseffekte hängen von dem Wert der Einkommenselastizitäten sowie von dem Wert der Preiselastizitäten der Import- und Exportgüternachfrage ab; sie hängen also auch davon ab, ob die Leistungsbilanz der Modellwirtschaft auf Änderungen der nationalen Preisniveaus des In- und Auslandes bzw, auf Änderungen der Weltmarktpreise und Wechselkurse "normal" reagiert oder nicht (3).

Hinsichtlich ihrer Bedeutung wird man vermuten, daß die funktionalen Verteilungseffekte der internationalen Warenverkehrsströme stärker sind als die institutionellen Verteilungseffekte der internationalen Besitzein-

(1) Unter internationalem Warenverkehr wird hier der internationale Austausch von Gütern und Diensten ausschließlich der privaten Kapitaldienste verstanden, deren Gegenwerte, die internationalen Besitzeinkommensströme, bereits im vorigen Paragraphen behandelt wurden.

(2) Vgl. A. Oberhauser, Zahlungsbilanzüberschüsse und Einkommensverteilung, Weltw. Archiv, 93, 1964, S. $286 \mathrm{ff}$.

(3) $\mathrm{Zu}$ den unterschiedlichen Reaktionen der Leistungs- bzw. Warenverkehrsbilanz vgl. K. Rose, Theorie der Außenwirtschaft, 2. Aufl., Berlin u. Frankfurt 1966, S. $64 \mathrm{ff}$. 
kommensströme, denn die Änderungen im Saldo der Warenverkehrsbilanz hatten in der Bundesrepublik im allgemeinen einen wesentlich größeren Umfang als die Änderungen im Saldo der Kapitalertragsbilanz. Dies gilt in erster Linie aber kurzfristig. Nach den Überlegungen des Paragraphen 4 ist damit zu rechnen, daß die vom internationalen Waren- und Dienstleistungsverkehr ausgehenden nachfragebedingten Verteilungseffekte wie alle sonstigen nachfragebedingten Verteilungseffekte durch den Anpassungsproze $\beta$ der privaten Produktion abgebaut werden und daß sich dieser Abbau in der funktionalen Verteilung zwischen Arbeits- und Besitzeinkommen verhältnismäßig rasch vollzieht.

\section{Der Warenimport}

Im Gegensatz zur Behandlung des internationalen Kapitalverkehrs und der durch ihn ausgelösten internationalen Besitzeinkommensströme geht die Behandlung des internationalen Warenverkehrs von einer Bruttobetrachtung der Warenverkehrsströme aus und nicht von den Salden dieser Ströme. Der internationale Warenverkehr der Modellwirtschaft wird also zerlegt in den Warenimport und den Warenexport; dabei werden die Werte beider Warenverkehrsströme noch einmal aufgespalten in ihre Mengenund ihre Preiskomponente. Für jede dieser vier Komponenten des internationalen Warenverkehrs wird eine eigene Verhaltensfunktion formuliert.

Die Mengenkomponente des Warenimports, der reale Import, $x_{I m}, t$, wird abhängig gemacht von dem Verhältnis, das zwischen dem um die durchschnittliche Importzollbelastung, $t_{I m, t}$, erhöhten Preisniveau für Importe, $p_{I m} t$, und dem inländischen Preisniveau, $p_{x, t}$, besteht (1). Da sich Wechselkursänderungen nicht voll in dem in heimischer Währung berechneten Preisniveau für Importe auswirken und teilweise zugunsten oder zulasten der ausländischen Anbieter gehen, wird als zusätzlicher Erklärungsfaktor die Meßziffer des Wechselkurses (Preis der Inlandswährung in ausländischen Währungseinheiten), $w_{t}$, berücksichtigt. Beide Preisargumente werden mit einer Verzögerung von einem Jahr angesetzt.

Die Importfunktion wird formuliert als

(11.1)

$$
x_{I m, t}=\mu_{1}^{*} \cdot x_{t}^{\mu_{2}} \cdot\left[\frac{p_{I m, t-1}}{p_{x, t-1}} \cdot\left(1+t_{I m, t-1}\right)\right]^{\mu_{3}} \cdot w_{t-1}^{\mu_{4}}
$$

(1) Vgl. dazu W. Krelle, D. Beckerhoff, H. G. Langer und H. Fuß, Ein Prognosesystem für die wirtschaftliche Entwicklung der Bundesrepublik Deutschland, a. a. O. , S. 277 ff. sowie H. König und V. Timmermann, Ein ökonometrisches Modell für die Bundesrepublik Deutschland 1950-1960, a. a. O., S. $615 \mathrm{f}$. 
Zur Vermeidung von Multikollinearität werden in (11.1) die absoluten Werte der Variablen durch Meßziffernwerte ersetzt:

$$
\frac{x_{I m, t}}{x_{I m, t-1}}=\mu_{1} \cdot\left[\frac{x_{t}}{x_{t-1}}\right]^{\mu_{2}} \cdot\left[\frac{\frac{p_{I m, t-1}}{p_{x, t-1}}\left(1+t t_{I m, t-1}\right)}{\frac{p_{I m, t-2}}{p_{x, t-2}}\left(1+t{ }_{I m, t-2}\right)}\right]^{\mu_{3}} \cdot\left[\frac{w_{t-1}}{w_{t-2}}\right]^{\mu_{4}}
$$

In dieser Form wurde die Warenimportfunktion nach einer logarithmischen Transformation für die Jahre 1958 bis 1970 geschätzt. Für die Schätzung wurden die Importwerte der volkswirtschaftlichen Gesamtrechnung der Bundesrepublik ohne die an das Ausland geleisteten Einkommen aus Unternehmertätigkeit und Vermögen verwendet; diese Werte wurden mit Hilfe des Preisindex für Importe der volkswirtschaftlichen Gesamtrechnung (1) deflationiert. Der Satz der durchschnittlichen Zollbelastung der Importe, $t_{I m}, t$, wurde berechnet als Quotient aus dem gesamten Zollaufkommen in der Bundesrepublik (2), $T_{\operatorname{Im}}, t$, und dem jeweiligen Importwert, $x_{I m}, t^{\bullet} p_{I m}, t \cdot$ Die Meßziffer des Wechselkurses, $\mathbf{w}_{t}^{\star}$, berücksichtigte im Gegensatz zum vorigen Paragraphen die umsatzsteuerliche Quasiaufwertung der DM von 1968, wurde aber ebenso als ein Durchschnitt aus zwölf Monatswerten gebildet.

Die Regressionsergebnisse lauten:

$$
\text { (11.3) } \begin{aligned}
\text { Ig } \mu_{1} & =-0,00499 \quad( \pm 0,00553) \\
\mu_{2} & = \pm 1,90151 \quad( \pm 0,22328) \\
\mu_{3} & =-0,33686 \quad( \pm 0,23945) \\
\mu_{4} & = \pm 0,85092 \quad( \pm 0,37149) \\
R_{1.2,3,4}^{2} & =0,91536 \quad \text { (Irrt.-Wahrsch.: } 0,18) \\
R_{2 \cdot 3,4}^{2} & =0,13987 \\
R_{3 \cdot 2,4}^{2} & =0,13162 \\
R_{4 \cdot 2,3}^{2} & =0,02226 \\
N N Q & =2,676
\end{aligned}
$$

(1) Der Index, berechnet nach den Angaben des Sachverständigenrates, bezieht sich auf die Gesamtheit der Importerlöse einschließlich der an das Ausland geleisteten Einkommen aus Unternehmertätigkeit und Vermögen. Vgl. die Jahresgutachten des Sachverständigenrates zur Begutachtung der gesamtwirtschaftlichen Entwicklung.

(2) Das Aufkommen an Exportzöllen ist vernachlässigbar gering. 
Der Schätzwert für die Einkommenselastizität der Importe, $\mu_{2}$, entspricht in etwa den Ergebnissen vorausgegangener Untersuchungen. Der Wert der Preiselastizität, $\mu_{3}$, der hier bei einer Irrtumswahrscheinlichkeit von $20 \%$ ermittelt wurde, ist dagegen vergleichsweise gering ausgefallen (1) (2). Schließlich deutet der hohe und signifikante Wert von $\mu_{4}$ Angebotsreaktionen der ausländischen Exporteure an. Wie man den folgenden Ergebnissen entnehmen kann, haben sich die Aufwertungen der DM von 1961 und 1968/69 nicht voll auf die in Inlandswährung berechneten Importpreise ausgewirkt; ein Teil des Aufwertungsgewinnes aus dem deutschen Importgeschäft ist den ausländischen Exporteuren in Form erhöhter Weltmarktpreise zugefallen und hat deren Angebotsreaktionen ausgelöst.

Die Entwicklung der Importgüterpreise wird in der Modellformulierung abhängig gemacht von der nationalen Preisentwicklung in den Partnerländern des internationalen Handels, die selbst exogen vorgegeben wird, und von der Meßziffer des Wechselkurses. Zur Formulierung der Preisfunktion wird der Index, $p_{I m}, t$, der in inländischen Währungseinheiten berechneten Importgüterpreise (3) mit der Meßziffer des Wechselkurses, w * multipliziert und ergibt einen Index für den Weltmarktpreis der Importgüter, (pIm, $\left.t^{*} w^{*}\right)$. Die Importpreisfunktion lautet dann (4):

$(11.4)$

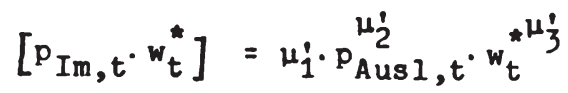

Für die Schätzung von (11.4) wurde das nominelle Bruttosozialprodukt zu Marktpreisen der 19 wichtigsten Welthandelspartner der Bundesre-

(1) Vgl. den kurzen Überblick bei W. Krelle, D. Beckerhoff, H. G. Langer und H. Fuß, Ein Prognosesystem..., a. a. O. , S. $271 \mathrm{f}$. Die von den Autoren selbst angestellten Schätzungen dürften nicht ganz zuverlässig sein; vgl. ebenda, S. $279 \mathrm{ff}$.

(2) Bei Vernachlässigung des Einflusses, der von den Änderungen des Wechselkurses ausging, bzw. unter der Annahme $\mu_{4}=0$ wäre der Schätzwert von $\mu_{3}$ absolut genommen noch geringer ausgefallen.

(3) In der volkswirtschaftlichen Gesamtrechnung werden die Importgüterpreise ab Grenze des exportierenden Landes und damit auch vor Zuschlag inländischer Importzölle ermittelt. Vgl. die Vorbemerkungen des Statist. Jahrbuchs f. d. BRD zu den Kapiteln "Außenhandel" und "Volkswirtschaftliche Gesamtrechnungen".

(4) Vgl. W. Krelle, D. Beckerhoff, H. G. Langer und H. Fuß, Ein Prognosesystem ..., a. a. O. , S. $300 \mathrm{f}$. 
publik (1) mit den offiziellen Währungsparitäten von Februar 1963 (2) in DM-Werte umgerechnet und aufaddiert. In gleicher Weise wurde mit dem realen Bruttosozialprodukt zu Marktpreisen (3) der Partnerländer verfahren, dessen aggregierter DM-Wert in der Modellformulierung das Realeinkommen des Auslandes, xAusl, t, repräsentiert. Als Quotient aus beiden Größen erhält man den Preisindex des aggregierten Bruttosozialprodukts der Partnerländer, der hier mit pAusl, $t$ bezeichnet wird und in (11.4) als Argument dient. Als Index der in inländischen Währungseinheiten ermittelten Importgüterpreise wurde der bereits genannte Preisindex für Importe der volkswirtschaftlichen Gesamtrechnung verwendet.

Die Regressionsschätzung lieferte für die Jahre 1958 bis 1970 das folgende Ergebnis:

$$
\text { (11.5) } \begin{aligned}
\text { Ig } \mu_{1}^{\prime} & =+0,01849 \quad( \pm 0,00446) \\
\mu_{2}^{\prime} & =+0,28208 \quad( \pm 0,09124) \\
\mu_{3}^{\prime} & =+0,48004 \quad( \pm 0,27079) \\
R_{1 \cdot 2,3}^{2} & =0,91069 \quad(\text { Irrt. -Wahrsch.: }<0,18) \\
r_{2}^{2} 3 & =0,78057 \\
\text { vNQ } & =1,525
\end{aligned}
$$

Nach (11.5) läßt sich für die von der Bundesrepublik vorgenommenen Wechselkurskorrekturen einschließlich der umsatzsteuerlichen QuasiAufwertung der DM von 1968 bei einer Irrtumswahrscheinlichkeit von $10 \%$ ein Einfluß auf die Weltmarktpreise der deutschen Importe behaupten. Dies deutet zusammen mit dem in (11.3) nachgewiesenen Importmengeneffekt der deutschen Aufwertungsmaßnahmen darauf hin, daß das Güter-

(1) Es handelt sich um die Länder Frankreich, Niederlande, USA, Italien, Belgien, Schweiz, Österreich, Schweden, Großbritannien, Dänemark, Japan, Südafrika, Norwegen, Finnland, Griechenland, Kanada,(Iran), Australien und Portugal. Die Reihe wurde von 1958 bis 1960 ohne und von 1960 bis 1970 mit dem Iran berechnet und 1960 verkettet. Die Angaben entstammen der internationalen Übersicht des Statist. Jahrbuchs f. d. BRD.

(2) Vgl. Monatsberichte der Deutschen Bundesbank, März 1963, S. 97 (für die Schweiz ebenda, S. 95)

(3) Der Berechnung lagen zwischen 1958 und 1960 überwiegend Werte mit Preisbasis 1958, zwischen 1960 und 1970 überwiegend Werte mit Preisbasis 1963 zugrunde. Die Reihen wurden verkettet. 
angebot des Auslandes an die Bundesrepublik keineswegs völlig preiselastisch war, und läßt vermuten, daß Änderungen der deutschen Importnachfrage grundsätzlich einen Einfluß auf den Weltmarktpreis der importierten Güter hatten. Ein Versuch, diesen Einfluß abseits der Wechselkurskorrekturen empirisch nachzuweisen, verlief nicht erfolgreich. Die Verwendung der Importpreisfunktion (11.4) mit dem Regressionsergebnis (11.5) stellt für die Modellformulierung daher eine notdürftige Kompromißlösung dar.

\section{Der Warenexport}

Die Warenexporte der Modellwirtschaft sind die Warenimporte ihres Auslandes. Die Funktionen zur Bestimmung des Warenexportwertes werden daher in voller Symmetrie zu den Funktionen zur Bestimmung des Warenimportwertes formuliert. Dazu werden die Warenexportwerte der Modellwirtschaft als erstes in ihrer Preis- und Mengenkomponente zerlegt. Die Preiskomponente, der Index, $\mathrm{pEx}, t$, der in inländischen Währungseinheiten berechneten Exportgüterpreise, wird mit der Meßziffer des Wechselkurses, $w_{t}^{*}$, multipliziert und ergibt einen Index für den Weltmarktpreis der Exportgüter, $\left(p_{E x}, t^{\bullet} w_{t}^{*}\right)$.

Dabei berücksichtigt die Meßziffer des Wechselkurses $w_{t}^{*}$, wiederum die umsatzsteuerliche Quasi-Aufwertung der DM von 1968. Die Exportwerte der Bundesrepublik (ohne die vom Ausland empfangenen Einkommen aus Unternehmertätigkeit und Vermögen) werden dann mit dem Preisindex für Exporte (einschließlich der vom Ausland empfangenen Einkommen aus Unternehmertätigkeit und Vermögen) der volkswirtschaftlichen Gesamtrechnung deflationiert und ergeben so die zugrunde gelegten realen Exportmengen, $x_{E x, t}$.

Die realen Exporte der Modellwirtschaft werden abhängig gemacht vom realen Bruttosozialprodukt des Auslandes, $x_{\text {Ausl, }}$, von der Relation, die in der Vorperiode zwischen dem Weltmarktpreis der Exporte und dem Bruttosozialproduktspreisindex des Auslandes bestand, $\left(\mathrm{p}_{\mathrm{Ex}}, \mathrm{t}-1^{\bullet} \mathrm{w}_{\mathrm{t}-1}^{\star}\right) /$ $p_{A u s l, t-1}$, sowie von der Meßziffer des Wechselkurses in der Vorperiode, $\mathrm{w}_{t-1}^{\star}$. Analog zur Warenimportfunktion (11.1) lautet die Warenexportfunktion:

$$
x_{\text {Ex }, t}=x_{1}^{x_{2}} \cdot x_{\text {Aus 1 }, t} \cdot\left[\frac{p_{E x, t-1} \cdot w_{t-1}^{*}}{p_{\text {Ausl }, t-1}}\right]^{x_{3}} \cdot w_{t-1}^{x_{4}}
$$

Aus dem im vorigen Abschnitt genannten Grund werden die absoluten Werte der Variablen auch hier durch Meßziffernwerte ersetzt: 


$$
\frac{x_{E x, t}}{x_{E x, t-1}}=x_{1} \cdot\left[\frac{x_{\text {Aus 1,t }}}{x_{\text {Aus 1 }, t-1}}\right]^{x_{2}} \cdot\left[\frac{\left(\frac{p_{E x, t-1} w_{t-1}^{*}}{p_{\text {Aus 1,t-1 }}}\right)}{\left(\frac{p_{E x, t-2} w_{t-2}}{p_{\text {Aus 1 }, t-2}}\right)}\right] \cdot\left[\frac{x_{t-1}}{w_{t-2}^{*}}\right]^{x_{4}}
$$

Nach logarithmischer Transformation lieferte die Kleinst-Quadrat-Schätzung für (11.7) mit den für die Bundesrepublik zusammengestellten Daten als Regressionsergebnis $(t=1958, \ldots, 1970)$ :

$$
\begin{aligned}
& \text { (11.8) } \quad \text { ig } x_{1}=+0,00375 \quad( \pm 0,00796) \\
& x_{2}=+1,12223( \pm 0,32987) \\
& x_{3}=-1,41630 \quad( \pm 0,32526) \\
& x_{4}=+0,74965 \quad( \pm 0,53305) \\
& R_{1 \cdot 2,3,4}^{2}=0,78695 \text { (Irrt.-Wahrsch.: }\langle 1 \% \text { ) } \\
& \mathrm{R}_{2 \cdot 3,4}^{2}=0,03790 \\
& \mathrm{R}_{3 \cdot 2,4}^{2}=0,49168 \\
& R_{4 \cdot 2,3}^{2}=0,49660 \\
& \text { VNQ } \quad=2,708
\end{aligned}
$$

Die Schätzwerte für die (Auslands-)Einkommenselastizität, $x_{2}$, und die Preiselastizität, $x_{3}$, der deutschen Exporte stimmen weitgehend mit den Schätzergebnissen einer anderen Untersuchung überein (1). Der bei einer Irrtumswahrscheinlichkeit von $20 \%$ ermittelte Schätzwert von $\mathrm{X}_{4}$ bringt Angebotsreaktionen der inländischen Exporteure zum Ausdruck, die versuchten, die durch die DM-Aufwertungen bedingten Verteuerungen im Exportangebot und die dadurch verursachten Exportrückgänge durch verstärkte Verkaufsanstrengungen nicht-preispolitischer Art wieder wettzumachen.

Die Exportpreisfunktion wird wie die Importpreisfunktion für die Weltmarktpreise der von der Modellwirtschaft exportierten Güter aufgestellt. Sie lautet analog zu (11.4):

$$
\left[p_{E x, t} \cdot w_{t}^{*}\right]=x_{1}^{\prime} \cdot p_{x, t}^{x_{2}^{\prime}} \cdot w_{t}^{* x_{j}^{\prime}}
$$

(1) Vgl. W. Krelle, D. Beckerhoff, H. G. Langer und H. Fuß, Ein Prognosemodell..., a. a. O. , S. $280 \mathrm{ff}$. 
macht also die Weltmarktpreise der von der Modellwirtschaft in der übrigen Welt abgesetzten Exportgüter abhängig vom inländischen Preisniveau der Modellwirtschaft und von dem Wechselkurs, den ihre staatlichen Institutionen festgelegt haben.

Die Schätzung, der der bereits genannte Preisindex für Exporte der volkswirtschaftlichen Gesamtrechnung und der Preisindex des privaten Bruttoinlandsprodukts der Bundesrepublik zugrunde lag, brachte als Ergebnis $(\mathrm{t}=1958, \ldots, 1970)$ :

$$
\begin{aligned}
& \text { (11.10) } 18 x_{i}^{\prime}=+0,00130 \quad( \pm 0,00207) \\
& x_{2}^{\prime}=+0,32225 \quad( \pm 0,04313) \\
& x_{3}^{\frac{1}{3}}=+0,97884 \quad( \pm 0,12772) \\
& R_{1 \cdot 2,3}^{2}=0,99067 \text { (Irrt.-Wahrsch.: }<0,17 \text { ) } \\
& \mathbf{r}_{2 \cdot 3}^{2}=0,79602 \\
& v N Q=1,989
\end{aligned}
$$

Das Ergebnis zeigt, daß die Exporteure der Bundesrepublik bei den Aufwertungen der DM im allgemeinen zu keinerlei Preiszugeständnissen bereit waren, sondern die ausländischen Importeure in vollem Umfang die Last der DM-Aufwertungen tragen ließen. Dem daraufhin einsetzenden Rückgang der Auslandsnachfrage nach deutschen Exportgütern begegneten sie, wie (11.8) zeigt, mit verstärkten Verkaufsanstrengungen nicht-preispolitischer Art. Doch konnten sie den Exportrückgang damit nur zum Teil wieder wettmachen.

Vergleicht man die für die Exportentwicklung geschätzten Parameterwerte mit den entsprechenden Parameterwerten für den Import, so fällt auf, daß die Einkommenselastizität der mengenmäßigen Importe, $\mu_{2}$, erheblich größer ist als die entsprechende Einkommenselastizität der mengenmäßigen Exporte, $x_{2}$, daß dafür aber die Preiselastizität der Exporte, $x_{3}$, absolut genommen wesentlich größer als die entsprechende Preiselastizität der Importe, $\mu_{3}$, ist. Diese Struktur der Elastizitäten muß im Zusammenhang mit der Tatsache gesehen werden, daß die Weltmarktpreise für deutsche Importe und Exporte während der sechziger Jahre wesentlich langsamer gestiegen sind als die heimischen Preisniveaus des In- und Auslandes und ist wahrscheinlich typisch. Nur aufgrund des im Vergleich zum Bruttosozialproduktspreisindex des Auslandes geringen Anstiegs der Exportgüterpreise und der hohen Preiselastizität der ausländischen Exportnachfrage gelang es der Bundesrepublik, ihre Exporte ebenso stark bzw. bis zu den Wechselkurskorrekturen von 1961 und 1968/69 sogar stärker ansteigen zu lassen als die wegen einer hohen Einkommenselastizität 
überproportional zum Inlandseinkommen steigenden Importe.

Die Struktur der Parameter, die für das Mengen- und Preisverhalten im internationalen Warenverkehr geschätzt wurden, impliziert im übrigen, daß kurzfristig, d. h. innerhalb des laufenden Jahres, mit "anomalen" Reaktionen der Warenverkehrsbilanz zu rechnen ist, weil die Importund Exportmengen nur verzögert auf Preisänderungen reagieren. Wirken sich die Preisänderungen im ersten Folgejahr auf die mengenmäßige Entwicklung der grenzüberschreitenden Warenverkehrsströme aus, stellen sich "normale" Leistungsbilanzreaktionen ein. Sieht man von den kurzfristigen Wirkungen einmal ab, kann man daher für die Modellwirtschaft davon ausgehen, daß eine im Vergleich zum Ausland starke Inflationierung im Inneren der Modellwirtschaft einen defizitären Einfluß auf die Ware nverkehrsbilanz ausübt und die funktionale Einkommensverteilung zwischen Arbeits - und Besitzeinkommen kurzfristig zugunsten der ersteren ändert. Das gleiche gilt für eine internationale Aufwertung der Inlandswährung.

4. Die Bestimmung der Wechselkurse und der Preise und Einkommen des Auslandes

Die drei Bestimmungsfaktoren des Außenwirtschaftsverkehr, deren Verhalten in der Modellwirtschaft bisher nicht bestimmt wurde, sind der Wechselkurs der Inlandswährung gegenüber der Auslandswährung, $w_{t}$ bzw, $w_{t}^{*}$, und die Preise und Realeinkommen des Auslandes, pAusl, $t$ bzw. xAusl, t. Der Verlauf dieser Größen wird für die ersten elf Jahre der Modellrechnung durch die für die vorausgegangenen Schätzungen empirisch ermittelten Werte der Jahre 1960 bis 1973 bzw. 75 exogen vorgegeben, um eine größere Annäherung an die während dieser Jahre in der Bundesrepublik herrschenden Verhältnisse zu gewinnen. Für die Jahre danach wird ihr Verhalten durch Funktionen bestimmt. Dabei wird der Wechselkurs nun als eine endogene Variable des Modellmechanismus angesehen, während die Preis - und Einkommensentwicklung des Auslandes durch Trendfunktionen allein vom Zeitablauf abhängig gemacht, im Prinzip also weiterhin exogen vorgegeben wird.

Die Wechselkurspolitik der staatlichen Instanzen, soweit sie nicht exogen vorgegeben wird, orientiert sich in der Modellwirtschaft allein am Saldo der internationalen Warenverkehrsbilanz, $Z_{\mathbf{X}}, t$. Dieser Saldo wird auf die Größe des privaten Bruttoinlandsprodukts bezogen und ergibt die Quote $Z_{\mathbf{X}, t} /\left(\mathbf{x t}^{\circ} \mathbf{p}_{\mathbf{x}}, \mathrm{t}\right)$. Fällt der kumulierte Wert der Quote in den letzten drei vergangenen Jahren außerhalb des Toleranzbereichs von $\pm 0,1$, wird eine Wechselkurskorrektur vorgenommen (1). Die Korrektur erfolgt

(1) Zur Veranschaulichung: Der kumulierte Wert der Quote betrug in den drei Jahren vor der DM-Aufwertung von 1961 (1958-1960) + 0,107 und 
jeweils zu Beginn eines Jahres und beträgt je nach der Richtung, in der der kumulierte Saldo der Warenverkehrsbilanz weist, stets plus oder minus fünf Prozent des bis dahin herrschenden Kursniveaus.

Wegen der Verzögerung in der Preisabhängigkeit der mengenmäßigen Exporte und Importe erzielt die zu Beginn einer Periode $t$ vollzogene Änderung des Wechselkurses ihre volle Wirkung erst während der Folgeperiode $t+1$. Um sicherzustellen, daß der während der laufenden Periode $t$ noch nicht abgebaute Saldo der Warenverkehrsbilanz nicht zu Beginn der Folgeperiode $t+1$ eine weitere Wechselkursänderung induziert, wird in der Modellformulierung eine $z$ weite Bedingung eingeführt. Sie besagt, daß eine Wechselkurskorrektur unterbleibt, wenn eine solche Korrektur bereits zu Beginn des Vorjahres vorgenommen wurde.

Das Verhalten der staatlichen Wechselkurspolitik ist in der Modellformulierung also bestimmt durch

$$
\frac{w_{t}}{w_{t-1}}=1,00 \begin{cases}\text { wenn } & \sum_{\tau=t-3}^{t-1}\left|\frac{z_{x, \tau}}{x_{\tau} \cdot p_{x, \tau}}\right| \leqslant+0,1 \\ \text { oder } & w_{t-1} \neq 1,00\end{cases}
$$

sowie

(11.12)

$$
\frac{w_{t}}{w_{t-1}}=\left\{\begin{array}{l}
1,05 \begin{cases}\text { wenn } & \sum_{\tau=t-3}^{t-1} \frac{z_{x, \tau}}{x_{\tau} \cdot p_{x, \tau}}>+0,1 \\
\text { und } & \frac{w_{t-1}}{w_{t-2}}=1,00\end{cases} \\
0,95 \begin{cases}\text { wenn } & \sum_{\tau=t-3}^{t-1} \frac{z_{x, \tau}}{x_{\tau} \cdot p_{x, \tau}}<-0,1 \\
\text { und } \quad \frac{w_{t-1}}{w_{t-2}}=1,00\end{cases}
\end{array}\right.
$$

in den drei Jahren vor der DM-Aufwertung von $1969(1966-1968)+0,099$. Die Angaben beziehen sich auf die für die Zwecke des Modells leicht geänderten Abgrenzungen der volkswirtschaftlichen Gesamtrechnung. 
Wechselkurskorrekturen, die von Seiten des Auslandes vorgenommen werden, bleiben in der Modellformulierung unberücksichtigt.

Die letzten Werte für das reale und nominelle Bruttosozialprodukt der neunzehn wichtigsten Handelspartnerländer der Bundesrepublik wurden für das Jahr 1973 ermittelt. Die Werte wurden mit den vom Deutschen Institut für Wirtschaftsforschung (1) geschätzten Wachstumsraten für die Jahre 1974 und 1975 fortgeschrieben. Die reale Einkommensentwicklung des Auslandes in den Jahren nach 1975 wird durch die Trendgleichung

$$
\text { (11.13) } \quad x_{\text {Aus 1, } t}=x_{1}^{\prime \prime} \cdot x_{2}^{\prime \prime} t-1976
$$

bestimmt, wobei $x_{1}^{\prime \prime}$ den gegenüber dem Jahr 1975 um $5 \%$ erhöhten Wert des realen Auslandseinkommens für das Jahr 1976 angibt und $\chi_{2}^{\prime \prime}$ den für die Folgezeit angenommenen Wachstumsfaktor.

\section{(11.14) $\quad x_{1}^{\prime \prime}=7.193,865$ Mrd. DM real $x_{2}^{\prime \prime}=1,043$}

Dem frei Hand bestimmten Prognosewert von $x_{2}^{\prime \prime}$ entspricht eine jährliche Wachstumsrate des realen Bruttosozialprodukts von 4,3\% in den Partnerländern des internationalen Handels.

Die voraussichtliche Entwicklung des Bruttosozialproduktspreisindex für die Außenhandelspartner der Bundesrepublik läßt sich kaum zuverlässig prognostizieren. Nachdem die jährlichen Preissteigerungsraten in den neunzehn wichtigsten Partnerländern der Bundesrepublik zuvor etwa 2, $5 \%$ betragen hatten, erhöhten sie sich von der zweiten Hälfte der sechziger Jahre an ständig und begannen erst Mitte der siebziger Jahre, wieder zu sinken; diese jüngste Entwicklung läßt sich nicht einfach extrapolieren. Für die Modellformulierung wird von der optimistischen Annahme ausgegangen, daß sich das Inflationstempo weiter verlangsamen wird.

Der letzte vom DIW geschätzte Wert für den Anstieg des Bruttosozialproduktspreisindex der Partnerländer gegenüber dem Vorjahr betrug 11,2\% (1975). Der Wert für 1976 wird noch einmal mit einer vermuteten Rate von 8,5\% angesetzt. Die anschließende Entwicklung des Index wird durch gleichmäßig auf ein Dauerniveau von 3,5\% absinkende Anstiegsraten festgelegt.

Die Trendgleichung für den Bruttosozialproduktspreisindex des Auslandes $(1963 \approx 1,000)$ lautet dementsprechend für die Jahre nach 1975:

$$
\text { (11.15) } \text { PAus I, }_{\text {A }}=x_{1}^{\prime \prime \prime} \cdot x_{2}^{\prime \prime \prime} t-1972 \cdot x_{3}^{\prime \prime \prime} \frac{1}{t-1972}
$$

(1) Vgl. Deutsches Institut für Wirtschaftsforschung, Grundlinien der Wirtschaftsentwicklung 1976, DIW-Wochenbericht $3 / 4$ vom 26.1.1976, S. 16 . 
wobei die zugehörigen Parameterwerte frei Hand bestimmt wurden:

$$
\text { (11.16) } \quad \begin{aligned}
x_{1}^{n_{1}} & =2,08299 \\
x_{2}^{m_{1}} & =1,03500 \\
x_{3}^{n_{1}} & =0,56771
\end{aligned}
$$




\section{Kapitel}

DIE VERHALTENSINSTRUMENTE DES STAATES

$\$ 12$ Die indirekten Steuern

\section{Die Verbrauchsteuer}

Nachdem das Verhalten des privaten Sektors und des Auslandes in der Modellformulierung bestimmt ist, müssen als letztes die Funktionen für das Verhalten des Staates aufgestellt werden. Begonnen wird mit den Verhaltensfunktionen, die sich auf die staatliche Mittelaufbringung bzw. auf die Finanzierung des Staatsbudgets beziehen, und hierbei entsprechend der Systematik der volkswirtschaftlichen Gesamtrechnung mit den indirekten Steuern.

Unter den indirekten Steuern der Modellwirtschaft hat die Verbrauchsteuer die aufkommens-bzw. belastungsmäßig größte Bedeutung. Sie wird in Form einer allgemeinen Nettoumsatzsteuer mit Vorabzug der Investitionen anstelle der Abschreibungen für Kapitalverzehr erhoben. Sie erfaßt in der aggregierten Betrachtung des Modells also alle Verkäufe des Unternehmenssektors, die zur Deckung des privaten Inlandskonsums und zur Deckung der gesamten staatlichen Güternachfrage dienen, gleich ob die verkauften Güter aus inländischer Produktion oder aus Importen stammen. Der Tarif der Verbrauchsteuer ist proportional ausgestaltet. Verbrauchsteuerliche Freibeträge werden den Unternehmern als Steuerschuldnern vom Staat nicht gewährt. Die Steuerschuld wird jeweils zum Monatsende fällig, ihr kassenmäßiges Aufkommen fällt im Staatshaushalt also praktisch unverzögert an. Die gesamtwirtschaftliche Aufkommensfunktion der Verbrauchsteuer lautet:

$$
T_{C, t}=t_{C 1}+t_{C 2} \cdot\left[C_{A, t}+C_{S, t}+A_{S t, t}{ }^{-L_{S t}, t}\right]
$$

wobei Parameter ${ }^{t} \mathrm{C} 1$ wegen des Fehlens allgemeiner Freibetragsregelungen a priori gleich null zu setzen ist und wobei $C_{A, t}$ bzw. $C_{S, t}$ die Konsumausgaben der Unselbständigen- bzw. Selbständigen-Haushalte, A St, t die Ausgaben des Staates für Käufe von Gütern und Diensten und $L_{S t, t}$ die öffentlichen Personalausgaben bezeichnen.

Zur Quantifizierung der Aufkommensfunktion (12.1) wurde das "Verbrauchsteuer"-Aufkommen als der Gesamtbetrag der indirekten Steuern (abzüglich indirekter Subventionen) entsprechend der volkswirtschaftlichen Gesamtrechnung minus dem Aufkommen der Gewerbesteuern, der Grund- 
steuer und der Zölle in der Bundesrepublik abgrenzt (1). Der Posten enthält infolge der Umsatzsteuer-Reform von 1968 eine Reihe Unregelmäßigkeiten, die nicht erfaßt werden konnten (2) und die wegen des Gewichts der Beträge in den Jahren 1967 bis 1970 erhebliche Abweichungen der modellmäßig beschriebenen Budgetentwicklung von der tatsächlichen Entwicklung des öffentlichen Haushalts in der Bundesrepublik verursachen.

Das Regressionsergebnis zu (12.1) für die Periode 1958 bis 1971 lautet:

$$
\begin{aligned}
& t_{C 1}=+0,36330( \pm 1,06136) \text { Mrd. DM } \\
& t_{C 2}=+0,13976( \pm 0,00327) \\
& \left.r_{1 \cdot 2}^{2}=0,99346 \text { (Irrt.-Wahrsch.: }<0,1 \%\right) \\
& \text { vNQ }=3,126
\end{aligned}
$$

Der Schätzwert von Parameter $t_{C 1}$ veranschaulicht mit einem nicht signifikant von null unterscheidbaren Wert von 0,363 Mrd. DM das Fehlen quantitativ erheblicher Freibetragsregelungen. Entsprechend errechnet sich im Anschluß an (12.1) und (12.2) für den Bereich der zeitlichen Variablen-Durchschnitte eine Aufkommenselastizität von 0,992 (3).

(1) In der Modellformulierung wird nicht zwischen der aggregierten Steuerschuld und den aggregierten Steuereinnahmen der verschiedenen Steuerarten unterschieden. Die Berechnung der Aufkommensfunktion stellt stets auf die kassenmäßigen Steuereinnahmen ab. Zum Aufkommen der einzelnen Steuern vgl. die Statistischen Jahrbücher für die Bundesrepublik Deutschland sowie die Finanzberichte des Bundesministeriums der Finanzen.

(2) Abgesehen von den Übergangswirkungen sind die Auswirkungen der Umsatzsteuer-Reform 1968 vor allem struktureller Natur und daher durch die Modellformulierung nicht zu beschreiben.

(3) Das Elastizitätsmaß ergibt sich durch Einsetzen des geschätzten Parameterwertes von $t_{\mathrm{C}}$ und der Variablendurchschnitte in die durch Differentiation von (12.1) abgeleitete Formel:

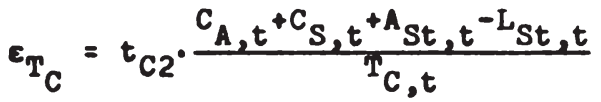

Die hier und im folgenden berechneten Aufkommenselastizitäten beziehen prozentuale Aufkommensänderungen auf prozentuale Änderungen der aggregierten Besteuerungsmenge. Sie bringen damit lediglich die aggregierten Auswirkungen der jeweiligen Steuertarife zum Ausdruck, vernachlässigen aber den Einfluß der allgemeinen Einkommensentwicklung auf die Größe der aggregierten Besteuerungsmenge, der bei der üblichen Definition der Aufkommenselastizitäten mit berücksichtigt wird. Vgl. 
Neben der allgemeinen Verbrauchsteuer in Form einer Nettoumsatzsteuer erhebt der Staat in der Modellwirtschaft eine spezielle Wertschöpfungsteuer, die sog. Gewerbeertragsteuer. Die Gewerbeertragsteuer erfaßt das im privaten Sektor entstandene Besitzeinkommen, unabhängig davon, welchen Gruppen der Modellwirtschaft es zufließt. Zur Vermeidung steuerlicher Kumulierungseffekte belastet sie das zu Marktpreisen bewertete Bruttobesitzeinkommen nach Bereinigung um Abschreibungen, Verbrauchsteuerlast und Zölle, das in dieser Abgrenzung weiterhin als "Gewerbeertrag" bezeichnet wird. Der Tarif der Steuer ist linear; es werden persönlich bemessene Freibeträge gewährt, die jedoch nicht für alle selbständigen Erwerbspersonen die gleiche Höhe haben (1). Da die Ermittlung

G. Hagemann, Aufkommenselastizitäten ausgewählter Steuern in der Bundesrepublik Deutschland 1958 - 1963, Tübingen 1968, S. 3 ff.

(1) Die modellmäßige Charakterisierung der deutschen Gewerbeertragsteuer stützt sich auf die Vorschriften des in der Bundesrepublik geltenden Gewerbesteuergesetzes (Anknüpfung an der einkommensteuerlichen Gewinnermittlung, anschließende Ergänzung um Fremdkapitalzinsen gemäß $₫ 7$ bis 9 Gewerbesteuergesetz), insbesondere auf die Gewährung persönlicher Freibeträge sowie ermäßigter Steuersätze in einer progressiven Eingangsstufe des Gewerbesteuertarifs ( $\$ 11$ Gewerbesteuergesetz) sowie auf den Sachverhalt, daß die Einkommen aus selbständiger landwirtschaftlicher und freiberuflicher Tätigkeit von der Gewerbesteuer pflicht freigestellt sind ( $\$ 1$ Ziffer 1 Gewerbesteuer-Durchführungsverordnung). Dabei wird davon ausgegangen, daß die gewährten Freibeträge und Tarifermäßigungen den Sinn haben, einen rechnerischen Unternehmerlohn von der Gewerbeertragsteuer freizuhalten, und insoweit im. Modell durch den Abzug des rechnerischen Unternehmerlohnes bei der Abgrenzung der aggregierten Besteuerungsmenge bereits abgedeckt wurden; vgl. Bundesministerium der Finanzen, Die steuerpolitischen Maßnahmen im Jahre 1961, Finanzbericht 1962 des BMF, Bonn, S. 151, wo sich das Ministerium diese Interpretation ausdrücklich zu eigen macht. Alle Ermäßigungen und Freistellungen, die über die steuerliche Entlastung des Unternehmerlohnes hinausgehen, werden als in den Tarif eingebaute gewerbesteuerliche Subventionen auf den Gewerbeertrag bzw. auf das Besitzeinkommen angesehen, die zur Förderung des Mittelstandes, der Landwirtschaft etc. gewährt werden und trotz ihrer personenbezogenen Bemessung keine eindeutig subjektiven Züge tragen. - Ein möglicher Unterschied zwischen der hier aufgestellten Modellbetrachtung und den Vorstellungen des deutschen Gewerbesteuerrechts liegt insoweit in der Höhe, in der der rechnerische Unternehmerlohn veranschlagt wird. 
des Gewerbeertrages mit der Gewinnermittlung der Unternehmen zusammenfällt, die Gewinnermittlung bzw. die Bilanzierung der Unternehmen aber erst ab Jahresende oder später erfolgt, fällt das Aufkommen der Gewerbeertragsteuer, abgesehen von trendmäßig veranschlagten Steuervorauszahlungen, mit einer gewissen Verzögerung im Staatshaushalt an.

Die Aufkommensfunktion wird in den aggregierten Größen (1) von Steueraufkommen und Gewerbeertrag formuliert und lautet:

$$
\begin{aligned}
T_{R G, 1}= & t_{R G 1}^{*}+ \\
& t_{R G 2}^{*} \cdot[\text { Gewe rbeertrag }]+ \\
& t_{R G 3}^{\star} \cdot[\text { Gewe rbeertrag } t-1]
\end{aligned}
$$

Hierbei bezeichnet $\mathrm{T}_{\mathrm{RG}, \mathrm{t}}$ das Aufkommen der Gewerbeertragsteuer in Milliarden DM; der Gewerbeertrag ist definiert als:

$$
\text { Gewerbeertrag }=
$$

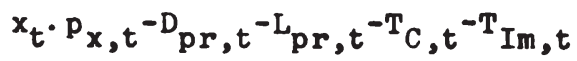

Die Aufkommensfunktion wird transformiert und ergibt als Regressionsansatz (2):

$$
\begin{aligned}
T_{R G, t}= & t_{R G 1}+ \\
& t_{R G 2} \cdot\left[\text { Gewerbeertrag }_{t}-\text { Gewerbeertrag } t-1\right]+ \\
& t_{R G 3} \cdot\left[\text { Gewe rbeertrag }{ }_{t-1}\right]
\end{aligned}
$$

Um (12.5) zu quantifizieren, wurde als erstes das Aufkommen der in der Bundesrepublik erhobenen Gewerbekapitalsteuer geschätzt (3). Durch

(1) Bei der Gewerbeertragsteuer ist es nicht sinnvoll, Steueraufkommen und aggregierte Besteuerungsmenge auf die Zahl der selbständigen Erwerbspersonen zu beziehen. Die Zahl der selbständigen Erwerbspersonen ist in ihrer Entwicklung vor allem durch die abnehmende Zahl der Selbständigen im landwirtschaftlichen Sektor bestimmt, die in der Bundesrepublik von der Gewerbesteuerpflicht freigestellt sind.

(2) Aufgrund des Transformationsprozesses gilt:

$$
\begin{aligned}
t_{R G 1} & =t_{R G 1}^{\star} \\
t_{R G 2} & =t_{R G 2}^{\star} \\
t_{R G 3} & =t_{R G 2}^{\star}+t_{R G 3}^{\star}
\end{aligned}
$$

(3) Vgl. Abschnitt 4 dieses Paragraphen 
Abzug des geschätzten Gewerbekapitalsteueraufkommens sowie der Lohnsummensteuer (1) von dem statistisch ausgewiesenen Gewerbesteueraufkommen insgesamt (2) ergaben sich Schätzwerte für das Aufkommen der Gewerbeertragsteuer.

Infolge des Steueränderungsgesetzes 1961, das die tariflichen Freibeträge der Gewerbeertragsteuer erheblich anhob, sind in der Entwicklung des so abgegrenzten Gewerbeertragsteueraufkommens zwei Phasen zu unterscheiden, die aufkommensmäßig zwischen den beiden Jahren 1963 und 1964 enden bzw. beginnen (3). Für beide Phasen wurde die Schätzung der Aufkommensfunktion getrennt vorgenommen.

Die erste Schätzperiode erstreckt sich von 1955 bis 1964; hierbei wurde der einbezogene Aufkommenswert 1964 um den Steuerausfall korrigiert, den das Bundesfinanzministerium für das erste Jahr der vollen Wirksamkeit der Reformmaßnahmen mit 0,630 Mrd. DM beziffert (4). Das zugehörige Regressionsergebnis lautet im Anschluß an (12.3):

$$
\begin{array}{ll}
t_{R G 1}=-3,06566 & ( \pm 0,22899) \text { Mrd. DM } \\
t_{R G 2}=+0,12798 & ( \pm 0,01813) \\
t_{R G 3}=+0,14495 & ( \pm 0,00372) \\
R_{1 \cdot 2,3}^{2}=0,99545 & \quad \text { (Irrt. Wahrsch.: }<0,18) \\
r_{2 \cdot 3}^{2}=0,00944 & \\
v N Q & =2,760
\end{array}
$$

Das Ergebnis zeigt, daß die Aufkommensentwicklung der Gewerbeertragsteuer in der Bundesrepublik bis 1963 sehr gut durch eine lineare Aufkommensfunktion wiedergegeben werden kann. Ein negativer Aufkommenseffekt der gewerbeertragsteuerlichen Freibeträge ist durch den

(1) Vgl. den folgenden Abschnitt 3 dieses Paragraphen

(2) Vgl. die Statistischen Jahrbücher für die Bundesrepublik Deutschland sowie die Finanzberichte des Bundesministeriums der Finanzen.

(3) Nach den Angaben des Bundesministeriums der Finanzen waren die vollen finanziellen Auswirkungen des Steueränderungsgesetzes 1961 im Bereich der Gewerbeertragsteuer für $1963 \mathrm{zu}$ erwarten. Vgl. Bundesministerium der Finanzen, Die steuerpolitischen Maßnahmen im Jahre 1961, Finanzbericht 1962 des BMF, Bonn, S. 155. - Tatsächlich stellten sie sich erst ein Jahr später, also 1964, ein.

(4) Vgl. ebenda, S. 154 
Schätzwert von $t_{R G 1}$ signifikant nachgewiesen (1). Außerdem stimmt der Schätzwert von Parameter $t_{R G 3}$ ziemlich genau mit der konstanten Grenzbelastung in der linearen Tarifzone der Gewerbeertragsteuer überein (2).

Um den Progressionscharakter der geschätzten Aufkommensfunktion in einer Weise zu kennzeichnen, die einen Vergleich mit den Aufkommensfunktionen der anderen Steuern zuläßt, wurden durch Einsetzen der zeitlichen Variablen-Durchschnitte der geschätzten Parameterwerte in die aus (12.5) abgeleiteten Elastizitätsformeln (3) die mittel- und kurzfristige Aufkommenselastizität der Gewerbeertragsteuer berechnet. Der Wert der mittelfristigen Aufkommenselastizität beträgt $+1,574$, der der kurzfristigen Aufkommenselastizität $+1,328$ (4).

Nach Wirksamwerden des Steueränderungsgesetzes 1961 im Jahre 1964 macht sich in der Aufkommensentwicklung der Gewerbeertragsteuer eine Erscheinung bemerkbar, die für die Schätzung von Steueraufkommensfunktionen allgemein von Bedeutung ist und auf die hier etwas näher

(1) Genau genommen heißt das, daß der aggregierte Freibetrag im Bereich der Gewerbeertragsteuer über die Höhe des rechnerischen Unternehmerlohnes hinausging, wie er hier bei der Ermittlung des aggregierten Besitzeinkommens in Abzug gebracht wurde. Die Tatsache, daß der aggregierte Freibetrag durch eine Konstante mit einem äußerst niedrigen Standardfehler erfaßt werden kann, bringt die fehlende Korrelation zur abnehmenden Gesamtzahl der selbständigen Erwerbspersonen (einschl. selbständiger Landwirte) zum Ausdruck.

(2) Diese tarifliche Grenzbelastung ergibt sich aus dem Produkt der in der linearen Tarifzone per Gesetz geltenden Steuermeßzahl von $5 \%$ und der von den Gemeinden fixierten Hebesätze, die im Durchschnitt des Bundesgebietes und im Durchschnitt der Jahre 1955 bis 1964 269, 1 \% betrugen; zur durchschnittlichen Höhe der Hebesätze vgl. die Statistischen Jahrbücher für die Bundesrepublik Deutschland.

(3) Die Formel für die mittelfristige Aufkommenselastizität wurde aus (12.5) durch partielle Differentiation nach dem Gewerbeertrag der laufenden und der vergangenen Periode abgeleitet und lautet:

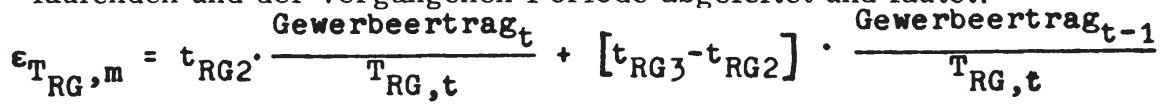

Für die kurzfristige Elastizität gilt entsprechend:

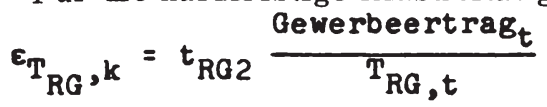

(4) Der Unterschied der Parameter $t_{R G 2}$ und $t_{R G 3}$ wie auch der beiden Elastizitätswerte deutet auf die Existenz nicht sehr erheblicher konjunktureller Unregelmäßigkeiten im Aufkommen der Steuer während dieser Periode. 
eingegangen werden muß: Man stelle sich eine beliebige Steuer vor, deren Bemessungsgrundlage im Laufe des wirtschaftlichen Wachstumsprozesses allgemein zu steigen tendiert. Der Tarif der Steuer sei linear, doch komme bei der Bemessung der Steuerschuld ein allgemeiner persönlicher Freibetrag zum Abzug. Ein Teil der Steuerpflichtigen liege mit dem Wert der Steuerbemessungsgrundlage über, ein anderer Teil unter der Freibetragsgrenze. Unter diesen Umständen ist das Aufkommen der Steuer zwei Entwicklungstendenzen ausgesetzt:

Als erstes steigt die Steuerschuld bei den Steuerpflichtigen, die schon zuvor mit dem Wert ihrer Steuerbemessungsgrundlage über der Freibetragsgrenze lagen. Die entsprechende Aufkommensänderung errechnet sich aus dem Produkt von konstantem Grenzsteuersatz und Änderung der aggregierten Bemessungsgrundlage dieser Gruppe. Gäbe es nur diesen Teil der Steuerpflichtigen, ließe sich die aggregierte Aufkommensentwicklung durch eine lineare Aufkommensfunktion darstellen. - Genau dies war im Bereich der Gewerbeertragsteuer die Situation bis 1963, da bis zu diesem Zeitpunkt die geltenden Freibeträge und die progressive Eingangszone der Gewerbeertragsteuer von den Steuerpflichtigen allgemein überschritten waren.

Hinzu kommt, daß von dem Teil der Steuerpflichtigen, die mit dem Wert ihrer Steuerbemessungsgrundlage unter der Freibetragsgrenze liegen, infolge des allgemeinen Wirtschaftswachstums ständig einige über die Freibetragsgrenze hinausgeraten und damit zum ersten Mal eine entsprechende Steuerschuld zu begleichen haben. Dies führt zu einer weiteren Aufkommenserhöhung, deren aggregierter Umfang davon abhängt, wieviele Steuerpflichtige von ihrer Bemessungsgrundlage gerade in den Bereich positiver Steuerschuld hineingewachsen sind.

Treffen beide Entwicklungstendenzen im Laufe des Wachstumsprozesses zusammen, hat dies zur Folge, daß bis zu dem Zeitpunkt, an dem die letzten Steuerpflichtigen mit dem Wert der Steuerbemessungsgrundlage über die Freibetragsgrenze hinausgeraten, das aggregierte Aufkommen der Steuer trotz des linearen Tarifverlaufs eine überlineare Funktion der aggregierten Besteuerungsmenge (1) ist. Erst wenn alle Steuerpflichtigen den Bereich positiver Steuerschuld erreicht haben, entwickelt sich das Aufkommen der Steuer linear (2).

Derartige aus der Tarif- und Einkommensstruktur resultierende Aufkommenseffekte, die der aggregierten Aufkommensfunktion meist einen zusätzlichen quasiprogressiven Charakter verleihen, spielen kurz- und langfristig bei allen nicht streng proportionalen Steuertarifen, vor allem im Zusammenhang mit Änderungen der Tarifstruktur, eine große Rolle

(1) Als solches wird hier und im folgenden der Wert der aggregierten Steuerbemessungsgrundlage gelegentlich bezeichnet.

(2) Zu der gesamten Erscheinung vgl. auch G. Hagemann, a. a. O. , S. 16 
und lassen sich in der Bundesrepublik nach einer Reihe von Steuerreformen als vorübergehende Anpassungsprozesse beobachten. Im Fall der Gewerbeertragsteuer machten sich die Effekte im Zusammenhang mit dem Steueränderungsgesetz 1961 bemerkbar. Dieses Gesetz erhöhte nämlich die Freibeträge der Gewerbeertragsteuer in einem solchen Umfang, daß ein Großteil der Steuerpflichtigen ab 1964 mit dem jeweiligen Gewerbeertrag unter die neue Freibetragsgrenze bzw. in die tarifliche Eingangszone mit ermäßigtem Steuersatz fiel, und setzte damit einen Anpassungsprozeß der beschriebenen Art in Gang, der durch die typisch überlineare Aufkommensentwicklung gekennzeichnet und bis zum Ende der Untersuchungsperiode noch nicht voll abgeklungen war.

Um diesen Anpassungsprozeß in der Zeit ab 1964 mit der aggregierten Aufkommensfunktion einzufangen, wurde (12.5) erweitert zu:

(12.7)

$$
\begin{aligned}
& T_{R G, t}= t_{R G 1}+ \\
&{ }_{R G 2} \cdot\left[\text { Gewe rbeertrag }_{t}-\text { Gewe rbeertrag }_{t-1}\right]+ \\
&{ }{ }_{R G 3} \cdot\left[\text { Gewe rbeertrag }_{t-1}\right]+ \\
& t_{R G 4} \cdot[\text { Gewe rbeertrag } \\
&t-1]
\end{aligned}
$$

wobei der Ausdruck $t_{\mathrm{RG}} \mathbf{}\left(\text { Gewerbeertrag }_{\mathrm{t}-1}\right)^{-1}$ der Aufkommensfunktion im Wachstumsprozeß eine abnehmende Krümmung verleiht und sie annähernd linear auslaufen läßt.

Wegen der Kürze der Schätzperiode und einiger erheblicher UnregelmäBigkeiten im Steueraufkommen, die sich rechnerisch nicht voll ausgleichen ließen (1), konnten mit einer Kleinst-Quadrat-Schätzung von (12.7) keine befriedigenden Regressionsergebnisse erzielt werden. Vor allem fiel der Schätzwert von Parameter $t_{R G 3}$ wesentlich kleiner aus als in der ersten Schätzperiode, obwohl der allgemeine Steuersatz jenseits der Eingangszone des Gewerbeertragsteuertarifs durch das Steuerände-

(1) Im Zuge der Neuverteilung des Gewerbesteueraufkommens zwischen den staatlichen Gebietskörperschaften der Bundesrepublik (Finanzreform 1970) verlangten die Gemeinden 1969 von den Unternehmen erhöhte Gewerbesteuervorauszahlungen, die das Bundesministerium der Finanzen auf zusätzliche 2,2 Mrd. DM schätzt. Vgl. Bundesministerium der Finanzen, Entwicklung des Steueraufkommens und Vorüberlegungen zur Steuerschätzung für 1971, Finanzbericht 1971 des BMF, Bonn, S. 44. Dieser Betrag wurde aus dem Gewerbeertragsteueraufkommen 1969 herausgerechnet und, was sich im nachhinein als nicht ganz korrekt erwies, dem Aufkommen 1970 zugeschlagen. Außerdem hoben die Gemeinden 1972 die Hebesätze der Gewerbesteuern kräftig an. Vgl. Deutsche Bundesbank, Neuere Tendenzen der Gemeindefinanzen, Monatsberichte der Deutschen Bundesbank, Juli 1973, S. 16 f. 
rungsgesetz 1961 keine Änderung erfahren hatte. Aus diesem Grund wurde der Wert von Parameter ${ }^{t}{ }_{R G 3}$ exogen mit $+0,145$, also mit dem in $(12.6)$ erzielten Wert, vorgegeben und anschließend nochmals eine bedingte Regression geschätzt. Das Regressionsergebnis lautet $(t=1964, \ldots, 1972)$ :

$$
\begin{aligned}
& \text { (12.8) } \quad t_{R G 1}=-13,18723( \pm 1,04698) \text { Mrd. DM } \\
& t_{\text {RG2 }}=+0,03264( \pm 0,03425) \\
& t_{R G 3}=+0,14500 \text { (exogen vorgegeben) } \\
& t_{\mathrm{RG} 4}=+747,83000 \quad( \pm 95,79000) \\
& \left.\mathrm{R}_{(1,3) \cdot 2,4}^{2}=0,91272 \text { (Irrt.-Wahrsch.: }<0,1 \%\right) \\
& \mathbf{r}_{2.4}^{2}=0,00241 \\
& \text { VNQ } \quad=2,810 \\
& R_{1 \cdot 2,(3), 4}^{2}=0,86853
\end{aligned}
$$

Die Korrelation $\mathrm{R}_{(1,3)}^{2}, 2,4$ und die angegebenen Standardfehler beziehen sich auf die Schätzung einer transformierten Version von (12.7), bei der die Linear-Kombination der abhängigen Variablen mit der Variablen, deren Parameter exogen vorgegeben wurde, auf die beiden übrigen Variablen von (12.7) regressiert wurde. $R_{1}^{2} \cdot 2,(3), 4$ bezeichnet die Korrelation zwischen den tatsächlichen Aufkommenswerten der Gewerbeertragsteuer und den hypothetischen Aufkommenswerten, die durch Einsetzen der vorgegebenen bzw. geschätzten Parameter sowie der jeweiligen Variablenwerte in die ursprüngliche Gleichung (12.7) ermittelt wurden; wegen der exogenen Vorgabe eines Parameterwertes handelt es sich um eine bedingte Korrelation.

Der Einfluß, der von kurzfristigen Änderungen des Gewerbeertrages ausging, bzw. der Wert von tRG2, konnte in (12.8) nicht signifikant geschätzt werden. Parameter ${ }^{t}{ }_{R G 4}$ bringt mit einem signifikant positiven Schätzwert die Überlinearität der Aufkommensentwicklung zum Ausdruck; da der reziproke Wert des aggregierten Gewerbeertrages im Wachstumsprozeß ständig sinkt, nähert sich die Aufkommensentwicklung nach (12.7) jedoch wieder einem linearen Verlauf, der praktisch gegen Ende der siebziger Jahre erreicht sein wird. Was nach Abschluß dieses Anpassungsprozesses als wesentliche Folge des Steueränderungsgesetzes 1961 verbleibt, ist nach (12.8) ein absolut gleichbleibender Niveaueffekt der Aufkommensentwicklung, der in dem vom Ergebnis (12.6) der ersten Schätzperiode stark abweichenden Wert des Parameters ${ }^{\text {RG1 }}{ }_{\text {zum Aus- }}$ 
druck kommt.

Der Schätzung entspricht für den Bereich der Variablendurchschnitte eine mittelfristige Aufkommenselastizität von +0,749 (1). Die Elastizität ist als Folge des Steueränderungsgesetzes 1961 wesentlich geringer als in der ersten Schätzperiode; sie steigt jedoch mit Ablauf des beschriebenen Anpassungsprozesses wieder erheblich an.

Figur 12. 1 illustriert mit der durchgezogenen Linie noch einmal modellmäßig die Entwicklung des Gewerbesteueraufkommens während der gesamten Untersuchungsperiode: Ihren linearen Anstieg zwischen 1955 und 1963, den in Form einer Treppenstufe in Erscheinung tretenden Aufkommensausfall für die ersten zwölf Monate nach Wirksamwerden der Steuerreform, den anschließenden überlinear verlaufenden Anpassungsprozeß und mit der rechten gestrichelten Linie die Asymptote dieses Anpassungsprozesses.

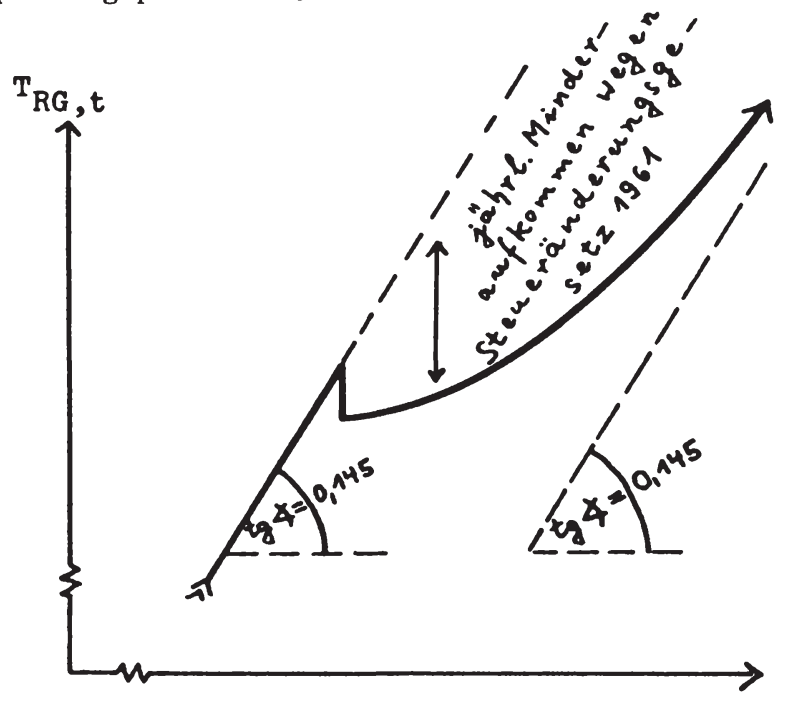

Gewerbe-

Figur 12.1 ertrag

(1) Die durch partielle Differentiation aus (12.7) abgeleitete Elastizitätsformel lautet:

$$
\begin{aligned}
\varepsilon_{T_{R G}, m}= & t_{R G 2} \cdot \frac{\text { Gewerbeertrag }- \text { Gewerbeertrag } t-1}{T_{R G, t}}+ \\
& {\left[t_{R G 3^{-t}}{ }_{R G 4} \cdot\left(\text { Gewerbeertrag }{ }_{t-1}\right)^{-2}\right] \cdot \frac{\text { Gewerbeertrag }-1}{T_{R G, t}} }
\end{aligned}
$$


Die Figur macht verständlich, daß das Bundesministerium der Finanzen den Steuerausfall, den das Steueränderungsgesetz $1961 \mathrm{im}$ Bereich der Gewerbeertragsteuer verursachte, für das erste Jahr der vollen Wirksamkeit mit 0,630 Mrd. DM angeben konnte. Der verursachte jährliche Steuerausfall betrug nach den hier vorgenommenen und mit Figur 12.1 erläuterten Schätzungen (1) im Jahre 1965 aber bereits 1,574 Mrd. DM und im Jahre 1970 3,967 Mrd. DM. Auf die Dauer müssen sich nach den angestellten Schätzungen jährliche Steuerausfälle ergeben, die der Differenz der in (12.6) und (12.8) erzielten Schätzwerte von Parameter $T_{R G 1}$ entsprechen; das sind jährlich 10 Mrd. DM. In Höhe dieser Steuerausfälle wird - aus der Sicht der Modellanalyse - die Kapitalnachfrage des Unternehmenssektors und damit das im privaten Sektor entstandene Besitzeinkommen, das zum größten Teil den inländischen SelbständigenHaushalten zufällt, steuerlich formal entlastet (2).

\section{Die Lohnsummensteuer}

Das Gegenstück zur Gewerbeertragsteuer bildet in der Modellwirtschaft eine zweite spezielle Wertschöpfungsteuer, die sog. Lohnsummensteuer. Die Lohnsummensteuer erfaßt abweichend von den Verhältnissen der Bundesrepublik, das gesamte im privaten Sektor entstandene Arbeitseinkommen einschließlich der rechnerischen Unternehmerlöhne. Sie ist linear ausgestattet und fällt ebenso wie die Gewerbeertragsteuer und die im folgenden Abschnitt besprochene Gewerbekapitalsteuer den kommunalen

(1) Mit Hilfe von (12.5) und (12.6) wurden hypothetische Aufkommenswerte für die Situation ohne Eintritt des Steueränderungsgesetzes 1961 errechnet und von den tatsächlichen Aufkommenswerten abgezogen.

(2) Der in Figur 12.1 geschilderte Verlauf des Gewerbeertragsteueraufkommens ist natürlich zum Teil Ausdruck des staatlichen Bemühens in der Bundesrepublik, durch eine Neuregelung der Freibeträge und der Eingangsprogressionen der Gewerbeertragsteuer eine angemessene Freistellung der im Wachstum steigenden Unternehmerlöhne zu erreichen, eine Neuregelung, die in regelmäßigen Zeitabständen wiederholt werden muß. Insofern ist es nicht gerechtfertigt, Auswirkungen auf die Kapitalnachfrage bzw, eineEntlastung der Besitzeinkommen anzunehmen. Man muß aber deutlich sehen, daß die nur diskontinuierliche Anpassung der Freibeträge im wirtschaftlichen Wachstum dazu führt, daß in der Zeit nach einer vollzogenen Anpassung ein über den Unternehmerlohn hinausgehender Teil des Gewerbeertrages (im Sinne des deutschen Steuerrechts) entlastet wird, während in der Zeit vor einer solchen Anpassung nicht einmal der volle Unternehmerlohn freigestellt wird. 
Gemeinden der Modellwirtschaft zu. Da die Lohnsummensteuer nicht von allen Gemeinden erhoben wird, belastet das Lohnsummensteueraufkommen das aggregierte Arbeitseinkommen wesentlich schwächer als die Gewerbeertragsteuer die Besitzeinkommen (1). Wegen der kontraktmäßigen Vorausbestimmung der Arbeitseinkommen, die der Bemessung der Steuerschuld zugrunde liegen, fällt das Aufkommen der Lohnsummensteuer unverzögert im Staatshaushalt an.

Die Aufkommensfunktion der Lohnsummensteuer lautet:

\section{(12.9) $\quad T_{L, t}=t_{L 1}+t_{L 2} \cdot L_{p r, t}$}

wobei $T_{L, t}$ das aggregierte Aufkommen der Lohnsummensteuer und $L_{p r, t}$ das im privaten Sektor der Modellwirtschaft entstandene aggregierte Arbeitseinkommen der Arbeitnehmer und Selbständigen bezeichnen.

Der Quantifizierung der Aufkommensfunktion wurde das Lohnsummensteuer-Aufkommen der Bundesrepublik in den Jahren 1955 bis 1972 zugrundegelegt. Dieses Aufkommen wurde für die Jahre 1955 bis 1962 im Anschluß an die Angaben des Statistischen Jahrbuchs für die Bundesrepublik als Produkt aus dem Lohnsummensteuergrundbetrag je Einwohner, dem durchschnittlichen Hebesatz der Lohnsummensteuer und der jeweiligen Einwohnerzahl berechnet. Für die Jahre 1963 bis 1972 ist es statistisch direkt ausgewiesen (2).

Bei der Entwicklung des Lohnsummensteueraufkommens ist zu beachten, daß die Gemeinden, denen in der Bundesrepublik das Recht, die Lohnsummensteuer zu erheben, zusteht, ab 1970 dazu übergingen, die Lohnsummensteuer neu einzuführen (3) oder ihre Hebesätze kräftig zu erhöhen (4). Die daraus resultierenden Aufkommenseffekte lassen sich bisher

(1) Wäre der Steuersatz bzw. die durchschnittliche Steuerbelastung im Fall der Gewerbeertragsteuer und der Lohnsummensteuer gleich hoch, hätte man es praktisch mit den beiden Teilsteuern einer allgemeinen Wertschöpfungsteuer zu tun. Hierin liegt auch der Grund, warum auf die getrennte Fassung der in der Bundesrepublik aufkommensmäßig schwachen Lohnsummensteuer Wert gelegt und der Unternehmerlohn in die aggregierte Bemessungsgrundlage der Lohnsummensteuer einbezogen wurde. Mit Hilfe der Gewerbeertragsteuer und der Lohnsummensteuer lassen sich in der Modellformulierung die Auswirkungen einer Nettoumsatzsteuer in der Form einer allgemeinen Wertschöpfungsteuer analysieren.

(2) Vgl. Monatsberichte der Deutschen Bundesbank, Juli 1973, S. 56

(3) In der Bundesrepublik hatte stets nur eine verhältnismäßig geringe Zahl von Gemeinden die Möglichkeit, die Lohnsummensteuer zu erheben, ausgenutzt. 
kaum in zuverlässiger Weise systematisch erfassen. Um ihren Einfluß dennoch miteinzubeziehen, wurde (12.9) erweitert zu

$$
\text { (12.10) } \begin{aligned}
T_{L, t}= & t_{L 1}+ \\
& t_{L 2 \cdot L_{p r, t}}+ \\
& t_{L 3} \cdot L_{p r, t_{2}}+ \\
& t_{L 4} \cdot \text { Dummy }_{t}
\end{aligned}
$$

$\mathrm{L}_{\text {pr }, t 1}$ gibt die im privaten Sektor entstandenen Arbeitseinkommen für die Jahre 1955 bis 1969 an und wird ab 1970 einschließlich gleich null gesetzt (1). $\mathrm{L}_{\mathrm{pr}, \mathrm{t} 2}$ wird vor 1970 gleich null gesetzt und gibt die im privaten Sektor entstandenen Arbeitseinkommen für die Jahre 1970 bis 1972 an. Die Dummy-Variable erhält bis 1969 den Wert null, ab 1970 den Wert eins.

Das Regressionsergebnis zu (12.10) für die Jahre 1955 bis 1972 lautet:

$$
\begin{aligned}
& \text { (12.11) } t_{L 1}=+0,12675 \quad( \pm 0,00949) \text { Mrd. DM } \\
& t_{L 2}= \pm 0,00330 \quad( \pm 0,00005) \\
& t_{L 3}= \pm 0,01012 \quad( \pm 0,00020) \\
& t_{L 4}=-2,42414 \quad( \pm 0,08305) \\
& \left.R_{1 \cdot 2,3,4}^{2}=0,99950 \quad \text { (Irrt.-Wahrsch.: } 0,18\right) \\
& R_{2 \cdot 3,4}^{2}=0,61921 \\
& R_{3 \cdot 2,4}^{2}=0,99261 \\
& R_{4 \cdot 2,3}^{2}=0,99281 \\
& v_{N Q}^{2}=1,939
\end{aligned}
$$

Die Aufkommenselastizität der Lohnsummensteuer, bezogen auf das im privaten Sektor entstandene Arbeitseinkommen beträgt für den Bereich

(4) Vgl. Deutsche Bundesbank, Neuere Tendenzen der Gemeindefinanzen, a. a. O. , S. 19

(1) Der Aufkommenswert 1970 liegt genau im Eckpunkt der unterschiedlichen Aufkommensentwicklung und kann sowohl dem ersten wie dem zweiten Entwicklungsabschnitt zugerechnet werden. 
der zeitlichen Variablen-Durchschnitte in den Jahren 1955 bis $1969+0,838$ und für die Jahre 1970 bis $1972+2,282$ (1). Da hierin die aggregierte Bemessungsgrundlage, anders als vom Gesetz vorgesehen, die rechnerischen Unternehmerlöhne einbezogen wurden und da vom Gesetz her keine Freibetragsregelungen gewährt werden (2), sind beide Werte lediglich Ausdruck für das jeweilige aggregierte Verhalten der Gemeinden hinsichtlich der Erhebung der Lohnsummensteuer und der Bemessung ihrer Hebesätze. In der Modellrechnung wird angenommen, daß die für die Periode 1970 bis 1972 geltenden Parameter dieses Verhalten auch über das Jahr 1972 hinaus kennzeichnen.

\section{Die Gewerbekapitalsteuer}

Auf das im Unternehmenssektor investierte Anlagevermögen erhebt der Staat der Modellwirtschaft die sog. Gewerbekapitalsteuer. Die Gewerbekapitalsteuer setzt am Nettoanlagevermögen zu Wiederbeschaffungspreisen des Unternehmenssektors (3) als Bemessungsgrundlage an und belastet es mit einem linearen Tarif. Wegen meist verspäteter jährlicher Vermögensfeststellung der Unternehmen fällt ihr Aufkommen mit einer Verzögerung von einem Jahr im Staatshaushalt an.

Die Aufkommensfunktion der Gewerbekapitalsteuer lautet:

$(12 \cdot 12)$

$$
T_{k, t}=t_{k 1}+t_{k 2} \cdot\left[k_{p r, t-1}^{n} \cdot p_{k, t-1}\right]
$$

wobei $T_{k, t}$ das aggregierte Aufkommen der Gewerbekapitalsteuer bezeichnet, $\mathrm{k}_{\mathrm{pr}, \mathrm{t}}^{\mathrm{n}}$ das reale Nettoanlagevermögen des Unternehmenssektors und $\mathrm{p}_{\mathrm{k}, \mathrm{t}}$ den Preisindex für Anlageinvestitionen, der in der Modellwirtschaft auch zur Bewertung des Anlagevermögens herangezogen wird.

(1) Berechnet nach der Elastizitätsformel

$$
\varepsilon_{T_{L}}= \begin{cases}t_{L 2} \cdot\left(L_{p r, t} / T_{L, t}\right) & \text { rur } t \leqslant 1969 \\ t_{L 3} \cdot\left(L_{p r, t} / T_{L, t}\right) & \text { rur } t \geqslant 1970\end{cases}
$$

(2) Das Gewerbesteuergesetz der Bundesrepublik sieht für die Lohnsummensteuer lediglich Freigrenzen vor ( $\$ 23$ Ziffer 2 Gewerbesteuergesetz)

(3) Es sei noch einmal daran erinnert, daß in der Modellformulierung die gesamte nicht in Kasse gehaltene Nominalvermögensbildung der privaten Haushalte im Unternehmenssektor angelegt wird und daß der Unternehmenssektor mit dem aufgenommenen Finanzkapital die gesamte Realvermögensbildung der Modellwirtschaft einschließlich des privaten Wohnungsbaus betreibt. 
Zur Quantifizierung von (12.12) wurde das Aufkommen der Grundsteuer und der Gewerbekapitalsteuer in der Bundesrepublik zu einem Aggregat zusammengefaßt und repräsentiert als solches das Gewerbekapitalsteueraufkommen der Modellwirtschaft. - Das Aufkommen der Grundsteuer wird in der Bundesrepublik statistisch gesondert ausgewiesen (1). Das Aufkommen der Gewerbekapitalsteuer wird nur zusammengefaßt mit der Gewerbeertragsteuer ausgewiesen und mußte daher geschätzt werden. Hierzu dienten als einzige Anhaltspunkte eine Angabe des Troeger-Gutachten, wonach das Aufkommen der Gewerbekapitalsteuer in der Bundesrepublik 1964 1,4 Mrd. DM betragen hat (2), sowie die gesetzlichen Vorschriften des Gewerbesteuerrechts, wonach der Tarif der Gewerbekapitalsteuer linear verläuft und lediglich eine verhältnismäßig niedrige Freigrenze von 6.000 DM vorsieht. Der vom Troeger-Gutachten angegebene Betrag wurde wegen des vermuteten Aufkommenslags auf das Nettoanlagevermögen zu Wiederbeschaffungspreisen des privaten Sektors zu Beginn des Jahres 1963 bezogen (3) und ergab für dessen Belastung einen Satz von 0,001941. Mit diesem konstant gehaltenen Satz und den jeweiligen Werten des privaten Nettoanlagevermögens wurde bei Berücksichtigung der angenommenen einjährigen Aufkommensverzögerung das Aufkommen der Gewerbekapitalsteuer für die gesamte Untersuchungsperiode geschätzt.

Bei der Quantifizierung von (12.12) mit Hilfe des zusammengefaßten Aufkommens der Grundsteuer und der Gewerbekapitalsteuer ist zu beachten, daß die unterschiedliche Bestands- und Preisentwicklung und im Zusammenhang damit die unterschiedlichen steuerlichen Bewertungsmaßstäbe beim Grund-, Bau - und Ausrüstungsvermögen die Parameterschätzung $\therefore$. einflussen und bei einer Aufkommensfortrechnung mit Hilfe der Regres-

(1) Vgl. die Finanzberichte des Bundesministeriums der Finanzen sowie die Monatsberichte der Deutschen Bundesbank

(2) Vgl. Kommission für die Finanzreform, Gutachten über die Finanzreform in der Bundesrepublik Deutschland (Troeger-Gutachten), Stuttgart 1966, Textziffer 391

(3) Wahrscheinlich wäre es sinnvoller gewesen, den oben genannten Betrag allein auf den aus Ausrüstungen bestehenden Teil des Nettoanlagevermögens zu beziehen und die Vor- und Rückrechnung allein mit Hilfe dieses Anteils vorzunehmen. Bauten werden durch die Gewerbekapitalsteuer der Bundesrepublik nicht belastet; die Preis- und Mengenentwicklung verlief bei Ausrüstungen und Bauten bzw. bei ihren abgeschriebenen Werten aber unterschiedlich. Die Folge der oben vorgenommenen Berechnungsart dürten daher gewisse Verzerrungen sein, die sich auch auf die Ermittlung des Gewerbeertragsteueraufkommens auswirken, während der Schätzperiode selbst aber aufkommensmäßig nicht sehr stark zu Buche schlagen. 
sionsergebnisse zu systematischen Prognosefehlern führen können (1).

Das Ergebnis der Kleinst-Quadrat-Schätzung zu (12.12) lautet $(t=1952 \ldots$, 1972):

$$
\text { (12.13) } \begin{aligned}
t_{k 1} & =+1,00408 \quad( \pm 0,02523) \text { Mrd. DM } \\
t_{k 2} & =+0,00335 \quad( \pm 0,00003) \\
r_{1 \cdot 2}^{2} & =0,99814 \text { (Irrt.-Wahrsch.: }<0,1 \%) \\
v N Q & =0,717
\end{aligned}
$$

Es ist wegen der Methode, mit der das Gewerbekapitalsteueraufkommen in der Bundesrepublik geschätzt wurde, nur begrenzt aussagefähig. Abgesehen davon deutet der niedrige von-Neumann-Quotient darauf, daß die Aufkommensfunktion (12.12) für den Bereich der im Aufkommen enthaltenen Grundsteuer nicht genau spezifiziert ist.

Nach (12.13) hat die Aufkommensfunktion als Folge der Tatsache, daß sich die Bemessungsgrundlage der Grundsteuer auf den nicht beliebig vermehrbaren Bestand von Grund und Boden erstreckt, der hier nicht zur Aufkommensbemessung herangezogen werden konnte, und als Folge der zunehmenden steuerlichen Unterbewertung des Grund- und Bauvermögens in der Bundesrepublik einen hohen positiven Niveauparameter und damit einen stark regressiven Verlauf. Die mittelfristige Elastizität der Aufkommensfunktion (2) im Bereich der zeitlichen Variablen-Durchschnitte beträgt $+0,688$.

\section{Die Importzölle}

Zölle werden in der Modellwirtschaft lediglich auf Importe erhoben. Es handelt sich um Wertzölle, die die nominellen Warenimporte aus dem Ausland mit einem linearen Tarif belasten.

(1) Dies gilt auch unabhängig von der in der vorletzten Fußnote besprochenen Verzerrung.

(2) Die Elastizitätsformel lautet im Anschluß an (12.12)

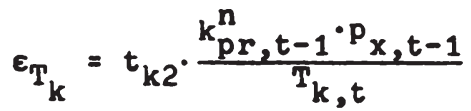

Die kurzfristige Aufkommenselastizität bezogen auf Änderungen des Nettoanlagevermögens in der laufenden Periode ist nach (12.12) ex-definitione gleich null. 
Die Aufkommensfunktion für die Zolleinnahmen, $T_{I m}, t$, des Staates lautet: (12.14) $\quad T_{I m, t}=t_{\operatorname{Im} 1}+t_{I m 2} \cdot\left[x_{I m, t} \cdot p_{I m, t}\right]$

Diese Funktion wurde wegen der in der Bundesrepublik im Jahre 1964 vorgenommenen Senkung der Zolltarife für die zwei Perioden 1950 bis 1963 und 1964 bis 1970 geschätzt (1).

Das Regressionsergebnis für die Periode 1950 bis 1963 lautet:

$$
\text { (12.15) } \begin{aligned}
t_{\operatorname{Im} 1} & =+0,27463 \quad( \pm 0,08425) \text { Mrd. DM } \\
t_{\operatorname{Im} 2} & =+0,04736 \quad( \pm 0,00211) \\
\mathbf{r}_{1 \cdot 2}^{2} & =0,97682 \quad \text { (Irrt.-Wahrsch.: }<0,1 \%) \\
\text { vNQ } & =0,861
\end{aligned}
$$

Das Ergebnis ist durch eine starke Autokorrelation der Residuen beeinträchtigt, die auf kleinere zollpolitische Maßnahmen des Staates und die dadurch bedingten Aufkommenseffekte zurückzuführen ist (2).

Das Ergebnis für die Periode 1964 bis 1972 lautet:

$$
\text { (12.16) } \begin{aligned}
t_{\text {Im } 1} & =+2,05443 \quad( \pm 0,27487) \text { Mrd. DM } \\
t_{\text {Im2 }} & =+0,00581 \quad( \pm 0,00268) \\
r_{1 \cdot 2}^{2} & =0,48481 \quad \text { (Irrt.-Wahrsch.: }<108) \\
\text { vNQ } & =2,669
\end{aligned}
$$

Dieses Ergebnis ist nicht sehr zuverlässig, deutet aber an, daß die zollpolitischen Maßnahmen von 1964 nicht nur zu einer Senkung der marginalen Importbelastung, sondern auch zu einer Erhöhung der von der allgemeinen Importentwicklung unabhängigen Zolleinnahmen führten.

(1) Die Angaben über die Entwicklung der staatlichen Zolleinnahmen wurden den Finanzberichten des Bundesministeriums der Finanzen und ergänzend dem Statistischen Jahrbuch für die Bundesrepublik Deutschland entnommen. Die Angaben über den nominellen Import wurden, wie bereits angegeben, um die an das Ausland geleisteten Vermögenserträge bereinigt. - Zu den im Jahre 1964 vorgenommenen Senkungen von Zolltarifen vgl. den Finanzbericht 1965 des Bundesministeriums der Finanzen, Bonn, S. 59 u. 62.

(2) Vgl. dazu die Vorüberlegungen zu den Steuerschätzungen in den verschiedenen Finanzberichten des Bundesministeriums der Finanzen. 
Wie bereits die positiven Werte des Niveauparameters $t_{I m} 1$ andeuten, hat die Aufkommensfunktion in beiden Schätzperioden einen regressiven Verlauf. Die Aufkommenselastizität beträgt für die zeitlichen Variablendurchschnitte der ersten Periode +0,861 und für die der zweiten Periode $+0,221$. 
1. Die Lohnsteuer auf den Arbeitnehmerlohn

Der Staat erhebt in der Modellwirtschaft vier Arten von direkten Steuern. Die erste ist die Lohnsteuer auf den Arbeitnehmerlohn. Sie erfaßt das Bruttoeinkommen aus unselbständiger Beschäftigung der Arbeitnehmer, $L_{A, t}$. Ihr Tarif enthält einen persönlichen Freibetrag; nach einer Eingangszone mit indirekter Progression bzw. mit konstantem marginalem Steuersatz ist er mit einer direkten Progression bzw. steigenden marginalen Steuersätzen ausgestattet. Da die Lohneinkommen durch Arbeitsverträge im voraus fest vereinbart sind und in wöchentlichen oder monatlichen Abständen zur Auszahlung gelangen, die Lohnsteuer andererseits im Quellenabzugsverfahren bei den Arbeitgebern erhoben wird, fällt das kassenmäßige Aufkommen der Steuer, $\mathrm{T}_{\mathrm{L}} \mathrm{A}, \mathrm{t}$, ohne merkliche Verzögerung im Staatshaushalt an.

Versucht man, die lohnsteuerliche Belastung der Einkommen aus unselbständiger Arbeit für die Gesamtheit oder den Durchschnitt aller Arbeitnehmer durch eine einzige Funktion zu erfassen, so ist die Formulierung dieser Funktion wie bei der Gewerbeertragsteuer durch Probleme belastet, die aus der Tarif- und Einkommensstruktur sowie dem vorgenommenen Aggregationsprozeß entstehen. Existiert nämlich ein Personenkreis, dessen Lohneinkommen unter der tariflich fixierten Freibetragsgrenze liegt und der den aus dem Freibetrag resultierenden Steuervorteil nicht voll ausnutzen kann, und nimmt dieser Personenkreis im Zuge des allgemeinen Einkommenswachstums zahlenmäßig ab, so hat dies zur Folge, daß die durchschnittliche Ausnutzung der lohnsteuerlichen Freibeträge im Wachstumsprozeß ansteigt und daß die Freibeträge in der aggregierten oder durchschnittlichen Aufkommensfunktion nicht eindeutig durch den Wert irgendeines Parameters zum Ausdruck gebracht werden können. Ähnliche Schwierigkeiten ergeben sich, sobald die Höhe der von den Arbeitnehmern verdienten Lohneinkommen aus der Zone der indirekten Progression mit konstantem marginalem Steuersatz in den Bereich der direkten Progression mit steigenden marginalen Steuersätzen überzuwechseln beginnt. Trotz dieser Probleme läßt sich für das aggregierte bzw. Pro-Kopf-Lohnsteueraufkommen eine verhältnismäßig einfache Aufkommensfunktion formulieren, die auch die verschiedenen Eigenschaften des gesetzlichen Lohnsteuertarifs noch deutlich erkennen läßt.

Bezeichnen $L_{A, t} / a_{t}$ das durchschnittliche Lohneinkommen und $T_{L A}, t / a_{t}$ die durchschnittliche Lohnsteuerzahlung eines Arbeitnehmers, dann lautet die Funktion für die durchschnittliche jährliche Lohnsteuerzahlung eines Arbeitnehmers in der Modellformulierung:

(13.1)

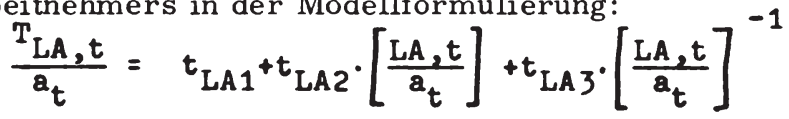


Hierbei ist wegen der Berücksichtigung eines persönlichen Freibetrags mit einem negativen Vorzeichen von $t_{L A} 1$ zu rechnen und wegen der in der Zone der direkten Progression ansteigenden marginalen Steuersätze mit einem positiven Vorzeichen von $t_{\text {LA 3 }}$. Die durchschnittliche Lohnsteuerbelastung der Arbeitnehmereinkommen, $\mathrm{T}_{\mathrm{LA}, \mathrm{t}} / \mathrm{L}_{\mathrm{t}}$, tendiert nach (13.1) mit wachsendem Pro-Kopf-Einkommen gegen den Wert von Parameter $\mathrm{t}_{\mathrm{LA} 2}$.

Die Lohnsteuerfunktion (13.1) wurde mit Hilfe der Daten über das Lohnsteueraufkommen in der Bundesrepublik für die beiden Teilperioden 1959 bis 1964 und 1965 bis 1972 getrennt geschätzt, weil zwischen den beiden Teilperioden die Einkommensteuerreform 1965 wirksam wurde, die sich auf die Freibeträge wie auf den marginalen Tarifverlauf der Einkommen- bzw. Lohnsteuer erstreckte und erhebliche Aufkommenseffekte mit sich brachte. Auf diese Weise wird der tatsächliche lohnsteuerliche Belastungsverlauf in der Bundesrepublik genauer abgebildet bzw. prognostiziert als mit einer einzigen Regression für die Gesamtperiode. Zu gleich kommt die getrennte Schätzung dem Versuch entgegen, die Auswirkungen der Steuerreform 1965 mit Hilfe der Modellrechnung zu analysieren.

Die entsprechenden Regressionsergebnisse lauten für die erste Teilperiode (1959 bis 1964):

(13.2) $\begin{aligned} t_{L A 1} & =+357,27000 \quad \text { (nicht signifikant) DM p.c. } \\ t_{L A 2} & =0,07873( \pm 0,01951) \\ t_{L A 3} & =3,60802 \cdot 10^{6}\left( \pm 1,21417 \cdot 10^{6}\right) \\ R_{1 \cdot 2,3}^{2} & =0,99916 \quad \text { (Irrt.-Wahrsch.: }<0,1 \%) \\ r_{2 \cdot 3}^{2} & =0,98624 \\ \text { vNQ } & =2,571\end{aligned}$

(1) Die Angaben über das jährliche Lohnsteueraufkommen in der Bundesrepublik wurden dem Jahresgutachten 1972 des Sachverständigenrates zur Begutachtung der gesamtwirtschaftlichen Entwicklung, Bundesratsdrucks. 612/72 vom 1. Dez. 1972, Bonn 1972, Tab. 69 sowie dem Monatsbericht der Deutschen Bundesbank, Juni 1973, S. 56 , entnommen. Der Aufkommensanteil des Saarlandes für 1959 läßt sich nach den Angaben der Finanzberichte 1961, S. 35, u. 1962, S. 45, des Bundesministeriums der Finanzen berechnen. 
Als Folge zahlreicher kleiner Tarifreformen und der mit ihnen verbundenen Aufkommenseffekte (1) haben die Parameterwerte nicht die vom gesetzlichen Tarifverlauf her zu erwartende Größe bzw. die zu erwartenden Vorzeichen. Für den Bereich der Variablen-Durchschnitte errechnet man mit Hilfe von (13.2) jedoch eine für die Lohnsteuer der Bundesrepublik durchaus typische, wenn auch etwas unterschätzte Aufkommenselastizität von $+2,037$ (2). Aus diesem Grund werden die geschätzten Parameter ungeachtet der von den kleinen Tarifreformen ausgehenden Einflüsse zur Lohnsteuerbemessung in den ersten fün Jahren der Modellrechnung, 1960 bis 1964, herangezogen (3).

(1) Es handelt sich überwiegend um die Neueinführung und Erhöhung von Freibeträgen. Zu den Aufkommenseffekten der Reformen vgl. G. Hagemann, Aufkommenselastizitäten ausgewählter Steuern in der Bundesrepublik Deutschland 1950 - 1963, Tübingen 1968, S. 189.

(2) Die Elastizität wurde durch Einsetzen der Durchschnittswerte von $\mathrm{T}_{\mathrm{LA}, t} / \mathrm{a}_{\mathrm{t}}$ und $\mathrm{L}_{\mathrm{A}, \mathrm{t}} / \mathrm{a}_{\mathrm{t}}$ in die durch partielle Differentiation aus der Lohnsteuerfunktion (13.1) abgeleitete Formel

$$
\varepsilon_{T_{L A}}=\left[t_{L A 2}{ }^{-t_{L A 3}}\left(\frac{L_{A, t}}{a_{t}}\right)^{-2}\right] \cdot\left[\frac{L_{A, t} / a_{t}}{T_{L A, t} / a_{t}}\right]
$$

berechnet. Sie gilt bei Annahme einer konstanten Zahl von abhängigen Erwerbstätigen auch für das aggregierte Steueraufkommen.

(3) Die Lohnsteueraufkommensbeträge lassen sich mit Hilfe der von G. Hagemann, Aufkommenselastizitäten ..., a. a. O., S. 189, angegebenen Korrekturbeträge, die für 1964 fortgeschrieben wurden, auf einen einheitlichen Rechtsstand mit Basis 1964 umrechnen. Bei der Schätzung mit diesen bereinigten Daten erhält man für Parameter $t_{L A 3}$ einen immer noch mit falschem Vorzeichen versehenen, aber nur bei einer hohen Irrtumswahrscheinlichkeit von $85 \%$ von null unterscheidbaren Wert. Setzt man den Parameter daher von vornherein gleich null, so lautet das Schätzergebnis:

$$
\text { (13.2a) } \begin{aligned}
t_{L A 1} & =-699,09644 \quad( \pm 9,69612) \\
t_{L A}=+0,14985 & ( \pm 0,00119) \\
r_{1.2}^{2} & =0,99975 \quad \text { (Irrt.-Wahrsch.: }<0,1 \%) \\
v N Q & =2,403
\end{aligned}
$$

Den Parameterwerten entspricht im Bereich der Variablen-Durchschnitte eine Elastizität von $+2,379$. 
Für die Zeit nach dem Inkraftreten der Einkommensteuerreform 1965, also für die Jahre 1965 bis 1972, lautet das Schätzergebnis:

$$
\text { (13.3) } \begin{aligned}
& \mathrm{t}_{\mathrm{LA} 1}=-2108,81000 \quad(\text { signifikant }) \mathrm{DM} \mathrm{p.c.} \\
& \mathrm{t}_{\mathrm{LA2}}=+0,20113 \quad( \pm 0,00452) \\
& \mathrm{t}_{\mathrm{LA3}}=+7,96115 \cdot 10^{6}\left( \pm 0,92078 \cdot 10^{6}\right) \\
& \mathrm{R}_{1 \cdot 2,3}^{2}=0,99991 \quad \text { (Irrt.-Wahrsch.:<0,18) } \\
& \mathrm{r}_{2 \cdot 3}^{2}=0,97614 \\
& \mathrm{vNQ}=2,972
\end{aligned}
$$

Anders als bei der Schätzung für die erste Teilperiode habèn die Regressionsparameter hier die vom gesetzlichen Lohnsteuertarif der Bundesrepublik her zu erwartende Größe bzw. die zu erwartenden Vorzeichen. So wird an dem negativen Vorzeichen und der Größe von ${ }^{L}$ A 1 der Einfluß der steuerlichen Freibeträge erkennbar; der Wert von tLA2 erinnert an den marginalen Steuersatz in der unteren Proportionalzone des ab 1965 geltenden gesetzlichen Steuertarifs (1); Vorzeichen und Größe von $t_{\text {LA3 }}$ bringen zum Ausdruck, daß die Arbeitnehmer der Bundesrepublik mit ihrem Lohneinkommen nach 1965 zunehmend in die direkte Progressionszone des Lohnsteuertarifs geraten sind und ihr Grenzsteuersatz allgemein zu steigen begonnen hat (2). Berechnet man mit Hilfe der geschätzten

(1) Der Satz beträgt $19 \%$.

(2) Die Lohneinkommen der Arbeitnehmer hielten sich während der ersten Teilperiode im allgemeinen im Bereich der Eingangsstufe des Lohnsteuertarifs, der lediglich mit einer indirekten Progression ausgestattet ist. Vgl. das Regressionsergebnis in Fußn. 3 von S. 205. Auch bei unveränderter Gültigkeit des vor 1965 wirksamen Tarifs über das Jahr 1965 hinaus wären sie zunehmend in die Zone der direkten Lohnsteuerprogression gefallen. Aus diesem Grund kann der Unterschied zwischen den für beide Teilperioden geschätzten Parameterwerten von (13.2 a) einerseits und (13.3) andererseits nicht allein auf die Einkommensteuerreform 1965 zurückgeführt werden. Aus diesem Grund ist es auch nicht möglich, die langfristigen finanziellen Auswirkungen der Einkommensteuerreform 1965 in der Modellrechnung dadurch einzufangen, daß man in der Zeit nach 1965 die für diese Jahre geschätzten Parameter durch die für die Jahre 1959 bis 1964 geschätzten Parameter ersetzt. Es ist lediglich möglich, für das Übergangsjahr 1965 mit Hilfe der Schätzung (13.2 a) die finanziellen Auswirkungen der Einkommensteuerreform 1965 im Bereich der Lohnsteuer abzuschätzen. 
Parameterwerte schließlich wie zuvor die Aufkommenselastizität der Lohnsteuer, so ist der im Vergleich zur ersten Teilperiode niedrige Wert von +1,753 (1) überwiegend die Folge der verzögerten Tarifprogression in der unteren Proportionalzone des Einkommensteuertarifs (2). Die Parameterwerte von (13.3) dienen in der Modellrechnung zur Lohnsteuerbemessung in den Jahren ab 1965. Mit der Annahme, daß die Parameterwerte auch über das Jahr 1972 hinaus gelten, wird in der Modellrechnung unterstellt, daß der Staat die durchschnittliche Lohnsteuerbelastung des Arbeitnehmerlohnes, die in der Bundesrepublik 1972 11,2\% betrug und aufgrund der direkten Lohnsteuerprogression erheblich steigen wird, nicht über $20 \%$ hinaus wachsen lassen wird.

\section{Die Lohnsteuer auf den rechnerischen Unternehmerlohn}

Bei der Einkommensbesteuerung der selbständigen Erwerbspersonen geht der Staat der Modellwirtschaft von dem Grundsatz aus, daß der rechnerische Unternehmerlohn, den sich die Selbständigen in Höhe des im privaten Sektor verdienten Arbeitnehmerlohnes als Vergütung für die zum Produktionsprozeß beigetragene eigene Arbeitsleistung zurechnen, steuerlich genau so zu behandeln ist wie das Lohneinkommen der Unselbständigen. Der rechnerische Unternehmerlohn der Selbständigen wird-daher ebenso wie das Lohneinkommen der Unselbständigen durch eine Lohnsteuer, die sog. Unternehmerlohnsteuer, erfaßt. Wegen der der spezifischen Ermittlung des Unternehmerlohnes läßt sich die Lohnsteuerschuld der Selbständigen im Gegensatz zu der auf die anderen Selbständigen-Einkommen entfallenden Steuer schnell bestimmen und führt zu einem unverzögerten Steueraufkommen.

Bezeichnen $\mathrm{L}_{S, t} / \mathrm{s}_{\mathrm{t}}$ den rechnerischen Unternehmerlohn und $\mathrm{T}_{\mathrm{LS}, \mathrm{t}} / \mathrm{s}_{\mathrm{t}}$ die durchschnittliche Lohnsteuerzahlung der Selbständigen, dann lautet die Funktion für das durchschnittliche Pro-Kopf-Aufkommen der Unternehmerlohnsteuer:

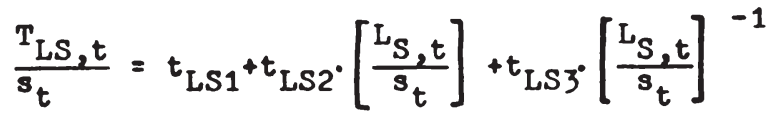

Die Reform verursachte danach Lohnsteuerausfälle in Höhe von 112.49 DM pro Kopf der abhängigen Erwerbstätigen bzw. von insgesamt 2,457 Mrd. DM.

(1) Mit Hilfe der in (13.2 a) angegebenen Parameterwerte für die erste Teilperiode hätte man für den Bereich der Variablendurchschnitte der zweiten Teilperiode eine Elastizität von $+1,539$ prognostiziert.

(2) Der signifikante Wert von Parameter $t_{L A 3}$ in (13.3) zeigt, daß die Lohnsteuerfunktion (12.1) einem einfachen linearen oder doppelt-logarithmischen Regressionsansatz überlegen ist. 
Die Parameterwerte der Funktion für die Jahre 1959 bis 1964 und für die Jahre ab 1965 bestimmen sich in der Modellrechnung nach (13.2) und (13.3), haben also die gleiche Größe wie die Werte der Parameter $t_{L A 1}$, $\mathrm{t}_{\mathrm{LA} 2}$ und $\mathrm{t}_{\mathrm{LA} 3}$ in der Lohnsteuerfunktion der Arbeitnehmer.

Für die Berechnungen des folgenden Abschnitts war es notwendig, die hypothetischen Aufkommenswerte zu bestimmen, die bei einer Erhebung der konstruierten Unternehmerlohnsteuer während der Untersuchungsperiode in der Bundesrepublik angefallen wären. Steuerfunktion (13.4) mit den Parameterwerten (13.2) und (13.3) wurde daher auf den für die Volkswirtschaftliche Gesamtrechnung der Bundesrepublik ermittelten rechnerischen Unternehmerlohn angewandt. Die Berechnung ging von den Pro-Kopf-Größen aus, die dann mit Hilfe der Zahl der selbständigen Erwerbspersonen in Aggregatgrößen umgerechnet wurden. Die hypothetischen Aufkommenswerte der für die Bundesrepublik fingierten Unternehmerlohnsteuer betrugen 1960 gut $20 \%$ und 1970 etwa $40 \%$ des Aufkommens der veranlagten Einkommensteuer.

\section{Die Steuer auf das Besitzeinkommen der Selbständigen}

Das Besitzeinkommen der Selbständigen unterliegt in der Modellwirtschaft einer gesonderten Steuer, die im modellmäßig dargestellten Investitionskalkül der Unternehmen die Rolle der Gewinnsteuer spielt. Diese Besitzeinkommensteuer ist im Prinzip ähnlich konstruiert wie die auf die Arbeitseinkommen der Selbständigen und Unselbständigen entfallende Lohnsteuer. Sie belastet das Besitzeinkommen der Selbständigen also progressiv; der Tarif enthält nur eine verzögerte Progression und geht mit wachsender Besteuerungsmenge zu einer Proportionalbesteuerung über. Wegen der Vermutung einer mit ihm verbundenen höheren steuerlichen Leistungsfähigkeit und aus verteilungspolitischen Gründen (1) wird das Besitzeinkommen der Selbständigen jedoch höher belastet als das Arbeitseinkommen der selbständigen und unselbständigen Erwerbspersonen. Außerdem macht sich im Aufkommen der Besitzeinkommensteuer bemerkbar, daß die Unternehmen die genaue Höhe der entstandenen Gewinne jeweils am Jahresende oder später ermitteln und die Steuerschuld daher, abgesehen von trendmäßig veranschlagten Steuervorauszahlungen, erst in der Folgeperiode exakt beglichen werden kann (2).

(1) Aus diesen verteilungspolitischen Gründen bleibt auch das Besitzeinkommen der Unselbständigen steuerfrei.

(2) Bei dem gegenwärtigen Stand der volkswirtschaftlichen Gesamtrechnung ist es innerhalb der Modellformulierung nicht möglich, die unterschiedliche Steuerbelastung von einbehaltenen und ausgeschütteten Körperschaftsgewinnen zu berücksichtigen. Wegen der schwerpunktmäßigen Verteilung des Aktien- und GmbH-Anteil-Besitzes auf die 
Um die Progressionseffekte der Besitzeinkommensteuer exakt herauszustellen, wird die Aufkommensfunktion wie im Fall der Lohnsteuer in ProKopf-Größen formuliert und lautet:

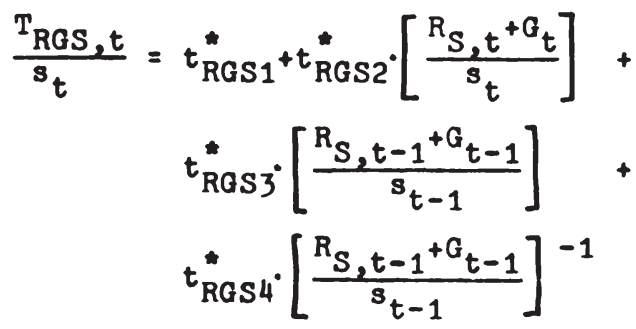

$T_{\text {RGS }} \mathrm{t}$ symbolisiert das aggregierte Steueraufkommen der Periode $\mathrm{t}$, $R_{S, t}$ den auf das gesamte Selbständigenvermögen verrechneten Kapitalzins $\left(F_{S, t-1} \cdot r_{t-1}\right)$ und $G_{t}$ die Summe der Unternehmensgewinne; $s_{t}$ gibt die Zahl der selbständigen Erwerbspersonen an.

Die Funktion wird transformiert und ergibt als Regressionsansatz (1):

oberen Einkommensbeziehergruppen und wegen der Nachbelastung der ausgeschütteten Körperschaftsgewinne im Rahmen der veranlagten Einkommensteuer sind die damit nicht erfaßten Aufkommenseffekte von begrenztem Gewicht. Außerdem bedient sich die einkommen- und körperschaftsteuerliche Gewinnermittlung im Modell abweichend vom deutschen Steuerrecht einer Abschreibungsrechnung, die sich wie die volkswirtschaftliche Gesamtrechnung an den Wiederbeschaffungswerten und an einem linearen Verlauf des realen Kapitalverzehrs orientiert. Die Bewertung der abzuschreibenden Anlagen zu Wiederbeschaffungs- statt zu Anschaffungswerten führt zu einer Unterschätzung, die Annahme eines linearen statt eines degressiven Kapitalverzehrs führt zu einer Überschätzung der steuerlichen Gewinngröße. Beide Ermittlungsfehler kompensieren sich in gewissem Umfang.

(1) Aufgrund der Transformation gilt

$$
\text { (13.6a) } \begin{aligned}
t_{\text {RGS1 }} & =t_{\text {RGS1 }}^{\star} \\
t_{\text {RGS2 }} & =t_{\text {RGS2 }}^{\star} \\
t_{\text {RGS } 3} & =t_{\text {RGS2 }}^{\star}+t_{\text {RGS3 }}^{\star} \\
t_{\text {RGS4 }} & =t_{\text {RGS } 4}^{\star}
\end{aligned}
$$




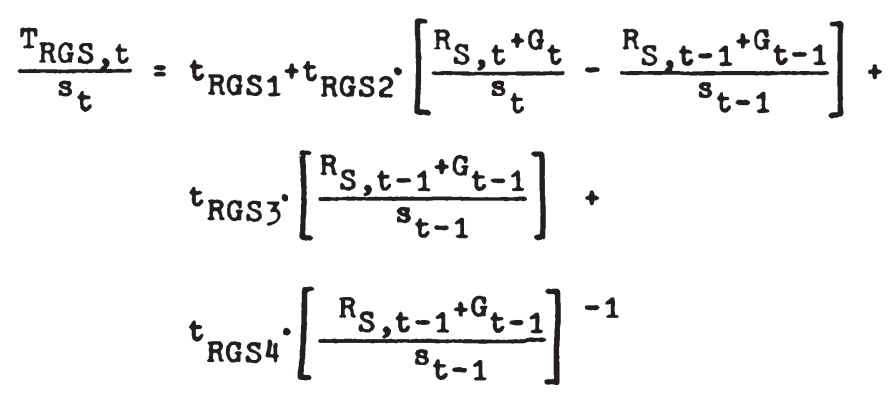

Zur Quantifizierung von (13.6) wurde das in der Bundesrepublik während der Jahre 1955 bis 1972 angefallene Aufkommen der veranlagten Einkommensteuer, der Körperschaftsteuer, der Kapitalertragsteuer und ab 1968 der Ergänzungsabgabe zur Einkommensteuer zusammengefaßt. Da die Einkommensteuerreform 1965 durch Abflachung der Progression im sog. "Mittelstandsbauch" des Einkommensteuertarifs einen Aufkommensverlust verursachte, der vom Bundesfinanzministerium für 1965 mit 0,600 Mrd. DM beziffert wurde (1), das zweite Steueränderungsgesetz von 1967 mit der Einführung der Ergänzungsabgabe zur Einkommensteuer aber wieder zu einem Mehraufkommen führte, das $1968 \quad 0,625$ Mrd. DM betrug, wurde das zusammengefaßte Aufkommen in den Jahren 1965, 1966 und 1967 um jeweils 0,600 Mrd. DM angenommener Steuerausfälle bereinigt, so daß die Kontinuität der rechnerischen Aufkommensentwicklung durch diese beiden Reformmaßnahmen nicht wesentlich gestört wurde (2). Anschließend wurden von dem bereinigten Gesamtaufkommen die hypothetischen Aufkommenswerte der für die Bundesrepublik fingierten Unternehmerlohnsteuer abgezogen, die im letzten Abschnitt ermittelt wurden (3). Die so erhaltenen Beträge repräsentieren das Aufkommen der Steuer auf das Besitzeinkommen der Selbständigen in der Modellwirtschaft (4).

Die Aufkommensentwicklung der hypothetisch abgegrenzten Besitzein-

(1) Vgl. Bundesministerium der Finanzen, Überblick über die Steuerrechtsänderungen in den Jahren 1964 und 1965, Finanzbericht 1966 des Bundesministeriums der Finanzen, Bonn, S. $208 \mathrm{ff}$.

(2) Wie sich im folgenden zeigt, sind damit die Auswirkungen der Einkommensteuerreform 1965 keineswegs völlig ausgeschaltet worden.

(3) Für die Jahre 1955 bis 1957 wurden die entsprechenden Beträge ohne Anwendung der im vorletzten Abschnitt ermittelten Lohnsteuerfunktion geschätzt.

(4) Die statistische Ermittlung der Größen $R_{S, t}$ und $G_{t}$ wurde bereits im Paragraphen 8 , Abschnitt 2 besprochen. 
kommensteuer weist zwischen 1955 und 1972 zwei deutliche Änderungstendenzen auf, die beide Male auf eine Abflachung der Aufkommensentwicklung hinauslaufen. Die erste Abflachung macht sich 1962/63 bemerkbar und ist wohl auf die Steueränderungsgesetze 1960 und 1961 zurückzuführen (1), die zweite Abflachung resultiert aus der Einkommensteuerreform 1965. Wegen dieser Tendenzumschwünge in der Aufkommensentwicklung wird die Schätzung von (13.6) trotz des damit verbundenen Verlustes von Freiheitsgraden für drei Teilperioden vorgenommen.

Die erste Teilperiode erstreckt sich von 1955 bis 1961; das zugehörige Regressionsergebnis lautet:

$$
\begin{aligned}
& \text { (13.7) } \quad t_{\text {RGS1 }}=-4089,17924 \text { (nicht signifikant) }{ }^{2} \text { DM p.c. } \\
& t_{\text {RGS2 }}=+0,30597( \pm 0,09313) \\
& t_{\text {RGS3 }}=+0,76597( \pm 0,12918) \\
& t_{\text {RGS } 4}=+7,31106 \cdot 10^{6}\left( \pm 4,07666 \cdot 10^{6}\right) \\
& R_{1.2,3,4}^{2}=0,99000 \quad \text { (Irrt.-Wahrsch.: }<1 \% \text { ) } \\
& R_{2 \cdot 3,4}^{2}=0,08809 \\
& R_{3 \cdot 2,4}^{2}=0,94381 \\
& \mathrm{R}_{4 \cdot 2,3}^{2}=0,94478 \\
& \text { VNQ }=3,294
\end{aligned}
$$

Diese Ergebnisse werden der Steuerfunktion in den drei ersten Jahren der Modellrechnung, 1960 bis 1962 , zugrunde gelegt.

Das hohe negative Absolutglied $\left(t_{R G S 1}\right)$ sowie der positive Schätzwert von $t_{\text {RGS4 }}$ in (13.7) verleihen der Steuerfunktion eine starke Aufkommens-

(1) Es ist unwahrscheinlich, daß diese erste Abflachung der Aufkommensentwicklung nach 1962 auf die einkommensteuerrechtliche Zulassung der degressiven Abschreibungsmöglichkeiten im Jahre 1958 zurückzuführen ist, die sich eher und allmählicher ausgewirkt haben müßte. Wahrscheinlich ist die Ursache in den steuerpolitischen Maßnahmen zur Förderung der Entwicklungshilfe bzw. der Auslandsinvestitionen zu suchen.

(2) Der Parameter ist erst bei einer Irrtumswahrscheinlichkeit von $7 \%$ signifikant von null zu unterscheiden. 
progression. Für den Bereich der Variablen-Durchschnitte ergibt sich nach (13.7) eine mittelfristige Aufkommenselastizität von +1, 896 (1). Andererseits zeigt der niedrige Schätzwert von tRGS2, daß das Steueraufkommen infolge der Aufkommensverzögerungen auf kurzfristige Schwankungen der steuerpflichtigen Besitzeinkommen schwächer reagiert als auf mittelfristige Schwankungen; für den Bereich der Variablen-Durchschnitte errechnet sich eine kurzfristige Aufkommenselastizität von +1, 101 (2). Der Unterschied zwischen der kurz- und mittelfristigen Aufkommenselastizität läßt vermuten, daß von der in der Modellwirtschaft erhobenen Besitzeinkommensteuer für die Dauer der Gültigkeit der in (13.7) geschätzten Parameter Wirkungen ausgehen, die etwaige konjunkturpolitische Bemühungen des Staates stören können.

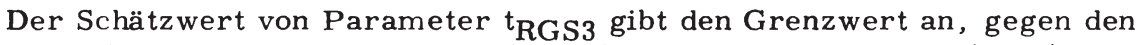
die Belastung der fundierten Selbständigen-Einkommen nach (13.6) auf die Dauer steigen müßte. Die Tatsache, daß dieser Wert über dem vom deutschen Einkommen- bzw. Körperschaftsteuerrecht her möglichen maximalen Grenzsteuersatz von $53 \%$ bzw. 51 \% liegt, illustriert die Konsequenzen, die mit der gewählten theoretischen Konstruktion einer Besitzeinkommensteuer verbunden sind. Da der ausgeschaltete Unternehmerlohn durch die ebenfalls ausgeschaltete Unternehmerlohnsteuer verhältnismäßig niedrig belastet wird, fällt die Belastung der verblei-

(1) Die mittelfristige Aufkommenselastizität wurde durch Einsetzen der Durchschnittswerte von $T_{R G S, t} / s_{t},\left(R_{S, t}+G_{t}\right) / s_{t}-\left(R_{S, t-1}+G_{t-1}\right) /$ $s_{t-1}$ und $\left(R_{S, t-1}+G_{t-1}\right) / s_{t-1}$ in die aus der steuerfunktion (13.6) abgeleitete Formel

$$
\begin{aligned}
& \epsilon_{T_{\text {RGS }}, m}= \\
& t_{R G S 2^{*}}\left[\frac{\left(R_{S, t}+G_{t}\right) / s_{t}-\left(R_{S, t-1}+G_{t-1}\right) / s_{t-1}}{T_{R G S, t} / s_{t}}\right]+ \\
& {\left[t_{R G S 3^{-}} t_{R G S 4^{0}}\left(\left\{R_{S, t-1}+G_{t-1}\right\} / s_{t-1}\right)^{-2}\right] \cdot\left[\frac{\left(R_{S, t-1}+G_{t-1}\right) / s_{t-1}}{T_{R G S, t} / s_{t}}\right]}
\end{aligned}
$$

berechnet. Sie gilt bei Annahme einer konstanten Zahl von selbständigen Erwerbspersonen auch für das aggregierte Steueraufkommen.

(2) Die kurzfristige Aufkommenselastizität ergibt sich durch Einsetzen der Durchschnittswerte von $T_{R G S, t} / s_{t}$ und $\left(R_{S, t}+G_{t}\right) / s_{t}$ in die durch partielle Differentiation aus $(13.6)$ abgeleitete Funktion

$$
\varepsilon_{T_{R G S} \cdot k}=t_{R G S 2^{\circ}}\left[\frac{\left(R_{S, t}+G_{t}\right) / s_{t}}{T_{R G S, t} t_{t}}\right]
$$


benden rechnerischen Besitzeinkommen der Selbständigen durch die hypothetisch abgegrenzte Besitzeinkommensteuer verhältnismäßig hoch aus; das trifft auf die durchschnittliche und die marginale Belastung der Bemessungsgröße zu, und zwar im Vergleich zu den Werten, die in der Bundesrepublik für die einkommen- und körperschaftsteuerliche Belastung der Selbständigen- und Unternehmenseinkommen insgesamt gelten. Aus diesem Grund, genau genommen wegen der niedrigen und nur schwach ansteigenden Grenzbelastung des Unternehmerlohnes, liegt auch der Schätzwert von ${ }_{\text {RGS3 }}$ über den maximalen Grenzsteuersätzen des deutschen Einkommen- und Körperschaftsteuerrechts. Bildet man aus dem hier geschätzten Parameter für den maximalen Grenzsteuersatz der Besitzeinkommensteuer, $t_{R G S 3}$, und dem entsprechenden Schätzwert für den (maximalen) Grenzsteuersatz der Unternehmerlohnsteuer, ${ }_{\text {LS2 }}$, einen gewogenen Durchschnitt, wobei die Größen des Besitzeinkommens und des Unternehmerlohnes als Gewichtungsfaktoren dienen, so erhält man jedoch wieder einen Durchschnittswert, der in der Nähe des einkommen- und körperschaftsteuerlichen Spitzensatzes der Bundesrepublik liegt. Ähnliches gilt für die Durchschnittsbelastung.

Daraus folgt: Für die Periode, auf die sich das zur Schätzung verwendete Material bezieht, und für die Parameterwerte, die bei der Schätzung erzielt wurden, ist die Konstruktion der Besitzeinkommensteuer weitgehend ein Problem der formalen Umrechnung auf eine modellmäßig geänderte Aggregatabgrenzung. Allerdings können aus der angenommenen Struktur zweier voneinander unabhängiger Steuern, die am Einkommen der Selbständigen ansetzen - hier eine Unternehmerlohnsteuer mit verhältnismäßig niedrigen Grenzsteuersätzen, dort eine Besitzeinkommensteuer mit verhältnismäßig hohen Grenzsteuersätzen - im Prinzip Aufkommenseffekte entstehen, die erheblich von der Aufkommensentwicklung in der Bundesrepublik abweichen, und zwar dann, wenn das Grössenverhältnis von rechnerischem Unternehmerlohn zu rechnerischem Besitzeinkommen der Selbständigen sich erheblich verschiebt (1).

(1) Um die Abweichungen zu vermeiden, hätte die rechnerische Besitzeinkommensteuer in der Modellformulierung anders konstruiert werden können: Als erstes wäre die Gesamtheit der auf die Selbständigen-Einkommen entfallenden direkten Steuern als eine Funktion der gesamten Selbständigen-Einkommen erfaßt worden. Die Rechnung wäre wieder in Pro-Kopf-Größen durchgeführt worden und hätte aufgrund der Schätzung eine hinreichend genaue Prognose des entsprechenden Pro-Kopf-Aufkommens für die Bundesrepublik erlaubt. Anschließend wäre das so bestimmte Steueraufkommen durch Abzug der fingierten Unternehmerlohnsteuer zerlegt worden, wobei der anfallende Restbetrag als Steuer auf das Besitzeinkommen der Selbständigen angesehen worden wäre. - Diese alternative Konstruktion hat $z$ weifellos den Vorteil größerer Übereinstimmung mit den 
Für die beiden folgenden Teilperioden konnte infolge der starken $\mathrm{Ab}$ flachung der Aufkommensentwicklung und der geringen Zahl der Freiheitsgrade Parameter ${ }_{\text {RGS3 }}$ der Aufkommensfunktion (13.6) nicht mehr signifikant geschätzt werden. Die vorausgegangenen steuerlichen Reformmaßnahmen hatten jedoch die Höhe der maximalen Grenzsteuer sätze nicht geändert. Aus diesem Grund wurde in den Schätzungen für beide Teilperioden der Parameterwert mit $+0,750$, praktisch also mit dem für die Jahre 1955 bis 1964 ermittelten Schätzwert, vorgegeben.

Die zweite Teilperiode erstreckt sich von 1962 bis 1966 (1); das Regressionsergebnis, das der Modellrechnung für die Jahre 1962 bis 1965 zugrunde gelegt wird, lautet:

empirischen Verhältnissen der Bundesrepublik; in Hinsicht auf die Modellanalyse läuft sie jedoch auch nur auf eine Kompromißlösung hinaus. Praktisch wird mit dem Vorgehen für die Modellwirtschaft die Annahme zweier voneinander unabhängiger Steuern aufgegeben; die Steuer auf die Besitzeinkommen der Selbständigen wird zu einem schwer kontrollierbaren steuerlichen Residuum, das sich für die modellmäßige Analyse spezieller steuerpolitischer Maßnahmen, insbesondere für die Analyse der Gewinnsteuerinzidenz wenig eignet. Es sei daher festgestellt, daß die Konstruktion einer Besitzeinkommensteuer zwangsläufig vor der Alternative einer stimmigen modellmäßigen Abbildung empirischer Verhältnisse oder einer gedanklich noch nachvollziehbaren theoretischen Analyse der Inzidenzproblematik stand. In dieser Wahl wurde die Entscheidung zugunsten der überschaubaren theoretischen Lösung getroffen.

(1) Es ist nicht eindeutig, ob das Jahr 1966 der zweiten oder der dritten Teilperiode zugeordnet werden soll. Zur Erhöhung der Zahl der Freiheitsgrade wurde es in beide Schätzungen einbezogen. 


$$
\begin{aligned}
& \text { (13.8) } \quad t_{\text {RGS1 }}=-8086,10322 \text { (nicht signifikant) DM p.c. } \\
& t_{\text {RGS2 }}=+0,25801 \quad( \pm 0,03662) \\
& t_{\text {RaS } 3}=+0,75000 \text { (exogen vorgegeben) } \\
& t_{\text {RGS }}=+42,16444.10^{6}\left( \pm 1,43678 \cdot 10^{6}\right) \\
& \mathbf{R}_{(1,3) \cdot 2,4}^{2}=0,99780 \text { (Irrt.-Wahrsch.: }<18 \text { ) } \\
& \mathbf{r}_{2 \cdot 4}^{2}=0,00007 \\
& \text { VNQ } \quad=4,504 \\
& R_{1 \cdot 2,(3), 4}^{2}=0,99419
\end{aligned}
$$

Für die dritte Teilperiode, die sich von 1966 bis 1972 erstreckt, lautet das Regressionsergebnis:

$$
\begin{aligned}
& \text { (13.9) } t_{\text {RGS1 }}=-15435,51955 \text { (nicht signifikant) DM p.c. } \\
& t_{\text {RaS2 }}=+0,11305( \pm 0,10780) \\
& t_{\text {RGS3 }}=+0,75000 \text { (exogen vorgegeben) } \\
& t_{\text {RGS }}=+125,52041 \cdot 10^{6}\left( \pm 11,75838 \cdot 10^{6}\right) \\
& R_{(1,3) \cdot 2,4}^{2}=0,96643 \text { (Irrt.-Wahrsch.: }<18 \text { ) } \\
& \mathbf{r}_{2 \cdot 4}^{2}=0,00001 \\
& \text { VNQ }=3,132 \\
& R_{1 \cdot 2,(3), 4=0,56333}^{2}
\end{aligned}
$$

In (13.8) und (13.9) beziehen sich die Korrelation $R_{(1,3)^{\bullet} 2,4}^{2}$ und die angegebenen Standardfehler auf die Schätzung einer transformierten Version von (13.6), bei der die Linearkombination der abhängigen Variablen mit der Variablen, deren Parameter exogen vorgegeben wurde, $T_{R G S, t} / s_{t}-t_{R G S 3}{ }^{*}\left(R_{S, t-1}+G_{T-1}\right) / s_{t-1}$, auf die beiden übrigen Variablen von (13.6) regressiert wurde. $R_{1}^{2} 2,(3), 4$ bezeichnet die Korrelation $z$ wischen den tatsächlichen Pro-Kopf-Aufkommenswerten der 
Besitzeinkommensteuer und den hypothetischen Pro-Kopf-Aufkommenswerten, die durch Einsetzen der vorgegebenen bzw. geschätzten Parameter sowie der jeweiligen Variablen-Werte in die ursprüngliche Gleichung (13.6) ermittelt wurden; es handelt sich wegen der exogenen Vorgabe eines Parameters um eine bedingte Korrelation.

Der wesentliche Unterschied zwischen den Regressionsergebnissen (13.8) und (13.9) einerseits und (13.7) andererseits besteht in der Größe der Schätzwerte von Parameter $t_{\text {RGS1 }}$ und $t_{\text {RGS4 }}$ Sowohl nach 1961 wie nach 1965 liegt der Schätzwert der Parameter absolut genommen merklich über dem vorherigen Niveau und bringt damit zum Ausdruck, daß die in der Bundesrepublik zu Beginn der zweiten und dritten Teilperiode einsetzenden steuerpolitischen Reformmaßnahmen (1) zu einer ganz erheblichen Entschärfung der Progressionseffekte führten, die bis 1961 mit der Aufkommensfunktion der hypothetisch abgegrenzten Besitzeinkommensteuer verbunden waren. Berechnet man für den Bereich der Variablen-Durchschnitte die Aufkommenselastizitäten, so erhält man für die Schätzperiode 1962 bis 1966 eine mittelfristige Aufkommenselastizität von +0,721 und eine kurzfristige Aufkommenselastizität von $+0,700$. Für die Schätzperiode 1966 bis 1972 beträgt die mittelfristige Aufkommenselastizität $+0,950$ und die kurzfristige Aufkommenselastizität t0, 424. Diese Werte liegen wesentlich unter den entsprechenden Werten für die erste Teilperiode und unter eins. Die Aufkommensentwicklung der hypothetisch abgegrenzten Besitzeinkommensteuer - ähnliches gilt für die Gesamtheit der auf die Selbständigen-Einkommen entfallenden direkten Steuern - verlief also nach 1962 quasi-regressiv (2). Die für

(1) $\mathrm{Zu}$ der Art der Reformmaßnahmen und dem Umfang der mit ihnen verbundenen finanziellen Auswirkungen vgl. die jährlichen Finanzberichte des Bundesministeriums der Finanzen sowie G. Hagemann, Aufkommenselastizitäten, a. a. O. , S. $107 \mathrm{ff}$, 121 ff., 186 und 191.

(2) Die Entwicklung ist, wie sich anhand der Veranlagungsstatistik für die Körperschaftsteuer nachweisen läßt, nicht auf die abnehmende Selbstfinanzierungsquote der Körperschaften in Zusammenhang mit dem gespaltenen Körperschaftsteuersatz zurückzuführen. Die auf diesen Tatbestand zurückzuführende Minderung des Körperschaftsteueraufkommens ist wesentlich geringer als die oben skizzierten Gesamtauswirkungen der Steuerreform. Vgl. Statistisches Jahrbuch für die Bundesrepublik Deutschland 1972, S. 415, woran anschlieBend sich die entsprechenden Körperschaftsteuerausfälle durch eine Standardisierungsrechnung ermitteln lassen. Hinzu kommt, daß wegen der einkommensteuerlichen Nachbelastung der ausgeschütteten Körperschaftsgewinne der Steuerausfall für den Gesamtbereich der Einkommensbesteuerung noch einmal wesentlich reduziert wird. 
die Jahre 1973 und 1974 vorliegenden Daten über das Aufkommen der verschiedenen Einkommensteuerarten bestätigen allerdings, was nach den Parameterwerten von (13.9) bereits zu erwarten ist: Nach der erneuten Abflachung der Entwicklung durch die Einkommensteuerreform 1965 tendiert das Aufkommen der hypothetisch abgegrenzten Besitzeinkommensteuer infolge des wirtschaftlichen Wachstums und der seit 1970 anhaltenden Inflation dazu, wieder zunehmend progressiv anzusteigen; ihre Aufkommenselastizitäten steigen merklich an.

Es soll nicht im voraus versucht werden, eine Aussage über die materielle Inzidenz der direkten Steuern abzuleiten. Doch können die Überlegungen dieses Abschnitts nicht abgeschlossen werden, ohne das modellmäßig beschriebene Verhalten des Staates im Bereich der Einkommensbesteuerung als solches einer ersten verteilungspolitischen Beurteilung zu unterziehen. Bei dieser Beurteilung darf nicht übersehen werden, daß infolge der direkten Progression und der Freibeträge die Einkommen aus unselbständiger Arbeit verhältnismäßig niedrig belastet wurden und die Vermögenserträge der Arbeitnehmer (weitgehend) (1) steuerfrei blieben. Auch darf nicht vergessen werden, daß die Reformen der sechziger Jahre nicht ausschließlich verteilungspolitischen Zielen dienten. Davon bleibt die verteilungspolitische Beurteilung der Reformmaßnahmen und ihrer besonderen Ausgestaltung jedoch unberührt. Die Tatsache, daß die Aufkommensentwicklung der hypothetisch abgegrenzten Besitzeinkommensteuer - ähnlich verhält es sich mit der Gesamtheit der an den Selbständigen(und Unternehmens-)Einkommen ansetzenden direkten Steuern - bis 1962 progressiv, dann aber bis 1970 regressiv, d.h. mit abnehmenden steuerlichen Durchschnittssätzen, verlief, während die Lohnsteuer und ähnlich die hypothetisch abgegrenzte Unternehmerlohnsteuer, abgesehen von den Jahren 1965 bis 1966, stets eine starke progressive Aufkommensentwicklung aufwies, zeigt, daß der Staat bei den während dieser Zeit durchgeführten Steuerreformen die fundierten Selbständigen-(und Unternehmens-) Einkommen bzw. die Selbständigen-(und Unternehmens-)Einkommen insgesamt wesentlich stärker als die Arbeits- bzw. Arbeitnehmereinkommen entlastete. Berechnet man mit Hilfe von (13.6) und mit Hilfe der in (13.7) für die Jahre 1955 bis 1961 geschätzten Parameter die jährlichen Steuerausfälle, die dem Staat durch diese Reformen allein im Bereich der hypothetisch abgegrenzten Steuer auf das Besitzeinkommen der Selbständigen entstanden sind, so erhält man für 1965 einen Wert von 4,1 Mrd. DM und für 1970 einen Wert von 24,4 Mrd. DM; das sind 1965 3,7\% und $197015,7 \%$ des gesamten Bruttoeinkommens der Selbständigen. Eine Vergleichsrechnung, bei der die Steuerfunktion (13.6) auf die Gesamtheit der Selbständigen-Einkommen (einschließlich Unternehmerlohn) und die

(1) Der Inhalt der Klammern gilt in den folgenden Überlegungen stets, sobald man die reinen Modellaussagen verläßt und sich den empirischen Verhältnissen der Bundesrepublik zuwendet. 
Gesamtheit der auf sie entfallenden Einkommensteuern (einschließlich Unternehmerlohnsteuer) bezogen wurde und bei der anschließend mit Hilfe der für die Periode 1955 bis 1961 geschätzten Parameter (1) das aggregierte Aufkommen von veranlagter Einkommensteuer, Körperschaftsteuer und Kapitalertragsteuer fortgerechnet wurde, führt für das Jahr 1965 zu entsprechenden Entlastungswerten von 5, 7 Mrd. DM bwz. 5, 0 \% und für das Jahr 1970 von 25,6 Mrd. DM bzw. 16,5\%. Die jährlichen Steuer ausfälle, die während der sechziger Jahre durch Reformen im Bereich der Arbeitnehmerlohnsteuer verursacht wurden, dürften demgegenüber nach einer überschlägigen Schätzung mit Rechtsstand 1960 als Vergleichsbasis im Jahre 1965 nicht mehr als 3,5 Mrd. DM und im Jahre 1970 nicht viel mehr als 6 Mrd. DM betragen haben; das sind jeweils 1,5 bzw. $1,6 \%$ des gesamten Arbeitnehmereinkommens. Gemessen an den steuerlichen Bestimmungen der Bundesrepublik von 1960 gilt die größere steuerliche Entlastung der Besitz- bzw. Selbständigen-Einkommen und die entsprechend geringere Entlastung der Arbeits-bzw. Arbeitnehmer-Einkommen also dem absoluten Betrag, $\Delta T$, wie der Prozentgröße, $\Delta t$, der Einkommensentlastung nach; sie besteht selbst dann noch, wenn die prozentuale Steuerentlastung, $\Delta t$, ins Verhältnis zur jeweils herrschenden steuerlichen Durchschnittsbelastung, $t$, gesetzt wird, also der relativen Änderung der steuerlichen Durchschnittsbelastung, $\Delta t / t$, nach (2) (3).

(1) Der zu t ${ }_{\text {RGS3 }}$ analoge Parameter für die maximale Grenzbelastung der Selbständigen - und Unternehmenseinkommen erhielt in der Schätzung einen Wert, der mit 0,56 nahe an den maximalen Grenzsteuersätzen des deutschen Einkommen- bzw. Körperschaftsteuerrechts liegt.

(2) Im Fall von Steuern mit direkter Progression bringen Erhöhungen steuerlicher Freibeträge oder allgemeine Tarifsenkungen für Steuerpflichtige mit einem hohen Wert der steuerlichen Bemessungsgrundlage stets eine höhere einkommensmäßige Steuerersparnis mit sich als für Steuerpflichtige mit einem niedrigen Wert der steuerlichen Bemessungsgrundlage. Die unterschiedliche Höhe der Entlastungseffekte braucht unter dem Gesichtspunkt der persönlichen Leistungsfähigkeit nicht dem Prinzip steuerlicher Gleichbehandlung zu widersprechen. Unter Verteilungsgesichtspunkten stellt sich aber die Frage, ob die Erhöhungen der Freibeträge oder - im Zusammenhang mit bestehenden Freibetragsregelungen - die allgemeinen Tarifsenkungen oder etwaige strukturelle Änderungen des progressiven Tarifverlaufs sich überhaupt am Kriterium der steuerlichen Leistungsfähigkeit, wie es sich zum Zeitpunkt der Reform unmittelbar darstellt, ausrichten und ob sie nicht falsch dosiert bzw. strukturiert sind. Diese Frage ist im Zusammenhang mit einer Beurteilung der genannten Reformmaßnahmen und der von ihnen ausgehenden formalen Umverteilungseffekte, wie sie oben festgestellt wurden, sicher einer genaueren Untersuchung wert.

(3) Vgl. auch Jahresgutachten 1972 des Sachverständigenrates zur Begutachtung der gesamtwirtschaftlichen Entwicklung, a. a. O. , Tz. 461. 
Man erinnert sich vom vorigen Paragraphen her, daß für die Zeit ab 1963 auch im Bereich der Gewerbeertragsteuer eine fühlbare Entlastung des (Unternehmens-bzw.) Selbständigen-Sektors einsetzte; außerdem bleibt noch im nächsten Abschnitt festzustellen, daß im Bereich der Vermögensteuer ab 1962 ebenfalls Steuererleichterungen wirksam wurden, die (hauptsächlich) den Selbständigen zugute gekommen sind. Alle diese Beobachtungen bestärken den Eindruck, daß die Steuerreformen, mit denen der Staat während der sechziger Jahre einen Anstieg der gesamtwirtschaftlichen Steuerlastquote vermied, in ihren unmittelbaren formalen Auswirkungen die Tendenz verfolgten, einen weiteren Anstieg der Einkommensbelastung, wie er durch die progressive Ausgestaltung des gesamten Steuersystems verursacht wird, bei den Selbständigen- bzw. fundierten Selbständigen-Einkommen zu verhindern, bei den Arbeitnehmer-Einkommen aber zuzulassen.

\section{Die Steuer auf das Selbständigen-Vermögen}

Zur Ergänzung der Besitzeinkommensteuer erhebt der Staat in der Modellwirtschaft eine Vermögensteuer. Diese Steuer erfaßt, da das Vermögen der Unselbständigen aus verteilungspolitischen Gründen steuerfrei bleibt, allein das Nominalvermögen der Selbständigen-Haushalte (1), F $\mathrm{S}_{\mathrm{S}} \mathrm{t}$, und belastet es mit einem bestimmten Prozentsatz der Bestandsgröße (Vermögensertragsteuer). Ihr Tarif verläuft infolge persönlicher Freibeträge stark progressiv. Ihr Aufkommen, $T_{F, t}$, folgt der Entwicklung der Bemessungsgröße wegen meist verspäteter Vermögensfeststellung mit einer Verzögerung von einem Jahr, d. h. die Bemessungsgröße bilden die Jahresendbestände des vorletzten Jahres.

Die Aufkommensfunktion der Vermögensteuer wird in Pro-Kopf-Größen formuliert und lautet:

(1) Diese modellmäßige Charakterisierung der deutschen Vermögensteuer stützt sich auf die Tatsache, daß das Steuergesetz vermögensteuerliche Freibeträge gewährt, deren Höhe in den sechziger Jahren weit über dem Durchschnittswert der steuerpflichtigen Arbeitnehmer-Vermögen gelegen haben dürfte. Vgl. hierzu $₫ 5$ des deutschen Vermögensteuergesetzes in der vor und nach dem Steueränderungsgesetz 1961 geltenden Fassung sowie Tabelle $24 \mathrm{X}$ in W. Krelle, J. Schunck u. J. Siebke, Überbetriebliche Ertragsbeteiligung der Arbeitnehmer, a. a. O. , Bd. II, S. 443, wo die Vermögensverteilung nach der Größe der Vermögen angegeben ist, die die privaten Haushalte 1960 in der Bundesrepublik besaßen. 
(13.10)

$$
\begin{aligned}
\frac{T_{F, t}}{s_{t}}= & t_{F 1}+ \\
& t_{F 2} \cdot\left[\frac{F_{S, t-2}}{s_{t-1}}\right]^{-1}+ \\
& t_{F 3} \cdot\left[\frac{F_{S, t-2}}{s_{t-1}}\right]
\end{aligned}
$$

Wegen der Bemessung der Vermögensteuer am Nominalvermögen der Selbständigen-Haushalte bleiben Bewertungsgewinne auf den bestehenden Vermögensbestand bzw. die Substanzerhaltungsrücklagen der Unternehmen anders als im Vermögensteuerrecht der Bundesrepublik (1) steuerlich außer Ansatz. Dies führt bei der Quantifizierung der Aufkommensfunktion zu einer gewissen Verzerrung der geschätzten Parameterwerte und verursacht im Fall sich ändernder Preissteigerurısraten Ungenauigkeiten bei der Fortrechnung der zu erwartenden Aufkommensbeträge, sobald die zeitlichen Grenzen der jeweiligen Schätzperiode überschritten oder die finanziellen Auswirkungen von Tarifänderungen untersucht werden.

Zur Quantifizierung von (13.10) wurde das Aufkommen der Vermögenund der Erbschaftsteuer (2) in der Bundesrepublik (3) zusammengefaßt und genauso wie das geschätzte Nominalvermögen der SelbständigenHaushalte durch die Zahl der selbständigen Erwerbspersonen dividiert. Dabei zeigte sich, daß das aggregierte Pro-Kopf-Aufkommen der beiden Steuern in seiner Entwicklung zwischen 1962 und 1963 einen merklichen Bruch aufweist. Dieser Bruch ist die Auswirkung des Steueränderungsgesetzes 1961, das zu einer wesentlichen Erhöhung der vermögensteuer-

(1) Die bewertungsrechtlichen Vorschriften des deutschen Vermögensteuerrechts laufen, wenn auch nicht exakt, auf eine steuerliche Erfassung des laufenden Vermögenswertes hinaus. Vgl. A. Oberhauser, Bewertungsprobleme im Steuerrecht, Handwörterbuch der Sozialwissenschaften, Bd. 12, S. $544 \mathrm{ff}$. Der vorhandene Bestand des Nominalvermögens, m. a. W. , das zeitliche Aggregat der vergangenen Nominalersparnis, bleibt dagegen von laufenden Preissteigerungen unberührt.

(2) Im Gegensatz zur mikroökonomischen Betrachtung kann die Erbschaftsteuer in der gesamtwirtschaftlichen Analyse als eine kontinuierliche Belastung des gesamtwirtschaftlichen Vermögensbestandes angesehen werden. Die dennoch verbleibenden Unterschiede zwischen den beiden Steuern hinsichtlich der Reaktionen der Steuerpflichtigen werden hier vernachlässigt.

(3) Die Angaben über das Aufkommen der Vermögen- und Erbschaftsteuer wurden dem Statist. Jahrbuch für die BRD sowie den Finanzberichten des Bundesministeriums der Finanzen entnommen. 
lichen Freibeträge führte und nach den Schätzungen des Bundesfinanzministeriums im Jahre 1963 erstmals einen Steuerausfall von 0, $235 \mathrm{Mrd}$. DM mit sich brachte (1). Wegen der nachhaltigen Aufkommenseffekte der Steuerreform 1961 wurde (13.10) ungeachtet sonstiger kleiner Tarifkorrekturen für zwei Schätzperioden quantifiziert.

Außerdem zeigte sich, daß das aggregierte Aufkommen der beiden Steuern deutlich durch den dreijährigen vermögensteuerlichen Veranlagungsrhythmus der Bundesrepublik geprägt ist. Aus diesem Grund wurde die Aufkommensfunktion um zwel Dummy-Variablen ergänzt. Statt (13.10) wurde die Aufkommensfunktion nun formuliert als:

$$
\begin{aligned}
\frac{T_{F, t}}{s_{t}}= & t_{F 1}+t_{F 2} \cdot\left[\frac{F_{S, t-2}}{s_{t-1}}\right]^{-1}+ \\
& {\left[t_{F 3}+t_{F 4} \cdot \text { Dummy }_{1, t}+t_{F 5} \cdot \text { Dummy }_{2, t}\right] \cdot\left[\frac{F_{S, t-2}}{s_{t-1}}\right] }
\end{aligned}
$$

Dummy $_{1, t}$ ist im ersten Jahr nach einer Vermögensteuerveranlagung und Dummy $2, \mathrm{t}$ im zweiten Jahr nach einer vermögensteuerlichen Veranlagung gleich eins; im Jahr einer vermögensteuerlichen Veranlagung selbst haben beide Dummy-Variablen den Wert null (2).

Gleichung (13.11) wurde auf beiden Seiten durch den Wert von $\mathrm{F}_{\mathrm{S}, \mathrm{t}-1} / \mathrm{s}_{\mathrm{t}-1}$ dividiert und ergab als Regressionsansatz:

$$
\begin{aligned}
\frac{T_{F, t} \cdot s_{t-1}}{s_{t} \cdot F_{S, t-2}}= & t_{P 1} \cdot\left[\frac{F_{S, t-2}}{s_{t-1}}\right]^{-1}+t_{F 2} \cdot\left[\frac{F_{S, t-2}}{s_{t-1}}\right]^{-2} \\
& t_{F 3}+t_{F 4} \cdot \text { Dummy }_{1, t}+ \\
& t_{F 5} \cdot \text { Dummy }_{2, t}
\end{aligned}
$$

Die erste Schätzperiode erstreckte sich von 1955 bis 1963 . Hierbei wurde

(1) Vgl. Bundesministerium der Finanzen, Die steuerpolitischen Maßnahmen im Jahr 1961, Finanzbericht 1962 des Bundesministeriums der Finanzen, Bonn, S. 154

(2) Während der ersten Schätzperiode wirkt sich störend aus, daß der vermögensteuerliche Veranlagungszeitraum in der Bundesrepublik zu Anfang, nämlich zwischen 1953 und 1957, einmal vier statt drei Jahre betrug. 
für das Jahr 1963 durch Zuschlag des vom Bundesfinanzministerium geschätzten Minderaufkommens zum tatsächlichen Steueraufkommen ein korrigierter, für den alten Steuerrechtsstand geltender Aufkommenswert gebildet. Das Regressionsergebnis, das in der Modellrechnung für die Jahre 1960 bis 1962 zugrundegelegt wird, lautet:

$$
\text { (13.13) } \begin{array}{ll}
t_{F 1}=-307,03613 & ( \pm 89,82929) \text { DM p.c. } \\
t_{F 2}=+3,83933 \cdot 106 & ( \pm 1,70680 \cdot 106) \\
t_{F 3}=+0,00984 & ( \pm 0,00111) \\
t_{F 4}=+0,00007 & ( \pm 0,00019) \\
t_{F 5}=+0,00059 & ( \pm 0,00018) \\
R_{1 \cdot 2,3,4,5}^{2}=0,96734 & \text { (Irrt.-Wahrsch.:<1\%) } \\
R_{2 \cdot 3,4,5}^{2}=0,98664 & \\
R_{3 \cdot 2,4,5}^{2}=0,98664 & \\
R_{4 \cdot 2,3,5}^{2}=0,32867 & \\
R_{5 \cdot 2,3,4}^{2}=0,27123 & \\
v N Q & =1,884
\end{array}
$$

Vernachlässigt man die Auswirkungen des vermögensteuerlichen Veranlagungsrhythmus, entspricht dem Regressionsergebnis für den Bereich der Variablen-Durchschnitte eine Aufkommenselastizität (1) von +1,687 bezogen auf das Wachstum des geschätzten Pro-Kopf-Vermögens der Selbständigen.

Die zwei te Schätzperiode erstreckt sich von 1962 bis 1971. Hierbei wurde das vom Bundesfinanzministerium für 1963 geschätzte Steuerminderaufkommen von dem tatsächlichen Steueraufkommen des Jahres 1962 abgezogen und so ein korrigierter, dem neuen Rechtsstand entsprechender Aufkommensbetrag für das Jahr 1962 berechnet. Das Regressionsergebnis, das der Modellrechnung für die Jahre ab 1963 zugrunde liegt, lautet:

(1) Die Berechnung der mittelfristigen Aufkommenselastizität im Anschluß an (13.10) verläuft abgesehen von der Aufkommensverzögerung wie bei der Lohnsteuer. 


$$
\begin{aligned}
& \text { (13.14) } \quad t_{\mathrm{P} 1}=-435,32031 \quad( \pm 154,56752) \mathrm{DM} \text { p.c. } \\
& t_{P 2}=+17,66575 \cdot 10^{6}\left( \pm 6,29707 \cdot 10^{6}\right) \\
& t_{\mathrm{P3}}=+0,00780 \quad( \pm 0,00092) \\
& t_{\mathrm{P4}}=+0,00039 \quad( \pm 0,00014) \\
& t_{\mathrm{F} 5}=+0,00005 \quad( \pm 0,00014) \\
& R_{1.2,3,4,5}^{2}=0,76474 \quad \text { (Irrt.-Wahrsch.: }<8 x \text { ) } \\
& \mathrm{R}_{2 \cdot 3,4,5}^{2}=0,98803 \\
& R_{3 \cdot 2,4,5}^{2}=0,98803 \\
& R_{4 \cdot 2,3,5}^{2}=0,30052 \\
& R_{5 \cdot 2,3,4}^{2}=0,35844 \\
& \text { vNQ }=2,336
\end{aligned}
$$

Dem Regressionsergebnis entspricht für den Bereich der VariablenDurchschnitte eine Aufkommenselastizität von $+1,081$ bezogen auf das Wachstum des geschätzten Pro-Kopf-Vermögens der Selbständigen.

Die Berücksichtigung des vermögensteuerlichen Veranlagungsrhythmus bzw. der beiden Dummy-Variablen diente lediglich einer genaueren Schätzung der Parameter $t_{F 1}$ bis $t_{F 3}$. In der Modellrechnung wird der Einfluß vernachlässigt, indem die beiden Parameter $t_{F 4}$ und $t_{F 5}$ selbst gleich null gesetzt werden, Parameter $t_{F 3}$ aber um ein Drittel des Wertes von $\left(t_{F} 4^{+t} F 5\right)$ erhöht wird (1) (2). Korrigiert man den Schätzwert von $t_{F} 3$ in dieser Weise, so liegt der Parameter in beiden Teilperioden mit 0,010 bzw, 0,008 in der Nähe des in der Bundesrepublik jenseits der persönlichen Freibeträge geltenden Vermögensteuersatzes von $1 \%$;

(1) Wenn nach (13.11) der Parameter von $F_{S, t-2} / s_{t-1}$ im Jahr einer Veranlagung $t_{F 3}$, im ersten Jahr nach einer Veranlagung $\left(t_{F 3}+t_{F 4}\right)$ und im zweiten Jahr nach einer Veranlagung $\left(t_{F 3} 3^{+t_{F 5}}\right)$ beträgt, so beträgt der Parameter im Durchschnitt der drei Jahre $t_{F 3}+\left(t_{F 4}{ }^{+t_{F}}\right) / 3$.

(2) Die unterschiedliche Struktur von $t_{F 4}$ und $t_{F 5}$ in den beiden Schätzperioden deutet auf unterschiedliche Auswirkungen des Veranlagungsrhythmus bzw. auf eine Verkürzung des vermögensteuerlichen Aufkommens-Lags. 
dabei hat der Zuschlag der Erbschaftsteuer infolge des verhältnismäßig geringen Aufkommenswertes nur einen geringen Einfluß auf den Wert des Parameters.

Der Schätzwert von $t_{F 1}$ gibt hauptsächlich den Einfluß der durch das Steueränderungsgesetz 1961 erhöhten Vermögensteuerfreibeträge wieder. Zusammen mit dem Schätzwert von $t_{F 2}$ bringt er eine Erscheinung zum Ausdruck, die ähnlich bereits bei der Aufkommensentwicklung der Gewerbeertragsteuer beobachtet wurde. Bei einem großen Teil der Selbständigen lag das Pro-Kopf-Vermögen vor 1962 erheblich unter der vermögensteuerlichen Freibetragsgrenze. Die Tatsache, daß von diesen Personen im Laufe des wirtschaftlichen Wachstums ständig etliche mit ihrem Pro-Kopf-Vermögen über die Freibetragsgrenze hinaus gerieten, verursachte einen überlinearen Verlauf der Aufkommensentwicklung, wobei die Marginalbelastung des durchschnittlichen Pro-Kopf-Vermögens nach (13.10) gegen den Schätzwert von Parameter tF3 strebte. Die von 1963 an wirksame Erhöhung der steuerlichen Freibeträge führte dazu, daß dieser Annäherungsprozeß, der sich 1962 bereits in starkem Umfang vollzogen hatte, erneut in Gang gesetzt wurde. Ab 1963 setzte also eine Aufkommensentwicklung mit niedrigerer, aber stark ansteigender Marginalbealstung des durchschnittlichen Pro-Kopf-Vermögens ein. Entsprechend waren mit der Erhöhung der Vermögensteuerfreibeträge anfangs die vom Bundesfinanzministerium geschätzten Steuerausfälle verbunden; die Steuerausfälle stiegen aber von 1963 an permanent an und nähern sich auf die Dauer einem absoluten Grenzwert, der durch das Produkt aus Erhöhung des Vermögensteuerfreibetrages, Zahl der steuerpflichtigen natürlichen Personen und Vermögensteuersatz bestimmt wird und in der Modellbetrachtung $t_{F 1} \cdot s_{t}$ betrăgt. Berechnet man mit Hilfe von (13.11) und (13.13) die Aufkommensbeträge, die nach 1962 ohne Erhöhung der Freibeträge angefallen wären, und zieht von ihnen die tatsächlichen Aufkommensbeträge ab, erhält man Schätzwerte für die verursachten jährlichen Steuerausfälle, die 1965 144,25 DM pro Kopf der selbständigen Erwerbspersonen oder aggregiert 0,766 Mrd. DM und 1970 244,32 DM pro Kopf der selbständigen Erwerbspersonen oder aggregiert 1,166 Mrd. DM betragen. 
1. Die Sozialversicherungsbeiträge

Um die finanzielle Absicherung laufender sozialer Risiken und altersbedingter Zukunftsbedürfnisse zu erzwingen und die Zahl der auf staatliche Fürsorge angewiesenen Personen gering zu halten, verpflichtet der Staat die Arbeitnehmer der Modellwirtschaft zur Mitgliedschaft in einer staatlichen Sozialversicherung. Mit der Mitgliedschaft ist die Zwangsabgabe von Sozialversicherungsbeiträgen verbunden, die sich nach der Höhe des jeweiligen Lohneinkommens der Arbeitnehmer richten. Aggregierte Bemessungsgrundlage des gesamten Aufkommens an Sozialversicherungsbeiträgen bildet also das Bruttoeinkommen aus unselbständiger Arbeit, das die Arbeitnehmer im privaten und staatlichen Sektor verdienen; die Aufkommensentwicklung ist eine lineare Funktion der aggregierten Bemessungsgrundlage.

Bezeichnen TSV, $t$ das Beitragsaufkommen der staatlichen Sozialversicherung und $\mathrm{L}_{\mathrm{A}}, \mathrm{t}$ das Bruttoeinkommen aus unselbständiger Arbeit der Modellwirtschaft, dann lautet die Aufkommensfunktion:

\section{(14.1) $\quad T_{S V, t}={ }^{t_{S V 1}}{ }^{+t_{S V 2}} \mathrm{~L}_{\mathrm{A}, \mathrm{t}}$}

wobei $\mathrm{t}_{\mathrm{SV}} 1$ wegen der tariflichen Proportionalität im allgemeinen einen Wert nahe null haben müßte.

Zur Quantifizierung wurde das Beitragsaufkommen der staatlichen Sozialversicherung erfaßt als die Summe der direkten Steuern und Sozialversicherungsbeiträge, die auf dem Umverteilungskonto der privaten Haushalte in der volkswirtschaftlichen Gesamtrechnung der Bundesrepublik ausgewiesen wird, minus dem Aufkommen von Lohnsteuer, veranlagter Einkommensteuer, Ergänzungsabgabe zur Einkommensteuer, Kapitalertragsteuer, Vermögensteuer und Erbschaftsteuer (1). Die Vermögensteuer und die Ergänzungsabgabe hätten für die privaten Haushalte an sich nur anteilig verrechnet werden dürfen; die Erbschaftsteuer hätte, da sie in der Volkswirtschaftlichen Gesamtrechnung unter den Vermögensübertragungen verbucht wird, korrekterweise gar nicht in Abzug gebracht werden dürfen. Da nach der Volkswirtschaftlichen Gesamtrechnung aber in den direkten Steuern der privaten Haushalte auch direkte Verbrauchsteuern

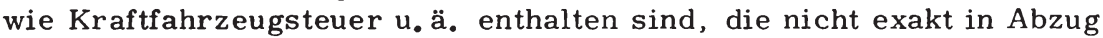

(1) Der errechnete Betrag liegt im allgemeinen geringfügig über der Summe der von den privaten Haushalten geleisteten Sozialversicherungsbeiträge, wie sie in den Ergänzungstabellen zur Volkswirtschaftlichen Gesamtrechnung im Statistischen Jahrbuch für die Bundesrepublik ausgewiesen wird. 
gebracht werden konnten, wurde mit der Verrechnung der Posten eine gewisse rechnerische Kompensation vorgenommen.

Wie im Bereich der meisten Steuern wirkten sich im Aufkommen der Sozialversicherungsbeiträge Reformmaßnahmen des Staates aus und zwangen dazu, die Aufkommensfunktion für mehrere Teilperioden zu schätzen. Die wichtigste Reformmaßnahme während der Untersuchungsperiode war die sukzessive Anhebung der Beitragssätze zur gesetzlichen Rentenversicherung $z$ wischen 1968 und 1973, mit der die aus der Änderung der Erwerbsquote resultierende Finanzierungslücke der Rentenversicherungsträger gedeckt werden sollte. Da schon zuvor in anderen Bereichen der Sozialversicherungsbeiträge Tariferhöhungen eintraten (1) und der Kreis der Pflichtversicherten ausgeweitet wurde, brach die Aufkommensentwicklung, die nach Wirksamwerden der Rentenversicherungs-Neuregelungs-Gesetze von 1957 ziemlich gleichmäßig verlaufen war, bereits nach 1965 ab und stieg anschließend wesentlich schneller als zuvor an.

Für die erste Teilperiode, die sich über die Jahre 1957 bis einschließlich 1965 erstreckt, wurde die Aufkommensfunktion (14.1) durch direkte Anwendung der Kleinst-Quadrat-Methode geschätzt. Das Ergebnis lautet:

$$
\text { (14.2) } \begin{aligned}
{ }^{t} t_{\text {SV1 }} & =+4,25152 \quad( \pm 0,43675) \text { Mrd. DM } \\
{ }_{\text {SV2 }}=+0,17788 \quad( \pm 0,00252) & \\
r_{1.2}^{2} & =0,99880 \quad \text { (Irrt.-Wahrsch.: }<0,18) \\
\text { vNQ } & =2,665
\end{aligned}
$$

Um die während der zweiten Periode eingetretenen sukzessiven Beitragserhöhungen als exogen vorgegebene staatliche Maßnahmen zu erfassen, wurde für die Jahre 1966 bis 1972 die durchschnittliche Beitragsbelastung der Arbeitnehmer-Löhne, $\mathrm{T}_{\mathrm{SV}, \mathrm{t}} / \mathrm{L}_{\mathrm{A}, \mathrm{t}}$, berechnet und auf die Zeit regressiert:

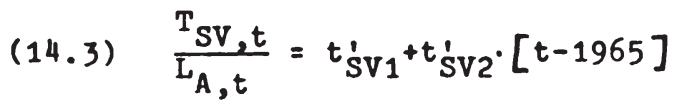

Das zugehörige Regressionsergebnis lautet:

(1) Vgl. Bundesministerium für Arbeit und Sozialordnung, Sozialbudget 1969/70 der Bundesrepublik Deutschland, Bonn 1970, S. 221 f. 


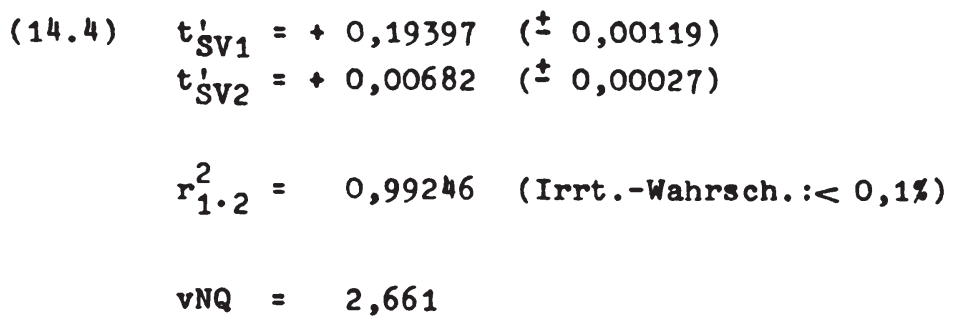

Mit Hilfe von (14.3) und (14.4) werden für die einzelnen Jahre der Teilperiode hypothetische Werte der durchschnittlichen Beitragsbelastung, $\mathrm{T}_{\mathrm{SV}, t} / \mathrm{L}_{\mathrm{A}, t}$, gebildet. Diese werden in der Modellformulierung als die jeweiligen staatliche fixierten Werte von Parameter tSV2 vorgegeben; gleichzeitig wird der Wert von Parameter tSV1 gleich null gesetzt.

Ab 1973 gelten in der Modellformulierung die Parameterwerte:

$$
\text { (14.5) } \quad \begin{aligned}
t_{\text {SV1 }} & =+4,25152 \text { Mrd. DM } \\
{ }^{t} & =+0,25800
\end{aligned}
$$

Damit wird unterstellt, daß ab 1973 die Phase der sukzessiven Beitragserhöhungen abgeschlossen ist und weiterhin wieder konstante tarifliche Beitragssätze gelten. Der Wert von Parameter $\mathrm{t}_{\mathrm{SV} 1}$ ist in der in (14.2) geschätzten Höhe angesetzt.

\section{Die Vermögenserträge des Staates}

Die Einkommen des Staates aus Unternehmertätigkeit und Vermögen werden in der Modellrechnung etwas anders als in der Volkswirtschaftlichen Gesamtrechnung der Bundesrepublik angesetzt. Es wird davon ausgegangen, daß diese Einkommen in der Modellwirtschaft ausschließlich aus dem Zinsertrag, $R_{S t, t}$, bestehen, den der Staat auf seinen Netto-

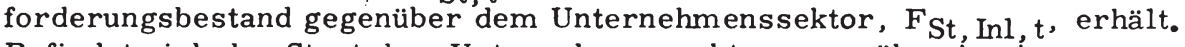
Befindet sich der Staat dem Unternehmenssektor gegenüber in einer Nettoschuldenposition, hat er selbst einen entsprechenden Kapitaldienst zu leisten; die Variablen $F_{S t, I n l, t}$ und $R_{S t, t}$ erhalten dann beide einen negativen Wert.

Die vom Unternehmenssektor empfangenen oder an diesen geleisteten Vermögenserträge des Staates errechnen sich durch die Verzinsung der staatlichen Nettoforderungs - oder -schuldenposition mit dem vollen Kapitalmarktzins (1). Wegen der Annahme einer nachschüssigen Verzin-

(1) Dies bedeutet implizit eine Abwicklung aller Kreditbeziehungen mit dem Unternehmenssektor in langfristigen Titeln. 
sung gilt:

$$
\text { (14.6) } \quad R_{S t, t}=r_{t-1} \cdot F_{S t, I n l, t-1}
$$

Gleichung (14.6) wurde bereits als Preisgleichung (2.51) im Paragraphen über die definitorischen Grundgleichungen der Modellformulierung aufgeführt. Dort wurde auch darauf hingewiesen, daß auf den stets positiven Kapitalexport des Staates in die übrige Welt keine Vermögenserträge anfallen, weil dieser Kapitalexport in der Modellwirtschaft als unentgeltliche Kapitalhilfe gewährt wird.

Da zum Zeitpunkt der Materialzusammenstellung keine zuverlässigen Angaben über die Höhe des staatlichen Nettoforderungsbestandes gegenüber dem privaten Sektor vorlagen, wurde der entsprechende Bestand für Anfang des Jahres 1950 verhältnismäßig willkürlich mit 5,0 Mrd. DM angesetzt (1). Von diesem Ausgangswert wurde mit Hilfe der im folgenden Abschnitt besprochenen Angaben über die staatliche Kreditaufnahme im privaten Sektor, $\Delta F_{S t}$, Inl, $t$, entsprechend Definition (2.58) eine hypothetische Zeitreihe für die Entwicklung des staatlichen Nettoforderungsbestandes in den Jahren 1950 bis 1971 berechnet. Durch Mulitplikation des jeweiligen Vorjahresendbestandes mit der Umlaufsrendite festverzinslicher Wertpapiere in der Vorperiode ergaben sich die jeweiligen Vermögenserträge des Staates. Die Summe dieser Erträge ist stets gröBer als das vom Unternehmenssektor empfangene Einkommen des Staates aus Unternehmertätigkeit und Vermögen in der Volkswirtschaftlichen Gesamtrechnung der Bundesrepublik. Die Differenz zwischen beiden Posten wurde daher bei der Aufbereitung des statistischen Materials als staatliche Zinssubvention an die Empfänger staatlicher Finanzinvestitionen oder als versteckte Dividendensubventionen an öffentliche Unternehmen angesehen und auf der Ausgabenseite des öffentlichen Haushaltes den Übertragungen des Staates an den Unternehmenssektor zugeschlagen.

(1) Mittlerweile hat die Deutsche Bundesbank in ihren Monatsberichten Angaben über das Geldvermögen und die Verpflichtungen der öffentlichen Haushalte veröffentlicht. Vgl. Deutsche Bundesbank, Entwicklung des Geldvermögens und der Verpflichtungen 1950 bis 1971 , a. a. O. , S. 37. Danach hätte statt der oben angenommenen Nettoforderungsposition von 5,0 Mrd. DM von einer Nettoschuldenposition des Staates gegenüber dem Unternehmenssektor von 8,0 Mrd. DM ausgegangen werden müssen, die vor allem dadurch entstand, daß der Staat bei der Währungsreform den Finanzinstitutionen des Unternehmenssektors gegenüber Ausgleichsverpflichtungen in Höhe von ca. $17 \mathrm{Mrd}$. DM übernahm. 
In der Modellformulierung werden alle Kreditbeziehungen, die der Staat mit dem privaten Sektor eingeht, zu einer einheitlichen Aggregatgröße zusammengefaßt. Es wird also nicht unterschieden zwischen staatlicher Schuldaufnahme und Darlehensrückflüssen aus dem privaten Sektor oder zwischen staatlicher Schuldentilgung und staatlicher Kreditvergabe an den privaten Sektor. Betrachtet wird lediglich die Änderung der staatlichen Nettoforderungsposition dem privaten Sektor gegenüber. Da der Staat in der Modellwirtschaft annahmegemäß keine Kassenbestände hält (1), ist diese Änderung identisch mit der Differenz aus dem Finanzierungsüberschuß des Staates in der Volkswirtschaftlichen Gesamtrechnung und dem staatlichen Nettokapitalexport in die übrige Welt.

Die Nettokreditaufnahme des Staates im privaten Sektor ist in der Modellwirtschaft ein integraler Bestandteil der staatlichen Budgetpolitik: Der Staat bestimmt den Umfang seines Leistungsangebotes an den privaten Sektor. Anschließend entscheidet er über die Finanzierung dieses Leistungsangebotes durch Steuern oder staatliche Verschuldung bzw. darüber, in welchem Umfang dem privaten Sektor aus den laufenden Staatseinnahmen Kredite (Finanzinvestitionen) zur Verfügung gestellt werden sollen. Die Summe aus laufenden Budgeteinnahmen und (positiver bzw. negativer) Nettokreditaufnahme im privaten Sektor ergibt das Budgetvolumen und bestimmt das laufende staatliche Ausgabevolumen.

Die Hypothese hinsichtlich des Verhaltens der staatlichen Kreditpolitik läßt sich für den allgemeinen Fall in folgender Weise präzisieren: Die staatliche Nettokreditaufnahme im privaten Sektor wird in einem bestimmten Verhältnis zum Umfang der bisher bereits besprochenen laufenden Staatseinnahmen geplant. Da sich die Haushaltsplanung in der jeweiligen Vorperiode und in Ungewißheit über die unmittelbare Zukunftsentwicklung vollzieht, orientiert sich die Kreditaufnahme an der zuverlässig abschätzbaren Einnahmesituation dieser Vorperiode. Zusätzlich macht der Staat die Nettokreditaufnahme im privaten Sektor aber von der kurzfristig angetroffenen konjunkturellen Situation der laufenden Periode abhängig, die er bei der Haushaltsplanung bereits in gewissem Umfang vorhergesehen haben mag. Dabei reduziert er die Nettokreditaufnahme

(1) Zur Rechtfertigung der Annahme sei darauf hingewiesen, daß das Verhältnis von Kassenbeständen zu Transaktionsvolumen im staatlichen Sektor der Bundesrepublik wesentlich niedriger ist als im privaten Sektor. Vgl. die konsolidierte Bilanz des Bankensystems und den Bilanzausweis der Deutschen Bundesbank in deren Monatsberichten. Die Hauptursache dafür liegt wahrscheinlich in dem gesetzlich verankerten Recht des Staates zur Inanspruchnahme von Kassenkrediten der Notenbank, wodurch der Staat dem Zwang, hohe Kassenbestände zu halten, enthoben ist. 
oder erhöht die Nettokreditvergabe bzw. die Staatsschuldentilgung in Zeiten überschäumender Konjunktur, in denen ihm bei gegebenen Steuertarifen auch ein über die mittelfristigen Aufkommenserwartungen hinausgehendes laufendes Einnahmevolumen zur Verfügung steht; und er erhöht die Nettokreditaufnahme oder schränkt die Nettokreditvergabe bzw. die Staatsschuldentilgung ein, sobald die Konjunktur abflaut und sich von der Entwicklung der laufenden Einnahmen her Finanzierungsengpässe in der Haushaltsrechnung ergeben. Diese Konjunkturabhängigkeit der staatlichen Kreditpolitik resultiert also aus den jeweiligen fiskalischen $\mathrm{Be}$ dürfnissen des Staates und aus der Anwendung der staatlichen Kreditpolitik als Instrument der gesamtwirtschaftlichen Nachfragestabilisierung (1).

Das beschriebene Verhalten des Staates im Kreditverkehr mit dem Unternehmenssektor wird im Modell formalisiert ausgedrückt durch Verhaltensfunktion (14.7):

$$
\text { (14.7) } \Delta F_{S t, \text { Inl }, t}=h_{F 1} \cdot H_{1, t-1}+h_{F 2} \cdot\left[x_{t} \cdot p_{x, t}-x_{0, t} \cdot p_{x, t-1}\right]
$$

Hierbei gibt $\Delta$ F St, Inl, $t$ die Änderung des Nettoforderungsbestandes gegenüber dem privaten Sektor an. Im Fall einer Nettokreditaufnahme erhält die Variable ein negatives, im Fall einer Kreditvergabe ein positives Vorzeichen. $\mathrm{H}_{1}$, $\mathrm{t}$ kennzeichnet das Haushaltsvolumen vor Berücksichtigung laufender Kreditabschlüsse mit dem privaten Sektor, umfaßt also das Aufkommen an indirekten und direkten Steuern, an Sozialversicherungsbeiträgen und staatlichen Vermögenserträgen.

(14.8)

$$
\begin{aligned}
H_{1, t}= & T_{C, t}+T_{R G, t}+T_{L, t}+T_{k, t}+T_{I m, t}{ }^{+} \\
& T_{L A, t}+T_{L S, t}+T_{R G S, t}{ }^{+T_{F, t}}+T_{S V, t}{ }^{+R_{S t}, t}
\end{aligned}
$$

$\left(\mathrm{xt}^{\bullet} \mathrm{px}, \mathrm{t}\right)$ ist die monetäre Gesamtnachfrage nach Produkten des Unternehmenssektors; $\left(\mathrm{x}_{\mathrm{O}}, \mathrm{t} \cdot \mathrm{p}_{\mathrm{x}, \mathrm{t}-1}\right)$ ist der Plan-output des Unternehmenssektors, bewertet mit den Absatzpreisen der Vorperiode. Die Differenz beider Größen, die auf der rechten Seite von (14.7) in eckigen Klammern auftritt, symbolisiert den Nachfrageüberhang im privaten

(1) Vgl. W. Albers, Staatsverschuldung und Geld- und Kreditpolitik, Finanzarchiv, NF 21, 1961, S. 25 ff. - Zu dem in der Literatur weniger abgehandelten Einsatz der Staatsschuldentilgung in der Hochkonjunktur vgl. A. Andel, Probleme der Staatsschuldentilgung, Berlin 1964; H. Hirsch, Die Finanzpolitik im Aufschwung, besonders im Hinblick auf das Problem der Staatsschuldentilgung, Finanzarchiv, NF 23, 1964, S. 394 ff. sowie A. Oberhauser, Staatsschuldentilgung als finanzpolitisches Instrument, Finanzarchiv, NF 24, 1965, S. $283 \mathrm{ff}$. 
Sektor (1).

Stellt man einmal weniger auf die fiskalische als auf die konjunkturpolitische Bedeutung der staatlichen Kreditpolitik ab, dann zeigt (14.7), daß der Staat mit seinem kreditpolitischen Instrumentarium nicht nur auf Abweichungen von der angemessenen Kapazitätsauslastung des Unternehmenssektors $\left(x_{t} \neq x_{0}, t\right)$ reagiert, sondern auch auf inflationäre Tendenzen $\left(\mathrm{p}_{\mathrm{x}, \mathrm{t}} \neq \mathrm{p}_{\mathrm{x}, \mathrm{t}-1}\right)$, die nicht mit konjunkturellen Schwankungen des Kapazitätsauslastungsgrades gekoppelt sein müssen, sondern die Folge anderer Ursachen wie z. B. der gewerkschaftlichen Lohnpolitik sein können. Die Gleichung zeigt aber auch das Dilemma, dem sich der Staat in Situationen gegenübersieht, die durch Kapazitätsunterauslastung mit inflationärem Preisauftrieb gekennzeichnet sind (Stagflation); ist $x_{t}<x_{0, t}$, aber auch $p_{x, t}>p_{x, t-1}$ und $x_{t} \cdot p_{x, t}=x_{0, t} \cdot p_{x, t-1}$, dann enthält sich der Staat des kreditpolitischen Mitteleinsatzes zur Bekämpfung dieser Situation und nimmt nur die für die konjunkturell ausgeglichene Situation geplanten kreditpolitischen Maßnahmen vor.

Zur Schätzung der Verhaltensfunktion (14.7) für die staatliche Kreditpolitik wurde der staatliche Finanzierungssaldo der volkswirtschaftichen Gesamtrechnung der Bundesrepublik um den Saldo des öffentlichen Kapitalverkehrs mit dem Ausland, wie er in der Zahlungsbilanzstatistik der Deutschen Bundesbank ausgewiesen wird (2), bereinigt und ergab auf diese Weise eine Zeitreihe für die Größe $\Delta F_{S t}$, Inl, t. Das Haushaltsvolumen $\mathrm{H}_{1}, \mathrm{t}$ wurde entsprechend Definition (14.8) aus dem bisher benutzten statistischen Material ermittelt. Für die Plankapazität bzw. den Planoutput des Unternehmenssektors, $x_{0, t}$, wurden die im vierten Paragraphen geschätzten Werte herangezogen.

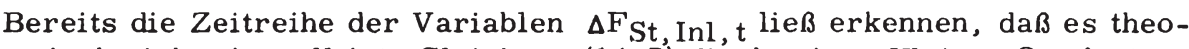
retisch nicht sinnvoll ist, Gleichung (14.7) direkt einer Kleinst-QuadratSchätzung zu unterziehen. Bis Mitte der sechziger Jahre hatte der Staat in der Bundesrepublik stets eine positive Geldvermögensbildung betrieben. Der Umfang dieser Geldvermögensbildung sank zwischen 1960 und 1965 absolut und im Verhältnis zum Haushaltsvolumen trendmäßig ab und deutet an, daß sich eine allmähliche Umorientierung in der staatlichen Kreditpolitik vollzog, die zweckmäßigerweise durch die Änderung eines der entsprechenden staatlichen Verhaltensparameter, hier des Parameters $h_{F 1}$ von (14.7), ausgedrückt werden sollte. Aus diesem Grunde wurde (14.7) auf beiden Seiten durch $\mathrm{H}_{1, t-1}$ dividiert:

(1) Wegen der Möglichkeit kosteninduzierter Preissteigerungen wird der Nachfrageüberhang durch die Differenz nicht exakt ausgedrückt.

(2) Vgl. Deutsche Bundesbank, Die Zahlungsbilanz der Bundesrepublik im Jahre 1972 nach Regionen, Statistische Beihefte zu den Monatsberichten der Deutschen Bundesbank, Reihe 3, Zahlungsbilanzstatistik, Nr. 7, Juli 1973, Beilage, S. 2 f. 
(14.9) $\frac{\Delta \mathrm{F}_{\mathrm{St}, \mathrm{In} 1, t}}{\mathrm{H}_{1, t-1}}=\mathrm{h}_{\mathrm{P} 1}+\mathrm{h}_{\mathrm{F} 2} \cdot\left[\frac{\mathrm{x}_{t} \cdot \mathrm{p}_{\mathrm{x}, \mathrm{t}}-\mathrm{x}_{0, t} \cdot \mathrm{p}_{\mathrm{x}, \mathrm{t}-1}}{\mathrm{H}_{1, t-1}}\right]$

und anschließend um eine Zeitvariable ergänzt:

(14.10)

$$
\begin{aligned}
\frac{\Delta F_{S t, I n 1, t}}{H_{1, t-1}}=h_{F 1}+h_{F 2} \cdot\left[\frac{x_{t} p_{x, t}-x_{0, t} p_{x, t-1}}{H_{1, t-1}}\right] \\
+h_{F 3} \cdot[t-1960]
\end{aligned}
$$

Gleichung (14.10) wurde für zwei Teilperioden geschätzt. Die erste Schätzung erstreckte sich auf die Jahre 1960 bis 1964. Das Regressionsergebnis lautet:

$$
\text { (14.11) } \begin{array}{ll}
\mathrm{h}_{\mathrm{F} 1}=+0,07002 & ( \pm 0,03309) \\
\mathrm{h}_{\mathrm{F} 2}=+0,02667 & ( \pm 0.18132) \\
\mathrm{h}_{\mathrm{F} 3}=-0.01536 & ( \pm 0,00582) \\
\mathrm{R}_{1.2 .3}^{2}=0,89171 & \text { (Irrt.-Wahrsch.:<11\%) } \\
\mathrm{r}_{2.3}^{2}=0,54140 & \\
\operatorname{vNQ} & =2,543
\end{array}
$$

Die Schätzwerte von Parameter $h_{\mathrm{F} 1}$ und $h_{\mathrm{F} 3}$ sind bei einer Irrtumswahrscheinlichkeit von 17 bzw. $12 \%$ signifikant. Der Wert von Parameter von $h_{F 2}$ ist verhältnismäßig gering und wegen der geringen $Z$ ahl von $z$ wei verbleibenden Freiheitsgraden erst bei einer Irrtumswahrscheinlichkeit von $90 \%$ von null zu unterscheiden, hat jedoch eine plausible Größenordnung. Die Werte zeigen insgesamt, daß die staatlichen Kreditoperationen während der frühen sechziger Jahre nicht bzw. nicht in erheblichem Umfang in den Dienst einer kurzfristigen Stabilisierungspolitik gestellt wurden.

Die zweite Teilperiode, die sich von 1965 bis 1971 erstreckt, in die also auch das Rezessionsjahr 1967 fällt, ist dadurch gekennzeichnet, daß die staatliche Kreditpolitik in der Bundesrepublik erstmals bewußt als Instrument zur Kompensation gesamtwirtschaftlicher Nachfrageschwankungen eingesetzt wurde (1). Zugleich ist nicht zu übersehen, daß sich im ersten Jahr dieser Periode, also 1965, ein abrupter Niveaubruch im Umfang der staatlichen Kreditoperationen vollzog, der auf eine kurzfristige Abnahme 
der staatlichen Kreditvergabe und/oder auf eine kurzfristige Zunahme der staatlichen Kreditaufnahme hinauslief und der wohl im Zusammenhang mit der Einkommensteuerreform 1965 und den durch sie verursachten Steuerausfällen gesehen werden muß. Vergleicht man den hypothetischen Wert des staatlichen Finanzierungssaldos $\Delta \mathrm{F}_{\mathrm{St}}$, Inl, $t$, wie er sich aufgrund von Regressionsschätzung (14.11) für das Jahr 1965 ergibt, mit dem tatsächlichen Wert, so erhält man diesem gegenüber einen Differenzbetrag von +1,529 Mrd. DM. Dieser plötzliche Abbau der staatlichen Nettokreditvergabe dürfte wesentlich mit zur Überhitzung der konjunkturellen Situation im Jahre 1965 beigetragen haben; es handelt sich allerdings nicht um die einzige destabilisierende Maßnahme der staatlichen Finanzpolitik im Jahre 1965, wie sich noch zeigen wird (2).

Bei der Kleinst-Quadrat-Schätzung für die Teilperiode 1965 bis 1971 erhielt Parameter $h_{\mathrm{F} 3}$ keinen signifikanten Schätzwert, weswegen man für diese zweite Teilperiode den in den frühen sechziger Jahren eingeleiteten Prozeß der kreditpolitischen Umorientierung der staatlichen Finanzpolitik als abgeschlossen ansehen kann. Die Regressionsschätzung von (14.10) unter der Annahme $h_{\mathrm{F} 3}=0 \mathrm{bzw}$. die Regressionsschätzung von (14.9) brachte als Ergebnis:

$$
\text { (14.12) } \begin{aligned}
\mathrm{h}_{\mathrm{F} 1}=-0,02621 & ( \pm 0,00513) \\
\mathrm{h}_{\mathrm{F} 2} & =+0,17644 \quad( \pm 0,03534) \\
r_{1.2}^{2} & =0,83293 \quad \text { (Irrt. - Wahrsch. : }<0,5 \%) \\
\text { vNQ } & =3,184
\end{aligned}
$$

Entsprechend finanzierte der Staat einen Anteil von gut 2,5\% seines gesamten Haushaltes unabhängig von der jeweiligen konjunkturellen Lage auf dem Wege der öffentlichen Verschuldung. Einem konjunkturellen Nachfrageausfall im Unternehmenssektor begegnet er mit einer zusätzlichen Nettokreditaufnahme in Höhe von etwa $18 \%$ des Nachfrageausfalls, einem entspre chenden Nachfrageüberhang mit einer zusätzlichen Nettokreditvergabe oder einer entsprechenden Schuldentilgung in Höhe von $18 \%$ des Nachfrageüberhangs. Um diese Angabe über die konjunkturpolitische Dosierung der öffentlichen Kreditpolitik richtig zu beurteilen, muß man sich vorstellen, daß die laufenden Einnahmen des Staates im Fall einer aggregierten Aufkommenselastizität von nahe eins sich in

(1) Unter dem Gesichtspunkt des bewußten Einsatzes wird man wohl eher die Verschuldung des Bundes während des Rezessionsjahres 1967 als die Tilgung der aufgenommenen Schuldbeträge sehen, die hauptsächlich in das Jahr 1969 fiel.

(2) Vgl. Paragraph 16, Abschnitt 3 über die staatliche Auslandshilfe. 
etwa proportional zum Umfang der monetären Gesam tnachfrage verhalten. Würde der Staat im Fall eines allgemeinen Nachfrageausfalls versuchen, sein mittelfristig geplantes Ausgabenvolumen aufrechtzuerhalten, müßte er sich mit einem Betrag verschulden, der dem Produkt aus aggregiertem Nachfrageausfall und mittelfristigem Anteil des Staates an der monetären Gesamtnachfrage gleichkäme. Dieser Staatsanteil an der gesamtwirtschaftlichen Nachfrage beträgt in der Bundesrepublik etwa ein Drittel und liegt über dem Wert von Parameter $h_{F 2}$ in (14.12). Die Annahme der Regressionsergebnisse (14.12) für die Modellrechnung bedeutet daher, daß der Staat der Modellwirtschaft sich bei konjunkturellen Nachfrageausfällen nicht genügend stark verschuldet, um sein mittelfristig geplantes Ausgabevolumen aufrecht halten zu können, und daß er sich vom Standpunkt einer konjunkturneutralen Ausgabenpolitik, etwa im Sinne des deutschen Sachverständigenrates zur Begutachtung der gesamtwirtschaftlichen Entwicklung, prozyklisch verhält. Dies wird hier als Folge des finanzpolitischen Verhaltens der untergeordneten staatlichen Gebietskörperschaften angesehen und im Modell als realistische Annahme über das aggregierte Verhalten der staatlichen Kreditpolitik angesehen (1).

(1) Die Art, in der hier das kreditpolitische Verhalten des Staates dargestellt wird, insbesondere die Annahme, der Staat halte keinerlei Kassenbestände, bedeutet für die Modellformulierung den Verzicht auf eine explizite Berücksichtigung von staatlichen Konjunkturausgleichsrücklagen. Da die Bildung bzw. Auflösung dieser Rücklagen zu dem modernen stabilitätspolitischen Instrumentarium des Staates gehört und in der Bundesrepublik seit 1969 angewandt wird, sei darauf aufmerksam gemacht, daß sich die von ihnen ausgehenden Wirkungen mit der vorliegenden Modellformulierung durchaus analysieren lassen. Die Bildung und Auflösung derartiger Rücklagen haben Einkommens - und Geldmengeneffekte. Ihre Einkommenseffekte sind die gleichen wie die der in diesem Abschnitt beschriebenen konjunkturabhängigen staatlichen Kreditpolitik. Ihre Liquiditätsoder Geldmengeeffekte sind ihrem Charakter nach die gleichen, die von der Liquiditätspolitik der Notenbank ausgehen. Abgesehen von der Verlagerung der Liquiditäts - bzw. Geldmengeneffekte aus dem Bereich des öffentlichen Haushalts in den Bereich der Notenbank lassen sich Bildung und Auflösung von staatlichen Konjunkturausgleichsrücklagen also durch eine kombinierte Änderung mehrerer Modellparameter, insbesondere des Parameters $h_{F 2}$ von (14.9) und des Parameters $\sigma_{1}$ der Liquiditätsversorgungsfunktion (9. 3), darstellen. 
1. Die Besoldung der öffentlichen Bediensteten

Nachdem auf der Grundlage der steuerpolitischen und kreditpolitischen Entscheidungen des Staates das gesamte Volumen der Budgeteinnahmen bestimmt ist, richten sich die weiteren staatlichen Verhaltensfunktionen auf die Bestimmung der einzelnen Budgetausgabeposten. Als erster Ausgabenblock werden die öffentlichen Personalausgaben behandelt, weil die Beschäftigung und Besoldung öffentlicher Bediensteter die wichtigste technische Voraussetzung für ein geordnetes Funktionieren des Staatsapparates ist (1). Die Entwicklung dieses Ausgabenblocks unterliegt zwei, im Modell voneinander unabhängigen, politischen Entscheidungen, der Festlegung der öffentlichen Besoldungspolitik und der Bestimmung des zu realisierenden öffentlichen Personalbedarfs.

Bei der Besoldung der öffentlichen Bediensteten orientiert sich der Staat an den Entlohnungsverhältnissen im privaten Sektor. Er übernimmt das Lohnniveau des privaten Sektors, gewährt auf dieses Lohnniveau aber als Entgelt für den im Durchschnitt höheren Ausbildungsgrad der öffentlichen Bediensteten, zur attraktiven Ausgestaltung des öffentlichen Dienstes oder aus ähnlichen Gründen ein positives Lohndifferential. Dieses Lohndifferential, das in Prozent des Lohnniveaus im privaten Sektor ausgedrückt wird, stellt den besoldungspolitischen Aktionsparameter des Staates dar.

Für den Lohnsatz im staatlichen Sektor, ${ }^{1} \mathrm{St}, t$, gilt in der Modellformulierung:

\section{(15.1) $\quad{ }^{1_{\text {St }, t}}=\mathrm{h}_{\text {LSt }, t^{\cdot}} \mathrm{I}_{\mathrm{pr}, \mathrm{t}}$}

Die modellmäßige Erfassung des besoldungspolitischen staatlichen Verhaltens reduziert sich also darauf, eine Funktion für die Entwicklung der Lohnrelation bzw. des staatlichen Aktionsparameters $\mathrm{h}_{\mathrm{LSt}, \mathrm{t}}$ aufzustellen. Da das besoldungspolitische Verhalten des Staates zu den modellexogenen Größen gehört, wird die Relation $\mathrm{h}_{\mathrm{LSt}, \mathrm{t}}$ als eine Funktion der Zeit angesehen.

(15.2) $\quad h_{\text {LSt }, t}=h_{\text {LSt } 1}+h_{\text {LSt } 2} \cdot[t-1960]+h_{L S t 3} \cdot[t-1962]^{-1}$

wobei $\mathrm{h}_{\mathrm{LSt1}}, \ldots, \mathrm{h}_{\mathrm{LSt3}}$ Verhaltenskonstanten darstellen.

(1) Von der Vorstellung einer staatlichen Produktionsfunktion her würde man hier auch einen Teil des staatlichen Anlagevermögens, also Verwaltungsbauten etc. anführen müssen. 
Zur Quantifizierung von (15.2) wurde folgendes statistisches Material herangezogen: Der volkswirtschaftlichen Gesamtrechnung für die Bundesrepublik wurden die Angaben über die vom Staat an inländische Arbeitskräfte gezahlten Einkommen aus unselbständiger Arbeit entnommen. Diese Werte wurden durch die Angaben des Statistischen Jahrbuchs über die Beschäftigtenzahl der staatlichen und kommunalen Gebietskörperschaften und der von ihnen abhängigen Wirtschaftsunternehmen ohne eigene Rechtsperson (1) dividiert und ergaben den Lohnsatz des staatlichen Sektors, $1_{S t, t}$. Die Division des staatlichen Lohnsatzes, $1_{S t, t}$, durch den bereits früher besprochenen Lohnsatz des privaten Sektors, $l_{\mathrm{pr}, \mathrm{t}}$, ergab die Werte der Lohnrelation $\mathrm{h}_{\mathrm{LSt}, \mathrm{t}}$.

Die erhaltene Zeitreihe für die Lohnrelation zwischen dem öffentlichen und dem privaten Sektor läßt für die Bundesrepublik zwei Phasen der Besoldungsentwicklung im öffentlichen Dienst erkennen. In den Jahren 1960 bis 1964 verlief die Lohnrelation im Trend linear und fast stationär. Die Schätzung wurde für diese Jahre unter der Annahme $\mathrm{h}_{\mathrm{LSt} 3}=0$ vorgenommen und führte zu den Schätzergebnissen (15.3):

$$
\text { (15.3) } \begin{array}{ll}
n_{\text {LSt } 1}=+1,75643 \quad & ( \pm 0,01421) \\
n_{\text {LSt2 }}=-0,00645 \quad( \pm 0,00580) & \\
r_{1.2}^{2}=0,29206 & (\text { Irrt.-Wahrsch.: }<35 \%) \\
\text { vNQ } & =4,338
\end{array}
$$

Zwischen 1964 und 1971 muß sich in der Bundesrepublik eine allgemeine Besserstellung der Beschäftigten des öffentlichen Dienstes im Vergleich zu denen des privaten Sektors und/oder eine Änderung der öffentlichen Beschäftigungsstruktur in Richtung auf einen zunehmenden Anteil qualifizierter Beschäftigter vollzogen haben. Die Lohnrelation $\mathrm{h}_{\mathrm{LSt}} \mathrm{t}$ näherte sich während dieser Zeit asymptotisch einem höheren Niveau. Es wurde angenommen, daß das angestrebte Niveau stationär verläuft und Parameter $\mathrm{h}_{\mathrm{LSt} 2}$ dementsprechend gleich null ist. Um ein starkes Abweichen der Lohnrelation von der allgemeinen Entwicklungstendenz im Jahre 1970 auszuschalten, wurde eine Dummy-Variable eingeführt, die 1970 den

(1) Die Angaben enthalten nicht die Beschäftigten der Bundesanstalt für Arbeit und der Sozialversicherungen sowie das militärische Personal der Bundeswehr und des Bundesgrenzschutzes. Vgl. Statistisches Jahrbuch für die Bundesrepublik Deutschland, Kapital Öffentliche Finanzwirtschaft. Sie sind insoweit im Modell zu gering angesetzt. Entsprechend ist auch der ermittelte Lohnsatz des staatlichen Sektors absolut und in Relation zum Lohnsatz des privaten Sektors zu hoch. 
Wert eins, ansonsten den Wert null erhielt und deren Regressionsparameter als $\mathrm{h}_{\mathrm{LSt} 4}$ bezeichnet wurde. Das Ergebnis der Kleinst-QuadratSchätzung für die Jahre 1964 bis 1971 lautet (1):

$$
\begin{aligned}
& \text { (15.4) } \quad h_{\text {LSt } 1}=+1,87210( \pm 0,00429) \\
& \mathrm{h}_{\text {LSt } 3}=-0,30551 \quad( \pm 0,01568) \\
& h_{\text {LSt } 4}=-0,03349 \quad( \pm 0,00585) \\
& \mathrm{R}_{1 \cdot 2,3}^{2}=0,98701 \quad \text { (Irrt. }- \text { Wahrsch.: }<0,18 \text { ) } \\
& r_{2 \cdot 3}^{2}=0,10086 \\
& \text { VNQ }=2,251
\end{aligned}
$$

Der Modellrechnung werden für die Jahre 1960 bis 1963 die in (15. 3) und für die Jahre ab 1964 die in (15.4) geschätzten Parameterwerte zugrunde gelegt.

\section{Das staatliche Beschäftigungsvolumen}

Bei der Bestimmung des öffentlichen Personalbedarfs geht der Staat der Modellwirtschaft von dem Anteil des öffentlichen Budgets aus, den die staatlichen Lohn- und Gehaltszahlungen in der Vergangenheit für sich beansprucht haben. Er überprüft, welche Ausweitung dieses Personalkostenanteils am Haushaltsvolumen sich nach Abstimmung mit der Einnahmeentwicklung und der Entwicklung des übrigen Bedarfs vertreten läßt bzw. welche Einschränkung des Anteil erforderlich wird.

Bezeichnet man den Personalkostenanteil am öffentlichen Haushaltsvolumen während der Beschäftigungsplanungsperiode $t-1$ mit $h_{A S t, t-1}$, dann gilt für diese Periode:

$$
\text { (15.5) }\left[a_{S t, t-1} \cdot 1_{S t, t-1}\right]=h_{\text {ASt }, t-1} \cdot H_{2, t-1}
$$

wobei a St, $t-1$ die Zahl der öffentlichen Bediensteten, $1_{S t, t-1}$ der Lohnsatz im öffentlichen Dienst und $\mathrm{H}_{2}, \mathrm{t}-1$ das staatliche Haushaltsvolumen nach Berücksichtigung der Neuabschlüsse im staatlichen Kreditverkehr mit dem privaten Sektor bezeichnet:

(1) Der Wert der Lohnrelation 1964 läßt sich beiden Schätzperioden zuordnen. Zur Erhöhung der Zahl der Freiheitsgrade wurde das Jahr in beide Schätzperioden einbezogen. 


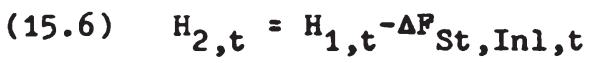

und $\mathrm{H}_{1}, \mathrm{t}-1$ wiederum gemäß (14.8) definiert ist.

Berücksichtigt man, daß grundlegende Änderungen der staatlichen Beschäftigung nicht von heute auf morgen, sondern allmählich und im Laufe einer Reihe von Jahren realisiert werden, können allmähliche Änderungen des Haushaltsanteils $h_{\text {LSt, } t}$ in (15.1) unterstellt werden, und statt (15.1) läßt sich als erstes eine Verhaltensfunktion

$$
\text { (15.7) } \quad\left[a_{\text {St }, t-1}{ }^{1}{ }_{\text {St }, t-1}\right]=h_{\text {ASt } 1}{ }^{+h_{A S t 2}}{ }^{*} H_{2, t-1}
$$

formulieren, in der $\mathrm{h}_{\mathrm{ASt} 1}$ und $\mathrm{h}_{\mathrm{ASt} 2}$ Verhaltenskonstanten darstellen.

In (15.7) wird eine Verzögerung eingeführt, indem der Zeitindex der Variablen ast, $t-1$ um ein Jahr erhöht wird. Danach gilt als'staatliche Arbeitsnachfrage- oder Beschäftigungsfunktion:

$$
\left[\mathrm{a}_{S t, t}{ }^{1_{S t}, t-1}\right]=\mathrm{h}_{\mathrm{ASt} 1}^{*}+\mathrm{h}_{\mathrm{ASt} 2}^{*} \mathrm{H}_{2, t-1}
$$

bzw. nach einer Umformung:

$$
a_{S t, t}=h_{A S t 1}^{* *} 1_{S t, t-1}^{-1} h_{A S t 2}^{* *} \cdot\left[H_{2, t-1} \cdot 1_{S t, t-1}^{-1}\right]
$$

Diese staatliche Beschäftigungsfunktion beruht auf der vom Staat vorgenommenen Planung eines bestimmten sich trendmäßig entwickelnden Personalkostenanteils am öffentlichen Haushalt (1) und macht die staatliche Beschäftigung vom Haushaltsvolumen und dem staatlichen Lohnsatz in der jeweils vorausgegangenen Planungsperiode abhängig. Die Funktion enthält eine nicht unerhebliche theoretische Vereinfachung, die hier in Kauf genommen wird; implizit wird nämlich angenommen, daß der Staat nach (15.9) genau die in der Vorperiode geplante Beschäftigung zu realisieren versucht und daß $\mathrm{ihm}$ dies wegen einer ausreichenden Attraktivität des öffentlichen Dienstes auch stets gelingt. Außerdem enthält die Funktion einen weiteren Schönheitsfehler, der im folgenden jedoch ausgemerzt werden soll.

Angenommen, der Staat beschäftige eine gleichbleibende Zahl von Arbeitnehmern und lasse das öffentliche Haushaltsvolumen unverändert wachsen. - Erhöht er in dieser Situation die Besoldung der öffentlichen Bediensteten im Vergleich zu denen des privaten Sektors nachhaltig um

(1) Der Personalkostenanteil hat damit bei der öffentlichen Arbeitsnachfrage in gewisser Weise eine ähnliche Bedeutung wie bei der grenzproduktivitätstheoretisch bestimmten privaten Arbeitsnachfrage. 
einen bestimmten Prozentsatz, dann steigt der staatliche Lohnsatz $1_{S t, t-1}$ auf der linken Seite von (15.8) stärker an, als er normalerweise im Laufe der wirtschaftlichen Entwicklung gestiegen wäre; ebenso muß auf der rechten Seite der Gleichung der Wert der beiden Parameter $\mathrm{h}_{\text {ASt1 }}$ und $\mathrm{h}_{\text {ASt2 }} \mathrm{zwangsläufig} \mathrm{steigen,} \mathrm{weil} \mathrm{sich} \mathrm{der} \mathrm{Personalkostenanteil} \mathrm{am} \mathrm{öffent-}$ lichen Haushaltsvolumen zugleich erhöht. Die beiden Parameter von (15.8) sind also nicht unabhängig von den besoldungspolitischen Entscheidungen des Staates; sie sind vielmehr als proportional zur Lohnrelation $\mathrm{h}_{\mathrm{LSt}, \mathrm{t}}$ zwischen staatlichem und privatem Sektor anzusehen:

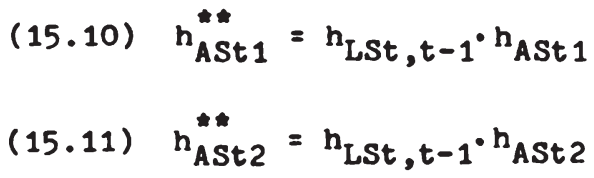

Setzt man (15.10) und (15.11) sowie (15.1) in (15.8) bzw. (15.9) ein, erhält man

$$
\text { (15.12) }\left[a_{\text {St }, t} \cdot 1_{\text {pr }, t-1}\right]=h_{\text {ASt } 1}{ }^{+h_{A S t 2}}{ }^{\cdot H_{2}, t-1}
$$

bzw.

$$
\text { (15.13) } a_{\text {St }, t}=h_{\text {ASt } 1} \cdot 1_{\mathrm{pr}, t-1}^{-1}+h_{\text {ASt } 2} \cdot\left[\mathrm{H}_{2, t-1} \cdot 1_{\mathrm{pr}, t-1}^{-1}\right]
$$

In dieser Form ist die staatliche Arbeitsnachfrage- bzw. Beschäftigungsfunktion abhängig vom öffentlichen Haushaltsvolumen und dem allgemeinen Lohnniveau bzw. dem Lohnniveau des privaten Sektors in der Vorperiode. Sie beruht wie zuvor auf der Planung bestimmter Personalausgabenanteile am öffentlichen Haushalt, ist aber unabhängig von den besoldungspolitischen Entscheidungen des Staates.

Die staatliche Beschäftigungsfunktion wurde der Einfachheit halber in der Version (15.12) geschätzt (1). Das zugrunde liegende Material (2) erstreckte sich auf die Jahre 1960 bis 1972. Es ließen sich wieder zwei Phasen mit unterschiedlicher Entwicklung identifizieren. Das Regressionsergebnis der Teilperiode 1960 bis 1967 lautet:

(1) Die Schätzung von Version (15.13) wäre durch das Problem hochgradiger Multikollinearität belastet gewesen.

(2) Vgl. Fußnote 1 von Seite 236. 


$$
\begin{aligned}
& \text { (15.14) } h_{\text {ASt } 1}=-1,63841( \pm 0,50330) \text { Mrd. DM } \\
& h_{\text {ASt2 }}=+0,14273( \pm 0,00392) \\
& \left.r_{1 \cdot 2}^{2}=0,99549 \text { (Irrt.-Wahrsch.: }<0,1 \not\right) \\
& \mathrm{vNQ}=2,745
\end{aligned}
$$

Das Regressionsergebnis für die Teilperiode 1968 bis 1972 lautet:

$$
\text { (15.15) } \begin{aligned}
\mathrm{h}_{\text {ASt } 1} & =-10,15067 \quad( \pm 1,43114) \text { Mrd. DM } \\
\mathrm{h}_{\text {ASt2 }} & =0,18922 \quad( \pm 0,00664) \\
r_{1.2}^{2} & =0,99632 \quad \text { (Irrt.-Wahrsch.: }<0,1 \%) \\
\text { vNQ } & =3,390
\end{aligned}
$$

Ökonometrisch mögen die beiden Ergebnisse (15.14) und (15.15) suspekt erscheinen, weil auf der linken und rechten Seite der Regressionsgleichung (15.12) zwei auf jeden Fall trendmäßig ansteigende Variable erscheinen und die entsprechende Variable der linken Seite, $l_{\mathrm{pr}, t}$, nicht die eigentlich abhängige Variable der staatlichen Beschäftigungsfunktion ist. Zur weiteren Information sei daher die Korrelation zwischen der tatsächlichen staatlichen Beschäftigung in der Bundesrepublik und der hypothetischen staatlichen Beschäftigung angegeben, die sich nach Einsetzen der geschätzten Regressionsparameter und der Werte für die unabhängigen Variablen aus $(15.13)$ ergibt. Die Korrelation beträgt während der ersten Teilperiode $r^{2}=0,910$ und während der zweiten Teilperiode $r^{2}=0,904$. Hinter beiden Ergebnissen verbirgt sich ein ständiges Wachstum der staatlichen Beschäftigung (1).

(1) Die staatliche Beschäftigung in der Bundesrepublik wuchs während der gesamten Beobachtungsperiode auch im Verhältnis zur privaten Beschäftigung ständig an. 
1. Die Übertragungen an die Arbeitnehmer-Haushalte

Die Übertragungen des Staates an die Arbeitnehmer-Haushalte bestehen in der Modellwirtschaft ausschließlich aus den öffentlichen Sozialleistungen, die der Staat den Arbeitnehmer-Haushalten im Gegenzug zu ihren Sozialversicherungsbeiträgen zahlt. Diese öffentlichen Sozialleistungen setzen sich aus einer Reihe von Teilleistungen zusammen, die an verschiedenen Tatbeständen wie Alter, Invalidität, Krankheit, Ar beitslosigkeit etc. anknüpfen. Allen Teilleistungen gemeinsam ist, daß sie direkt - durch einkommensbezogene Ansprüche der Empfänger oder indirekt - durch mit dem Einkommensanstieg verbundene Kostenerhöhungen (z. B. im Krankheitsfall) - mit dem allgemeinen Lohnniveau ansteigen. Wegen der besonderen Bemessung der Rentenzahlungen (1), die einen Großteil aller Sozialleistungen ausmachen, folgt der Wert des Gesamtaggregates dem allgemeinen Lohnniveau mit einem zeitlich verteilten Lag.

Die Funktion für die Übertragungen des Staates an die ArbeitnehmerHaushalte wird im Modell formuliert als:

$$
\begin{aligned}
U_{S t, A, t}= & h_{U A 1^{*}}^{*} h_{U A 2^{*}}^{*}\left[\sum_{\tau=1}^{3} \frac{1}{3} L_{A, t-\tau}\right]+ \\
& h_{U A 3^{*}}^{*} L_{A, t^{+}}+h_{U A 4^{*}}^{*}\left[\left(e_{v, t}-e_{t}\right) \cdot l_{p r, t}\right]
\end{aligned}
$$

d. h. die Übertragungen an die Arbeitnehmer-Haushalte, U St, A, $t$, werden abhängig gemacht vom aggregierten Bruttoeinkommen aus unselbständiger Arbeit im Durchschnitt der drei vorausgegangenen Perioden, vom aggregierten Bruttoeinkommen aus unselbständiger Arbeit der laufenden Periode und vom Lohnausfall der Arbeitslosen in der laufenden Periode.

Zur Ausschaltung von Mulitikollinearität wird (16.1) transformiert (2):

(1) Vgl. Sozialenquête-Kommission, Soziale Sicherung in der Bundesrepublik Deutschland. Sozialenquête, erstattet von W. Bogs, H. Achinger, H. Meinhold, L. Neundörfer und W. Schreiber, Stuttgart u. a. , o. J. , Textziffer 215 und 219. - Es sei darauf verwiesen, daß der rentenbezogene Verzögerungsmechanismus in (16.1) im Vergleich zum Verzögerungsmechanismus der Rentenbemessung in der Bundesrepublik um ein Jahr verschoben ist. 
(16.2) $U_{S t, A, t}=h_{U A 1}+h_{U A 2} \cdot\left[\sum_{\tau-1}^{3} \frac{1}{3} L_{A, t-\tau}\right]+$

$$
\begin{aligned}
& h_{U A 3^{*}} \cdot\left[L_{A, t}-\sum_{\tau=1}^{3} \frac{1}{3} L_{A, t-\tau}\right]+ \\
& h_{U A 4} \cdot\left[\left(e_{v, t}-e_{t}\right) \cdot 1_{p r, t}\right]
\end{aligned}
$$

Bei der Quantifizierung der Funktionsparameter wurden die Übertragungen $U_{S t}, A, t$ statistisch repräsentiert durch den Wert der an die privaten Haushalte geleisteten laufenden und Vermögensübertragungen des Staates in der volkswirtschaftlichen Gesamtrechnung abzüglich der von den privaten Haushalten an den Staat geleisteten laufenden Übertragungen. Das zugehörige Regressionsergebnis lautet: $(t=1960, \ldots$, 1971):

$$
\text { (16.3) } \begin{array}{ll}
h_{U A 1}=-1,00426 & ( \pm 1,07493) \text { Mrd. DM } \\
h_{U A 2}=+0,29808 & ( \pm 0,01152) \\
h_{U A 3}=+0,09705 & ( \pm 0,02431) \\
h_{U A 4}=+0,81833 & ( \pm 0,42934) \\
R_{1.2,3,4}^{2}=0,99832 \quad(\text { Irrt. -Wahrsch.: }<0,18) \\
R_{2 \cdot 3,4}^{2}=0,82301 \\
R_{3 \cdot 2,4}^{2}=0,73427 \\
R_{4 \cdot 2,3}^{2}=0,65351 \\
\text { VNQ }=1,700
\end{array}
$$

(2) Aufgrund der Transformation gilt:

$$
\begin{aligned}
n_{U A 1} & =h_{U A 1}^{\star} \\
n_{U A 2} & =h_{U A 2}^{*}+h_{U A 3}^{*} \\
n_{U A 3} & =h_{U A 3}^{*} \\
n_{U A 4} & =h_{U A 4}^{*}
\end{aligned}
$$


Leider konnte durch die Transformation die Multikorrelation der unabhängigen Variablen nur zu einem Teil ausgeschaltet werden. Die Parameter stimmen aber nach Rückrechnung auf die für (16.1) geltenden Werte in etwa mit den Sätzen der Sozialversicherungsleistungen in der Bundesrepublik überein und können daher auf jeden Fall akzeptiert werden.

2. Die Übertragungen des Staates an den Unternehmenssektor

Bei den Übertragungen des Staates an den Unternehmenssektor handelt es sich in der Modellwirtschaft um finanzielle Hilfen, die aus strukturellen oder konjunkturellen Gründen gewährt werden. Von den struktu rellen Hilfsleistungen wird angenommen, daß sie gleichmäßig mit der Wertschöpfung des Unternehmenssektors, $x_{t} \cdot p_{x, t}$, ansteigen, von den konjunkturellen Förderungsmaßnahmen, daß sie an dem Wachstum der realen Produktion, $x_{t}-x_{t-1}$, im Unternehmenssektor, jeweils bewertet zu Vorperiodenpreisen, ausgerichtet werden.

Die Funktion für die Übertragung des Staates an den Unternehmenssektor, USt, Ut, lautet:

$$
\text { (16.4) } \begin{aligned}
\mathrm{U}_{\text {St }, U, t}= & { }_{\mathrm{h}_{U 1}+h_{U U 2} \cdot\left[x_{t} \cdot p_{x, t}\right]+} \\
& { }_{h_{U U 3}} \cdot\left[x_{t}-x_{t-1}\right] \cdot p_{x, t-1}
\end{aligned}
$$

Als statistisches Material für die Variable $U_{S t, U} t$ wurde aus der volkswirtschaftlichen Gesamtrechnung der Bundesrepublik der Wert der vom Staat an die Unternehmen geleisteten Vermögensübertragungen abzüglich der von den Unternehmen an den Staat geleisteten laufenden und Vermögensübertragungen herangezogen. Dem Gesamtbetrag wurde die Differenz $\mathrm{z}$ wischen den hypothetisch angesetzten staatlichen Vermögenserträgen (1) und dem Saldo der vom privaten Sektor empfangenen staatlichen Einkommen aus Unternehmertätigkeit und Vermögen (2) zugeschlagen, die hier als Zinssubvention des Staates an den Unternehmenssektor angesehen wird.

Die Parameterschätzung wurde für zwei Teilperioden vorgenommen. Für die erste Teilperiode, die sich von 1955 bis 1962 erstreckt, lautet das Ergebnis der Kleinst-Quadrat-Schätzung:

(1) Vgl. Paragraph 14, Abschnitt 3.

(2) Diese werden wiederum der volkswirtschaftlichen Gesamtrechnung der Bundesrepublik Deutschland entnommen. 


$$
\text { (16.5) } \begin{aligned}
& \mathrm{h}_{\mathrm{UU1}}=-2,83408( \pm 1,20258) \text { Mrd. DM } \\
& \mathrm{h}_{\mathrm{UU} 2}=+0,02909( \pm 0,00402) \\
& \mathrm{h}_{\mathrm{UU} 3}=-0,15421 \quad( \pm 0,04314) \\
& \left.\mathrm{R}_{1 \cdot 2,3}^{2}=0,92758 \quad \text { (Irrt.-Wahrsch.: }<0,1 \%\right) \\
& \mathrm{r}_{2 \cdot 3}^{2}=0,00049 \\
& \text { VNQ }=2,650
\end{aligned}
$$

Diese Ergebnisse liegen der Modellrechnung für die Jahre 1960 bis 1962 zugrunde.

Das Ergebnis für die zweite Teilperiode, die sich von 1963 bis 1971 erstreckt, lautet:

$$
\text { (16.6) } \begin{aligned}
& \mathrm{h}_{\mathrm{UU} 1}=-1,02839( \pm 0,57705) \text { Mrd. DM } \\
& \mathrm{h}_{\mathrm{UU} 2}=+0,01400( \pm 0,00122) \\
& \mathrm{h}_{\mathrm{UU} 3}=-0,04151( \pm 0,01001) \\
& \left.\mathrm{R}_{1 \cdot 2,3}^{2}=0,95627 \quad \text { (Irrt.-Wahrsch.: }<0,1 \%\right) \\
& \mathrm{r}_{2 \cdot 3}^{2}=0,11764 \\
& \mathrm{VNQ}=3,232
\end{aligned}
$$

Diese Ergebnisse gelten in der Modellrechnung ab 1963 und deuten eine Änderung des staatlichen Verhaltens in Richtung auf ein im Trend weniger stark als bisher steigendes Engagement der staatlichen Suventionspolitik an. Zugleich läßt der geringere Wert von Parameter $h_{U U} 3$ erkennen, daß der Staat das Instrument direkter finanzieller Hilfen an den Unternehmenssektor weniger als bisher zu konjunkturpolitischen $Z$ wecken einsetzte. Es liegt nahe, einen Zusammenhang zu den parallelen Änderungen im Bereich der staatlichen Kreditpolitik zu sehen (1) und davon auszugehen, daß die staatliche Stabilisierungspolitik Mitte der sechsziger Jahre die beschäftigungspolitischen Konsequenzen aus der Keynes'schen Beschäftigungstheorie zog und sich aus dem Bereich der direkten finanziel-

(1) Vgl. Paragraph 14, Abschnitt 3. 
len Hilfen an den Unternehmenssektor in den Bereich der antizyklisch eingesetzten Kreditpolitik verlagerte.

3. Die Kapitalhilfe des Staates an die übrige Welt

Die Kapitalhilfe des Staates an die übrige Welt, $\mathrm{U}_{\mathrm{St}}$, Ausl, $t$, besteht in der Modellwirtschaft aus Übertragungen im engeren Sinn, U Ut, Ausl, $t$, und aus unverzinslichen Krediten, $\Delta F_{S t}$, Ausl, $t$. Beide Posten werden hier zusammengefaßt, weil sie in der Modellformulierung eine ähnliche Bedeutung haben (1):

$$
U_{\text {St, Aus I, } t}^{*}=U_{S t, A u s l, t}{ }^{+\Delta F_{S t, A u s l, t}}
$$

Der Betrag des Gesamtaggregates wird vom Staat als ein bestimmter Budgetanteil festgelegt, der jedoch in aufkommensschwachen Haushaltsjahren, praktisch also in Rezessionsjahren, niedriger bemessen wird als in aufkommensstarken Haushaltsjahren. Die Funktion der staatlichen Kapitalhilfe an das Ausland läßt sich daher formulieren als:

$$
\begin{aligned}
& U_{\text {St,Aus 1, } t}^{\star}=h_{\text {UAus 11 }}^{\star}{ }^{\star} \\
& \mathrm{h}_{\text {UAus } 12}^{\star} \cdot \mathrm{H}_{2, \mathrm{t}}+ \\
& h_{\text {UAus } 13}^{\star H_{2}, t-1}
\end{aligned}
$$

bzw. nach einer Transformation (2) zur Ausschaltung oder Abschwächung von Multikollinearität:

(1) Die staatlichen Kredite an das Ausland ergeben in der aggregierten Betrachtung wegen der begrenzten Laufzeit einen revolvierenden Bestand. In der Modellformulierung werden lediglich die positiven oder negativen Änderungen dieses Bestandes berücksichtigt; der Bestand als solcher bleibt ebenso wie die per Annahme ausgeschlossene Verzinsung des Bestandes außer acht.

(2) Aufgrund der Transformation gilt:

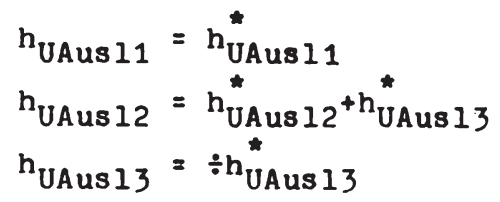




$$
\begin{aligned}
& U_{\text {St,Aus } 1, t}^{\star}=h_{\text {UAus } 11+}+ \\
& { }^{h_{\text {UAus } 12}} \cdot \mathrm{H}_{2, \mathrm{t}}+ \\
& h_{\text {UAus } 13^{\circ}}\left[\mathrm{H}_{\left.2, t^{-H_{2}}, t-1\right]}\right.
\end{aligned}
$$

Die der staatlichen Kapitalhilfe bei der Schätzung von (16.9) zugrunde gelegten Beträge wurden der Zahlungsbilanzstatistik der Deutschen Bundesbank entnommen (1). Die Zeitreihe ließ erkennen, daß das Verhalten des Staates im Bereich der Auslandshilfe ab 1961/62 eine gewisse Wandlung vollzogen hat. Während der Staat den Umfang der Hilfe bis dahin Jahr für Jahr kräftig aufstockte, sah er 1961 das erreichte Niveau offensichtlich als angemessen bzw. schon als zu hoch an. Möglicherweise spielte hierbei eine Rolle, daß der Staat nach der DM-Aufwertung vom Frühjahr 1961 keine Notwendigkeit mehr sah, Außenhandelsüberschüsse bzw. daraus resultierende gesamtwirtschaftliche Nachfrageeffekte durch staatlichen Kapitalexport und staatliche Unterstützungszahlungen an das Ausland zu kompensieren. Jedenfalls wurde der Umfang der Auslandshilfe 1962 gekürzt (2) und in den folgenden Jahren nur noch langsam bzw. unterproportional zum Haushaltsvolumen ausgeweitet.

Für die Teilperiode 1955 bis 1961 lautet das Ergebnis der Kleinst-QuadratSchätzung:

(1) Es handelt sich um den Gesamtbetrag aus dem Saldo der staatlichen Übertragungen und dem Saldo des staatlichen Kapitalexportes. Vgl. Deutsche Bundesbank, Die Zahlungsbilanz der Bundesrepublik im Jahre 1972, nach Regionen, a. a. O., S. 2 f.

(2) Die dadurch 1962 frei werdenden Haushaltsmittel kamen vor allem der staatlichen Nachfrage nach Gütern und Diensten (ohne Personalausgaben) im Inland zugute, die in diesem $\mathrm{Jahr}$ außergewöhnlich stark expandierten. Die Ergebnisse von Proberechnungen mit dem Simulationsmodell legen die Vermutung nahe, daß diese kurzfristig vollzogene Umschichtung der Staatsausgaben eine der Hauptursachen des konjunkturellen Zwischenhochs 1962 war. 


$$
\begin{aligned}
& \text { (16.10) } h_{\text {UAus } 11}=-4,83908 \quad( \pm 2,41234) \text { Mrd. DM } \\
& h_{\text {UAUS12 }}=+0,09581 \quad( \pm 0,04158) \\
& \left.h_{\text {UAus 13 }}=+0,18952 \text { ( } \pm 0,19431\right) \\
& R_{1 \cdot 2,3}^{2}=0,82300 \text { (Irrt.-Wahrsch.: }<5 \% \text { ) } \\
& \mathbf{r}_{2 \cdot 3}^{2}=0,49364 \\
& \text { VNQ }=3,508
\end{aligned}
$$

Der Wert von Parameter $h_{U}$ Ausl3 ist geringer als sein Standardfehler. Nach dem bisherigen Vorgehen wäre deswegen die Variable $\left(\mathrm{H}_{2, t}-\mathrm{H}_{2}, \mathrm{t}-1\right)$ vernachlässigt worden. Die Ausschaltung der Variablen verringert aber das Bestimmtheitsmaß um 0,04 auf 0,781 und wurde daher unterlassen.

Bei der Regressionsschätzung für die Teilperiode 1962 bis 1972 wurde ähnlich verfahren. Hier fiel infolge hoher Multikollinearität der Schätzwert von Parameter hUAusl2 geringer aus als sein Standardfehler, die Ausschaltung der Variablen hätte aber das Bestimmtheitsmaß auf 0,762 vermindert und gleichzeitig den von-Neumann-Quotienten auf 3,039 erhöht. Außerdem wurde hier eine Dummy-Variable mit dem Parameter hUAusl4 in die Regressionsgleichung eingeführt, um eine punktuelle Kürzung der staatlichen Kredithilfe an das Ausland im Jahre $1965 \mathrm{zu}$ erfassen; die Variable hat 1965 den Wert eins, ansonsten den Wert null. Das Regressionsergebnis lautet:

$$
\text { (16.11) } \begin{aligned}
\mathrm{h}_{\text {UAus 11 }}=+5,16290 \quad( \pm 0,82558) \text { Mrd. DM } \\
\mathrm{n}_{\text {UAus 12 }}=-0,00684 \quad( \pm 0,00713) \\
\mathrm{n}_{\text {UAus 13 }}=+0,09039 \quad( \pm 0,03685) \\
\mathrm{n}_{\text {UAus 14 }}=-1,62118 \quad( \pm 0,57130) \\
\mathrm{R}_{1 \cdot 2,3,4}^{2}=0,78975 \quad \text { (Irrt.-Wahrsch.:<1\%) } \\
\mathrm{R}_{2 \cdot 3,4}^{2}=0,83942 \\
\mathrm{R}_{3 \cdot 2,4}^{2}=0,83576 \\
\mathrm{R}_{4 \cdot 2,3}^{2}=0,05456 \\
\mathrm{vNQ}=2,806
\end{aligned}
$$


In dem Ergebnis schlägt sich das im Trend nur sehr geringe Wachstum der staatlichen Auslandshilfe nach 1962 nieder. Der im Vergleich zu hUAusl2 hohe Wert von Parameter hUAusl3 deutet ähnlich wie in Regressionsergebnis $(16.10)$ an, daß die kurzfristige Entwicklung der staatlichen Auslandshilfe stark vom kurzfristig verfügbaren finanziellen Spielraum des Staatshaushaltes abhing, was sich konjunkturpolitisch als durchaus von Vorteil erwies. Parameter hUAus14 bestimmt den Umfang, um den die staatliche Auslandshilfe im Jahre 1965 gekürzt wurde, mit 1, 62 Mrd. DM. Bei der genannten Abhängigkeit der Auslandshilfe von der jeweiligen finanziellen Anspannung des Staatshaushaltes ist diese Kürzung wohl auf die Einkommensteuerreform 1965 und die durch sie bedingten Steuerausfälle zurückzuführen. Sie hat wie die gleichzeitig vorgenommene Kürzung der staatlichen Nettokreditvergabe dazu beigetragen, der Einkommensteuersenkung 1965 in der gerade herrschenden Boomsituation einen konjunkturverschärfenden Charakter zu verleihen. 


\section{Der Gesamtumfang}

Das, was der Staat von seinen Haushaltseinnahmen nicht für staatliche Lohn- und Gehaltszahlungen sowie für die staatlichen Übertragungen verausgabt, dient ihm zur Finanzierung der Güterkäufe. Die Bestimmung der staatlichen Güterkäufe, $A_{S t,} t^{-L_{S t} t}$, ergibt sich in der Modellformulierung also implizit aus der Budgetgleichung

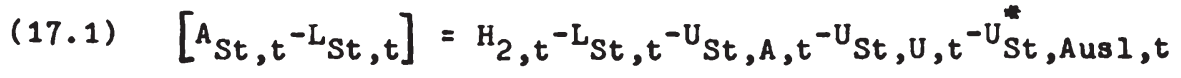

und bedarf keiner eigenen Verhaltensfunktion.

Dieses Verfahren zur Bestimmung der staatlichen Güterkäufe ist rein formal bedingt und beruht auf der Tatsache, daß der öffentliche Haushalt in Einnahmen und Ausgaben formal stets ausgeglichen ist und daß, wenn alle Haushaltsposten bis auf einen explizit durch Verhaltensfunktionen bestimmt sind, auch dieser letzte Haushaltsposten ex-definitione bestimmt ist. Materiell geht die gesamte Darstellung des öffentlichen Haushaltsgebarens natürlich davon aus, daß der Staat für jeden einzelnen Haushaltsposten bestimmte, inhaltlich präzisierte Verhaltensvorstellungen hegt. Im Prinzip läßt sich daher für jeden Haushaltsposten, auch für die staatlichen Güterkäufe, explizit eine eigene Verhaltensfunktion aufstellen. Sämtliche staatlichen Einnahme- und Ausgabepläne werden aber stets aufeinander abgestimmt und müssen unter dem Gesichtspunkt des formalen Haushaltsausgleichs konsistent sein, und dies gilt auch für die entsprechenden Verhaltensfunktionen der Modellformulierung. In dieser Hinsicht ist (17.1) die formal einfachste modellmäßige Darstellung des staatlichen Verhaltens im Bereich der Güterkäufe.

Die Darstellungsweise (17.1) sagt für sich genommen nichts über die Art des entsprechenden staatlichen Verhaltens im Bereich der Güterkäufe aus. Es sei deswegen kurz angedeutet, daß die Ausgaben des Staates für die Käufe von Gütern und Diensten, $A_{S t, t}$, in der Bundesrepublik im allgemeinen einen verhältnismäßig stabilen Anteil am gesamten öffentlichen Haushalt, $\mathrm{H}_{2}$, , ausmachten (1). Der Anteil stieg während der ersten Hälfte der sechziger Jahre - wohl im Zuge der vorgenommenen Steuersenkungen - an und lag, wie man sowohl in der zweiten Hälfte der fünfziger als auch in der zweiten Hälfte der sechziger Jahre beob-

(1) Der Anteil betrug während der Jahre 1955 bis 1971 aufgrund des für die Modellzwecke leicht frisierten Materials 0,296 bei einer durchschnittlichen Abweichung von $\pm 0,025$. 
achten kann, in Rezessionsphasen deutlich unter und in Jahren der Hochkonjunktur über dem mittelfristigen Normalniveau. Diese Entwicklungstendenzen müßten sich auch in dem Standardablauf der Modellrechnung feststellen lassen.

In Hinsicht auf die modellmäßige Analyse von Änderungen des staatlichen Einnahme- und Ausgabeverhaltens sei darauf aufmerksam gemacht, daß die gewählte Darstellung des öffentlichen Haushaltsgebarens und im Zusammenhang damit insbesondere Gleichung (17.1) für die Art der insgesamt unterstellten Budgetannahmen von Bedeutung sind. Ändert sich ein einzelner staatlicher Einnahmeparameter, ohne daß sich irgendein $\mathrm{Pa}-$ rameter auf der Ausgabenseite des öffentlichen Budgets ändert, dann kommt es zu einer entsprechenden Änderung des Haushaltsvolumens und damit der öffentlichen Ausgaben. Die Analyse der Inzidenzeffekte, die von Reformen auf der Einnahmeseite des öffentlichen Haushalts ausgehen, wird also, wenn nicht zugleich weitere Parameteränderungen auf der Einnahmeseite berücksichtigt werden, auotmatisch unter der Annahme einer Verausgabung (Einsparung) der resultierenden Mehr-(Minder-) Einnahmen durch den Staat durchgeführt. Diese Grundeinstellung der Modellformulierung ist wegen der besonderen Problematik der makroökonomischen Überwälzungstheorie, die sich auf die Budgetinzidenz steuerpolitischer Maßnahmen richtet, sinnvoll. - Ändert sich dagegen ein einzelner staatlicher Ausgabenparameter (1), ohne daß sich irgendein Parameter auf der Einnahmenseite des öffentlichen Budgets ändert, dann bleibt das Haushaltsvolumen des Staates unverändert und es kommt nach $(17.1) \mathrm{zu}$ einer Substitution verschiedener Ausgabearbeiten. Die Inzidenzeffekte, die von Änderungen staatlicher Ausgabeparameter ausgehen, werden also, sofern nicht zugleich weitere Parameteränderungen auf der Einnahmenseite des Budgets berücksichtigt werden, automatisch unter dem Gesichtspunkt der Differentialinzidenz analysiert (2). - Diese Bemerkungen gelten natürlich unscharf. Die vielfältigen interdependenten Beziehungen zwischen Staatseinnahmen und Staatsausgaben sowie $z$ wischen staatlichem und privatem Sektor machen eine genaue Kontrolle der Budgetentwicklung auf Änderungen der staatlichen Verhaltensparameter hin sehr schwer. Bei einer beliebigen Steuererhöhung und gleichzeitiger Verausgabung der Steuermehreinnahmen reagiert z. B. nach (14.7) die staatliche Kreditpolitik auf die verursachte Änderung der (Über-)Nachfrage und erlaubt daher keine ganz isolierte Analyse der

(1) Das gilt nicht nur für die in den Paragraphen 15 und 16 genannten $\mathrm{Pa-}$ rameter, sondern, wie im folgenden Abschnitt dieses Paragraphen noch ersichtlich wird, auch für die Parameter der staatlichen Infrastrukturinvestitionen.

(2) $\mathrm{Zu}$ den Begriffen der Budget- und Differentialinzidenz vgl. R. A. Musgrave, Finanztheorie, Tübingen 1966, S. $158 \mathrm{ff}$. 
Budgetinzidenz der Steuererhöhung (1). Andererseits verwendet der Staat nach (16.8) von vornherein einen kleinen Teil der Steuermehreinnahmen zur Auslandshilfe, so daß die marginale inlandswirksame Ausgabenneigung des Staates kleiner als eins ist und von dort her die Grundvoraussetzungen für das Haavelmo-Theorem und das makroökonomische Überwälzungstheorem nur mit Einschränkung erfüllt sind.

\section{Der Anteil der öffentlichen Infrastrukturinvestitionen}

Unter den staatlichen Güterkäufen sind die Käufe, die der Erstellung öffentlicher Infrastrukturanlagen dienen, von besonderer Bedeutung, da die öffentlichen Infrastrukturanlagen dem Produktionsprozeß im privaten Sektor unmittelbar als Produktionsfaktor dienen. Die Infrastrukturinvestitionen des Staates, $\mathrm{I}_{\mathrm{St}, t}^{\mathrm{br}}$, werden daher in der Modellformulierung stets gesondert erfaßt, während die sonstigen Investitionen des Staates, $\mathrm{I}_{\text {St, sonst, }} \mathrm{t}$, die in der volkswirtschaftlichen Gesamtrechnung des Modells noch explizit aufgeführt wurden, in der weiteren Modellformulierung mit den Güterkäufen für den unmittelbaren Staatskonsum zusammengefaßt und nur noch mit diesen zusammen berücksichtigt werden.

Die Investitionen des Staates in die öffentliche Infrastruktur werden in der Modellformulierung als ein bestimmter Anteil an der Gesamtheit der staatlichen Güterkäufe geplant. Dieser Anteil wird bestimmt durch Funktion (17.2):

$$
\text { (17.2) } \quad I_{S t, t}^{\text {br }}=h_{I 1}{ }^{+h_{I 2}} \cdot\left[A_{S t, t}{ }^{-L_{S t, t}}\right]
$$

Zur Schätzung der Funktion wurden die staatlichen Infrastrukturinvestitionen im Anschluß an die Anlagevermögensrechnung des Statistischen Bundesamtes berechnet (2), die auf die Stromrechnung der Volkswirtschaftlichen Gesamtrechnung abgestimmt ist. Die Summe aus der jährlichen Änderung des dort angegebenen staatlichen Bruttoanlagevermögens und dem ebenfalls angegebenen Anlagenabgang ergab gemäß Definition (2.65) die realen Bruttoanlageinvestitionen des Staates in der Bundesrepublik. Die Inflationierung mit dem Preisindex für Anlageinvestitionen der Volkswirtschaftlichen Gesamtrechnung führte zu dem entsprechenden Nominalwert der staatlichen Bruttoanlageinvestitionen. Von diesem Gesamtbetrag wurde entsprechend den Annahmen des Paragraphen 4 eine Hälfte als staatliche Infrastrukturinvestition, die verbleibende

(1) Vgl. zu diesen konzeptionellen Schwierigkeiten R. A. Musgrave, a. a. O., S. $164 \mathrm{f}$ sowie C. S. Shoup, Public Finance, Chicago 1969, S. $7 \mathrm{ff}$.

(2) Vgl. H. Lützel, Das reproduzierbare Anlagevermögen in Preisen von 1962 , a. a. O. , S. 608 f. 
andere Hälfte als sonstige Investition des Staates angesehen.

Die Kleinst-Quadrat-Schätzung zu (17.2) wurde für die Jahre 1960 bis 1971 durchgeführt. Das Ergebnis lautet:

$$
\begin{aligned}
& h_{I 1}=-0,09652 \quad( \pm 0,52221) \text { Mrd. DM } \\
& h_{I 2}=+0,18494 \quad( \pm 0,00985) \\
& \left.r_{1 \cdot 2}^{2}=0,97241 \quad \text { (Irrt.-Wahrsch.: }<0,18\right) \\
& \text { vNQ }=1,807
\end{aligned}
$$

3. Anlagenabgang und Abschreibungen für Kapitalverzehr des Staates

Um die Entwicklung des Gesamtbestandes an öffentlichen Infrastrukturanlagen realistisch zu erfassen, müssen neben den durch die staatliche Investitionstätigkeit bestimmten Neuzugängen explizit die Abgänge alter Anlagen berücksichtigt werden. Der staatliche Anlagenabgang wird in der Modellformulierung ähnlich behandelt wie der Anlagenabgang im Unternehmenssektor. Er wird wie dort exogen vorgegeben. Zu diesem Zweck wird eine einfache Trendfunktion aufgestellt, in der der Abgang

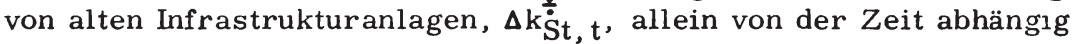
gemacht wird:

$$
\text { (17.4) } \quad \Delta k_{S t, t}^{\div}=h_{k 1} \cdot h_{k 2}^{t-1960}
$$

Bei der Schätzung der Funktionsparameter wurde der Abgang von alten Infrastrukturanlagen mit der Hälfte des gesamten staatlichen Anlagenabgangs angesetzt, wie er in der Anlagenvermögensrechnung des Statistischen Bundesamtes ausgewiesen wird (1).

Das Regressionsergebnis der in die Logarithmen transformierten Gleichung (17.4) lautet $(\mathrm{t}=1960, \ldots, 1972)$ :

(1) Vgl. H. Lützel, Das reproduzierbare Anlagevermögen in Breiten von 1962 , a. a. O. , S. $608^{*}$ sowie derselbe, Das reproduzierbare Sachvermögen zu Anschaffungs- und zu Wiederbeschaffungspreisen, a. a. O., S. $689^{\star}$. 


(17.5) $\begin{aligned} & l_{8} h_{k 1}=-0,60641 \quad( \pm 0,00863) \text { Ig Mrd. DM } \\ & l_{8} h_{k 2}=+0,03199 \quad( \pm 0,00122) \\ & \left.r_{1 \cdot 2}^{2}=0,98423 \quad \text { (Irrt.-Wahrsch.: }<0,1 \%\right) \\ & \text { vNQ }=3,600\end{aligned}$

Wie man bereits dem statistischen Material entnehmen konnte, stieg der Anlagenabgang im staatlichen Sektor der Bundesrepublik anders als im Unternehmenssektor mit einer verhältnismäßig stabilen jährlichen Wachstumsrate (1), die nach (17.5) 7,6 \% betrug.

Ähnlich wurde mit den staatlichen Abschreibungen verfahren. Die Abschreibungen des Staates, $D_{S t, t}$, werden wie die des Unternehmenssektors linear von dem zu Wiederbeschaffungspreisen bewerteten Bruttoanlagevermögen ermittelt. Der Einfachheit halber werden sie in der Modellformulierung allein auf die öffentlichen Infrastrukturanlagen verrechnet, größenmäßig aber so veranschlagt, daß sie den gesamten staatlichen Abschreibungen in der Volkswirtschaftlichen Gesamtrechnung der Bundesrepublik entsprechen (2). Es gilt also:

(17.6) $\quad D_{S t, t}=\left[\frac{d_{S t, t}}{k_{S t, t}}\right] \cdot k_{S t, t} \cdot p_{k, t}$

wobei $\mathrm{d}_{\mathrm{St}, \mathrm{t}} / \mathrm{k}_{\mathrm{St}, \mathrm{t}}$ den jeweils geltenden staatlichen Abschreibungssatz bezeichnet.

Der staatliche Abschreibungssatz $d_{S t, t} / k_{S t, t}$ wurde als Quotient aus den gesamten staatlichen Abschreibungen der Volkswirtschaftichen Gesamtrechnung und dem staatlichen Infrastrukturanlagenbestand, also der Hälfte des gesamten staatlichen Bruttoanlagevermögens in der Anlagenvermögensrechnung des Bundesamtes, errechnet. Die errechneten

(1) Natürlich beruht der Unterschied auf den Annahmen der Anlagenvermögensrechnung des Statistischen Bundesamtes. Zur Entwicklung im Unternehmenssektor vgl. Paragraph 6, Abschnitt 1.

(2) Da Infrastrukturanlagen und -investitionen des Staates ohnehin mit gleichbleibend $50 \%$ des gesamten staatlichen Anlagenbestandes bzw. der gesamten staatlichen Anlageinvestition in der Bundesrepublik angesetzt wurden, bedeutet dieses Vorgehen keine zusätzliche Restriktion. Es ist nur darauf zu achten, daß der durchschnittliche Abschreibungssatz infolge der Berechnungsweise um $100 \%$ über dem vom Statistischen Bundesamt angesetzten Wert liegt und damit die durchschnittliche Lebensdauer der staatlichen Anlagen zu gering erscheinen läßt. 
Werte werden in der Modellrechnung für die Jahre 1960 bis 1972 exogen vorgegeben. Für die Jahre nach 1972 wird die Entwicklung des staatlichen Abschreibungssatzes durch die Trendfunktion

(17.7) $\left[\frac{d_{S t, t}}{k_{S t, t}}\right]=h_{D 1}+h_{D 2} \cdot[t-1970]$

bestimmt, die aufgrund der $d_{S t, t} / k_{S t, t}$-Werte der Jahre 1969 bis 1972 geschätzt wurde:

$$
\begin{aligned}
& \text { (17.8) } \quad h_{D 1}=+0,00937 \quad( \pm 0,00037) \\
& h_{D 2}=+0,00088 \quad( \pm 0,00003) \\
& \left.r_{1 \cdot 2}^{2}=0,99726 \text { (Irrt.-Wahrsch.: }<0,18\right) \\
& V N Q=2,685
\end{aligned}
$$

Für die Zeit nach 1972 wird also eine unvermindert anhaltende Erhöhung des staatlichen Abschreibungssatzes bzw. eine unvermindert anhaltende Verkürzung der für die staatlichen Anlagen geltenden Lebensdauer angenommen (1).

(1) Es sei darauf aufmerksam gemacht, daß die staatlichen Abschreibungen für die Modellrechnung eine funktionslose Mitläufer-Variable sind, die nur der genaueren Bestimmung des staatlichen BIP-Beitrags dient. 


\section{Kapitel}

\section{LÖSUNG UND AUSWERTUNG DER MODELLERGEBNISSE}

$§ 18$ Der rechnerische Lösungsweg

\section{Zur Berechnungsmethode}

Die Art, in der das System von Definitions- und Verhaltensgleichungen der vorausgegangenen Kapitel gelöst wurde, läßt sich hier nicht in allen Einzelschritten vorführen. Der gesamte Lösungsweg ist dem Rechenprogramm des folgenden Abschnitts zu entnehmen, das in FORTRAN IV formuliert und mit der Maschine durchgerechnet wurde. In diesem Abschnitt werden nur die wichtigsten Grundzüge und Probleme der Modellrechnung sowie einige nachträgliche Modifikationen angesprochen.

Die ausführliche Berücksichtigung des staatlichen Verhaltens, wie es im letzten Kapitel beschrieben wurde, bedeutet für die Lösung des aufgestellten Verteilungsmodells eine erhebliche Komplikation. Dennoch ist es im Prinzip nicht der Einbau der staatlichen Aktivität, ebensowenig die Berücksichtigung des Auslandes, was der Modellösung die eigentlichen Schwierigkeiten bereitet, sondern die Art der Verhaltensfunktionen, mit denen das Verhalten der verschiedenen Sektoren in der Bundesrepublik zu erfassen versucht wurde.

Läßt man einmal die Gleichungen des Modells Revue passieren, die auch in einer vereinfachten Version des Modells für eine geschlossene Wirtschaft ohne staatliche Aktivität hätten berücksichtigt werden müssen, so begegnet man bereits in einer solchen vereinfachten Version drei verschiedenen Funktionstypen: Das definitorische System der Volkswirtschaftlichen Gesamtrechnung und eine Reihe von Verhaltensfunktionen bestehen aus einfachen algebraischen Funktionen:

$$
a_{11} \cdot x_{1}+\ldots+a_{1 n} \cdot x_{n}=0
$$

Hierzu gesellt sich der Funktionstyp, der die speziellen Annahmen hinsichtlich des Verhaltens der Produktion mit konstanten Substitutionselastizitäten zum Ausdruck bringt:

$$
b_{j 1} \cdot x_{1}^{c_{j 1}}+\ldots+b_{j n} \cdot x_{n}^{c_{j n}}=0
$$

Schließlich treten im Bereich der Kapazitätsplanung, des Kreditmarktverhaltens etc. Funktionen auf, die linear in den Logarithmen sind: 


$$
d_{k 1} \cdot 1 g x_{1}+\ldots+d_{k n} \cdot 1 g X_{n}=0
$$

Unter den Verhaltensgleichungen erscheinen noch einige Funktionen gemischten Typs. Diese lassen sich jedoch ohne schwerwiegende Informationsverlust auf einen der drei Grundtypen (18.1) bis (18.3) reduzieren (1).

Das Problem der Modellrechnung ist, daß sich ganz unabhängig von der zusätzlichen Schwierigkeit, die sich daraus ergibt, daß in der Rechnung auch verzögert-endogene Variable auftreten, ein interdependentes System von Gleichungen des Typs (18.1) bis (18.3) mathematisch nicht exakt lösen läßt. Man ist daher gezwungen, entweder zugunsten einer allgemeinen mathematischen Lösung das ganze Gleichungssystem zu linearisieren und damit den Geltungsbereich der Modellaussagen stark einzugrenzen oder, was im vorliegenden Fall unternommen wurde und was sich angesichts der großen Zahl der gesamtwirtschaftlichen Zusammenhänge auch im Falle mathematischer Lösbarkeit als sinnvoll angeboten hätte, bei Vorgabe numerischer Parameterwerte die gesamte Modellösung für eine Reihe aufeinanderfolgender Perioden näherungsweise durchzurechnen, um schließlich für verschiedene Parameterkonstellationen die numerisch bestimmten zeitlichen Modellabläufe miteinander zu vergleichen. Die hier gewählte Methode, das aus Funktionen des Grundtyps (18.1) bis (18.3) zusammengesetzte Gleichungssystem zu analysieren, besteht also in einer numerischen Sequenzenrechnung, bei der der interdependente Teil des Modells iterativ gelöst wird. Bei einem solchen Vorgehen ist die Ausweitung der Modellformulierung auf eine offene Wirtschaft mit staatlicher Aktivität abgesehen von dem größeren Umfang des Modells technisch unproblematisch.

Während der vielen Proberechnungen mit dem Modell tauchte im $\mathrm{Zu}$ sammenhang mit der iterativen Lösung ein Problem auf, dessen Charakterisierung einen gewissen Einblick in den Lösungsweg des Rechenprogramms gibt: Bei der Auflösung des gesamten Gleichungssystems wurde so vorgegangen, daß zuerst die Einkommensentstehungsgleichung der Volkswirtschaftlichen Gesamtrechnung durch Einsetzen von Gleichungen für das Nachfrageverhalten nach der Variablen $1_{p r}, t$ aufgelöst wurde. Dabei wurden erst die linearen interdependenten Zusammenhänge berücksichtigt, dann die verzögert-endogenen Abhängigkeiten und exogenen Einflüsse, schließlich alle nicht-linearen Zusammenhänge. Die Gleichsetzung mit der ebenfalls nach $l_{\mathrm{pr}}, \mathrm{t}$ aufgelösten Gleichung für das bewertete Produktionsergebnis ergab eine Gleichung für die kurzfristige Überschußnachfrage auf dem privaten output-Markt. Diese Überschußnachfrage

(1) Zum Beispiel läßt sich die Funktion für das Verhalten der gewerkschaftlichen Lohnpolitik bzw. die Philipps-Kurve durch den Funktionstyp (18.2), der den CES-Produktionsfunktionen zugrunde liegt, angemessen darstellen. 
muß im kurzfristigen Einkommensgleichgewicht des Modells gleich null sein. Dabei sei daran erinnert, daß der kurzfristige Gleichgewichtsbegriff stark formal im Sinne einer mathematischen Lösbarkeit des Systems ist und keinerlei Bedeutung für die Existenz oder Nicht-Existenz eines Gleichgewichtszustandes bzw. einer Gleichgewichtsentwicklung in einem längerfristigen Sinn oder auch nur in einem konjunkturellen Sinn hat (1). Die genannte Überschußnachfragefunktion ist die Iterationsfunktion FF, deren nächstliegende Nullstelle mit Hilfe der Newton-Iteration berechnet werden sollte (2).

Während der Proberechnungen ließ sich anhand von Zwischenergebnissen (3) der Modellrechnung der Verlauf der Iterationsfunktion bzw. der Überschußnachfragefunktion überprüfen. Dabei ließ sich feststellen, daß die FF-Funktion in vielen Fällen tatsächlich eine Nullstelle im relevanten Bereich (4) von $l_{\mathrm{pr}, t}$ hatte. In diesem Falle verlief die Funktion folgendermaßen:

(1) Allerdings muß zugegeben werden, daß auf dieser Ebene eine Klasse von Stabilitätsproblemen auftaucht bzw. verbleibt, die durch den dynamischen Charakter des Gesamtmodells nicht ausgeräumt ist und die sich auf die noch kürzere Frist als die eines Jahres bezieht.

(2) Bei der Überarbeitung des Modells wurde nachträglich der Investitionsgüterpreis als zusätzlich endogene Variable in die Modellformulierung aufgenommen. Um die Berechnungen und die Programm-Formulierung nicht ein zweites Mall vornehmen zu müssen, wurde der Investitionsgüterpreis mit Hilfe einer zweiten Iteration, dieses Mal auf der Basis der Regula Falsi, bestimmt, so daß der Kern der Modellrechnung im Endeffekt aus zwei ineinander verschachtelten Iterationen besteht.

(3) Zum Ausweis der Zwischenergebnisse wird die logische Variable KONTROL in Anweisung 393 des Rechenprogramms gleich . FALSE. gesetzt.

(4) Der relevante Bereich wurde im Programm mit dem $5^{ \pm 1}$-fachen des $1_{\mathrm{pr}, \mathrm{t}-1^{- \text {Wertes }}}$ angesetzt. 


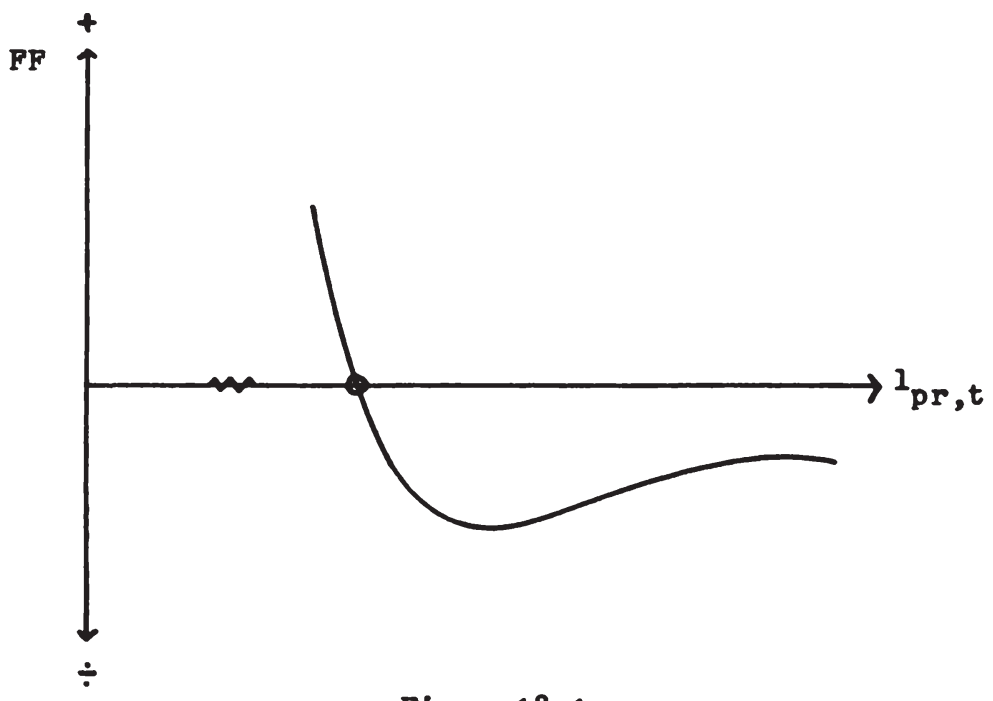

Plgur 18.1

In einer Reihe von Fällen traten jedoch Situationen auf, in denen die Iterationsfunktion keine Nullstelle hatte. Es handelte sich dann geometrisch um folgenden, aufwärts verschobenen Verlauf der Iterationsfunktion FF:

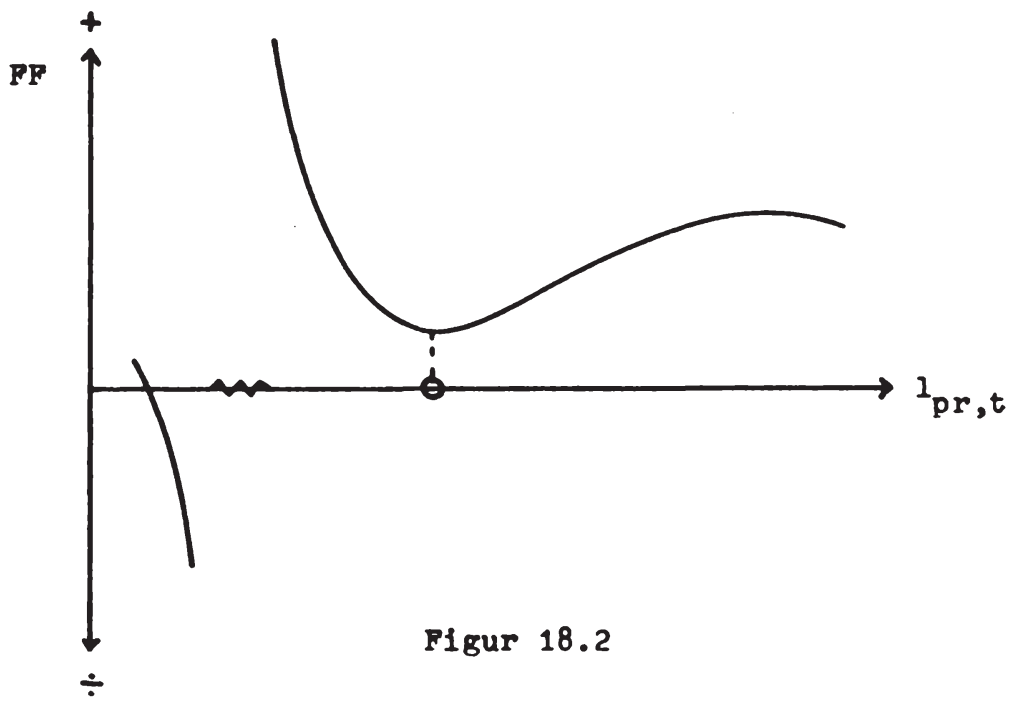


wobei sich im relevanten Bereich von $l_{\mathrm{pr}}, \mathrm{t}$ statt der gesuchten Nullstelle nur ein Funktionsminimum oberhalb der Ábszisse von Figur 18.2 einstellte (1).

In diesen Fällen hatte das formulierte Modell im relevanten Bereich von $l_{\mathrm{pr}, \mathrm{t}}$ offensichtlich keine exakte Lösung, was ökonomisch gesehen bedeutet, daß in einer geometrischen Darstellung des privaten outputMarktes der Modellwirtschaft die Angebotsfunktion keinen Schnittpunkt mit der Nachfragefunktion hatte und damit kein Abbau der Überschußnachfrage auf diesem Markt bzw. kein kurzfristiges Modellgleichgewicht erreicht wurde. Derartige Modellsituationen, die durch das Nicht-Vorhandensein kurzfristiger Gleichgewichtssituationen gekennzeichnet sind, versagen in der Beschreibung der hier abzubildenden wirtschaftlichen Tatbestände und müssen durch Änderung der Modellstruktur ausgeschaltet werden.

$\mathrm{Zu}$ diesem Zweck wurde das Programm zur Berechnung des Modells erst einmal so geändert, daß in den genannten pathologischen Fällen (2)

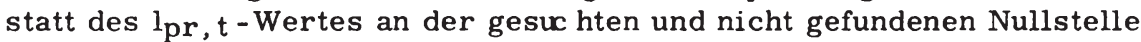
der $l_{p r}, t-W e r t$ im Minimum der Iterationsfunktion FF berechnet, als provisorische Lösung akzeptiert und zur approximativen Berechnung aller übrigen Variablen herangezogen wurde. Dabei wurde zugleich der entsprechende Wert der Überschußnachfrage, absolut (FF) und als Anteil an der Bruttowertschöpfung des privaten Sektors (FFQ), ausgewiesen.

In dem anschließenden Suchprozeß gelang es dann, die Ursache für das Auftreten der pathologischen Situation herauszufinden. Es zeigte sich, daß die bis dahin verwendete Funktion, nach der das Wachstum des Vorratsbestandes im Unternehmenssektor durch das Wachstum der realen Produktion und die Änderung der Inflationsrate bestimmt wurde,

(18.4) $\frac{v_{t}}{v_{t-1}}=n_{1}^{*} \cdot\left[\frac{x_{t}}{x_{t-1}}\right]^{n} \cdot\left[\frac{p_{x, t^{\prime}} x, t-1}{p_{x, t-1} / p_{x}, t-2}\right]^{n \frac{*}{3}}$

der Nachfrage nach Produkten des privaten Sektors über einen hohen positiven Wert von $\eta_{3}^{\star}$ eine positive Preiselastizität verlieh - eine an sich simple Erklärung. Die Funktion für den Vorratsbestand wurde

(1) Unterhalb des relevanten Bereichs bzw. im Bereich $1_{p r}, t<0,1 \cdot 1 p r, t-1$ ließ sich ein zweiter Funktionsast mit einer weiteren Nullstelle feststellen. Vgl. Figur 18.2.

(2) Der Rechen-Output weist in den genannten Fällen den Text "Keine Nullstelle im relevanten Bereich der FF-Funktion" aus. 
daher durch die fast gleich gute Funktion (6.11) ausgetauscht (1), so daß von jetzt an im relevanten Bereich der Modellrechnung stets nur noch exakte kurzfristige Gleichgewichtslösungen anfielen.

\section{Nachträgliche Korrekturen und Vereinfachungen}

Der Auflösung des gesamten Gleichungssystems bis zur Iterationsfunktion FF $\left(l_{\mathrm{pr}, \mathrm{t}}\right)$ kamen im übrigen von der Grundstruktur der Modellannahmen einige Vereinfachungen zugute. Die wichtigste dieser Vereinfachungen ist wohl, was von monetaristischer Seite kritisiert werden mag, daß weder Kapitalmarktzins noch Geldmenge einen Einfluß auf die Bestimmung der laufenden Nachfrageströme haben (2). Darüber hinaus wurden zur Lösung des Modells und zur Anpassung an die empirischen Verhältnisse in der Bundesrepublik einige Vereinfachungen und Modifikationen nachträglich eingeführt, die im folgenden vorgestellt werden sollen.

Eine erste Vereinfachung diente dazu, die Rechnung nicht allzu unübersichtlich werden zu lassen. Nach (4.46) bis (4.49) wird die Beschäftigungsplanung, nach (5.6) bzw. (5.28) die kurzfristige Arbeitsnachfrage des Unternehmenssektors durch den Arbeitskostensatz $1_{\mathrm{pr}} \mathrm{t}$ beeinflußt. Gemäß (4.14) gehen in den Arbeitskostensatz steuerliche Árbeitskosten-

(1) Theoretisch hätte einer positiven Preiselastizität der Vorratsinvestition auch ein real-balance-Effekt im Bereich der Konsumnachfrage oder eine kurzfristige Zinsabhängigkeit der Bruttoanlageinvestitionen als Kompensation entgegengesetzt werden können, vorausgesetzt, daß diese eine gewisse Mindeststärke aufgewiesen hätten. Angesichts der Höhe des Schätzwertes von $\eta_{3}^{\star}(z+0,63)$ ist mit ziemlicher Sicherheit davon auszugehen, daß der real-balance-Effekt in seinen Auswirkungen auf die Konsumnachfrage die Voraussetzung für sich genommen nicht erfüllt.

(2) Kreditzins und Geldversorgung spielen im Modell kurzfristig eine passive Rolle. Kurzfristige Änderungen des Geldumlaufs sind hier vor allem die Folge des Finanzierungsbedarfs der Unternehmen, der aus der Realisierung der Investitionspläne und Durchsetzung gewerkschaftlicher Lohnforderungen entsteht. Zu den monetären Folgen von Lohnerhöhungen vgl. P. Davidson und S. Weintraub, Money as Cause and Effect, Economic Journal, 83, 1973, S. $1117 \mathrm{ff}$. Es wurde bereits im Paragraphen 9 darauf hingewiesen, daß die Notenbank diese monetären Folgen der Lohnerhöhungen mit Rücksicht auf sonst eintretende Beschäftigungseinbußen kurzfristig nicht bekämpft. Insofern dürfte die Modellkonstruktion trotz monetaristischer Kritik zu rechtfertigen sein. 
bestandteile ein, die selbst gemäß (4.12) und (4.13) definiert sind. Die Vereinfachung besteht darin, daß im Fall der kurzfristigen Arbeitsnachfragefunktionen die steuerlichen Arbeitskostenbestandteile abweichend von $(4.12)$ und $(4.13)$ als

$$
t_{L, t}^{*}=\frac{t_{L 1}+t_{L 2} \cdot L_{p r, t-1}}{L_{p r, t-1}}
$$

und

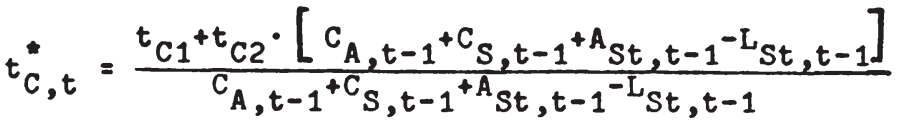

definiert werden. Die Variablen auf der rechten Gleichungs seite wirken sich also anders als in (4.12) und (4.13) verzögert aus. Bei der Berechnung gelten aber die steuerlichen Aufkommensparameter $t_{L 1}, t_{L} 2, t_{C 1}$

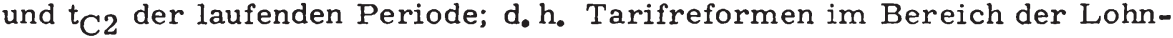
summen- und Verbrauchsteuer wirken sich unmittelbar auf den bei der kurzfristigen Arbeitsnachfrage wirksamen Arbeitskostensatz aus. Die Auswirkungen der Vereinfachung auf das Ergebnis der Modellrechnung hängt von dem Gewicht der absoluten Aufkommensparameter tL1 bzw. tC1 im Vergleich zum Gesamtaufkommen der Lohnsummen- bzw. Verbrauchsteuer ab. Praktisch ist dieses Gewicht so gering, daß mit wesentlichen Veränderungen der Modellergebnisse nicht gerechnet zu werden braucht.

Einige für die Konsistenz des Modells notwendige Modifikationen beziehen sich auf Werte von Parametern: Parameter $B_{0}$ der Beschäftigungsplanungsfunktion (4.49) wurde im Anschluß an (4.48) mit dem Wert

$$
\text { (18.7) } \quad \beta_{0}=\mu_{a} \cdot a_{1} \cdot a_{2}
$$

angesetzt. Aus dem Vergleich von (4.50) mit (5.32) ist zu ersehen, daß diese Modifikation eine Änderung des Parameters von weniger als ein Prozent verursacht.

Zusätzlich wurde Parameter $\mu_{a}$ statt mit dem im Anschluß an (5. 32) ermittelten Wert von 0,893 mit einem Wert von 0,931 angesetzt. Diese Modifikation wurde notwendig, weil der Arbeitskostensatz bei den Regressionsschätzungen noch auf der Basis der Erhebung einer Bruttoumsatzsteuer kalkuliert wurde, während er in der Modellrechnung unter der Annahme der Erhebung einer Nettoumsatzsteuer in Form der indirekten Verbrauchsteuer kalkuliert wird (1).

Schließlich sei noch einmal an die Korrektur des Parameters $\varphi_{1}$ der Kassenhaltungsfunktion (9.14) erinnert, die bereits im Paragraphen 9

(1) Vgl. S. 83 . 
begründet worden war (1).

Mit einer letzten Serie von Korrekturen wurde schließlich versucht, die zeitliche Entwicklung der Modellergebnisse zu stabilisieren, was nur zum geringen Teil gelang. Eine solche Abstimmung erwies sich als unumgänglich, weil gleich im ersten Jahr der Modellrechnung (1960) in den Ergebnissen ein erheblicher Nachfrageüberhang in Erscheinung trat, auf den die Modellrechnung bereits im ersten Folgejahr mit systemsprengenden Ungleichgewichtstendenzen reagierte. Um die rechnerische Ausgangssituation zu stabilisieren und damit zugleich eine Anpassung an die Volkswirtschaftliche Gesamtrechnung der Bundesrepublik im Jahr $1960 \mathrm{zu}$ erreichen, wurde ein einfacher Algorithmus entwickelt, mit dessen Hilfe die Werte von mehreren Parametern simultan so geändert wurden, daß im Prinzip, bei Verwendung geeigneter Parameter, eine entsprechende $\mathrm{Zahl}$ endogener Variable beliebig gesteuert bzw. eine entsprechende Zahl kritischer Abweichungen von den empirischen Verhältnissen in der Bundesrepublik zum Verschwinden gebracht werden konnte. Mit Hilfe dieses Algorithmus sowie der Kontrolle von Schätzergebnissen und Datenmaterial ließ sich herausfinden, daß der Nachfrageüberhang im Ausgangsjahr auf eine ungenaue bzw. überhöhte Ermittlung der verfügbaren Einkommen und Konsumausgaben im Bereich der Arbeitnehmer - und Selbständigen-Haushalte zurückzuführen ist, die sich auf die Schätzung der entsprechenden Konsumparameter in (7.11) und (8.6) ausgewirkt haben muß und die ganze weitere Entwicklung der Modellrechnung hätte beeinflussen müssen. Überwiegend aus diesem Grund mußten alle Konsumparameter der Arbeitnehmer- und SelbständigenHaushalte um gut $3 \%$ vermindert werden. Der entsprechende Korrekturparameter ist PKOR(1).

Regressionsschätzung (3.2) über die Entwicklung der Erwerbsbevölkerung hat einen statistischen Erklärungswert von nur $71 \%$. Aus diesem Grund wurde versuchsweise auch der exogen vorgegebene $e_{v}, t^{-W e r t}$ für das Jahr 1959 mit Hilfe des Korrekturfaktors PKOR(2) abgeändert. Der ermittelte Umfang der erforderlichen Korrektur stimmte fast vollständig mit dem Residuum der Regressionsschätzung für das Jahr 1960 überein, das in diesem Jahr einen besonders hohen Wert hatte.

Zur Stabilisierung der rechnerischen Ausgangssituation bzw. zur Abstimmung auf die konjunkturelle Situation der Bundesrepublik im Jahr 1960 wurden auch die entsprechenden Jahreswerte der Planbeschäftigung und der Plankapazität mit Hilfe der Korrekturparameter PKOR(3) und PKOR(4) geringfügig abgeändert.

Die in diesem Zusammenhang ferner vorgenommene Korrektur des Lohnniveaus in den Jahren 1960 und 1961 mit Hilfe von PKOR11 und PKOR12 sowie die Korrektur des Import- und Exportvolumens 1960

(1) Vgl. S. $164 \mathrm{f}$. 
um die entsprechenden Schätzfehler der Regressionsschätzungen stellen nur eine unwesentliche Korrektur der Modellergebnisse dar, die allein zur Anpassung an die empirischen Verhältnisse in der Bundesrepublik dienen.

In den weiteren Berechnungen zeigte sich, daß die zeitliche Entwicklung der Modellergebnisse trotz der Beseitigung des anfänglichen Nachfrageüberhanges weiterhin ausgesprochen instabil verlief. Nach wenigen Perioden erreichte die Entwicklung die Grenzen an denen die zyklischen Schwankungen in kumulative expansive oder kontraktive Prozesse übergingen und an denen die Ergebniswerte dann bald in den Bereich außerhalb der Kapazität der Rechenmaschine fielen. Alle Versuche, mit Hilfe des entwickelten Algorithmus Hauptursachen dieser Instabilität ausfindig zu machen, und anschließend die Modellentwicklung für eine größere Zahl von Jahren zu stabilisieren, scheiterten. Lediglich in einer Berechnungsserie schienen sich Chancen für eine wirksame Stabilisierung der Modellentwicklung aufzutun. In dieser Berechnungsserie konnte die Modellentwicklung über $18 \mathrm{Jahre}$ hinweg berechnet werden. Da die Ergebnisse auch hier noch nicht zufriedenstellen, wird auf die Ergebnisse dieser Berechnungsserie im folgenden nur am Rande eingegangen.

Im weiteren wird aus den zahlreichen Proberechnungen nur die dargestellt und behandelt, die mit einem Minimum an weiteren korrigierenden Eingriffen auskam und die die Standardentwicklung für die Jahre 1960 bis $1971 \mathrm{zu}$ ermitteln erlaubte. In diesem Fall wurde im zweiten Jahr der Entwicklung, also 1961, ein auf dieses Jahr begrenztes zusätzliches Nachfragedefizit erzeugt, das die Arbeitslosenquote um 0,8\% erhöhte. Das zusätzliche Nachfragedefizit wurde durch eine Senkung des exogen vorgegebenen Kapitalkostensatzes 1959 um 2, 5 \% des ursprünglichen Niveaus erreicht, wodurch der Umfang der Bruttoanlageninvestitionen im Jahre 1961 reduziert wurde. Der entsprechende Korrekturparameter für den Kapitalkostensatz des Jahres 1959 ist symbolisiert durch PKOR(5). Ferner wurde mit Hilfe des Korrekturparameters PKOR(6) der Effizienzparameter $a_{3}$ der langfristigen Produktionsfunktion geändert. Schließlich wurde mit Hilfe des Korrekturparameters PKOR(7) eine nochmalige Korrektur aller Konsumparameter für die Jahre ab 1961 vorgenommen.

\section{Die Liste der Symbole}

Auf den folgenden Seiten findet der Leser eine Liste der Symbole aller Variablen und Parameter, die in der Modellformulierung aufgetreten sind und auch im Rechenprogramm berücksichtigt werden. Die Liste enthält neben den Symbolen und ihrer Kennzeichnung die "Namen", die die entsprechenden Größen im Rechenprogramm haben. Die Symbolbezeichnungen der Variablen und Parameter stimmen zwischen Textteil und Programm weitgehend überein. Die Symbole der Variablen 
enthalten im Programm als erstes Zeichen ein " $\mathrm{X}$ ", die Symbole der Parameter ein "P".

Lediglich im Fall der Parameter für die Aufkommensfunktion (14.1) und (14.3) der Sozialversicherungsbeiträge und für die Funktion (14.10), die das staatlic he Kreditverhalten kennzeichnet, wurde eine kleine Änderung in der Symbolik vorgenommen. Im Programm wird Parameter ${ }^{\mathrm{S} V} 2$ von (14.2) bzw. (14.5) für die Jahre vor 1966 bzw. nach 1972 als PTSV21 eingelesen und über die Anwei sung

\section{PTSV2=PTSV $21+\mathrm{PTSV} 22+(\mathrm{T}-15.0)$}

bei Nullsetzen von PTSV22 dem Parameter PTSV2 zugewiesen. In den Jahren 1966 bis 1972 werden für PTSV21 und PTSV22 die Parameter

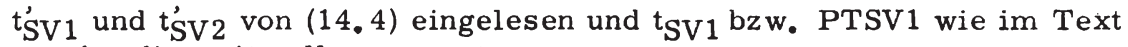
angekündigt mit null angesetzt.

Ebenso wurden die Parameter $h_{F 1}$ und $h_{F 3}$ im Programm als PHF11 und PHF12 eingelesen und über die Anweisung

129 PHF $1=$ PHF $11+$ PHF $2+(\mathrm{T}-10,0)$

intern dem Parameter PHF1 eingespeichert. 
Liste der Symbole von Variablen

Variable

$a_{t}$

${ }^{a}$ St, t

$\left[\frac{e^{e}, t^{-e_{t}}}{a_{t}+\left(e_{v, t}-e_{t}\right)}\right]$

${ }^{\text {A }}$, , t

$\mathrm{C}_{\mathrm{A}, \mathrm{t}}$

$\mathrm{C}_{\mathrm{S}, \mathrm{t}}$

$\mathrm{d}_{\mathrm{pr}, \mathrm{t}}$

$\left[\mathrm{d}_{\mathrm{pr}, \mathrm{t}} / \mathrm{k}_{\mathrm{pr}, \mathrm{t}}\right]$ XDPRQ

$\mathrm{d}_{\text {St }, \mathrm{t}}$

$\left[\mathrm{d}_{\mathrm{St}, \mathrm{t}} / \mathrm{k}_{\mathrm{St}, \mathrm{t}}\right]$ XDSTQ

$\mathrm{D}_{\mathrm{pr}, \mathrm{t}}$

$\mathrm{D}_{\text {St }, \mathrm{t}}$

$e_{t}$

e pr, t

$e_{o, p r, t}$

$e_{v, t}$

$\mathrm{F}_{\mathrm{t}}$ *

$\mathrm{F}_{\mathrm{A}, \mathrm{t}}$
XDSTR

XDPR

X DST

Programm -

Code

$\mathrm{XA}$

XEST

$\mathrm{XAQ}$

XAST

$\mathrm{XCA}$

$\mathrm{XCS}$

XDPRR

XPR

$\mathrm{XE}$

XEPR

XEOPR

XEV

XFX

XFA
Kennzeichnung

beschäftigte Arbeitnehmer, insgesamt

öffentliche Bedienstete

Arbeitslosenquote

staatliche Käufe von Gütern und Diensten

Arbeitnehmer-Konsum

Selbständigen-Konsum

Abschreibungen, real, des privaten Sektors

Abschreibungsquote des privaten Sektors

Abschreibungen, real, des Staates

Abschreibungsquote des Staates

Abschreibungen, nominal, des privaten Sektors

Abschreibungen, nominal, des Staates

Erwerbstätige, insgesamt

Erwerbstätige im privaten Sektor

Planbeschäftigung des privaten Sektors

Erwerbspersonen

Finanzkapital des Unternehmenssektors

Forderungsbestand der Arbeitnehmer 


$\begin{array}{lll}\text { Variable } & \text { Programm- } & \text { Kennzeichnung } \\ \text { Code }\end{array}$

\begin{tabular}{|c|c|c|}
\hline $\mathrm{F}_{\mathrm{Anl}, \mathrm{t}}^{\star}$ & XFXAN & $\begin{array}{l}\text { zur Anlagenfinanzierung aufgenom- } \\
\text { menes Finanzkapital des Unter- } \\
\text { nehmenssektors }\end{array}$ \\
\hline $\mathrm{F}_{\text {Ausl, } \mathrm{t}}$ & XFAUS & $\begin{array}{l}\text { Forderungen des Auslandes an } \\
\text { inländische Unternehmen }\end{array}$ \\
\hline$\Delta \mathrm{F}_{\text {Ausl, }}$ & XFAUSZU & $\begin{array}{l}\text { Zugang von Forderungen beim Aus- } \\
\text { land }\end{array}$ \\
\hline $\mathrm{F}_{\text {Bkt }}$ & XFBK & $\begin{array}{l}\text { Forderungen des Bankensektors an } \\
\text { Produktionsunternehmen }\end{array}$ \\
\hline$\Delta_{\mathrm{Bk}, \mathrm{t}}$ & XFBKZU & $\begin{array}{l}\text { Zugang von Forderungen beim } \\
\text { Bankensektor }\end{array}$ \\
\hline $\mathrm{F}_{\mathrm{S}, \mathrm{t}}$ & XFS & Forderungsbestand der Selbständigen \\
\hline $\mathrm{F}_{\mathrm{St}, \mathrm{Inl}, \mathrm{t}}$ & XFST & $\begin{array}{l}\text { Forderungen des Staates an inlän- } \\
\text { dische Unternehmen }\end{array}$ \\
\hline$\Delta \mathrm{F}_{\mathrm{St}, \mathrm{Inl}, \mathrm{t}}$ & XFSTZU & $\begin{array}{l}\text { Zugang von Inlandsforderungen beim } \\
\text { Staat }\end{array}$ \\
\hline $\mathrm{g}_{\mathrm{k}^{*}, \mathrm{t}}$ & $\mathrm{XGKX}$ & Wachstumsrate von $\mathrm{k}_{\mathrm{St}, \mathrm{t}}$ \\
\hline$g_{1, t}$ & XGLX & $\begin{array}{l}\text { Wachstumsrate des Arbeitskosten- } \\
\text { satzes } l_{\mathrm{pr}, \mathrm{t}}\end{array}$ \\
\hline$g_{p, t}$ & XGP & $\begin{array}{l}\text { Wachstumsrate des Preisniveaus } \\
\mathrm{p}_{x, t}\end{array}$ \\
\hline $\mathrm{g}_{\mathrm{r}, \mathrm{t}}^{\star}$ & XGRX & $\begin{array}{l}\text { Wachstumsrate des Kapitalkosten- } \\
\text { satzes } r_{t}\end{array}$ \\
\hline$g_{x, t}$ & XGX & $\begin{array}{l}\text { Wachstumsrate der privaten Pro- } \\
\text { duktion } \mathrm{x}_{\mathrm{t}}\end{array}$ \\
\hline$G_{t}$ & XG & $\begin{array}{l}\text { Bruttounternehmensgewinne (vor } \\
\text { der Umverteilung) }\end{array}$ \\
\hline Gew'Ertrag ${ }_{t}$ & XGERT & Gewerbeertrag \\
\hline $\mathrm{H}_{1, \mathrm{t}}$ & $\mathrm{XH1}$ & $\begin{array}{l}\text { staatliche Haushaltsvolumen } 1 \\
\text { (= Einnahmen ohne Einnahmen aus } \\
\text { öffentlicher Kreditaufnahme) }\end{array}$ \\
\hline
\end{tabular}




$\begin{array}{ll}\text { Variable } & \begin{array}{l}\text { Programm- Kennzeichnung } \\ \text { Code }\end{array}\end{array}$

\begin{tabular}{|c|c|c|}
\hline $\mathrm{H}_{2, \mathrm{t}}$ & $\mathrm{XH} 2$ & $\begin{array}{l}\text { staatliches Haushaltsvolumen } 2 \\
\text { (= Einnahmen einschließlich Ein- } \\
\text { nahmen aus inländischer Netto- } \\
\text { kreditaufnahme) }\end{array}$ \\
\hline $\mathrm{I}_{\mathrm{pr}, \mathrm{t}}^{\mathrm{br}}$ & XIPR & Bruttoinvestition der Unternehmen \\
\hline $\mathrm{I}_{\mathrm{pr}, \mathrm{Anl}, \mathrm{t}}^{\mathrm{br}}$ & XIPRAN & $\begin{array}{l}\text { Bruttoanlageinvestition der Unter- } \\
\text { nehmen }\end{array}$ \\
\hline$I_{S t, t}^{b r}$ & XIST & Infrastrukturinvestition des Staates \\
\hline$I_{v, t}$ & XIV & Lagerinvestitionen der Unternehmen \\
\hline $\mathrm{k}_{\mathrm{pr}, \mathrm{t}}^{\mathrm{n}}$ & XKPR & $\begin{array}{l}\text { Nettoanlagevermögen der Unter- } \\
\text { nehmen, real }\end{array}$ \\
\hline${ }_{\mathrm{pr}, \mathrm{t}}$ & XKPR & $\begin{array}{l}\text { Bruttoanlagevermögen der Unter- } \\
\text { nehmen, real }\end{array}$ \\
\hline$\Delta_{\mathrm{k}}^{+}$ & XKPRZU & $\begin{array}{l}\text { Zugang von neuen Anlagen im } \\
\text { privaten Sektor }\end{array}$ \\
\hline$\Delta \mathrm{k}_{\mathrm{pr}, \mathrm{t}}^{\circ}$ & XKPRAB & $\begin{array}{l}\text { Abgang von alten Anlagen im } \\
\text { privaten Sektor }\end{array}$ \\
\hline $\mathbf{k}_{\mathrm{o}, \mathrm{St}, \mathrm{t}}$ & XKOST & $\begin{array}{l}\text { erwarteter öffentlicher Infrastruk- } \\
\text { turanlagenbestand }\end{array}$ \\
\hline $\mathrm{k}_{\mathrm{o}, \mathrm{St}, \mathrm{t}}^{\star}$ & XKXOST & $\begin{array}{l}\text { zur Kennzeichnung vgl. Gleichung } \\
(4.3) \mathrm{bzw} .(4.53)\end{array}$ \\
\hline${ }^{k}$ St, t & $\mathrm{XKST}$ & $\begin{array}{l}\text { öffentlicher Infrastrukturanlagen- } \\
\text { bestand }\end{array}$ \\
\hline$\Delta \mathrm{k}_{\mathrm{St}, \mathrm{t}}^{+}$ & XKSTZU & $\begin{array}{l}\text { Zugang von neuen öffentlichen } \\
\text { Infrastrukturanlagen }\end{array}$ \\
\hline$\Delta \mathrm{k}_{\mathrm{St}, \mathrm{t}}^{\dot{\circ}}$ & XKSTAB & $\begin{array}{l}\text { Abgang von alten öffentlichen } \\
\text { Infrastrukturanlagen }\end{array}$ \\
\hline $\mathrm{k}_{\mathrm{St}, \mathrm{t}}^{\star}$ & XKXST & $\begin{array}{l}\text { zur Kennzeichnung vgl. Gleichung } \\
(4.3)\end{array}$ \\
\hline$l_{p r, t}$ & XLOHN & Lohnsatz des privaten Sektors \\
\hline $1 \stackrel{p r, t}{*}$ & XLOHNX & $\begin{array}{l}\text { Arbeitskostensatz des privaten } \\
\text { Sektors }\end{array}$ \\
\hline $\mathrm{L}_{\mathrm{t}}$ & $\mathrm{XL}$ & Arbeitseinkommen, insgesamt \\
\hline
\end{tabular}




$\begin{array}{lll}\text { Variable } & \text { Programm- Kennzeichnung } \\ \text { Code }\end{array}$

$\begin{array}{ll}\mathrm{L}_{\mathrm{A}, \mathrm{t}} & \text { XLA } \\ {\left[\mathrm{L}_{\mathrm{A}, \mathrm{t}} / \mathrm{Y}_{\mathrm{t}}\right]} & \text { XLAQ }\end{array}$

Einkommen aus unselbständiger Beschäftigung

Anteil der Einkommen aus unselbständiger Beschäftigung am Volkseinkommen
$\mathrm{L}_{\mathrm{pr}, \mathrm{t}}$
XLPR
$\left[\mathrm{L}_{\mathrm{pr}, \mathrm{t}} / \mathrm{Y}_{\mathrm{pr}, \mathrm{t}}^{\star}\right]$ XLPRQ

im privaten Sektor entstandenes

Arbeitseinkommen

Anteil der Arbeitseinkommen an der Bruttowertschöpfung des privaten Sektors
$\mathrm{L}_{\mathrm{S}, \mathrm{t}}$
${ }^{\mathrm{L}} \mathrm{St}, \mathrm{t}$
XLS
XLST

$\mathrm{M}_{\mathrm{t}}$

$\left[\mathrm{M}_{\mathrm{t}} / \mathrm{Y}_{\mathrm{t}}^{\mathrm{br}}\right]$

$\mathrm{M}_{\mathrm{A}, \mathrm{t}}$

$\left[\frac{M_{A, t}+M_{S, t}}{L_{A, t}{ }^{+L}}\right]$

$\mathrm{M}_{\text {Dev , }}$

$M_{S, t}$

${ }^{M_{U, t}}$

$\mathrm{N}_{\mathrm{t}}$

P Ausl, t $_{\text {A }}$

$\mathrm{p}_{\mathrm{Ex}, \mathrm{t}}$

$\mathrm{p}_{\text {Im }, \mathrm{t}}$

$\mathrm{P}_{k, t}$
XM

XMQ

XMA

XMHHQ

XMDEV

XMS

XMU

$\mathrm{XN}$

XPAUS

XPEX

XPIM

XPK aggregierter Unternehmerlohn

im staatlichen Sektor entstandenes Arbeitseinkommen

Geldumlauf

gesamtwirtschaftlicher Kassenhaltungsquotient

Kassenbestand der ArbeitnehmerHaushalte

Kassenhaltungsquotient der privaten Haushalte

Devisenbestand

Kassenbestand der SelbständigenHaushalte

Kassenbestand der Unternehmen

Einlagenvolumen der Geschäftsbanken

BSP-Preisindex des Auslandes

Preisindex für Exporte

Preisindex für Importe

Preisindex für Anlageinvestitionen 


$\begin{array}{ll}\text { Variable } & \text { Programm- Kennzeichnung } \\ \text { Code }\end{array}$

\begin{tabular}{|c|c|c|}
\hline$p_{v, t}$ & XPV & Preisindex für Lagerinvestitionen \\
\hline$p_{x, t}$ & $\mathrm{XPX}$ & $\begin{array}{l}\text { Preisindex des privaten BIP- } \\
\text { Beitrages }\end{array}$ \\
\hline$Q_{t}$ & $\mathrm{XQ}$ & $\begin{array}{l}\text { Liquiditätsreserven der Geschäfts- } \\
\text { banken }\end{array}$ \\
\hline$r_{t}$ & XZINS & Zinssatz \\
\hline $\mathrm{r}_{\mathrm{t}}^{\star}$ & XZINSX & Kapitalkostensatz \\
\hline$r_{\text {Ausl, } t}$ & XZINSAU & Auslandszins \\
\hline$R_{t}$ & $\mathrm{XR}$ & Zinseinkommen der Inländer \\
\hline $\mathrm{R}_{\mathrm{t}}^{\star}$ & $\mathrm{XRX}$ & $\begin{array}{l}\text { im Unternehmenssektor entstan- } \\
\text { denes Zinseinkommen }\end{array}$ \\
\hline $\mathrm{R}_{\mathrm{A}, \mathrm{t}}$ & $\mathrm{XRA}$ & $\begin{array}{l}\text { Zinseinkommen der Arbeitnehmer- } \\
\text { Haushalte }\end{array}$ \\
\hline${ }^{R}, t$ & XRS & $\begin{array}{l}\text { Zinseinkommen der Selbständigen- } \\
\text { Haushalte }\end{array}$ \\
\hline $\mathrm{R}_{\text {St, } t}$ & XRST & Zinseinkommen des Staates \\
\hline$s_{t}$ & $\mathrm{XS}$ & Selbständige \\
\hline${ }^{S} \mathrm{~A}, \mathrm{t}$ & $\mathrm{XSA}$ & $\begin{array}{l}\text { Ersparnisse der Arbeitnehmer- } \\
\text { Haushalte }\end{array}$ \\
\hline$S_{S, t}$ & XSS & $\begin{array}{l}\text { Ersparnis der Selbständigen- } \\
\text { Haushalte }\end{array}$ \\
\hline $\mathrm{S}_{\mathrm{U}, \mathrm{t}}$ & XSU & Ersparnis der Unternehmen \\
\hline$t_{c, t}$ & XTCQ & $\begin{array}{l}\text { Prozentbelastung der Verbrauchs- } \\
\text { ausgaben durch die Verbrauchsteuer }\end{array}$ \\
\hline $\mathrm{t}_{\mathrm{Im}, \mathrm{t}}$ & XTIMQ & $\begin{array}{l}\text { Prozentbelastung der Importwerte } \\
\text { durch Zölle }\end{array}$ \\
\hline$t_{k, t}$ & XTKQ & $\begin{array}{l}\text { Prozentbelastung des bewerteten } \\
\text { Nettoanlagevermögens durch die } \\
\text { Gewerbekapitalsteuer }\end{array}$ \\
\hline$t_{L, t}$ & XTLQ & $\begin{array}{l}\text { Prozentbelastung der vom Unterneh- } \\
\text { menssektor verteilten Arbeitsein- } \\
\text { kommen durch die Lohnsummensteuer }\end{array}$ \\
\hline
\end{tabular}




$\begin{array}{ll}\text { Variable } & \begin{array}{l}\text { Programm- Kennzeichnung } \\ \text { Code }\end{array}\end{array}$

${ }^{t} R G, t$

$\mathrm{t}_{\text {RGS, }} \mathrm{t}$

XTRGSQ

$\mathrm{T}_{\mathrm{C}, \mathrm{t}}$

$T_{\text {dir, }} t$

$T_{F, t}$

$\mathrm{T}_{\text {Im, } \mathrm{t}}$

$T_{\text {ind, }} \mathrm{t}$

$T_{k, t}$

$\mathrm{T}_{\mathrm{L}, \mathrm{t}}$

$\mathrm{T}_{\text {LA, t }}$

$\mathrm{T}_{\text {LS, }}$

$\mathrm{T}_{\text {RG, }}$

$T_{\text {RGS, } t}$

$\mathrm{T}_{\text {SV }, \mathrm{t}}$

$\mathrm{U}_{\mathrm{A}, \text { Ausl, } \mathrm{t}}$

$\mathrm{U}_{\text {St, } \mathrm{t}}$

$\mathrm{U}_{\mathrm{St}, \mathrm{A}, \mathrm{t}}$

$\mathrm{U}_{\mathrm{St}}^{\star}$, Ausl, $\mathrm{t}$

$\mathrm{U}_{\mathrm{St}, \mathrm{U}, \mathrm{t}}$

XUSTU
Prozentbelastung des Gewerbeertrags durch die Gewerbeertragsteuer

Prozentbelastung der fundierten Selbständigen-Einkommen durch die auf diese entfallende direkte Steuer

Aufkommen der Verbrauchsteuer

Aufkommen an direkten Steuern

Aufkommen der Vermögensteuer

Zollaufkommen

Aufkommen an indirekten Steuern

Aufkommen der Gewerbekapitalsteuer

Aufkommen der Lohnsummensteuer

Aufkommen der Lohnsteuer

Aufkommen der Unternehmerlohnsteuer

Aufkommen der Gewerbeertragsteuer

Aufkommen der Steuer auf fundierte Selbständigen-Einkommen

Aufkommen an Sozialversicherungsbeiträgen

Auslandsübertragungen der Arbeitnehmer

geleistete Übertragungen des Staates, insgesamt (einschließlich Kreditgewährung an das Ausland)

Sozialleistungen des Staates

staatliche Kapitalhilfe an das Ausland (einschließlich Kreditgewährung)

Subventionen an den Unternehmenssektor 


$\begin{array}{lll}\text { Variable } & \begin{array}{l}\text { Programm- Kennzeichnung } \\ \text { Code }\end{array}\end{array}$

\begin{tabular}{|c|c|c|}
\hline$\ddot{U}_{W S A, t}$ & XUEWSA & $\begin{array}{l}\text { Vermögensübertragungen zwischen } \\
\text { den Sektoren der Selbständigen- und } \\
\text { Unselbständigen-Haushalte }\end{array}$ \\
\hline $\mathrm{v}_{\mathrm{t}}$ & XV & Vorratsbestand, real \\
\hline $\mathrm{V}_{\mathrm{S}, \mathrm{t}}$ & XVS & $\begin{array}{l}\text { vom Unternehmenssektor an die } \\
\text { Selbständigen-Haushalte ausge- } \\
\text { schüttete Besitzeinkommen }\end{array}$ \\
\hline $\mathbf{w}_{\mathrm{t}}$ & XW & Wechselkurs \\
\hline$w_{t}^{\star}$ & XwX & $\begin{array}{l}\text { Wechselkurs (b. Berücksichtigung } \\
\text { der Quasiaufwertung von 1968) }\end{array}$ \\
\hline $\mathbf{w}_{\mathrm{A}, \mathrm{t}}$ & XWA & Nominalvermögen der Arbeitnehmer \\
\hline$\left[\mathrm{w}_{\mathrm{A}, \mathrm{t}} / \mathrm{w}_{\mathrm{HH}, \mathrm{t}}\right]$ & XWAQ & $\begin{array}{l}\text { Anteil der Arbeitnehmer am Nomi- } \\
\text { nalvermögen des privaten Sektors }\end{array}$ \\
\hline $\mathbf{w}_{\mathrm{HH}, \mathrm{t}}$ & XWHH & $\begin{array}{l}\text { Nominalvermögen des privaten } \\
\text { Sektors }\end{array}$ \\
\hline$\left[w_{H H, t} / w_{p r, t}\right]$ & XWHHQ & $\begin{array}{l}\text { Anteil des privaten Nominalvermö- } \\
\text { gens am Gesamtbetrag von privatem } \\
\text { Nominalvermögen plus Substanzer- } \\
\text { haltungsrücklage der Unternehmen }\end{array}$ \\
\hline $\mathrm{w}_{\mathrm{pr}, \mathrm{t}}$ & XWPR & $\begin{array}{l}\text { Nominalvermögen des privaten Sek- } \\
\text { tors plus Substanzerhaltungsrück- } \\
\text { lage der Unternehmen }\end{array}$ \\
\hline $\mathbf{w}_{\mathrm{S}, \mathrm{t}}$ & XWS & Nominalvermögen der Selbständigen \\
\hline $\mathrm{w}_{\mathrm{U}, \mathrm{t}}$ & XWU & $\begin{array}{l}\text { aggregierte Substanzerhaltungsrück- } \\
\text { lage des Unternehmenssektors }\end{array}$ \\
\hline$x_{t}$ & $\mathrm{XX}$ & $\begin{array}{l}\text { BIP-Beitrag des privaten Sektors, } \\
\text { real }\end{array}$ \\
\hline${ }^{\mathbf{x}}$ Ausl, $\mathrm{t}$ & XXAUS & BSP des Auslandes, real \\
\hline $\mathbf{x}_{E x, t}$ & XXEX & Exportvolumen, real \\
\hline $\mathrm{x}_{\mathrm{Im}, \mathrm{t}}$ & XXIM & Importvolumen, real \\
\hline$x_{0, t}$ & $\mathrm{XXO}$ & Plankapazität \\
\hline
\end{tabular}


Kapitalkoeffizient des privaten Sektors

\begin{tabular}{ll}
\hline$\left[x_{t} / k_{p r, t}\right]$ & XKKOEF \\
{$\left[x_{t} / x_{o, t}\right]$} & XKAPAZ \\
$Y_{t}$ & $X Y$ \\
$Y_{t}^{b r}$ & $X Y B R$ \\
$Y_{t}^{n}$ & $X Y N$ \\
$Y_{A, t}$ & XYA \\
{$\left[Y_{A, t} / Y_{t}\right]$} & XYAQ \\
$Y_{A, t}^{n}$ & XYAN \\
$Y_{A, t}^{n}$ & XYANX \\
{$\left[Y_{A, t}^{n^{*}} / Y_{p r, t}^{n}\right]$} & XYANQ
\end{tabular}

Kapazitätsauslastungsgrad

Volkseinkommen

BSP z. Marktpreisen

NSP z. Marktpreisen

Einkommen der ArbeitnehmerHaushalte

Anteile der Arbei tnehmer-Einkommen am Volkseinkommen

verfügbares Einkommen der Arbeitnehmer-Hau shalte

verfügbares Einkommen der Arbeitnehmer-Haushalte zuzüglich der Auslandsübertragungen der Arbeitnehmer

Anteil der verfügbaren ArbeitnehmerEinkommen (zuzüglich der Auslands übertragungen der Arbeitnehmer) am verfügbaren Einkommen des privaten Sektors (zuzüglich der Auslandsübertragungen der Arbeitnehmer)

$\mathrm{Y}_{\mathrm{pr}, \mathrm{t}}^{\star}$

XYPRX

$\mathrm{Y}_{\mathrm{pr}, \mathrm{t}}^{\mathrm{n}}$

XYPRN

$\mathrm{Y}_{\mathrm{pr}, \mathrm{t}}^{\mathrm{n}}$

XYPRNX

$\left[\frac{\mathrm{Y}_{\mathrm{pr}, \mathrm{t}}^{\mathrm{n}^{\star}}}{\mathrm{Y}_{\mathrm{t}}+\mathrm{T}_{\text {ind }, t}}\right]$

XYPRNQ

$\mathrm{Y}_{\mathrm{S}, \mathrm{t}}$

XYS im privaten Sektor entstandene Bruttowertschöpfung

verfügbares Einkommen des privaten Sektors

verfügbares Einkommen des privaten Sektors zuzüglich der Auslandsüber tragungen der Arbeitnehmer

Anteil des verfügbaren Einkommens des privaten Sektors (zuzüglich der Auslandsübertragungen der Arbeitnehmer) am NSP zu Marktpreisen Einkommen der Selbständigen-Haushalte 


$\begin{array}{ll}\text { Variable } & \begin{array}{l}\text { Programm- Kennzeichnung } \\ \text { Code }\end{array}\end{array}$

\begin{tabular}{|c|c|c|}
\hline$Y_{S, t}^{n}$ & XYSN & $\begin{array}{l}\text { verfügbares Einkommen der Selb- } \\
\text { ständigen-Haushalte }\end{array}$ \\
\hline $\mathrm{Y}_{\text {St, t }}$ & XYST & Einkommen des Staates \\
\hline $\mathrm{Y}_{\mathrm{St}, \mathrm{t}}^{\mathrm{n}}$ & XYSTN & $\begin{array}{l}\text { verfügbares Einkommen des Staates } \\
\text { (nach Abzug der Kreditgewährung } \\
\text { an das Ausland) }\end{array}$ \\
\hline${ }^{Y_{U, t}}$ & XYU & Einkommen der Unternehmen \\
\hline$Y_{U, t}^{n}$ & XYUN & $\begin{array}{l}\text { verfügbares Einkommen der Unter- } \\
\text { nehmen }\end{array}$ \\
\hline$Z_{\text {Dev, } t}$ & XZDEV & Saldo der Devisenbilanz \\
\hline${ }^{Z} R, t$ & $\mathrm{XZR}$ & $\begin{array}{l}\text { Saldo der Vermögenserträge im } \\
\text { Kreditverkehr mit dem Ausland }\end{array}$ \\
\hline$z_{x, t}$ & $\mathrm{XZX}$ & $\begin{array}{l}\text { Außenbeitrag im internationalen } \\
\text { Warenverkehr }\end{array}$ \\
\hline
\end{tabular}


Parameter für das Verhalten des privaten Sektors
Programm Code
Gleichungs -No. im Text $a_{1}$

$a_{2}$

a 3

f

$\operatorname{antilog} \boldsymbol{\alpha}_{1}^{\prime \prime}$

$\alpha_{2}^{\prime \prime}$

$a_{3}^{\prime \prime}$

${ }^{B} 0$

$\beta_{1}$

$\mathrm{B}_{2}$

$\mathrm{B}_{3}$

$\gamma_{1}$

$r_{2}$

$\sigma_{1}$

$\delta_{2}$

$\delta_{3}$

${ } 4$

$\varepsilon_{1}$

$\varepsilon_{2}$

E 3

$\varepsilon_{4}$

$\varepsilon^{\prime} 1$

$\varepsilon^{\prime}$

$\varepsilon^{\prime} 3$

$\varepsilon^{\prime}$

$\zeta 1$
PALFA1

PALFA2

PALFA3

PF

PALFAX1

PALFAX2

PALFAX3

PBETAO

4.50

PBETA1

PBETA2

PBETA3

PGAM1

PGAM2

PDELT1

PDELT2

PDELT3

PDELT4

PEPSI1

PEPSI2

PEPSI3

PEPSI4

PEPSIX1

PEPSIX2

PEPSIX3

PEPSIX4

PZET1
4. 44

4.45

4. 49

4. 3

4. 51

4. 54

4

5.27

5. 33

5.28

5.33

5.13

5.14

5. 15

5.27

5. 32

3. 1

3. 2

3. 3

3.4

6.5

6. 6 
Parameter für das Verhalten des privaten Sektors
ProgrammCode
Gleichungs-No. im Text

5

PZET2

$n$

PETA1

6. 11

6.12

$n_{2}$

PETA2

$n_{3}$

PETA3

$n_{4}$

PETA4

$\vartheta_{1}$

PTHET1

6.13

6.14

$\vartheta_{2}$

$\checkmark 3$

$\vartheta_{4}$

PTHET2

PTHET 3

PTHET4

$\iota_{1}$

PJOT1

7.5

7. 6

$\iota_{2}$

PJOT2

$x_{1}$

PKAP1

7.1

7.2

$x_{2}$

PKAP2

$x_{3}$

PKAP3

$x_{4}+x_{7}$

PKAP4

$x_{5}+x_{7}$

PKAP5

$x_{6}+x_{7}$

$x_{7}$

$x_{8}$

PKAP6

PKAP7 bzw. PKAP8

PKAP9

$\lambda_{1}$

PLAM1

9.4

9.9

$\lambda_{2}$

PLAM2

$\lambda_{3}$

PLAM3

$\lambda_{4}$

PLAM4

$\lambda_{5}$

PLAM5

$\mu_{\mathrm{a}}$

PMUEA

5.30

5. 32

$\mu_{1}$

PMUE1

11.2

11.3

$\mu_{2}$

PMUE2 
Parameter für das Verhalten des privaten Sektors
Programm -

Code

Gleichungs - No.

im Text

\begin{tabular}{|c|c|c|c|}
\hline$\mu_{3}$ & PMUE3 & & \\
\hline$\mu_{4}$ & PMUE4 & & \\
\hline$\mu_{1}^{\prime}$ & PMUEX1 & 11.4 & 11.5 \\
\hline$\mu_{2}^{\prime}$ & PMUEX2 & & \\
\hline$\mu_{3}^{\prime}$ & PMUEX3 & & \\
\hline$v_{1}$ & PNUE1 & 7.7 & 7.8 \\
\hline$v_{2}$ & PNUE2 & & \\
\hline $\operatorname{antilog} \boldsymbol{\xi}_{1}$ & PXIX1 & 6.2 & 6.3 \\
\hline antilog $\boldsymbol{\xi}_{2}$ & PXIX2 & & \\
\hline $\operatorname{antilog} \boldsymbol{\xi}_{3}$ & PXIX3 & & \\
\hline${ }^{\circ} \mathrm{A} 1$ & POMIA1 & 7.9 & 7.11 \\
\hline${ }^{\circ} \mathrm{A} 2$ & POMIA2 & & \\
\hline${ }^{\circ} \mathrm{A3}$ & POMIA3 & & \\
\hline${ }^{\circ} \mathrm{A} 4$ & POMIA4 & & \\
\hline${ }^{\circ} \mathrm{S} 1$ & POMIS1 & 8.5 & 8.6 \\
\hline${ }^{\circ} \mathrm{S} 2$ & POMIS2 & & \\
\hline${ }^{\circ} \mathrm{S} 3$ & POMIS3 & & \\
\hline$\pi_{1}$ & PPII & 8.3 & 8.4 \\
\hline$\pi_{2}$ & PPI2 & & \\
\hline$\pi_{3}$ & PPI3 & & \\
\hline$o_{1}$ & PRHO1 & 9.2 & 9.7 \\
\hline $\mathrm{o}_{2}$ & PRHO2 & & \\
\hline$\rho_{3}$ & PRHO3 & & \\
\hline $\mathrm{P}_{4}$ & PRHO4 & & \\
\hline${ }^{\circ} 5$ & PRHO5 & & \\
\hline$\rho_{6}$ & PRHO6 & & \\
\hline
\end{tabular}


Parameter für das Verhalten des privaten Sektors
Programm -

Code

PRHOX1

PRHOX2

PSIG1

PSIG2

PSIG 3

PSIG 4

PSIG5

PTAU1

9.5

9.10
Gleichungs-No. im Text $\tau_{1}$

$\tau_{2}$

$\tau_{3}$

$\tau_{4}$

$\tau_{5}$

$\tau_{6}$

$u_{1}$

$u_{2}$

$u_{3}$

$u_{4}$

$\varphi_{1}$

$\varphi_{2}$

$\varphi_{3}$

${ }^{\oplus} 4$

$x_{1}$

$x_{2}$

$x_{3}$

$x_{4}$

$x_{1}^{\prime}$

$x^{\prime}$
PTAU2

PTAU 3

PTAU 4

PTAU5

PTAU6

PYPSI1

6. 9

6.10

PYPSI2

PYPSI3

PYPSI4

P PHI1

9.12

9.13

PPHI2

PPHI3

PPHI4

PCHI1

11.7

11.8

$\mathrm{PCHI} 2$

PCHI3

PCHI4

PCHIX1

11.9

11. 10

PCHIX2 
Parameter für das Verhalten des privaten Sektors

$x_{3}^{\prime}$

$x_{1}$

$x_{2}^{\prime \prime}$

$x^{\prime \prime} 1$

$\times \frac{m}{2}$

$\times \frac{10}{3}$

$\Psi_{1}$

$\Psi_{2}$

$\Psi_{3}$

$\Psi_{4}$

$\omega_{1}$

$\omega_{2}$

$\omega_{3}$

$\mathrm{r}_{\text {Ris }}$
Programm -

Code

PCHIX3

PCHI2X1

PCHI2X2

PCHI3X1

PCHI3X2

PCHI3X3

PPSI1

PPSI2

PPSI3

PPSI4

POMEG 1

POMEG2

POMEG 3

PRISIKO

4. 11
Gleichungs-No. im Text
10.4

10.5

$11.13 \quad 11.14$

11.15

11.16

10.1

10.3

10.5 


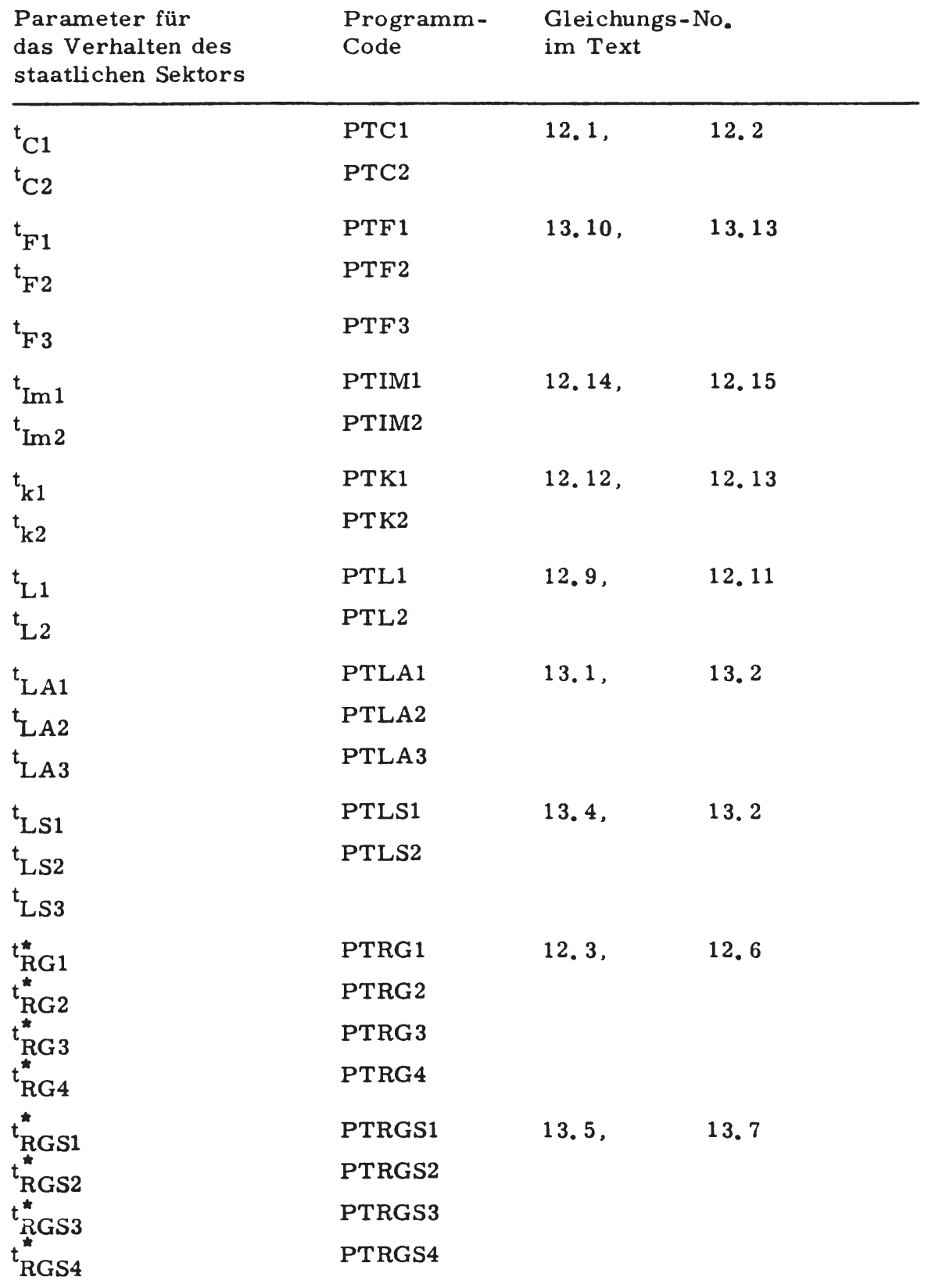


Parameter für das Verhalten des staatlichen Sektors
(1)

(1)

$\mathrm{t}_{\mathrm{SV} 2}$

-.-

${ }^{\mathrm{h}}$ ASt1

$\mathrm{h}_{\text {ASt2 }}$

${ }^{\mathrm{D} 1}$

D2

-.-

$\mathrm{h}_{\mathrm{F} 1}$ (1)

$\mathrm{h}_{\mathrm{F} 3}$ (1)

-.-

$\mathrm{h}_{\mathrm{J} 1}$

$\mathrm{h}_{\mathrm{J} 2}$

$\mathrm{h}_{\mathrm{k} 1}$

$\mathrm{h}_{\mathrm{k} 2}$

$\mathrm{h}_{\text {LSt, t }}$

$\mathrm{h}_{\text {LSt1 }}$

h LSt2

$h_{\text {LSt3 }}$

$\mathrm{h}_{\mathrm{LSt} 4}$

$\mathrm{h}^{\star}$ UA1

$\mathrm{h}^{\star}$ UA2
Programm- Gleichungs-No.

Code im Text
PTSV1

PTSV2

PTSV 21

PTSV22

PHEST 1

15.12,

15.14

PHEST2

PHD1

17. 7 ,

17.8

PHD2

PHF1

14. 10 ,

14.11

PHF11

PHF 12

PHF2

PHII

17. 2 ,

17.3

PHI2

PHK1

17. 4 ,

17.5

PHK2

PHLST

15. 2 ,

15. 3

PHLST1

PHLST2

PHLST 3

PHLST4

PHUA1

16. 1 ,

16. 3

PHUA2

(1) Vgl. die Bemerkungen zur Codierung zu Beginn dieses Abschnitts. 
Parameter für das Verhalten des staatlichen Sektors
Programm- Gleichungs - No.

Code im Text

\begin{tabular}{|c|c|c|c|c|}
\hline $\mathrm{h}^{*} \mathrm{UA3}$ & PHUA3 & & & \\
\hline $\mathrm{h}^{\star}$ UA4 & PHUA4 & & & \\
\hline $\mathrm{h}^{\star}$ UAusl1 & PHUAUS1 & 16. 8 & 16.10 & \\
\hline $\mathrm{h}^{\star}$ UAus12 & PHUAUS2 & & & \\
\hline $\mathrm{h}^{\star}$ UAus13 & PHUAUS3 & & & \\
\hline $\mathrm{h}^{\star}{ }_{\text {UAusl4 }}$ & PHUAUS4 & & & \\
\hline $\mathrm{h}_{\mathrm{UU} 1}$ & PHUU 1 & 16. 4 , & 16.5 & \\
\hline$h_{U U 2}$ & PHUU2 & & & \\
\hline$h_{U U 3}$ & PHUU 3 & & & \\
\hline$\Delta t_{C 1}$ & DTC1 & 12.1 & 12,2 & \\
\hline${ }^{\Delta t} \mathrm{C} 2$ & DTC2 & & & \\
\hline$\Delta t_{F 1}$ & DTF1 & 13.10 & 13. 12 & 13.13 \\
\hline$\Delta t_{F 2}$ & DTF2 & & & \\
\hline$\Delta t_{F 3}$ & DTF 3 & & & \\
\hline$\Delta \mathrm{t}_{\operatorname{Im} 1}$ & DTIM1 & 12.14 & 12.5 & 12.16 \\
\hline$\Delta \mathrm{t}_{\operatorname{Im} 2}$ & DTIM2 & & & \\
\hline & DT K1 & 17.4 & 17.5 & \\
\hline & DT K2 & & & \\
\hline$\Delta \mathrm{t}$ & DTL1 & 12.10 & 12.11 & \\
\hline$\Delta t$ & DTL2 & & & \\
\hline$\Delta t_{L A 1}$ & DTLA1 & 13. 1 , & 13.2 , & 13.3 \\
\hline$\Delta \mathrm{t}_{\mathrm{LA2}}$ & DTLA2 & & & \\
\hline$\Delta t_{L A 3}$ & DTLA3 & & & \\
\hline${ }^{\Delta t}{ }_{R G 1}$ & DTRG1 & 12. 3 , & 12.8 & \\
\hline$\Delta t_{R G 2}$ & DTRG2 & & & \\
\hline$\Delta t_{\text {RG3 }}$ & DTRG3 & & & \\
\hline
\end{tabular}


Parameter für

das Verhalten des staatlichen Sektors
Programm- Gleichungs-No.

Code im Text

$\Delta \mathrm{t}_{\mathrm{RG} 4}$

DTRG4

$\Delta_{1} t_{\text {RGS1 }}$

DTRGS1

13. 5 ,

13. 7 ,

13.8

$\Delta_{1} t_{\text {RGS2 }}$

DTRGS2

$\Delta_{1}{ }^{t}$ RGS3

DTRGS3

$\Delta_{1}{ }^{t}$ RGS4

DTRGS4

$\Delta{ }_{2}{ }^{\mathrm{t}} \mathrm{RGS} 1$

D1TRGS1

13. 5 ,

13. 8 ,

13. 9

$\Delta{ }^{\mathrm{t}}{ }^{\mathrm{RGS} 2}$

D1 TRGS2

$\Delta_{2}{ }^{t}$ RGS3

D1TRGS3

$\Delta_{2}{ }^{\mathrm{t}}$ RGS4

D1 TRGS4

$\Delta \mathrm{t}_{\mathrm{SV} 1}$ (1)

DTSV1

14. 1 ,

14.4

$\Delta \mathrm{t}_{\mathrm{SV} 21}$

$\Delta \mathrm{t}_{\mathrm{SV} 22}$

DTSV 21

DTSV22

$\Delta_{1}{ }^{\mathrm{t}} \mathrm{SV} 1$

D1TSV1

14. 1 ,

14.4

$\Delta_{1} t_{S V} 21$

D1 TSV21

$\Delta_{1}{ }^{t}$ SV 22

D1TSV22

$\Delta \mathrm{h}_{\text {ASt } 1}$

DHEST 1

15.12,

15.15

$\Delta \mathrm{h}_{\mathrm{ASt} 2}$

DHEST2

$\Delta \mathrm{h}_{\mathrm{F} 11}(1)$

DHF 11

14. 7 ,

14.12

$\Delta \mathrm{h}_{\mathrm{F} 12}$

DHF 12

$\Delta \mathrm{h}_{\mathrm{F} 2}$

DHF 2

$\Delta \mathrm{h}_{\mathrm{J} 1}$

DHJ 1

17.2

$\Delta \mathrm{h}_{\mathrm{J} 2}$

DHJ 2

$\Delta \mathrm{h}_{\mathrm{k} 1}$

$\Delta \mathrm{h}_{\mathrm{k} 2}$

DHK1

17.4

DHK2

(1) Vgl. die Bemerkungen zur Codierung zu Beginn dieses Abschnitts. 
Parameter für Cas Verhalten des staatlichen Sektors
Programm- Gleichungs-No.

Code im Text

\begin{tabular}{|c|c|c|c|}
\hline$\Delta h_{\text {LST1 }}$ & DHLST1 & 15.2, & 15.4 \\
\hline$\Delta \mathrm{h}_{\mathrm{LST} 2}$ & DHLST2 & & \\
\hline$\Delta h_{\text {LST3 }}$ & DHLST 3 & & \\
\hline$\Delta_{\mathrm{hST}^{\prime}}$ & DHLST4 & & \\
\hline$\Delta_{\mathrm{h}} \mathrm{UA1}_{1}$ & DHUA 1 & 16.1 & \\
\hline$\Delta \mathrm{h}_{\mathrm{UA} 2}$ & DHUA2 & & \\
\hline$\Delta \mathrm{h}_{\mathrm{UA3}}$ & DHUA3 & & \\
\hline $\mathrm{h}_{\mathrm{UA}} 4$ & DHUA4 & & \\
\hline$\Delta_{h_{\text {UAus } 1}}$ & DHUAUS1 & 16. 8 & 16. \\
\hline$\Delta h_{\text {UAus } 2}$ & DHUAUS2 & & \\
\hline$\Delta \mathrm{h}_{\text {UAus3 }}$ & DHUAUS3 & & \\
\hline $\mathrm{U} 1$ & DHUU1 & 16. 4 & 16.6 \\
\hline$\Delta \mathrm{h}_{\mathrm{UU} 2}$ & DHUU2 & & \\
\hline$\Delta h_{\text {UU }}$ & DHUU 3 & & \\
\hline
\end{tabular}


Liste der Parametersymble

Jahr einer staatlichen

Programm-

Reform im Bereich

Code

Sparförderung

$\mathrm{J} 1$

$T_{C, t}$

$T_{F, t}$

$T_{\text {Im, } t}$

$T_{k, t}$

$\mathrm{T}_{\mathrm{L}, \mathrm{t}}$

$T_{\text {LA, } t}$

$T_{\text {RG, }}$

$T_{\text {RGS, } t}$

$T_{\text {RGS, } t}$

$T_{\text {SV , } t}$

$T_{\text {SV, } t}$

J2

J 3

J4

J5

J 6

J7

J8

J 9

J 10

J 11

$\mathrm{J} 12$

${ }^{a}$ St, t.

J 13

$F_{\text {St, Inl, } t}$

J 14

$I_{S t, t}^{b r}$

J 15

$k_{S t, t}$

J 16

hLSt, $t$

J 17

$\mathrm{U}_{\text {St, }} \mathrm{A}, \mathrm{t}$

J 18

$U_{\text {St, Ausl, t }}$

J 19

$\mathrm{U}_{\mathrm{St}, \mathrm{U}, \mathrm{t}}$

J20 


\section{Das Rechenprogramm INZID (FORTRAN IV)}

Im folgenden findet sich das in FORTRAN IV formulierte Rechenprogramm INZID zur Lösung des Modells samt dem eingelesenen Datenpaket. Der Programmtext ist wenig elegant und infolge des Verzicnts auf eine Schachtelung in Unterprogramme leider auch etwas schwer zu überschauen. Außerdem war es auch nicht möglich, den Text bis zum Beginn der zweiten Iterationsschleife durch ausführlichere Kommentierung gedanklich nachvollziehbar zu machen, so daß die Art, in der das gesamte Gleichungssystem im Programm aufgelöst wurde, kaum kontrolliert werden kann. Nachvollziehbar wird der Programmtext erst mit Beginn der Newton-Iteration und vor allem in Anschluß an das Ende der Iteration 2, wo es im wesentlichen darum geht, nach der Lösung der reduzierten Lohn-bzw. Iterationsgleichung und mit Hilfe der bei der Lösung direkt angefallenen Ergebnisse das Definitionsgleichungssystem der Volkswirtschaftlichen Gesamtrechnung von Paragraph 2 mit numerischen Werten zu füllen. Die Output-Anweisungen des Programms dienen einer ersten Auswertung des Modells und erstrecken sich nur auf die wichtigsten Modellgrößen. 
PROGRAMM GELUCHT IM CODE IBM $2 h$. STAND: 1.NOVEMBER 1977

PROGRAM INZIDIINPUT, OUTPUT, TAPE 5=INPUT, TAPE 6=OUTPUTI DIMENSION

IXA $(40), \times[S T(40), \times A O(40), \times A S T(40), \times C A(40), \times(S(40), \times D P R R(40)$, $2 \times D P R Q(40)$, XNSTR $(40), X D S T O(40), X D P R(40), \times D S T(40)$, 3 XE $(40), X E P R(40), X E M P P(40), X E V(40), X F X(40), X F A(40), X F X A N(40)$, 4 XFAUS $(40)$, XFAUSZU (40), XFBK(40), XFBKZU(40), XFS $(40)$, XFST $(40)$, 5XFSTZU (40), XGKX(40), XGLX(40), XGP(40),XGRX(40), XGX(40), XG(40), 6 XGERT $(40), x H I(40), x H 2(40), x I P R(40), x I P R A N(40), x I S T(40), x I V(40)$, 7 XKPP (40), XKNPR( 40$)$, XKPRZU(40), XKPRAB $(40)$, XKOST $(40)$, XKXOST $(40)$, $8 \times K S T(40), X K S T Z U(40), X K S T A B(40), X K X S T(40), X \operatorname{OH}(40), X L O H N \times(40)$, $9 \times L(40), X L A(40), X L A O(40), X L P R(40), X L P R O(40): X L S(40), X L S T(40)$, $9 \times M(40), X M U(40), X M A(40), X M H H O(40), X M D E V(40), X M S(40), X M U(40)$, IXPAUS $(40), X P E X(40), X P I M(40), X P V(40), X P X(40), \times 0(40), X Z I N S(40)$, $2 \times Z I N S \times(40), \times Z I N S A U(40), \times R(40), X R A(40), X R S(40), \times R S T(40), \times S(40)$, $3 \times S A(40), \times S S(40), \times S U(40), \times T C O(40), X T I M O(40), \times T K O(40), \times T L O(40)$,

INZIO

INZIIO

INZIO

INIIO

INZIO

INZIO

INZIO

IN IID

IN IID

INZID

INZIO

INZID

INZID

IN 210

INZIO

INZ ID

INZ IO

IN $Z$ I D

INZIO

INZIO

IN 2 ID

IN $Z$ II

4 XTRGO(4U), XTRGSO $(40), X T C(40), x T O I R(40), \times T F(40), \times T I M(40), X T I N D(40)$, INZID $5 \times T K(40), X T L(40), \times T L A(40), X T L S(40), X T R G(40), \times T R G S(40), X T S V(40)$,

INZIO 6XUAAUS $(40)$, XUST $(40)$, XUSTA $(40)$, XUSTAUS $(40)$, XUSTU $(40)$, XUEWSA $(40)$ INZIO $7 \times V(40), X V S(40), X W(4 U), X W X(4 U), X W A(40), X W A O(40), X W H H(40), X W H H O(40)$, INZIO RXWPR(40), XWS (40), XWU(40), XX(40), XXAUS $(40), \times X E \times(40), \times X I M(40)$

INZIO DIMENSIIIN

ЭXXO(40), XY(4U), XYBR $(40), X Y A(40), X Y A O(40), X Y A N(40), X Y A N O(40)$, GXYPRX(40), XYPPN(40), XYPRNO $(40)$, XYS $(40), X Y S N(40), X Y S T(40)$,

INZIO

INZI D

IN 2 I 0

IXYSTN(40), XYU(40), XYUN $(40), \times 2 D E V(40), X Z R(40), \times 2 \times(40)$

NACHTRAG:

DIMENSION

1FORINZ(5U), JAHR $(50)$, XEKOEF (50), XGL $(50), X I P R O(50)$, XKAPAZ(50)

2 XKKDEF $(50), X N(50)$, XPK $(50), X R X(50), X T H Y P(50), X Y N(50)$

DIMENSION

$1 D F(18), F(18), U(18), X(50), Y(30), Z(30)$

DIMENSIDN

1 PANP( 21$)$, PKOR (20), PKORC (50), PKOPEO)(50), PKOREV (50), PKURL $(50)$. $2 P K O R \times O(5(1), S S S(20), \operatorname{VVV}(20,20), \times x \times(20,21), 222(20)$

INZID

IN 2 ID

INZIO

INZID

INZIO

IN? In

IN 210

INZIO

INZ IO

INZIO 
LUGICAL KUNTROL, NULL, IANPAS

KONTROL= FALSE.

EINGABE DER AUSGANGJWERTE VIN ENDOGENEN VARIABLEN

DIMENSIONIERUNG OER EINGEGEBENEN DATEN:

INZIO

NOMINALWERT VON AGGREGATEN IN MILLIONEN OM

REALWERT VON AGGREGATEN IN MILLIONEN DM IPREISE VON 19621

INZIO

INZID

PREISE ALS INOEXWEPTE $(1962=1,00000)$

INZIO

INZID

LOHISSAETZE IN DM PRD KUPF

ZINSSAETZE UNU KAPITALKOSTENSATZ IN PROZENT/100

BLCCHAEFTIGIE BZW. NICHT-BESCH. ER'NERBSPERSONEN IN MILLIONEN

IN 2 ID

INZID

IN?ID

INZIT

KEAD $(5,1)$ XEST(10),XASI $(9), X C A(9), X C S(9), X D P R R(1),), X O S T R(10)$,

INZID $2 \times F S(9)$, XFST $(7)$, XGKX (9), XGX(9),XG(9), XSERT $(9), X H Z(9), \times K P R(10), \quad$ INZID 3 XKPR $(11), X K N P P(9)$, XKHPR $(10)$, XKNPK(11), XKPRZU(10),

4 XKOST(1U), XKXกST (9), XKXOST $(10)$, XKJT(10), XKXST(9), XKXST $(10)$,

INZIID

INZI $D$

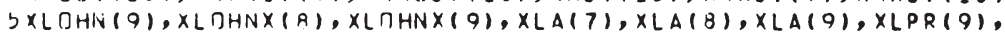

IN 210

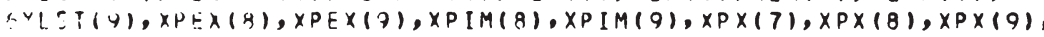

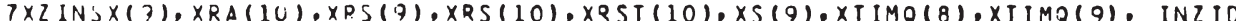

oXTC $(y), X][M(3), X T L(3), X V(9), X W A(9), X W S(9), x \times(7), x \times(3), x \times(9)$,

INZID

$\exists \times X E \times(9), X \times I M(9), \times X O(10), X Z R(10), \times 2 \times(8), \times 2 \times(9), X A O(9), X K P R 7 \cup(9), \quad$ INZID

QXKSTZU(9), XPK(9), XPX(4), XPX(5), XPX(A), XHI(9)

INZID

1 FIPMATITF10.0)

EINGABE DER ZEI FEJHEN VON FXOGEN VORGEGEBENFN VARIABLEN

INZII

IN 210

$\operatorname{IN} 210$

INZIO

JENSFITS DER FNUPERIUDE J01,J02,... DER ZFITREIMFN WERDEN

$\ln 210$

DIE LXOGENFN VARIADLEN ALS FUNKTIONEN DER ZEIT BESTIMMT.

INZIO

INZIO

READ $(5,2) \quad J 01, J 02, J 03, J 04, J 05, J 06, J 07, J 08$

INZID

FDRMATIRII $2,1 \times 1)$

INZID

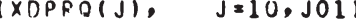

INZIT

READ $(5,1)$

$(x D 2 T 0(7)$

$J=10, j 021$

READ $(5,1)$

$(x \operatorname{CAUS}(J)$,

$J=8, J 031$

READ(5,1) (XLINSAU(J), J=9, JO4)

INZID

P.EAD $(5,1)$

(XW(J), J=8,J05)

INZID

IN Z ID 
STANDARCABLAIJF DER MUDELLENTWICKLUNG

\section{EINGABE deR parameter und parameteraenderungen (STANDARDablauf)}

REA!) $(5,1)$ PALFA1, PALFA2, PALFA3, PF, PALFAX1, PALFAX2, PALFAX3,

INZID

IPBETA1, PPETA2, PBETA3, PGAM1, PGAM2,

PDELT4, 2PEPSII,PFPSI 2, PFPSI3, PFPSI 4, PEPSIXI, PEPSIX2, PEPSIX3,PEPSIX4,PZET1, INZID 3P 7ET2, PF.TA1, PFTA2, PETA 3, PTHET1, PTHET2, PTHET 3, PJOT1, PJOT2, PKAP1, INZID 4PKAP2, PKAP3, PKAF4, PMUEA, PMUE1, PMUE 2, PMUE 3 , PMUE 4, PMUFX1,

INZID 5 PMUEX 2 , PMUE $X 3, P N U E 1, P N U F 2, P \times[\times 1, P \times 1 \times 2, P \times 1 \times 3, P O M I A 1, P O M I A 2, P O M 151$, INZID 5PUMIS?, PPI1, PPI 2, PPI 3, PRHOI, PPHO2, PRHI 3, PPHD4, PRHO5, PRHOS, 7PSIG2,PSIG 3,PSIG4,PSI ,5, PIAU1, PIAU2, PIAU3, PTAU4, PTAU5,

IN 210 CPPHI1, PPHI ?, PPHI3, PPHI4, PCHI1, PCH ? , PCHI3, PCHI 4, PCHIXI, PCHIX2, $3 P C H 1 \times 3, P C H I 2 \times 1, P C H 12 \times 2, P C H I 3 \times 1, P C H I 3 \times 2, P C H 13 \times 3$, PPSI1, PPS I 2, PPSI 3, 7PPS I4, POMEG1, PIMEG2, POMEG3, PIC1.PTC 2, PTF 1, PIF 3, PTIM1, PTIM2 IPTK1, PTK2, PTL1, PILL, PTLA1, PTLA?, PTRG1, PTRG2, PTRG3, PTRG4.

3 PHD1, PHL2, PHF 11, PHF 1?, PHF 2, PHI1, PHI2, PHK 1, PHK?, PHLST1, PHLST2,

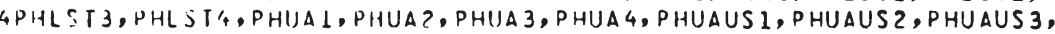
5PHUU1, PHUU2, PHUU3, OTC1, DTC2, DTF1, DIF3, OTIM1, DTIM2, DIK1, DTK2, SDTL1, OTL2, TTLA1, UTLA2, OTRG1,DTRG2, OTRG3, OTRGS1. TOTRGS?,DTPRS 3 , U 1

30TSV22, D1TSV1, DITSV21, DITSV??, OHFST1, DHEST2, DHF11, DHF1?, DHF2, 9OHI1, UHI2, DHK1, DHK2, DHLST1, DHLSTL, DHLST3, DHLST4, OHUA1, DHUA2, 9 DHUA3, OHUA4, DHUAIJS 1, UHUAUS 2, DHUAUS 3, DHUU1, DHUU2, DHUU3, PRISIKO, 1 PKAPE, PYPSI 1, PYPSI2, PYPSI 3, PTAU6, PLAM 2, PLAM 3, PLAM4, PLAM5, PRHOX1, 2PR HIOX2, PKAPG,PKAP 7, PKAPR, PKAP9, POMIA 3, POMIA4, POMIS 3, PE TA 4 PTF2, PTLA3, PTRGS4, DTF2, DTLA3, OTRG4, UTRGS4, 01TRGS4,

3 FORMAT( 4 E 15.5 ) PSIGI, PLAMI

INZIO

INZIO

IN 7 I

IN 210

INZ In

INZIO

INZ ID

INZIO

IN ZID

INZ I D

I N Z I D

INZID 
$\operatorname{KEAD}(5,4) \quad \mathrm{J} 1, \mathrm{~J} 2, \mathrm{~J} 3, \mathrm{~J} 4, \mathrm{~J} 5, \mathrm{~J} 6, \mathrm{~J} 7, \mathrm{~J} 8, \mathrm{~J} 9, \mathrm{~J} 10, \mathrm{~J} 11, \mathrm{~J} 12, \mathrm{~J} 13, \mathrm{~J} 14, \mathrm{~J} 15$,

IN ZI I

INZID

4 FORMAT(20I3) J16,J17,J18,J19,J20

C

C

INPUT-KONTROLLE
IF (KONTROL)
GO TO 9

WR I TE $(6,1 \cup)$

10 FURMAT////10X,15HINPUT-KONTROLLE///)

WRITE $(6,5) \times E S T(10), X A S T(9), X C A(9), X C S(9), X D P R R(10), X D S T R(10)$,

IN 2 I 0

INZID

INZID

IN 210

INZIIO

INZIO

INZID

INZIO

INZIO

IXERTT(I0), XAST(9),XXCA(9),XCS(9),XOPRR(IO),XOSTR(10), INZID IXOPR (9), XEPR (9), XEOPR (10), XEV(9),XFX(9), XFXAN(9),XFAUS(9),XFS(8), INZID $2 \times F S(9), X F S T(9), X G K X(9), X G \times(9), \times G(9), X G E R T(9), X H Z(9), X K P R(10)$, INZIO $3 \times K P R(11), X K N P R(9), X K N P R(10), X K N P R(11), X K P R Z U(10)$,

4XKOST $(10), X K X U S T(9), X K X O S T(10), X K S T(10), X K X S T(9), X K X S T(10)$, $5 \times \operatorname{LHN}(9), X \operatorname{OHN} X(4), X L O H N X(9), X L A(7), X L A(8), X L A(9), X L P R(9)$, $6 \times L S T(9), X P E X(8), X P E X(9), X P I M(8), X P I M(9), X P X(7), X P X(8), X P X(9)$, $8 \times T C(9), X T I M(9), X T L(9), X V(9), X W A(9), X W S(9), X X(7), \times X(8), X X(9)$, INZIO $9 \times X E X(9), X X I M(9), X X O(10), X Z R(10), \times Z \times(8), \times Z X(9), X A 0(9), X K P R Z U(9)$, $9 \times K S(Z \cup(9), X P K(9), X P X(4), X P \times(5), \times P \times(6), \times H 1(9)$

5 FORMAT $(5(5 X, F 13.5))$

WRITE (6,6) J01,J02,J03,J04,J05,J06,J07,J08

( FORMAT $(5 X, R(I 2,1 X))$

WRITE $(t, 5)$ (XDPRO(J), J=10,J01)

WRITE $(6,5) \quad(X D S T O(J), \quad J=10, J 02)$

WRITE $(0,5)$ (XPAUS $(J), \quad J=8, J 03)$

WRITE $(0,5) \quad(\times 2 I N S A U(J), \quad J=9, J 04)$

INZI ID

INZIO

INZIO

INZI D

INZIO

INZID

$W R \perp T L(6,5) \quad(X W(J), \quad J=8, J 05)$

WRITE $(6,5)$ (XWX(J), J=8,J06)

WRITE $(0,5) \quad(\times X A U S(J), \quad J=9, J 07)$

WRITE $(6,5) \quad(X K P R A B(J), \quad J=10, J 0 B)$

WRITE $(6,5)$ PALFAI, PALFA2, PALFA3, PF, PALFAX1, PALFAX2, PALFAX3,

INZID

IN 210

IN 2 IO

INZIO

INZID

IN $2 I 0$ IPHETA1, PBETA2, PUETA3, PGAM1, PGAM2,

PDELT4,

2PEPSI 1, PEPS 12, PEPS 1 3, PEPSI 4, PEPS IX1, PEPS $1 \times 2$, PEPS $1 \times 3, P E P S I \times 4, P Z E T 1$, 3PZET2, PETA1, PETA2, PETA3, PTHET1, PTHET2, PTHET3, PJOT1, PJOT2, PKAPI,

IN 2 I 
TDTSV22, D1TSV1, O1TSV21, D1TSV22, DHEST1, DHEST2, DHF 11, UHF 12, DHF 2, 9 UH1 1, DHI 2, OHK1, DHK2, DHLST1, DHLST2, OHLST3, OHLST4, DHUA1, DHUA2, QDHIIA 3, UHUA 4, UHUAUS 1, UHUAUS ?. OHUAUS 3, DHUIJ1, DHUU2, DHUU3, PRI SIKO, 1 PKAPS, PYPSI1, PYPSI 2, PYPS I 3, PIAUI6, PLAM2 , PLAM3, PLAM4, PLAM5, PRHOX1, CPKHDX2, PKAPK,PKAP7,PKAPR, PKAPQ, POMIA3, POMIA4,POMIS3, PETA4 1

W'RITE (b, 7) PTF2, PTLA3, PTRGS4, OTF 2, OTLA3, OTRG4, OTRGS4, DITRGS4, PSIGI,PLAMI

7 FUPMAT $(5(5), F 15,51)$

WRITE $(6,8) \mathrm{Jl}, \mathrm{J} 2, \mathrm{~J} 3, \mathrm{J4}, \mathrm{J5}, \mathrm{Jh}, \mathrm{J} 7, \mathrm{~J} 9, \mathrm{~J} 9, \mathrm{~J} 10, \mathrm{~J} 11, \mathrm{~J} 12, \mathrm{~J} 13, \mathrm{~J} 14, \mathrm{~J} 15$, $1 \mathrm{J16,J17,J18,J19,J20}$

a FRRMATISX,20I3, /

Q CONTINUE

ANPASSUNG DFS MOUELLS AN DIE VOLKSWIRTSCHAFTLICHF GESAMTRECHIIUNG, DER BUNUESREPUBLIK

$12 A+H L:$

PANP(JZ):

ZAHL UFR PARTIELL ZU AENDERNDEN PARAMETER MESSZIFFER VON PKOR(JZ) NACH AENDERUNG (BASISWFRT $=1.0000001$

PKUR (JZ):

$S(I Z):$ KIJREK TURFAKTOREN FUER MDOELLPARAMETER

INZID

INZIO

IN II

INZIO

INZIO

INZID

INZID

I 2 I 0

IN I ID

INZIO

IN 2 I 0

IN 2 ID

INZID

INZ IO

IN $I$ IO

INZID

INZID

IN 2 ID

IN $Z$ I 0

INZI IO

I NZI 0

INZI

I 2 I D

INZID

$X X X(I Z, J Z):$ EFFEKTIVE WERTE DER ZIELVAPIAQLEN ENDDGEN ERMITTFLTF PARAMETER

INZID

$\operatorname{ZzZ}(12):$

ZILLWEKTE DER ZIELVARIABLEN

VVVIIZ,JZ): FNDOGEN ERMITTELTE PARAMETFR 
$J Z A H L=12 A H L+2$

PKOR (1) $=0.96906902$

PKOR $(3)=0.17702653$

PKOP $(4)=0.98794479$

PKחR (5) $=0.06649705$

IN ZID

PKOR $(6)=1.01436600$

IN I I D

211

$P K D R(7)=1.023211 \mathrm{sJ}$

INZI I

212

$Z Z Z(1)=3(15290.0$

INZIR

$722(2)=0.013204$

$27.2(?)=(. \cdots 2) 33$

INZID

$Z Z Z(4)=0.574505$

$7 Z Z(5)=0.00 P 655$

$2 Z Z(5)=0.01651$

INZIO

IN Z I D

INZIO

$72 Z(4)=1.028137$

$7 Z Z(7)=3458 C 0.0$

PKतर $7=1.0$

PKחR $11=1.0048726$

$P K \cap D 12=0.4047795$

$X \times E X(9)=1.0118404 * X \times F \times(9)$

INZIIO

IN $Z 10$

INZIO

INZIO

IN I. IO

IN Z ID

INZI I

$X X I M(9)=1.0022637 \% X X I M(9)$

IN 210

DI 11 $J Z=1,1 Z A H L$

PANP(JZ) =PKOD (JZ)

11 CIJNT

IR(;SI $=$ PTRGSI

TRGSZ = PTRGS?

TRG $3=P$ TRGS 3

IRG $54=P T R G ' 54$

UAUS $1=$ PHUAUS 1

UAUS? = P!U UUS 2

UAUS $2=$ PHUAUS 3

$1 F 1=P T F_{1}$

TF $2=P$ TF 2

TF $3=P$ TF 3

HIIII $=$ PHIIUI

HUIJ? = PHUU2

HIJU $3=$ PHUU

TIHI $=P T J M I$

$T I M ?=P T I M Z$

$T R G 1=P T R G 1$ 
$H L S T 2=P H L S T 2$

HLST $3=$ PHLST 3

HLST $4=$ PHLST

$T L A I=P T L A I$

$T L A Z=P T L A 2$

INZID

$T L A 3=P T L A 3$

$H F 11=P H F 11$

IN?IO

$H F 12=P H F 12$

INZIO

$H F 2=P H F 2$

IN 2 IO

$T S V I=P T S V I$

$T S V ? .1=P T S V 21$

INZIO

TSVZ2:PISVZ2

INZID

HESTI=PHESTI

INZIID

IN 210

IN Z IO

$T L 1=P T L 1$

IN 2 I D

$I L ?=P T L$ ?

NOCHMAL $=0$

INZID

IN ZID

NICHIAAL $=$

$\mathrm{NTCH}=\mathrm{O}$

12 CINTINUt

IF (NTCH.NH. NOCHMAL) GO TO 17

INZIO

IN 2 I

INZID

IOA $=1$

INIIO

IN 210

GI In 15

17 CONTINUE

DO 702 IDAEI, JZAHL

IN?IO

255

256

257

258

259

260

261

262

263

264

265

266

267

268

269

270

271

IF(IDA.FB.1) GO TO 15

INZII

273

IFIIUA.NE.2) S,O TO 14

INZID

274

$P \triangle N P(1)=1$. ULUN1U

OCI $13 \quad J Z=$ ?, I I.AHL

INZ ID

276

INIII 277

INTIO 278

PANP $(J Z)=1.000000$

INZIO

INZIn

270

3 CINTIINUE

GO TO 15

14 T.TNTINUE

$J Z=I D A-1$

$P A N P(J Z)=1.000010$

PANP(JZ-1)=1.000010**1-1.0)

INZIO

290

281

INZII

282

IN TI 10

IN 210

INZII

PANP(JZ-2) $=1.000000$

INZIO

INZIO

INZII

5 CINTINUE 
$P$ (]) I A $=P A N P(1) * P O M I A 2$

$P U M I A 3=P A N P(1) * P O M I A 3$

POMIA $=$ PANP $(1)$ \&OMIAG

INZID

$P O M I S I=A A N P(1)$ \#UMISI

IN 2 IO

PUMIS3 $=P A N P(1)$ PUMI 53

INZID

$X E V(9)=P A N P(2) * X E V(9)$

$X F U P R(10)=P \triangle N P(3)$ XEUPR $(10)$

INZIO

$X \times 0(10)=(P A N P(3)$ * *PALFA2) \#PANP(4) \#XXO(10)

INZID

$x Z I N S \times(9)=P$ ANP $(5) * X 2$ INS $\times(9)$

245

HALFA3 $=P A N P(6) * P A L F A 3$

IN 2 I I

PKIIR $7=F A N P(7) * P K U R 7$

IF(IUA.NE.JZAHL)

Go in 16

IN 2 IO

IN $N$ I D

PANP $(J 7-1)=1 \cdot$ UUJUOO

GO TO 701

it CONTINUE

PTRGS $1=T R G S 1$

PIRGS 2 = TRGS2

PTRGS3 $=$ TFGS 3

PTRGS4=TRGS4

$P T H Y P 1=P T R G S 1$

$P$ THYP $2=P T R G S 2$

PTHYP3 $=$ TTRGS 3

PTHYP $=P$ TRGS 4

PHUAUS $1=$ UAUS 1

INZIO

INZIO

INZID

INZID

IN ZID

IN Z ID

IN 2 ID

IN T.ID

IN ZID

IN Z ID

INZIO

PHUAUS $2=$ UAUS 2

INZIO

P HUAUS $3=$ UAIIS 3

INZID

$P T F 1=T F 1$

$P T F ?=T F 2$

IN 2 I 0

IN 2 I D

PTF $3=$ TF 3

PHUU $1=$ HUUI

IN 2 ID

INZID

PHUU2 $=\mathrm{HUU2}$

INT.ID

DHUIJ $3=$ HUU 3

$P T I M I=T T M I$

$P I I M 2=T I M 2$

$P T R G 1=T P G 1$

$P T D G 2=T R G 2$

$P[R G 3=T 0 R .3$

PIKG $4=$ TRG 4

PHL STI $=$ HLST 1

PHLST $2=H L S T 2$

PHLST $3=$ HLST 3

INZID 
PHLST4 =HLSTA

$P T L A L=T L A I$

IN 210

$P T L A 2=T L A 2$

IN?ID

$P T L A 3=T L A 3$

INZID

PHF $11=H F 11$

PHF12 $=H F 12$

PHF? =HF 2

IN 210

336

$P T \subseteq V]=T S V I$

PTSV $21=T S V 21$

INZIO

PTSV22=IJV?

INZID

338

DHFSTI $=$ HESTI

IN 2 IO

339

PHF'T2=HLST2

340

$P T L I=T L .1$

INZIO

INZID

INZIID

$P T L ?=T L L$

INZID

342

UMIAI $=P U M I A I$

IN ZID

UMIAZ $=P O M I A Z$

INZID

343

$J M I A 3=P U M I A 3$

INZIO

344

345

346

IN 210

347

UJII $A G=D$ JMI $\triangle 4$

IN T. ID

348

I)MISI=POMISI

INZID

349

OMISZ=PIJMISZ

IN 2 ID

350

UMIS $3=P$ PUIS 3

IN 2 IO

351

352

BESINN DER MDUELLRECHNUNG

IN 210

353

354
355

INZIO $\quad 356$

$J=9$

$T=9.0$

$X$ IDUMZF $=0.0$

INZID

INZIO

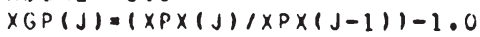

IN IIO

IN 2 IO

$\sin =11$

INZIO

$J M A X=12$

$J M A X=14$

$J M A X=15$

IN $Z 10$

IN 210

INZIO

$\operatorname{JiA} A x=15$

$\operatorname{JMAX}=17$

$J M A X=13$

IN 210

IN ZID

INZID

IF ( (NOCH.NE . NOCHMAL) , DR , (IDA.NE, I))

GO TO 151

INZID

INZIO

INZIO

INZIO

IN 2 ID

356
357

358

339

360

361

362 
JMAX $=16$

I MAX $=17$

$\operatorname{JMAX}=18$

$J M A X=19$

JMAX $=22$

JMAX $=23$

JMAX $=? .4$

JMAX $=25$

JMAX $=26$

JMAX $=? 7$

JMAX $=24$

JMAX $=29$

JMAX $=30$

$J M \Delta X=20$

$J M A X=21$

151 CTNTINUE

DIJ 5999

$J J=1 U, J M A X$

$\mathrm{J}=\mathrm{J}+1$

INZIID

389

IN I ID

INZID

IF ( $(N O C H . N E \cdot O) \cdot O K .(I D A \cdot N E \cdot 1) \cdot O R \cdot(J \cdot N E .9))$ GU TO 149

INZID

390

INZI I

391

144 KONTROL = .FALSE.

GO TO 150

149 KIJNTROL = .TRUE.

150 CONTINUE

POMIAI $=U M I A I+O M I A 3 * X C A(J-1) /(1 \times F V(J-1)-\times S(J-1)) \times 0 \times(J-1))$

393

IN 210

INZII $\quad 396$

INZID $\quad 397$

$P \cap M I S I=[1 M I S I+011 I S 3 * X C S(J-1) /(\times S(J-1) * X P \times(J-1)$ PKORC ( $)=$ PKURT

PKCJRC (IU) $=1.000000$

POMIAI $=$ RKORC (J) \#PUMIAI

PUMIA? $=P K O R C(J) * P O M I A 2$

PUMIAZ $=$ PKחPC (J) PUMIA

POMIA $4=$ PKURC (J) $\#$ POMIA4

$P O M I S I=P K I P P C(J) * P O M I S I$

$P O M I S 2=P K O R C(J) * P U M I S 2$

INZID

398

INT.ID $\quad 399$

INZII $\quad 400$

INZID 401

INZTO $4 \mathrm{CZ}$

INZIO 403

INZID $\quad 404$

INZIO 405

INZIID 406

INZID 407

INZIO 408

PUMI $₹ 3=P K O R C(J) \neq P$ IIIS 3 
11 S PIPGI $=P T R G 1+D T R G 1$

$P T R G ?=P T R G 2+D T R G 2$

$P T R O B=P T R G 3+D T R G 3$

PTRT, $4=P T P G 4+D T P G 4$ 
117 IF(J-J9) $117,113,119$

11R PTRGSI $=$ PIPGS1+DTPISS

PTRGS? = PTRGS? + DTRGS

PTRGS3 $=$ PTPGS3 +DTFGS 3

PTRGS4=PIRGS4+DTRGS4

119 If $(J-J 10) \quad 121,120,121$

1 1 0 PTPGS $1=P$ TPCS $1+D 1$ TRGS 1

PIRGS2 $=P T P G S 2+D 1 T P G S 2$

PTFGS $3=P$ TRGS 3+D1TRG 3

PTRGS4 =PTPGS4+DITRGS4

121 If $(J-J 11) \quad 123,122,123$

122 PTSVI $=P T S V I+0 T S V I$

PTSV $21-P T S V ? 1+D I S V 21$

PTSVZ2=PISV $22+015 V 22$

123 If $(J-J 12) \quad 125,124,125$

$1 ? 4$ PTSV1 $=$ PTSV $1+0115 V 1$

PTSV $21=P T S V 21+01$ TSV? 1

PTSV 2? = PISV $25+0115 V 2 z$

125 PTSVZ=PTSV21+PTSVZ2*(T-15.0)

IF $(J-J 13) \quad 127,12 \%, 127$

INZIT

IN 2 ID

IN ZID

INZIO

INZID

IN 210

INZ IO

I 7 II

IN 210

IN 710

IN 210

INZIO

INZII

INZID

IN $2 I 0$

IN 2 I D

IN 2 I 0

126 PHEST $1=$ PHEST $1+$ DHESTI

PHEST $2=$ PHEST $2+$ DHEST?

127 IF $(J-J 14) \quad(29,128,127$

INZIO

IN IID

IN 710

12P $P H F 11=P H F 1 \downarrow+D H F 11$

PHF $12=P H F 1 ?+D H F 12$

$P H F 2=P H F 2+U H F 2$

129 PHF $1=P H F 11+P H F 12 *(T-10.0)$

IF $(J-J 1:) \quad 131,136,131$

13 () $P H I 1=P H I 1+D H 11$

$P H I 2=P H I 2+D H I 2$

131 I $F(J-J 16) \quad 133,132,133$

13 ? PHK $1=P H K 1+D H K$

$P H K 2=P H K 2+D H K 2$

IN 210

IN 2 ID

IN 210

IN 7.10

IN? ID

INZID

INZIO

INZID

IN ZID

IN! 2 IO

INZIO

PARAMETFR PHLST3 DAPF ERST AB JAHR J 313 EINSCHL. UNGLEICH NULL

INZID

SEIN. OIESE VIRSCHRIFT WIRD BEI NICHT-BEACHTEN AUTOMATISCH

INZIO

$I N Z 10$

INZID

133 IF $(J-J 17) \quad 135,134,135$

INZID

$134 \mathrm{PHLSTI}=\mathrm{PHLST} 1+$ DHLSTI

IN 210

PHLST2 $=$ PHLST2+DHLST2 
135 I $F(J-20)$

$$
\begin{aligned}
& \text { XDUMLST } \\
& \text { GU TO }
\end{aligned}
$$

137 XDUMLST $=1.0$

145 IF (J-12) $146,146,147$

INZIT

INZIO

146 PHLST $30=0.0$

$$
\text { 60) TO } 13 \text { R }
$$

147 PHLST3U $=$ PHLST3/(T-12.0)

138 PHLST $=$ PHLST $1+P H L S T 2 *(T-10.0)+P H L S T 30+P H L S T 4 *$ XDUMLST

INZIO

132 PHUA I $=$ PHUA I +DHUAI

PHUA 2 = PHUA? + DHUA 2

INZII

IN 210

IN Z ID

PHUA $4=0$

INZID

INTIO

INZII

141 PHUAUS1 = PHUAUS1 + 141 HUUS

IN 710

PHUAUS $2=$ PHUAUS $2+$ DHUAUS?

INZIO

INZII PHUAUS $3=$ PHUAUS $3+$ DHUAIJS 3

INZID

PHUAUS $4=6.00000$

IF(J.NE.15) GD TO 156

IN I I

PHUAUS $4=-1621.18$

156 CONTINUF.

$$
\text { IF(J-J?0) } 144,143,144
$$

INZID

IN 2 In

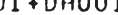

PHUU2 $=$ PHUU2 + OHIJUI?

INZIO

AUFLOE SUNG DER FINKUMMENSENTSTEHUNGSGLFICHUNG NACH XLOHN(J) 
$U E=-(P O M I S 2 * P P I 3) *(1.0-P T Q G S 2) /(1.0-U 1)$

$U 7=P T C ? /(1.0-U 1)$

IN Z I 0

UIR $=1.0$

INZIO

540

$U 9=(U 1-P U M I S 2 * P P I 3 * P T C 2) *(1.0-P T F G S 2) /(1.0-U 1)$

INZID

UI $=(U 1-P O M I S 2 * P P I 3 * P T C 2) /(1.0-U 1)$

INZIO

$U 11=-P T C 2 * P I P G ? /(1,0-U 1)$

UI? $=-P T R G 2$

$U 13=(1.0-(U 1-P O M I S 2 * P P I 3 * P T C 2) *(1.0-P T R G S 2)) /(1.0-U 1)$

INZIO

$U 14=-(U 1-P O M I S 2 * P P I 3 * P T C 2) * P T R G ?(11.0-U 1)$

U1 $5=-P T C 2 * P T P G S 2 *(1.0-P T R G 2) /(1.0-U 1)$

IN 2 ID

IN T. IO

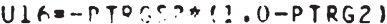

$U 17=-P T R G S 2 / 11.0-U 1)$

$U 1 H=(1.0-(U 1-P T M 1 S 2 * P P I 3 * P T C 2) *(1.0-P T R G 2)) /(1.0-U 1)$

IN Z ID

INZ ID

$U 19=(1.0-P H U A U S 2) *(1.0-P H F 1)$

$U 2 n=(U 3+U 19 *(U 7+U 11+U 15)) / U 2$

INZIO

INZIO

IN I I D

$U 21=(U 4+U 19 *(U 8+U 12+U 1 h)) / U 2$

IN 210

$U 22=(U 5+U 19 *(U 9+U 13+U 17)) / U 2$

$U 23=(U 6+U 19 *(U 10+U 14+U 18)) / U 2$

INZIO

$U 24=1.0+U 21 * P T C 2 /(1.0-U 1)$

IN 7 I D

INZID ID

$U 2 b=1120 * P D M I S 2 * P P I 3$

$U ? 6=U 21 * U 1 /(1.0-U 1)$

$\mathrm{U} 27=U 22 * P T R G 2$

$1128=U 23 * P T R G S 2$

U? $9=P O M I A 2 *$ ( PHUAI-PTSV1-PNUE 1 )*U24+U20*POMIS 2 *PPII

+U21*P $[C 1 /(1.0-U 1)-U 24$ (PHUAUS $1+P H U A U S 4)+U 2 ?$ * TRG1

IN 2 ID

IN TID

IN 2 I D

INZIO

IN Z I D

IN ZID $U 30=U 25-1124 *(1.0-P$ HUAUS 2$) * P H F 2+1127+U 28$

U $31=1124 *(1.0-P H U A U S 2) *$ PHF 2

$U 32=-U 25-U 27-U 28$

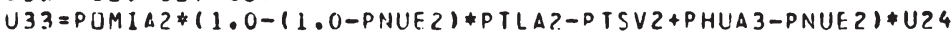

INZTD

IN 7 ID

IN ZIO

$1134=-U 24$

$U 35=U 20 * P O M I S 2$

$U 36=P O M I A Z * P H U A 2 * U 24$

$1137=-P O M J A 2 *(1.0-P N U E 2) * P$ TLA $3 *$ U24

U $3=P O M I A P * P H U A 4 * U 24$

INZID

IN IID

IN IID

INZIO

IN IID

INZIO

$1139=-P O M I A Z *(1.0-P N U E 2) * P T L A 1 * U 24$

$U 40=P U M I A I * U 24$

INZID

$U 41=U 20 * P O M I S 1$

INZIO

$1142=P U M I A Z * U 24-U 25-U 28$ 
$U_{4} 4=-U 25+U 26-U 2 R+1119$

$U 45=U 25+U 28$

$U 4 h=U 2 ? * P T R G 3$

$U 47=U 22$ PTRG 4

$U 4 R=U P .3 * P$ TP.GS 3

$U 49=U ? 3$ ? PTRGS 4

$U E(1=-U 25+U 26-U 27-U 28+1 U 19$

$U 51=U 26+U 19$

$U E ?=-U 20 * P D M I 52+U 2++U 19$

$U 53=-U 25+U 26+U 19$

$U 54=U 25-U 24$

$U 55=-U 24 * P H U A U S 3$

U5 $5 A=(1, C-U 24) *(1,0-P H U A U S 2) *$ FHF 1

LINEARER TEIL MIT VERZOEGERT-ENDOGENEN ABHAENGIGKFITEN

UND EXUGENEN EINFLUESSEN

NICHT-LINFARER TELL

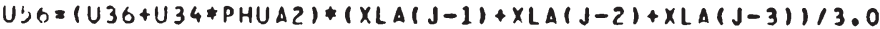

$U 57=(146 *(X X)(J-1) * X F X(J-1)-X D P R(J-1)-X L P P(J-1)-X T C(J-1)-X T 1 M(J-1)$

$U F B=U 47 *(U 46 / U 57)$

$1159=114 a *(\times R S(J-1)+\times(J)(J-1)) / \times S(J-1)$

$U() \cap=U 49 *(U 48 / U 59)$

$U S 1=U 55 * X+2(J-1)$

U $61 A=U 55 A * X H 1(J-1)$

$1162=(144 * P T K 2 * X K N P R(J-1) * X P K(J-1)$

$163=U 53 * P T F 3 * X F S(J-2) / X S(J-1)$

$U 64=U 53 * P$ TF $2 * \times 5(J-1) / \times F S(J-2)$

U $65=-1 J 54$ PHUU3*XX(J-1)*XPX(J-1)

USh $=U 29+U 44 *(P T L 1+P T K 1)+U 50 * P$ TIMI

U67 $=U 65+U 51$ *PTSV $1+U 34$ *PHUA $1+U 54$ * PHUU1

$U 6 B=U 67+U j 6+U 57+U 5 P+U 61+U B 1 A+U 62+U 65$

U69:U23*PTRGS 1+UE 2*PTLS1+U53*PTF 1

$U 70=U 64+U 59+U 60+U 63+U 64$

$U 71=U 30+U S 4 * P H U I J 2$

$U 7 ?=U 54 * P H \cup U 3 * \times P \times(J-1)$

$U 73=U 33+U 51 *(P T L A 2+P T S V 2)+U 34$ PHUA 3

$1174=1132+1144 * P T L 2$

$U 7:=U 35+U 52 * P T L S 2$ 
$U 76=U 37+U 51 * P$ TLA3

$1177=U 39+U 34 * P$ H!IA 4

$U 78=U 37+U^{r} 1 * P T L A 1$

$U 79=U 52 * P T L S 3$

$U A O=U S O * P T I M Z-1.0$

$U 91=U R 8+U 42 * \times P A(J)+U 43 * \times R S(J)+U 44 * \times R S T(J)+U 45 * \times Z R(J)+U 78 * \times E S T(J)$

IIE? $=U 31 * \times \times \square(J)$

I) $3=U 73+U 74-U 77$

$U 1+4=U 74+U 7 b-U 77$

$U 85=(1134+1) 73) \neq P H L S T-1) 77$

$U B R=U 70-U 7 R$

$U R 7=U 41-U 40$

U $88=U 8.4-1183$

$X T L X Q=(P T L 1+P T L 2 \times X L P R(J-1)) / X L P R(J-1)$

$X T C X O=(P T C 1+P T C 2 *(X C A(J-1)+X C S(J-1)+X A S T(J-1)-X L S T(J-1))$

1 $((X C A(J-1)+X C S(J-1)+X A S T(J-1)-X(S T(J-1))$

$\theta=(1 \cdot 0+X T L \times 0) /(1 \cdot U-X T C \times 0)$

UUก $=P G A M 1 /(P G A M) *(1.0+P D E L T 4))$

U? $1=-U 90 * P D E L T 4$

PDEL11 =PALFA1*PALFA2*XKXOST(J)

U92 = (PMUE $A$ PDELT 1$) *$ U9O

$\ln 1=1 \cdot U-U 90$

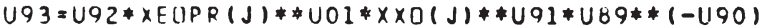

$U O ?=1.0 /(T)$

PKCREV $(J)=1.000000$

UO $3=(X P \times(J-1) / X P X(J-2)) * P E P S I 4$ \&KDREV (J)

$U 94=X E V(J-1) * P E P S I 1 * P E P S I 2 * * U 02 * X \operatorname{LUHN}(J-1) * *(-P E P S I 3) * U 03$ U95 $=P E P S I \times I * P E P S I \times 2 * *(T-10.0)$

1 (XEPR $(J-1) * X L O H N(J-1) /(X \times(J-1) * \times P \times(J-1))) * P E P S I \times 4$

$U 96=U 94 * U 95$

$X P I M(J)=P M U E \times 1 * X P A U S(J) * P M U F \times 2 * \times W \times(J) *(P M U E \times 3-1.0)$

U97:PTHETI*XPIM(J) * \# THET 3

Un $4=(\times P \times(J-1) / \times P \times(J-2)) *$ PETA3

UO5 $=U 0_{4} *(x \operatorname{LPR}(J-1) /(\times \times(J-1) * \times P \times(J-1))) *$ *PETAG

U98 $=\times V(J-1) * P E T A 1 * \times V(J-1) *(-P E T A 2) * U 05$

U99:U97

$\mathrm{U} 1 \mathrm{CO}=\mathrm{U97} * \mathrm{~V} \times(\mathrm{J}-1)$

$U 1 \cap 1=P T H E T ?$

UnI $=((\operatorname{XPEX}(\mathrm{J}-1) * \operatorname{XPAUS}(\mathrm{J}-2)) /(\operatorname{XPEX}(\mathrm{J}-2) * \operatorname{XPAUS}(\mathrm{J}-1)) *$ PCHI 3 
GLF ICHSETZEN DER EINKOMMENSENTSTEHUNGSGLEICHUNG MIT DER NACH XI.UHNI (J) AUFGFLUESTEN FUNKTION FUER UAS PELERTETE PRODUKTIONSERGEBNIS.

$U 117=U 71-1 \cdot 0$

U1 $18=-P$ OELT $4 / P G A M 1$

U1 $19=-1$. U/PDELT 4

$U 120=(U 113 / P G A M 1) * X X O(J) *(-P D E L T 4)$

I) $121=1.0+$ PDFLT4

$U 12 ?=U 118-1.0$

U123=PDELT4 (1.0-PGAM?)/PGAM2

U124=PDELT4/PGAM?

$U 125=\times \times 0(J) * *(-U 123)$

$U 126=U 114 * \times \times 0(J) *(-U 124)$

U1 $27=-U 115-1.0$

$1129=P G A M I / P G A M 2$

$U 129=1.0-1140$

$U 13.3=1.0+U 90$

$U 131=1 . U+P E P S I 3$

$U 132=1.0 / P K A P 2$

KDNTRULLLERGEBNISSE

IF(KONTRUL) GO TO 298

WRITE (h, 297) JAHR(J)

277 FORMAT $/ / 1 / / 10 X, 32$ HKDNTROLLFRGEBNISSE FUFR DAS JAHR, $1 \times, 141111$

WRITE $(6,299)$ U1, U2,U3,U4,U5,U6,U7, U8, U9,U10

WRITE(6,299) UI1,U12,U13,U14,U15,U16,U17,U18,U19,U20

WRITE(6,299) U21,U22,U23,U24,U25,U?,6,U27,U28,U29,U30

WRITE $(6,299) \cup 31,1332, \cup 33, \cup 34, \cup 35, \cup 36, \cup 37, \cup 38, \cup 39, \cup 40$

WRITE $(6,299)$ U41, 142, U43,U44,U45, U46, U47,U48, U49,U50

WRITE(6,299) U51,U52,U53,U54,U55,U56,U67,U59,U59,U60

WRITE $(5,299)$ U61,1162,U63,U64,U65,1166,U67,U63,U69,U70

WRIIF 16,2991 U71,U72,U73,U74,U75,U76,U77,U78,U79, U80

WRITE $(6,299)$ U81, U४2, U83,UR4, U85, U96,U87, U88, U89, U90

WRITE $(6,297)$ U91,U92,U93,U94,U95,U96,U97,U98,U99,U100

WRITE(h,299) UI01,UIU2,U103,U104,U105,U106,U10\%,U108,U109,U110

WRITE $(0,299)$ U111,U112,U113,U114,U115,U116,U117,U11R,U119,U120

WRITE(4,279) U121,U122,U123,U124,U125,U126,J127,U128,U129,U130 
WRITE $(6,279)$ U131, U132

DIE ITERATION APPROXIMIERT DEN WERT DES INVESTITIONSGUETERPREISES XPK(J) BZW. DEN WERT OER HIFRZU BENDETIGTEN VARIAB-

$J T=0$

$Y(1)=1.065 * \times K S T Z U(J-1)$

$2.999 \mathrm{JT}=\mathrm{JT}+1$

$U O I=(X K P R Z U(J)+U 32 * X D P R R(J)) * P Y P S I 1$

$U 02=(X K P F Z U(J)+2.0 * Y(J T)) * \# P Y P S I Z$

$U U 3=((X K P R Z U(J-1)+2.0 * X K S T Z U(J-1)) / X X(J-1)) *$ PYDSI 3

INZIO

752

INZIN $\quad 753$

IN 710

INZID $\quad 755$

INZID $\quad 756$

INZID $\quad 757$

INZID 759

IN $7.10 \quad 759$

INZID $\quad 760$

$U 13.3=\cup 101 * U \cup 2 * \cup 03$

INZID

BEGINN DER ITERATION 2

DIE ITERATION LOEST DIE NACH OER VARIABLEN $X(I T)=X L O H N(J)$

INZIO

INZI ID

INZID

AUFGELUESTE FUNKTION FUER DIE MDNETAERE UEBERSCHUS SNACHFRAGE

IN 2 ID

$\triangle U F$ DEM TUTPUT-MARKT DES PRIVATEN SEKTORS.

DIESE IJERERSCHUSSNACHFRAGE HAT IM KURZFRISTIGEN GLEICH-

INZID

INZIO

INZIO

GEWICHT DEN WERT NULL.

DIF UEREKSCHUSSNACHFRAGEFUNKTION LAUTET DAHER IM FOLGENDEN:

INZID

$F F=0.0$ : SUMME (PARAMETER U(TN) * FUNKTION F(IN)),

INZID

INZID

WOBEI DIF SUMME UEBER ALLE WERTE DER INDEXVARIABLEN IN IIN=1,..

INZID -..18I GEBILDET WIRD.

F(IN) IST JEWFILS EINE FUNKTION DFR VARIABLEN XIIT)

INZIIO

INZID UND 11:T TF(IN) ALS ERSTE ABLEITUNG.

EXISTIERT IM RELEVANTEN BEREICH DER FF-FUNKTION KEINE NULL-

INZID

IN Z ID

STELLL, WIRD OER NAECHST-LIEGENDE EXTREMWEPT DER FF-FUNKTION

IN ZID APPRTXIMIERT. DER DEM EXTREMWERT ZUGEHUERIGE XIITI-WERT WIRD

INZIO ALS NAEHERUNGSLOFSUNG FUFR DAS ZU ERMITTELNDE LOHNNIVEAU 
$x(1)=1.05 * x L$ กHN $(J-1)$

INZIO

$300 \quad I T=I T+1$

IF IIT-2E) $330,330,315$

INZID

795

INZIO

330 CDNTINUE

$$
\begin{aligned}
& F(1)=1.0 \\
& D F(1)=0.0
\end{aligned}
$$$$
1 F(x(1 T)-0.2 * x \operatorname{DHN}(J-1)) \quad 315,313,329
$$

INZID

IN 2 ID

INZ ID

INZIO

IN ZIO

329 IF $(X(I T)-5.0 * \times$ L חHN $(J-1)) \quad 302,315,315$

INZIO

IN $I I 0$

IN ZID

$F 0 ?=10109 * x(1 \mathrm{~T}) * \forall(1)$

FU $3=U$ IIU*XIIT)*\#UIII

$\mathrm{FO} 4=\mathrm{FO} 1-\mathrm{FO} 2+\mathrm{FO} 3-X \mathrm{FST}(\mathrm{J})$

INZIO

INZII

IF $(F \cap 4-1.0)$

IN I ID

INZIn

IN 7.10

$F(2)=\times \times 0(J) *(U 113 * F 05+U 114) * * U 119$

IN I ID

DFO $1=U 94$ *PFPS $13 * x(I I) *$ (PEPS $13-1.0)$

INZIO

$D F C 2=U 109 * U 111 * x(11) * *(1) 111-1.0)$

INZID

DFO $4=0 F 01-D F 02+D F 03$

INZIO

$D F(?)=U 120 * F(2) *$ U $121 * F 04 *$ U122*0FO4

INZID

$F O B=U 12 A * F(2) * 0124$

INZIO

IF (F07-FUE) 315,315,320

IN 2 IO

INZID

IN Z ID

$D F O 7=1123 * F O 7 * D F(2) / F(2)$

IN $Z$ IO $U F() R=U 124 * F \cup R * D F(2) / F(2)$

IN Z IO 
INZIO

IN $T$ I $D$

INZID

IN Z I D $-(P I H F T 2 * D F(3) / F(3)) * F 010$

IN 2 I 0

INZIO

IN Z I D $F(15)=11102 * F(3) * P C H I \times 2$ $D F(15)=P C H[\times 2 * F(15) * D F(3) / F(3)$

$F(10)=1103 * F(2) *$ PMUE?

$U F(1 h)=(P M U E 2 * D F(2) / F(2)) * F(16)$

$F C 11=F(t)-F(11)+X F . T(J)$

$F O 12=F(5)-F(10)+X E S T(J) * P H L S T * X(I T)$

$F(17)=F 011 * 2.0 / F 012$

$U+O 11=U F(3)-D F(11)$

DFU12=DF( $)-D F(10)+X E S I(J) \neq P H L S T$

UF $(17)=(2.0 * D F \cup 11 / F 011-D F 012 / F C 1 ?) * F(17)$

$F(14)=11133 * F(2) *(-P Y P S I 2) * F(3)$

() $F(1 R)=(-P Y P S I 2 * U F(2) / F(2)+D F(3) / F(3)) * F(18)$

INZID

INZI IO

INZID

IN 7 ID

IN 7 ID

INZIO

INZIO

INZIO

INZID

IN 2 IO

INZIO

$U(1)=U R 1$

$U(2)=U 72$

I) $(3)=U 82$

IN ZID

INZIO

INZID

IN $Z$ ID

$U(4)=U 117$

INZID 
$U(8)=U A 5$

$U(Y)=U 79$

INZIO

INIIO

$U(11)=(18 R$

$U(12)=1187$

IN Z IO

$U(13)=U 77$

$11(14)=1.0$

() $(15)=1.0$

$U(16)=U 90$

$U(17)=U 75$

$U(18)=1.0$

$F F=U .0$

$U F F=U .0$

J) $3 \cap 1$

I $N=1,18$

$F F=F F+U(I N) * F(1 N)$

$301 D F F=D F F+U(I N) * D F(I N)$

IF (KI)NTROL) GD) TO 306

IF $(\mathrm{JT}-1) \quad 300,304,304$

3) 4 WRITE $(6,305)$ JAHR (J),JT,IT,X(IT),FF. DFF

305 F DPMATIICX,I4,3X,3HJT $=I 2,3 X, 3 H I T=, I 2,5 X, 6 H X(I T)=, E 15,8$, 1 $3 x, 3 H F F=, E 15.5,3 x, 4 H D F F=, E 15.81$ 
311 ITHB :TSR+I

IF(ITDB-1) $312, ? 13,312$

$312 C==A R E(F F)-A R S(P F)$

$$
\text { If }(\mathrm{CC}-\mathrm{C}, \mathrm{O}) \quad 313,313.314
$$

$313 B B=F F$

$D B H=U F F$

$X 5 B=X(1 T)$

$3 ! 4$ CTNTINUF

IF (ITHPAT-0) 320.320 .315

INZIO

INZIO

90 ค

IN 2 I 0

909

IN 2 ID

910

IN ZID

911

$3 \perp \cdot I F(I T-2)$

$323,323,327$

IN 2 In

INZIO

IE RUM = ITF PUM+

IF (ITERUM-1) 32h,324,326

INZID

IN 2 ID

INZID

IF $(K !) N T P L)$

INZIO

WR ITE $(6,3$ ? 5$)$

25 FIPMAT $16 x, 4$ IHVFRR INGERUNG DES AUSSANT. WERTES VON X(ITI)

INZIO

INZID

IN 2 IO

IN 210 SO TO 302

327 ITERAT ITERAT+1

IF (ITFRAT-1) $310,316,31$ A

31 h CONTINUE

IFIKUNTROL) GU TD 322

WRITT $(6,317)$

317 FJRMATI/1OX, 4 IHSTATT DER NULLSTELLE WIRD DIE STELLE DER, 3ZHFF-FUNKTIDN MIT DFF=0 BERECHNET. $/ 1$

322 CJNTINUE

IF $((A A, E O \cdot 0.0) \cdot A N D,(E R, G T \cdot 0.0))$

IF $((B B, E Q, 0.0), A H D \cdot(A A, G T \cdot 0.0))$

IF ( (AA.EO.U.O) . AND. (B,B.LT.U.O )

GO TO 331

SO TO 332

IF $((B B, E 0,0,0)$. AND. (AA.LT.0.0)

6ก TO 334

(s) $T 0 \quad 335$

$331 \times \Delta A=0.95 * 3 B$

$\because 0 \quad T \cap 335$

$332 \times B R=1.05 * \times A A$

G? TO 335

$333 \times A A=1.05 \div 3 B$

G) TO 335

$334 \times A B=0.75 \div \times A A$

333 C TNTINUE

$X(I T)=0.5 *(X A A+X B B)$

IN 2 II

INZIO

IN 2 ID

IN 2 I D

INZIO

INZIO

IN IIO

IN Z I D

INZID

IN $Z 10$

IN 2 ID

IN 2 ID

INZID

IN 210

INZID

IN Z IO

INZIO

INZIO

INZID

INZIO

INZID

INZIO

Gu TO 302

IN Z I 
$31 P D D=U A A *(B B * D B B+X Y B)$

$E F=U B B *(\triangle A+D A A+X \triangle A$

$C, G=D A A-[\cdot B A$

$x(I T+1)=(D D-E E) / G G$

$P R U L F=X(I T+1)-X(T T)$

$P R U F F=\triangle B S(P R U E F)$

$X K D I T=1 . O E-8 * X(I T)$

NULL $=$ OFALSE.

IF (PRUEF-XKRIT) 321.319 .319

$319 I T=I T+1$

$$
\text { Gח TI } 302
$$

320 CONTINUE

$X(I T+I)=X(I T)-(F F / O F F)$

PRUEF $=X(I T+1)-X(I T)$

$P R U E F=A B S(P R U E F)$

$X K R I T=1 . O E-R * X(I T)$

NULL $=. T R U E$.

IF (PRUFF-XKKIT) $321,300,300$

DIt MODELLERGESNISSE

IN I ID

968

$X \operatorname{LPR}(J)=F(5)$

$X F F R(J)=F(6)$

XEKDFF (J) $=F($ (S)/XESTPR(J)

$X L S \Gamma(J)=F(B) * P H L S T$

$X(S(J)=F(10)$

$X S(J)=F(11)$

$X E V(J)=F(13) / X(I T)$

$X I V(1)=F(14)$ 
$x A(1)=F 011$

BERECHNUNG OER VOLKSWIRTSCHAFTLICHEN GESAMTRECHNUNG

GOI=PYPJII*( (XKPRZU(J)+2.0*Y(JT) $) / X X(J)) *$ PYPSI

INZID

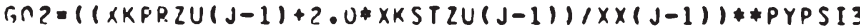

INZIIO

INZIO $x B K(7)=(201 * 1,05 * x B \times(7)$

X?ET:Si=XUSIR(J)*XPK(J)

$X Y R K(J)=F(4)+X(S I(J)+X D S T(J)+X Z R(J)$

INZIO

INZID

IN IIO

$X D P R(J)=X D P R R(J) * X P K(J)$

$x[P R A N(J)=X K P R Z U(J) \notin X P K(J)$

IN ZIO

$X T P R(J)=X[P R A N(J)+F(I \zeta)$

$X I P S O(J)=X I P R(J) / X Y B R(J)$

$X K K J E F(J)=X K P R(J) / X X(J)$

$X T C(J)=P T C 1+P T C 2 *(F(4)-X T P R(J)-F(15)+F(16))$

$X I C U(J)=X T C(J) /(F(4)-X J P R(J)-F(15)+F(16))$

$X I I M(J)=P T I M I+P T I M 2 * F(16)$

$X I I M U(J)=X I I M(J) / F(16)$

$X G E P T(J)=F(4)-X \cap P R(J)-F(5)-X T C(J)-X T I M(J)$

$X T R G(J)=P T P G 1+P T R G 2 * \times G E R I(J)+P T R G 3 * \times G E R T(J-1)$

$+P T R G 4 *(1.0 / \times G E R T(J-1))$

$X I L(J)=P T L 1+P T L 2 * F(5)$

$X T L O(J)=X T L(J) / F(5)$

$x \operatorname{LOHNX}(J)=X \operatorname{LUHN}(J) *(1.0+X T L O(J)) /(1.0-X T C O(J))$

IN 210

IN ZIO

INZID

IN ZIO

IN ZID

INZIO

IN 210

IN 210

INZ IO

IN 210

INZIO

IN $Z 10$

INZIO

$x[K(J)=P T K 1+P T K 2 *(X K N P R(J-1) * x P K(J-1))$

$X T I N D(J)=X T C(J)+X I I M(J)+X T R G(J)+X T L(J)+X T K(J)$

IN 2 I 0

IN 2 I 0

INZID

$X Y N(J)=X Y B R(J)-X D P R(J)-X D S T(J)$

$X Y(J)=X Y N(J)-X T I N O(J)$

$X L A \supset(J)=X L A(J) / X Y(J)$

$X(P R O(J)=F(5) / F(4)$

$X K(J)=X \operatorname{RA}(J)+X P S(J)+X \operatorname{RS}(\mathrm{X})$

$X R \times(J)=X \bar{R}(J)-X Z R(J)$

$X G(J)=F(4)-X \cap P R(J)-X T I N O(J)-F(5)-X R X(J)$

INZII

INZIT

IN 210

INZIIO

INZI!

IN 2 II

$X Y A(J)=X L A(J)+X R A(J)$

IN 2 In

$X Y A D(J)=X Y A(J) / X Y(J)$

IN 2 I

$x \cup S T U(J)=$ PHUU $1+$ PHUU $2 * F(4)+$ PHUU3 $*(F(2)-x \times(J-1)) * x P \times(J-1)$

INZID

IN 7 ID 
$X T R G S(J)=P T R G S 1 * F(11)+P T R G S 2 *(X R S(J)+X G(J))+G 01+G 02$

GOOI = PTHYP3*(XRS $(J-1)+X G(J-1)) * F(11) / \times S(J-1)$

INZID

1030

GONZ=PTHYP4*((XS (J-1)*F(11))/(XDS(J-1)+XG(J-1))

IN 2 IO

XTHYP $(J)=P$ THYP $* F(1)+P$ THYP $*(X R S(J)+X G(J))+G 001+G 002$

IN 210

RIJKINZ $(7)=X T P G J(J)-X T H Y P(J)$

GU $3=P$ PF $2 * \times S(J-1) * F(11) / \times F S(J-2)$

IN 2 ID

$r, 04=P$ IF $3 * x F S(1-2) * F(11) / \times 5(1-1)$

$X T F(J)=P T F 1+F(11)+G 03+C 04$

$G 05=X G(J)+X U S T U(J)-X T R G S(J)-X T F(J)$

INZIO

IN 210

IN 210

$X Y S(J)=P P I 1+P P I 2 * X P S(J)+P P I 3 * G 05$

IN ZID

$X Y S(J)=X L S(J)+X V S(J)$

$X Y U(J)=X \operatorname{RS}(J)+X G(J)-X V S(J)$

$X Y S T(J)=X R \leq T(J)$

$X T L A(J)=P T L A I * X A(J)+P T L A Z * X L A(J)+P T L A 3 * F(17)$

IN IID

IN Z ID

IN 2 ID

$X T S V(J)=P I S V I+P T S V Z * X L A(J)$

$G \cap(=P H U A 2 *(X L A(J-1)+X(A(J-2)+X L A(J-3)) / 3.0$

GO $7=$ PHUA $4 *(F(13)-X E(J) * X(I T))$

$X U S T A(J)=P H U A 1+G O R+P H U A 3 * X(A)(J)+G 07$

XUAAUS $(J)=$ PNUE $1+P$ PUEE $Z(X L A(J)-X T L A(J))$

$X Y A N(J)=X Y A(J)-X T L A(J)-X T S V(J)+X U S T A(J)-X U A A U S(J)$

IN $2 I 0$

IN ZIO

IN 2 ID

IN T. ID

IN 2 IO

IN 210

$X T L S(J)=P T L S 1 * F(11)+P T L S 2 * F(10)+P T L S 3 * F(0)$

$\operatorname{XYSN}(J)=X Y S(J)-X T L S(J)$

$X Y U N(J)=X Y U(J)-X T R G S(J)-X T F(J)+X U S T U(J)$

$X T O I R(J)=X T L A(J)+X T L S(J)+X T R G S(J)+X T F(J)$

$X H I(J)=X T I N D(J)+X T D I D(J)+X T S V(J)+X R S T(J)$

$X F S T Z U(J)=P H F 1 * X H I(J-1)+P H F Z *(F(4)-X X O(J) * X P X(J-1)$

$X H z(J)=X H 1(J)-X F S T Z U(J)$

XUSTAUS $(J)=$ PHUAUS $1+$ PHUAUS $2 * x H 2(J)+P H U A U S 3 * \times H 2(J-1)+$ PHUAUS 4

IN 210

IN 2 ID

IN IID

$1 N 210$

INZID

IN 2 ID

IN 210

INZID

IN 2 IO

INZID

$X U S T(J)=X U S T A(J)+X U S T U(J)+X U S T A U S(J)$

$X Y S I N(J)=X Y S T(J)+X I I N D(J)+X T D I R(J)+X T S V(J)-X U S T(J)$

INZIO

$X Y P R N(J)=(X Y A N(J)+X U A A U S(J))+X Y S N(J)+X Y U N(J)$

INIID

IN 210

$X Y A N \partial(J)=(X Y A N(J)+X U A A U S(J)) / X Y P P N(J)$

INZIO

XYPRNO $(J)=X Y P P N(J) /(X Y Y(J)+X T I N D(J)$

INIID

$X C A(J)=P O M I A I *(X E V(J)-X S(J)) * F(3)+P O M I A Z * X Y A N(J)$

INZID

$X S A(J)=X Y A N(J)-X C A(J)$

$1 N 210$

$X C S(J)=P O M I S 1 * F(12)+P O M I S 2 * X Y S N(J)$

IN 710

$\operatorname{XSS}(J)=\operatorname{XYSN}(J)-X C S(J)$ 
$G 09=(G 08-F(10) / F(13) 1 /(1.0-G 08)$

$X W A(J)=X W A(J-J)+X S A(J)+X U F W S A(J)$

INZID

$X W S(J)=x W S(J-1)+x S S(J)+X S U(J)-X U E W S A(J)$

IN IID

$X W H H(J)=X W A(J)+X W S(J)$

$X_{W A O}(J)=X_{W A}(J) / X W H H(J)$

$X A Q(J)=U 104 * X(I T) *$ UI 32

IN I I

GUIU $=F(4) /(x \times(J-]) \neq \times P \times(J-1))$

INZI

GO $11=F(3) / \times P \times(J-1)$

$I F(J-10)$

$409,407,409$

$400 \times[01 \mathrm{JMQ}=10.0$

$$
\text { Gत TO } 410
$$

$400 \times D U M O=1.0$

410 CDNTINUE

GOOO7 = XOUIM 2 *PRHOS

$P P H \cap \cap \geq P F H D I * X \triangle 2(\mathrm{~J}) * P R H O 4 * X 7 I N S A U(\mathrm{~J}) * P R H O 5 * 60097$

INZID

IN IID

INZ IO

IN Z ID

INZ ID

INZID

INZID

INZID

INZIO

GUOZO $=X D U M O * P S I G 5$

$P S 1 G O=P S I G I * A O U(J) * P S I G, 4 G 0098$

$G O \cap Q 9=X D(I M U * P L A M I 5$

$P L A M C 3=P L A M 3-P L A M 4$

$P L A M O=P L A M I * X Y R R(J) * P L A M O 3 *(X W A(J-1)+Y W S(J-1)) * P L A M 4 * G 0099$

INZII

IN ZID

INIID

INZIO

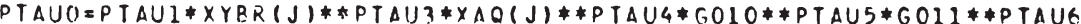

$G U I \cap 0=1.0 /(P P H O 2+P K H O 3 *(P S T G 2+P S I G 3 * P L A M 2)-P$ TAU2)

$G O 1 U 1=P R H U 3 * P S I G 3$

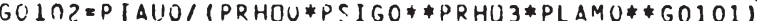

$X Z I N S(J)=G O 10 Z *$ GOIOO

$X N(J)=P L A M O * X I I N S(J) * P L A M 2$

$X O(J)=P S I G U * X Z I N S(J) * P S I G Z * X N(J) * P S J G 3$

$X M(J)=P$ IAUO*XZINS(J)*PTAU?

$X M O(J)=X M(J) / X Y B K(J)$

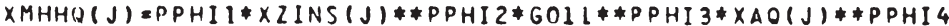

$X M A(J)=X M H H O(J)+X L \Delta(J)$

$x M S(J)=X M H H O(J) * X L S(J)$

$X M U(J)=X M(J)-X M A(J)-X M S(J)$

$X F A(J)=X W A(J)-X M A(J)$

$X F S(J)=X W S(J)-X M S(J)$

$X F S T(J)=X F S T(J-1)+X F S T Z U(J)$

$X F X(J)=X F X(J-1)+X I O R(J)+(X M U(J)-X M U(J-1))-X U P R(J)$

$X F \times A N(J)=X F \times A N(J-1)+X I P D A N(J)-X D P D(J)$

$X F A U S Z U(J)=(P P S I 1+P D S I 3 *(X O(J) / X N(J))+P P S I 4 * X D U M 7 F) * F(4)$ 
1

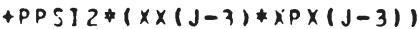

$X Z D E V(J)=x Z X(J)+X Z R(J)+X F A U S Z U(J)-X U A A U S(J)-X U S T A U S(J)$

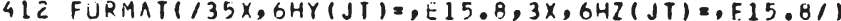

1118

४U4 CONTINUE

XPV $(J)=U 97$ * $(3)$ *PTHET2

1120

$X V(J)=U 98 * F(2) * P E T A 2$

$X X I M(J)=F(16) / X P I M(J)$

$X P E X(J)=F(15) / X \times E X(J)$

$X W U(J)=X M(J(J)+X V(J) * X P V(J)+X K N P R(J+1) \neq X P K(J)-X F X(J)$

IN ZID

1121

INZID $\quad 1122$

INZID $\quad 1123$

IN $210 \quad 1124$

INZID 1125

$X W F D(J)=X W H H(J)+X W U(J)$

$X W H H O(J)=X W H H(J) / X W P R(J)$

$P Q U E F=Y(J T)-Z(J T)$

$P R U E F=A R S(P R U E F)$

$X K P I T=1 . O F-E \$ Y(J T)$

I $(P R U E F-X K R I T) \quad 403,403,400$

400 IF $(J T-1) \quad 401,401,402$

4n1 $Y(J T+1)=2(J T)$

$$
\text { Go } 10 \quad 2979
$$

$4(12$ DIFFI $=2(\mathrm{JT})-2(\mathrm{JT}-1)$

DLFF? $=Y(J T)-Y(J T-1)$

RFGULA $=2(J T)-Y(J T) * D I F F 1 / D I F F 2$

$F A L S I=1.0-U I F+1 / D I F F 2$

$Y(J T+1)=R E G U L A / F A L S I$

$$
\text { G० }
$$

4u? XKSTZU(J)= L (JT)

KIJNIRULLERGERNISSE

IF(KUNTFOL) GO IO 411

WRITF $(6,297)$ JAHP(J)

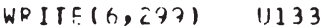

INZID $\quad 1126$

INZID 1127

INZID II2A

INZID $\quad 1129$

INZID 1130

INZIO 1131

INZID $\quad 1132$

INZIO $\quad 1133$

INIID $\quad 1134$

IN ZID $\quad 1135$

INZIO $\quad 1136$

IN ZIO 1137

INZID 1138

INLID 1139

IN 2 I

INIID 1141

INZID $\quad 1142$

IN $2 I 0 \quad 1143$

INZIO 1144

INZID 1145

INZIO 1146

INZID 1147

INZID $\quad 114 \mathrm{P}$

WPITE(6.4C5) X(IT). JT, IT

INZID $\quad 1140$

INZID $\quad 1150$


WRITE $(6,407)$ FF, DFF

4(1) FOPMATI/1NX, BHFF=,F15.R, 5X, 4HDFF=, F15. P

INZIO

1154

411 CONTINUP

EXDGENF UND VERZOEGERT-ENODGENE GROESSEN DER FOLGFPERITDE

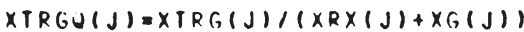

$X \operatorname{TRGSO}(J)=X T P G S(J) /(X P S(J)+X G(J))$

GUIZ=XIINS $(7) \neq(1.0+x T P G O(J))$

GUITEPRISIKO* $(1.0+X T P G U(J)+X T R G S O(J))$

$X Z J A S X(J)=((G 012+0013) * X F X A N(J-1)+X T K(J)+X D P R(J))$

1 $($ (XKDR (1) \#XPK(J))

$X K X S T(J)=(1.0 / P A L F A 1) *(1.0-(1.0-P A L F A 1) * X X(J) / X K S T(J))$

$x \cdot x \times(7)=(x \times(7) / x \times(7-1))-1.0$

$x \in K \times(J)=(x K \times S T(J) / X K \times S T(J-1))-1.0$

$X G R \times(J)=(\times 2 I N S \times(1) / \times 2 J N S \times(J-1))-1.0$

$6017=(x \times(J) \neq X K X S I(J)) /(X K P R(J) * X 7.1 N S X(J))$

$G \cap 19=(1.0+x G \times(J)) *(1.0+x G K X(J)) /(1.0+X G R \times(J))$

$X K P K(J+2)=P A L F A X 1 * X K P R(J+1) * G 017$ * $P A L F A X 2 * G J 18 *$ *PALFAX3

INZIn

INZI In

1162

1163

INZIO $\quad 1165$

INZIO $\quad 1166$

IN ZID $\quad 1167$

INZID $116 R$

INZID IIRO

INZID $\quad 1170$

INZID 1171

IN $110 \quad 1172$

INZID $\quad 1173$

IN2IO 1174

INIID $\quad 1175$

IF $(J-J 08) \quad 501,500,500$

$500 \quad r, 01=1-9.0$

$G 02=1.0 /(T-14.0)$

XKPRAB $(J+1)=P X I X 1 * P X I \times 2 * G 01 * P X I \times 3 *$ * 002

$5 J 1$ PRUEF $3=X K P R(J+?)-(X K P R(J+1)-X K P R A R(J+1)$

Ir (PPUEF 3-0.0) $525,326,526$

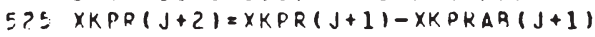

$526 \times K K P R Z U(J+1)=X K P D(J+2)-X K P R(J+1)+X K P R A B(J+1)$

I $F(J-J \cup 1) \quad 503.502,502$

502 XUPRU $(J+1)=P 2 E T 1+P Z E T ? *(1.01(T-? .0))$

:0 $3 \times X P R R(J+1)=\times D P P O(J+1) * X K P R(J+1)$

$\operatorname{XKNPR}(J+2)=X K N P P(J+1)+X K P R T U(J+1)-X D P R R(J+1)$

INZID

INZIID

INZIO

INZI ID

INZIO

IN 210

IN 2 ID

IN 210

INZID

INZIO

INIID

XKSTAP $(J)=P H K 1 * P H K 2 *(T-10.0)$

$X K S T(J+1)=X K S T(J)+X K J I Z U(J)-X K S T A R(J)$

INZIO

INZID

IF(J-JOC) S,U5,504,504 
$504 \times D S T O(J+1)=P H D I+P H C Z *(T-19.0)$

$X G L(J)=(X L \cap H N(J) / X \operatorname{LH} H N(J-1))-1.0$

INZIIO

$X G L X(J-1)=(X \operatorname{LHHN} \times(J-1) / X L$ OHN $X(J-2))-1.0$

INZII

$G \cap I Y=X P P R(J) / X F \cdot T P P(J)$

INZIO $\quad 1197$

$G 021=(1.0+X G \times(J-1)) *(1.0+X G F(J-1)) *(1.0+X G K X(J-1))(1,0+x G L \times(J-1)$ INZID $X F() P R(J+1)=x F .0 P P(J) * C, 019 *$ *BETA1*GU20*PBETA2*G021**PBETA3

INZID

PKIIPL:O $(j+1)=1.000000$

$X E Q P R(J+1)=P K U P E Q(J+1) * X F \cap P R(J+1)$

$X K X U S T(J+1)=X K X U S T(J) *(1.0+x G K X(J-1))$

INZID

IN 2 I 0

* $($ XKXST $(J-1) / X K \times O S T(J-1)) * P A L F A \times 2$

$6022=P A L F A 3 * \times E O P P(J+1) *(1.0+P F) *(T-9.0)$

$\times X O(J+1)=G 022$ *PALFA2*XKPP $(J+1) *(1.0-P A L F A 2) * \times K \times O S T(J+1)$

$X K O S T(J+1)=(1.0-P A L F A 1) * X \times[)(J+1) /(1.0-P A L F A L * X K X O S T(J+1))$

$X E S T(J+1)=(P H E S T 1+P H E S T 2 * X H 2(J)) / X L O H N(J)$

$X P A(J+1)=(P J \cup T 1+P J U I 2 /(T-4.0)) \times Z I N S(J) * X F A(J)$

$x K S(J+1)=x Z \operatorname{InS}(J) * x F S(J)$

$x \operatorname{RST}(\mathrm{J}+1)=x \operatorname{TinS}(\mathrm{J}) * x F S T(\mathrm{X})$

$G 053=\times 5 \times(7) /(\times \times(7) \div \times 0 \times(7))$

$G \cap P 4=\times 2 \times(J-1) /(\times \times(J-1) * \times P \times(J-1))$

$G \cap 25=x \geq \times(J-2)((x \times(J-2) * x \times(J-2))$

$6026=(6,023+6024+60<5)$

I $F(J-J 05)$

$513,506,50 h$

.U6 IF $(X N(J)-X W(J-1)) \quad 509.507 .509$

507 IF (GU26-0.1) 508,509,510

$5 \cap 8$ IF $(G 026+0.1) \quad 511,503,509$

$509 \times W(J+1)=x W(J)$

(i) TO 512

$510 X W(J+1)=X W(J) * 1.05$

GO TI 512

INZIO

IN Z IO

INZID

INZID

INZID

INZID

IN 2 ID

IN Z I D

INZIO

IN Z ID

INZII

INZIO

IN T. I0

IN 210

IN $Z 10$

INZIO

INZID

IN Z I I

IN 2 I

INZI!

IN 2 ID

$511 \times W(J+1)=x W^{\prime}(J) / 1.05$

, $12 \times W \times(.1+Z)=x_{n}(J+1)$

$513 \times 2 R(J+1)=P O M E G 1+P O M E G Z *(X Z \operatorname{INS}(J) \neq X F A U S(J))+P O M E G 3 * X W(J+1)$

INZID

INZIO

I $F(x \operatorname{in}(J)-x w(J-1))$

$515,527,515$

527 IF $(X W(J+1)-X W(J)) \quad 514,515,516$

IN 210

INZ ID

INZID

INZI I

1198

1199

1200

1201

1202

1203

1204

1205

1206

1207

1208

1209

1210

1211

1212

1213

1214

1215

1216

1217

1218

1219

1220

1221

1222

1223

1224

1225 
$516 \times 0 O U M Z F=+1.0$

217 IF $(\mathrm{J}-\mathrm{J} 04)$

$519,518,518$

IN 2 ID

5 I $X Z I N S A U(J+1)=P R H C \times 1+P R H U \times 2 * X Z I N S A U(J)$

210 if $(j-j, 7)$

$321,520,520$

INZID

$520 \times \times A U S(J+1)=P C H I ? \times 1 * P C H I 2 \times 2 * *(T-25.0)$

INZID 1237

521 If $(J-J 03) \quad 523,522,522$

5.2. $G(0)=1.0 /(T-21.0)$

INZID

INZ10 $\quad 1239$ XPAUS $(\mathrm{J}+1)=P(H I 3 \times 1 * P C H I 3 \times 2 * *(T-21.0) * P C H I 3 \times 3 * * 601$

523 CDNIINUE

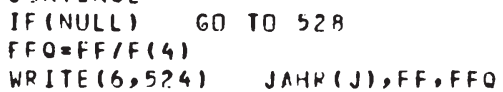

224 F[IRMATIIOX,27HBERECHNUNG FFRTIG FULR JAHR, 1X, I4,

INZIO

INZID $\quad 1243$

INZID

INZIO $\quad 1245$

1 57 . KEINE NULLSTELLE IM RELEVANTEN BEREICH DER FF-FUNKTION., $5 H$ FF $=, E 13.6,190 \times$, 5H FFO $=0$ E 13.61 GO TO 5999

5) PH WRITE $(5,529)$ JAHR(J)

a?9 PIPMATIICX,27HRERECHNUNG FERTIG FUER JAHR, $1 \mathrm{X}, 14,1 \mathrm{H}, 1$

PUMIAI $=$ TMIAI

POMIAIETMIAL

POMIL3= TMIA

POMIA4=OMIA

PUMISI:DMISI

PUMISZ $=0 M I S 2$

P.JMIS3 $=D M I S 3$

Q99 CONTINUE

TF. ILAUSORUCK DER MODELLERGEBNISSE

IN IIO

IN 210

INZID

INZIID

INZID

IN $Z I O$

INIID

INZII

INZID

INZI ID

INZ ID

INZI IO

IN 210

IN 2 ID

INZID

INZIIO

INZ TD

INZID

IF ( ( NOCH.NE.NDCHMAL) , OR. (IDA.NE . I) )

1 . AND. ( (NOCH.NE.0),OR. (IDA.NE. 11$))$

WRITE $(6,600)$

IN ZI I

INZID

INZID

1245

1247

1248

1249

1250

1251

1252

1253

1254

12.55

1256

1257

1258

1259

1260

1261

1262

1263

1264

hUN TJRMATI/////11X, 4HJAHR, 1X,14HBSP Z.MARKTPR,, 5X,10HPREISINDEX,

$13 X, 12 H P R F I S A N S T I E G, / 44 X, 14 H I N$ PRUZENT/100 ///1

WRITE $(6,(0))$ (JAHP(J), XYRR(J),XPX(J),XGP(J)

$J=10, J M A X 1$

1265

1267

GU1 FURMAT $(11 x, 14,2 x, F 13.6,2 x, E 13.6,2 x, E 13.6)$

WRITE $(5,602)$

1270

INTIO 127

hO2 FOPMAT ( / / /11X, 4HJAHR, 6X, 1OHBIP(PRIV), ,3X,11HKAFAZ,AUSL,

INZID

1271
1272 
1 IX, I 4HARBEITSL.OUTTE/21X, 4HRFAL, 9X, RHOUOTIENT///

603 IOP:AAT /////11X, 4H JAHR, 1X, 14HBESCHAEFTIGTE, 1X, I 4HABWEICHUNG VON, $11 X, 14 H A N T E I L$ PRIVATE, $116 X, 14 H D$. PRIV. SEKTORS, $1 X, 14 H P L A N B E S C H A E F-$ $21 X, 14 H B R U T T U I N V E S T I-, 131 X, 14 H T I G U N G$ D.PRIV., 1X, 14HTION A.RSP, , $3 / 31 X, 14$ HSEKTOPS $|1| 1$

WRITE (G,601) (JAHR(J),XFPR(J),XEKDEF(J),XIPRO(J), J=10,JMAX) WRITF $(6,610)$

610 FOPMAT $/ / / / / 11 \mathrm{X}, 4 \mathrm{HJAHR,} 1 \mathrm{X}, 14 \mathrm{HPRIV,KAPITAL}$ -

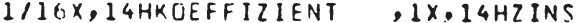

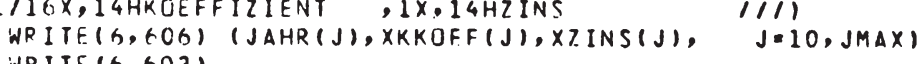

IN 2 I D

IN $Z$ IO

1278

1279

1280

1281

128 ?

1283 WRITE $(6,603)$

INZIO

INZID

I N Z I D

IN 2 ID

INZI ID

1284

1285

BO3 FURMAT $/ / / / 111 X, 4 H J A H R, 1 X, 13 H A U S S E N B E I T R A G, 5 X, 11 H D E V$. ZUFLUSS, $4 X$,

1 IOHWECHS.KUP.J/16X,11HO.SALDO RER/16X,13HKAP.ERTR,BIL, / / I

IN ZID

WPITE $(6,601)$ (JAHR (J), XZX(J), XZDEV J J), XW(J), J=10,JMAX)

INZID WR ITE $(6,612)$

612 FOPMATI/1/1/1X, 4HJAHR, 1X, 14HLDHNSAT? IM , 1X, 14HLOHNANSTIEG D.I

INZIO

INZIO

1286

1287

1288

1289

1290

1291

214 HIN PPOZENT/ICO/II

WRITE(6,606) (JAHR $(J), X L O H N(J), X G L(J), \quad J=10, J M A X)$ WRITE $(6,604)$

INZID

INZIO

INZIO

INZID

6)4 FQRMAT $/ / 1 / 111 \times, 4 H J A H R, 1 \times, 14$ HARBEITSEINKOM-, 1X, 14HANTEIL ARPEIT-, INZIO

1 IX, 14HANTEIL ARBEIT-, $116 x, 14$ HMENSOUUTE AN, 1X, 14HNEHMFRLDHN AN, INZIN

$21 \times, 14$ HNEHMERE INKOM-, $116 \times, 14 \mathrm{HBRUTIOWERTSCH,} 1 \times, 14$ HVOLKSEINKOMMEN, INZIU

$31 \times, 14$ HMEN AN VDLKS-, $116 x, 14 H D$.PRIV.SEKTDRS, $16 x$,

4 I 4 HEINKOIMEN $1 / /$ ) WRITE $(6,601)$ (JAHR $(J), X L P R Q(J), X L A D(J), X Y A O(J), \quad J=10, J M A X)$
WRITE $(6,605)$

INII $I 0$

IN $2 I 0$

INZID

IN 210

605 FJHMAT $/ / / / 111 X, 4 H J A H P, 1 X, 14 H A N T F I L$ D.VERF, , IX, 14HANTEIL D.VFRF., INZID

$11 X, 14$ HISP.L.MARKT- , /16X,14HARB.NEHMEREIN-, 1X, 14HEINK.D.PRIV., I I $21 \times, 14$ HPREISEN , $116 X, 14 H K O M M E N$ A.VERF $, 1 X, 14 H S E K T O R S$ A. NSP, 3 /IAX, 14HEINK.D.PRIV., IX, 14HZ.MARKTPREISEN/16X,

414 HEKTORS $\$ 1,1 \times, 14 \mathrm{H}$ WRITE(6.6OI) (JAHP(J), XYANO(J),XYPRNO(J),XYN(J), JEIO,JMAX)

INZIO

INZII

INZIO

INZID

IN 210

ROG FORMAT(11X, I4, 2X,E13,5,2X,F13.6) W'L ITE $(6,014)$

314 F IRMAT(/11X,31H**) VERFUEG?. FINKTMMEN ENTHAL-116X, 12 ZHTEN HJER AUCH AUSL.JEPER-116X, 2 SHWEISUNGEN D.APBEITNEHMER / / / WRITF(b, AUT)

INZIID

IN Z ID

INZII

IN? IO

1292

1293

1294

1295

1296

1297

1298

1299

1300

1301

1302

1303

1304

1305

1306

1307

$13 \cup 8$

1309

1310

1311

INZID 1312 
1 1X, 14HKUMUL NIJMI- $116 x_{0} 14$ HNFHMER A.KUMU- $1 x_{0} 14$ HNOMINALFRSHAR

$21 x, 14$ HMINALERSPARNIS $16 x, 14$ HLIFRTER NDMI - $1 x, 14$ HNIS D.PRIV. $\therefore$ INZII

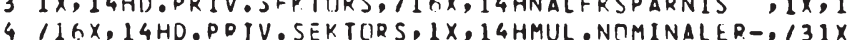

1316

I IHSPARNIS PLUS , $131 x, 14$ HBEWERTUNGS- $, 131 x, 10 H G E W I N N E$

IN IID WPITE $(6$, CUI)
WRITF $(6$, AD

1111 INZID

INZID

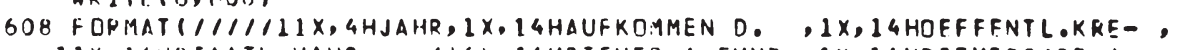

IN 210

$11 x, 14 H S I A A T L$.HAUS - , $16 x, 14$ HSIEUER A. TUND, $11 x, 14$ HDITVERGABE A.,

$2.1 x, 14$ HHALTSVOLUMEN $1,116 x, 14$ HSELAST,EINK, ,1X,

314 HPRIV.SEKTOR O1X,14H(LAUFENDE FIN-146x,14HNAHMEN)

IN 210

WR ITE (6, 001$)$ (

$J=10, J M A X I$

$(1 / 1$

INZIO

INZI ID

WRITE $(5,(.13)$

413 FURMAT $/ / \| / 11 X$, 4HJAHR, 1 X, 14 HS TEUERMEHRAUF,$- 1 X$,

1 14HPERSONAL - , /16X,14HKOMMEN INFOLGE, 1X, 14HAUSGABEN DFS

TRM $1 X, 14 H S T A A T E S$ / $16 x$,

? 16X,14HSTEUERREFTRM , 1X, 14HSTAATES

3 I 4 H(FURMALRECHN.) / $/ 11$

WRITE $(6,6 C B)$ (JAHR (J),FDRINZ(J), XLST(J), J=10,JMAX)

BII CDNTINUE.

IF (NOCH.FO.NOCHMAL) GN TI 707

C

NFIIRFD CIIIUINU UL? KUPREKTURFAKTORFN PKUP(1), PKOR(2), .

ZUP ANPASSUNG DES MODFLLS AN DIE VILKSWIRTSCHAFTLICHE

GE SAMTRLCHNUNG DER BUNDE SREPIJBLIK

$J z=10 A$

$x \times x(1, J 2)=x \times(10)$

$x \times x(2, J Z)=x A O(10)$

$X \times X(3, J Z)=X P \times(10)$

$x \times x(4, J Z)=x L P R \cup(10)$

$x \times x(5, J 2)=x \Delta 0(11)$

$x \times x(6, J 2)=x P \times(13)$

$\times \times \times(7, J 2)=x \times(13)$

IF (IUA.EO.I) GO TO 701

DI $700 \quad I Z=1, I 2 \mathrm{AHL}$

VVV (IZ,JZ-1) $=(\times \times \times(12, J Z) / \times \times \times(I Z, 1)-1.0) /(P A N P(J Z-1)-1.0)$

IN 2 I I

IN IID

INZID

IN II IN

INZ IO

CIRRFC

CORREC

INZID

IN I In

INZIO

INZID

IN?IIO

IN IID

INZID

INZID

IN 2 IO

INIID

IN ZID

IN IID

IN 2 ID

IN I ID

INIID

INIID

IN 210

INZII

INZIID

IN 210

IN IID 


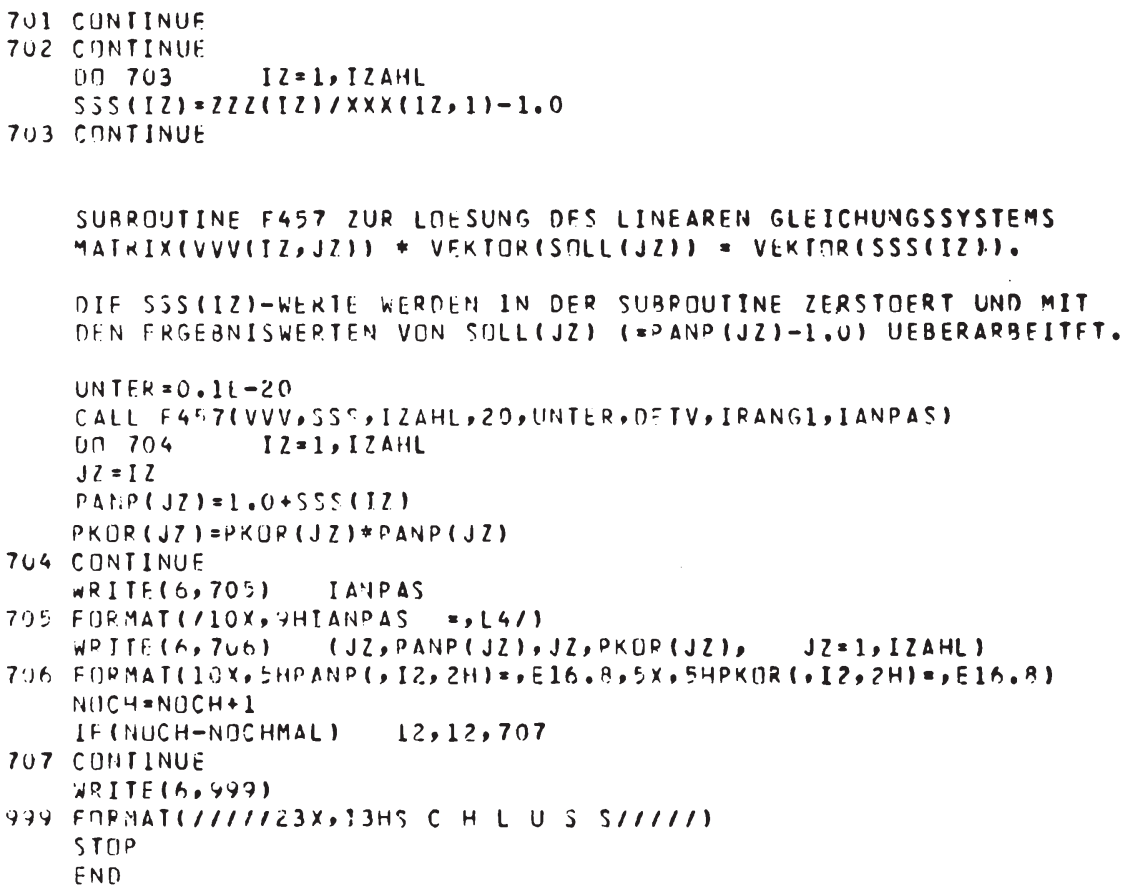


I. HOUT-KINTPOLI.E

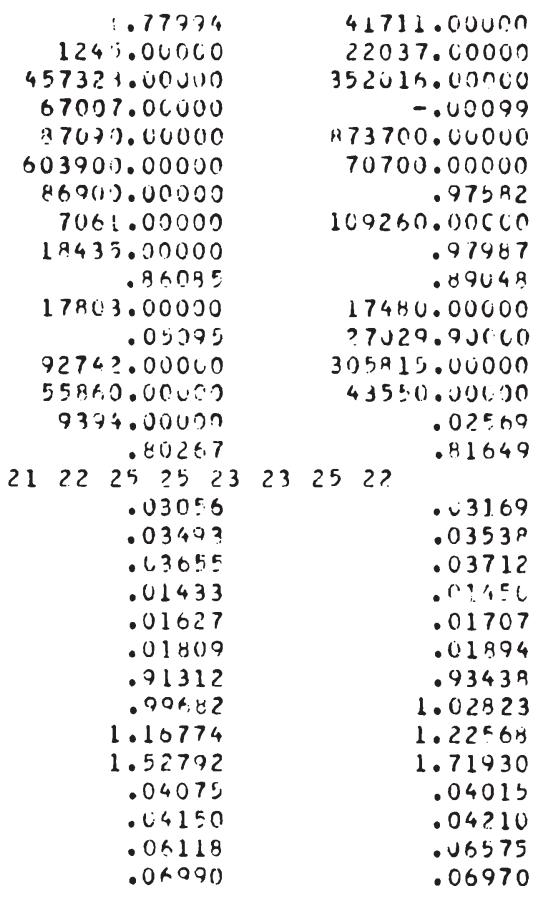

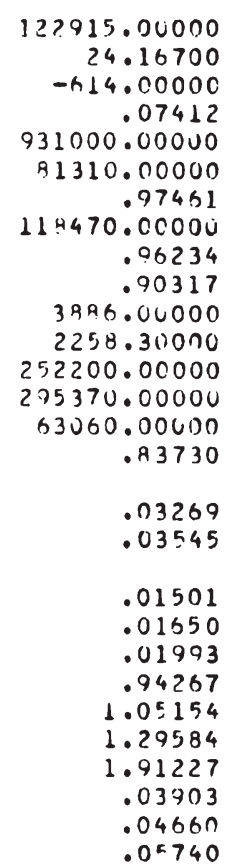

40370.00000

24.14925

273900.00000

23327.00000

519700.00000

.97288

5013.00000

126990.00000

1.04372

.06799

6.11500

614.00000

2.50780.00uno

$-1340.00000$

4610.00000

88931.00000

.03351

$.0356 ?$

.01547

.01725

.95563

1.08119

1.36217

.03953

.04353

.05530
26700.00000

26.31400

301393.00000

56602.00000

$5+0000.00000$

.97341

6630.00000

145320.00000

1.01860

2200.00000

.05215

82490.00000

280110.60000

9052.00000

.86600

.03433

.03599

.01596

.01730

.97833

1.12403

1.42571

.04000 .05258

.06300 


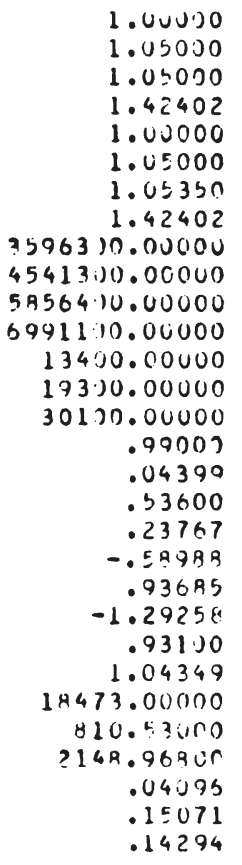

1.00000
1.05000
1.95717
1.05000
1.05000
1.07217

3741500.00000
4.05000 .00000
5994700.00000
5951300.00000
14200.00000
21201.09000
32100.00000
.75000
.02900
.80055
-.14709
.04073
.93203
.19399
.99869
.28209
1.05200
.53253
2.02975
-2.61753
-.12930
-.02975

1.00000
1.05000
1.14765
1.00000
1.05000
1.14765

3895100.00000
5143700.01000
6234200.00000
15000.00000
23500.00000
34200.00000
6817.77000
.02000
1.31538
.18537
-.07177
.29884
-.03445
1.90151
.48004
.03527
-12553.03000
1.04224
.9712130
-1.34791

1.04167
1.05000
1.14765
1.04167
1.05000
1.14765

4007200.00000
5312600.00000
6600000.00000
16200.00000
25600.06000

.04458
.04000
.97843
.97528
.14419
.04556
-2.81775
-.033486
-2734.95000
369.44279
.88872
-.19815
.27519
-.01592
.01766

$4309300.0000 n$ $56 \cap 4000.00000$ 7005100.00000

17600.00000 27900.00000

.99371 .69000

1.14995

.02843

.84523

.70714

1.04125

.85092

$.0 ? 557$

.73948

.62164

$-.55046$

$-.03243$

$-.35063$

1. 00867 


\begin{tabular}{|c|c|c|c|c|c|}
\hline 1.12223 & $-1.41+3 v$ & .74965 & 1.00300 & .32275 & \\
\hline .77984 & 7193965.60000 & $1.0430 n$ & 2.08290 & $1.03=00$ & \\
\hline .04771 & .27034 & -.31172 & -.13181 & -.01968 & \\
\hline 5923.72000 & $-.4 \cup 561$ & -7308.07006 & 363.30000 & .13976 & \\
\hline$-3 \cup 7.03613$ & .01006 & 274.63000 & .04736 & 1004.08000 & \\
\hline .00335 & 126.75000 & .01370 & 357.270 Uu & .07873 & \\
\hline-3055.060000 & .12798 & .01697 & 0.00000 & $-40 R 9.17920$ & \\
\hline . 3.577 & - istinu & 4251.52000 & .17788 & $0.0000 n$ & \\
\hline$-1+38.41600$ & .14273 & .00937 & .00088 & .07042 & \\
\hline-.01536 & . $0 ? 26+7$ & -96.52000 & .18494 & 247.51000 & \\
\hline 1.07644 & 1.75643 & -.00645 & 0.00000 & 0.00000 & \\
\hline$-1 \cup 0.4 .26000$ & .20103 & .00705 & .81833 & -4839.08000 & \\
\hline .25533 & $-.1345 ?$ & -2834.08000 & .02909 & -.15421 & \\
\hline 0.0 ouun & 0.00000 & $-128 \cdot 2.841 \%$ & -.00211 & 1779.80000 & \\
\hline-.04155 & $0.00 u(10$ & 0.00000 & -2424.14000 & .00682 & \\
\hline$\cdot 24 \mathrm{hH.} 48000$ & .12240 & -10121.57000 & -.09534 & .09539 & \\
\hline .03995 .92400 & -.04796 & .03199 & -7349.41630 & -.14496 & \\
\hline $.1440 \mathrm{~h}$ & & & & & \\
\hline-4251.52000 & . 41680 & .00482 & 4251.52000 & .06403 & \\
\hline$-.0 n t 82$ & -8512.26000 & .04649 & -.09623 & .01536 & \\
\hline .14477 & 0.00000 & 0.00000 & 0.00000 & 0.00000 & \\
\hline .11567 & .00645 & -.30551 & -.03349 & 0.00000 & \\
\hline 0.0 טujuט & $0 . \operatorname{coc} 00$ & 0.00000 & 9901.98000 & -.20179 & \\
\hline .09713 & $186,5.690 .90$ & -.01509 & .11270 & .01400 & \\
\hline .57723 & 1.76076 & .36411 & .08498 & -.96055 & \\
\hline .01978 & $1.2650 B$ & 1.0 .5783 & -.02822 & .02500 & \\
\hline .50000 & .36443 & $-.78 \times 73$ & -.78873 & -.01694 & \\
\hline $.16 ? 03$ & -137.07530 & .18046 & -1.67348 & & \\
\hline $.38393 E+07$ & $-.360 \times 1) 5+07$ & $.73 i 11 E+07$ & .1 & & - $11569 E+\cap B$ \\
\hline $.74783 E+07$ & $.34853 E+09$ & $.83356 E+08$ & .4 & & $.69933 E-02$ \\
\hline
\end{tabular}


1. Der Standardablauf der Modellentwicklung

Der hier dargestellte Standardablauf der Modellentwicklung berücksichtigt abgesehen von den im Paragraphen 18 genannten Vereinfachungen und Korrekturen die wirtschaftlichen Verhältnisse so, wie sie sich in dem statistischen Datenmaterial für die Bundesrepublik darboten und wie sie aufgrund dieses Materials mit Hilfe der Regressionsschätzungen erfaßt wurden. Insbesondere berücksichtigt der Standardablauf alle staatlichen Verhaltensreformen so, wie sie mit Teilschätzungen für verschiedene Zeitabschnitte, Dummy-Variablen etc. erfaßt wurden.

Wie man an den Ergebnissen der folgenden Übersicht, insbesondere an den entscheidenden Indikatoren der wirtschaftlichen Entwicklung, etwa der Arbeitslosenquote, dem Kapazitätsauslastungsquotienten oder der Preissteigerungsrate ablesen kann, weichen die wirtschaftlichen Verhältnisse, wie sie durch die Modellrechnung nachgebildet werden, erheblich von den empirischen Verhältnissen der Bundesrepublik ab. Bei der Kürze der Gesamtperiode läßt sich gerade noch eine deutliche Talwelle des Konjunkturzyklus zwischen 1960 und 1966 mit einem konjunkturellen Zwischenhoch im Jahre 1962 erkennen. Im Jahre 1972 ist die Modellentwicklung aufgrund ihrer geringen Stabilität bereits wegexplodiert. Die Ergebnisse der Modellrechnung für den Standardablauf werden daher im folgenden ohne weiteren Kommentar tabellarisch dargestellt. 
Inlielle 1n.1. Norlellergelmlage dre Standardrechnung

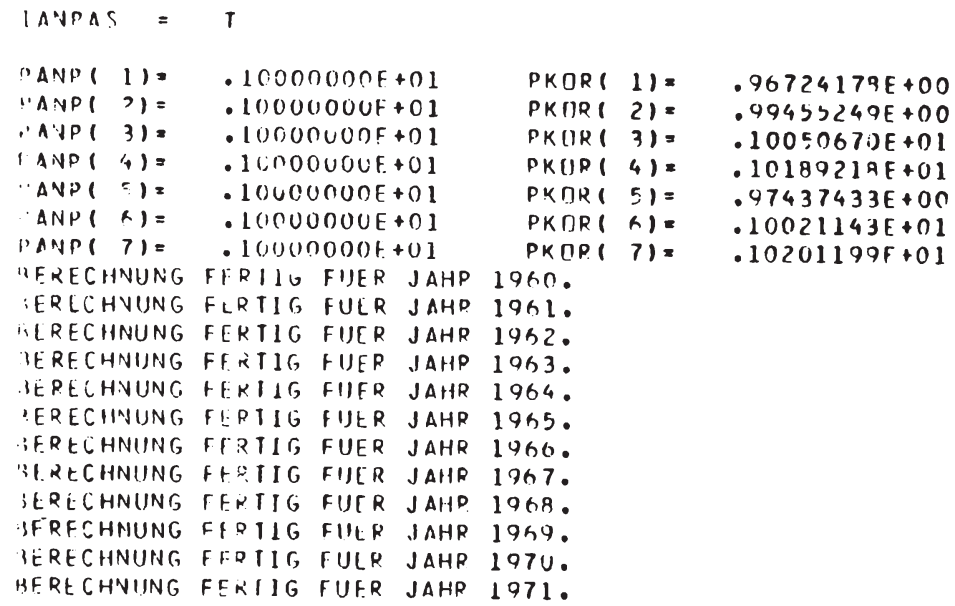

JAIR RSP T.MARKTPR.

PRE IS INDEX

PREI SANSTIFG

IN PRDZENT/100

$\begin{array}{ll}196 U & .302255 E+06 \\ 1961 & .317443 E+06 \\ 1742 & .354592 E+06 \\ 1763 & .354472 E+116 \\ 1764 & .41606 U t+06 \\ 1755 & .450379 E+06 \\ 1966 & .52344 t+06 \\ 1367 & .071044 E+16 \\ 1960 & .754955 E+06 \\ 1769 & .924943 E+C 6 \\ 1970 & .96942 U E+06 \\ 1771 & .747704 E+06\end{array}$

$.422993 \mathrm{~F}+0 \mathrm{C}$

$.219494 E-01$

$.95596 t+100$

$.357455 \mathrm{E}-01$

$.793361 t+00$

- $390962 F-01$

$.102814 E+01$

- 33UORIE-U1

- $100725 E+01$

. $380423 \mathrm{E}-01$

. $109931 \mathrm{~F}+01$

. 30037 AF-01

$.117626 E+01$

- 699984F-01

$.132369 f+01$

. $1 ? 5341 \mathrm{~F}+00$

$.154322 E+01$

$.165848 \mathrm{E}+00$

$.16589 A E+00$

. $179170 \mathrm{E}+01$

. 10635 A +00

$.165931 t+01$

-.161 AG6F+OO 
Tabelle 19.1: Morlellorgebnlsse Her Standardreclunung

\begin{tabular}{|c|c|c|c|}
\hline JAHR & $\begin{array}{l}\text { Z1P(PRIV), } \\
\mathrm{kCAl}\end{array}$ & $\begin{array}{l}\text { KAPAZ .AUSL. } \\
\text { DUNTIFNT }\end{array}$ & AP.BEITSL. UUDTE \\
\hline 1960 & $.305200 F+06$ & $.101055 F+01$ & $.132040 F-01$ \\
\hline 1761 & . $311: 34 t+r 6$ & $.0854175+00$ & . IA510OF-01 \\
\hline 1962 & $.33210 r t+06$ & $.1002+44 t+01$ & $.50729] \mathrm{F}-02$ \\
\hline 1003 & $.345300 E+O 6$ & $.979797[+00$ & $.154674[-0]$ \\
\hline 176.4 & - $341108 E+100$ & $.98749 .7 t+00$ & $.197984 E-01$ \\
\hline 1365 & . $3130364 E+06$ & $.102073 F+01$ & $.100701 E-01$ \\
\hline 1966 & $.409755 E+U S$ & $.102644 F+01$ & $.682623 \mathrm{E}-02$ \\
\hline 1767 & $.431574 E+n 6$ & $.100933 E+01$ & - $505603 F-02$ \\
\hline 1703 & - 45.5734t+ub & .1 UC:R58E+ 01 & $.8600311-02$ \\
\hline 1369 & $.400005 t+06$ & $.9 B O 530 E+J 0$ & $.228335 F-01$ \\
\hline 1770 & $.4354 P 6 E+C O$ & $.9752 \mathrm{BSF}+\cup 0$ & $.102649 F+00$ \\
\hline 1471 & $.39442 A \mathrm{~F}+U \mathrm{~B}$ & . $8130801+00$ & $.177440 \mathrm{Ft}$ \\
\hline
\end{tabular}

JAHK RESCHAFFIIGTE U.PRIV.SFKTIRS
ABWEICHUNG VIJN ANTEIL PRIVATE PLANBF SCHAEF - BPUTTRINVFSTITIGUNG D.PPIV. TION A.RSP SFKTOPS

\begin{tabular}{|c|c|}
\hline 1960 & $.244590 E+02$ \\
\hline $1+4$ & $.24482 A E+U 2$ \\
\hline $17+?$ & $.7474428+02$ \\
\hline 1703 & . $P^{4}=14 v t+U 2$ \\
\hline 1764 & $.245373 t+0 ?$ \\
\hline 1วด5 & $.2470 ; \cup \cup F+C 2$ \\
\hline 1360 & $.2497 A 3 E+02$ \\
\hline $17 t, 7$ & .?ᄂ4I8BF+ก? \\
\hline $17 \theta, 2$ & $.257510 E+02$ \\
\hline 1969 & $.254990 E+02$ \\
\hline 1970 & . $231036 t+02$ \\
\hline 1971 & - 2 ปUน $3 F+02$ \\
\hline
\end{tabular}

$\begin{array}{ll}.100608 E+01 & .240560 F+00 \\ .987152 E+0 U & .222738 F+00 \\ .948962 E+00 & .230337 F+00 \\ .927172 E+00 & .235657 F+00 \\ .987864 E+00 & .224476 F+00 \\ .101247 E+01 & .222824 E+00 \\ .101699 E+01 & .226514 F+00 \\ .100455 E+01 & . .229187 E+00 \\ .100421 E+01 & .232169 F+00 \\ .984020 E+00 & .218560 F+00 \\ .907224 E+00 & .166205 F+00 \\ .862313 E+0 C & .107399 F+00\end{array}$


Tahelle 19.1: Modellergehnisse der Standardrechnung

IAHR PRIV.KAPITAL KOEFFIZITNT

KAPITALMAPKT7. INS

\begin{tabular}{|c|c|c|}
\hline 1950 & $.240147 t+n 1$ & $.03 K 707 F-U 1$ \\
\hline 1961 & $.29+915 F+01$ &.$: 560,36 \mathrm{~F}-01$ \\
\hline 1942 & $.297 \times 25 E+C 1$ & $.5+3620 E-01$ \\
\hline 1363 & $.304 \angle 3 B t+01$ & $.592734 E-U 1$ \\
\hline 1364 & $.309751 \mathrm{~F}+01$ & .5032 USE-01 \\
\hline 1765 & - $301+5 \leq 2 E+01$ & $.639645 F-01$ \\
\hline 1766 & -306?27E+U1 & $.724712 f-41$ \\
\hline 1467 & $.307 t, 93 t+0 t$ & . 91 tangE-01 \\
\hline 1965 & $.307150 F+01$ & $.104711 E+00$ \\
\hline 1969 & $.314103[+61$ & $.16 \theta=34 F+00$ \\
\hline 1270 & . $3 \cup 7233 t+01$ & $.201505 C+0 C$ \\
\hline 1971 & $.411162 t+U 1$ & $.137643 F+00$ \\
\hline
\end{tabular}

JAH? AIJSSENGFIIRAG ?) SALUT UEP $\angle A P, E R T R, B I L$.
DFV. IUFLUSS

WECHS. KURS $\begin{array}{ll}1360 & .977395 E+04 \\ 1961 & .113154 E+05 \\ 1962 & .556731 E+04 \\ 1363 & .586723 E+04 \\ 1964 & .647471 t+04 \\ 1965 & .451759 t+04 \\ 1966 & .577416 E+04 \\ 1967 & .347178 E+04 \\ 1969 & .105193 t+04 \\ 1769 & .143518 E+04 \\ 1970 & .171241 E+05 \\ 1771 & .303335 t+03\end{array}$
- $50064 E+04$ -.3950म1E+04

- 1 J6UOUN + 04

$-.259810 E+04$

-.306631E+03

$.112653 t+04$

$.926175 t+04$

$.198534 t+05$

. $388365 E+05$

$.371493 E+03$

$.415855 F+05$

$-.4303: 6 E+05$
. $100000 E+01$

$.104167 E+01$

$.10,000 E+01$

$.105000 E+01$

$.105000 F+01$

$.105000 \mathrm{~F}+\mathrm{C} 1$

$.105000 E+01$

- $10500 U E+01$

. $105000 E+01$

$.105717 E+01$

$.114765 E+01$

$.114755 F+01$ 
'Tahelle 19.1: Morlellergemiase der Standardrechmung

JAHR LQHNSAT? IM

PRIV.SEKTOP
LIHNANSTIEG D. PRIV.SEKIIJRS

IN PPIZENT/100

\begin{tabular}{|c|c|c|}
\hline 1760 & $.661843 t+114$ & $.100687 t+00$ \\
\hline 1751 & $.708035 t+04$ & $.097929 E-01$ \\
\hline 1762 & $.159774 E+04$ & $.871974[-01$ \\
\hline 1903 & $.839949 F+04$ & $.911641 E-01$ \\
\hline 1964 & . 91๖75RL+04 & $.902536 E-01$ \\
\hline $1+65$ & $.987,22 L+04$ & $.7 \times 3 \times 5 A F-01$ \\
\hline 1060 & - ILCS:AE+OS & $.119043 \mathrm{~F}+00$ \\
\hline 170,7 & $.1 ?$. $8>7 F+115$ & $.175091 t+00$ \\
\hline はナ6? & $.153134 f+03$ & $.2177596+00$ \\
\hline $176: 9$ & $.194011 t+0 s$ & $.226419 E+00$ \\
\hline 1770 & $.22 B 521 F+03$ & .17834 AF +00 \\
\hline 1271 & $.206224 F+C b$ & $-.0794631-01$ \\
\hline
\end{tabular}

JAHR ARBEITSTINKUMMENSUUUTE AN ARUTTIIWERTSCH. O.PRIV.SEKTIRS
ANTFIL ARHEITNEHMFRLUHN $\triangle N$ VIJLKSF INKTMMEN
ANITIL ARAEITNE HME RE INK DMMFN AN VOLKSEINKOMMEN

\begin{tabular}{|c|c|c|c|}
\hline 130.0 & $. \triangle 7450 E E+U U$ & $.503149 t+J 0$ & $.31244 R E+00$ \\
\hline 1961 & . SH19प्र9t +0n & $.019244 F+00$ & $.630422 F+00$ \\
\hline 1962. & $.5778361+00$ & $.632042 E+00$ & $.642728 F+00$ \\
\hline 1963 & $.579151 t+00$ & $.635131 \%+00$ & $.647133 E+00$ \\
\hline 1964 & -5t3096t+cuv & $.642002 t+00$ & $.655807 E+00$ \\
\hline 1965 & $.574105 t+00$ & $.641614 t+00$ & $.653400 F+00$ \\
\hline 1764 & $.5726: 8 E+00$ & $.642972 t+00$ & $.159978 \mathrm{~F}+00$ \\
\hline 1967 & $.577 .34 \mathrm{~F}+100$ & $.650401 E+00$ & .60 RU1 $17 E+00$ \\
\hline 1968 & $.577743 E+00$ & $.653932 E+00$ & $.672360 F+00$ \\
\hline 1969 & $.585906 t+00$ & . $\$ 7010 P E+00$ & $.693469 \mathrm{~F}+00$ \\
\hline $\begin{array}{l}1970 \\
1971\end{array}$ & $\begin{array}{l}.614756 t+00 \\
.632353 t+0 J\end{array}$ & $\begin{array}{l}.714357 E+0 C \\
.706352 F+U n\end{array}$ & $\begin{array}{l}.754305 E+00 \\
.839980 E+n D\end{array}$ \\
\hline
\end{tabular}


Tabelle 19.1: Modellergebnisse der Standardrechnung

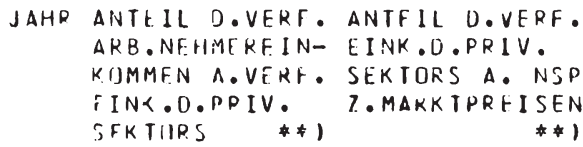

$* 1$

\begin{tabular}{|c|c|c|c|}
\hline 1360 & $.671455 F+00$ & $.780569 E+\cup 0$ & $.277059 E+06$ \\
\hline 1961 & $.58,7044 E+(10$ & $.7 B 4780 E+03$ & $.290620 E+06$ \\
\hline 1362 & $.704357 E+00$ & . $76+237 E+00$ & $.321239 E+06$ \\
\hline 1763 & - lurnalituv & $.775122 t+0(1$ & $.346595 \mathrm{~F}+06$ \\
\hline 1354 & $.71: 652[+00$ & $.7 B 1060 t+40$ & $.374304 E+06$ \\
\hline 1965 & $.714737 \mathrm{~F}+0 \mathrm{U}$ & $.75322 \cup E+00$ & $.413262 \mathrm{E}+06$ \\
\hline 1966 & $.707357 t+00$ & $.782488 E+00$ & $.469495 E+06$ \\
\hline 1967 & $.704079 E+0 ?$ & $.775675 E+00$ & $.556032 E+06$ \\
\hline $190 \mathrm{H}$ & $.700748 \mathrm{BE}+00$ & $.763471 E+00$ & $.683040 E+06$ \\
\hline 1763 & $.725004 t+00$ & $.750351 E+00$ & $.824361 E+00$ \\
\hline 1970 & - $5 \cup 44 U 4 E+00$ & $.781346 \mathrm{~L}+00$ & . $854550 E+06$ \\
\hline 1771 & $.933278 E+00$ & $.839089 F+00$ & $.649484 E+06$ \\
\hline
\end{tabular}

* ) VERFIIEGa.finKuMMEN ENTHAL TEN HIER AUCH AUSL.UEBERWEISUNGEN D.ARBE I TNEHMER .

JAHK ANTEIL D.ARBNEHMER A.KUMUILIERTER NOMINALERSPARN IS D.PKIV.SEKTUKS
ANTEIL KUMUL. NTMJNALERSPARNIS D.PRIV. SEKTOPS A.KUL MUL . NOMINALER SPARNIS PLUS BEWERTUNGSGLWINNE

$\begin{array}{ll}1760 & .244157 E+00 \\ 1761 & .252760 E+00 \\ 1962 & .264717 L+00 \\ 1763 & .276397 E+00 \\ 1464 & .287198 E+0 U \\ 1765 & .278905 E+00 \\ 1766 & .307010 E+00 \\ 1767 & .314461 E+0 U \\ 1764 & .321173 E+00 \\ 1969 & .333327 E+00 \\ 197 C & .363562 E+0 U \\ 1971 & .414761 E+0 U\end{array}$

$$
\begin{aligned}
& .780365 E+00 \\
& .769762 E+00 \\
& .745197 E+00 \\
& .732842 E+00 \\
& .725698 E+00 \\
& .724520 E+00 \\
& .699044 t+00 \\
& .644290 E+00 \\
& .571653 E+00 \\
& .516883 E+00 \\
& .500769 t+00 \\
& .625761 E+i) 0
\end{aligned}
$$

NSP. Z.MARKTPREISEN
KUMUL - NOMI -

MINALFRSPARNIS O.PRIV.SEKTORS
$.441462 E+06$ $.450946 E+06$ $.5 ? ? .835 E+06$ $.571330 E+06$ $.+20 \leq 30 \mathrm{~F}+06$ . $T 7$ DOOE+06 $.741080 E+06$ $.815016 \mathrm{~F}+06$ $.903338 E+06$ . $996 h 44 F+06$ $.100452 E+07$ $.109073 \mathrm{E}+07$ 
Tabelle 19.1: Modellergebnisse der Standardrechnung

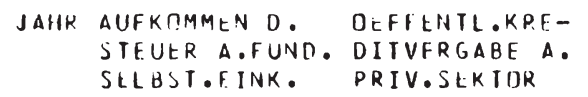

STAATL.HAUS HAL ISVULIJMEN 1 (LAUFENDE EINNAHMEN )

\begin{tabular}{|c|c|c|c|}
\hline $1+60$ & $.152136 E+05$ & $.646515 E+04$ & $.100730 F+06$ \\
\hline 1901 & $.166151 F+05$ & $.566654 t+04$ & $.109359 E+06$ \\
\hline 1962 & $.197192 t+C 5$ & $.465286 f+04$ & $.123605 F+06$ \\
\hline $17 \times 3$ & $.191791 \mathrm{~F}+\mathrm{U}$ & $.327796 t+04$ & $.132197 \mathrm{~F}+06$ \\
\hline 1964 & $.195396 t+05$ & $.138559 F+04$ & $.141984 \mathrm{~F}+06$ \\
\hline 1965 & -? $14944 E+05$ & $-.568240 E+02$ & $.154004 E+06$ \\
\hline 1960 & $.223 \supset 34 E+05$ & $.357433 E+04$ & $.173792 E+06$ \\
\hline 1967 & $.222600 E+05$ & $.750220 t+04$ & $.207557 E+06$ \\
\hline 1968 & - $255470 \mathrm{t}+05$ & $.131589 E+05$ & $.261624 E+06$ \\
\hline 1369 & $.300549 E+05$ & $.118509 E+05$ & $.333466 F+06$ \\
\hline 1970 & $.232639 E+U 5$ & $-.136+60 E+O S$ & $.373122 E+06$ \\
\hline 1771 & $.27312 \mathrm{at}+05$ & $-.640802 t+05$ & $.315079 E+06$ \\
\hline
\end{tabular}

JAHK STEUERMEHRAUF - PERSONAL KUMMEN INFOLGE AUSGABEN DES SIEUERRFFURM (FURMALRECHN.)

\section{STAATES}

\footnotetext{
1961

$176 ?$

1963

1964

1955

1766

1767

I9RR

1969

1970

1971

0 .

. $238435 E+04$

- .154531t+04

-.196834E+04

-. $34 \pi 4 \cup 3 E+04$

-.10V964E+ 5

$-.190567 E+05$

-.26574PE+0S

-.301695E+05

-. 1740 Ut + U5

. 362062E+US

$.221209 F+05$

$.249486 \mathrm{E}+05$

$.240753[+05$

$.314211 \mathrm{E}+0 \mathrm{~J}$

$.351812 E+05$

$.408945 E+05$

. $48.2175 E+03$

$.596 .921 \mathrm{E}+05$

. $826791 \mathrm{E}+0 \mathrm{~S}$

$.107573 E+06$

.104 b $23 F+06$
}

n.
$.206915 E+05$ 
2. Die Steuerreformen 1962 und 1965 im Bereich der Steuer auf fundierte Selbständigen-Einkommen und ihre Inzidenzeffekte.

Im Bereich der Steuer auf fundierte Selbständigen-Einkommen wurden bei den Schätzungen des Paragraphen 13 die Aufkommenswirkungen zweier Steuerreformen in der Bundesrepublik erfaßt. Das Steueränderungsgesetz 1961/62 fand aufkommensmäßig seinen Niederschlag erstmals im Jahre 1962; die Einkommensteuerreform 1965 wirkte sich infolge der Verzögerungen im Bereich der Einkommensteuerzahlungen erstmals im Jahre 1966 aus. Da die Steuer auf die fundierten Selbständigen-Einkommen unter den hier abgegrenzten Steuerarten die größte Verwandtschaft mit einer Gewinnsteuer hat, werden die Steuerreformen im Bereich dieser Steuer dazu benutzt, erste inzidenztheoretische Schlußfolgerungen hinsichtlich der Gewinnsteuerüberwälzung aus der Modellrechnung zu ziehen. Dabei werden die Auswirkungen beider Reformen getrennt analysiert.

Um die Auswirkungen der Steuerreform $1962 \mathrm{zu}$ ermitteln, wurde in der Modellrechnung anders als im Standardlauf des vorigen Abschnitts Parameter J9, der den Jahrestermin der Steuerreform bestimmt, mit dem Wert 0 statt 12 vorgegeben, so daß die Reform ausfiel. Die Ergebnisse, die im folgenden mit den entsprechenden Werten der Standardrechnung verglichen werden, werden hier selbst nicht noch einmal vorgeführt; statt dessen wird gleich auf Relationen und Differenzbeträge $z$ wischen den Werten beider Rechnungen abgestellt.

Das auffallende allgemeine Ergebnis der Neuberechnung ist, daß die Modellentwicklung infolge der Reformausschaltung innerhalb von sechs Jahren, also innerhalb eines noch kürzeren Zeitraumes als im Standardlauf, wegexplodiert. Dies ist die Folge der enormen Instabilität des Modells.

Betrachtet man die Einzelergebnisse, so ist als nächstes die formale Inzidenz der Steuerreform zu beachten. Als formale Inzidenz wird der Einfachheit halber der formal geschätzte Aufkommenseffekt der Steuerreform in Prozent des Nettosozialprodukts zu Marktpreisen bezeichnet. Die formale Inzidenz gibt also an, wie sioh der Anteil der verfügbaren Einkommen der Selbständigen-Haushalte und Unternehmen am verfügbaren Einkommen insgesamt (Nettosozialprodukt zu Marktpreisen) aufgrund der Steuerreform geändert hätte, wenn keine sonstigen Änderungen der wirtschaftlichen Verhältnisse, insbesonder auch keine Änderungen der aggregierten Steuerbemessungsgrößen und ebenso wenig Überwälzungseffekte eingetreten wären. Diese hypothetische Rechnung basiert hier auf den wirtschaftlichen Verhältnissen der Standardrechnung und wurde in den Ergebnissen des vorigen Abschnitts bereits in Form der absoluten "Steuermehraufkommen infolge Steuerreform" ausgewiesen.

Nach der formalen Inzidenzberechnung erbrachte die Steuer $\mathrm{T}_{\mathrm{RGS}}$ infolge der Reform 1962 im ersten Jahr nach Wirksamwerden, also 1962, ein Mehraufkommen von 2, $384 \mathrm{Mrd}$. DM. Aufkommensausfälle machen sich erst 1963, von da an aber in absolut und quotal zunehmendem Maße bemerkbar. 
Tabelle 19.2:

Jahr

Formal geschätztes Steuermehraufkommen an $T$

i. Verh. zu NSP 1 RGS
Tatsächliches Steuermehraufkommen an $T_{\text {RGS }}$

i. Verh. zu NSP ${ }_{\text {MP }}(1)$
(1)

1961

1962

1963

1964

1965
(2)

$0,000.000$
$+0,007.422$
$-0,004.461$
$-0,005.258$
$-0,008.430$

(3)

$$
\begin{array}{r}
0,000.000 \\
+0,011.751 \\
+0,014.072 \\
+0,030.959 \\
+0,035.531
\end{array}
$$

(1) NSP ${ }_{\text {MP }}$ der Standardrechnung von Abschnitt 1. 
Dem formal geschätzten Steuermehraufkommen entspricht im Jahre 1962 ein effektives Steuermehraufkommen. Da dieses Steuermehraufkommen vom Staat zum größten Teil nachfragewirksam verausgabt wurde, verursachte die Reform 1962 einen expansiven Nachfrageeffekt, der auch die Bruttogewinne des Unternehmenssektors steigen ließ. Als Folge des Gewinnanstiegs ist das tatsächlich resultierende Steuermehraufkommen bzw. der Differenzbetrag zwischen dem tatsächlichen TRGS-Aufkommen in der Rechnung mit Reform und dem tatsächlichen TRGS-Aufkommen in der Rechnung ohne Reform mit einem Wert von 3,775 Mrd. DM um gut die Hälfte größer als das formal geschätzte Mehraufkommen. Bezieht man auf das tatsächliche Steuermehraufkommen den durch die Reform im Jahre 1962 verursachten Anstieg des nominellen Bruttosozialprodukts, so erhält man einen "Multiplikator" von 4,550 und damit einen Eindruck von der im Modell übertriebenen Stärke des 1962 bewirkten expansiven Nachfrageeffektes. Dieser Nachfrageeffekt überkompensiert in seinen Auswirkungen auf das Investitionsniveau des Jahres 1963 die Bremswirkungen des gleichzeitig eingetretenen Zinsanstiegs. Der Expansionsprozeß setzt sich daher in den Folgejahren fort und läßt das tatsächliche Steuermehraufkommen im Bereich von $\mathrm{T}_{\text {RGS }}$ anders als das auf Basis der Standardrechnung formal geschätzte Mehraufkommen absolut und quotal steigen (1).

Zur Beurteilung der nicht-formalen Inzidenzeffekte bzw. der Überwälzungseffekte, die zusammen mit den formalen Inzidenzeffekten die effektive Inzidenz der Steuerreform ausmachen, muß auf die Art des ausgelösten und instabil verlaufenden Expansionsprozesses eingegangen werden, denn nach den theoretischen Ableitungen des 3. Kapitels sind kreislaufbedingte steuerliche Utberwälzungsprozesse immer von einer Nachfrageexpansion bzw. von einer Höherauslastung der privaten Anlagenkapazitäten abhängig. In Tabelle 19. 3 wurden daher einige Meßziffern zusammengestellt, die den Expansionsprozeß kennzeichnen und zwar wurden die Werte für den realen BIP-Beitrag des privaten Sektors, für die Kapazitätsauslastung der Unternehmen, für den Preisindex des privaten BIP und für den Lohnsatz des privaten Sektors in der Rechnung mit Reform (Standardlauf) auf die jeweiligen Werte in der Rechnung ohne Reform basiert. Die Meßziffern lassen deutliche Unterschiede in den realen und monetären Eigenschaften des ausgelösten Expansionsprozesses erkennen: Das private Produktionsniveau weitet sich bis in das zweite Folgejahr nach der Reform, also 1964, aus; anschließend zeichnet sich eine Wiederannäherung an das Produktionsniveau in der Rechnung ohne Steuerreform ab. Das Preisniveau, ähnlich das private Lohnniveau, steigt dagegen infolge des Expansionsprozesses kumulativ an. Dividiert man die Meßziffern für Lohnsatz und Preisniveau, so zeigt sich auch in der Entwicklung dieses Quotienten (2) nach dem zweiten Folgejahr eine Umkehr der ausgelösten Entwicklung.

(1) Vgl. Tabelle 19.2

(2) Vgl. Tabelle 19. 3, Spalte (6) 


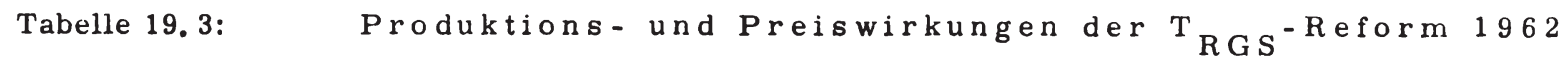

$\begin{array}{lllll}\text { Jahr } & \begin{array}{l}\text { BIPreal des } \\ \text { priv. Sektors (1) }\end{array} & \begin{array}{l}\text { Kapazitätsaus- } \\ \text { lastung der } \\ \text { Unternehmen (1) }\end{array} & \begin{array}{l}\text { Preisindex des } \\ \text { priv. BIP (1) }\end{array} & \begin{array}{l}\text { Lohnniveau des } \\ \text { priv. Sektors (1) }\end{array}\end{array}$

\begin{tabular}{llllll}
\hline$(1)$ & $(2)$ & $(3)$ & $(4)$ & $(5)$ & $(6)$ \\
\hline 1961 & $1,000.000$ & $1,000.000$ & $1,000.000$ & $1,000.000$ & $1,000.000$ \\
1962 & $1,017.684$ & $1,017.684$ & $1,034.038$ & $1,029.924$ & $0,996.021$ \\
1963 & $1,052.285$ & $1,043.977$ & $1,142.323$ & $1,129.621$ & $0,988.880$ \\
1964 & $1,075.869$ & $1,035.599$ & $1,418.598$ & $1,391.661$ & $0,981.012$ \\
1965 & $1,038.656$ & $0,963.552$ & $1,789.867$ & $1,766.239$ & $0,986.799$
\end{tabular}

(1) Jeweils Quotient aus Wert mit Reform und Wert ohne Reform. 
Aus dieser Beobachtung darf nicht der Schluß gezogen werden, die Instabilität des Modells sei in erster Linie eine monetäre Instabilität bzw. eine Instabilität des im Modell enthaltenen Preissystems, die sich im weiteren auf den Bereich der Realgrößen überträgt. Ein Blick auf Spalte (1) und (6) von Tabelle 19. 3 zeigt, daß der durch die Reform ausgelöste Prozeß der Produktionsausweitung mit einem tendenziellen Sinken des Reallohnes einhergeht. Gerade diese Gegenläufigkeit von kurzfristiger Produktions und Reallohnentwicklung war eine typische Eigenschaft des neoklassiscnen Modells, die im Paragraphen 5 mit Hilfe der Modifikation der kurzfristigen Grenzproduktivitätstheorie ausgeschaltet werden sollte. Das Ziel dieser Modifikation ist offensichtlich nicht erreicht worden. In einer weiteren hier nur andeutungsweise beschriebenen Berechnungsserie wurde daher die Modifikation der kurzfristigen Grenzproduktivitätstheorie über eine proportionale Änderung der Parameter $\gamma_{1}$ und $\gamma_{2}$ verstärkt. Auf diese Weise konnte der Standardlauf für eine Reihe zusätzlicher Jahre stabilisiert werden. Auf Ausschaltung der hier betrachteten Steuerreformen entwickelten sich aber ähnlich starke Instabilitätstendenzen wie zuvor. Nach Ausschaltung der $\mathrm{T}_{\mathrm{RGS}}$-Reform 1962 lief die Modellrechnung wiederum nur vier weitere Jahre; dabei verteilten sich die Instabilitätseigenschaften jedoch gleichmäßiger auf Real- und Nominalgrößen. - Es findet sich also wohl eine gewisse Erklärung für die Verteilung der Instabilitäten auf den realen und monetären Bereich. Die eigentlichen Ursachen der Instabilität sind damit aber noch nicht gefunden.

Die nicht-formalen Inzidenz - bzw. Überwälzungseffekte der analysierten Steuerreformen werden im folgenden anhand zweier Größen dargestellt: Die erste Größe ist der Anteil der Arbeitseinkommen an der Bruttowertschöpfung des privaten Sektors. Sie bringt die Funktionalverteilung im Unternehmensseiktor zum Ausdruck. - Die zweite Größe ist der Anteil der verfügbaren Arbeitnehmereinkommen am verfügbaren Einkommen der privaten Haushalte insgesamt (1) bzw. am Nettosozialprodukt zu Marktpreisen. Diese Größe bringt die institutionelle Eịkommensverteilung im privaten Sektor bzw. in der Gesamtwirtschaft zum Ausdruck. - Für beide Quoten wird in Tabelle 19.4 der Differenzbetrag zwischen den Ergebnissen der Rechnung mit Reform und der Rechnung ohne Reform ausgewiesen.

Betrachtet man die Auswirkungen der Steuerreform auf die Funktionalverteilung, so fügen sich die Ergebnisse in das bisher skizzierte Bild ein. Der durch die Steuerreform kurzfristig bewirkte expansive Effekt läßt 1962 die Unternehmensgewinne steigen; die Arbeitseinkommensquote an der Bruttowertschöpfung des privaten Sektors sinkt. Insoweit kann von einer Überwälzung der 1962 eingetretenen Steuermehrbelastung der fundierten Selbständigen- und (Unternehmens-)Einkommen gesprochen

(1) Verfügbare Einkommen werden hier also einschließlich der Übertragungen der Arbeitnehmer an das Ausland erfaßt. 
Tabelle 19.4: Einkommensverteilungswirkungen der T RGS $^{- \text {Reform } 1962}$

$\begin{array}{llll}\text { Jahr } & \text { Anteil Arbeitsein- } & \text { Anteil verfügb. Arbeit- } & \text { Anteil verfügb. Arbeit- } \\ \text { kommen a. Brutto- } & \text { nehmereinkommen a. } & \text { nehmereinkommen a. } \\ \text { wertschöpfung des } & \text { verfügb. Einkommen des } & \text { NSP }_{\text {MP }}(1) \\ \text { priv. Sektors (1) } & \text { priv. Sektors (1) (2) } & \end{array}$

(1)

(2)

(3)

(4)

\begin{tabular}{rrrr}
\hline 1961 & $0,000.000$ & $0,000.000$ & $0,000.000$ \\
1962 & $-0,005.006$ & $+0,004.253$ & $-0,009.398$ \\
1963 & $-0,011.849$ & $-0,017.329$ & $-0,035.947$ \\
1964 & $-0,009.429$ & $-0,033.405$ & $-0,062.712$ \\
1965 & $+0,011.726$ & $-0,034.505$ & $-0,034.834$
\end{tabular}

(1) Jeweils Differenzbetrag aus Wert mit Reform und Wert ohne Reform

(2) Die verfügbaren Einkommen enthalten hier auch die Auslandsübertragungen der Arbeitnehmer. 
werden. Der Überwälzungseffekt beläuft sich aber auf weniger als die Hälfte der effektiven Steuermehrzahlung bzw. des entsprechenden Anteils am NSP $M P(1)$. - Da der ausgelöste expansive Prozeß über das Jahr 1962 hinaus anhält, setzt sich das Sinken der Arbeitseinkommensquote im ersten Folgejahr (1963) fort, wobei sich der Überwälzungseffekt im Vergleich zu dem steigenden Betrag der effektiven Steuermehrzahlung erhöht. Anschließend schlägt die Entwicklung jedoch um, was im Widerspruch zu den bisherigen Ergebnissen der makroökonomischen Überwälzungstheorie Föhl'scher Prägung steht. Während das effektive Steuermehraufkommen infolge der Steuerprogression sogar als Quote am NSP $M P$ weiterhin stark ansteigt, nähert sich die Arbeitseinkommensquote im ersten Folgejahr (1964) wieder dem Wert in der Rechnung ohne Reform und liegt im dritten Folgejahr bereits unter diesem Wert. Alle Anzeichen deuten auf eine zyklische Entwicklung des mit der Steuermehrbelastung einhergehenden Überwälzungsprozesses, was hier nicht weiter verfolgt werden kann, da die Modellrechnung infolge der Instabilität abbricht. Die Entwicklung verläuft offensichtlich ähnlich wie die Entwicklung der realen Nachfrage bzw. des Produktionsniveaus im privaten Sektor. Genau genommen wird sie, entsprechend der im Paragraph 5 aufgestellten Theorie, durch die Entwicklung der Kapazitätsauslastung im Unternehmenssektor bestimmt, die im Vergleich zur Berechnung ohne Reform ihren höchsten Wert bereits ein Jahr eher als die absolute Produktionsentwicklung, nämlich 1963 , erreicht hat.

Die durch die $\mathrm{T}_{\mathrm{RGS}}$-Reform 1962 bewirkte Verschiebung der institutionellen Einkommensverteilung weicht von der Verschiebung der Funktionalverteilung erheblich ab. Daß der Anteil der verfügbaren ArbeitnehmerEinkommen am verfügbaren Einkommen der privaten Haushalte bzw. des privaten Sektors insgesamt (2) 1962 höher liegt als in der Rechnung ohne Reform, ist Ausdruck dafür, daß die durch die Steuerreform bewirkte Mehrbelastung der fundierten Selbständigen-Einkommen nicht voll überwälzt werden konnte, und ist damit noch in engem Zusammenhang zu den funktionalen Verteilungswirkungen der Steuerreform zu sehen. Die Verschiebung der institutionellen Verteilung in den Folgejahren ist dann aber vor allem durch die Art der Umverteilungsvorgänge zwischen dem privaten und dem staatlichen Sektor geprägt. Infolge des ausgelösten Expansionsprozesses sinkt die Arbeitslosigkeit bzw. der Betrag, den der Staat für Arbeitslosenunterstützung aufzubringen hat; andererseits gewährt der Staat infolge der verbesserten Haushaltssituation vermehrt Subventionen an den Unternehmenssektor. Beides u. a. m. führt dazu, daß der Anteil der Arbeitnehmer am verfügbaren Einkommen des privaten Sektors sinkt.

(1) Vgl. Tabelle 19.4 Spalte (2) mit Tabelle 19.2 Spalte (3).

(2) Es sei hier noch einmal darauf verwiesen, daß in der Modellformulierung die Unternehmen eigentumsmäßig voll den Selbständigen-Haushalten zugerechnet werden. 
Tabelle 19. 5:

Jahr

Formal geschätztes Steuer-

mehraufkommen an TRGS

i. Verh. zu NSP ${ }_{\text {MP }}$ (1)
Tatsächliches Steuer mehraufkommen an $T_{R G S}$

i. Verh, zu NSP MP (1)
(1)

1965

1966

1967

1968

1969

\section{(2)}

$$
\begin{array}{r}
0,000.000 \\
-0,007.839 \\
-0,016.835 \\
-0,021.360 \\
-0,020.247
\end{array}
$$

(3)

(1) NSP ${ }_{\text {MP }}$ der Standardrechnung von Abschnitt 1 
Tabelle 19.6: Die Produktions-und Preiswirkungen der T RGS-Reform $1965 / 66$

\begin{tabular}{llllll} 
Jahr & BIP & \multicolumn{1}{c}{ des } & Kapazitätsaus- & Preisindex des & Lohnniveau des (5) / (4) \\
priv. Sektors (1) & $\begin{array}{l}\text { lastung der } \\
\text { Unternehmen (1) }\end{array}$ & priv. BIP (1) & priv. Sektors (1)
\end{tabular}

\begin{tabular}{llllll}
\hline$(1)$ & $(2)$ & $(3)$ & $(4)$ & $(5)$ & $(6)$ \\
1965 & $1,000.000$ & $1,000.000$ & $1,000.000$ & $1,000.000$ & $1,000.000$ \\
1966 & $0,991.386$ & $0,991.386$ & $0,981.214$ & $0,983.499$ & $1,002.328$ \\
1967 & $0,965.297$ & $0,969.149$ & $0,884.299$ & $0,892.733$ & $1,009.537$ \\
1968 & $0,915.235$ & $0,937.455$ & $0,666.658$ & $0,684.705$ & $1,027.070$ \\
1969 & $0,862.536$ & $0,926.716$ & $0,403.579$ & $0,421.961$ & $1,045.547$
\end{tabular}

(1) Jeweils Quotient aus Wert mit Reform und Wert ohne Reform. 
Tabelle 19.7: Einkommensverteilungswirkungen der T RGS-Reform $1965 / 66$

\begin{tabular}{llll} 
Jahr & Anteil Arbeitsein- & Anteil verfügb. Arbeit- & Anteil verfügb. Arbeit- \\
kommen a. Brutto- & nehmereinkommen a. & nehmereinkommen a. \\
wertschöpfung des & verfügb. Einkommen d. & NSP MP (1) \\
priv. Sektors (1) & priv. Sektors (1) (2) & \\
\hline
\end{tabular}

(1)

(3)

(4)

\begin{tabular}{rrrr}
\hline 1965 & $0,000.000$ & $0,000.000$ & $0,000.000$ \\
1966 & $+0,002.580$ & $-0,003.823$ & $+0,005.324$ \\
1967 & $+0,009.250$ & $-0,000.218$ & $+0,023.747$ \\
1968 & $+0,019.673$ & $+0,019.994$ & $+0,060.801$ \\
1969 & $+0,023.079$ & $+0,042.519$ & $+0,101.097$
\end{tabular}

(1) Jeweils Differenzbetrag aus Wert mit Reform und Wert ohne Reform.

(2) Die verfügbaren Einkommen enthalten hier auch die Auslandsübertragungen der Arbeitnehmer. 
Setzt man in der Modellrechnung den Zeitparameter J10 anstelle von J9 gleich null und schaltet damit die Auswirkungen der Einkommensteuersenkung 1965 im Bereich der Steuer TRGS aus, so erhält man keine wesentlichen Unterschiede zu den bisherigen Ergebnissen (1). Da die Steuersenkung von Anfang an, d. h. ab 1966, zu einem Minderaufkommen an $\mathrm{T}_{\text {RGS }}$ führt, stellt sich jetzt aufgrund der gleichzeitigen staatlichen Ausgabensenkung ein kontraktiver Prozeß ein. Die ganze Entwicklung verläuft also in etwa mit umgekehrten Vorzeichen. Der Prozeß ist anfangs weniger stark als im Fall der Reform 1962 - das Verhältnis zwischen verursachter absoluter BSP-Änderung und effektivem Steuerminderaufkommen ergibt 1962 einen "Multiplikator" von 3,015. Anschließend entwickelt sich der Prozeß jedoch stärker als der durch die Reform 1962 ausgelöste expansive Prozeß. Die anfangs ausgelösten Änderungstendenzen im Preissystem bleiben wiederum bestehen und verstärken sich kumulativ in Richtung Deflation. Im Bereich der realen Nachfrageentwicklung bzw. der Kapazitätsauslastung sowie im Bereich der Verteilungswirkungen abseits der formalen Inzidenz lassen sich die gleichen Anzeichen für einen zyklischen Verlauf feststellen. Dabei erstreckt sich der ausgelöste Zyklus dieses Mal offensichtlich über eine größere Zahl von Jahren; da die Modellrechnung infolge der großen Instabilität wieder im fünften Jahr nach Wirksamwerden der Reform abbricht, kann nicht eindeutig ausgemacht werden, ob der zyklische Prozeß 1969 bereits den Punkt des ersten Umschwungs erreicht hat.

Die magere Ausbeute der rechnerischen Modellauswertung läßt sich wie folgt zusammenfassen:

1. Das Modell verfügt über starke Instabilitätseigenschaften, die sich bei exogenen Störungen wie den betrachteten Steuerreformen bereits kurzfristig bemerkbar machen. Die Ursachen der Instabilität sind nicht abgeklärt worden. Die schon kurzfristige Erkennbarkeit der Instabilitätseigenschaften läßt jedoch vermuten, daß deren Ursachen nicht unbedingt im Bereich des Kapitalmarktmechanismus und der verzögert reagierenden privaten Anlageinvestitionen zu suchen sind.

2. Die im Paragraphen 5 vorgenommene Modifikation der kurzfristigen Grenzproduktivitätstheorie muß zumindest was die Schätzwerte der Parameter $\gamma_{1}$ und $\gamma_{2}$ angeht, als unzulänglich angesehen werden.

3. Bei einer Erhöhung der Steuer auf die fundierten Selbständigen- (und Unternehmens)Einkommen setzt infolge der Verausgabung des Steuermehraufkommens durch den Staat kurzfristig ein expansiver Prozeß ein. Der durch die Steuererhöhung ausgelöste expansive Prozeß läßt die Unternehmensgewinne brutto steigen und die Arbeitseinkommensquote an der Bruttowertschöpfung der Unternehmen sinken. Den Unternehmen gelingt in der Modellwirtschaft also eine Überwälzung der

(1) Vgl. die Tabellen 19.5 bis 19.7. 
Steuermehrbelastung, allerdings nicht voll, sondern nur in begrenztem Umfang.

4. Während im theoretischen Teil ein Abbau der eingetretenen Überwälzungseffekte vermutet wurde und dabei stillschweigend eher an einen asymptotischen Verlauf dieses Abbaus gedacht wurde, verlaufen die durch die Steuererhöhung ausgelösten Verschiebungen der Funktionalverteilung bzw. die Überwälzungseffekte in der Modellrechnung tatsächlich zyklisch, wobei sie sich in den ersten Folgejahren erst einmal verstärken. Infolge der Instabilität der Modellrechnung kann nicht ausgemacht werden, ob diese zyklische Entwicklung mit abnehmenden Schwingungen ausläuft.

5. Da die verursachten Einkommenseffekte in ihren Auswirkungen auf das private Investitionsniveau der Folgezeit stärker sind als die gleichzeitig verursachten Änderungen des Kapitalmarktzinses, beide Änderungen im Modell aber in engem Verhältnis zueinander stehen, ist $z u$ vermuten, daß bei einer erfolgreichen Ausschaltung der Instabilitätseigenschaften die Entwicklung des Überwälzungseffektes ebenfalls zyklisch verläuft, es sei denn, die Quellen der Instabilität lägen innerhalb des Modells im Bereich des Kapitalmarktmechanismus und/ oder im Verhalten der privaten Anlageninvestitionen.

6. Die im Zuge der beiden Steuerreformen ausgelösten Umverteilungsvorgänge $z$ wischen dem privaten und staatlichen Sektor sind in ihren Auswirkungen auf die institutionelle Einkommensverteilung zwischen Arbeitnehmer - und Selbständigen-Haushalten der Modellwirtschaft z. T. erheblich stärker ausgefallen als die funktionalen Überwälzungseffekte. 
$\$ 20$ Versuch einer abschließenden Beurteilung des makroökonomischen Überwälzungstheorems

Obwohl die Modellrechnung aufgrund ihrer Instabilität nicht in vollem Umfang die erhofften Auskünfte über die Inzidenz einer Gewinnsteuer erbrachte, sei hier zum Abschluß aus der Sicht der Modellformulierung der Versuch einer zusammenfassenden Beurteilung des makroökonomischen Überwälzungstheorems unternommen.

Das Kreislauftheorem der (Gewinn-)Steuerüberwälzung ist eine distributive und zugleich finanzwissenschaftliche Anwendung keynesianischer Multiplikatortheorie. Es ist aber wohl mehr als nur partielle Multiplikatortheorie. Nach den hier erzielten Untersuchungsergebnissen muß davon ausgegangen werden, daß die für die Gültigkeit des Theorems notwendigen Voraussetzungen, wie sie sich aus der Föhl'schen Überwälzungsdebatte herausgeschält haben, in der kurzen Frist weitgehend erfüllt sind. Die Einführung oder Erhöhung einer Gewinnsteuer z. B. beeinträchtigt die Realisierung der im vorhinein aufgestellten Investitionspläne im Bereich der Anlageinvestition nicht. Die Pläne werden in ihrem realen Umfang voll realisiert. Der dadurch bedingte kurzfristig zinsunelastische Finanzierungsbedarf der Investoren wird über ein stark zinselastisches Kreditangebot der Banken auf jeden Fall abgedeckt. Hinzukommt, daß die Einführung bzw. Erhöhung der Gewinnsteuer keinen direkten Einfluß auf die kurzfristige Arbeitsnachfrage des privaten Sektors bzw. die Entlohnung der Arbeitnehmer hat, der sich auf den Konsum bzw. die Ersparnis der Arbeitnehmer auswirken müßte, und daß die staatliche Ausgabeneignung bei voller Verausgabung des Steuermehraufkommens über der konsumtiven Ausgabeneigung der Selbständigen-Haushalte, bezogen auf die im Unternehmenssektor entstandenen und den Selbständigen-Haushalten zufallenden Gewinneinkommen, liegt. Mit der zusätzlichen Belastung der Unternehmensgewinne und der nachfragewirksamen Verausgabung des Steuermehraufkommens durch den Staat ist daher eine Expansion der monetären Gesamtnachfrage verbunden, wie das auch vom Haavelmo-Theorem behauptet wird. Dieser monetäre Expansionsprozeß ist nach der Modellformulierung von einer Änderung der Einkommensverteilung in Richtung auf einen höheren Einkommensanteil der Selbständigen-Haushalte bzw. Unternehmen vor Berücksichtigung der steuerlichen Umverteilung verbunden. Insofern muß aus der Sicht der Modellformulierung das Ergebnis des Kreislauftheorems der Gewinnsteuerüberwälzung für die kurze Frist nach wie vor als realistisch angesehen werden. Allerdings ergab die Auswertung der Modellberechnung, daß die Überwälzung kurzfristig nicht in vollem Umfang der "Gewinn-" Steuererhöhung gelingt.

Daß die Aussage einer kurzfristigen Überwälzbarkeit der Gewinnsteuern im Laufe der bisherigen Diskussion nicht allgemein akzeptiert wurde, hat seine Ursachen darin, daß einige Prämissen dieser Aussage nicht 
überzeugten. Die Kritik richtet sich vor allem gegen die zentrale Investitionshypothese. Diese Hypothese wurde hier durch eine neoklassisch abgeleitete Investitionsfunktion präzisiert, wonach die realen Bruttoanlageinvestitionen des privaten Sektors ausschließlich durch Vergangenheitseinflüsse bestimmt sind. Die These von der ausschließlichen Vergangenheitsabhängigkeit der privaten Anlageinvestitionen konnte im $\mathrm{Pa}-$ ragraphen 4 noch nicht ausreichend empirisch abgesichert werden. Immerhin erklärt diese Funktion die unterschiedlichen Wachstumsraten des privaten Anlagebestandes während der Referenzperiode zu $80 \%$ und nachträglich bei einer weitergehenden Berücksichtigung der Absatz- und Investitionsgüterpreise zu $92 \%$. Selbst wenn eine gewisse Abhängigkeit der privaten Anlageinvestitionen von den laufenden wirtschaftlichen Ereignissen angenommen werden müßte, wäre außerdem zu erwarten, daß dabei die Stärke der Einkommensabhängigkeit die Stärke der Kapitalkostenabhängigkeit unmittelbar überwiegt, wie das hier auch bei den Vergangenheitseinflüssen beobachtet werden konnte.

Erheblich für die Ablehnung ist wohl auch der Einwand, daß die Kreislauftheorie der Gewinnsteuerüberwälzung in ihrer bisherigen Formulierung das Arbeitsnachfrageverhalten des Unternehmenssektors bzw. die Entlohnung des Produktionsfaktors Arbeit nicht in ihre Überlegungen einbezogen hat und damit eine ausgesprochene Lücke in der kreislauftheoretischen Argumentationskette enthält. Diese Lücke wiegt um so schwerer, als die Lohnbildung auf dem Arbeitsmarkt nach der Investitionstätigkeit der zweite wichtige Faktor bei der Bestimmung der gesamtwirtschaftlichen monetären Expansionsprozesse zu sein scheint. Nach den hier angestellten neoklassischen Überlegungen ist diese Lücke jedoch zu schließen, ohne daß das Ergebnis der Kreislauftheorie der Steuerüberwälzung grundsätzlich verändert wird - freilich nur für die kurze Frist.

Schließlich dürfte die rein nachfragetheoretische Argumentationsweise für die Ablehnung von Bedeutung gewesen sein, weswegen hier eine Verknüpfung der postkeynesianisch-nachfragetheoretischen und neoklassisch-produktionstheoretischen Verteilungsansätze vorgenommen wurde. Wenn man nun sieht, wie gerade die neoklassische Theorie nach einiger Modifikation das makroökonomische Steuerüberwälzungstheorem für die kurze Frist stützt, wundert man sich, daß die vor allem in der amerikanischen Literatur verbreitete neoklassische Inzidenztheorie bisher nicht selbst einen besseren Zugang zu dem Problem der Gewinnsteuerinzidenz gefunden hat. Angesichts der finanzpolitischen Bedeutung einer möglichen Gewinnsteuerüberwälzung nimmt es sich ein wenig hilflos aus, wie sich Vertreter einer rein neoklassischen Inzidenztheorie mit Modellen, die mehr oder weniger stillschweigend für die lange Frist konzipiert sind, dem Problem der Gewinnsteuerinzidenz annehmen. Hier wird nicht berücksichtigt, daß die Substitutionselastizität im Einsatz der Produktionsfaktoren kurz- und langfristig einen unterschiedlichen Wert hat; es bleibt bei dem klassischen Fehler, daß die Ungültig- 
keit des Say'schen Theorems mißachtet wird; und mit letzterem wird übersehen, daß am Anfang einer Einführung oder Erhöhung der Gewinnsteuer entsprechend den Überlegungen des Haavelmo-Theorems ein expansivel Prozeß steht, der über eine hier nicht näher anzugebende Folge von Perioden andauert. Für die kurze Frist sind die Ergebnisse derartiger Ableitungen, kurz gesagt, falsch.

Nach der Verknüpfung der beiden inzidenztheoretischen Richtungen wird man für die kurze Frist etwa folgendermaßen argumentieren. Da die Unternehmen für die laufende Periode eine mittelfristige Optimalkapazität festgelegt haben und diese Optimalkapazität kurzfristig nicht verändert werden kann, sind die bei einer Gewinnsteuererhöhung kurzfristig in Erscheinung tretenden Überwälzungseffekte bedingt durch einen Nachfrageüberhang. Ein solcher Nachfrageüberhang tritt auch dann ein, wenn der Staat eine Ausgabenerhöhung mit Hilfe einer zusätzlichen Kreditaufnahme finanziert, obwohl in diesem Zusammenhang nicht von Überwälzung gesprochen werden kann. Im Falle einer Erhöhung von Kostensteuern sehen die Schlußfolgerungen anders aus. Trotz des mit der Erhöhung der Kostensteuern und ihrer Wiederverausgabung einhergehenden monetären Expansionsprozesses kommt es wegen der preispolitischen Reaktion auf der Güterangebotsseite zu keinem Nachfrageüberhang; im Zweifel kann man hier von einem kurzfristig unveränderten Beschäftigungsniveau bzw. Kapazitätsauslastungsgrad ausgehen, was nach den hier angestellten Überlegungen bedeutet, daß sich der Anteil der Arbeitskosten an der Bruttowertschöpfung nicht verändert hat. In diesem Fall wird man jedoch bei konstantem Faktorkostenanteil wegen der formalen Inzidenz mit einem Rückgang der Faktoreinkommensquoten rechnen; m. a. W. es tritt eine Rückwälzung der entsprechenden Faktorkostensteuern auf den formal belasteten Faktor ein.

Schwieriger als für die kurze Frist, für die sich von postkeynesianischer und neoklassischer Seite her übereinstimmende Aussagen ableiten lassen, ist die Frage, wie es sich mit der Inzidenz der Steuern, insbesondere der Gewinnsteuer, auf mittlere und lange Sicht verhält. Die Beantwortung dieser Frage wird dadurch bestimmt, ob der bei der Einführung oder Erhöhung der Gewinnsteuer eintretende Nachfrageüberhang im Zeitablauf abgebaut wird oder nicht. Im Laufe der theoretischen Ableitungen wurden zwei Mechanismen aufgezeigt, von denen erwartet wurde, daß sie im Gegensatz zu den Ansichten der postkeynesianischen Überwälzungstheoretiker zu einem Abbau der Überwälzungseffekte führen.

Der erste Mechanismus ist der Kapitalmarktmechanismus: Die Erhöhung der Steuerzahlung und der durch die Wiederverausgabung des Steueraufkommens ausgelöste expansive Prozeß erhöhen den gesamtwirtschaftlichen Finanzierungsbedarf; der dadurch verursachte Zinsanstieg wirkt sich auf die Dauer hemmend auf die Investitionstätigkeit aus. - Die in dieser Hinsicht von postkeynesianischer Seite vorgetragenen Argumente, die darauf hinauslaufen, daß mit einer derartigen Investitionshemmung von Seiten des Kapitalmarktzinses nicht zu rechnen sei, sind nicht akzeptabel. 
Hier liegt in der Tat die schwache Stelle der makroökonomischen Überwälzungstheorie postkeynesianischen Typs, sofern sie langfristig argumentiert. Im Gegensatz zu den Ansichten von Keynes hat die postkeynesianische Theorie die Vermutung einer derartigen Investitionshemmung dadurch herunterzuspielen versucht, daß sie behauptete, die Zinselastizität der Investition sei äußerst gering und damit vernachlässigbar. Dem liegt jedoch abseits von der fehlenden Unterscheidung $\mathbf{z}$ wischen kurzer und langer Anpassungsfrist eine falsche Interpretation der von ökonometrischen Untersuchungen erzielten Schätzergebnisse über die Zinselastizität der Investitionstätigkeit zugrunde. Setzt man diese Zinselastizität einmal in einer über die Jahresperiode hinausgehenden Betrachtung mit dem geringen Wert von 0,1 an, und unterstellt außerdem einen Einkommensmultiplikator der Investitionstätigkeit von $\mathrm{z}$ wei, dann muß eine durchaus realistische Änderung des Kapitalmarktzinses von fünf auf sechs Prozent, also um zwanzig Prozent des Ausgangszinses, ceteris paribus zu einer einprozentigen Erhöhung der gesamtwirtschaftlichen Nachfrage führen; es tritt also ein beträchtlicher Nachfrageeffekt ein. Läge die Zinselastizität der Investitionstätigkeit wesentlich über einem derart niedrigen Wert, hätte das Wirtschaftssystem nicht die konjunkturelle Stabilität, die es tatsächlich aufweist. - Nach den hier angestellten und auch nach anderen Untersuchungen muß jenseits der kurzen Frist grundsätzlich mit einer Zinsreagibilität der Investitionstätigkeit gerechnet werden, und dieses muß in einer kreislauftheoretischen Analyse der Steuerüberwälzung für die längere Frist berücksichtigt werden. Allerdings muß nach den theoretischen Ableitungen und nach der Auswertung der Rechenergebnisse zugegeben werden, daß es, wenn dies der einzige Mechanismus wäre, wegen der gleichzeitigen Einkommenseffekte des Expansiorsprozesses u. U. einige Zeit dauerte, bis sich die vom Kapitalmarkt ausgehenden Investitionshemmungen durchsetzten und damit den Abbau der Überwälzungseffekte einleiteten.

Der zweite Mechanismus besteht in der relativ schnell eintretenden Anpassung der Beschäftigungsplanung an den ausgelösten Nachfrageüber hang. In den theoretischen Ableitungen wurde vermutet, daß die Anpassungsreaktion die im Zuge der Gewinnsteuerüberwälzung eingetretene Minderung der Arbeitseinkommensquote in der relativ kurzen Zeit von wenigen Jahren wieder zum Verschwinden bringt. Die Auswertung der Rechenergebnisse legt die Vermutung nahe, daß dieser Abbau, wenn er sich vollzieht, zyklisch verläuft.

Ob die Überwälzungseffekte aufgrund beider Mechanismen im Zeitablauf voll abgebaut werden, kann hier infolge der Instabilität der Modellrechnung nicht exakt ausgemacht werden. Es müßten hierzu erst einmal die Ursachen der Instabilität herausgefunden werden bzw. geprüft werden, welche destabilisierenden Kräfte in der Modellrechnung zu stark und/oder welche stabilisierenden Kräfte zu schwach ausgestaltet sind, und, wieso die gesamtwirtschaftliche Ausgabeneigung in der Modellrechnung so hoch ist, daß kurzfristige Steuer-"Multiplikatoren" von 
$4 \mathrm{bzw}$. 3 ermittelt werden konnten. Das abzuklären, ist hier trotz langer Suche nicht gelungen. Es sei deswegen nur auf eine Überlegung abseits der Modellrechnung verwiesen. In Wirklichkeit wird man nämlich davon ausgehen, daß die Notenbank eine nachhaltige Erhöhung der Preisanstiegsraten zu verhindern suchen wird. Wenn ihr das gelingt, d, h. wenn sich das Preisniveau infolge der Steuererhebung und infolge des dadurch ausgelösten expansiven Prozesses auf einem höheren Niveau einspielt, sich im Endeffekt aber mit derselben Anstiegsrate und in etwa gleichen konjunkturellen Schwankungen wie vor Einsetzen der Steuererhöhung entwickelt, dann muß nach den Ergebnissen der Paragraphen 4 und 5 auf jeden Fall damit gerechnet werden, daß die Überwälzungseffekte im Fall einer zusätzlichen steuerlichen Gewinnbelastung auf Dauer verschwunden sind.

Insgesamt kommt man zu dem Ergebnis, daß sich im Fall einer zusätzlichen Gewinnsteuerlast kurz - und langfristig unterschiedliche Verteilungseffekte einstellen (1). Dies ist ein Ergebnis, das in gewisser Analogie zu den Ergebnissen der Krelle'schen Verteilungstheorie (2) steht. Wenn es zutrifft, wäre zu überlegen, ob die kurzfristig sich vollziehende Überwälzung neu eingeführter oder erhöhter Gewinnsteuerlasten letzten Endes nicht von Vorteil für das gesamte Wirtschaftssystem ist, weil sie während einer Übergangszeit die Anpassung des Unternehmenssektors an die neue steuerliche Belastungssituation erleichtert, die durch eine Minderung der verfügbaren Selbständigen-bzw. Unternehmenseinkommen bei weitgehend unverändertem Anteil der Bruttobesitzeinkommen an der Bruttowertschöpfung gekennzeichnet ist. Unwillkürlich denkt man an die alte Canard'sche Steuerregel, wonach eine neue Steuer eine schlechte Steuer sei, weil sie die Wirtschaftssubjekte zu Anpassungsprozessen und Änderungen des eingespielten Verhaltens zwinge. Das wäre eine sehr optimistische Sicht des makroökonomischen Überwälzungstheorems, aber sie ist wahrscheinlich angebracht. Die Debatte um die Überwälzung der Gewinnsteuer, die im Grunde eine späte Nachwirkung des postkeynesianischen Gedankengutes im Bereich der Finanzwissenschaft, insbesondere im Bereich der Steuerlehre ist, hat in der Vergangenheit zu Zweifeln an der verteilungspolitischen Eignung der progressiven Einkommensteuer geführt. Im Nachhinein erweisen sich diese Zweifel als für die Dauer unbegründet.

(1) Im Endeffekt wird das durch die Größe der beiden Substitutions elastizitäten bestimmt.

(2) Vgl. W. Krelle, Verteilungstheorie, Tübingen 1962, S. $251 \mathrm{ff}$. 
A N H A NG 


\section{Anhang 1}

Bruttoanlagevermögen, Nettoanlagevermögen und Anlagenfinanzierung im langfristigen Wachstumsgleichgewicht des Unternehmenssektors

Für den Unternehmenssektor der Modellwirtschaft läßt sich theoretisch ein langfristiges Wachstumsgleichgewicht vorstellen, in welchem die Änderungsraten aller nominellen Aggregatgrößen konstant und einander gleich sind, ebenso die Änderungsraten aller realen Aggregatgrößen und der Preise. In diesem Wachstumsgleichgewicht herrschen in der Vermögensbestandsrechnung des Unternehmenssektors einige stabile Strukturbeziehungen:

Die Wachstumsrate des nominellen bzw. zu Wiederbeschaffungspreisen bewerteten Nettoanlagevermögens ist für unendlich kleine Perioden definiert als:

$$
\frac{d\left[k_{p r, t}^{n} \cdot p_{k, t}\right]}{k_{p r, t}^{n} \cdot p_{k, t}}=\frac{d k_{p r, t}^{n} \cdot p_{k, t}+k_{p r, t}^{n} \cdot d p_{k, t}}{k_{p r, t}^{n} \cdot p_{k, t}}
$$

Der Ausdruck $d k_{p r}^{n}, t^{\bullet} p_{k, t}$ in (1) entspricht innerhalb der untersuchten Modellwirtschaft genau dem zur Anlagefinanzierung aus Ersparnis bzw. Kreditschöpfung neu aufgenommenen Finanzkapital $d F_{A n l, t} t$ bzw. dem Nominalwert der entsprechenden neu-emittierten Forderungstitel. Daher gilt:

$$
g_{n o m}=\frac{d P_{A n l, t}^{k}}{k_{p r, t}^{n} \cdot p_{k, t}}+g_{p}
$$

wobei

$$
\begin{aligned}
& g_{\text {nom }}=\text { Wachstumsrate der nominellen Aggregate } \\
& g_{p}=\text { Anderungsrate der Preise } \\
& g_{\text {real }}=g_{\text {nom }}-g_{p}
\end{aligned}
$$

bzw.

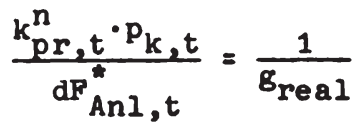


Die Wachstumsrate des nominellen bzw. zu Wiederbeschaffungspreisen bewerteten Bruttoanlagevermögens ist für unendlich kleine Perioden definiert als:

$$
\frac{d\left[k_{p r, t} \cdot p_{k, t}\right]}{k_{p r, t} p_{k, t}}=\frac{d k_{p r, t} \cdot p_{k, t}+k_{p r, t} \cdot d p_{k, t}}{k_{p r, t} \cdot p_{k, t}}
$$

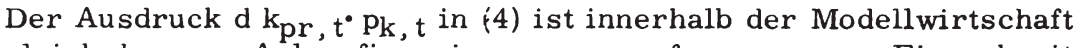
gleich dem zur Anlagefinanzierung neu-aufgenommenen Finanzkapital $d$ FÂnl, $t$ plus dem Überschuß der Abschreibungserlöse über die Ersatzinvestitionen. Es gilt also:

$$
g_{\text {nom }}=\frac{d F_{\text {Anl }, t^{+}}^{+D_{p r, t}}{ }^{-I_{t}^{r}}}{k_{p r, t} \cdot p_{k, t}}+g_{p}
$$

Wegen der Konstanz der Wachstums - und Preissteigerungsraten lassen sich die nominellen Ersatzinvestitionen auf die Summe von d FAnl, $t$ und $\mathrm{D}_{\mathrm{pr}, \mathrm{t}}$ umrechnen (1):

$$
I_{t}^{r}=\left[d F_{A n l, t}^{*}+D_{p r, t}\right] \cdot\left[1+g_{\text {real }}\right]^{-T}
$$

wobei $\mathrm{T}$ die als konstant angenommene Lebensdauer der Anlagen darstellt. Nach Einsetzen von (6) lautet (5):

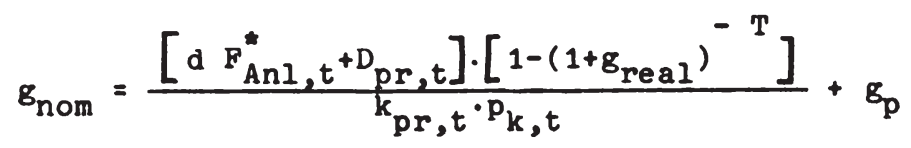

Die Abschreibungen für Kapitalverzehr werden in der Modellwirtschaft linear von dem zu Wiederbeschaffungspreisen bewerteten Bruttoanlagevermögen berechnet:

$$
D_{p r, t}=\frac{1}{T} \cdot k_{p r, t} \cdot p_{k, t}
$$

Aus (7) und (8) folgt:

(9)

$$
\frac{k_{p r, t} \cdot p_{k, t}}{d F_{A n l, t}^{*}}=\left[\frac{g_{\text {real }}}{1-\left(1+g_{\text {real }}\right)^{-T}}-\frac{1}{T}\right]^{-1}
$$

(1) Vgl. R. F. Harrod, Replacements, Net Investment, Amortisation Funds, Economic Journal, 80, 1970, S. $24 \mathrm{ff}$. 
Division von (3) durch (9) ergibt:

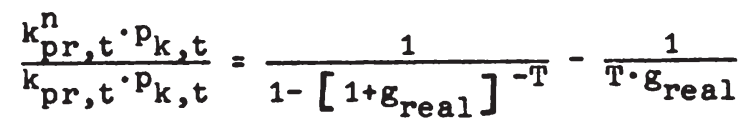

Die für das langfristige Wachstumsgleichgewicht des Unternehmenssektors aufgestellte Gleichung (10) kann dazu benutzt werden, um das Verhältnis von Nettoanlagevermögen zu Bruttoanlagevermögen des privaten Sektors in der Bundesrepublik zu schätzen. Für die nominelle Wachstumsrate gnom wird die durchschnittliche Wachstumsrate der nominellen Bruttoanlageinvestitionen zwischen 1960 und 1970 von 9,2\% und für $g_{p}$ die durchschnittliche Steigerungsrate der Investitionsgüterpreise während der gleichen Zeit von 3,5 \% benutzt; die durchschnittliche Lebensdauer der Anlagen zwischen 1960 und 1970 wird im Anschluß an die Anlagevermögensrechnung des Statistischen Bundesamtes mit 29 Jahren angesetzt. Dann gibt Gleichung (10) das Verhältnis von Netto- zu Bruttoanlagevermögen mit 0,645 an. Aufgrund der Anlagevermögensrechnung des Statistischen Bundesamtes müßte der Wert im Jahre 1970 0,665 betragen (1). Für einen nicht zu unglei chmäßigen Verlauf der längerfristigen wirtschaftlichen Entwicklung hat (10) also durchaus einen gewissen aproximativen Aussagegehalte, zumal der Wert des Quotienten im relevanten Bereich nur wenig auf Änderungen von $\mathrm{T}$ reagiert.

Aufgrund von (2) gilt:

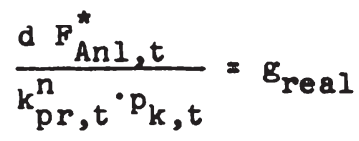

und nach Zerlegung des Quotienten auf der linken Seite von (11):

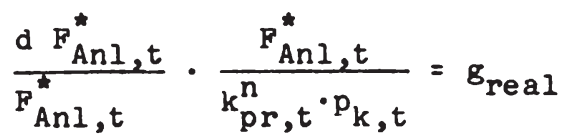

Da die Wachstumsrate des zur Anlagenfinanzierung aufgenommenen Finanzkapitals, $d F_{A n l, t}^{\star} / F_{A n l, t}^{\star}$, wiederum gleich $g_{\text {nom }}$ ist, gilt nach (12):

$$
\frac{F_{\text {Anl,t }}^{*}}{k_{p r, t}^{n} \cdot p_{k, t}}=\frac{g_{\text {real }}}{B_{\text {nom }}}
$$

(1) Vgl. H. Lützel, Das reproduzierbare Anlagevermögen in Preisen von 1962 , a. a. O. , S. 601 
Verläuft das langfristige Wachstum des Unternehmenssektors ohne Anstieg der Wiederbeschaffungspreise, ist der Quotient gleich eins; das Nettoanlagevermögen entspricht dann in der Modellwirtschaft dem aufgenommenen Finanzkapital $F_{A}^{\star} n l, t$. Steigen während des Wachstums prozesses die Wiederbeschaffungspreise, beträgt das zur Anlagenfinanzierung aufgenommene Finanzkapital nur $100 \cdot \mathrm{g}_{\mathrm{real}} / \mathrm{g}_{\text {nom }}$ Prozent des zu Wiederbeschaffungspreisen bewerteten Nettoanlagevermögens. 100 • $g_{p} / g_{\text {nom }}$ Prozent des nominellen Nettoanlagevermögens werden in der aggregierten Anlagevermögensrechnung des Unternehmenssektors finanzierungsmäßig durch die Substanzerhaltungsrücklage gedeckt.

Entspricht der nominellen Wachstumsrate z. B. ein Wert von 9,2 \% und der Preisanstiegsrate ein Wert von $3,5 \%$, wie das oben für die durchschnittliche Entwicklung der nominellen Bruttoanlageinvestitionen und der Investitionsgüterpreise in der Bundesrepublik zwischen 1960 und 1970 angegeben wurde, dann beträgt das zur Anlagenfinanzierung aufgenommene Finanzkapital $\mathrm{F}_{A \mathrm{n} l, t}^{\star} 100 \cdot 0,619$ Prozent und die Substanzerhaltungsrücklage für den Anlagenbestand 100 0, 381 Prozent des jeweiligen nominellen Nettoanlagevermögens. Um zu einer realistischen Relation für die Modellwirtschaft zu gelangen, wurde dieses Größenverhältnis in Hinsicht auf die Währungsumstellung von 1948 und die geringeren Preisanstiegsraten in den Fünfziger Jahren der Bundesrepublik auf $0,70: 0,30$ für das Jahr 1960 abgeändert; d. h. das zur Anlagefinanzierung aufgenommene Finanzkapital $F_{A n l, t}$ wurde für 1960 mit $70 \%$ des nominellen Nettoanlagevermögens bemessen.

Die Bedeutung von Gleichung (13) geht über den bisher angedeuteten Rahmen hinaus. Sie kann auch benutzt werden, um das zur Finanzierung der Vorratsinvestitionen aufgenommene Finanzkapital zu schätzen. Hierzu muß lediglich bekannt sein, welchen Wiederbeschaffungswert der vorhandene Vorratsbestand hat und wie hoch der langfristige Anstieg der Vorratsinvestitionen und der Preise für Vorratsinvestitionen in der Vergangenheit war. Da die Preise für Vorratsinvestitionen in der Bundesrepublik bis 1970 mittelfristig fast konstant waren, wurde das zur Vorratsfinanzierung aufgenommene Finanzkapital für das Jahr 1960 mit $100 \%$ des Wiederbeschaffungswertes der Vorratsbestände angesetzt.

Verteilungstheoretisch wichtig ist die Anwendung von (13) auf die Vermögensposition der Unselbständigen-Haushalte, die ihre Ersparnisbildung innerhalb der Modellwirtschaft ausschließlich in Forderungstiteln angelegt haben, darüberhinaus aber keine eigentumsrechtlichen Ansprüche an den Unternehmenssektor bzw. auf die Substanzerhaltungsrücklage des Unternehmenssektors haben. Der Realwert ihrer Forderungstitel nimmt jährlich mit der Inflationsrate ab. Wachsen die Nominalersparnisse der Unselbständigen-Haushalte mit der konstanten Rate $g_{\text {nom }}$ und steigt das allgemeine Preisniveau mit der konstanten Rate $g_{p}$, dann gibt (13) den Prozentsatz des akkumulierten Nominalvermögens an, der den Unselbständigen-Haushalten nach Abzug aller im Zeitablauf erlittenen Inflationsverluste real verbleibt. $g_{p} / g_{\text {nom }}$ ist also ein Quotient für die 
inflationistische Erosion ihres Forderungsbestandes. Allerdings ist für die verteilungsmäßige Beurteilung Vorsicht geboten: Ein niedriger Wert des Quotienten ist für den Unternehmenssektor bei konstantem Kapitalmarktzins gleichbedeutend mit einem vergleichsweise niedrigen Realzins bzw. mit einem vergleichsweise niedrigen realen Kapitalkostensatz $r_{t}^{\star}$ Veranlaßt das niedrige Niveau des Kapitalkostensatzes die Unternehmen zu erhöhter Investitionstätigkeit, steigt je nach Verhalten des Kreditangebotes der Kapitalmarktzins, was den Gläubigern festverzinslicher Wertpapiere und damit den Unselbständigen-Haushalten über die variable Verzinsung der Titel wieder zugute kommt. - Insofern kann der Quotient $\mathrm{g}_{\text {real }} / \mathrm{g}_{\text {nom }}$ in der Modellwirtschaft nicht ohne weiteres als eine Art Ausbeutungsquotient angesehen werden. Sollen die Verteilungswirkungen der Inflation beurteilt werden, müssen letzten Endes die inflationsbedingten Verteilungseffekte sowohl im Bereich der Vermögensbestände als auch im Bereich der laufenden Einkommensströme erfaßt und einander gegenüber gestellt werden.

Realwert der kumulierten Nominalersparnis im langfristigen Wachstumsgleichgewicht (Nominalwert $=1,000$ )

$$
\text { Realwert }=g_{\text {real }} /\left(g_{\text {real }}+g_{p}\right)
$$

$g_{p}=\quad g_{\text {real }}=$
0,035
0,045
0,055

0,000

0,010

1,000

1,000

1,000

0,020

0,777

0,818

0,846

0,636

0,692

0,733

0,030

0,538

0,600

0,647

0,035

0,500

0,562

0,611

0,040

0,466

0,529

0,578

0,050

0,411

0,473

0,523

0,060

0,368

0,428

0,478

0,070

0,333

0,391

0,440

0,080

0,304

0,360

0,407

0,090

0,280

0,333

0,379

0,100

0,259

0,310

0,354 


\section{Anhang 2:}

Bemessung der Risikoprämie durch Ansatz einer verkürzten pay-off-Periode bei der Anlagenamortisation

Angenommen: Die realen Bruttoanlageinvestitionen, $\Delta \mathrm{k}_{\mathrm{pr}}^{+} \mathrm{t}-1$, des Unternehmenssektors wachsen mit einer konstanten jährlichen Rate $g_{\text {real }}$. Die im Zeitablauf investierten Anlagen haben eine konstante Lebensdauer von T Jahren.

Dann beträgt der im Jahr $\mathrm{t}$ im Unternehmenssektor tatsächlich vorhandene Anlagenbestand bei einer Gestationsperiode von einem Jahr:

$$
k_{p r, t}=\Delta k_{p r, t-1}^{+} \cdot \frac{1-q^{T}}{1-q} \quad\left(q=\frac{1}{1+g_{\text {real }}}\right)
$$

Bei linearem Verlauf des tatsächlichen Kapitalverzehrs entfallen auf diesen Bestand im Jahre $t$ reale Abschreibungen in Höhe von:

$$
d_{p r, t}=\frac{1}{T} \cdot \Delta k_{p r, t-1}^{+} \cdot \frac{1-q^{T}}{1-q}
$$

Die Unternehmen gehen in ihrer Kalkulation vorsichtshalber von einer verkürzten Lebensdauer der Anlagen von \$.T Jahren aus. Haben Anlagen einmal diese kalkulatorische Lebensdauer erreicht, scheiden sie praktisch aus der Vermögensrechnung des Unternehmenssektors aus bzw. werden dort nur noch mit einem Erinnerungswert verbucht. Das noch nicht restlos abgeschriebene reale Bruttoanlagevermögen beträgt daher in der Vermögensrechnung des Unternehmenssektors:

$$
k_{p r, t}^{\prime}=\Delta k_{p r, t-1}^{+} \cdot \frac{1-q^{\text {Ir }}}{1-q}
$$

Auf diesen Bestand berechnen die Unternehmen bei linearem Verlauf des überhöht angesetzten Kapitalverzehrs reale Abschreibungen in Höhe von:

$$
d_{p r, t}^{\prime}=\frac{1}{\Im \cdot T} \cdot \Delta k_{p r, t-1}^{+} \cdot \frac{1-q^{\vartheta T}}{1-q}
$$

Für die Analyse der Modellwirtschaft wird versucht, von dem tatsächlichen Anlagenbestand und dem tatsächlichen Kapitalverzehr auszugehen, wie ihn die Volkswirtschaftliche Gesamtrechnung der Bundesrepublik schätzt, und die überhöhten kalkulatorischen Abschreibungen des Unternehmenssektors gesondert zu erfassen. Zu diesem Zweck werden die überhöhten Abschreibungen (4) des Unternehmenssektors auf das tatsächlich vorhandene bzw. am Produktionsprozeß beteiligte Bruttoanlagevermögen (1) bezogen, gleich ob die damit erfaßten Anlagen in der kalkulatorischen 
Abschreibungsrechnung des Unternehmenssektors bereits voll abgeschrieben sind oder nicht:

$$
\frac{d_{p r, t}^{\prime}}{k p r, t}=\frac{1}{\$ T} \cdot \frac{1-q^{\text {IT }}}{1-q^{T}}
$$

(5) ist der Abschreibungssatz des Unternehmenssektors in der Volkswirtschaftlichen Gesamtrechnung nach Berücksichtigung der kalkulatorisch bedingten Mehrabschreibungen.

Setzen die Unternehmen die pay-off-Periode mit einem Jahr al:, also mit dem $1 / \mathrm{T}$-fachen der tatsächlichen Lebensdauer, dann bedeutet dies, daß alle im Jahre t-1 investierten Anlagen im Jahr t voll abgeschrieben werden. Der Anteil der überhöht angesetzten Abschreibungen an der Bruttowertschöpfung des Unternehmenssektors entspricht dann der Quote der Bruttoanlageninvestitionen an der Bruttowertschöpfung, und der überhöhte Abschreibungssatz $d_{p r}, t / k_{p r}, t$ in der Volkswirtschaftlichen Gesamtrechnung beträgt:

$$
\frac{d_{p r, t}^{\prime}}{k_{p r, t}}=\left[\frac{\Delta k_{p r, t-1}^{+}}{x_{t}}\right] \cdot\left[\frac{x_{t}}{k_{p r, t}}\right]
$$

Vernachlässigt man die unterschiedliche Periodisierung der Variablen in (6), so besagt die Gleichung, daß der Abschreibungssatz des Unternehmenssektors in der Volkswirtschaftlichen Gesamtrechnung trotz überhöhter Kalkulation praktisch eine Obergrenze hat, die durch die Quote der Bruttoanlageinvestitionen an der Bruttowertschöpfung und durch den Kapitalkoeffizienten bestimmt ist. Diese Obergrenze gibt zusammen mit dem Wert $1 / T$, dem Abschreibungssatz zur Bemessung des tatsächlichen realen Kapitalverzehrs, den Spielraum an, innerhalb dessen eine vorsichtige Abschreibungspolitik der Unternehmen sich auf den Abschreibungssatz des Unternehmenssektors in der Volkswirtschaftlichen Gesamtrechnung auswirken kann, innerhalb dessen sich also der Wert von (5) bewegt:

Die Anlagevermögensrechnung des Statistischen Bundesamtes impliziert für die sechziger Jahre eine durchschnittliche Lebensdauer der Anlagen von $29 \mathrm{Jahren}$, weswegen $1 / \mathrm{T}=0,034$. Während dieser Zeit liegt die nach (6) berechnete Obergrenze des Abschreibungssatzes für den Unternehmenssektor der Bundesrepublik bei 0,078. Um für den Unternehmenssektor der Modellwirtschaft den zwischen beiden Grenzen liegenden Wert von (5) zu berechnen, wurde angenommen, daß die Unternehmen überhöhte Abschreibungen nur bei den Ausrüstungen, nicht bei den Bauten vornehmen und daß in Hinsicht auf das bestandsmäßige Größenverhältnis zwischen Ausrüstungen und Bauten von $1: 2$ die durchschnittliche kalkulatorische Lebensdauer aller Anlagen $21 \mathrm{Jahr}$, also ca. $75 \%$ der tatsächlichen Lebensdauer, beträgt. Bei einer Wachstumsrate der realen Bruttoanlage- 
investitionen von 5,7\% ergibt (5) dann einen Wert des durchschnittlichen kalkulatorischen Abschreibungssatzes von 0,041 bzw. eine Erhöhung des Abschreibungssatzes um 0,7 Prozentpunkte.

Bezieht man den absoluten und zu Wiederbeschaffungspreisen angesetzten Wert der kalkulatorisch bedingten Mehrabschreibungen nicht auf das Bruttoanlagevermögen, sondern auf das zu seiner Finanzierung aufgenommene Finanzkapital, $F_{A n l}^{\star} t$, so gelangt man zu einem Satz von 0,015. Dieser Satz wird von den Unternehmen der Modellwirtschaft in konstanter Höhe als Risikoprämie dem Kapitalmarktzins zugeschlagen; allerdings wird nach der Schätzung der Anlagenplanungsfunktion in Paragraph 4 noch eine Anpassung des Satzes an das endgültige Niveau von 0,014 vorgenommen. 

T A B E L L E N A HA NG 
Tabelle 1

Jahr

realer (nomineller) Kapitalkostensatz je Einheit des m.S. m. B. realen (zu Wiederbeschaffungspreisen bewerteten) Bruttoanlagevermögens (1) im privaten Sektor der Bundesrepublik

$$
r_{\text {Ris }}=0,015 \quad r_{\text {Ris }}=0,014
$$

1950

4

$\begin{array}{rll}55 & 0,068.720 & 0,068.192 \\ 6 & 0,071.802 & 0,071.258 \\ 7 & 0,076.508 & 0,075.928 \\ 8 & 0,072.263 & 0,071.672 \\ 9 & 0,070.607 & 0,069.990 \\ 60 & 0,074.050 & 0,073.424 \\ 1 & 0,073.187 & 0,072.543 \\ 2 & 0,074.596 & 0,073.940 \\ 3 & 0,076.070 & 0,075.412 \\ 4 & 0,077.128 & 0,076.541 \\ 65 & 0,080.596 & 0,079.950 \\ 6 & 0,086.532 & 0,085.874 \\ 7 & 0,084.357 & 0,083.673 \\ 8 & 0,082.080 & 0,081.436 \\ 9 & 0,082.993 & 0,082.357 \\ 70 & 0,085.239 & 0,084.676 \\ 1 & 0,083.754 & 0,083.208\end{array}$

(1) Zur Berechnung vgl. Paragraph 4 Abschn. 3, insbesondere die Definition (4.11) des Kapitalkostensatzes. 
Tabelle 2

Jahr

Anteil der Arbeitseinkommen (1) am Beitrag des privaten Sektors zum Bruttoinlandsprodukt (2) der Bundesrepublik Deutschland (3)

$\begin{array}{rc}1950 & 0,615 \\ 1 & 0,602 \\ 2 & 0,575 \\ 3 & 0,582 \\ 4 & 0,584 \\ 55 & 0,575 \\ 6 & 0,579 \\ 7 & 0,579 \\ 8 & 0,584 \\ 9 & 0,574 \\ 60 & 0,575 \\ 1 & 0,580 \\ 2 & 0,589 \\ 3 & 0,587 \\ 4 & 0,581 \\ 65 & 0,580 \\ 6 & 0,583 \\ 7 & 0,576 \\ 8 & 0,565 \\ 9 & 0,565 \\ 70 & 0,585 \\ 1 & 0,594(\mathrm{p}) \\ 2 & -- \\ & \end{array}$

(1) Bruttoeinkommen aus unselbständiger Arbeit der Inländer minus vom Staat an Inländer gezahlte Bruttoeinkommen aus unselbständiger Arbeit; der Betrag wurde durch den Anteil der unselbständigen Beschäftigten an der Gesamtzahl der im privaten Sektor Beschäftigten dividiert und dadurch um ein rechnerisches Arbeitseinkommen der Selbständigen erhöht, das dem durchschnittlichen Arbeitseinkommen eines Unselbständigen entspricht.

(2) Zur Abstimmung des Materials auf die Modellformulierung wurde der nominelle Bruttoinlandsproduktbeitrag des privaten Sektors um die per Saldo vom Ausland empfangenen Einkommen aus unselbständiger Arbeit ergänzt.

(3) Mit Saar und West-Berlin 
Quelle: von 1950 bis 1959 geschätzt; anschließend berechnet nach Statistisches Jahrbuch für die Bundesrepublik Deutschland sowie Jahresgutachten des Sachverständigenrates zur Begutachtung der gesamtwirtschaftlichen Entwicklung. 
Tabelle 3

\begin{tabular}{|c|c|c|c|}
\hline \multirow[t]{3}{*}{ Jahr } & $\begin{array}{l}\text { Beschäftigte im } \\
\text { privaten Sektor der } \\
\text { Bundesrepublik (1) }\end{array}$ & $\begin{array}{l}\text { Planbeschäftigung } \\
\text { im privaten Sektor } \\
\text { der Bundesrepublik } \\
\text { (2) }\end{array}$ & $\begin{array}{l}\text { Verhältnis von } \\
\text { tatsächlicher zur } \\
\text { Planbeschäftigung }\end{array}$ \\
\hline & (in Tsd.) & (in Tsd.) & \multirow[b]{2}{*}{$\mathrm{e}_{\mathrm{pr}, \mathrm{t}} / \mathrm{e}_{\mathrm{o}, \mathrm{pr}, \mathrm{t}}$} \\
\hline & $\mathrm{e}_{\mathrm{pr}, \mathrm{t}}$ & $\mathrm{e}_{\mathrm{o}, \mathrm{pr}, \mathrm{t}}$ & \\
\hline 1950 & $19.757,000$ & $\ldots$ & $\ldots$ \\
\hline 1 & $20.305,000$ & $-\infty$ & -- \\
\hline 2 & $20.713,000$ & $20.713,000$ & $1,000.000$ \\
\hline 3 & $21.253,000$ & $21.251,407$ & $1,000.075$ \\
\hline 4 & $21.851,000$ & $22.153,759$ & $0,986.334$ \\
\hline 55 & $22.679,000$ & $22.060,502$ & $1,028.036$ \\
\hline 6 & $23.274,000$ & $22.933,998$ & $1,014.825$ \\
\hline 7 & $23.766,000$ & $24.048,721$ & $0,988.244$ \\
\hline 8 & $23.932,000$ & $24.183,375$ & $0,989.605$ \\
\hline 9 & $24.167,000$ & $24.434,975$ & $0,989,033$ \\
\hline 60 & $24.460,059$ & $24.189,249$ & $1,011.195$ \\
\hline 1 & $24.754,294$ & $24.838,308$ & $0,996.618$ \\
\hline 2 & $24.903,845$ & $25.028,924$ & $0,995.003$ \\
\hline 3 & $24.935,162$ & $24.945,948$ & $0,999.568$ \\
\hline 4 & $24.983,826$ & $24.765,187$ & $1,008.828$ \\
\hline 65 & $25.107,482$ & $25.099,875$ & $1,000,303$ \\
\hline 6 & $24.990,608$ & $25.250,625$ & $0,989.703$ \\
\hline 7 & $24.150,233$ & $25.195,233$ & $0,958.524$ \\
\hline 8 & $24.210,292$ & $24.193,634$ & $1,000.689$ \\
\hline 9 & $24.630,721$ & $23.770,834$ & $1,036.174$ \\
\hline 70 & $24.942,312$ & $25.056,427$ & $0,995.446$ \\
\hline 1 & $24.828,391$ & $25.130,020$ & $0,987,997$ \\
\hline
\end{tabular}

(1) Quelle: berechnet nach Jahresgutachten des Sachverständigenrates zur Begutachtung der gesamtwirtschaftlichen Entwicklung (Tabellenteil) sowie Statistisches Jahrbuch für die Bundesrepublik Deutschland (Teil Finanzen und Steuern, Angaben über den Personalstand der staatlichen Gebietskörperschaften und der öffentlichen Wirtschaftsunternehmen ohne eigene Rechtsperson). Für die Jahre vor 1960 wurde die staatliche Beschäftigung grob geschätzt.

(2) Die Modellwerte wurden mit Gleichung (4.49) und den Parameterwerten (4.50) berechnet. Die Bestimmung der Parameterwerte erfolgte nach dem im Paragraphen 4 Abschnitt 6 angegebenen Annäherungsverfahren. 
Tabelle 4

$\mathrm{Jahr}$

m.S. m. B.
Bruttoinlandsproduktbeitrag $\mathrm{x}_{\mathrm{t}}$ des privaten Sektors in der Bundesrepublik im Verhältnis zur vorausgeplanten Produktionskapazität $x_{0, t}(1)(2)$

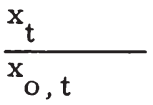

$\begin{array}{rl}1950 & - \\ 1 & -- \\ 2 & 0,921.761 \\ 3 & 0,943.123 \\ 4 & 0,944.001 \\ 55 & 1,021.279 \\ 6 & 1,014.984 \\ 7 & 0,988.373 \\ 8 & 0,971.548 \\ 9 & 0,987.961 \\ 60 & 1,033.574 \\ 1 & 1,019.471 \\ 2 & 1,003.702 \\ 3 & 0,990.173 \\ 4 & 1,015.134 \\ 65 & 1,010.758 \\ 6 & 0,985.184 \\ 7 & 0,936.998 \\ 8 & 0,990.889 \\ 9 & 1,037.721 \\ 70 & 1,006.629 \\ 1 & 0,986.160\end{array}$

(1) Zur Berechnung der Modellwerte vgl. Paragraph 4 Abschnitt 7, insbesondere Gleichung (4.51)

(2) In der gesamtwirtschaftlichen Anlagevermögensrechnung des Statistischen Bundesamtes wird der private Anlagenbestand der unmittelbaren Nachkriegszeit trotz noch vorhandener Kriegsschäden in vollem Umfang als Bruttoanlagevermögen aktiviert. Dies geschieht aus methodischen Gründen. Die Werte des Bruttoanlagevermögens weisen daher die tatsächlich für produktive Zwecke verfügbare Anlagenkapazität im Umfang der noch vorhandenen Schäden zu hoch aus. Der Bewertungs - 
effekt wirkt sich über Verwendung der Bruttoanlagevermögenswerte in Produktionsfunktion (4.51) auf die Schätzung von $x_{0, t}$ und $x_{t} / x_{0, t}$ aus. Die Anpassungsbewegung, die der Quotient $x_{t} / x_{0}, t z$ wischen 1952 und $1957 / 58$ an den langfristigen Trendwert von 1,0 beschreibt, ist deutlicher Ausdruck dieser zerstörungsbedingten Kapazitätsunterauslastung. Von der Modellformulierung her wäre eine entsprechende Bereinigung des Bruttoanlagevermögens sinnvoll gewesen, weil erst dann eine korrekte Schätzung von $x_{0}, t$, der tatsächlichen Produktionskapazität, erzielt worden wäre und weil erst dann der Quotient $x_{t} / x_{0}, t$ ebenso wie der Quotient $e_{\mathrm{pr}, \mathrm{t}} / \mathrm{e}_{\mathrm{o}, \mathrm{pr}, \mathrm{t}}$ stets um den Wert eins herum geschwankt und damit der kurzfristigen Produktionsfunktion (5.1) bzw. (5.18) Genüge getan hätte. Stattdessen wurde $x_{t} / x_{0}, t^{-R e i h e, ~}$ sobald sie zur Schätzung irgendwelcher Funktionen herangezogen wurde, nur für die Jahre $1960 \mathrm{ff}$. verwandt, in denen der Wiederaufbauprozeß der Nachkriegszeit als abgeschlossen gelten kann. 
Tabelle 5

\begin{tabular}{|c|c|}
\hline $\begin{array}{l}\text { Jahr } \\
\text { m.S. m. B. } \\
\text { (Jahresanfang) }\end{array}$ & $\begin{array}{l}\text { Nominalvermögen (1) des privaten Sektors in der } \\
\text { Bundesrepublik Deuts chland (2) } \\
\qquad \text { Mrd. DM }\end{array}$ \\
\hline 1950 & 170,550 \\
\hline 1 & 184,271 \\
\hline 2 & 199,944 \\
\hline 3 & 216,718 \\
\hline 4 & 223,125 \\
\hline 55 & 242,661 \\
\hline 6 & 267,894 \\
\hline 7 & 294,534 \\
\hline 8 & 325,876 \\
\hline 9 & 361,168 \\
\hline 60 & 398,557 \\
\hline 1 & 440,429 \\
\hline 2 & 483,909 \\
\hline 3 & 527,729 \\
\hline 4 & 571,714 \\
\hline 65 & 625,033 \\
\hline 6 & 686,486 \\
\hline 7 & 745,931 \\
\hline 8 & 803,399 \\
\hline 9 & 878,907 \\
\hline 70 & 951,117 \\
\hline 1 & $1.029,567$ \\
\hline
\end{tabular}

(1) Nominalvermögen $=$ Summe der in der Vergangenheit gebildeten Nominalersparnis

(2) Zur Berechnung der Modellwerte vgl. die Bemerkungen im Paragraphen 7 Abschnitt 2 sowie Textanhang 1 
Tabelle 6

Jahr

m.S. m. B.

(Jahresanfang)
Modellwerte für das Nominalvermögen der Unselbständigen-Haushalte in der Bundesrepublik Deutschland (1)
Anteil am Nominalvermögen des privaten Sektors

\begin{tabular}{rrll}
\hline & & Ausgangs \\
1950 & 42,637 & annahme: & $0,250.000$ \\
1 & 44,356 & $0,240.712$ \\
2 & 46,207 & $0,231.100$ \\
3 & 49,369 & $0,227.804$ \\
4 & 53,211 & $0,238.484$ \\
55 & 57,678 & $0,237.691$ \\
6 & 62,085 & $0,231.754$ \\
7 & 66,924 & $0,227.220$ \\
8 & 74,435 & $0,228.417$ \\
9 & 83,000 & $0,229.812$ \\
60 & 92,742 & $0,232.695$ \\
1 & 103,628 & $0,235.290$ \\
2 & 115,918 & $0,239.545$ \\
3 & 129,449 & $0,245.296$ \\
4 & 146,793 & $0,256.760$ \\
65 & 167,486 & $0,267.963$ \\
6 & 192,968 & $0,281.095$ \\
7 & 217,309 & $0,291.326$ \\
8 & 241,536 & $0,300.642$ \\
9 & $268,924(2)(\mathrm{p})$ & $0,305.976(2)(\mathrm{p})$ \\
70 & $300,397(2)(\mathrm{p})$ & $0,315.836(2)(\mathrm{p})$ \\
1 & $336,491(2)(\mathrm{p})$ & $0,326.827(2)(\mathrm{p})$ \\
& &
\end{tabular}

(1) Zur Berechnung der Modellwerte vgl. Paragraph 7 Abschnitt 2

(2) Mit Hilfe der Funktionen (7.5) für die Rendite des nicht in Kasse angelegten Unselbständigen-Vermögens und (7.9) für den Unselbständigen-Konsum sowie der Regressionsergebnisse (7.6a) und (7.11) fortgerechnete Werte. 
Tabelle 7

\begin{tabular}{rll} 
Jahr & $\begin{array}{l}\text { Ertragsrate des nicht } \\
\text { in Kasse gehaltenen } \\
\text { Nominalvermögens der } \\
\text { Unselbständigen-Haus- } \\
\text { halte }(1)\end{array}$ & $\begin{array}{l}\text { dito i. v. Her Umlaufs- } \\
\text { rendite festverzinslicher } \\
\text { Wertpapiere in der } \\
\text { laufenden Periode }\end{array}$ \\
\hline 1950 & $0,003.840$ & \\
1 & $0,004.512$ & $0,048.000$ \\
2 & $0,006.183$ & $0,053.082$ \\
3 & $0,007.593$ & $0,081.355$ \\
4 & $0,009.846$ & $0,099.907$ \\
55 & $0,013.736$ & $0,134.876$ \\
6 & $0,016.394$ & $0,202.000$ \\
7 & $0,018.455$ & $0,230.901$. \\
8 & $0,021.003$ & $0,239.675$ \\
9 & $0,023.346$ & $0,323.123$ \\
60 & $0,028.707$ & $0,402.517$ \\
1 & $0,029.341$ & $0,455.666$ \\
2 & $0,031.278$ & $0,497.305$ \\
3 & $0,033.115$ & $0,521.300$ \\
4 & $0,034.866$ & $0,542.868$ \\
65 & $0,038.089$ & $0,562.354$ \\
6 & $0,042.106$ & $0,560.132$ \\
7 & $0,040.507$ & $0,539.820$ \\
& & $0,578.671$ \\
& &
\end{tabular}

(1) Zur Berechnung der Modellwerte vgl. Paragraph 7 Abschnitt 2 
Albers, W.

Allen, R. G. D.

Andel, N.

Asimakopulos, A.

Burbridge, J. B.

Bartels, $\mathrm{H}_{\text {. }}$

Raabe, K-H.

Spieker, H.

u. a.

Baumgarten, $\mathbf{P}$.

Bombach, G.

Bombach, G.

Brown, T. M.

Bundesministerium für

Arbeit und Sozialordnung

Bundesministerium der Finanzen

Bundesministerium der Finanzen
Staatsverschuldung und Geld- und Kreditpolitik, Finanzarchiv, NF 21, 1961 , S. $25 \mathrm{ff}$.

Macro-Econimic Theory. A Mathematical Treatment, London 1968

Probleme der Staatsschuldentilgung, Berlin 1964

The Short-Period Incidence of

Taxation, Economic Journal, 84, 1974 , S. $267 \mathrm{ff}$.

Die Verteilung des Volkseinkommens in der Bundesrepublik Deutschland 1950 bis 1965 , Wirtschaft und Statistik, 10,1958 , S. $135 \mathrm{ff}$.

Selbstfinanzierung und Einkommensverteilung, Tübingen 1971

Staatshaushalt und Volkswirtschaftliche Gesamtrechnung, Finanzarchiv, NF 17, 1956/57, S. $344 \mathrm{ff}$.

Preisstabilität, wirtschaftliches Wachstum und Einkommensverteilung,

Schweiz. Zeitschrift für Volkswirtschaft und Statistik, 95, 1959, S. $1 \mathrm{ff}$.

Habit Persistence and Lags in

Consumer Behavior, Econometrica, 20,1952 , S. $355 \mathrm{ff}$.

Sozialbudget 1969/70 der Bundesrepublik Deutschland, Bonn 1970

Das Steueränderungsgesetz 1960 , Finanzbericht 1961, Bonn, S. $138 \mathrm{ff}$.

Die steuerlichen Maßnahmen im Jahre 1961, Finanzbericht 1962, Bonn,

S. $150 \mathrm{ff}$. 
Bundesministerium der Finanzen

Bundesministerium der Finanzen

Bundesministerium der Finanzen

Clark, S.

Davidson, $\mathbf{P}$.

Weintraub, S.

Deutsche Bundesbank

Deutsche Bundesbank

Deutsche Bundesbank

Deutsche Bundesbank

Deutsche Bundesbank

Deutsche Bundesbank

Deutsches Institut für

Wirtschaftsforschung

Domar, E.
Überblick über die Steuerrechtsänderungen seit 1961 und über den Stand der Steuergesetzgebung, Finanzbericht 1965, Bonn, S. $161 \mathrm{ff}$.

Überblick über die Steuerrechtsänderungen in den Jahren 1964 und 1965 , Finanzbericht 1966, Bonn, S. 206 ff.

Überblick über die Steuerrechtsänderungen 1965/66 und 1967, Finanzbericht 1968, Bonn, S. $171 \mathrm{ff}$.

Labor Hoarding in Durable Goods Industries, American Economic Review, 63,1973 , S. $811 \mathrm{ff}$.

Money as Cause and Effect, Economic Journal, 83, 1973, S. $1117 \mathrm{ff}$.

Erläuterungen zur Liquiditätsanalyse der Bundesbank, Monatsbericht Juli 1970 , S. $28 \mathrm{ff}$.

Gesamtwirtschaftliche Finanzierungsrechnung sowie Bestand an Geldvermögen und Verpflichtungen 1970, Monatsbericht Mai 1971, S. $20 \mathrm{ff}$.

Längerfristige Entwicklung des Geldvolumens, Monatsbericht Juli 1971, S. $11 \mathrm{ff}$.

Entwicklung des Geldvermögens und der Verpflichtungen von 1950 bis 1971 , Monatsbericht März 1973, S. 24 ff.

Neuere Tendenzen der Gemeindefinanz, Monatsbericht Juli 1973, S. $15 \mathrm{ff}$.

Die Zahlungsbilanz der Bundesrepublik im Jahre 1972 nach Regionen, Beilage zu den Statistischen Beiheften zu den Monatsberichten der Deutschen Bundesbank, Reihe 3, Zahlungsbilanzstatistik, Nr. 7, Juli 1973

Grundlinien der Wirtschaftsentwicklung 1976, Wochenbericht des DIW, Heft $3 / 4$ vom 26.1.1976, S. $16 \mathrm{ff}$.

Depreciation, Replacement and Growth, Economic Journal, 63, 1953, S. $1 \mathrm{ff}$. 
Dosser, D.

Durbin, J.

Ehrlicher, W.

Eisner, R.

Eisner, R.

Enthoven, A. C.

Erbé, Rene

Föhl, C.

Föhl, C.

Friedman, M.

Friedman, M.

Fritsch, B.

Gehrig, G.

Kuhlo, K. G.
Tax Incidence and Growth, Economic Journal, 71, 1961, S. $572 \mathrm{ff}$.

Testing for Serial Correlation in LeastSquares Regressions when Some of the Regressors are Lagged Dependent Variables, Econometrica, 38, 1970, S. $410 \mathrm{ff}$.

Die Wirkungen der Unternehmenssteuern auf Investitionen, Gewinne und Löhne, Finanzarchiv, NF 18, 1957/58, S. $373 \mathrm{ff}$.

Tax Policy and Investment Behavior: Comment, American Economic Review, 59,1969 , S. $379 \mathrm{ff}$.

Tax Policy and Investment Behavior:

Further Comment, American Economic Review, 60, 1970, S. 746

A Neo-classical Model of Money, Debt, and Economic Growth, in: J.G. Gurly u. E. S. Shaw, Money in a Theory of Finance, Washington 1960

Causes and Effects of Private Cap:tal Movements in Germany 1955-69 Kyklos, 23, 1970, S. $927 \mathrm{ff}$.

Kritik der progressiven Einkommensbesteuerung, Finanzarchiv, NF 14, $1953 / 54$ S. $88 \mathrm{ff}$.

Das Steuerparadoxon, Finanzarchiv, $\mathrm{NF} 17,1956, \mathrm{~S} .1 \mathrm{ff}$.

The Role of Monetary Policy, American Economic Review, 58, S. $1 \mathrm{ff}$.

Die Geldnachfrage: einige theoretische und empirische Erbebnisse, in: ders. Die optimale Geldmenge und andere Essays, München 1970 , S. $157 \mathrm{ff}$.

Die Simulation als Instrument makroökonomischer Prognosen, Schweiz. Zeitschrift für Volkswirtschaft und Statistik, 102,1966 , S. $416 \mathrm{ff}$.

Ökonometrische Analyse des Produktionsprozesses, Ifo-Studien, 7, 1961, S. $222 \mathrm{ff}$. 
Godley, W. A. H.

Nordhaus, W. D.

Haavelmo, T.

Hagemann, G.

Hall, R. E.

Haller, $\mathrm{H}$.

Haller, $\mathrm{H}$.

Hamer, G.

Häuser, K.

Richter, R.

Harrod, R. F.

Heubes, J.

Heubes, J.

Heuß, E.

Heuß, E.
Pricing in the Trade Cycle, Economic Journal, 82, 1972, S. $853 \mathrm{ff}$.

A Study in the Theory of Investment, Chicago 1960

Aufkommenselastizitäten ausgewählter Steuern in der Bundesrepublik Deutschland 1950-1963, Tübingen 1968

Tax Policy and Investment Behavior, American Economic Review, 57, 1967 , S. $389 \mathrm{ff}$.

Nettoumsatzsteuer versus Gewinnsteuer, Finanzarchiv, NF 16, 1955, S. 201

Probleme der progressiven Besteuerung, Tübingen 1970

Die Behandlung der Umsatz- (Mehrwert-) steuer in den Volkswirtschaftlichen Gesamtrechnungen, Wirtschaft und Statistik, 1968, S. $439 \mathrm{ff}$.

Zum Problem der Einkommensteuerüberwälzung, Finanzarchiv, NF 16, 1955, S. $258 \mathrm{ff}$. sowie NF 17, 1956, S. $38 \mathrm{ff}$.

Replacement, Net Investment, Amortisation Funds, Economic Journal, 80, 1970, S. $24 \mathrm{ff}$.

CES-Produktionsfunktionen für Industriezweige, Westdeutschland 1950-1965, Jahrbücher für Nationalökonomie und Statistik, 183, 1969/70, S. $510 \mathrm{ff}$.

Investitionsverhalten in der Industrie der Bundesrepublik Deutschland 1950-1970, Zeitschrift für die gesamte Staatswissenschaft, 129,1973 , S. $685 \mathrm{ff}$.

Krelles Verteilungstheorie, Zeitschrift für die gesamte Staatswissenschaft, 122 , 1966 , S. $163 \mathrm{ff}$.

Eine vereinfachte Version der Verteilungstheorie, Zeitschrift für die gesamte Staatswissenschaft', 122, 1966, S. $254 \mathrm{ff}$. 
Hirsch, H.

Hövelmann, P.

Jeck, A.

Jeck, A.

Johnston, J.

Jorgensen, D. W.

Jorgensen, D. W.

Kaiser, W.

Zerwas, A.

Kaldor, N.

Kalecki, M.

Kirner, W.

König, $\mathrm{H}$.

Timmermann, V.
Die Finanzpolitik im Aufschwung, besonders im Hinblick auf das Problem der Schuldentilgung, Finanzarchiv, NF 23, $1963 / 64$, S. $394 \mathrm{ff}$.

Die Verteilungseffekte von Gewinnsteuern in angebots - und nachfragetheoretischen Modellen, Diss., Freiburg 1973

The Trends of Income Distribution in West Germany, in: J. Marchal u. B. Ducros (Hrsg.), The Distribution of National Income, London u. a. 1968, S. $78 \mathrm{ff}$.

Wachstum und Verteilung des Volkseinkommens, Tübingen 1970

Econometric Methods, New York/San Francisco/Toronto/London 1963

Capital Theory and Investment Behavior, American Economic Review, 53, 1963, PaP, S. $247 \mathrm{ff}$.

Econometric Studies of Investment Behavior: A Survey, Journal of Economic Literature, 4, 1971, $1111 \mathrm{ff}$.

Die Struktur des Sparens in der Bundesrepublik Deutschland von 1950-1967, Berlin 1970

Alternative Theories of Distribution, Review of Economic Studies, 23, 19551956, wiederabgedruckt in: ders., Essays on Value and Distribution, London 1960, S. $209 \mathrm{ff}$.

The Theory of Economic Dynamics. An Essay on Cyclical and Long-Run Changes in Capitalist Econimy, London 1954

Zeitreihen für das Anlagevermögen der Wirtschaftsbereiche in der Bundesrepublik Deutschland, DIW-Beiträge zur Strukturforschung, Heft 5, Berlin 1968

Ein ökonomisches Modell für die Bundesrepublik Deutschland 1950 bis 1960 , Zeitschrift für die gesamte Staatswissenschaft, 118,1962 , S. $598 \mathrm{ff}$. 
König, H.

Klein, L. R.

Kommission für die Finanzreform

Koshal, R. K.

Gallaway, L. E.

Koyck, L. M.

Krelle, W.

Krelle, W.

Krelle, W.

Krelle, W.

Krelle, W.

Krelle, W.

Krelle, W.

Krelle, W. Schunk, J. Siebke, J.
Einkommenskreislaufgeschwindigkeit des Geldes und Zinssatzveränderungen: Eine ökonometrische Studie über die Geldnachfrage in der Bundesrepublik Deutschland, Zeitschrift für die gesamte Staatswissenschaft, 124,1968 , S. $70 \mathrm{ff}$.

Issues in Econometric Studies of

Investment Behavior, Journal of Economic Literatur, 7, 1974, S. $43 \mathrm{ff}$.

Gutachten über die Finanzreform in der Bundesrepublik Deutschland (TroegerGutachten), Stuttgart 1966

The Phillips Curve for West Germany, Kyklos, 24, 1971, S. $346 \mathrm{ff}$.

Distributed Lags and Investment Analysis, Amsterdam 1954

Die Grenzproduktivitätstheorie des Lohnes, Jahrbücher für Nationalökonomie und Statistik, 1962,1950, S. $1 \mathrm{ff}$.

Zur Verteilung der progressiven Einkommensbesteuerung, Finanzarchiv, NF 16, 1955 , S. $22 \mathrm{ff}$.

Bestimmungsgründe der Einkommensver teilung, in: Einkommensbildung und Einkommensverteilung, Schriften des Vereins für Socialpolitik, NF Bd. 13, Berlin 1957, S. $55 \mathrm{ff}$.

Verteilungstheorie, Tübingen 1962

Eine vereinfachte Version der "Verteilungstheorie" Zeitschrift für die gesamte Staatswissenschaft, 122, 1966, S. $511 \mathrm{ff}$.

Produktionstheorie, Tübingen 1969

Macht und ökonomisches Gesetz in der Verteilung, in: Macht und ökonomisches Gesetz, Schriften des Vereins für Socialpolitik, NF 74, Berlin 1973, S. $77 \mathrm{ff}$.

Überbetriebliche Ertragsbeteilung der Arbeitnehmer, Tübingen 1968 
Krelle, W.

Beckerhoff, D. Langer, H. G. Fuß, H.

Krelle, W. Siebke, J.

Krupp, H-J.

Krzyzaniak, M.

Krzyzaniak, M.

Krzyzaniak, M.

Krzyzaniak, M.

Krzyzaniak, M.

Läufer, N. K. A.

Lüdecke, $R$.

Lützel, $\mathrm{H}$.
Ein Prognosesystem für die wirtschaftliche Entwicklung der Bundesrepublik

Deutschland, Meisenheim a. G. 1969

Vermögensverteilung und Vermögenspolitik in der Bundesrepublik Deutschland. Ein Überblick, Zeitschrift für die gesamte Staatswissenschaft, 129, 1973,

S. $478 \mathrm{ff}$.

Theorie der personellen Einkommensverteilung, Berlin 1968

Effects in Profits Taxes: Duduced from Neoclassical Growth Models, in: ders. (Hrsg.), Effects of Corporation Income Tax, Detroit 1966, S. $17 \mathrm{ff}$.

Long Run Burden of a General Tax on Profits in a Neo-Classical World, Public Finance, 22, 1967, S. $473 \mathrm{ff}$.

The Burden of a Differential Tax on Profits in Neoclassical World, Public Finance, 23, 1968, S. $477 \mathrm{ff}$.

Factor Substitution and the General Tax on Profits, Public Finance, 25, 1970, S. $489 \mathrm{ff}$.

The Differential Incidence of Taxes on Profits and Factor Incomes, Finanzarchiv, 30,1972 , S. $464 \mathrm{ff}$.

Der revisionstheoretische Gegensatz zwischen Loanable-Funds - und Liquiditäts-Theorie des Zinses mit ökonometrischem Test, in: Studien zur Geldtheorie und monetäre Ökonometrie, Schriften des Vereins für Socialpolitik, NF Bd. 66, Berlin 1972, S. $199 \mathrm{ff}$.

Synthesemöglichkeiten des nachfrage- und angebotstheoretischen Ansatzes in der makroökonomischen Verteilungstheorie, Jahrbücher für Nationalökonomie und Statistik, 186, 1972, S. $309 \mathrm{ff}$.

Das reproduzierbare Anlagevermögen in Preisen von 1962, Wirtschaft und Statistik 1971, S. $593 \mathrm{ff}$. 
Lützel, H.

Meade, J. E.

Meade, J. E.

Meissner, W.

Menges, G.

Goßmann, J.

Mieszkowsky, P.

Miller, R.L.

Mückl, W.

Baumgarten, P. Schmidt, K. D.

Musgrave, R. A.

Niehans, J.

Niehans, J.

Oberhauser, A.
Das reproduzierbare Sachvermögen zu Anschaffungs - und Wiederbeschaffungs preisen, Wirtschaft und Statistik 1972, S. $611 \mathrm{ff}$.

A Neo-Classical Theory of Economic Growth, London $2^{\circ}-1962$

The Rate of Profit in a Growing Economy, Economic Journal, 73, 1963, S. $565 \mathrm{ff}$.

Zur Methodologie der Simulation, Zeitschrift für die gesamte Staatswissenschaft, 126,1970 , S. $385 \mathrm{ff}$.

Ökonometrische Untersuchungen der Preisentwicklung in der Bundesrepublik Deutschland, Schriftenreihe der Wirtschaftsvereinigung Eisen- und Stahlindustrie zur Wirtschafts - und Industriepolitik, Heft 9, Düsseldorf 1968

Tax Incidence Theory: The Effect of Taxes on the Distribution of Income, Journal of Economic Literature, 7, 1969, S. $1103 \mathrm{ff}$.

The Reserve Labour Hypothesis: Some Tests of Implications, Economic Journal, 81,1971 , S. $17 \mathrm{ff}$.

Die Beziehungen zwischen Wirtschaftsstruktur und Einkommensverteilung, Tübingen 1968

Finanztheorie, Tübingen 1968

Die Wirkungen von Lohnerhöhungen, technischen Fortschritten, Steuern und Spargewohnheiten auf Preise, Produktion und Einkommensverteilung, in: Einkommensverteilung und technischer Fortschritt, Schriften des Vereins für Socialpolitik, NF Bd. 17, Berlin 1959

Ein Beitrag zum Verständnis von Krelles Makromodell, Weltwirtschaftliches Archiv, 92, 1964 I, S. 238 ff.

Finanzpolitik und private Vermögensbildung, Köln u. Opladen 1963 
Oberhauser, A.

Oberhauser, A.

Ott, A. E.

Pasinetti, L. L.

Pasinetti, L. L.

Patinkin, D.

Phillips, A. W.

Preiser, E.

Preiser, P.

Recktenwald, H. C.

Richter, R.
Zahlungsbilanzüberschüsse und Einkommensverteilung, Weltwirtschaftliches Archiv, 93, 1964, S. $286 \mathrm{ff}$.

Staatsschuldentilgung als finanzpolitisches Instrument, Finanzarchiv, NF 24, 1965, S. $283 \mathrm{ff}$.

Einführung in die dynamische Wirtschaftstheorie, Göttingen 1963

Rate of Profit and Income Distribution in Relation to the Rate of Economic Growth, Review of Economic Studies, 29,1962 , S. $267 \mathrm{ff}$.

New Results in an Old Framework. Comment on Samuelson and Modigliani, Review Economic Studies, 33, 1966, S. $303 \mathrm{ff}$.

Money, Interest and Prices. An Integration of Monetary and Value Theory, New York $2^{\circ}-1965$

The Relation between Unemployment and the Rate of Change of Money Wages in the United Kingdom, 1861-1957, Economica, 25, 1958, S. $283 \mathrm{ff}$.

Erkenntniswert und Grenzen der Grenzproduktivitätstheorie, Schweiz. Zeitschrift für Volkswirtschaft und Statistik, 89,1953 , S. $25 \mathrm{ff}$. wiederabgeschrieben in ders., Bildung und Verteilung des Volkseinkommens, Göttingen $2^{\circ}-1961$, S. 265 ff.

Wachstum und Einkommensverteilung, Sitzungsberichte der Heidelberger Akademie der Wissenschaften, philosophischhistorische Klasse, Jahrgang 1961, Abhandlung 5

Bedeutung, Grenzen und neuere Entwicklung der Steuerüberwälzungslehre, Finanzarchiv, NF 16, 1955, S. $274 \mathrm{ff}$.

Die Rolle der Banken in makroökonomischen Modellen, Jahrbücher für Nationalökonomie und Statistik, 180, 1967 , S. $441 \mathrm{ff}$. 
Rose, K.

Roskamp, K.

Gurcharan, S. L.

Sachverständigenrat zur Begutachtung der gesamtwirtschaftlichen Entwicklung

Samuelson, P. A. Modigliani, F.

Scheele, E.

Scheper, W.

Shoup, C. S.

Siebke, J.

Willms, M.

Siebke, J.

Willms, M.

Solow, R. M.

Solow, R. M.

Sozialenquete-Kommission
Theorie der Außenwirtschaft, Berlin und Frankfurt/M. $2^{\circ}-1966$

The Demand for Monetary Asset in the West Germany Economy: Evidence from Short-run Data, Zeitschrift für die gesamte Staatswissenschaft, 126, 1970, S. $468 \mathrm{ff}$.

Jahresgutachten 1972 ,

Bundesrat-Drucksache 612/72

v. 6. 12.1972

The Pasinetti Paradox in Neoclassical and More General Models, Review of Economic Studies, 33, 1966, 269 ff.

Einkommensverteilung und Wirtschafts wachstum, Tübingen 1965

Produktionsfunktionen mit konstanten Substitutionselastizitäten, Jahrbücher für Nationalökonomie und Statistik, 177,1965 , S. $1 \mathrm{ff}$.

Public Finance, Chicago 1969

Das Geldangebot in der Bundesrepublik Deutschland. Eine empirische Analyse für die Periode 1958 bis 1968, Zeitschrift für die gesamte Staatswissenschaft, 126,1970, S. $55 \mathrm{ff}$.

Zinsniveau, Geldpolitik und Inflation, Kredit und Kapital, 5, 1972, S. $171 \mathrm{ff}$.

A Contribution to the Theory of Economic Growth, Quarterly Journal of Economics, $70,1956,65 \mathrm{ff}$.

Distribution in the Long and Short Run, in: J. Marchal u. B. Ducros (Hrsg.) The Distribution of National Income, London, New York u. a. 1968, S. 449 ff.

Soziale Sicherung in der Bundesrepublik Deutschland. Sozialenquete. Bericht der Sozialenquete-Kommission erstattet von W. Bogs, H. Achinger, H. Meinhold, L. Neundörfer und W. Schreiber, Stuttgart u. a., o. J. 
Statistisches Bundesamt

Statistisches Bundesamt

Statistisches Bundesamt

Stein, J. L.

Stobbe, A.

Streißler, E.

Stucken, R.

Ehrlicher, W.

Timmermann, V.

Tintner, G.

United Nations

Uzawa, $\mathrm{H}$.

Weintraub, S.

Weintraub, S.

Westphal, U.
Volkswirtschaftliche Gesamtrechnungen 1972. Erste vorläufige Ergebnisse, Wirtschaft und Statistik 1973, S. $69 \mathrm{ff}$.

Statistisches Jahrbuch für die Bundesrepublik Deutschland, fortlaufend

Fachserie L, Finanzen und Steuern

International Short-Term Capital

Movements, American Economic Review, 55,1965 , S. $40 \mathrm{ff}$.

Untersuchungen zur makroökonomischen Theorie der Einkommensverteilung, Tübingen 1962

Long Term Structural Changes in the Distribution of Income, Zeitschrift für Nationalökonomie, Wien, 29, 1969 , $39 \mathrm{ff}$.

Zur Frage der Überwälzung der Unternehmenssteuern, Finanzarchiv, NF 14, $1953 / 54$, S. $367 \mathrm{ff}$.

Zur Refinanzierung der Banken beim Zentralbankensystem. Eine empirische Analyse für die Bundesrepublik Deutschland, 1958-1968, Jahrbücher für Nationalökonomie und Statistik, 185, 1971 , S. $328 \mathrm{ff}$.

Econometrics, New York 1965

Statistical Yearbook, New York, fortlaufend

Production-Funktions with Constant Elasticities of Substitution, Review of Economic Studies, 29, 1962, S. $294 \mathrm{ff}$.

An Approach to the Theory of Income Distribution, Philadelphia 1958

Cost Inflation and the State of Economic Theory - A Comment, Economic Journal, 84,1974 , S. $379 \mathrm{ff}$.

Theoretische und empirische Untersuchungen zur Geldnachfrage und zum Geldangebot, Tübingen 1970 
Willgerodt, $\mathrm{H}$.

Bartel, K.

Schillert, U.

u. a.

Wilson, W. G.

Wilson, Th. A.

Eckstein, $\mathrm{O}$.

Wissenschaftlicher Beirat beim Bundesministerium

für Wirtschaft

Wissenschaftlicher Beirat beim Bundesministerium der Finanzen

Wittmann, W.

Woll, A.
Vermögen für alle. Probleme der Bildung, Verteilung und Werterhaltung des Vermögens in der Marktwirtschaft, Düsseldorf 1971

Relationship between Output and Employment, Review of Economics and Statistics, 42,1960 , S. $37 \mathrm{ff}$.

Short Run Productivity Behavior in U.S. Manufacturing, Review of Economics and Statistics, 46,1964 , S. $41 \mathrm{ff}$.

Sicherung der wirtschaftlichen Expansion, Gutachten vom 18.1.1953, in: ders., Sammelband der Gutachten von 1948 bis 1972, Göttingen 1973, S. $169 \mathrm{ff}$. Problem einer Netto-Umsatzbesteuerung, Schriftenreihe des BMF, Heft 2, Bonn o.J.

Die Staatsausgaben in der markoökonomischen Produktionsfunktion, Kyklos, 22, 1969 , S. $297 \mathrm{ff}$.

Zur Theorie der Geldnachfrage: Analytische Ansätze und statistische Ergebnisse für die Bundesrepublik Deutschland, Zeitschrift für die gesamte Staatswissenschaft, 125,1969 , S. $56 \mathrm{ff}$. 